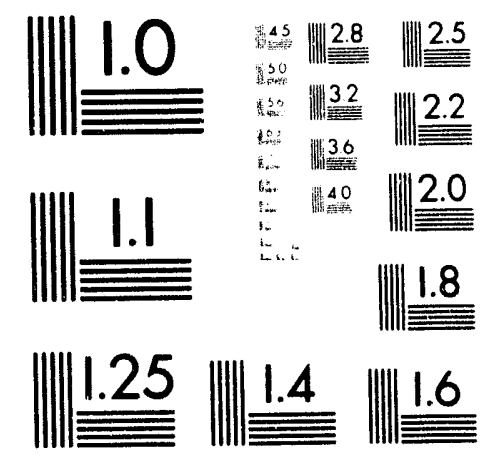



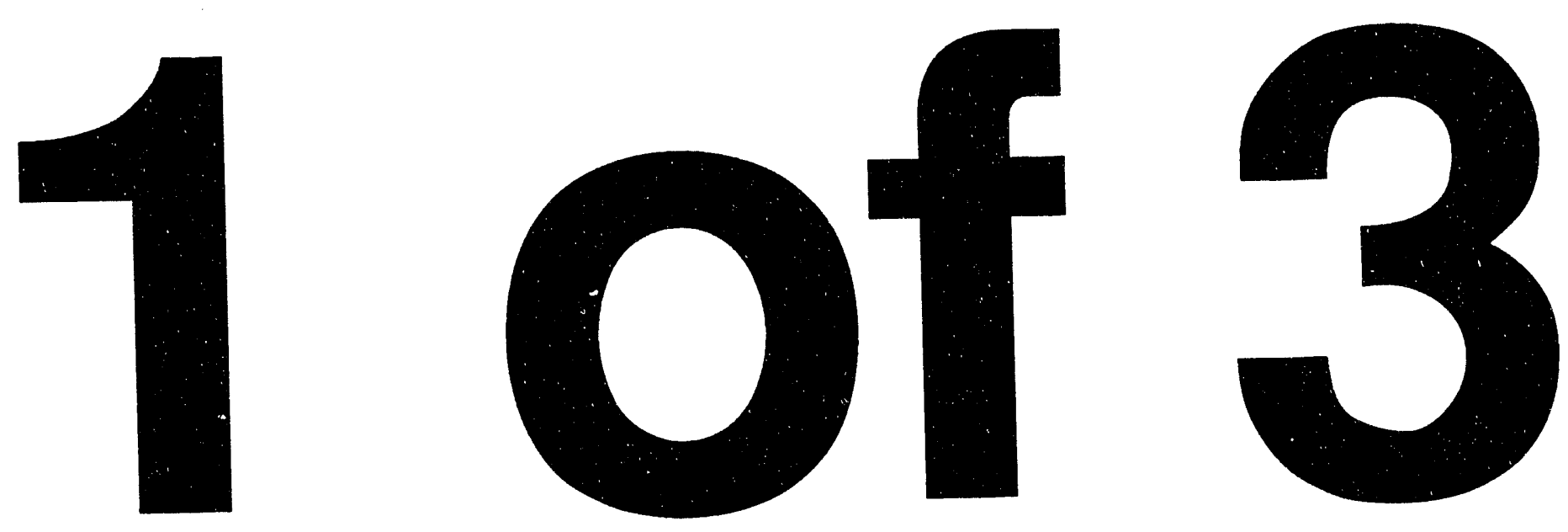


\title{
COMPATIBILITY OF REFRIGERANTS AND LUBRICANTS WITH MOTOR MATERIALS
}

\author{
Effects of Refrigerant Exposures on Motor Materials \\ Volume II \\ Robert Doerr and Stephen Kujak

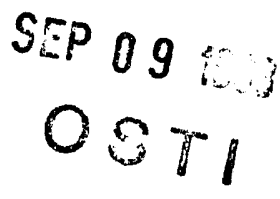

The Trane Company

3600 Pammel Creek Road

La Crosse, Wisconsin 54601-7599

May 1993

Prepared for

The Air-Conditioning and Refrigeration Technology Institute Under

ARTI MCLR Project Number 650-50400

This research project is supported, in whole or in part, by U.S. Department of Energy grant DE-FG02-91CE23810: Materials Compatibility and Lubricants Research (MCLR) on CFC-Refrigerant Substitutes. Federal funding supporting this project constitutes $93.67 \%$ of allowable costs. Funding from non-government sources supporting this project consists of direct cost sharing of $6.33 \%$ of allowable costs; and in-kind contributions from the air-conditioning and refrigeration industry.

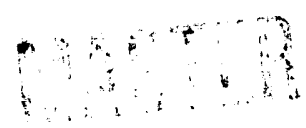




\section{DISCLAIMER}

The U.S. Department of Energy's and the air-conditioning industry's support for the Materials Compatibility and Lubricants Research (MCLR) program does not constitute an endorsement by the U.S Department of Energy, nor by the air-conditioning and refrigeration industry, of the views expressed herein.

\section{NOTICE}

This report was prepared as an account of work sponsored by the United States Government. Neither the United States nor the Department of Energy, nor the AirConditioning and Refrigeration Technology Institute, nor any of their employees, nor any of their contractors, subcontractors, or their employees, makes any warranty, completeness, or usefuiness of any information, apparatus, product or process disclosed or represents that its use would not infringe privately-owned rights.

\section{COPYRIGHT NOTICE \\ (for journal publication submissions)}

By acceptance of this article, the publisher and/or recipient acknowledges the rights of the U.S. Government and the Air-Conditioning and Refrigeration Technology Institute, Inc. (ARTI) rights to retain a nonexclusive, royalty-free license in and to any
copyrights covering this paper. 


\title{
COM:PATIBILITY OF REFRIGERANTS AND LUBRICANTS WITH MOTOR MATERIALS
}

\author{
Effects of Refrigerant Exposures on Motor Materials \\ Volume II
}

Because of the large scope of this project and the large amount of data recorded, the final report is divided into four volumes.

Volume II contains all the recorded measurements from the tests on the motor materials after exposures to the 11 pure refrigerants and to nitrogen at same temperatures. The motor materials are identified by the codes listed on page iv. A letter coding system was used to identify the motor materials in the data tables. Data for each pure refrigerantlubricant are listed in a separate appendix.

Volume I contains the abstract, scope, discussion of results, charts of motor material compatibility, test procedures, material identifications and 84 pages of data summary tables. This volume provides results of the study and other information of interest to most users of the information.

Volume III contains all the recurded measurements from the tests on the motor materials after exposure to the 17 refrigerant-lubricant combinations and to nitrogen at the same temperatures.

Volume IV contains the photographs of motor materials after exposures to a pure refrigerants or to refrigerant-lubricant combinations. 


\section{TABLE OF CONTENTS}

Effect of Refrigerant Exposures on Motor Materials Volume II

CODE CHART FOR MOTOR MATERIAL EXPOSURES

UNITS OF MEASURE

ABBREVIATIONS

APPENDIXES(Experimental data for 500 hour exposures to)

Appendix $A$

Appendix $B$

Appendix $C$

Appendix D

Appendix $E$ Appendix $F$

Appendix $G$

Appendix $\mathrm{H}$ Appendix 1 Appendix J

Appendix $\mathrm{K}$ Appendix $L$ Appendix $M$
Nitrogen Exposure @ $60^{\circ} \mathrm{C}\left(140^{\circ} \mathrm{F}\right)$

Nitrogen Exposure @ $90^{\circ} \mathrm{C}\left(194^{\circ} \mathrm{F}\right)$

HCFC-22 Exposure @ 90 $\mathrm{C}\left(194^{\circ} \mathrm{F}\right)$

HCFC-123 Exposure @ $90^{\circ} \mathrm{C}\left(194^{\circ} \mathrm{F}\right)$

HCFC-124 Exposure @ 90 $\mathrm{C}\left(194^{\circ} \mathrm{F}\right)$

HCFC- $142 \mathrm{~b}$ Exposure @ $90^{\circ} \mathrm{C}\left(194^{\circ} \mathrm{F}\right)$

HFC-152a Exposure @ $90^{\circ} \mathrm{C}\left(194^{\circ} \mathrm{F}\right)$

HFC-134a Exposure @ $90^{\circ} \mathrm{C}\left(194^{\circ} \mathrm{F}\right)$

HFC-134 Exposure @ $90^{\circ} \mathrm{C}\left(194^{\circ} \mathrm{F}\right)$

HFC-125 Exposure @ $60^{\circ} \mathrm{C}\left(140^{\circ} \mathrm{F}\right)$

HFC-32 Exposure @ $60^{\circ} \mathrm{C}\left(140^{\circ} \mathrm{F}\right)$

HFC-143a Exposure @ $60^{\circ} \mathrm{C}\left(140^{\circ} \mathrm{F}\right)$

HFC-245ca Exposure @ $121^{\circ} \mathrm{C}\left(250^{\circ} \mathrm{F}\right)$
Pages

iv

$\checkmark$

vi

$A-1$ to $A-18$

$B-1$ to $B-18$

C-1 to $C-18$

D-1 to D-1 8

$E-1$ to $E-18$

$F-1$ to $F-18$

G-1 to G-18

$\mathrm{H}-1$ to $\mathrm{H}-18$

$\mathrm{H}-1$ to $\mathrm{L}-18$

$\mathrm{J}-1$ to $\mathrm{J}-18$

$\mathrm{K}-1$ to $\mathrm{K}-18$

$L-1$ to $L-18$

$M-1$ to $M-18$ 


\section{Code Chart For Motor Material Exposures}

\section{Magnet Wire}

Code

A -Modified polyester overcoated with polyamide imide, as described in Section MW 73 of NEMA Standard MW 1000.

B - Modified polyester overcoated with polyamide imide and epoxy saturated glass as described in Section MW 73 and MW 46 of NEMA Standard MW 1000.

C -Polyester imide over coated with polyamide imide.

\section{Varnishes}

Code
A $-U-475 E H$ solvent epoxy
B -Y-390PG solvent epoxy-phenolic
C -ER-610 93\% solids epoxy
D $-Y-833 \quad 100 \%$ solids VPI epoxy
E -923 solvent epoxy
F -isopoxy 800 water-borne epoxy

\section{Sheet Insulation, Slot Liners and Phase Separators}

Code
A -Nomex/Mylar/Nomex
B -Dacron/Mylar/Dacron
C -Mylar MO
D -Nomex 410
E -Nomex Mica 418
F -Melinex 228

\section{Spiral Wrapped Sleeving Insulation}

\section{Code}

$\begin{array}{ll}\text { A } & \text {-Nomex } \\ \text { B } & \text {-Mylar } \\ \text { C } & \text {-Nomex/Mylar }\end{array}$

\section{Lead Wire Insulation}

\section{Code}
A -Dacron/Mylar/Dacron
B -Dacron/Teflon/Mylar/Dacron

\section{Tapes}

\section{Code}

A - Heat Cleaned Glass

B - Heat Shrinkable Braided Polyester

C -Permacel P247 glass/acrylic

\section{Tie Cords}

Code

A-Polyester

\#1-after 500 hour exposure to refrigerant or refrigerant/lubricant. \#2-after 500 hour exposure plus 24 hours at $127^{\circ} \mathrm{C}\left(302^{\circ} \mathrm{F}\right)$. 


\section{Units of Measure}

Magnet Wire

Test Performed

Weight Change

Burnout Strength

Dielectric Strength

\section{Varnish}

Test Performed

Weight Change

Bond Strength

Sheet Insulation

Test Performed

Weight Change

Tensile Strength

Elongation

Dielactric Strength

Spiral Wirapped Sleeving

Test Performed

Weight Change

\section{Lead Wire}

Test Performed

Weight Change

Dielectric Strength

Tapes and Tie Cords

Test Performed

Weight Change

Break Load Strength

Elongation
Experimental Units

grams $(g)$

seconds(sec)

Kilovolts(Kv)

Experimental Units

grams $(g)$

pounds(lbs)

Experimental Units

grams $(g)$

$1000 \mathrm{lbf}$ per square inch(Ksi)

inches

Kilovolts(kV)

Experimental Units

$\operatorname{grams}(\mathrm{g})$

Experimental Units

grams(g)

Kilovolts(kV)

Experimental Units

$\operatorname{grams}(\mathrm{g})$ pounds(lbs)

inches 


\section{Abbreviations}

WT=weight

EXP=exposed or experimental

BRN OUT=burnout

VIS=visual inspection

DIE=dielectric

VARN=varnish

BND STRENGTH=bond strength

COIL=helical coil

FLEX=flexibility test

$\mathrm{N} / \mathrm{C}=$ no change 


\section{Appendix A}

Experimental Data for Nitrogen Exposure at $60^{\circ} \mathrm{C}\left(140^{\circ} \mathrm{F}\right)$ 


\begin{tabular}{|c|c|c|c|c|c|c|c|c|c|c|}
\hline & 500 HRS IN & Nitrogen@ & $140 \mathrm{~F}$ & & & & & & & \\
\hline ID & WT & EXPWT & $\begin{array}{c}\text { WT \% } \\
\text { CHANGE }\end{array}$ & EXP VIS & $\begin{array}{c}\text { BASE BRN } \\
\text { OUT } \\
\text { (AVE) }\end{array}$ & $\begin{array}{c}\text { EXP BRN } \\
\text { OUT }\end{array}$ & $\begin{array}{c}\text { BRN OUT } \\
\% \\
\text { CHANGE }\end{array}$ & $\begin{array}{c}\text { BASE DIE } \\
\text { (AVE) }\end{array}$ & EXPDIE & $\begin{array}{c}\text { DIE \% } \\
\text { CHANGE }\end{array}$ \\
\hline$A 1$ & 23.2173 & 23.2139 & $-0.015 \%$ & $\mathrm{~N} / \mathrm{C}$ & 576 & 564 & & 15.80 & 13.87 & \\
\hline & & & & & 576 & 530 & $-4.3 \%$ & 15.80 & 17.40 & $2.3 \%$ \\
\hline & & & & & 576 & 560 & & 15.80 & 17.23 & \\
\hline$B 1$ & 25.9485 & 25.9454 & $-0.012 \%$ & $\mathrm{~N} / \mathrm{C}$ & 736 & 730 & & 11.62 & 11.77 & \\
\hline & & & & & 736 & 728 & $-0.9 \%$ & 11.62 & 12.63 & $6.7 \%$ \\
\hline & & & & & 736 & 730 & & 11.62 & 12.81 & \\
\hline C1 & 23.0471 & 23.0433 & $-0.016 \%$ & $\mathrm{~N} / \mathrm{C}$ & 579 & 510 & & 16.58 & 14.85 & \\
\hline & & & & & 579 & 549 & $-6.3 \%$ & 16.58 & 15.39 & $-3.8 \%$ \\
\hline & & & & & 579 & 568 & & 16.58 & 17.63 & \\
\hline & $-->24$ HRS & @ 302 F & & & & & & & & \\
\hline A2 & 23.2749 & 23.2680 & $-0.030 \%$ & $N / C$ & 576 & 588 & & 15.80 & 16.01 & \\
\hline & & & & & 576 & 596 & $-1.7 \%$ & 15.80 & 17.49 & $9.4 \%$ \\
\hline & & & & & 576 & 515 & & 15.80 & 18.37 & \\
\hline$B 2$ & 25.8003 & 25.7984 & $-0.007 \%$ & $\mathrm{~N} / \mathrm{C}$ & 736 & 729 & & 11.62 & 11.47 & \\
\hline & & & & & 736 & 729 & $-1.0 \%$ & 11.62 & 11.43 & $0.0 \%$ \\
\hline & & & & & 736 & 728 & & 11.62 & 11.97 & \\
\hline $\mathrm{C} 2$ & 23.0630 & 23.0568 & $-0.027 \%$ & $\mathrm{~N} / \mathrm{C}$ & 579 & 550 & & 16.58 & 16.90 & \\
\hline & & & & & 579 & 561 & $-3.4 \%$ & 16.58 & 16.31 & $-2.7 \%$ \\
\hline & & & & & 579 & 567 & & 16.58 & 15.21 & \\
\hline
\end{tabular}




\begin{tabular}{|c|c|c|c|c|c|c|c|c|c|c|c|}
\hline \multicolumn{12}{|c|}{500 HOURS IN Nitrogen @ 140 F } \\
\hline & VARN & $\begin{array}{l}\text { TWISTED } \\
\text { PAIR WT }\end{array}$ & $\begin{array}{c}\text { EXP T } \\
\text { PAIR WT }\end{array}$ & $\begin{array}{c}\text { WT \% } \\
\text { CHANGE }\end{array}$ & $\begin{array}{l}\text { EXP } \\
\text { VIS }\end{array}$ & ||BASE DIE & EXPDIE & $\begin{array}{c}\text { DIE \% } \\
\text { CHANGE }\end{array}$ & $\begin{array}{c}\text { BASE } \\
\text { BURN } \\
\text { OUT }\end{array}$ & $\begin{array}{c}\text { EXP } \\
\text { BURN } \\
\text { OUT }\end{array}$ & $\begin{array}{c}\text { BRNOUT } \\
\% \\
\text { CHANGE }\end{array}$ \\
\hline \multirow{3}{*}{$\begin{array}{l}P \\
0\end{array}$} & \multirow{3}{*}{$\begin{array}{l}U-475 \\
\text { A } 1\end{array}$} & \multirow[t]{3}{*}{23.8376} & \multirow[t]{3}{*}{23.8466} & \multirow[t]{3}{*}{$0.038 \%$} & $\mathrm{~N} / \mathrm{C}$ & 16.24 & 15.70 & & 430 & 337 & \\
\hline & & & & & & 16.24 & 15.73 & $-6.47 \%$ & 430 & 353 & $-20.70 \%$ \\
\hline & & & & & & 16.24 & 14.14 & & 430 & 333 & \\
\hline \multirow{3}{*}{$\begin{array}{l}Y \\
E \\
S\end{array}$} & \multirow{3}{*}{$\begin{array}{l}Y-390 \\
B 1\end{array}$} & \multirow[t]{3}{*}{21.9774} & \multirow[t]{3}{*}{21.9624} & \multirow[t]{3}{*}{$.0 .068 \%$} & $\mathrm{~N} / \mathrm{C}$ & 18.77 & 10.10 & & 510 & 442 & \\
\hline & & & & & & 18.77 & 20.00 & $-22.45 \%$ & 510 & 440 & $-13.86 \%$ \\
\hline & & & & & & 18.77 & 13.57 & & 510 & 436 & \\
\hline \multirow{4}{*}{$\begin{array}{l}T \\
E \\
R\end{array}$} & \multirow{3}{*}{$\begin{array}{l}\text { ER-610 } \\
\text { C1 }\end{array}$} & \multirow[t]{3}{*}{22.2632} & \multirow[t]{3}{*}{22.2666} & \multirow[t]{3}{*}{$0.015 \%$} & $\mathrm{~N} / \mathrm{C}$ & 15.57 & 16.37 & & 442 & 403 & \\
\hline & & & & & & 15.57 & 16.71 & $2.14 \%$ & 442 & 475 & $-2.79 \%$ \\
\hline & & & & & & 15.57 & 14.63 & & 442 & 411 & \\
\hline & \multirow{3}{*}{$\begin{array}{l}Y-833 \\
D 1\end{array}$} & \multirow[t]{3}{*}{21.5453} & \multirow[t]{3}{*}{21.5442} & \multirow[t]{3}{*}{$-0.005 \%$} & $\mathrm{~N} / \mathrm{C}$ & 12.04 & 11.80 & & 578 & 577 & \\
\hline$P$ & & & & & & 12.04 & 11.81 & $-2.16 \%$ & 578 & 555 & $2.13 \%$ \\
\hline 0 & & & & & & 12.04 & 11.73 & & 578 & 565 & \\
\hline \multirow{3}{*}{\multicolumn{2}{|c|}{$\begin{array}{l}923 \\
E 1\end{array}$}} & \multirow[t]{3}{*}{22.6395} & \multirow[t]{3}{*}{22.6448} & \multirow[t]{3}{*}{$0.023 \%$} & $\mathrm{~N} / \mathrm{C}$ & 16.76 & 20.00 & & 606 & 560 & \\
\hline & & & & & & 16.76 & 20.00 & $4.79 \%$ & 606 & 441 & $-22.00 \%$ \\
\hline & & & & & & 16.76 & 12.69 & & 606 & 417 & \\
\hline$M$ & ISO-800 & 21.6169 & 21.6172 & $0.001 \%$ & $\mathrm{~N} / \mathrm{C}$ & 19.08 & 17.50 & & 580 & 567 & \\
\hline 1 & & & & & & 19.08 & 17.97 & $-17.30 \%$ & 580 & 605 & $-1.44 \%$ \\
\hline$D$ & & & & & & 19.08 & 11.87 & & 580 & 543 & \\
\hline$E$ & & $24 \mathrm{HOURS}$ & S AT $302 \mathrm{~F}$ & & & & & & & & \\
\hline & $U-475$ & 23.9045 & 23.9028 & $-0.007 \%$ & $\mathrm{~N} / \mathrm{C}$ & 16.24 & 15.24 & & 430 & 361 & \\
\hline 1 & A2 & & & & & 16.24 & 17.30 & $-11.02 \%$ & 430 & 292 & $-21.01 \%$ \\
\hline$M$ & & & & & & 16.24 & 10.81 & & 430 & 366 & \\
\hline 1 & $Y-390$ & 21.5635 & 21.5614 & $-0.010 \%$ & $\mathrm{~N} / \mathrm{C}$ & 18.77 & 11.86 & & 510 & 452 & \\
\hline$D$ & $B 2$ & & & & & 18.77 & 20.00 & $-12.40 \%$ & 510 & 428 & $-6.80 \%$ \\
\hline$E$ & & & & & & 18.77 & 17.47 & & 510 & 546 & \\
\hline & ER-610 & 22.3508 & 22.3444 & $-C .029 \%$ & $\mathrm{~N} / \mathrm{C}$ & 15.57 & 11.79 & & 442 & 434 & \\
\hline & $\mathrm{C} 2$ & & & & & 15.57 & 13.14 & $-19.37 \%$ & 442 & 401 & $-5.20 \%$ \\
\hline & & & & & & 15.57 & 12.73 & & 442 & 422 & \\
\hline & $Y-833$ & 21.4100 & 21.4094 & $-0.003 \%$ & $\mathrm{~N} / \mathrm{C}$ & 12.04 & 10.63 & & 578 & 500 & \\
\hline & $D 2$ & & & & & 12.04 & 10.13 & $-9.27 \%$ & 578 & 579 & $-4.73 \%$ \\
\hline & & & & & & 12.04 & 12.01 & & 578 & 573 & \\
\hline & 923 & 22.5382 & 22.5370 & $-0.005 \%$ & $\mathrm{~N} / \mathrm{C}$ & 16.76 & 12.50 & & 606 & 449 & \\
\hline & E2 & & & & & 16.76 & 9.11 & $-30.19 \%$ & 606 & 404 & $-26.62 \%$ \\
\hline & & & & & & 16.76 & 13.49 & & 606 & 481 & \\
\hline & $150-800$ & 21.4983 & 21.4960 & $-0.011 \%$ & $\mathrm{~N} / \mathrm{C}$ & 19.08 & 15.90 & & 580 & 573 & \\
\hline & $\mathrm{F} 2$ & & & & & 19.08 & 16.63 & $-11.84 \%$ & 580 & 594 & $-1.15 \%$ \\
\hline & & & & & & 19.08 & 17.93 & & 580 & 553 & \\
\hline
\end{tabular}




\begin{tabular}{|c|c|c|c|c|c|c|c|c|c|c|c|}
\hline 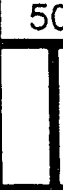 & VARN & $\begin{array}{l}\text { TWISTED } \\
\text { PAIR WT }\end{array}$ & $\begin{array}{c}\text { EXPT } \\
\text { PAIR WT }\end{array}$ & $\begin{array}{c}\text { WT \% } \\
\text { CHANGE }\end{array}$ & $\begin{array}{l}\text { EXP } \\
\text { VIS }\end{array}$ & $\begin{array}{l}\text { BASE } \\
\text { DIE }\end{array}$ & $\begin{array}{l}\text { EXP } \\
\text { DIE }\end{array}$ & $\begin{array}{c}\text { DIE \% } \\
\text { CHANGE }\end{array}$ & $\begin{array}{l}\text { BASE } \\
\text { BURN } \\
\text { OUT }\end{array}$ & $\begin{array}{c}\text { EXP } \\
\text { BURN } \\
\text { OUT }\end{array}$ & $\begin{array}{c}\text { BRNOUT } \\
\% \\
\text { CHANGE }\end{array}$ \\
\hline \multirow{3}{*}{$\begin{array}{l}P \\
O \\
L\end{array}$} & \multirow{3}{*}{$\begin{array}{l}\text { U-475 } \\
\text { A } 1\end{array}$} & \multirow[t]{3}{*}{26.6666} & \multirow[t]{3}{*}{26.6744} & \multirow[t]{3}{*}{$0.03 \%$} & $\mathrm{~N} / \mathrm{C}$ & 13.32 & 15.45 & & 746 & 728 & \\
\hline & & & & & & 13.32 & 12.47 & $9.38 \%$ & 746 & 731 & $-3.35 \%$ \\
\hline & & & & & & 13.32 & 15.79 & & 746 & 704 & \\
\hline \multirow{3}{*}{$\begin{array}{l}Y \\
E \\
S\end{array}$} & $Y-390$ & \multirow[t]{3}{*}{26.7664} & \multirow[t]{3}{*}{26.7696} & \multirow[t]{3}{*}{$0.01 \%$} & $\mathrm{~N} / \mathrm{C}$ & 12.28 & 14.43 & & 755 & 732 & \\
\hline & \multirow[t]{2}{*}{81} & & & & & 12.28 & 12.67 & $4.29 \%$ & 755 & 727 & $-3.49 \%$ \\
\hline & & & & & & 12.28 & 11.32 & & 755 & 727 & \\
\hline \multirow{3}{*}{$\begin{array}{l}T \\
E \\
R\end{array}$} & \multirow{3}{*}{$\begin{array}{l}\text { ER-610 } \\
\text { C1 }\end{array}$} & \multirow[t]{3}{*}{24.1810} & \multirow[t]{3}{*}{24.1680} & \multirow[t]{3}{*}{$-0.05 \%$} & $\mathrm{~N} / \mathrm{C}$ & 12.73 & 10.77 & & 734 & 718 & \\
\hline & & & & & & 12.73 & 12.39 & $-9.45 \%$ & 734 & 731 & $-1.18 \%$ \\
\hline & & & & & & 12.73 & 11.42 & & 734 & 727 & \\
\hline & \multirow{3}{*}{$\begin{array}{l}Y-833 \\
D 1\end{array}$} & \multirow[t]{3}{*}{21.3290} & \multirow[t]{3}{*}{21.3299} & \multirow[t]{3}{*}{$0.00 \%$} & $\mathrm{~N} / \mathrm{C}$ & 12.49 & 11.17 & & 734 & 710 & \\
\hline$P$ & & & & & & 12.49 & 11.10 & $-8.78 \%$ & 734 & 680 & $-3.91 \%$ \\
\hline 0 & & & & & & 12.49 & 11.91 & & 734 & 726 & \\
\hline \multirow{3}{*}{$\begin{array}{l}\mathrm{L} \\
\mathrm{Y} \\
\mathrm{A}\end{array}$} & \multirow{3}{*}{$\begin{array}{l}923 \\
E 1\end{array}$} & \multirow[t]{3}{*}{28.2001} & \multirow[t]{3}{*}{28.2112} & \multirow[t]{3}{*}{$0.04 \%$} & $\mathrm{~N} / \mathrm{C}$ & 14.38 & 15.65 & & 742 & 734 & \\
\hline & & & & & & 14.38 & 12.27 & $-6.86 \%$ & 742 & 732 & $-0.90 \%$ \\
\hline & & & & & & 14.38 & 12.26 & & 742 & 740 & \\
\hline$M$ & $1 S O-800$ & 25.6680 & 25.6647 & $-0.01 \%$ & $\mathrm{~N} / \mathrm{C}$ & 12.29 & 12.30 & & 747 & 741 & \\
\hline 1 & $\mathrm{~F} 1$ & & & & & 12.29 & 13.10 & $3.88 \%$ & 747 & 736 & $-0.85 \%$ \\
\hline$D$ & & & & & & 12.29 & 12.90 & & 747 & 745 & \\
\hline$E$ & & Nitrogen & $>24$ hours & S@302 & $F$ & & & & & & \\
\hline & $U-475$ & 23.3957 & 23.3984 & $0.01 \%$ & $\mathrm{~N} / \mathrm{C}$ & 13.32 & 13.76 & & 746 & 727 & \\
\hline 1 & $A 2$ & & & & & 13.32 & 13.40 & $-3.10 \%$ & 746 & 726 & $-2.50 \%$ \\
\hline$M$ & & & & & & 13.32 & 11.56 & & 746 & 729 & \\
\hline 1 & $Y-390$ & 26.8449 & 26.8416 & $-0.01 \%$ & $\mathrm{~N} / \mathrm{C}$ & 12.28 & 12.31 & & 755 & 735 & \\
\hline$D$ & B2 & & & & & 12.28 & 11.31 & $-1.06 \%$ & 755 & 729 & $-3.05 \%$ \\
\hline$E$ & & & & & & 12.28 & 12.83 & & 755 & 732 & \\
\hline & ER-610 & 24.3963 & 24.3952 & $0.00 \%$ & $\mathrm{~N} / \mathrm{C}$ & 12.73 & 11.65 & & 734 & 729 & \\
\hline$E$ & C2 & & & & & 12.73 & 12.81 & $-13.75 \%$ & 734 & 730 & $-0.64 \%$ \\
\hline$P$ & & & & & & 12.73 & 8.48 & & 734 & 729 & \\
\hline 0 & $Y-833$ & 21.4484 & 21.4467 & $-0.01 \%$ & $\mathrm{~N} / \mathrm{C}$ & 12.49 & 12.11 & & 734 & 706 & \\
\hline$x$ & D2 & & & & & 12.49 & 11.75 & $-5.63 \%$ & 734 & 652. & $-5.50 \%$ \\
\hline$Y$ & & & & & & 12.49 & 11.50 & & 734 & 723 & \\
\hline & 923 & 27.4130 & 27.4125 & $0.00 \%$ & $\mathrm{~N} / \mathrm{C}$ & 14.38 & 12.50 & & 742 & 726 & \\
\hline$G$ & E2 & & & & & 14.38 & 14.17 & $-6.72 \%$ & 742 & 727 & $-2.16 \%$ \\
\hline$L$ & & & & & & 14.38 & 13.57 & & 742 & 726 & \\
\hline$A$ & $150-800$ & 25.4652 & 25.4628 & $-0.01 \%$ & & 12.29 & 12.54 & & 747 & 747 & \\
\hline$S$ & $\mathrm{~F} 2$ & & & & $\mathrm{~N} / \mathrm{C}$ & 12.29 & 11.59 & $-1.14 \%$ & 747 & 732 & $-1.00 \%$ \\
\hline$S$ & & & & & & 12.29 & 12.32 & & 747 & 746 & \\
\hline
\end{tabular}


500 HOURS IN Nitrogen @ 140 F

\begin{tabular}{|c|c|c|c|c|c|c|c|c|c|c|}
\hline VARN & $\begin{array}{l}\text { TWISTED } \\
\text { PAIR WT }\end{array}$ & \begin{tabular}{|c|} 
EXPT \\
PAIR WT
\end{tabular} & $\begin{array}{c}\text { WT \% } \\
\text { CHANGE }\end{array}$ & $\begin{array}{l}\text { EXP } \\
\text { VIS }\end{array}$ & $\begin{array}{c}\text { BASE } \\
\text { DIE }\end{array}$ & $\begin{array}{l}\text { EXP } \\
\text { DIE }\end{array}$ & $\begin{array}{c}\text { DIE \% } \\
\text { CHANGE }\end{array}$ & $\begin{array}{c}\text { BASE } \\
\text { BURN } \\
\text { OUT }\end{array}$ & $\begin{array}{c}\text { EXP } \\
\text { BURN } \\
\text { OUT }\end{array}$ & $\begin{array}{c}\text { BRNOUT } \\
\% \\
\text { CHANGE }\end{array}$ \\
\hline \multirow{3}{*}{$\begin{array}{l}\text { U-475 } \\
\text { A } 1\end{array}$} & 24.1266 & 24.1319 & $0.022 \%$ & $\mathrm{~N} / \mathrm{C}$ & 15.10 & 8.63 & & 469 & 279 & \\
\hline & & & & & 15.10 & 7.62 & $-37.53 \%$ & 469 & 259 & $-39.66 \%$ \\
\hline & & & & & 15.10 & 12.05 & & 469 & 311 & \\
\hline \multirow{3}{*}{$\begin{array}{l}Y-390 \\
B 1 \\
\end{array}$} & 21.9228 & 21.9312 & $0.038 \%$ & $\mathrm{~N} / \mathrm{C}$ & 18.24 & 10.88 & & 473 & 398 & \\
\hline & & & & & 18.24 & 20.00 & $.7 .02 \%$ & 473 & 405 & $-5.07 \%$ \\
\hline & & & & & 18.24 & 20.00 & & 473 & 544 & \\
\hline \multirow{3}{*}{$\begin{array}{l}\text { ER-610 } \\
\text { C1 }\end{array}$} & 22.4758 & 22.4809 & $0.023 \%$ & $\mathrm{~N} / \mathrm{C}$ & 14.53 & 8.16 & & 494 & 283 & \\
\hline & & & & & 14.53 & 12.00 & \begin{tabular}{|l|}
$-37.16 \%$ \\
\end{tabular} & 494 & 390 & $-30.36 \%$ \\
\hline & & & & & 14.53 & 7.23 & & 494 & 359 & \\
\hline \multirow{3}{*}{$\begin{array}{l}\mathrm{Y}-833 \\
\mathrm{D} 1\end{array}$} & 22.0466 & 22.0549 & $0.038 \%$ & $\mathrm{~N} / \mathrm{C}$ & 11.38 & 12.27 & & 557 & 318 & \\
\hline & & & & & 11.38 & 20.00 & $27.42 \%$ & 557 & 366 & $-41.29 \%$ \\
\hline & & & & & 11.38 & 11.23 & & 557 & 297 & \\
\hline \multirow{3}{*}{$\begin{array}{l}923 \\
E 1\end{array}$} & 23.1337 & 23.1408 & $0.031 \%$ & $N / C$ & 15.85 & 16.22 & & 503 & 520 & \\
\hline & & & & & 15.85 & 17.90 & $-0.76 \%$ & 503 & 520 & $-4.44 \%$ \\
\hline & & & & & 15.85 & 13.07 & & 503 & 402 & \\
\hline \multirow{3}{*}{$\begin{array}{l}150-800 \\
F 1\end{array}$} & 21.6704 & 21.6726 & $0.010 \%$ & $\mathrm{~N} / \mathrm{C}$ & 14.75 & 17.84 & & 632 & 578 & \\
\hline & & & & & 14.75 & 16.97 & $18.71 \%$ & 632 & 549 & $-10.50 \%$ \\
\hline & & & & & 14.75 & 17.72 & & 632 & 570 & \\
\hline L & $\rightarrow>24 h o$ & ours@302 & & & & & & & & \\
\hline \multirow{3}{*}{$\begin{array}{l}U-475 \\
A 2\end{array}$} & 24.2843 & 24.2840 & $-0.001 \%$ & $\mathrm{~N} / \mathrm{C}$ & 15.10 & 16.09 & & 469 & 351 & \\
\hline & & & & & 15.10 & 11.63 & $-19.56 \%$ & 469 & 343 & $-27.93 \%$ \\
\hline & & & & & 15.10 & 8.72 & & 469 & 320 & \\
\hline \multirow{3}{*}{ D } & 21.9349 & 21.9345 & $-0.002 \%$ & $\mathrm{~N} / \mathrm{C}$ & 18.24 & 16.69 & & 473 & 478 & \\
\hline & & & & & 18.24 & 13.64 & $-36.92 \%$ & 473 & 481 & $1.20 \%$ \\
\hline & & & & & 18.24 & 9.37 & & 473 & 477 & \\
\hline \multirow{3}{*}{$\begin{array}{l}\text { ER-610 } \\
\text { C2 }\end{array}$} & 22.6978 & 22.6996 & $0.008 \%$ & $\mathrm{~N} / \mathrm{C}$ & 14.53 & 13.63 & & 494 & 353 & \\
\hline & & & & & 14.53 & 12.55 & $-11.81 \%$ & 494 & 325 & $-28.07 \%$ \\
\hline & & & & & 14.53 & 12.26 & & 494 & 388 & \\
\hline \multirow{3}{*}{$\begin{array}{l}\mathrm{Y}-833 \\
D 2\end{array}$} & 21.7187 & 21.7150 & $-0.017 \%$ & $\mathrm{~N} / \mathrm{C}$ & 11.38 & 18.55 & & 557 & 344 & \\
\hline & & & & & 11.38 & 13.66 & $51.96 \%$ & 557 & 343 & $-39.74 \%$ \\
\hline & & & & & 11.38 & 19.67 & & 557 & 320 & \\
\hline \multirow{3}{*}{$\begin{array}{l}923 \\
\text { E2 }\end{array}$} & 23.2099 & 23.2093 & $-0.003 \%$ & $\mathrm{~N} / \mathrm{C}$ & 15.85 & 13.16 & & 503 & 401 & \\
\hline & & & & & 15.85 & 12.26 & $-11.19 \%$ & 503 & 472 & $-12.33 \%$ \\
\hline & & & & & 15.85 & 16.81 & & 503 & 450 & \\
\hline \multirow{3}{*}{$\begin{array}{l}\text { ISO-800 } \\
\text { F2 }\end{array}$} & 21.8631 & 21.9472 & $.0 .073 \%$ & $\mathrm{~N} / \mathrm{C}$ & 14.75 & 15.20 & & 632 & 570 & \\
\hline & & & & & 14.75 & 13.83 & $6.51 \%$ & 632 & 556 & $-11.45 \%$ \\
\hline & & & & & 14.75 & 18.10 & & 632 & 553 & \\
\hline
\end{tabular}




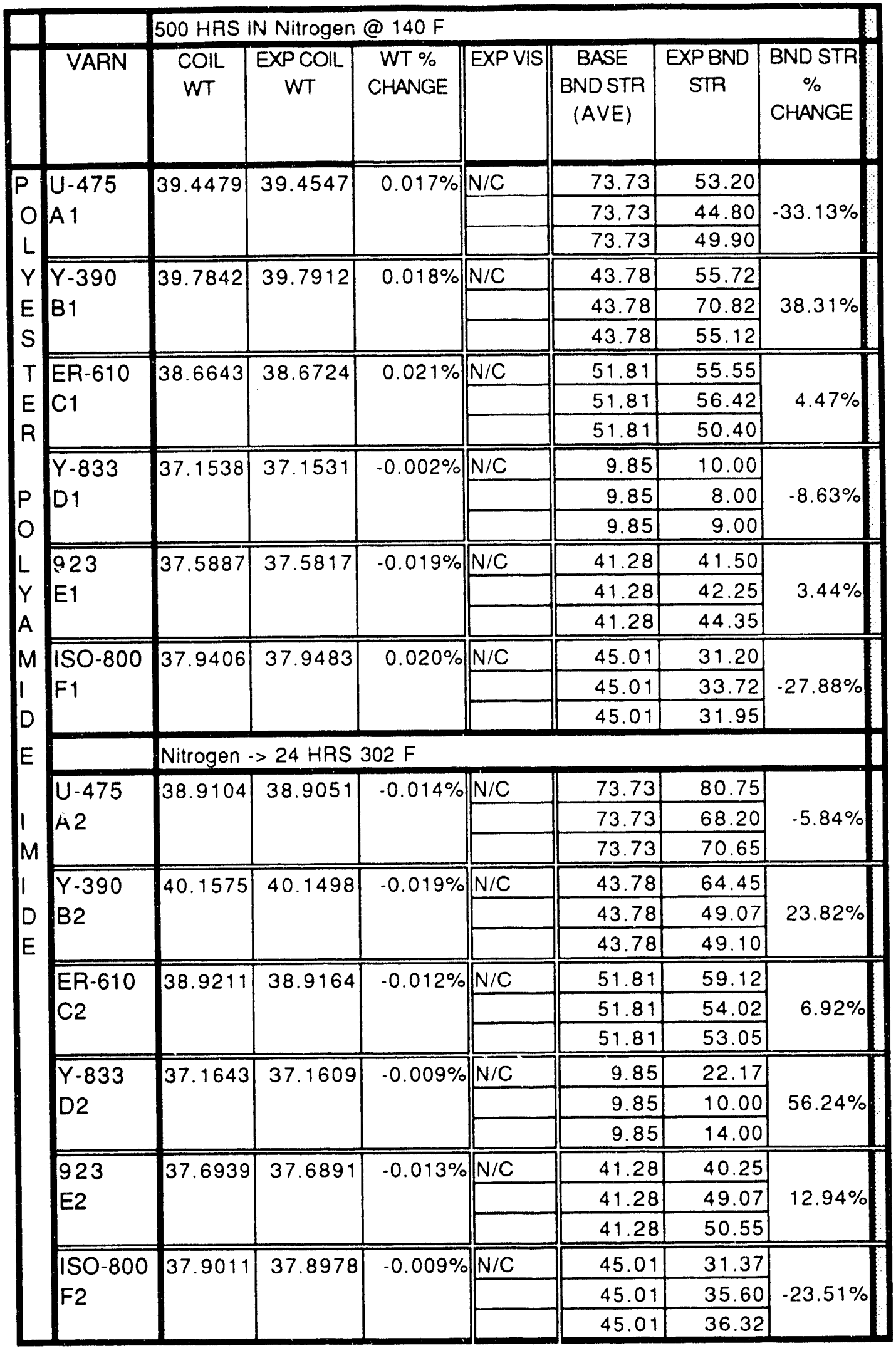




\begin{tabular}{|c|c|c|c|c|c|c|c|c|}
\hline & & $500 \mathrm{HRS}$ & IN Nitroge & @ 140F & & & & \\
\hline & VARN & $\begin{array}{l}\text { COIL } \\
\text { WT }\end{array}$ & $\begin{array}{c}\text { EXP COIL } \\
W T\end{array}$ & $\begin{array}{c}\text { WT \% } \\
\text { CHANGE }\end{array}$ & EXP VIS & $\begin{array}{c}\text { BASE } \\
\text { BND STR } \\
\text { (AVE) }\end{array}$ & $\begin{array}{c}\text { EXP BND } \\
\text { STR }\end{array}$ & $\begin{array}{c}\text { BND STR } \\
\% \\
\text { CHANGE }\end{array}$ \\
\hline$P$ & U-475 & 38.1533 & 38.1567 & $0.009 \%$ & $\mathrm{~N} / \mathrm{C}$ & 40.14 & 32.05 & \\
\hline 0 & A 1 & & & & & 40.14 & 36.62 & $-14.99 \%$ \\
\hline$L$ & & & & & & 40.14 & 33.70 & \\
\hline$Y$ & $Y-390$ & 36.9446 & 36.9541 & $0.026 \%$ & $\mathrm{~N} / \mathrm{C}$ & 36.12 & 27.85 & \\
\hline$E$ & B1 & & & & & 36.12 & 37.22 & $-10.56 \%$ \\
\hline S & & & & & & 36.12 & 31.85 & \\
\hline$T$ & ER-610 & 36.5143 & 36.5194 & $0.014 \%$ & $\mathrm{~N} / \mathrm{C}$ & 35.96 & 35.17 & \\
\hline$E$ & C1 & & & & & 35.96 & 32.55 & $-9.42 \%$ \\
\hline $\mathrm{R}$ & & & & & & 35.96 & 30.00 & \\
\hline & $Y-833$ & 36.8104 & 36.8171 & $0.018 \%$ & $\mathrm{~N} / \mathrm{C}$ & 33.14 & 33.57 & \\
\hline$P$ & D1 & & & & & 33.14 & 14.30 & $-20.57 \%$ \\
\hline 0 & & & & & & 33.14 & 31.10 & \\
\hline$L$ & 923 & 35.7969 & 35.8063 & $0.026 \%$ & $\mathrm{~N} / \mathrm{C}$ & 40.52 & 36.27 & \\
\hline$Y$ & E1 & & & & & 40.52 & 39.42 & $-7.28 \%$ \\
\hline A & & & & & & 40.52 & 37.02 & \\
\hline$M$ & ISO-800 & 35.4549 & 35.4568 & $0.005 \%$ & $\mathrm{~N} / \mathrm{C}$ & 20.20 & 18.65 & \\
\hline I & F1 & & & & & 20.20 & 19.67 & $-3.48 \%$ \\
\hline D & & & & & & 20.20 & 20.17 & \\
\hline$E$ & & Nitrogen & $\rightarrow 24 \mathrm{HRS}$ & $302 \mathrm{~F}$ & & & & \\
\hline & U-475 & 37.9895 & 37.9820 & $-0.020 \%$ & $\mathrm{~N} / \mathrm{C}$ & 40.14 & 38.62 & \\
\hline 1 & A2 & & & & & 40.14 & broken & $.7 .05 \%$ \\
\hline$M$ & & & & & & 40.14 & 36.00 & \\
\hline 1 & $Y-390$ & 37.3761 & 37.3713 & $-0.013 \%$ & Varnish & 36.12 & 32.97 & \\
\hline D & B2 & & & & Pockets & 36.12 & 32.80 & $-9.50 \%$ \\
\hline$E$ & & & & & & 36.12 & 32.30 & \\
\hline & ER-610 & 37.1049 & 37.1018 & $-0.008 \%$ & $\mathrm{~N} / \mathrm{C}$ & 35.96 & 38.02 & \\
\hline$E$ & C2 & & & & & 35.96 & 35.67 & $2.21 \%$ \\
\hline$P$ & & & & & & 35.96 & 36.57 & \\
\hline 0 & $Y-833$ & 36.9632 & 36.9617 & $-0.004 \%$ & $\mathrm{~N} / \mathrm{C}$ & 33.14 & 27.75 & \\
\hline$x$ & D2 & & & & & 33.14 & 24.80 & $-9.98 \%$ \\
\hline$Y$ & & & & & & 33.14 & 36.95 & \\
\hline & 923 & 36.9032 & 36.8978 & $-0.015 \%$ & $\mathrm{~N} / \mathrm{C}$ & 40.52 & 35.90 & \\
\hline G & E2 & & & & & 40.52 & 39.40 & $.9 .37 \%$ \\
\hline$L$ & & & & & & 40.52 & 34.87 & \\
\hline A & ISO-800 & 35.4208 & 35.4168 & $-0.011 \%$ & $\mathrm{~N} / \mathrm{C}$ & 20.20 & 19.92 & \\
\hline$S$ & F2 & & & & & 20.20 & 19.67 & $-4.19 \%$ \\
\hline$S$ & & & & & & 20.20 & 18.47 & \\
\hline
\end{tabular}




\begin{tabular}{|c|c|c|c|c|c|c|c|c|}
\hline & & $0 \mathrm{HRS}$ & $\pi v$ & $40 \mathrm{~F}$ & & & & \\
\hline & VARN & $\begin{array}{l}\text { COIL } \\
\text { WT }\end{array}$ & $\begin{array}{c}\text { EXP COIL } \\
W T\end{array}$ & $\begin{array}{c}\text { WT \% } \\
\text { CHANGE }\end{array}$ & EXP VIS & \begin{tabular}{|c|} 
BASE \\
BND STR \\
(AVE)
\end{tabular} & $\begin{array}{c}\text { EXPBND } \\
\text { STR }\end{array}$ & $\begin{array}{c}\text { BND STR } \\
\% \\
\text { CHANGE }\end{array}$ \\
\hline$P$ & U.475 & 37.5011 & 37.5052 & $0.011 \%$ & $\mathrm{~N} / \mathrm{C}$ & 51.21 & 65.77 & \\
\hline 0 & $A 1$ & & & & & 51.21 & 66.77 & $30.73 \%$ \\
\hline$L$ & & & & & & 51.21 & 68.30 & \\
\hline$Y$ & $Y-390$ & 39.2874 & 39.2822 & $-0.013 \%$ & $\mathrm{~N} / \mathrm{C}$ & 50.72 & 60.07 & \\
\hline$E$ & B1 & & & & & 50.72 & 54.05 & $2.14 \%$ \\
\hline$S$ & & & & & & 50.72 & 41.30 & \\
\hline$T$ & ER-610 & 37.3294 & 37.3346 & $0.014 \%$ & $\mathrm{~N} / \mathrm{C}$ & 58.33 & 54.92 & \\
\hline$E$ & C1 & & & & & 58.33 & 62.92 & $-0.66 \%$ \\
\hline$R$ & & & & & & 58.33 & 56.00 & \\
\hline & $Y-833$ & 36.8415 & 36.8416 & $0.000 \%$ & $\mathrm{~N} / \mathrm{C}$ & 5.84 & 9.00 & \\
\hline 1 & D1 & & & & & 5.84 & 5.00 & $54.11 \%$ \\
\hline M & & & & & & 5.84 & 13.00 & \\
\hline I & 923 & 38.6136 & 38.6166 & $0.008 \%$ & $\mathrm{~N} / \mathrm{C}$ & 49.26 & 41.25 & \\
\hline$D$ & E1 & & & & & 49.26 & 38.07 & $-19.28 \%$ \\
\hline$E$ & & & & & & 49.26 & 39.97 & \\
\hline & ISO-800 & 38.5336 & 38.5334 & $-0.001 \%$ & $\mathrm{~N} / \mathrm{C}$ & 36.08 & 43.35 & \\
\hline$P$ & $\mathrm{~F} 1$ & & & & & 36.08 & 55.65 & $12.85 \%$ \\
\hline $\mathrm{O}$ & & & & & & 36.08 & 23.15 & \\
\hline$L$ & & Nitrogen-> & 24 HRS 3 & $02 \mathrm{~F}$ & & & & \\
\hline$Y$ & U.475 & 37.5624 & 37.5515 & $-0.029 \%$ & $\mathrm{~N} / \mathrm{C}$ & 51.21 & 71.87 & \\
\hline$A$ & A2 & & & & & 51.21 & 74.97 & $42.71 \%$ \\
\hline$M$ & & & & & & 51.21 & 72.40 & \\
\hline 1 & Y-390 & 39.7222 & 39.7177 & $-0.011 \%$ & $N / C$ & 50.72 & 58.70 & \\
\hline D & B2 & & & & & 50.72 & 55.10 & $9.79 \%$ \\
\hline$E$ & & & & & & 50.72 & 53.25 & \\
\hline & ER-610 & 37.6181 & 37.6148 & $-0.009 \%$ & $\mathrm{~N} / \mathrm{C}$ & 58.33 & 57.00 & \\
\hline I & $\mathrm{C} 2$ & & & & & 58.33 & 51.57 & $-8.41 \%$ \\
\hline$M$ & & & & & & 58.33 & 51.70 & \\
\hline I & $Y-833$ & 37.1415 & 37.1361 & $-0.015 \%$ & $\mathrm{~N} / \mathrm{C}$ & 5.84 & 24.45 & \\
\hline$D$ & D2 & & & & & 5.84 & 21.40 & $190.24 \%$ \\
\hline$E$ & & & & & & 5.84 & 5.00 & \\
\hline & $\overline{923}$ & 38.4291 & 38.4248 & $-0.011 \%$ & $\mathrm{~N} / \mathrm{C}$ & 49.26 & 43.42 & \\
\hline & E2 & & & & & 49.26 & 49.97 & $.8 .13 \%$ \\
\hline & & & & & & 49.26 & 42.37 & \\
\hline & $150-800$ & 38.5579 & 38.5534 & $-0.012 \%$ & $\mathrm{~N} / \mathrm{C}$ & 36.08 & 58.92 & \\
\hline & $\mathrm{F} 2$ & & & & & 36.08 & 52.12 & $45.43 \%$ \\
\hline & & & & & & 36.08 & 46.37 & \\
\hline
\end{tabular}




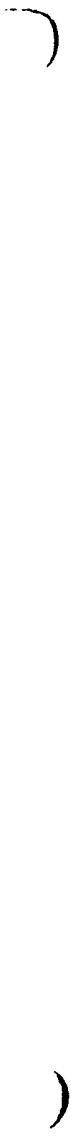

\begin{tabular}{|c|c|c|c|c|c|}
\hline & 500 HRS IN & Nitrogen@ & $140 \mathrm{~F}$ & & \\
\hline ID & WT & EXPWT & $\begin{array}{l}\text { WT \% } \\
\text { CHANGE }\end{array}$ & EXP VIS & EXP FLEX \\
\hline A1 & 4.7448 & 4.7437 & $-0.023 \%$ & $\mathrm{~N} / \mathrm{C}$ & \\
\hline & & & & & YES \\
\hline $\mathrm{B1}$ & 6.2039 & 6.2061 & $0.035 \%$ & $\mathrm{~N} / \mathrm{C}$ & \\
\hline & & & & & YES \\
\hline $\mathrm{Ct}$ & 55886 & 55881 & $-0.009 \%$ & $\overline{\mathrm{N} / \mathrm{C}}$ & \\
\hline & & & & & YES \\
\hline & & & & & \\
\hline & Nitrogen & $302 F 24$ & & & \\
\hline A2 & 4.7642 & 4.7618 & $-0.050 \%$ & $\mathrm{~N} / \mathrm{C}$ & \\
\hline & & & & & YES \\
\hline B2 & 6.2798 & 6.2829 & $0.049 \%$ & $\mathrm{~N} / \mathrm{C}$ & \\
\hline & & & & & YES \\
\hline$\overline{C 2}$ & 5.6049 & 5.6001 & $-0.086 \%$ & $\mathrm{~N} / \mathrm{C}$ & \\
\hline & & & & & YES \\
\hline & & & & & \\
\hline
\end{tabular}




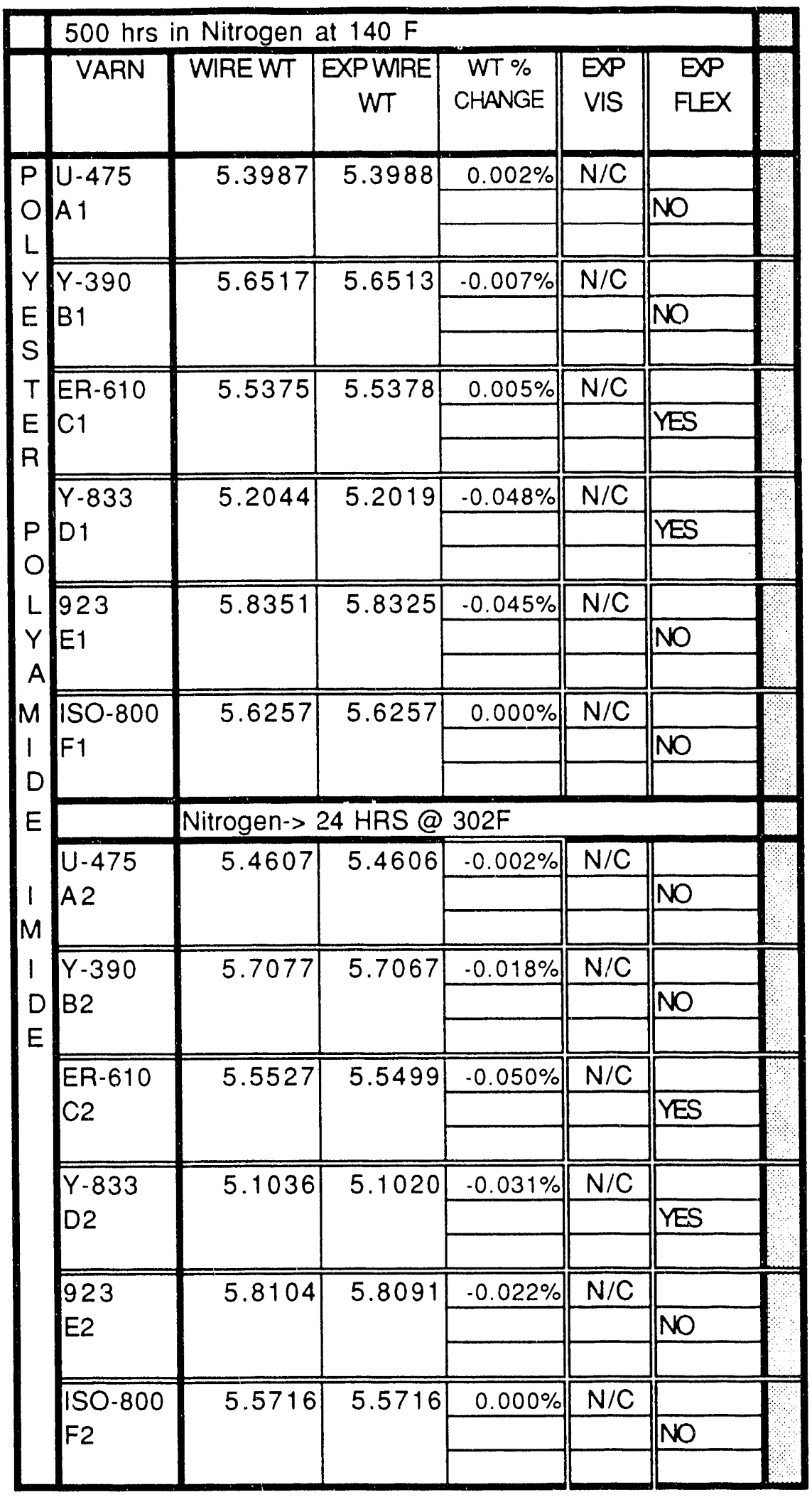




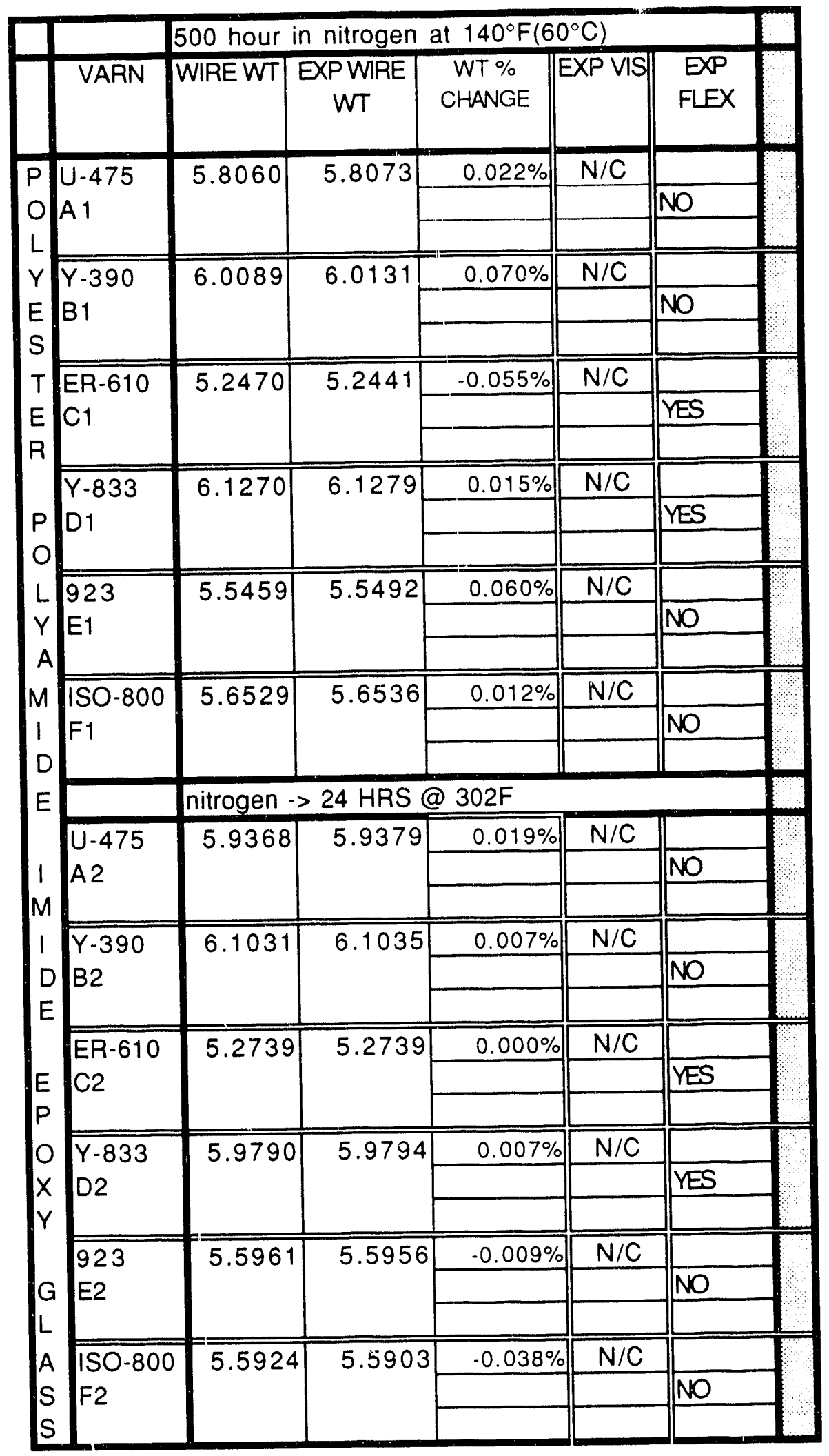




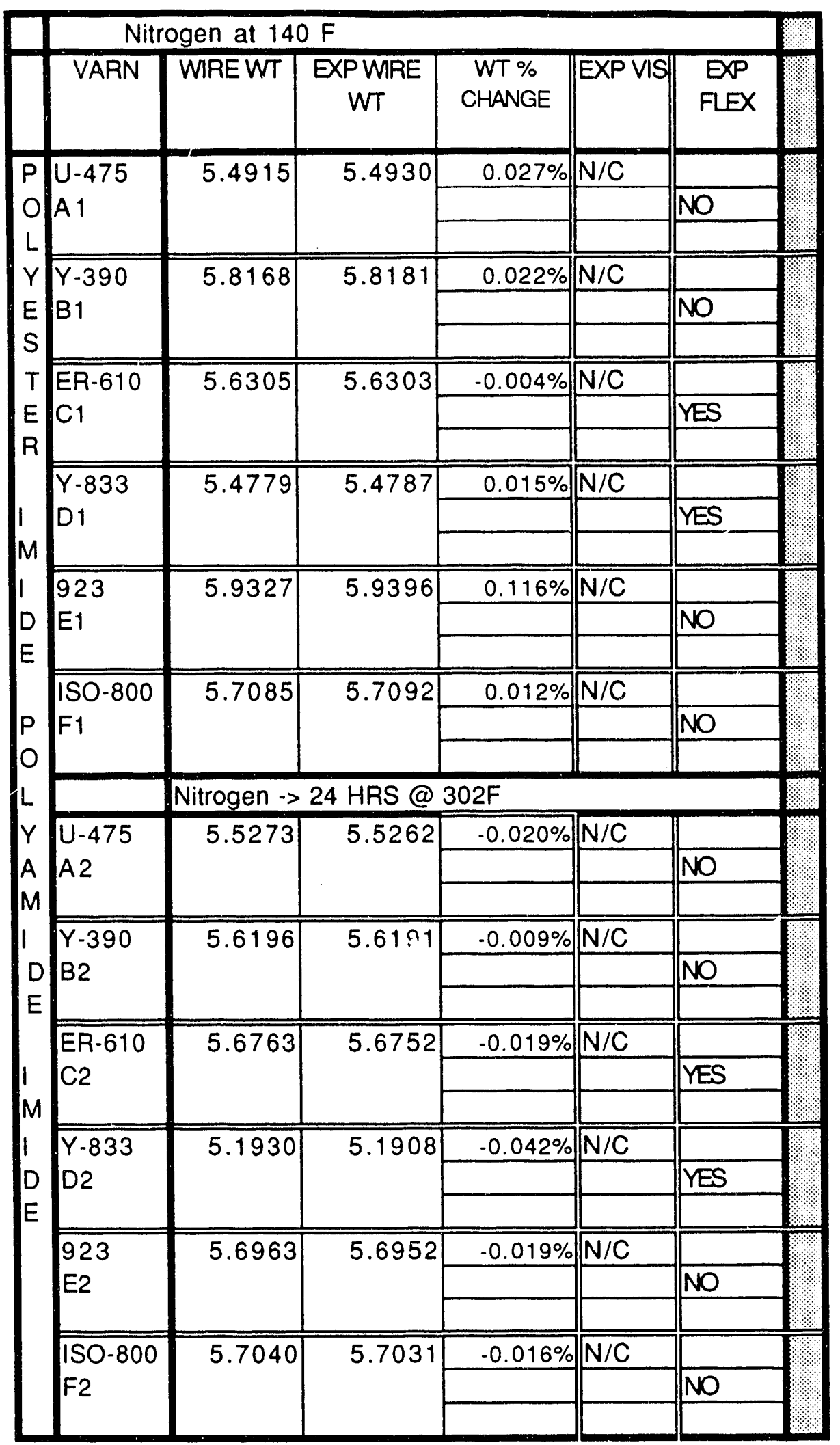




\begin{tabular}{|c|c|c|c|c|c|}
\hline & 500 HRS IN & Jitrogen@1 & $140 \mathrm{~F}$ & & \\
\hline$\overline{D D}$ & WT & EXPWT & \begin{tabular}{c|} 
WT $\%$ \\
CHANGE
\end{tabular} & EXP VIS & EXPFLEX \\
\hline $\begin{array}{l}\text { U-475 } \\
\text { A1 }\end{array}$ & 2.2051 & 2.2120 & $0.31 \%$ & $\mathrm{~N} / \mathrm{C}$ & $\mathrm{N} / \mathrm{C}$ \\
\hline $\begin{array}{l}Y-390 \\
B 1\end{array}$ & 2.1027 & 2.1088 & $0.29 \%$ & \begin{tabular}{|l|} 
slightly \\
warped \\
\end{tabular} & $\mathrm{N} / \mathrm{C}$ \\
\hline ER-610 & 2.5004 & 2.5099 & $0.38 \%$ & $\mathrm{~N} / \mathrm{C}$ & $N / C$ \\
\hline$Y-833$ & 2.3183 & 2.3224 & $0.18 \%$ & $\mathrm{~N} / \mathrm{C}$ & $N / C$ \\
\hline 923 & 1.7684 & 1.7734 & $0.28 \%$ & $\mathrm{~N} / \mathrm{C}$ & $\mathrm{N} / \mathrm{C}$ \\
\hline & & & & & \\
\hline $150-800$ & 1.0921 & 1.0942 & $0.19 \%$ & Slightly & $\mathrm{N} / \mathrm{C}$ \\
\hline & & & & warped & \\
\hline & Nitrogen at & $>302 \mathrm{~F} 24 \mathrm{H}$ & & & \\
\hline U-475 & 2.4477 & 2.4291 & $-0.76 \%$ & Darkened & $N / C$ \\
\hline A2 & & & & Slightly & \\
\hline & & & & Warped & \\
\hline$Y-390$ & 2.4425 & 2.4091 & $-1.37 \%$ & slightly & $\mathrm{N} / \mathrm{C}$ \\
\hline & & & & warped & \\
\hline ER-610 & 1.9845 & 1.9786 & $-0.30 \%$ & Darkened & $\mathrm{N} / \mathrm{C}$ \\
\hline $\mathrm{C} 2$ & & & & & \\
\hline Y-833 & 2.7184 & 2.7198 & $0.05 \%$ & $\mathrm{~N} / \mathrm{C}$ & $N / C$ \\
\hline D2 & & & & & \\
\hline & 17253 & 17250 & $-0.02 \%$ & & $N / C$ \\
\hline $\begin{array}{l}923 \\
\mathrm{E} 2\end{array}$ & & & & & \\
\hline & & & 1640 & Warned & INUS \\
\hline $\begin{array}{l}\text { ISO-800 } \\
\text { F2 }\end{array}$ & 1.1148 & 1.0965 & $-1.64 \% \|$ & Warped & N/C \\
\hline & & & & & \\
\hline
\end{tabular}




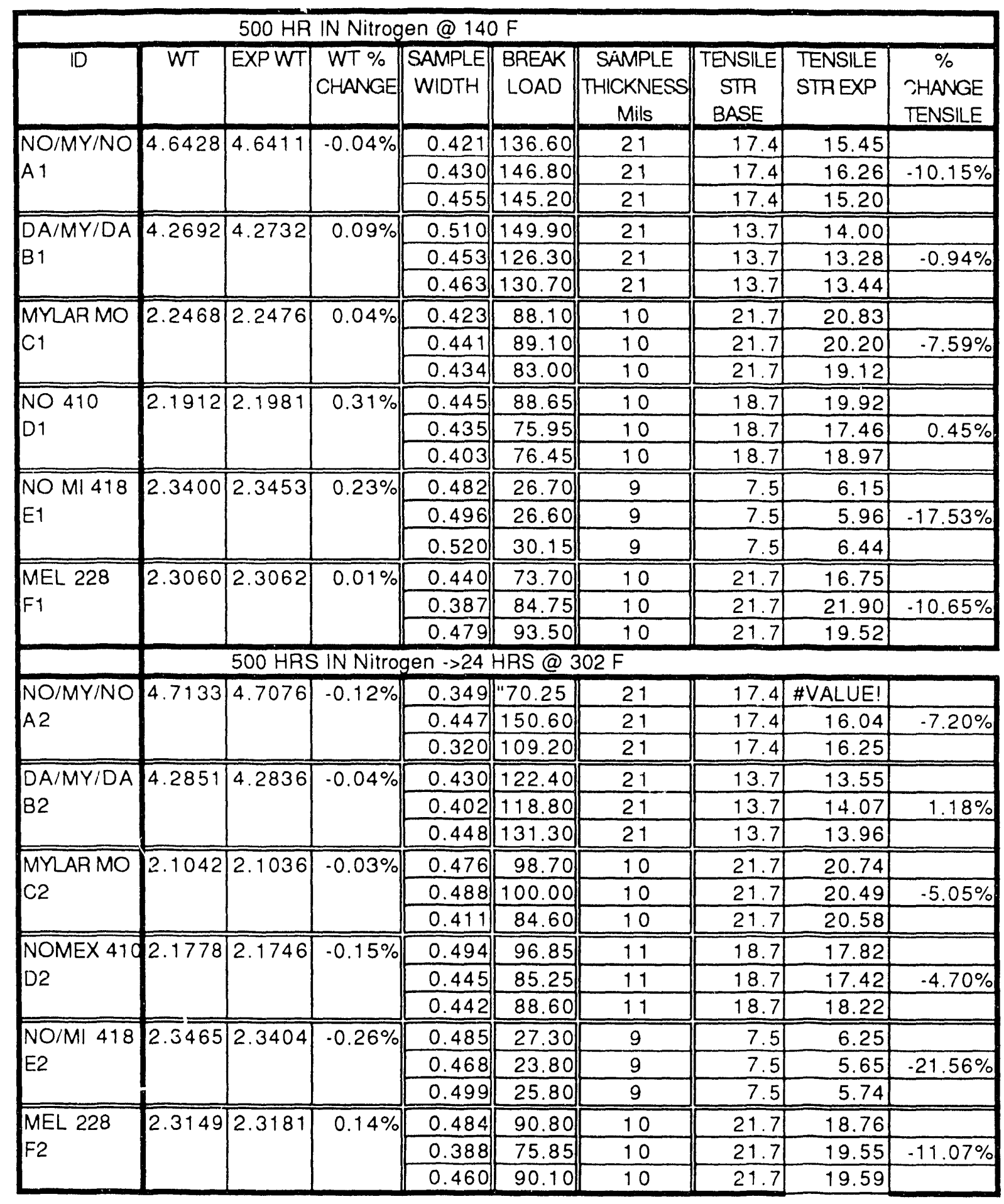




\begin{tabular}{|c|c|c|c|c|c|c|c|c|}
\hline \multicolumn{8}{|c|}{ After 500 hour exposure at $140^{\circ} \mathrm{F}\left(60^{\circ} \mathrm{C}\right)$} & \multirow[b]{2}{*}{$\begin{array}{c}\text { VISUAL } \\
\text { EXP }\end{array}$} \\
\hline ID & $\begin{array}{c}\text { STRETCH } \\
\text { (inch) }\end{array}$ & $\begin{array}{c}\% \\
\text { ELONG }\end{array}$ & $\begin{array}{c}\text { BASE } \\
\text { ELONG } \\
\text { (AVE) }\end{array}$ & $\begin{array}{c}\text { ELONG } \\
\% \\
\text { CHANGE }\end{array}$ & $\begin{array}{c}\text { BASE } \\
\text { DEE } \\
\text { (AVE) }\end{array}$ & EXP DIE & $\begin{array}{c}\text { DIE \% } \\
\text { CHANGE }\end{array}$ & \\
\hline \multirow{3}{*}{$\begin{array}{l}\mathrm{NO} / \mathrm{MY} / \mathrm{NO} \\
\mathrm{A} 1\end{array}$} & 0.33 & $8.3 \%$ & $20.0 \%$ & & $>18.97$ & $>13.39$ & & \multirow[t]{3}{*}{$N / C$} \\
\hline & 0.65 & $16.3 \%$ & $20.0 \%$ & $.40 .0 \%$ & $>18.97$ & $>13.96$ & flash & \\
\hline & 0.46 & $11.5 \%$ & $20.0 \%$ & & $>18.97$ & $>15.12$ & & \\
\hline \multirow{3}{*}{$\begin{array}{l}\mathrm{DA} / \mathrm{MY} / \mathrm{DA} \\
\mathrm{B} 1\end{array}$} & 0.61 & $30.5 \%$ & $46.0 \%$ & & $>15.27$ & $>12.56$ & & \multirow[t]{3}{*}{$N / C$} \\
\hline & 0.56 & $28.0 \%$ & $46.0 \%$ & $-36.2 \%$ & $>15.27$ & $>14.63$ & flash & \\
\hline & 0.59 & $29.5 \%$ & $46.0 \%$ & & $>15.27$ & $>13.63$ & & \\
\hline \multirow{3}{*}{$\begin{array}{l}\text { MYLAR MO } \\
\text { C1 }\end{array}$} & 2.96 & $148.0 \%$ & $131.0 \%$ & & $>14.91$ & $>12.31$ & & \multirow[t]{3}{*}{$N / C$} \\
\hline & 2.86 & $143.0 \%$ & $131.0 \%$ & $5.5 \%$ & $>14.91$ & $>13.09$ & flash & \\
\hline & 2.47 & $123.5 \%$ & $131.0 \%$ & & $>14.91$ & $>13.21$ & & \\
\hline \multirow{3}{*}{$\begin{array}{l}\text { NO } 410 \\
\text { D1 }\end{array}$} & 0.68 & $17.0 \%$ & $17.0 \%$ & & 10.67 & 11.27 & & \multirow[t]{3}{*}{$N / C$} \\
\hline & 0.46 & $11.5 \%$ & $17.0 \%$ & $-11.8 \%$ & 10.67 & 9.92 & $0.9 \%$ & \\
\hline & 0.66 & $16.5 \%$ & $17.0 \%$ & & 10.67 & 11.12 & & \\
\hline \multirow{3}{*}{$\begin{array}{l}\text { NO MI } 418 \\
\text { E1 }\end{array}$} & 0.08 & $2.0 \%$ & $4.0 \%$ & & 10.23 & 8.47 & & \multirow[t]{3}{*}{$N / C$} \\
\hline & 0.09 & $2.3 \%$ & $4.0 \%$ & $-43.8 \%$ & 10.23 & 9.46 & $-14.5 \%$ & \\
\hline & 0.10 & $2.5 \%$ & $4.0 \%$ & & 10.23 & 8.31 & & \\
\hline \multirow{4}{*}{$\begin{array}{l}\text { MEL } 228 \\
F 1\end{array}$} & 3.24 & $162.0 \%$ & $160.0 \%$ & & $>14.22$ & $>13.03$ & & \multirow[t]{3}{*}{$\mathrm{N} / \mathrm{C}$} \\
\hline & 3.26 & $163.0 \%$ & $160.0 \%$ & $2.9 \%$ & $>14.22$ & $>11.73$ & flash & \\
\hline & 3.38 & $169.0 \%$ & $160.0 \%$ & & $>14.22$ & $>13.20$ & & \\
\hline & \multicolumn{7}{|c|}{ After 500 hour exposure plus 24 hour air bake @ $302^{\circ} \mathrm{F}\left(150^{\circ} \mathrm{C}\right.$} & C) \\
\hline \multirow{3}{*}{$\begin{array}{l}\mathrm{NO} / \mathrm{MY} / \mathrm{NO} \\
\mathrm{A} 2\end{array}$} & 0.11 & $2.8 \%$ & $20.0 \%$ & & $>18.97$ & $>14.26$ & & \multirow[t]{3}{*}{$N / C$} \\
\hline & 0.46 & $11.5 \%$ & $20.0 \%$ & $-42.5 \%$ & $>18.97$ & $>13.64$ & flash & \\
\hline & 0.46 & $11.5 \%$ & $20.0 \%$ & & $>18.97$ & $>14.31$ & & \\
\hline \multirow{3}{*}{$\begin{array}{l}\mathrm{DA} / \mathrm{MY} / \mathrm{DA} \\
\mathrm{B} 2\end{array}$} & 0.52 & $26.0 \%$ & $46.0 \%$ & & $\geq 15.27$ & $>13.18$ & & \multirow[t]{3}{*}{$\mathrm{N} / \mathrm{C}$} \\
\hline & 0.57 & $28.5 \%$ & $46.0 \%$ & $-40.2 \%$ & $>15.27$ & $>12.97$ & flash & \\
\hline & 0.56 & $280 \%$ & $46.0 \%$ & & $>15.27$ & $7>14.06$ & & \\
\hline \multirow{3}{*}{$\begin{array}{l}\text { MYLARMO } \\
\text { C2 }\end{array}$} & 2.82 & $141.0 \%$ & $131.0 \%$ & & $>14.91$ & $1>12.05$ & & \multirow[t]{3}{*}{$\sqrt{N / C}$} \\
\hline & 3.08 & $154.0 \%$ & $131.0 \%$ & $12.7 \%$ & $>14.91$ & $>10.62$ & flash & \\
\hline & 2.96 & $148.0 \%$ & $131.0 \%$ & & $>14.91$ & $1>11.47$ & & \\
\hline \multirow{3}{*}{$\begin{array}{l}\text { NOMEX 410 } \\
\text { D2 }\end{array}$} & 0.54 & $13.5 \%$ & $17.0 \%$ & & 10.67 & 9.64 & & \multirow[t]{3}{*}{$\sqrt{\mathrm{N} / \mathrm{C}}$} \\
\hline & 0.46 & $11.5 \%$ & $17.0 \%$ & $-23.5 \%$ & 10.67 & 10.04 & $-4.0 \%$ & \\
\hline & 0.56 & $14.0 \%$ & $17.0 \%$ & & 10.6 & $11 . n 1$ & & \\
\hline \multirow{3}{*}{$\begin{array}{l}\mathrm{NO} / \mathrm{MI} 418 \\
\mathrm{E2}\end{array}$} & 0.10 & $2.5 \%$ & $4.0 \%$ & & 10.23 & 9.90 & & \multirow[t]{3}{*}{$\sqrt{N / C}$} \\
\hline & 0.08 & $2.0 \%$ & $4.0 \%$ & $-47.9 \%$ & 10.23 & 8.46 & $-12.6 \%$ & \\
\hline & 0.07 & $1.8 \%$ & $4.0 \%$ & & 10.23 & 8.45 & & \\
\hline MEL 228 & 3.14 & $157.0 \%$ & $160.0 \%$ & & $>14.22$ & $2>13.09$ & & $\sqrt{N / C}$ \\
\hline F2 & 3.28 & $164.0 \%$ & $160.0 \%$ & $0.3 \%$ & $>14.22$ & $>13.46$ & 6 flash & \\
\hline & 3.21 & $160.5 \%$ & $160.0 \%$ & & $>14.22$ & $2>11.99$ & & \\
\hline
\end{tabular}


SLEEVING

\begin{tabular}{|c|c|c|c|c|}
\hline & \multicolumn{4}{|c|}{500 HRS IN Nitrogen @ 140 F } \\
\hline & WT & EXPWT & $\begin{array}{c}\% \\
\text { CHANGE }\end{array}$ & EXPVIS \\
\hline A 1 & 0.5893 & 0.5871 & $-0.37 \%$ & $\mathrm{~N} / \mathrm{C}$ \\
\hline & 0.4572 & 0.4577 & $0.11 \%$ & $\mathrm{~N} / \mathrm{C}$ \\
\hline$\overline{\mathrm{C} 1}$ & $0 . \overline{3972}$ & 0.3996 & $0.60 \%$ & $\mathrm{~N} / \mathrm{C}$ \\
\hline & Nitrogen - & 24 hrs@3 & $302 \mathrm{~F}$ & \\
\hline & 0.5430 & 0.5417 & $-0.24 \%$ & $\mathrm{~N} / \mathrm{C}$ \\
\hline & 0.4604 & 0.4608 & $0.09 \%$ & $\mathrm{~N} / \mathrm{C}$ \\
\hline & & & & \\
\hline C2 & 0.4039 & 0.4039 & $0.00 \%$ & Pockects where \\
\hline & & & & "see photo \\
\hline
\end{tabular}




\begin{tabular}{|c|c|c|c|c|c|c|c|c|c|}
\hline & $500 \mathrm{HRS}$ & IN Nitroge & n@140 F & & & & & & \\
\hline D & WT & EXPWT & $\begin{array}{c}\text { WT \% } \\
\text { CHANGE }\end{array}$ & $\begin{array}{l}\text { BREAK } \\
\text { LOAD } \\
\text { (AVE) }\end{array}$ & $\begin{array}{l}\text { BREAK } \\
\text { LOAD } \\
\text { EPP }\end{array}$ & $\begin{array}{c}\% \\
\text { CHANGE } \\
\text { BPK } \\
\text { LOAD }\end{array}$ & $\begin{array}{c}\text { STRETCH } \\
(\mathrm{INCH})\end{array}$ & $\%$ ELONG & EXPVIS \\
\hline$A 1$ & 1.4977 & 1.4967 & $-0.07 \%$ & 39.02 & 43.85 & & 0.04 & $2.00 \%$ & $\mathrm{~N} / \mathrm{C}$ \\
\hline Glass & & & & 39.02 & 37.65 & $9.56 \%$ & 0.05 & $2.50 \%$ & \\
\hline & & & & 39.02 & 46.75 & & 0.05 & $2.50 \%$ & \\
\hline $\mathrm{B1}$ & 0.6202 & 0.6232 & $0.48 \%$ & 56.12 & 55.75 & & 0.68 & $34.00 \%$ & $\mathrm{~N} / \mathrm{C}$ \\
\hline Polyester & & & & 56.12 & 60.00 & $5.40 \%$ & 0.57 & $28.50 \%$ & \\
\hline & & & & 56.12 & 61.70 & & 0.49 & $24.50 \%$ & \\
\hline & 1.4650 & 1.4702 & $0.35 \%$ & 88.50 & 42.55 & & 0.10 & $5.00 \%$ & $\mathrm{~N} / \mathrm{C}$ \\
\hline Permacel & & & & 88.50 & 79.48 & $.31 .44 \%$ & 0.10 & $5.00 \%$ & \\
\hline & & & & 88.50 & 60.00 & & 0.11 & $5.50 \%$ & \\
\hline & & $500 \mathrm{HRS}$ & in Nitroger & $\rightarrow 302 \mathrm{~F}$ & 24 Hins & & & & \\
\hline A2 & 1.3523 & 1.3512 & $-0.08 \%$ & $39.0 ?$ & 41.20 & & 0.05 & $2.50 \%$ & $\mathrm{~N} / \mathrm{C}$ \\
\hline Glass & & & & 39.02 & 28.65 & $-3.94 \%$ & 0.04 & $2.00 \%$ & \\
\hline & & & & 39.02 & 42.60 & & 0.05 & $2.50 \%$ & \\
\hline & 0.5681 & 0.5690 & $0.16 \%$ & 56.12 & 54.55 & & 0.49 & $24.50 \%$ & $\mathrm{~N} / \mathrm{C}$ \\
\hline Polyester & & & & 56.12 & 60.35 & $1.48 \%$ & 0.66 & $33.00 \%$ & \\
\hline & & & & 56.12 & 55.95 & & 0.57 & $28.50 \%$ & \\
\hline & 1.3383 & 1.3228 & $-1.16 \%$ & 88.50 & 109.50 & & 0.13 & $6.50 \%$ & Slightly \\
\hline Permacel & & & & 88.50 & 114.00 & $20.41 \%$ & 0.11 & $5.50 \%$ & darker in \\
\hline & & & & 88.50 & 96.20 & & 0.11 & $5.50 \%$ & color \\
\hline
\end{tabular}


TIE CORDS

\begin{tabular}{|c|c|c|c|c|c|c|c|c|c|}
\hline \multicolumn{10}{|c|}{500 HRS IN Nitrogen @ 140 F } \\
\hline ID & WT & EXPWT & $\begin{array}{c}\text { WT \% } \\
\text { CHANGE }\end{array}$ & $\begin{array}{l}E X P \\
V I S\end{array}$ & $\begin{array}{l}\text { BREAK } \\
\text { LOAD } \\
\text { (AVE) }\end{array}$ & $\begin{array}{l}\text { BREAK } \\
\text { LOAD } \\
\text { EXP } \\
(\operatorname{av} \theta)\end{array}$ & $\begin{array}{c}\% \\
\text { CHANGE } \\
\text { BRK } \\
\text { LOAD }\end{array}$ & $\begin{array}{c}\text { STRETCH } \\
\text { (Inch) }\end{array}$ & $\begin{array}{c}\% \\
\text { ELONG }\end{array}$ \\
\hline \multirow[t]{4}{*}{ A1 } & \multirow[t]{3}{*}{0.2686} & \multirow[t]{3}{*}{0.27} & \multirow[t]{3}{*}{$0.52 \%$} & $\mathrm{~N} / \mathrm{C}$ & 28.36 & 33.15 & & 0.24 & $12.0 \%$ \\
\hline & & & & & 28.36 & 36.30 & \multirow{2}{*}{$20.00 \%$} & 0.27 & $13.5 \%$ \\
\hline & & & & & 28.36 & 32.65 & & 0.24 & $12.0 \%$ \\
\hline & \multicolumn{9}{|c|}{500 HRS IN Nitrogen -> 24 HRS @ 302F } \\
\hline \multirow[t]{3}{*}{$\mathrm{A} 2$} & \multirow[t]{3}{*}{0.2794} & \multirow[t]{3}{*}{0.2801} & \multirow[t]{3}{*}{$0.251 \%$} & $\mathrm{~N} / \mathrm{C}$ & 28.36 & 37.95 & & 0.43 & $21.5 \%$ \\
\hline & & & & & 28.36 & 37.35 & $29.29 \%$ & 0.55 & $27.5 \%$ \\
\hline & & & & & 28.36 & 34.70 & & 0.40 & $20.0 \%$ \\
\hline
\end{tabular}




\begin{tabular}{|c|c|c|c|c|c|c|c|}
\hline & 500 HRS IN I & trogen@1 & $40 \mathrm{~F}$ & & & & \\
\hline $\mathbb{1 D}$ & WT & EXPWT & $\begin{array}{c}\text { WT \% } \\
\text { CHANGE }\end{array}$ & EXP VIS & $\begin{array}{c}\text { BASE DIE } \\
(A \vee E)\end{array}$ & EXPDIE & $\begin{array}{c}\text { DIE\% } \\
\text { CHANGE }\end{array}$ \\
\hline DMAD & 3.9798 & 3.9775 & $-0.06 \%$ & $\mathrm{~N} / \mathrm{C}$ & 9.61 & 9.00 & \\
\hline$A 1$ & & & & & 9.61 & 9.69 & $-0.28 \%$ \\
\hline & & & & & 9.61 & 10.06 & \\
\hline DTMD & 4.2369 & 4.2366 & $-0.01 \%$ & $N / C$ & 9.95 & 9.57 & \\
\hline 31 & & & & & 9.95 & 9.11 & $-1.21 \%$ \\
\hline & & & & & 9.95 & 10.81 & \\
\hline & $\rightarrow 24$ HRS & D $302 F$ & & & & & \\
\hline DMD & 4.1012 & 4.0980 & $-0.08 \%$ & $\mathrm{~N} / \mathrm{C}$ & 9.61 & 9.10 & \\
\hline$\overline{A 2}$ & & & & & 9.61 & 9.61 & $-0.73 \%$ \\
\hline & & & & & 9.61 & 9.91 & \\
\hline DTMD & 4.2288 & 4.2271 & $-0.04 \%$ & $\mathrm{~N} / \mathrm{C}$ & 9.95 & 7.93 & \\
\hline B2 & & & & & 9.95 & 10.01 & $-7.47 \%$ \\
\hline & & & & & 9.95 & 9.68 & \\
\hline
\end{tabular}




\section{Appendix B}

Experimental Data for Nitrogen Exposure at $90^{\circ} \mathrm{C}\left(194^{\circ} \mathrm{F}\right)$ 


\begin{tabular}{|c|c|c|c|c|c|c|c|c|c|c|}
\hline 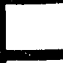 & \multicolumn{3}{|c|}{500 HRS IN Nitrogen@194 F } & \multirow[b]{2}{*}{ EXP VIS } & \multirow[b]{2}{*}{$\begin{array}{c}\text { BASE BRN } \\
\text { OUT } \\
(A \vee E)\end{array}$} & \multirow[b]{2}{*}{$\begin{array}{c}\text { EXP BRN } \\
\text { OUT }\end{array}$} & \multirow[b]{2}{*}{$\begin{array}{c}\text { BRN OUTT } \\
\% \\
\text { CHANGE }\end{array}$} & \multirow[b]{2}{*}{$\begin{array}{c}\text { BASE DIE } \\
\text { (AVE) }\end{array}$} & \multirow[b]{2}{*}{ EXPDIE } & \multirow[b]{2}{*}{$\begin{array}{c}\text { DIE \% } \\
\text { CHANGE }\end{array}$} \\
\hline ID & WT & EXPWT & $\begin{array}{c}\text { WT \% } \\
\text { CHANGE }\end{array}$ & & & & & & & \\
\hline \multirow[t]{3}{*}{$\bar{A} 1$} & \multirow[t]{3}{*}{25.0173} & \multirow[t]{3}{*}{25.0131} & \multirow[t]{3}{*}{$-0.017 \%$} & $\mathrm{~N} / \mathrm{C}$ & 576 & 561 & & 15.80 & 15.07 & \\
\hline & & & & & 576 & 563 & $-2.3 \%$ & 15.80 & 14.51 & $-7.9 \%$ \\
\hline & & & & & 576 & 565 & & 15.80 & 14.09 & \\
\hline \multirow[t]{3}{*}{ B1 } & \multirow[t]{3}{*}{23.8352} & \multirow[t]{3}{*}{23.8317} & \multirow[t]{3}{*}{$-0.015 \%$} & $\mathrm{~N} / \mathrm{C}$ & 736 & 729 & & 11.62 & 12.10 & \\
\hline & & & & & 736 & 721 & $-1.3 \%$ & 11.62 & 12.07 & $5.1 \%$ \\
\hline & & & & & 736 & 730 & & 11.62 & 12.46 & \\
\hline \multirow[t]{4}{*}{$\overline{C 1}$} & \multirow[t]{3}{*}{24.4929} & \multirow[t]{3}{*}{24.4932} & \multirow[t]{3}{*}{$0.001 \%$} & $\mathrm{~N} / \mathrm{C}$ & 579 & 589 & & 16.58 & 17.25 & \\
\hline & & & & & 579 & 582 & $0.5 \%$ & 16.58 & 18.15 & $6.2 \%$ \\
\hline & & & & & 579 & 575 & & 16.58 & 17.41 & \\
\hline & \multicolumn{10}{|c|}{ Nitrogen $\cdots>24$ hours at $302^{\circ} \mathrm{F}\left(150^{\circ} \mathrm{C}\right)$} \\
\hline \multirow[t]{3}{*}{ A2 } & \multirow[t]{3}{*}{25.519} & \multirow[t]{3}{*}{25.4979} & \multirow[t]{3}{*}{$-0.083 \%$} & $\mathrm{~N} / \mathrm{C}$ & 576 & 570 & & 15.80 & 15.34 & \\
\hline & & & & & 576 & 540 & $-3.0 \%$ & 15.80 & 10.06 & $-14.2 \%$ \\
\hline & & & & & 576 & 566 & & 15.80 & 15.29 & \\
\hline \multirow[t]{3}{*}{$B 2$} & \multirow[t]{3}{*}{22.5205} & \multirow[t]{3}{*}{22.5086} & \multirow[t]{3}{*}{$-0.053 \%$} & $\mathrm{~N} / \mathrm{C}$ & 736 & 733 & & 11.62 & 11.81 & \\
\hline & & & & & 736 & 734 & $-0.4 \%$ & 11.62 & 12.56 & $5.5 \%$ \\
\hline & & & & & 736 & 733 & & 11.62 & 12.39 & \\
\hline \multirow[t]{3}{*}{$\mathrm{C2}$} & \multirow[t]{3}{*}{24.5618} & \multirow[t]{3}{*}{24.5611} & $-0.003 \%$ & $\mathrm{~N} / \mathrm{C}$ & 547 & 450 & & 16.58 & 12.27 & \\
\hline & & & & & 591 & 337 & $-29.8 \%$ & 16.58 & 17.69 & $-5.1 \%$ \\
\hline & & & & & 581 & 457 & & 16.58 & 17.25 & \\
\hline
\end{tabular}


500 HOURS IN Nitrogen @ 194 F

\begin{tabular}{|c|c|c|c|c|c|c|c|c|c|c|c|}
\hline & VARN & $\begin{array}{l}\text { TWISTED } \\
\text { PAIR WT }\end{array}$ & $\begin{array}{l}\text { Exp Pair } \\
\text { weight }\end{array}$ & $\begin{array}{c}\text { WT \% } \\
\text { CHANGE }\end{array}$ & $\begin{array}{l}\text { EXP } \\
\text { VIS }\end{array}$ & BASE DIE & EXP DIE & $\begin{array}{c}\text { DIE \% } \\
\text { CHANGE }\end{array}$ & $\begin{array}{c}\text { BASE } \\
\text { BURN } \\
\text { OUT }\end{array}$ & $\begin{array}{c}\text { EXP } \\
\text { BURN } \\
\text { OUT }\end{array}$ & $\begin{array}{c}\text { BRNOUT } \\
\% \\
\text { CHANGE }\end{array}$ \\
\hline \multirow{3}{*}{$\begin{array}{l}P \\
0 \\
L\end{array}$} & $U-475$ & 22.6684 & 22.6703 & $0.008 \%$ & $\mathrm{~N} / \mathrm{C}$ & 16.24 & 20.00 & & 430 & 333 & \\
\hline & A1 & & & & & 16.24 & 17.78 & $11.12 \%$ & 430 & 310 & $-24.73 \%$ \\
\hline & & & & & & 16.24 & 16.36 & & 430 & 328 & \\
\hline \multirow{3}{*}{$\begin{array}{l}Y \\
E \\
S\end{array}$} & $Y-390$ & 22.8231 & 22.8185 & $-0.020 \%$ & $\mathrm{~N} / \mathrm{C}$ & 18.77 & 20.00 & & 510 & 500 & \\
\hline & B1 & & & & & 18.77 & 15.64 & $-1.19 \%$ & 510 & 510 & $-0.52 \%$ \\
\hline & & & & & & 18.77 & 20.00 & & 510 & 512 & \\
\hline \multirow{4}{*}{$\begin{array}{l}T \\
E \\
R\end{array}$} & ER-610 & 21.7013 & 21.7000 & $-0.006 \%$ & $\mathrm{~N} / \mathrm{C}$ & 15.57 & 17.00 & & 442 & 429 & \\
\hline & & & & & & 15.57 & 15.67 & $2.76 \%$ & 442 & 416 & $-5.58 \%$ \\
\hline & & & & & & 15.57 & 15.33 & & 442 & 407 & \\
\hline & $Y-833$ & 23.0665 & 23.0643 & $-0.010 \%$ & $\mathrm{~N} / \mathrm{C}$ & 12.04 & 10.33 & & 578 & 567 & \\
\hline \multirow{2}{*}{$\begin{array}{l}P \\
O\end{array}$} & D1 & & & & & 12.04 & 12.27 & $-7.23 \%$ & 578 & 533 & $-5.42 \%$ \\
\hline & & & & & & 12.04 & 10.91 & & 578 & 540 & \\
\hline \multirow{3}{*}{$\begin{array}{l}\mathrm{L} \\
\mathrm{Y} \\
\mathrm{A}\end{array}$} & 923 & 23.0186 & 23.0192 & $0.003 \%$ & $N / C$ & 16.76 & 5.94 & & 606 & 465 & \\
\hline & $E 1$ & & & & & 16.76 & broke & $-22.61 \%$ & 606 & 544 & $-17.60 \%$ \\
\hline & & & & & & 16.76 & 20.00 & & 606 & 489 & \\
\hline \multirow{3}{*}{$\begin{array}{l}M \\
1 \\
D\end{array}$} & ISO-800 & 21.6766 & 21.6741 & $-0.012 \%$ & $\mathrm{~N} / \mathrm{C}$ & 19.08 & 20.00 & & 580 & 508 & \\
\hline & $\mathrm{F} 1$ & & & & & 19.08 & 8.89 & $-26.73 \%$ & 580 & 480 & $-10.40 \%$ \\
\hline & & & & & & 19.08 & 13.05 & & 580 & 571 & \\
\hline \multirow[t]{2}{*}{$E$} & & 24 HOUR & S AT $302 \mathrm{~F}$ & & & & & & & & \\
\hline & $U-475$ & 22.4152 & 22.4114 & $-0.017 \%$ & $\mathrm{~N} / \mathrm{C}$ & 16.24 & 16.32 & & 430 & 379 & \\
\hline \multirow{2}{*}{$\begin{array}{l}1 \\
M\end{array}$} & $A 2$ & & & & & 16.24 & 16.54 & $8.50 \%$ & 430 & 370 & $-12.64 \%$ \\
\hline & & & & & & 16.24 & 20.00 & & 430 & 378 & \\
\hline \multirow{3}{*}{$\begin{array}{l}1 \\
D \\
E\end{array}$} & \multirow{6}{*}{$\begin{array}{l}Y-390 \\
B 2 \\
E R-610 \\
C 2\end{array}$} & 22.8138 & 22.8077 & $-0.027 \%$ & $\mathrm{~N} / \mathrm{C}$ & 18.77 & 20.00 & & 510 & 545 & \\
\hline & & & & & & 18.77 & 18.50 & $-11.15 \%$ & 510 & 488 & $9.15 \%$ \\
\hline & & & & & & 18.77 & 11.53 & & 510 & 637 & \\
\hline & & 21.6518 & 21.6522 & $0.002 \%$ & $N / C$ & 15.57 & 17.14 & & 442 & 380 & \\
\hline & & & & & & 15.57 & 16.89 & $10.17 \%$ & 442 & 392 & $-12.22 \%$ \\
\hline & & & & & & 15.57 & 17.43 & & 442 & 392 & \\
\hline \multirow{3}{*}{\multicolumn{2}{|c|}{$\begin{array}{l}Y-833 \\
D 2\end{array}$}} & 23.2459 & 23.2422 & $-0.016 \%$ & $\mathrm{~N} / \mathrm{C}$ & 12.04 & 11.33 & & 578 & 466 & \\
\hline & & & & & & 12.04 & 11.57 & $-5.73 \%$ & 578 & 569 & $-9.69 \%$ \\
\hline & & & & & & 12.04 & 11.15 & & 578 & 531 & \\
\hline \multirow{3}{*}{\multicolumn{2}{|c|}{$\begin{array}{l}923 \\
E 2\end{array}$}} & 22.7316 & 22.7253 & $-0.028 \%$ & $\mathrm{~N} / \mathrm{C}$ & 16.76 & 16.95 & & 606 & 496 & \\
\hline & & & & & & 16.76 & 13.71 & $.15 .41 \%$ & 606 & 406 & $-24.15 \%$ \\
\hline & & & & & & 16.76 & 11.87 & & 606 & 477 & \\
\hline \multirow{3}{*}{\multicolumn{2}{|c|}{$\begin{array}{l}\text { ISO-800 } \\
\text { F2 }\end{array}$}} & 21.7101 & 21.7050 & $-0.023 \%$ & $\mathrm{~N} / \mathrm{C}$ & 19.08 & 20.00 & & 580 & 609 & \\
\hline & & & & & & $\frac{19.08}{1908}$ & $\frac{14.02}{18.89}$ & $-7.56 \%$ & 580 & $\frac{559}{570}$ & $-0.11 \%$ \\
\hline & & & & & & 19.08 & 18.89 & & 580 & 570 & \\
\hline
\end{tabular}




\begin{tabular}{|c|c|c|c|c|c|c|c|c|c|c|c|}
\hline \multicolumn{12}{|c|}{500 HOURS IN Nitrogen @ 194 ${ }^{\circ} \mathrm{F}$} \\
\hline & VARN & $\begin{array}{l}\text { TWISTED } \\
\text { PAIR WT }\end{array}$ & $\begin{array}{c}\text { EXPT } \\
\text { PAIR WT }\end{array}$ & $\begin{array}{c}\text { WT \% } \\
\text { CHANGE }\end{array}$ & $\begin{array}{l}\text { EXP } \\
\text { VIS }\end{array}$ & $\begin{array}{c}\text { BASE } \\
\text { DIE }\end{array}$ & $\begin{array}{l}\text { EXP } \\
\text { DIE }\end{array}$ & $\begin{array}{c}\text { DIE \% } \\
\text { CHANGE }\end{array}$ & $\begin{array}{l}\text { BASE } \\
\text { BURN } \\
\text { OUT }\end{array}$ & \begin{tabular}{|l} 
EXP \\
BURN \\
OUT
\end{tabular} & $\begin{array}{c}\text { BRNOUT } \\
\% \\
\text { CHANGE }\end{array}$ \\
\hline \multirow{3}{*}{$\begin{array}{l}P \\
O \\
L\end{array}$} & \multirow{3}{*}{$\begin{array}{l}\text { U-475 } \\
\text { A } 1\end{array}$} & 27.0452 & 27.0473 & $0.01 \%$ & $\mathrm{~N} / \mathrm{C}$ & 13.32 & 12.92 & & 746 & 730 & \\
\hline & & & & & & 13.32 & 13.27 & $-0.85 \%$ & 746 & 731 & $-2.01 \%$ \\
\hline & & & & & & 13.32 & 13.43 & & 746 & 732 & \\
\hline$Y$ & \multirow{3}{*}{$\begin{array}{l}Y-390 \\
B 1\end{array}$} & 23.3779 & 23.3763 & $-0.01 \%$ & $\mathrm{~N} / \mathrm{C}$ & 12.28 & 12.40 & & $\overline{755}$ & 742 & \\
\hline E & & & & & & 12.28 & 10.31 & $-6.51 \%$ & 755 & 747 & $-1.81 \%$ \\
\hline$s$ & & & & & & 12.28 & 11.73 & & 755 & 735 & \\
\hline \multirow{4}{*}{$\begin{array}{l}T \\
E \\
R\end{array}$} & \multirow{3}{*}{$\begin{array}{l}\text { ER-610 } \\
\text { C1 }\end{array}$} & 20.2273 & 20.2199 & $.0 .04 \%$ & $\mathrm{~N} / \mathrm{C}$ & 12.73 & 11.96 & & 734 & 732 & \\
\hline & & & & & & 12.73 & 11.71 & $-7.03 \%$ & 734 & 732 & $-0.27 \%$ \\
\hline & & & & & & 12.73 & broke & & 734 & 732 & \\
\hline & \multirow{3}{*}{$\begin{array}{l}Y-833 \\
D 1\end{array}$} & 21.3295 & 21.3282 & $-0.01 \%$ & $\mathrm{~N} / \mathrm{C}$ & 12.49 & 12.12 & & 734 & 713 & \\
\hline \multirow{2}{*}{$\begin{array}{l}P \\
O\end{array}$} & & & & & & 12.49 & 10.77 & $-6.06 \%$ & 734 & 725 & $-1.73 \%$ \\
\hline & & & & & & 12.49 & 12.31 & & 734 & 726 & \\
\hline \multirow{3}{*}{$\begin{array}{l}L \\
Y \\
A\end{array}$} & \multirow{3}{*}{$\begin{array}{l}923 \\
E 1\end{array}$} & 26.2462 & 26.2433 & $-0.01 \%$ & $\mathrm{~N} / \mathrm{C}$ & 14.38 & 11.74 & & 742 & 733 & \\
\hline & & & & & & 14.38 & 11.57 & $-19.52 \%$ & 742 & 747 & $-0.72 \%$ \\
\hline & & & & & & 14.38 & 11.41 & & 742 & 730 & \\
\hline \multirow{3}{*}{$\begin{array}{l}M \\
1 \\
D\end{array}$} & \multirow{3}{*}{$\begin{array}{l}150-800 \\
F 1\end{array}$} & 29.1508 & 29.1528 & $0.01 \%$ & $\mathrm{~N} / \mathrm{C}$ & 12.29 & 10.26 & & 747 & 747 & \\
\hline & & & & & & 12.29 & 12.87 & $-3.91 \%$ & 747 & 741 & $-0.71 \%$ \\
\hline & & & & & & 12.29 & 12.30 & & 747 & 737 & \\
\hline \multirow[t]{2}{*}{$E$} & \multirow[b]{2}{*}{$U-475$} & \multicolumn{10}{|c|}{ Nitrogen $->>24$ hours @ 302 F } \\
\hline & & 27.0249 & 27.0230 & $-0.01 \%$ & $\mathrm{~N} / \mathrm{C}$ & 13.32 & 12.79 & & 746 & 728 & \\
\hline \multirow{2}{*}{$\begin{array}{l}1 \\
M\end{array}$} & \multirow[t]{2}{*}{ A2 } & & & & & 13.32 & 9.76 & $-7.38 \%$ & 746 & 730 & $-2.32 \%$ \\
\hline & & & & & & 13.32 & 14.46 & & 746 & 728 & \\
\hline 1 & \multirow{3}{*}{$\begin{array}{l}Y-390 \\
B 2\end{array}$} & 23.2621 & 23.2573 & $-0.02 \%$ & $\mathrm{~N} / \mathrm{C}$ & 12.28 & 10.82 & & $7 \overline{755}$ & 736 & \\
\hline$D$ & & & & & & 12.28 & 13.09 & $-0.65 \%$ & 755 & 731 & $-2.85 \%$ \\
\hline $\begin{array}{l}\text { I } \\
D \\
E\end{array}$ & & & & & & 12.28 & 12.69 & & 755 & 736 & \\
\hline \multirow{3}{*}{\multicolumn{2}{|c|}{$\begin{array}{l}\text { ER-610 } \\
\text { C2 }\end{array}$}} & 23.5619 & 23.5703 & $0.04 \%$ & $\mathrm{~N} / \mathrm{C}$ & 12.73 & 10.96 & & 734 & 652 & \\
\hline & & & & & & 12.73 & 11.51 & $1.86 \%$ & 734 & 729 & $-5.93 \%$ \\
\hline $\mathrm{P}$ & & & & & & 12.73 & 16.43 & & 734 & lost & \\
\hline \multirow{4}{*}{$\begin{array}{l}0 \\
X \\
y\end{array}$} & \multirow{3}{*}{$\begin{array}{l}Y-833 \\
D 2\end{array}$} & 21.4654 & 21.4615 & $-0.02 \%$ & $\mathrm{~N} / \mathrm{C}$ & 12.49 & 11.11 & & 734 & 698 & \\
\hline & & & & & & 12.49 & 11.41 & $-6.62 \%$ & 734 & 728 & $-2.72 \%$ \\
\hline & & & & & & 12.49 & 12.47 & & 734 & 716 & \\
\hline & \multirow{3}{*}{$\begin{array}{l}923 \\
E 2\end{array}$} & 26.7630 & 26.7555 & $-0.03 \%$ & $\mathrm{~N} / \mathrm{C}$ & 14.38 & 20.00 & & 742 & 736 & \\
\hline$G$ & & & & & & 14.38 & 20.00 & $34.33 \%$ & 742 & 741 & $-0.81 \%$ \\
\hline$L$ & & & & & & 14.38 & 17.95 & & 742 & 727 & \\
\hline \multirow{3}{*}{$\begin{array}{l}A \\
S \\
S\end{array}$} & $150-800$ & 28.1556 & 28.1523 & $-0.01 \%$ & & 12.29 & 12.85 & & 747 & 737 & \\
\hline & $F 2$ & & & & $N / C$ & 12.29 & 10.52 & $-1.93 \%$ & 747 & 742 & $-1.00 \%$ \\
\hline & & & & & & 12.29 & 12.79 & & 747 & 730 & \\
\hline
\end{tabular}




\begin{tabular}{|c|c|c|c|c|c|c|c|c|c|c|c|}
\hline & VARN & $\begin{array}{l}\text { TWISTED } \\
\text { PAIR WT }\end{array}$ & $\begin{array}{c}\text { EXP T } \\
\text { PAIR WT }\end{array}$ & $\begin{array}{c}\text { WT \% } \\
\text { CHANGE }\end{array}$ & $\begin{array}{l}\text { EXP } \\
\text { VIS }\end{array}$ & $\begin{array}{c}\text { BASE } \\
\text { DIE }\end{array}$ & $\begin{array}{l}\text { EXP } \\
\text { DIE }\end{array}$ & $\begin{array}{c}\text { DIE \% } \\
\text { CHANGE }\end{array}$ & $\begin{array}{l}\text { BASE } \\
\text { BUPN } \\
\text { OUT }\end{array}$ & $\begin{array}{c}\text { EXP } \\
\text { BURN } \\
\text { OUT }\end{array}$ & $\begin{array}{c}\text { BRNOUT } \\
\% \\
\text { CHANGE }\end{array}$ \\
\hline & \multirow{3}{*}{$\begin{array}{l}U-475 \\
\text { A } 1\end{array}$} & \multirow[t]{3}{*}{23.0730} & \multirow[t]{3}{*}{23.0658} & \multirow[t]{3}{*}{$-0.031 \%$} & $\mathrm{~N} / \mathrm{C}$ & 15.10 & 12.40 & & 469 & 539 & \\
\hline \multirow{2}{*}{$\begin{array}{l}P \\
O \\
L\end{array}$} & & & & & & 15.10 & 10.11 & $-19.82 \%$ & 469 & 540 & $18.69 \%$ \\
\hline & & & & & & 15.10 & 13.81 & & \begin{tabular}{|l|}
469 \\
\end{tabular} & 591 & \\
\hline \multirow{3}{*}{$\begin{array}{l}Y \\
E \\
S\end{array}$} & \multirow{3}{*}{$\begin{array}{l}\bar{Y}-390 \\
B 1\end{array}$} & \multirow[t]{3}{*}{21.8142} & \multirow[t]{3}{*}{21.8014} & \multirow[t]{3}{*}{$-0.059 \%$} & $\mathrm{~N} / \mathrm{C}$ & 18.24 & broke & & 473 & 509 & \\
\hline & & & & & & 18.24 & 12.41 & $-23.27 \%$ & \begin{tabular}{|l|}
473 \\
\end{tabular} & 427 & $-2.33 \%$ \\
\hline & & & & & & 18.24 & 15.58 & & 473 & 450 & \\
\hline \multirow{3}{*}{$\begin{array}{l}T \\
E \\
R\end{array}$} & ER-610 & \multirow[t]{3}{*}{22.1527} & \multirow[t]{3}{*}{22.1880} & \multirow[t]{3}{*}{$0.159 \%$} & $\mathrm{~N} / \mathrm{C}$ & 14.53 & 15.46 & & 494 & 401 & \\
\hline & \multirow[t]{2}{*}{ C1 } & & & & & 14.53 & 14.33 & $3.10 \%$ & 494 & 449 & $-11.40 \%$ \\
\hline & & & & & & 14.53 & 15.15 & & 494 & 463 & \\
\hline & \multirow{3}{*}{$\begin{array}{l}\mathrm{Y}-833 \\
\mathrm{D} 1\end{array}$} & \multirow[t]{3}{*}{25.3475} & \multirow[t]{3}{*}{25.3368} & \multirow[t]{3}{*}{$-0.042 \%$} & $\mathrm{~N} / \mathrm{C}$ & 11.38 & 14.46 & & 557 & 308 & \\
\hline & & & & & & 11.38 & 16.30 & $44.02 \%$ & 557 & 346 & $-42.01 \%$ \\
\hline$M$ & & & & & & 11.38 & 18.41 & & 557 & 315 & \\
\hline \multirow{3}{*}{$\begin{array}{l}D \\
E\end{array}$} & 923 & \multirow[t]{3}{*}{25.0774} & \multirow[t]{3}{*}{25.0643} & \multirow[t]{3}{*}{$-0.052 \%$} & $\mathrm{~N} / \mathrm{C}$ & 15.85 & 20.00 & & 503 & 443 & \\
\hline & E1 & & & & & 15.85 & 20.00 & $20.74 \%$ & 503 & 420 & $-13.65 \%$ \\
\hline & & & & & & 15.85 & 17.41 & & 503 & 440 & \\
\hline & $1 S O-800$ & 22.0673 & 22.0612 & $-0.028 \%$ & $\mathrm{~N} / \mathrm{C}$ & 14.75 & 15.80 & & 632 & \begin{tabular}{|l|l|}
576 \\
\end{tabular} & \\
\hline$P$ & $F 1$ & & & & & 14.75 & 14.62 & $-10.96 \%$ & 632 & 593 & $.9 .07 \%$ \\
\hline 0 & & & & & & 14.75 & 8.98 & & 632 & 555 & \\
\hline L & & Nitrogen & $\rightarrow 24$ hour & s@302 & $\mathrm{F}$ & & & & & & \\
\hline Y & U-475 & 24.0089 & 24.0039 & $-0.021 \%$ & $\mathrm{~N} / \mathrm{C}$ & 15.10 & 10.72 & & 469 & 516 & \\
\hline A & $A 2$ & & & & & 15.10 & 10.72 & $-28.57 \%$ & 469 & 579 & $10.23 \%$ \\
\hline$M$ & & & & & & 15.10 & 10.92 & & 469 & \begin{tabular}{l|l}
456 \\
\end{tabular} & \\
\hline 1 & $Y-390$ & 21.9070 & 21.8924 & $-0.067 \%$ & $\mathrm{~N} / \mathrm{C}$ & 18.24 & 8.21 & & 473 & 620 & \\
\hline$D$ & $\mathrm{~B} 2$ & & & & & 18.24 & 19.84 & $-5.40 \%$ & 473 & 426 & $9.30 \%$ \\
\hline$E$ & & & & & & 18.24 & 14.67 & & 473 & 505 & \\
\hline & ER-610 & 22.0712 & 22.0632 & $-0.036 \%$ & $\mathrm{~N} / \mathrm{C}$ & 14.53 & 15.61 & & 494 & 379 & \\
\hline ' & $\mathrm{C} 2$ & & & & & 14.53 & 16.45 & $5.12 \%$ & 494 & 409 & $20.58 \%$ \\
\hline$M$ & & & & & & 14.53 & 13.76 & & 494 & 389 & \\
\hline 1 & $Y-833$ & 25.0422 & 24.9320 & $-0.440 \%$ & $\mathrm{~N} / \mathrm{C}$ & 11.38 & 16.39 & & 557 & \begin{tabular}{|l|}
369 \\
\end{tabular} & \\
\hline 0 & D2 & & & & & 11.38 & 17.45 & $36.58 \%$ & 557 & \begin{tabular}{|l|}
332 \\
\end{tabular} & $-35.85 \%$ \\
\hline$E$ & & & & & & 11.38 & 12.79 & & 557 & 371 & \\
\hline & 923 & 25.7509 & 25.7329 & $-0.070 \%$ & $\mathrm{~N} / \mathrm{C}$ & 15.85 & 16.95 & & 503 & 551 & \\
\hline & E2 & & & & & 15.85 & 13.71 & $-10.68 \%$ & 503 & 441 & $-4.84 \%$ \\
\hline & & & & & & 15.85 & 11.81 & & 503 & 444 & \\
\hline & ISO-800 & 22.0732 & 22.0619 & $-0.051 \%$ & $\mathrm{~N} / \mathrm{C}$ & 14.75 & broke & & 632 & 594 & \\
\hline & $\mathrm{F} 2$ & & & & & 14.75 & 13.27 & $-4.41 \%$ & 632 & \begin{tabular}{|l|}
574 \\
\end{tabular} & $-8.18 \%$ \\
\hline & & & & & & 14.75 & 14.93 & & 632 & 573 & \\
\hline
\end{tabular}




\section{7}

\begin{tabular}{|c|c|c|c|c|c|c|c|c|}
\hline & & $500 \mathrm{HRS}$ & N Nitrogen & @ 194. $\mathrm{F}$ & & & & \\
\hline & VARN & $\begin{array}{l}\text { COIL } \\
\text { WT }\end{array}$ & $\begin{array}{c}\text { EXP COIL } \\
W T\end{array}$ & $\begin{array}{c}\text { WT \% } \\
\text { CHANGE }\end{array}$ & EXPVIS & \begin{tabular}{|c|} 
BASE \\
BND STR \\
(AVE)
\end{tabular} & $\begin{array}{c}\text { EXP BND } \\
\text { STR }\end{array}$ & $\begin{array}{c}\text { BND STR } \\
\% \\
\text { CHANGE }\end{array}$ \\
\hline$P$ & U.475 & 40.1089 & 40.1165 & $0.019 \%$ & $N / C$ & 73.73 & 73.57 & \\
\hline 0 & A 1 & & & & & 73.73 & 58.95 & $-11.04 \%$ \\
\hline$L$ & & & & & & 73.73 & 64.25 & \\
\hline$Y$ & $Y-390$ & 39.0368 & 39.0439 & $0.018 \%$ & $\mathrm{~N} / \mathrm{C}$ & 43.78 & 48.47 & \\
\hline$E$ & B1 & & & & & 43.78 & 51.20 & $6.78 \%$ \\
\hline$S$ & & & & & & 43.78 & 40.57 & \\
\hline $\mathrm{T}$ & ER-610 & 40.2526 & 40.3003 & $0.119 \%$ & $\mathrm{~N} / \mathrm{C}$ & 51.81 & 66.32 & \\
\hline$E$ & & & & & & 51.81 & 54.35 & $10.45 \%$ \\
\hline$R$ & & & & & & 51.81 & 51.00 & \\
\hline & Y-833 & 38.4237 & 38.4234 & $-0.001 \%$ & $\mathrm{~N} / \mathrm{C}$ & 9.85 & 17.50 & \\
\hline$P$ & D1 & & & & & 9.85 & 19.92 & $188.22 \%$ \\
\hline $\mathrm{O}$ & & & & & & 9.85 & 47.75 & \\
\hline L & 923 & 38.9578 & 38.9568 & $-0.003 \%$ & $\mathrm{~N} / \mathrm{C}$ & 41.28 & 36.97 & \\
\hline Y & E1 & & & & & 41.28 & 33.32 & $0.16 \%$ \\
\hline$A$ & & & & & & 41.28 & 53.75 & \\
\hline$M$ & ISO-800 & 38.4408 & 38.4489 & $0.021 \%$ & $\mathrm{~N} / \mathrm{C}$ & 45.01 & 52.27 & \\
\hline 1 & F1 & & & & & 45.01 & 46.92 & $10.19 \%$ \\
\hline$D$ & & & & & & 45.01 & 36.52 & \\
\hline$E$ & & Nitrogen & $>24 \mathrm{HRS}$ & $302^{\circ} \mathrm{F}$ & & & & \\
\hline & U.475 & 40.3451 & 40.3410 & $-0.010 \%$ & $\mathrm{~N} / \mathrm{C}$ & 73.73 & 58.85 & \\
\hline 1 & $A 2$ & & & & & 73.73 & 60.50 & $-15.88 \%$ \\
\hline$M$ & & & & & & 73.73 & 63.55 & \\
\hline 1 & $Y-390$ & 38.9613 & 38.9589 & $-0.006 \%$ & $\mathrm{~N} / \mathrm{C}$ & 43.78 & 54.20 & \\
\hline D & B2 & & & & & 43.78 & 53.70 & $27.99 \%$ \\
\hline$E$ & & & & & & 43.78 & 60.20 & \\
\hline & ER-610 & 38.7011 & 38.6978 & $-0.009 \%$ & $\mathrm{~N} / \mathrm{C}$ & 51.81 & 63.45 & \\
\hline & $\mathrm{C} 2$ & & & & & 51.81 & 75.05 & $37.75 \%$ \\
\hline & & & & & & 51.81 & 75.60 & \\
\hline & Y-833 & 38.5377 & 38.5355 & $-0.006 \%$ & $\mathrm{~N} / \mathrm{C}$ & 9.85 & 64.85 & \\
\hline & D2 & & & & & 9.85 & 7.90 & $353.47 \%$ \\
\hline & & & & & & 9.85 & 61.25 & \\
\hline & 923 & 39.8244 & 39.8249 & $0.001 \%$ & $\mathrm{~N} / \mathrm{C}$ & 41.28 & 37.77 & \\
\hline & E2 & & & & & 41.28 & 41.12 & $7.93 \%$ \\
\hline & & & & & & 41.28 & 54.77 & \\
\hline & ISO-800 & 37.2253 & 37.2218 & $-0.009 \%$ & $\mathrm{~N} / \mathrm{C}$ & 45.01 & 56.62 & \\
\hline & F2 & & & & & 45.01 & 29.17 & $-1.44 \%$ \\
\hline & & & & & & 45.01 & 47.30 & \\
\hline
\end{tabular}


HELICAL COILSMIRE B---Nitrogen @ $194^{\circ} \mathrm{F}\left(90^{\circ} \mathrm{C}\right)$

\begin{tabular}{|c|c|c|c|c|c|c|c|c|}
\hline & & $500 \mathrm{HRS}$ & IN Nitroger & @ $194^{\circ} \mathrm{F}$ & & & & \\
\hline & VARN & $\begin{array}{l}\text { COIL } \\
\text { WT }\end{array}$ & $\begin{array}{c}\text { EXP COIL } \\
W T\end{array}$ & $\begin{array}{c}\text { WT \% } \\
\text { CHANGE }\end{array}$ & EXP VIS & $\begin{array}{c}\text { BASE } \\
\text { BND STR } \\
\text { (AVE) }\end{array}$ & $\begin{array}{c}\text { EXPBND } \\
\text { STR }\end{array}$ & $\begin{array}{c}\text { BND STR } \\
\% \\
\text { CHANGE }\end{array}$ \\
\hline$P$ & U.475 & 37.6309 & 37.6430 & $0.032 \%$ & $\mathrm{~N} / \mathrm{C}$ & 40.14 & 34.92 & \\
\hline o & A 1 & & & & & 40.14 & 34.60 & $-13.70 \%$ \\
\hline$L$ & & & & & & 40.14 & 34.40 & \\
\hline Y & $Y-390$ & 38.5257 & 38.5365 & $0.028 \%$ & $\mathrm{~N} / \mathrm{C}$ & 36.12 & 30.82 & \\
\hline$E$ & B1 & & & & & 36.12 & 39.25 & $-0.76 \%$ \\
\hline S & & & & & & 36.12 & $37.47 \mid$ & \\
\hline $\mathrm{T}$ & ER-610 & 37.4404 & 37.4485 & $0.022 \%$ & $\mathrm{~N} / \mathrm{C}$ & 35.96 & 29.80 & \\
\hline$E$ & C1 & & & & & 35.96 & 30.45 & $-17.58 \%$ \\
\hline $\mathrm{R}$ & & & & & & 35.96 & 28.67 & \\
\hline & $Y-833$ & 37.7714 & 37.7799 & $0.023 \%$ & $\mathrm{~N} / \mathrm{C}$ & 33.14 & 12.20 & \\
\hline$P$ & D1 & & & & & 33.14 & 29.87 & $-39.08 \%$ \\
\hline 0 & & & & & & 33.14 & 18.50 & \\
\hline$L$ & 923 & 38.3050 & 38.3152 & $0.027 \%$ & $\mathrm{~N} / \mathrm{C}$ & 40.52 & 29.15 & \\
\hline$Y$ & E1 & & & & & 40.52 & 31.50 & $-20.08 \%$ \\
\hline A & & & & & & 40.52 & 36.50 & \\
\hline$M$ & |SO-800 & 37.1400 & 37.1404 & $0.001 \%$ & $\mathrm{~N} / \mathrm{C}$ & 20.20 & 23.57 & \\
\hline 1 & F1 & & & & & 20.20 & 22.50 & $16.57 \%$ \\
\hline D & & & & & & 20.20 & 24.57 & \\
\hline$E$ & & Nitrogen - & $\rightarrow 24 \mathrm{HRS}$ & $302^{\circ} \mathrm{F}$ & & & & \\
\hline & U-475 & 36.7209 & 36.7184 & $-0.007 \%$ & $\mathrm{~N} / \mathrm{C}$ & 40.14 & 33.22 & \\
\hline 1 & A2 & & & & & 40.14 & 31.15 & $-22.36 \%$ \\
\hline$M$ & & & & & & 40.14 & 29.12 & \\
\hline 1 & Y-390 & 38.5696 & 38.5832 & $0.035 \%$ & $\mathrm{~N} / \mathrm{C}$ & 36.12 & 36.27 & \\
\hline$D$ & B2 & & & & & 36.12 & 35.85 & $-1.84 \%$ \\
\hline$E$ & & & & & & 36.12 & 34.25 & \\
\hline & ER-610 & 37.6121 & 37.6106 & $-0.004 \%$ & $\mathrm{~N} / \mathrm{C}$ & 35.96 & 35.37 & \\
\hline$E$ & C2 & & & & & 35.96 & 29.45 & $-7.64 \%$ \\
\hline$P$ & & & & & & 35.96 & 34.82 & \\
\hline 0 & $Y-833$ & 36.0136 & 36.0118 & $-0.005 \%$ & $N / C$ & 33.14 & 30.77 & \\
\hline$x$ & D2 & & & & & 33.14 & 9.00 & $-40.06 \%$ \\
\hline$Y$ & & & & & & 33.14 & 19.82 & \\
\hline & 923 & 38.1740 & 38.1714 & $-0.007 \%$ & $\mathrm{~N} / \mathrm{C}$ & 40.52 & 32.80 & \\
\hline$G$ & E2 & & & & & 40.52 & 28.10 & $-27.67 \%$ \\
\hline$L$ & & & & & & 40.52 & 27.02 & \\
\hline A & ISO-800 & 36.5539 & 36.5885 & $0.095 \%$ & $\mathrm{~N} / \mathrm{C}$ & 20.20 & 14.65 & \\
\hline$S$ & F2 & & & & & 20.20 & 19.60 & $-10.45 \%$ \\
\hline$S$ & & & & & & 20.20 & 20.02 & \\
\hline
\end{tabular}


HELICAL COILSMIRE C---Nitrogen @ 194 $\mathrm{F}\left(90^{\circ} \mathrm{C}\right)$

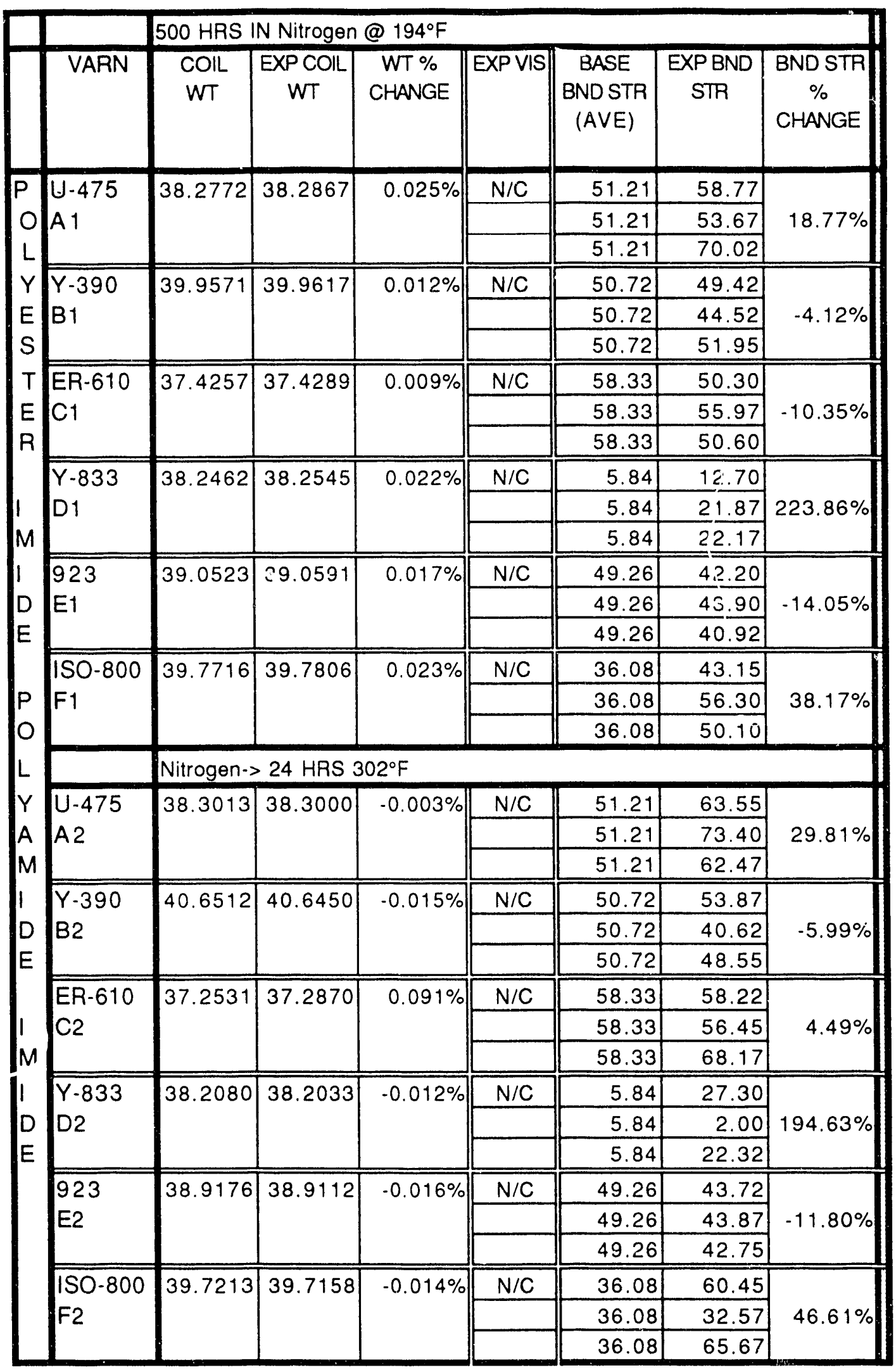


SINGLE MAG WIRE WITHOUT VARNISH--Nitrogen@ @ $194^{\circ} \mathrm{F}\left(90^{\circ} \mathrm{C}\right)$

\begin{tabular}{|c|c|c|c|c|c|}
\hline & 500 HRS IN & Vitrogen@ & $194^{\circ} \mathrm{F}$ & & \\
\hline (D) & $\overline{W T}$ & EXPWT & $\begin{array}{c}\text { WT \% } \\
\text { CHANGE }\end{array}$ & EXP VIS & EXPFLEX \\
\hline$A 1$ & 4.3772 & 4.3778 & $0.014 \%$ & $\mathrm{~N} / \mathrm{C}$ & \\
\hline & & & & & YES \\
\hline$B 1$ & 4.5737 & 4.5689 & $-0.105 \%$ & $\mathrm{~N} / \mathrm{C}$ & \\
\hline & & & & & YES \\
\hline$\overline{C 1}$ & 4.5382 & 4.5381 & $-0.002 \%$ & $\mathrm{~N} / \mathrm{C}$ & \\
\hline & & & & & YES \\
\hline & Nitrogen -> & $302^{\circ} \mathrm{F}$ for & 4 HRS & & \\
\hline$A 2$ & 4.3384 & 4.3374 & $-0.023 \%$ & $\mathrm{~N} / \mathrm{C}$ & \\
\hline & & & & & YES \\
\hline$\overline{B 2}$ & 4.4635 & 4.4621 & $-0.031 \%$ & $\mathrm{~N} / \mathrm{C}$ & \\
\hline & & & & & YES \\
\hline $\mathrm{C2}$ & 4.6678 & 4.6687 & $0.019 \%$ & $\overline{N / C}$ & \\
\hline & & & & & YES \\
\hline & & & & & \\
\hline
\end{tabular}




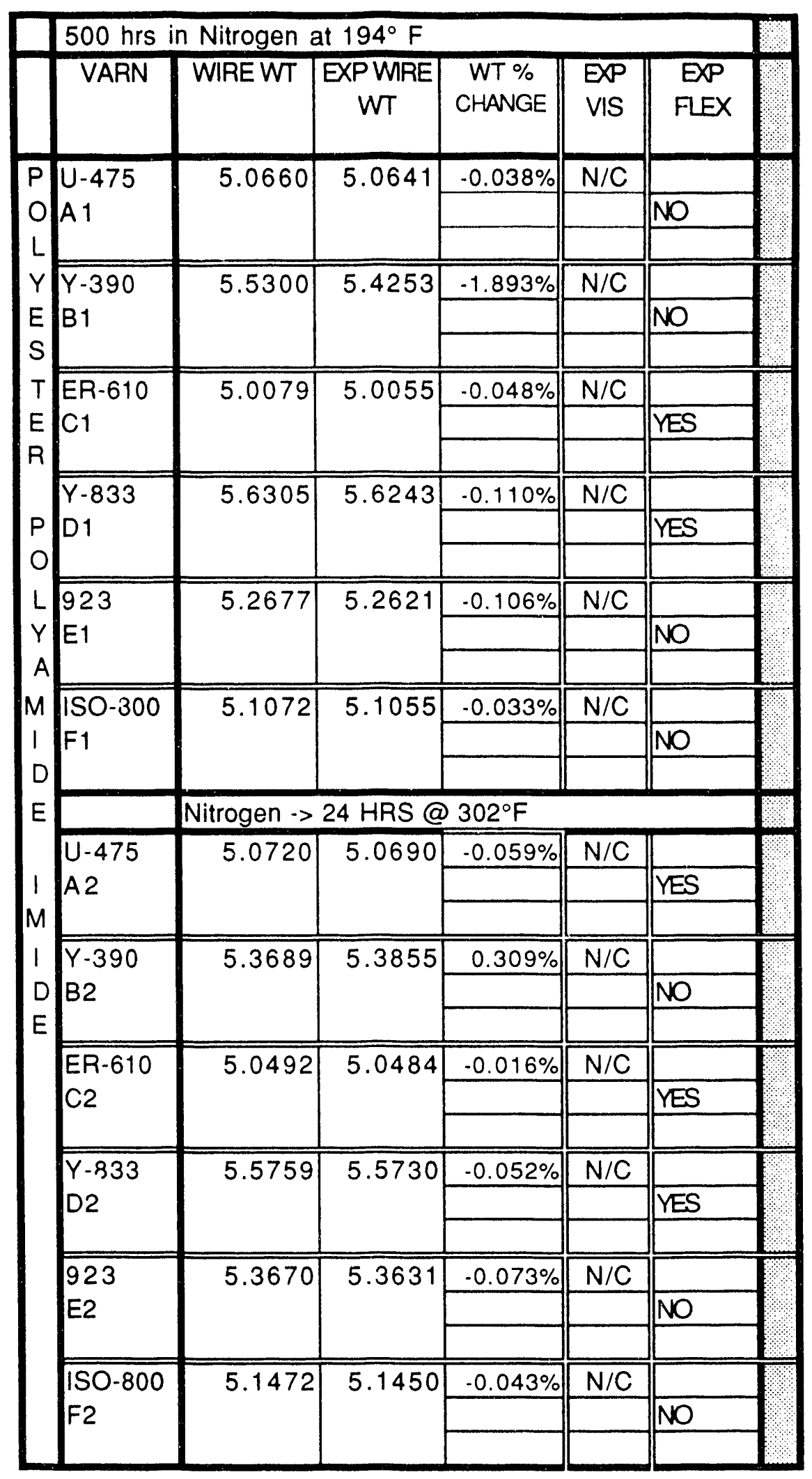




\begin{tabular}{|c|c|c|c|c|c|c|c|}
\hline & \multicolumn{6}{|c|}{ Nitrogen at $194 \mathrm{~F}$} & \\
\hline & VARN & WIRE WT & $\begin{array}{c}\text { EXPWRE } \\
W T\end{array}$ & $\begin{array}{l}\text { WT \% } \\
\text { CHANGE }\end{array}$ & EPPVIS: & $\begin{array}{l}\text { EXP } \\
\text { FLEX }\end{array}$ & \\
\hline \multirow{4}{*}{$\begin{array}{l}P \\
\\
O \\
L \\
V\end{array}$} & \multirow{3}{*}{\begin{tabular}{|l|}
-475 \\
$A 1$
\end{tabular}} & \multirow{3}{*}{5.5438} & \multirow{3}{*}{5.5422} & $-0.029 \%$ & $\mathrm{~N} / \mathrm{C}$ & & \\
\hline & & & & & & NO & \\
\hline & & & & & & & \\
\hline & \multirow{3}{*}{$\begin{array}{l}Y-390 \\
B 1\end{array}$} & \multirow[t]{3}{*}{ 5.5548 } & \multirow[t]{3}{*}{5.5899} & $0.632 \%$ & $\mathrm{~N} / \mathrm{C}$ & & \\
\hline \multirow{2}{*}{\begin{tabular}{l|l}
$Y$ & $Y$ \\
$E$ & $E$ \\
$S$ &
\end{tabular}} & & & & & & NO & \\
\hline & & & & & & & \\
\hline \multirow{3}{*}{$\begin{array}{l}T \\
E \\
R\end{array}$} & \multirow{2}{*}{$\begin{array}{l}\text { ER-610 } \\
\text { C1 }\end{array}$} & \multirow{2}{*}{4.9519} & \multirow{2}{*}{4.9487} & $-0.005 \%$ & 10 & YES & \\
\hline & & & & & & & \\
\hline & $\overline{Y-833}$ & \multirow[t]{3}{*}{4.5588} & \multirow[t]{3}{*}{4.5559} & $-0.064 \%$ & $\mathrm{~N} / \mathrm{C}$ & & \\
\hline \multirow{2}{*}{$\begin{array}{l}\bar{Y} \\
\\
\end{array}$} & \multirow{2}{*}{ D1 } & & & & & YES & \\
\hline & & & & & & & \\
\hline \multirow{3}{*}{\begin{tabular}{l|l}
$L$ & 9 \\
$Y$ & $E$ \\
$A$
\end{tabular}} & \multirow{3}{*}{$\begin{array}{l}923 \\
E 1\end{array}$} & \multirow[t]{3}{*}{4.5577} & \multirow[t]{3}{*}{5.2928} & $16.129 \%$ & $\mathrm{~N} / \mathrm{C}$ & & \\
\hline & & & & & & NO & \\
\hline & & & & & & & \\
\hline \multirow{3}{*}{$\begin{array}{c}M \\
1 \\
D\end{array}$} & \multirow{3}{*}{\begin{tabular}{|l} 
ISO-800 \\
$\mathrm{F} 1$
\end{tabular}} & \multirow{3}{*}{ 5.4781 } & \multirow[t]{3}{*}{5.4760} & $-0.038 \%$ & $\mathrm{~N} / \mathrm{C}$ & & \\
\hline & & & & & & NO & \\
\hline & & & & & & & \\
\hline E & & Nitrogen - & $>24 \mathrm{HRS}$ & D $302 \mathrm{~F}$ & & & \\
\hline & U-475 & 5.5460 & 5.5450 & $.0 .018 \%$ & $\mathrm{~N} / \mathrm{C}$ & & \\
\hline 1 & A2 & & & & & NO & \\
\hline M & & & & & & & \\
\hline 1 & $Y-390$ & 5.6724 & 5.6688 & $-0.063 \%$ & $\mathrm{~N} / \mathrm{C}$ & & \\
\hline D & 82 & & & & & NO & \\
\hline $\mathrm{E}$ & & & & & & & \\
\hline & ER-610 & 4.9674 & 4.9645 & $.0 .058 \%$ & $\mathrm{~N} / \mathrm{C}$ & & \\
\hline$E$ & & & & & & YES & \\
\hline $\mathrm{P}$ & & & & & & & \\
\hline 0 & $Y-833$ & 5.2594 & 5.2423 & $-0.325 \%$ & $\mathrm{~N} / \mathrm{C}$ & & \\
\hline$x$ & D2 & & & & & YES & \\
\hline$r$ & 923 & 5.3007 & 5.2965 & $.0 .079 \%$ & $N / C$ & & \\
\hline$G$ & E2 & & & & & NO & \\
\hline L & & & & & & & \\
\hline$A$ & $150-800$ & 5.4540 & 5.4906 & $0.671 \%$ & $\mathrm{~N} / \mathrm{C}$ & & \\
\hline$s$ & F2 & & & & & NO & \\
\hline$s$ & & & & & & & \\
\hline
\end{tabular}




\begin{tabular}{|c|c|c|c|c|c|c|c|}
\hline & & ogen at 19 & & & & & \\
\hline & VARN & WIREWT & $\begin{array}{c}\text { EXPWRE } \\
W T\end{array}$ & $\begin{array}{l}\text { WT \% } \\
\text { CHANGE }\end{array}$ & EXP VIS & $\begin{array}{l}\text { EXP } \\
\text { FLEX }\end{array}$ & \\
\hline $\mathrm{P}$ & U-475 & 5.3162 & 5.3144 & $-0.034 \%$ & $\mathrm{~N} / \mathrm{C}$ & & \\
\hline 0 & & & & & & NO & \\
\hline & & & & & & & \\
\hline$Y$ & $Y-390$ & 5.5719 & 5.5672 & $-0.084 \%$ & $N / C$ & & \\
\hline$E$ & B1 & & & & & $\mathrm{NO}$ & \\
\hline$S$ & & & & & & & \\
\hline$T$ & ER-610 & 5.5502 & 5.5493 & $-0.016 \%$ & $N / C$ & & \\
\hline \begin{tabular}{|l|}
$E$ \\
$R$
\end{tabular} & & & & & & YES & \\
\hline & Y-833 & 5.2695 & 5.2877| & $0.345 \%$ & $\sqrt{N / C}$ & & \\
\hline 1 & D1 & & & & & YES & \\
\hline$M$ & & & & & & & \\
\hline 1 & $\overline{923}$ & 5.6581 & 5.6539 & $-0.074 \%$ & $\mathrm{~N} / \mathrm{C}$ & & \\
\hline D & E1 & & & & & NO & \\
\hline$E$ & & & & & & & \\
\hline$P$ & $\begin{array}{l}\text { ISO-800 } \\
\text { F1 }\end{array}$ & 5.5788 & 5.5772 & $-0.029 \%$ & $\mathrm{~N} / \mathrm{C}$ & NO & \\
\hline 0 & & & & & & & \\
\hline$L$ & & Nitrogen $\rightarrow$ & 24 HRS @ & $302 \mathrm{~F}$ & & & \\
\hline Y & $U-475$ & 5.3712 & 5.3686 & $.0 .048 \%$ & $N / C$ & & \\
\hline A & A2 & & & & & NO & \\
\hline$M$ & & & & & & & \\
\hline 1 & $Y-390$ & 5.7256 & 5.7209 & $-0.082 \%$ & $N / C$ & & \\
\hline$D$ & & & & & & NO & \\
\hline 나 & FR-610 & 5.5384 & 55362 & $0040 \%$ & $\sqrt{N / C}$ & & \\
\hline 1 & $\mathrm{C} 2$ & & & & & YES & \\
\hline$M$ & & & & & & & \\
\hline & $Y-833$ & 5.3576 & 5.3558 & $-0.034 \%$ & $N / C$ & & \\
\hline D & & & & & & YES & \\
\hline & 923 & 57306 & 5.7243 & $.0110 \%$ & $\sqrt{N / C}$ & & \\
\hline & E2 & & & & & NO & \\
\hline & & & & & & & \\
\hline & $150-800$ & 5.5759 & 5.5722 & $-0.066 \%$ & $N / C$ & & \\
\hline & & & & & & NO & \\
\hline & & & & & & & \\
\hline
\end{tabular}


Varnish Disks----Nitrogen @ $194^{\circ} \mathrm{F}\left(90^{\circ} \mathrm{C}\right)$

\begin{tabular}{|c|c|c|c|c|c|}
\hline & 500 HRS IN & Vitrogen @ & $194 \mathrm{~F}$ & & \\
\hline$\overline{I D}$ & WT & EXPWT & $\begin{array}{c}\text { WT \% } \\
\text { CHANGE } \\
\end{array}$ & EXPVIS & EXPFLEX \\
\hline $\begin{array}{l}\text { U-475 } \\
\text { A1 }\end{array}$ & 2.4523 & 2.4523 & $0.00 \%$ & $\mathrm{~N} / \mathrm{C}$ & $\mathrm{N} / \mathrm{C}$ \\
\hline $\begin{array}{l}-390 \\
B 1\end{array}$ & 2.1089 & 2.1019 & $0.33 \%$ & $\begin{array}{l}\text { slightly } \\
\text { warped }\end{array}$ & $\mathrm{N} / \mathrm{C}$ \\
\hline ER-610 & 2.3522 & 2.3597 & $0.32 \%$ & $\mathrm{~N} / \mathrm{C}$ & $N / C$ \\
\hline & & & & & \\
\hline$Y-833$ & 2.2590 & 2.2599 & $0.04 \%$ & $\mathrm{~N} / \mathrm{C}$ & $N / C$ \\
\hline D1 & & & & & \\
\hline 923 & 1.7369 & 1.7356 & $-0.07 \%$ & $\mathrm{~N} / \mathrm{C}$ & $N / C$ \\
\hline E1 & & & & & \\
\hline $150-800$ & 1.9567 & 1.9510 & $-0.29 \%$ & slightly & $\mathrm{N} / \mathrm{C}$ \\
\hline$F 1$ & & & & warped & \\
\hline & Nitrogen at & $\cdots>24$ hour & at $302^{\circ} \mathrm{F}$. & & \\
\hline U-475 & 2.3780 & 2.3613 & $-0.70 \%$ & $N / C$ & $N / C$ \\
\hline A2 & & & & & \\
\hline$Y-390$ & 2.2201 & 2.1908 & $-1.32 \%$ & slightly & $N / C$ \\
\hline & & & & warped & \\
\hline ER-610 & 2.7084 & 2.6998 & $-0.32 \%$ & darkened & $\mathrm{N} / \mathrm{C}$ \\
\hline C2 & & & & & \\
\hline$Y-833$ & 2.4352 & 2.4360 & $0.03 \%$ & $N / C$ & $\sqrt{N / C}$ \\
\hline D2 & & & & & \\
\hline & & & & & \\
\hline $\begin{array}{l}923 \\
\end{array}$ & 1.7797 & 1.7739 & $-0.33 \%$ & & \\
\hline E2 & & & & $\mathrm{N} / \mathrm{C}$ & $N / C$ \\
\hline $150-800$ & 2.0535 & 1.9782 & $-3.67 \%$ & warped & \\
\hline & & & & & $N / C$ \\
\hline & & & & & \\
\hline
\end{tabular}




\begin{tabular}{|c|c|c|c|c|c|c|c|c|c|}
\hline \multicolumn{10}{|c|}{500 HR IN Nitrogen @ $194^{\circ} \mathrm{F}$} \\
\hline ID & W' & EXPWT & $\begin{array}{c}\text { WT \% } \\
\text { CHANGE }\end{array}$ & $\begin{array}{l}\text { SAMPLE } \\
\text { WIDTH }\end{array}$ & $\begin{array}{l}\text { BREAK } \\
\text { LOAD }\end{array}$ & $\begin{array}{c}\text { SAMPLE } \\
\text { THICKNESS } \\
\text { Mils } \\
\end{array}$ & $\begin{array}{c}\text { TENSILE } \\
\text { STR } \\
\text { BASE } \\
\end{array}$ & $\begin{array}{l}\text { TENSILE } \\
\text { STREXP }\end{array}$ & $\begin{array}{c}\% \\
\text { CHANGE } \\
\text { TENSILE }\end{array}$ \\
\hline \multirow{3}{*}{$\begin{array}{l}\text { NO/MY/N(S) } \\
\text { A1 }\end{array}$} & \multirow[t]{3}{*}{4.6148} & \multirow[t]{3}{*}{4.6174} & \multirow[t]{3}{*}{$0.06 \%$} & 0.370 & 128.70 & 21 & 17.4 & 16.56 & \\
\hline & & & & 0.425 & 146.80 & 21 & 17.4 & 16.45 & $-8.47 \%$ \\
\hline & & & & 0.507 & 157.20 & 21 & 17.4 & 14.76 & \\
\hline \multirow{3}{*}{$\begin{array}{l}\mathrm{DA} / \mathrm{MY} / \mathrm{DA} \\
\mathrm{B} 1\end{array}$} & \multirow[t]{3}{*}{4.3227} & \multirow[t]{3}{*}{4.3277} & \multirow[t]{3}{*}{$0.12 \%$} & 0.369 & 97.20 & 21 & 13.7 & 12.54 & \\
\hline & & & & 0.435 & 119.80 & 21 & 13.7 & 13.11 & $.6 .47 \%$ \\
\hline & & & & 0.450 & 120.80 & 21 & 13.7 & 12.78 & \\
\hline \multirow{3}{*}{$\begin{array}{l}\text { MYLAR MO } \\
\text { C1 }\end{array}$} & \multirow[t]{3}{*}{2.2027} & \multirow[t]{3}{*}{2.2077} & \multirow[t]{3}{*}{$0.23 \%$} & 0.499 & 101.60 & 10 & 21.7 & 20.36 & \\
\hline & & & & 0.489 & 97.05 & 10 & 21.7 & 19.85 & $-6.84 \%$ \\
\hline & & & & 0.502 & 102.60 & 10 & 21.7 & 20.44 & \\
\hline \multirow{3}{*}{$\begin{array}{l}\text { NO } 410 \\
\text { D1 }\end{array}$} & \multirow[t]{3}{*}{2.0630} & \multirow[t]{3}{*}{2.0669} & \multirow[t]{3}{*}{$0.19 \%$} & 0.484 & 91.50 & 10 & 18.7 & 18.90 & \\
\hline & & & & 0.522 & 96.35 & 10 & 18.7 & 18.46 & $1.14 \%$ \\
\hline & & & & 0.466 & 90.30 & 10 & 18.7 & 19.38 & \\
\hline \multirow{3}{*}{$\begin{array}{l}\text { NO MI } 418 \\
\text { E1 }\end{array}$} & \multirow[t]{3}{*}{2.1118} & \multirow[t]{3}{*}{2.1165} & \multirow[t]{3}{*}{$0.22 \%$} & 0.502 & 27.75 & 9 & 7.5 & 6.14 & \\
\hline & & & & 0.490 & 27.25 & 9 & 7.5 & 6.18 & $-18.72 \%$ \\
\hline & & & & 0.528 & 28.35 & 9 & 7.5 & 5.97 & \\
\hline \multirow{4}{*}{$\begin{array}{l}\text { MEL } 228 \\
F 1\end{array}$} & \multirow[t]{3}{*}{2.5339} & \multirow[t]{3}{*}{2.5397} & \multirow[t]{3}{*}{$0.23 \%$} & 0.540 & 107.10 & 10 & 21.7 & 19.83 & \\
\hline & & & & 0.470 & 92.50 & 10 & 21.7 & 19.68 & $-10.17 \%$ \\
\hline & & & & 0.507 & 96.15 & 10 & 21.7 & 18.96 & \\
\hline & & 500 HRS & IN Nitrog & jen@ @ 194 & $4^{\circ} \mathrm{F} \cdot>24$ & HRS@302 & & & \\
\hline NO/MY/NO & 4.1604 & 4.1435 & $-0.41 \%$ & $0.40 ?$ & 140.00 & 21 & $\therefore .4$ & 16.38 & \\
\hline A2 & & & & 0.414 & 151.10 & 21 & 17.4 & 17.38 & $-2.29 \%$ \\
\hline & & & & 0.475 & 172.00 & 21 & 17.4 & 17.24 & \\
\hline $\mathrm{DA} / \mathrm{MY} / \mathrm{DA}$ & 4.1479 & 4.1382 & $-0.23 \%$ & 0.380 & 107.10 & 21 & 13.7 & 13.42 & \\
\hline $\mathrm{B} 2$ & & & & 0.476 & 135.30 & 21 & 13.7 & 13.54 & $-1.30 \%$ \\
\hline & & & & 0.380 & 108.60 & 21 & 13.7 & 13.61 & \\
\hline MYLAR MO & 2.2383 & 2.2379 & $-0.02 \%$ & 0.389 & 77.20 & 10 & 21.7 & 19.85 & \\
\hline $\mathrm{C} 2$ & & & & 0.415 & 79.95 & 10 & 21.7 & 19.27 & $-9.42 \%$ \\
\hline & & & & 0.390 & 77.45 & 10 & 21.7 & 19.86 & \\
\hline NOMEX 410 & 2.2687 & 2.2720 & $0.15 \%$ & 0.516 & 104.20 & 10 & 18.7 & 20.19 & \\
\hline D2 & & & & 0.483 & 101.90 & 10 & 18.7 & 21.10 & $9.15 \%$ \\
\hline & & & & 0.508 & 101.30 & 10 & 18.7 & 19.94 & \\
\hline NO/MI 418 & 2.1342 & 2.1213 & $-0.60 \%$ & 0.510 & 28.55 & 9 & 7.5 & 6.22 & \\
\hline E2 & & & & 0.517 & 28.70 & 9 & 7.5 & 6.17 & $-19.20 \%$ \\
\hline & & & & 0.470 & 24.50 & 9 & 7.5 & 5.79 & \\
\hline MEL 228 & 2.2689 & 2.2686 & $-0.01 \%$ & 0.478 & 94.70 & 10 & 21.7 & 19.81 & \\
\hline$F 2$ & & & & 0.515 & 99.30 & 10 & 21.7 & 19.28 & $-9.92 \%$ \\
\hline & & & & 0.500 & 97.75 & 10 & 21.7 & 19.55 & \\
\hline
\end{tabular}




\begin{tabular}{|c|c|c|c|c|c|c|c|c|}
\hline \multicolumn{9}{|c|}{ After 500 hour exposure to Nitrogen @ $194^{\circ} \mathrm{F}\left(90^{\circ} \mathrm{C}\right)$} \\
\hline $\mathbb{D}$ & $\begin{array}{c}\text { STRETC. } \\
\text { (inch) }\end{array}$ & $\begin{array}{c}\% \\
\text { ELONG }\end{array}$ & $\begin{array}{l}\text { BASE } \\
\text { ELONG } \\
\text { (AVE) }\end{array}$ & $\begin{array}{c}\text { ELONG } \\
\% \\
\text { CHANGE }\end{array}$ & $\begin{array}{c}\text { BASE } \\
\text { DIE } \\
\text { (AVE) }\end{array}$ & EXPDIE & $\begin{array}{c}\text { DIE \% } \\
\text { CHANGE }\end{array}$ & VISUAL EXP \\
\hline \multirow{3}{*}{$\begin{array}{l}\text { NO/MY/NO } \\
\text { A1 }\end{array}$} & 0.73 & $18.3 \%$ & $20.0 \%$ & & $>18.97$ & $>16.0$ & & \multirow[t]{3}{*}{$\mathrm{N} / \mathrm{C}$} \\
\hline & 0.84 & $21.0 \%$ & $20.0 \%$ & $-1.9 \%$ & $>18.97$ & $>15.49$ & flash & \\
\hline & .25 & \#\#\#\#\# & $20.0 \%$ & & $>18.97$ & $>15.05$ & & \\
\hline \multirow{3}{*}{$\begin{array}{l}\mathrm{DA} / \mathrm{MY} / \mathrm{DA} \\
\mathrm{B1}\end{array}$} & 0.51 & $25.5 \%$ & $46.0 \%$ & & $>15.27$ & $>14.8$ & & \multirow[t]{3}{*}{$\mathrm{N} / \mathrm{C}$} \\
\hline & 0.54 & $27.0 \%$ & $46.0 \%$ & $.43 .1 \%$ & $>15.27$ & $>13.91$ & flash & \\
\hline & 0.52 & $26.0 \%$ & $46.0 \%$ & & $>15.27$ & $>13.11$ & & \\
\hline \multirow{3}{*}{$\begin{array}{l}\text { MYLAR MO } \\
\text { C1 }\end{array}$} & 3.09 & $154.5 \%$ & $131.0 \%$ & & $>14.91$ & $>13.68$ & & \multirow[t]{3}{*}{$\mathrm{N} / \mathrm{C}$} \\
\hline & 2.99 & $149.5 \%$ & $131.0 \%$ & $15.8 \%$ & $>14.91$ & $>13.12$ & flash & \\
\hline & 3.02 & $151.0 \%$ & $131.0 \%$ & & $>14.91$ & $>13.41$ & & \\
\hline \multirow{3}{*}{$\begin{array}{l}\text { NO } 410 \\
\text { D1 }\end{array}$} & 0.54 & $13.5 \%$ & $17.0 \%$ & & 10.67 & 10.46 & & \multirow[t]{3}{*}{$\mathrm{N} / \mathrm{C}$} \\
\hline & 0.40 & $10.0 \%$ & $17.0 \%$ & $-24.0 \%$ & 10.67 & 9.36 & $-9.2 \%$ & \\
\hline & 0.61 & $15.3 \%$ & $17.0 \%$ & & $10.6 i$ & 9.26 & & \\
\hline \multirow{3}{*}{$\begin{array}{l}\text { NC Ml } 418 \\
\text { Et }\end{array}$} & 0.07 & $1.8 \%$ & $4.0 \%$ & & 10.23 & 9.11 & & \multirow[t]{3}{*}{$\mathrm{N} / \mathrm{C}$} \\
\hline & 0.06 & $1.5 \%$ & $4.0 \%$ & $-60.4 \%$ & 10.23 & 9.85 & $-7.3 \%$ & \\
\hline & 0.06 & $1.5 \%$ & $4.0 \%$ & & 10.23 & 9.50 & & \\
\hline \multirow{3}{*}{$\begin{array}{l}\text { MEL } 228 \\
\text { F1 }\end{array}$} & 3.76 & $188.0 \%$ & $160.0 \%$ & & $>14.22$ & $>14.01$ & & \multirow[t]{3}{*}{$\mathrm{N} / \mathrm{C}$} \\
\hline & 3.51 & $175.5 \%$ & $160.0 \%$ & $9.8 \%$ & $>14.22$ & $>14.13$ & flash & \\
\hline & 3.27 & $163.5 \%$ & $160.0 \%$ & & $>14.22$ & $>13.43$ & & \\
\hline & \multicolumn{7}{|c|}{ After 500 hour Exposure plus a 24 hour airbake @ $302^{\circ} \mathrm{F}\left(150^{\circ}\right.$} & $\left.{ }^{\circ} \mathrm{C}\right)$ \\
\hline \multirow{3}{*}{$\begin{array}{l}\text { NO/MY/NO } \\
\text { A2 }\end{array}$} & 0.68 & $17.0 \%$ & $20.0 \%$ & & $>18.97$ & $>15.90$ & & \multirow[t]{3}{*}{$\mathrm{N} / \mathrm{C}$} \\
\hline & 0.86 & $21.5 \%$ & $20.0 \%$ & $-6.7 \%$ & $>18.97$ & $>15.55$ & flash & \\
\hline & 0.70 & $17.5 \%$ & $20.0 \%$ & & $>18.97$ & $>16.40$ & & \\
\hline \multirow{3}{*}{$\begin{array}{l}D A / M Y / D A \\
B 2\end{array}$} & 0.57 & $28.5 \%$ & $46.0 \%$ & & $>15.27$ & $>14.11$ & & \multirow{3}{*}{$\begin{array}{l}\text { slighlty } \\
\text { warped }\end{array}$} \\
\hline & 0.56 & $28.0 \%$ & $46.0 \%$ & $-38.8 \%$ & $>15.27$ & $>13.27$ & flash & \\
\hline & 0.56 & $28.0 \%$ & $46.0 \%$ & & $>15.27$ & $>12.5$ & & \\
\hline \multirow{3}{*}{$\begin{array}{l}\text { MYLAR MO } \\
\text { C2 }\end{array}$} & 3.19 & $159.5 \%$ & $131.0 \%$ & & $>14.91$ & $>13.61$ & & \multirow[t]{3}{*}{$N / C$} \\
\hline & 2.94 & $147.0 \%$ & $131.0 \%$ & $17.8 \%$ & $>14.91$ & $>13.26$ & flash & \\
\hline & 3.13 & $156.5 \%$ & $131.0 \%$ & & $>14.91$ & $>12.94$ & & \\
\hline \multirow{3}{*}{$\begin{array}{l}\text { NOMEX 410 } \\
\text { D2 }\end{array}$} & 0.56 & $14.0 \%$ & $17.0 \%$ & & 10.67 & 10.50 & & \multirow[t]{3}{*}{$N / C$} \\
\hline & 0.58 & $14.5 \%$ & $17.0 \%$ & $-21.1 \%$ & 10.67 & 10.70 & $3.7 \%$ & \\
\hline & 0.47 & $11.8 \%$ & $17.0 \%$ & & 10.67 & 12.00 & & \\
\hline \multirow{3}{*}{$\begin{array}{l}\mathrm{NO} / \mathrm{Ml} 418 \\
\mathrm{E2}\end{array}$} & 0.07 & $1.8 \%$ & $4.0 \%$ & & 10.23 & 9.12 & & \multirow[t]{3}{*}{$\mathrm{N} / \mathrm{C}$} \\
\hline & 0.07 & $1.8 \%$ & $4.0 \%$ & $.56 .3 \%$ & 10.23 & 8.76 & $-12.1 \%$ & \\
\hline & 0.07 & $1.8 \%$ & $4.0 \%$ & & 10.23 & 9.10 & & \\
\hline \multirow{3}{*}{$\begin{array}{l}\text { MEL } 228 \\
\text { F2 }\end{array}$} & 3.57 & $178.5 \%$ & $160.0 \%$ & & $>14.22$ & $>13.12$ & & $N / C$ \\
\hline & 3.44 & $172.0 \%$ & $160.0 \%$ & $9.4 \%$ & $>14.22$ & $>13.7$ & flash & \\
\hline & 3.49 & $174.5 \%$ & $160.0 \%$ & & $>14.22$ & $>14.41$ & & \\
\hline
\end{tabular}


SLEEVING----Nitrogen @ $194^{\circ} \mathrm{F}\left(90^{\circ} \mathrm{C}\right)$

\begin{tabular}{|c|c|c|c|c|}
\hline & \multicolumn{4}{|c|}{500 HRS IN Nitrogen @ 194. F } \\
\hline & WT & EXPWT & $\begin{array}{c}\% \\
\text { CHANGE }\end{array}$ & EXPVS \\
\hline $\begin{array}{l}\text { A1 } \\
\text { NOMEX }\end{array}$ & 0.5660 & 0.5650 & $-0.18 \%$ & $\mathrm{~N} / \mathrm{C}$ \\
\hline \begin{tabular}{|l} 
B1 \\
MYLAR
\end{tabular} & 0.4429 & 0.4467 & $0.86 \%$ & $\mathrm{~N} / \mathrm{C}$ \\
\hline $\begin{array}{l}\mathrm{C1} \\
\text { NO/MY }\end{array}$ & 0.4056 & 0.4065 & $0.22 \%$ & $\overline{\mathrm{N} / \mathrm{C}}$ \\
\hline & Nitrogen@ & $94^{\circ} \mathrm{F} \cdots>>24$ & hrs @302 & \\
\hline $\begin{array}{l}\mathrm{A} 2 \\
\text { NOMEX }\end{array}$ & 0.5615 & 0.5632 & $0.30 \%$ & $N / C$ \\
\hline $\begin{array}{l}\text { B2 } \\
\text { MYLAR }\end{array}$ & 0.5047 & 0.5042 & $-0.10 \%$ & $\mathrm{~N} / \mathrm{C}$ \\
\hline $\begin{array}{l}\mathrm{C} 2 \\
\mathrm{NO} / \mathrm{MY}\end{array}$ & 0.3935 & 0.3934 & $-0.03 \%$ & $\mathrm{~N} / \mathrm{C}$ \\
\hline
\end{tabular}




\begin{tabular}{|c|c|c|c|c|c|c|c|c|c|}
\hline & \multicolumn{9}{|c|}{500 HRS IN Nitrogen @ 194 F } \\
\hline D & WT & EXPWT & $\begin{array}{c}\text { WT \% } \\
\text { CHANGE }\end{array}$ & $\begin{array}{l}\text { BREAK } \\
\text { LOAD } \\
\text { (AVE) }\end{array}$ & $\begin{array}{c}\text { BREAK } \\
\text { LOAD } \\
\text { EPP }\end{array}$ & $\begin{array}{c}\% \\
\text { CHANGE } \\
\text { BPK } \\
\text { LOAD } \\
\end{array}$ & $\begin{array}{c}\text { STRETCH } \\
(\mathrm{INCH})\end{array}$ & $\%$ ELONG & EXPVIS \\
\hline \multirow{3}{*}{$\begin{array}{l}\text { A1 } \\
\text { Glass }\end{array}$} & \multirow[t]{3}{*}{1.5187} & \multirow[t]{3}{*}{1.5217} & \multirow[t]{3}{*}{$0.20 \%$} & 39.02 & 35.60 & & 0.05 & $2.50 \%$ & $\mathrm{~N} / \mathrm{C}$ \\
\hline & & & & 39.02 & 30.85 & $5.52 \%$ & 0.06 & $3.00 \%$ & \\
\hline & & & & 39.02 & 44.15 & & 0.05 & $2.50 \%$ & \\
\hline \multirow{3}{*}{$\begin{array}{l}\text { B1 } \\
\text { Polyester }\end{array}$} & \multirow[t]{3}{*}{0.6247} & \multirow[t]{3}{*}{0.6276} & \multirow[t]{3}{*}{$0.46 \%$} & 56.12 & 48.20 & & 0.41 & $20.50 \%$ & $\mathrm{~N} / \mathrm{C}$ \\
\hline & & & & 56.12 & 58.40 & $1.61 \%$ & 0.65 & $32.50 \%$ & \\
\hline & & & & 56.12 & 59.05 & & 0.63 & $31.50 \%$ & \\
\hline \multirow{4}{*}{$\begin{array}{l}\text { C1 } \\
\text { Permacel }\end{array}$} & \multirow[t]{3}{*}{1.4638} & \multirow[t]{3}{*}{1.4569} & \multirow[t]{3}{*}{$-0.47 \%$} & 88.50 & 121.20 & & 0.15 & $7.50 \%$ & $\mathrm{~N} / \mathrm{C}$ \\
\hline & & & & 88.50 & 79.95 & $.6 .89 \%$ & 0.11 & $5.50 \%$ & \\
\hline & & & & 88.50 & 46.05 & & 0.11 & $5.50 \%$ & \\
\hline & \multicolumn{7}{|c|}{500 HRS in Nitrogen $->302^{\circ} \mathrm{F} 24 \mathrm{HRS}$} & & \\
\hline \multirow{3}{*}{$\begin{array}{l}\text { A2 } \\
\text { Glass }\end{array}$} & \multirow[t]{3}{*}{1.5927} & \multirow[t]{3}{*}{1.5968} & \multirow[t]{3}{*}{$0.26 \%$} & 39.02 & 40.20 & & 0.05 & $2.50 \%$ & $\mathrm{~N} / \mathrm{C}$ \\
\hline & & & & 39.02 & 39.75 & $-0.01 \%$ & 0.06 & $3.00 \%$ & \\
\hline & & & & 39.02 & 37.10 & & 0.06 & $3.00 \%$ & \\
\hline \multirow{3}{*}{$\begin{array}{l}\text { B2 } \\
\text { Polyester }\end{array}$} & \multirow[t]{3}{*}{0.5795} & \multirow[t]{3}{*}{0.5812} & \multirow{3}{*}{$0.29 \%$} & 56.12 & 63.60 & & 0.95 & $47.50 \%$ & $\mathrm{~N} / \mathrm{C}$ \\
\hline & & & & 56.12 & 58.75 & $8.46 \%$ & 0.70 & $35.00 \%$ & \\
\hline & & & & 56.12 & 60.25 & & 0.69 & $34.50 \%$ & \\
\hline \multirow{3}{*}{$\begin{array}{l}\text { C2 } \\
\text { Permacel }\end{array}$} & \multirow[t]{3}{*}{1.4067} & \multirow[t]{3}{*}{1.3776} & \multirow[t]{3}{*}{$-2.07 \%$} & 88.50 & 53.85 & & 0.20 & $10.00 \%$ & slightly \\
\hline & & & & 88.50 & 142.40 & $4.12 \%$ & 0.12 & $6.00 \%$ & Darkened \\
\hline & & & & 88.50 & 80.20 & & 0.14 & $7.00 \%$ & \\
\hline
\end{tabular}


TIE CORD-.--Nitrogen @ $194^{\circ} \mathrm{F}\left(90^{\circ} \mathrm{C}\right)$

\begin{tabular}{|c|c|c|c|c|c|c|c|c|c|}
\hline \multicolumn{10}{|c|}{500 HRS IN Nitrogen @ 194. F } \\
\hline ID & WT & EXPWT & $\begin{array}{c}\text { WT \% } \\
\text { CHANGE }\end{array}$ & \begin{tabular}{|l|}
$E X P$ \\
$V I S$
\end{tabular} & $\begin{array}{l}\text { BREAK } \\
\text { LOAD } \\
\text { (AVE) }\end{array}$ & $\begin{array}{c}\text { BREAK } \\
\text { LOAD } \\
\text { EPP } \\
(\operatorname{av} \theta)\end{array}$ & $\begin{array}{c}\% \\
\text { CHANGE } \\
\text { BPK } \\
\text { LOAD }\end{array}$ & $\begin{array}{c}\text { STRETCH } \\
\text { (Inch) }\end{array}$ & $\begin{array}{c}\% \\
\text { ELONG }\end{array}$ \\
\hline \multirow{4}{*}{ A1 } & \multirow[t]{3}{*}{0.2540} & \multirow[t]{3}{*}{0.2539} & \multirow[t]{3}{*}{$-0.04 \%$} & $\mathrm{~N} / \mathrm{C}$ & 28.36 & 31.40 & & 0.24 & $12.0 \%$ \\
\hline & & & & & 28.36 & 36.75 & $23.47 \%$ & 0.27 & $13.5 \%$ \\
\hline & & & & & 28.36 & 36.90 & & 0.26 & $13.0 \%$ \\
\hline & \multicolumn{9}{|c|}{500 HRS IN Nitrogen $>24$ HRS @ 302 $\mathrm{F}$} \\
\hline \multirow[t]{3}{*}{ A2 } & \multirow[t]{3}{*}{0.2667} & \multirow[t]{3}{*}{0.2663} & \multirow[t]{3}{*}{$-0.150 \%$} & $\mathrm{~N} / \mathrm{C}$ & 28.36 & 32.25 & & 0.40 & $20.0 \%$ \\
\hline & & & & & 28.36 & 32.15 & $16.01 \%$ & 0.49 & $24.5 \%$ \\
\hline & & & & & 28.36 & 34.30 & & 0.26 & $13.0 \%$ \\
\hline
\end{tabular}




\begin{tabular}{|c|c|c|c|c|c|c|c|}
\hline & 500 HRS IN & trogen@1 & $94^{\circ} \mathrm{F}$ & & & & \\
\hline ID & $\overline{\mathrm{WT}}$ & EXPWT & $\begin{array}{l}\text { WT \% } \\
\text { CHANGE }\end{array}$ & EXPVIS & $\begin{array}{c}\text { BASE DIE } \\
\text { (AVE) }\end{array}$ & EXP DIE & $\begin{array}{c}\text { DIE\% } \\
\text { CHANGE }\end{array}$ \\
\hline$D M D$ & 4.0428 & 4.0446 & $0.04 \%$ & $\mathrm{~N} / \mathrm{C}$ & 9.61 & 7.45 & \\
\hline$A 1$ & & & & & 9.61 & 10.23 & $-5.45 \%$ \\
\hline & & & & & 9.61 & 9.58 & \\
\hline DTMD & 4.3351 & 4.3370 & $0.04 \%$ & $\mathrm{~N} / \mathrm{C}$ & 9.95 & 11.03 & \\
\hline$B 1$ & & & & & 9.95 & 9.00 & $8.34 \%$ \\
\hline & & & & & 9.95 & 12.31 & \\
\hline & Nitrogen@1 & $4^{\circ} \mathrm{F}->24 \mathrm{HF}$ & RS @ 302 & & & & \\
\hline$D M D$ & 4.0622 & 4.0642 & $0.05 \%$ & $\mathrm{~N} / \mathrm{C}$ & 9.61 & 8.18 & \\
\hline A2 & & & & & 9.61 & 9.46 & $5.86 \%$ \\
\hline & & & & & 9.61 & 9.50 & \\
\hline DTMD & 4.3780 & 4.3754 & $-0.06 \%$ & $\mathrm{~N} / \mathrm{C}$ & \begin{tabular}{l|l}
9.95 \\
\end{tabular} & 10.75 & \\
\hline B2 & & & & & 9.95 & 9.65 & $-0.07 \%$ \\
\hline & & & & & 9.95 & 9.43 & \\
\hline
\end{tabular}




\section{Appendix C}

Experimental Data for HCFC-22 Exposure at $90^{\circ} \mathrm{C}\left(194^{\circ} \mathrm{F}\right)$ 


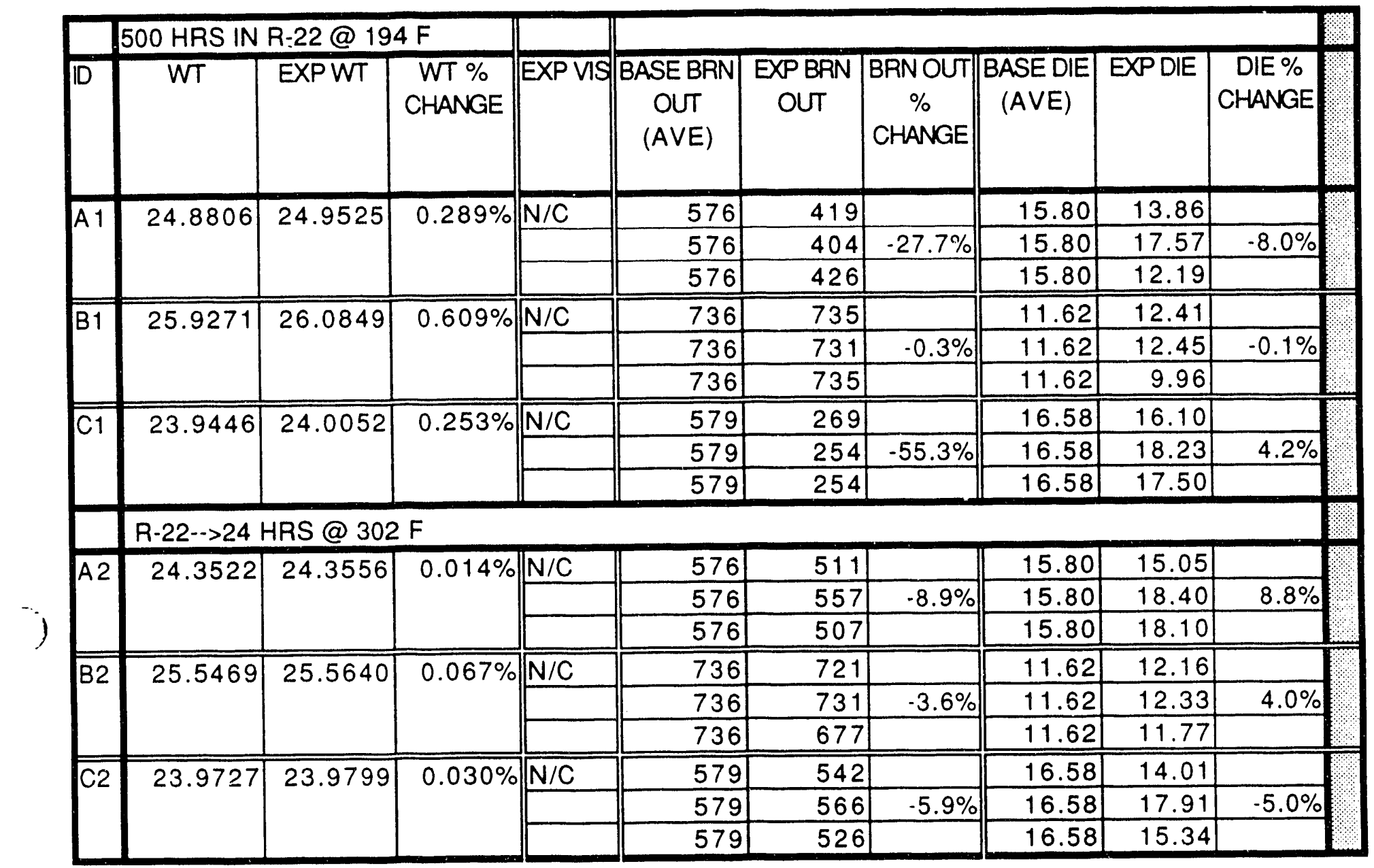




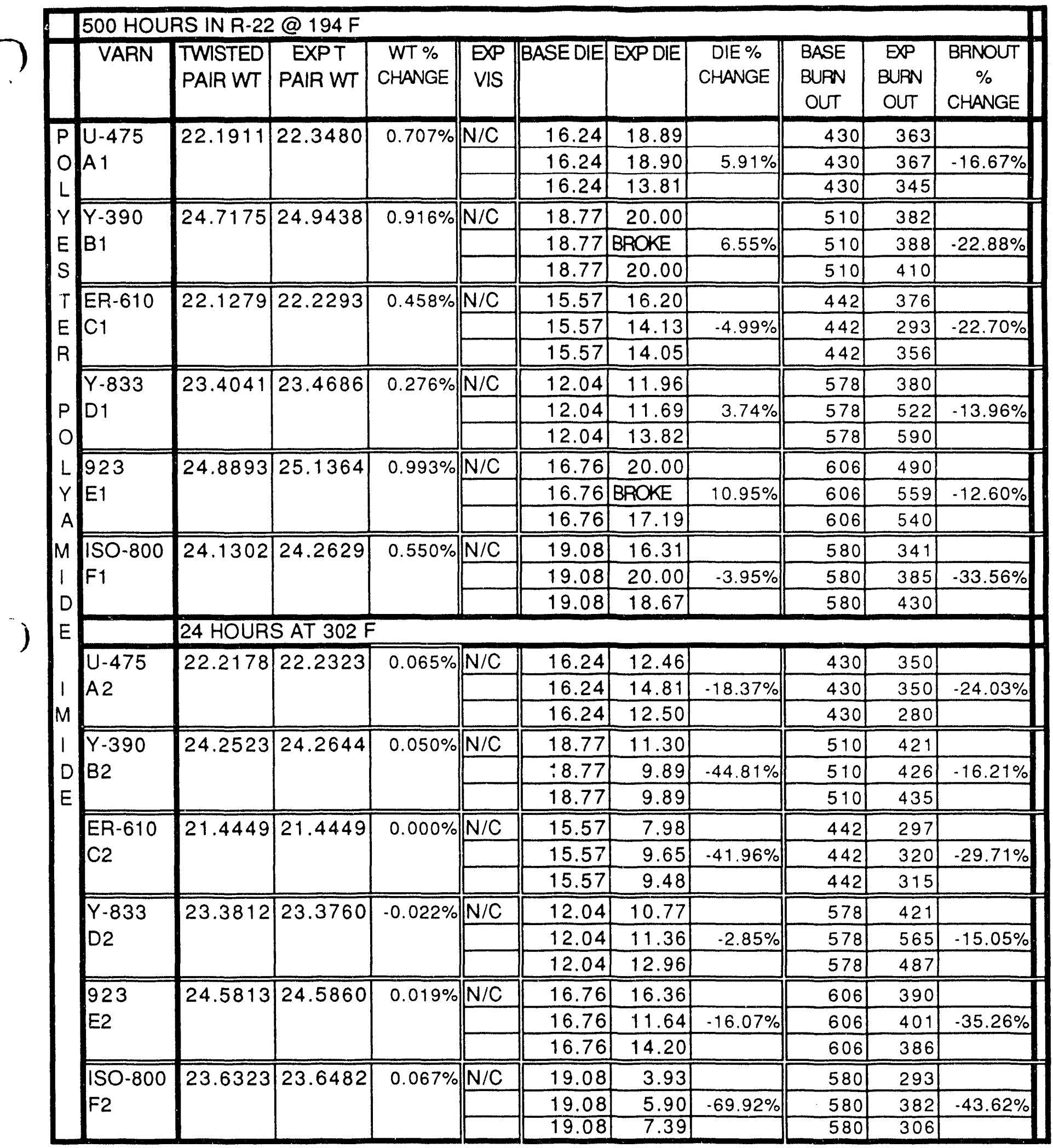




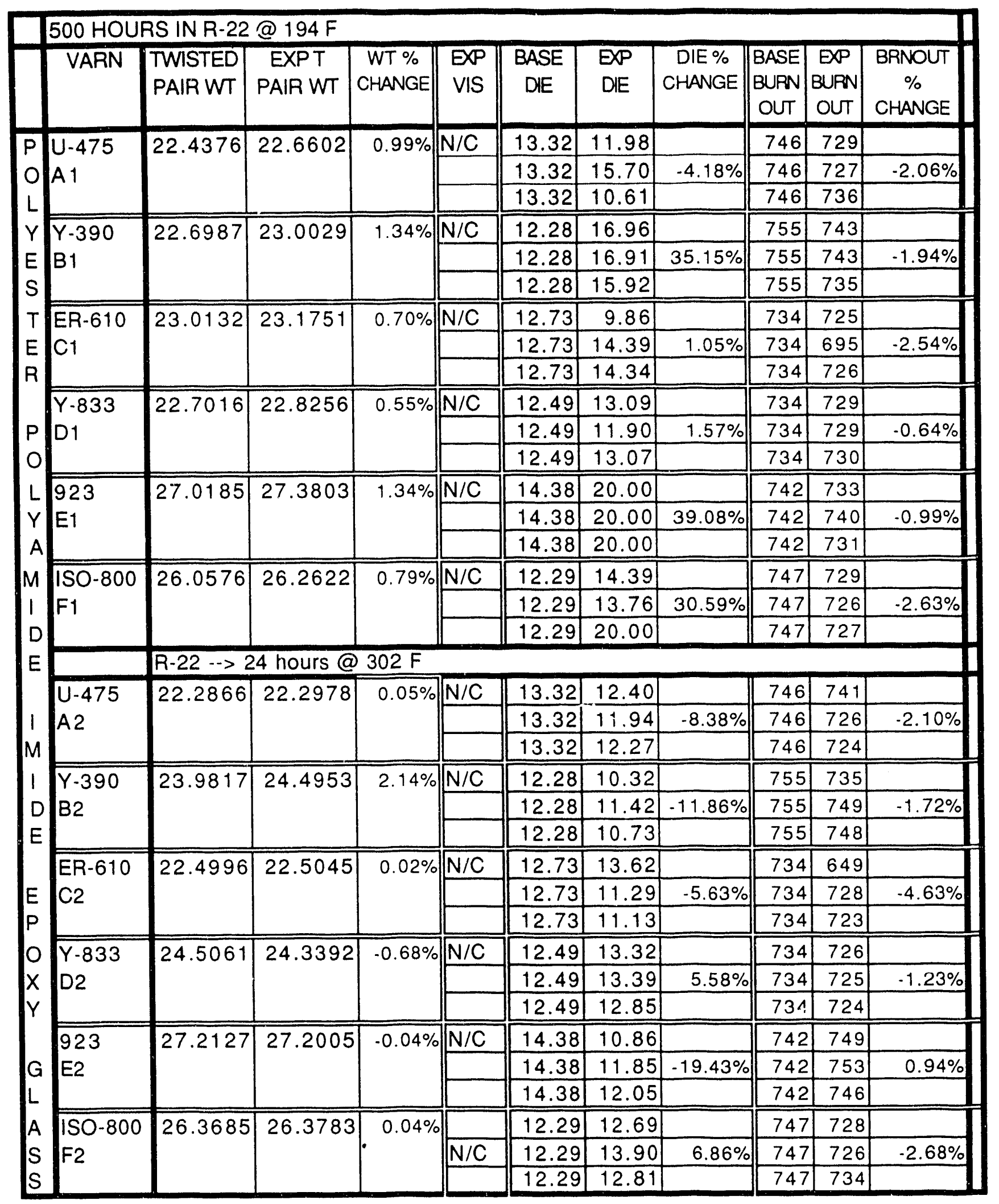




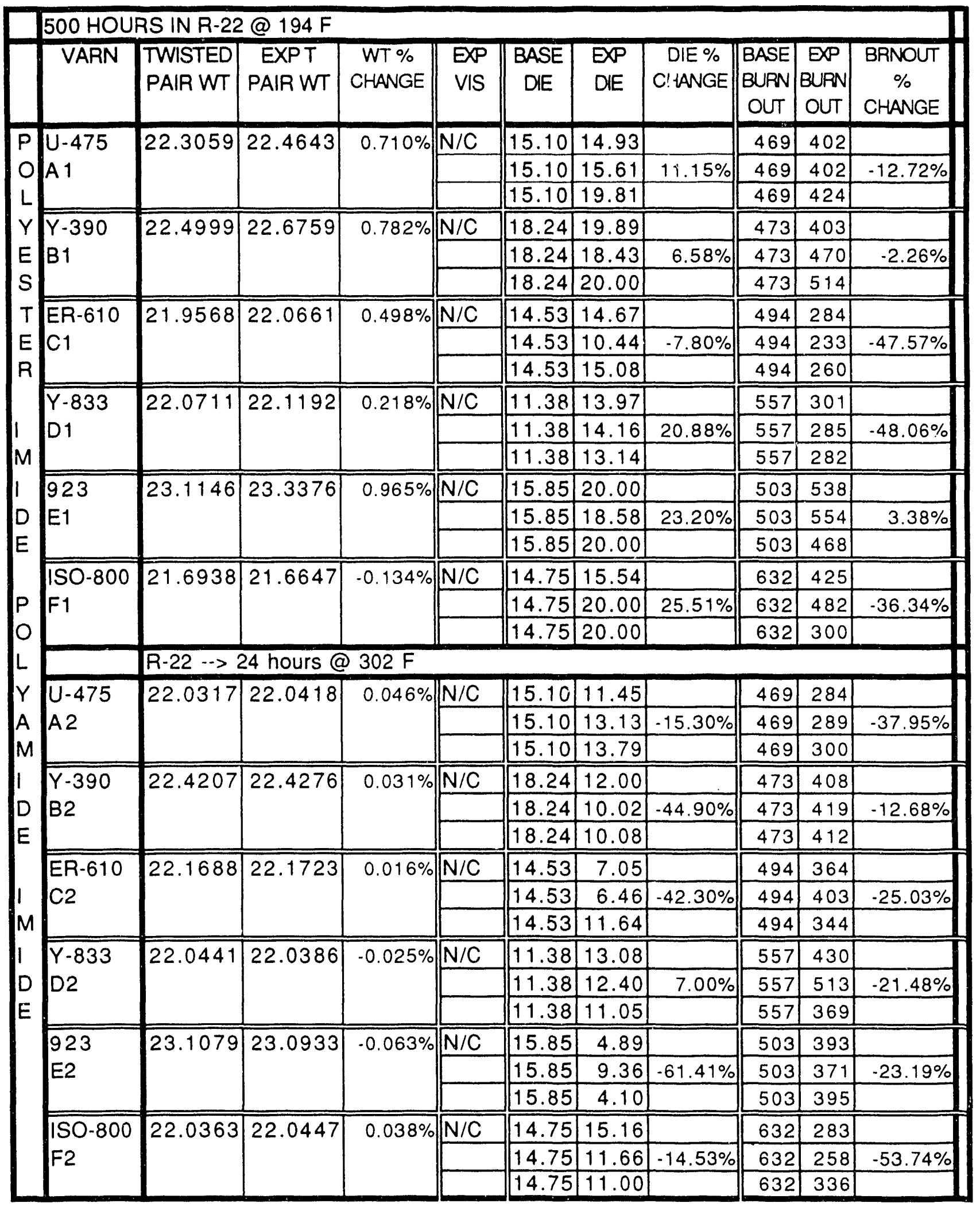




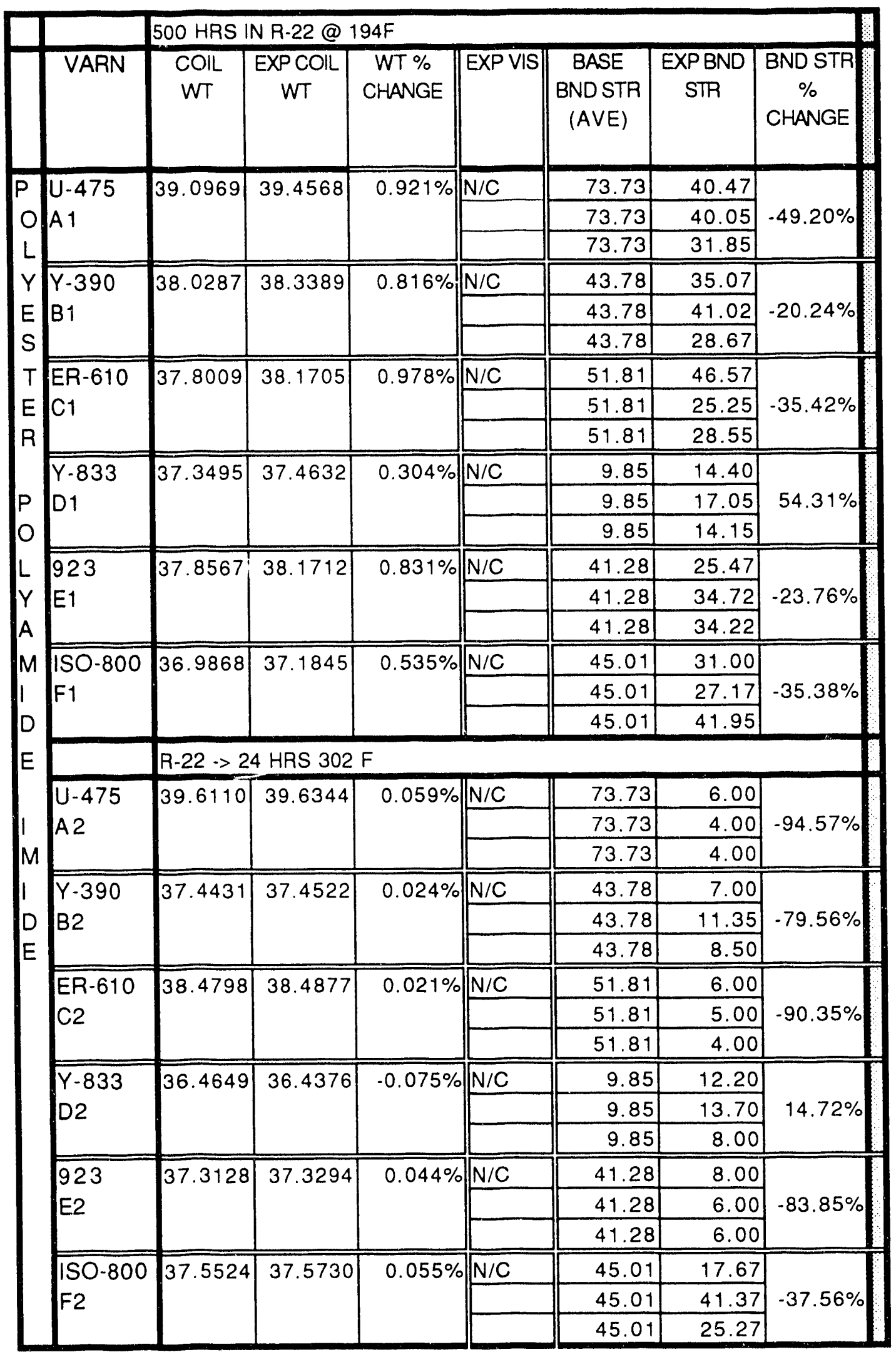


HELICAL COILSNWIRE B

\begin{tabular}{|c|c|c|c|c|c|c|c|c|}
\hline & & 500 HRS I & IN R-22 @ & $194 \mathrm{~F}$ & & & & \\
\hline & VARN & $\begin{array}{l}\text { COIL } \\
\text { WT }\end{array}$ & $\begin{array}{c}\text { EXP COII } \\
W T\end{array}$ & $\begin{array}{c}\text { WT \% } \\
\text { CHANGE }\end{array}$ & EXP VIS & $\begin{array}{c}\text { BASE } \\
\text { BND STR } \\
\text { (AVE) }\end{array}$ & $\begin{array}{c}\text { EXPBND } \\
\text { STR }\end{array}$ & $\begin{array}{c}\text { BND STR } \\
\% \\
\text { CHANGE }\end{array}$ \\
\hline$P$ & U-475 & 37.1905 & 37.6554 & $1.250 \%$ & $\mathrm{~N} / \mathrm{C}$ & 40.14 & 65.22 & \\
\hline 0 & A 1 & & & & & 40.14 & 64.75 & $59.52 \%$ \\
\hline $\mathrm{L}$ & & & & & & 40.14 & 62.12 & \\
\hline$Y$ & $Y-390$ & 36.8661 & 37.3769 & $1.386 \%$ & $\mathrm{~N} / \mathrm{C}$ & 36.12 & 55.95 & \\
\hline$E$ & B1 & & & & & 36.12 & 53.22 & $60.50 \%$ \\
\hline s & & & & & & 36.12 & 64.75 & \\
\hline$T$ & ER-610 & 37.4839 & 37.9241 & $1.174 \%$ & $\mathrm{~N} / \mathrm{C}$ & 35.96 & 49.22 & \\
\hline$E$ & C1 & & & & & 35.96 & 44.17 & $28.74 \%$ \\
\hline$R$ & & & & & & 35.96 & 45.50 & \\
\hline & $Y-833$ & 36.6558 & 37.0888 & $1.181 \%$ & $\mathrm{~N} / \mathrm{C}$ & 33.14 & 19.10 & \\
\hline $\mathrm{P}$ & D1 & & & & & 33.14 & 14.85 & $-51.05 \%$ \\
\hline 0 & & & & & & 33.14 & 14.72 & \\
\hline$L$ & 923 & 36.1828 & 36.7126 & $1.464 \%$ & $\mathrm{~N} / \mathrm{C}$ & 40.52 & 47.77 & \\
\hline$Y$ & E1 & & & & & 40.52 & 62.10 & $31.20 \%$ \\
\hline A & & & & & & 40.52 & 49.62 & \\
\hline$M$ & ISO-800 & 35.3699 & 35.6681 & $0.843 \%$ & $\mathrm{~N} / \mathrm{C}$ & 20.20 & 23.02 & \\
\hline 1 & F1 & & & & & 20.20 & 26.67 & $29.27 \%$ \\
\hline D & & & & & & 20.20 & 28.65 & \\
\hline$E$ & & $R \cdot 22>2$ & 4 HRS 302 & & & & & \\
\hline & U -475 & 36.9206 & 36.9429 & $0.060 \%$ & $\mathrm{~N} / \mathrm{C}$ & 40.14 & 31.35 & \\
\hline 1 & A2 & & & & & 40.14 & 11.00 & $-51.71 \%$ \\
\hline$M$ & & & & & & 40.14 & 15.80 & \\
\hline 1 & $Y-390$ & 36.0476 & 36.0726 & $0.069 \%$ & Varnish & 36.12 & 18.52 & \\
\hline$D$ & B2 & & & & Pockets & 36.12 & 10.92 & $-58.71 \%$ \\
\hline$E$ & & & & & & 36.12 & 15.30 & \\
\hline & ER-610 & 37.3783 & 37.3906 & $0.033 \%$ & $\mathrm{~N} / \mathrm{C}$ & 35.96 & 27.80 & \\
\hline$E$ & C2 & & & & & 35.96 & 29.32 & $-19.92 \%$ \\
\hline$P$ & & & & & & 35.96 & 29.27 & \\
\hline 0 & $Y-833$ & 36.7545 & 36.7548 & $0.001 \%$ & $\mathrm{~N} / \mathrm{C}$ & 33.14 & 4.00 & \\
\hline$x$ & D2 & & & & & 33.14 & 14.87 & $-77.00 \%$ \\
\hline$Y$ & & & & & & 33.14 & 4.00 & \\
\hline & 923 & 36.5749 & 36.6066 & $0.087 \%$ & $\mathrm{~N} / \mathrm{C}$ & 40.52 & 20.85 & \\
\hline$G$ & E2 & & & & & 40.52 & 18.00 & $-51.71 \%$ \\
\hline$L$ & & & & & & 40.52 & 19.85 & \\
\hline$A$ & $1 \mathrm{SO}-800$ & 35.4294 & 35.4367 & $0.021 \%$ & $\mathrm{~N} / \mathrm{C}$ & 20.20 & 9.00 & \\
\hline$S$ & F2 & & & & & 20.20 & 8.00 & $-58.75 \%$ \\
\hline S & & & & & & 20.20 & 8.00 & \\
\hline
\end{tabular}

C-6 


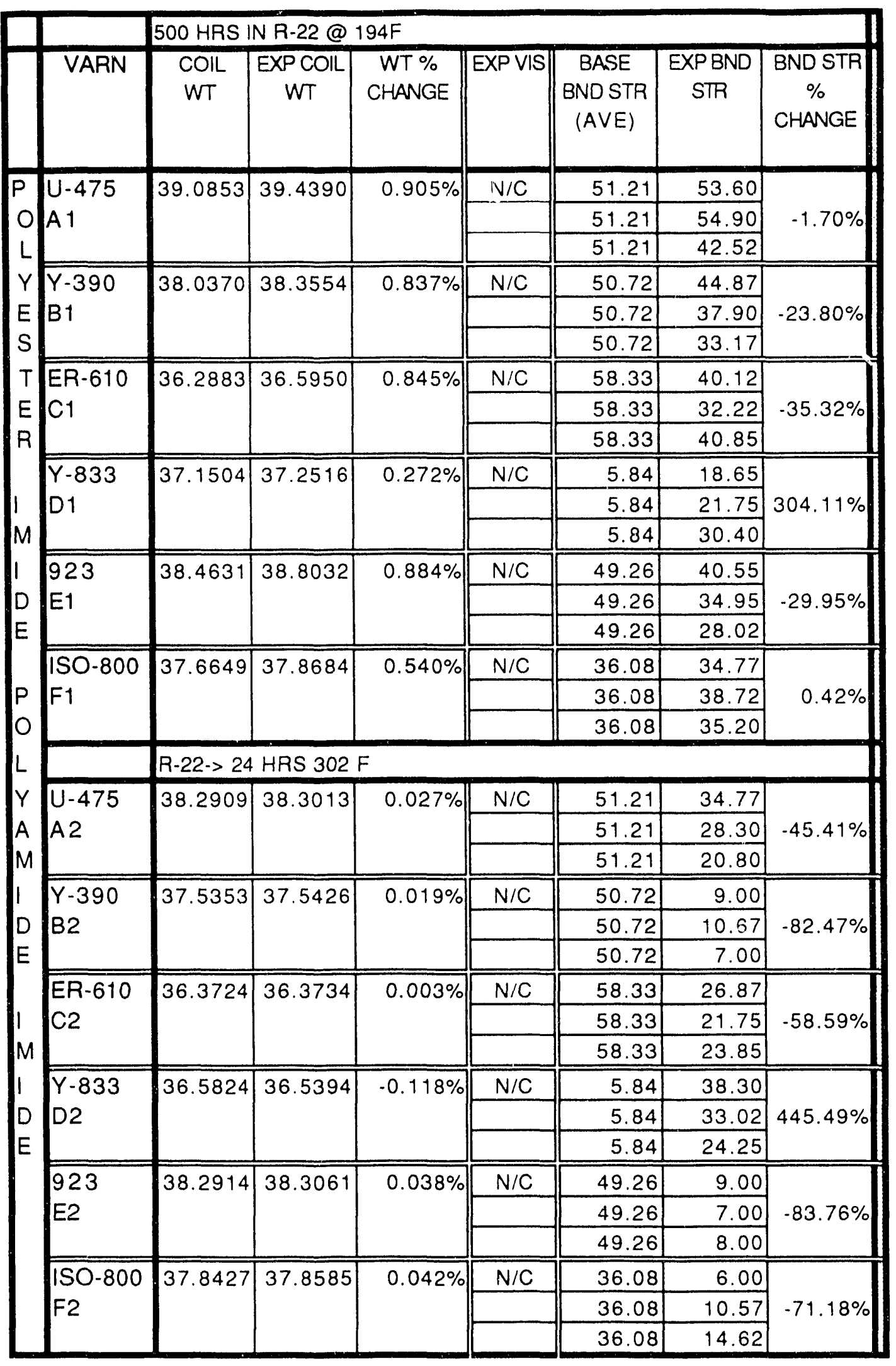




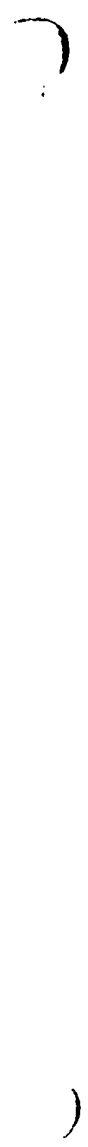

\begin{tabular}{|c|c|c|c|c|c|}
\hline & 500 HRS IN & 2.22@19 & & & \\
\hline ID & WT & EXPWT & $\begin{array}{c}\text { WT \% } \\
\text { CHANGE }\end{array}$ & EXP VIS & EXPFLEX \\
\hline A1 & 5.4558 & 5.4656 & $0.180 \%$ & $\mathrm{~N} / \mathrm{C}$ & \\
\hline & & & & & YES \\
\hline$B 1$ & 5.5924 & 5.6285 & $0.646 \%$ & $\mathrm{~N} / \mathrm{C}$ & \\
\hline & & & & & YES \\
\hline$\overline{c 1}$ & 5.3986 & 5.4115 & $0.239 \%$ & $N / C$ & \\
\hline & & & & & YES \\
\hline & R-22 $>30$ & IF $24 \mathrm{HRS}$ & & & \\
\hline$A 2$ & 5.424 & 5.4224 & $-0.029 \%$ & $\mathrm{~N} / \mathrm{C}$ & \\
\hline & & & & & YES \\
\hline$\overline{\mathrm{B2}}$ & 5.7027 & 5.703 & $0.005 \%$ & $\mathrm{~N} / \mathrm{C}$ & \\
\hline & & & & & YES \\
\hline$\overline{\mathrm{C} 2}$ & 5.4548 & $\overline{5.4526}$ & $-0.040 \%$ & $N / C$ & \\
\hline & & & & & YES \\
\hline & & & & & \\
\hline
\end{tabular}




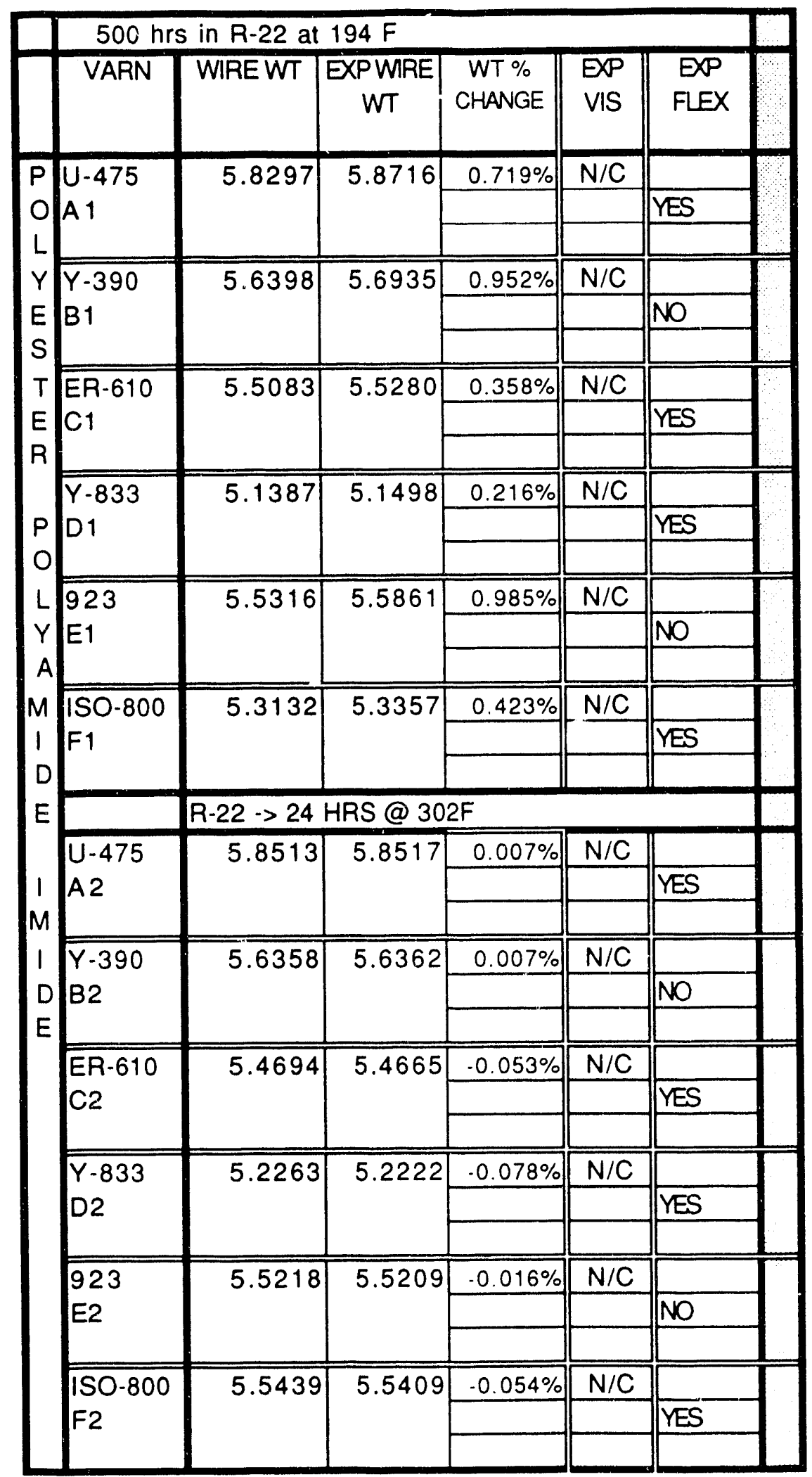




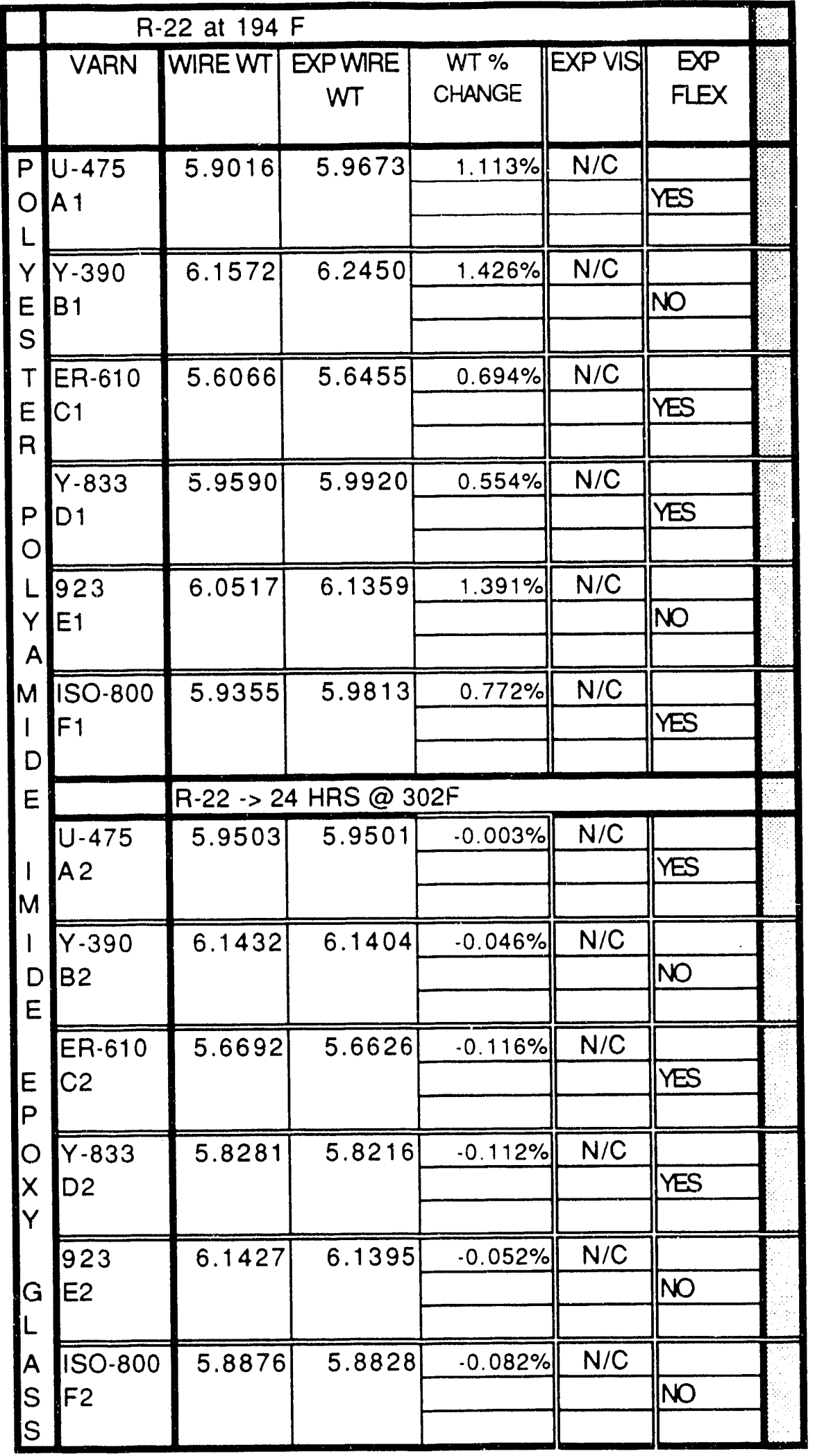




\begin{tabular}{|c|c|c|c|c|c|c|c|}
\hline & & -22 at 194 & & & & & \\
\hline & VARN & WIREWT & $\begin{array}{c}\text { EXPWRE } \\
\text { WT }\end{array}$ & $\begin{array}{c}\text { WT \% } \\
\text { CHANGE }\end{array}$ & |EXPVIS| & $\begin{array}{l}\text { EXP } \\
\text { FLEX }\end{array}$ & \\
\hline$P$ & U-475 & 5.3509 & 5.3913 & $0.755 \%$ & $N / C$ & & \\
\hline 0 & & & & & & YES & \\
\hline L & & & & & & & \\
\hline $\begin{array}{l}Y \\
E\end{array}$ & $Y-390$ & 6.0023 & 6.0676 & $1.088 \%$ & $N / C$ & & \\
\hline $\begin{array}{l}E \\
S\end{array}$ & B1 & & & & & $\mathrm{NO}$ & \\
\hline $\mathrm{T}$ & ER-610 & 5.6873 & 5.7103 & $0.404 \%$ & $\widehat{N / C}$ & & \\
\hline$E$ & & & & & & YES & \\
\hline $\mathrm{m}$ & & & & & & & \\
\hline 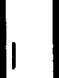 & $\begin{array}{l}Y-833 \\
D 1\end{array}$ & 5.6649 & 5.6790 & $0.249 \%$ & $N / C$ & VES & \\
\hline$M$ & & & & & & & \\
\hline I & 923 & 5.8157 & 5.8774 & $1.061 \%$ & $N / C$ & & \\
\hline D & E1 & & & & & NO & \\
\hline E & & & & & & & \\
\hline & ISO-800 & 5.7820 & 5.8090 & $0.467 \%$ & $N / C$ & & \\
\hline $\mathrm{P}$ & & & & & & YES & \\
\hline LL & & $R-22->24$ & HRS @ 302 & & & & \\
\hline$Y$ & $U-475$ & 5.2919 & 5.2933 & $0.026 \%$ & $N / C$ & & \\
\hline A & A2 & & & & & NO & \\
\hline$M$ & & & & & & & \\
\hline- & $Y-390$ & 6.0783 & 6.0796 & $0.021 \%$ & $N / C$ & & \\
\hline D & & & & & & NNO. & \\
\hline & ER-610 & 5.6561 & 5.6559 & $-0.004 \%$ & $N / C$ & & \\
\hline | & & & & & & YES & \\
\hline$M$ & & & & & & & \\
\hline 1 & $Y-833$ & 5.6405 & 5.6366 & $-0.069 \%$ & $N / C$ & & \\
\hline D & D2 & & & & & YES & \\
\hline & 923 & 5.9076 & 5.9190 & $0.193 \%$ & $N / C$ & & \\
\hline & E2 & & & & & NO & \\
\hline & & & & & & & \\
\hline & ISO-800 & 5.8217 & 5.8199 & $-0.031 \%$ & $N / C$ & & \\
\hline & F2 & & & & & NO & \\
\hline & & & & & & & \\
\hline
\end{tabular}




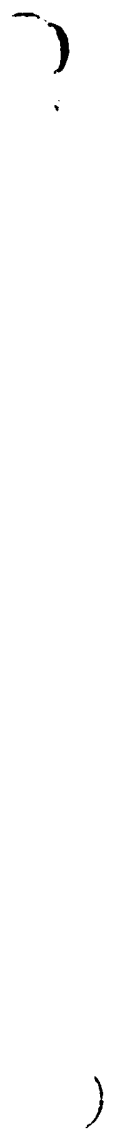

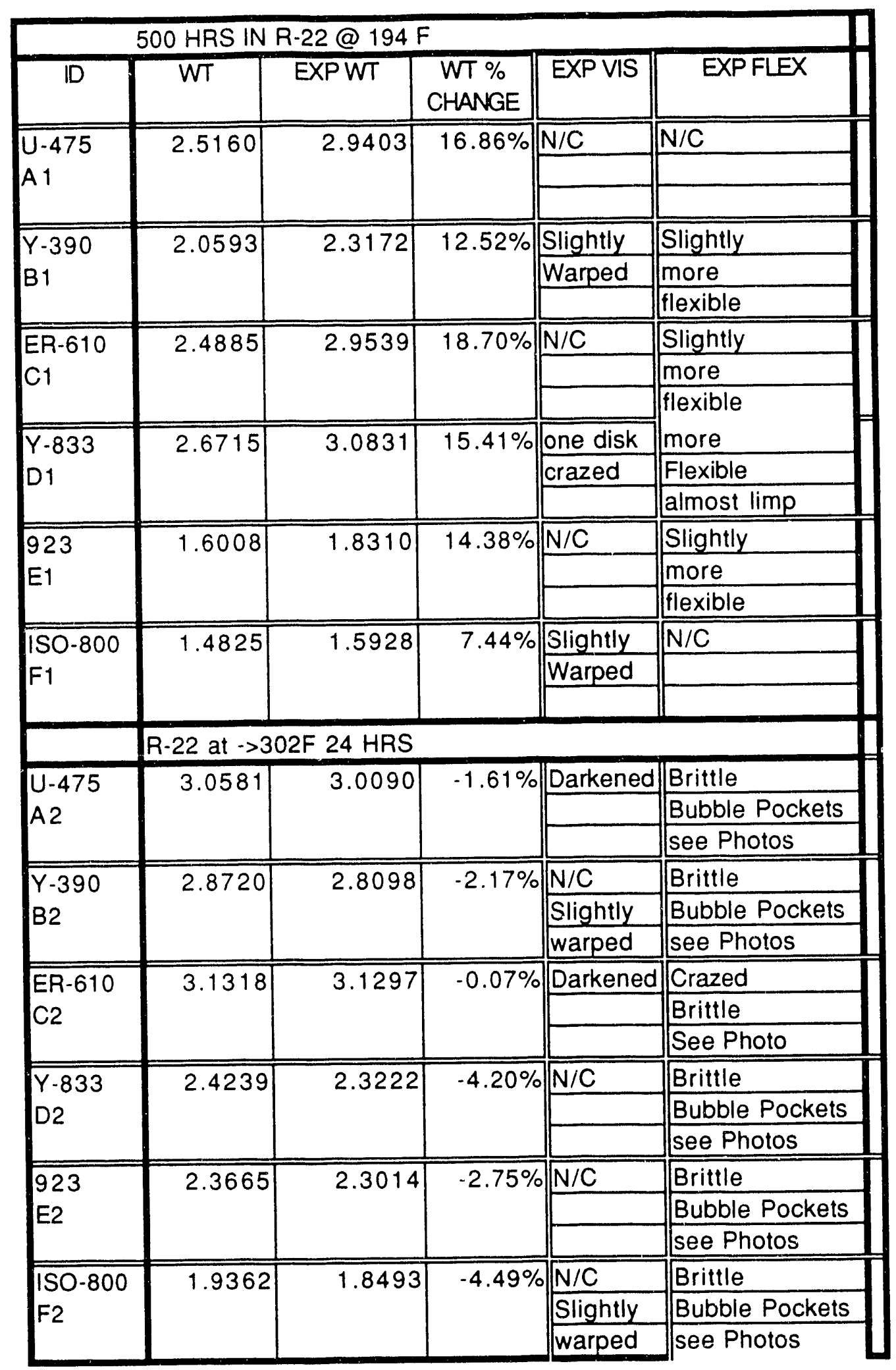




\begin{tabular}{|c|c|c|c|c|c|c|c|c|c|}
\hline & & $500 \mathrm{H}$ & IR IN R-2 & $2 @ 194 \mathrm{~F}$ & & & & & \\
\hline 10 & WT & EXPWT & $\begin{array}{c}\text { WT \% } \\
\text { CHANGE }\end{array}$ & $\begin{array}{l}\text { SAMPLE } \\
\text { WIDTH }\end{array}$ & $\begin{array}{l}\text { BREAK } \\
\text { LOAD }\end{array}$ & $\begin{array}{c}\text { SAMPLE } \\
\text { THICKNESS } \\
\text { Mils }\end{array}$ & $\begin{array}{c}\text { TENSILE } \\
\text { STR } \\
\text { BASE }\end{array}$ & $\begin{array}{l}\text { TENSILE } \\
\text { STREXP }\end{array}$ & $\begin{array}{c}\% \\
\text { CHANGE } \\
\text { TENSILE }\end{array}$ \\
\hline NO/MYINO & 5.2242 & 5.5758 & $6.73 \%$ & 0.442 & 137.80 & 22 & 17.4 & 14.17 & \\
\hline A1 & & & & 0.442 & 137.20 & 22 & 17.4 & 14.11 & $-17.95 \%$ \\
\hline & & & & 0.422 & 135.10 & 22 & 17.4 & 14.55 & \\
\hline$D A / M Y / D A$ & 4.9109 & 5.2170 & $6.23 \%$ & 0.447 & 115.50 & 22 & 13.7 & 11.74 & \\
\hline $\mathrm{B1}$ & & & & 0.435 & 89.90 & 22 & 13.7 & 9.39 & $-23.05 \%$ \\
\hline & & & & 0.481 & 111.00 & 22 & 13.7 & 10.49 & \\
\hline MYLAR MO & 2.7834 & 2.9524 & $6.07 \%$ & 0.498 & 84.45 & 10 & 21.7 & 16.96 & \\
\hline$C_{1}$ & & & & 0.474 & 91.40 & 10 & 21.7 & 19.28 & $-15.09 \%$ \\
\hline & & & & 0.502 & 95.55 & 10 & 21.7 & 19.03 & \\
\hline NO 410 & 2.8119 & 2.9835 & $6.10 \%$ & 0.476 & 85.15 & 11 & 18.7 & 16.26 & \\
\hline D1 & & & & 0.497 & 97.45 & 11 & 18.7 & 17.83 & $-9.60 \%$ \\
\hline & & & & 0.499 & 91.25 & 11 & 18.7 & 16.62 & \\
\hline NO MI 418 & 2.8244 & 2.9051 & $2.86 \%$ & 0.476 & 25.10 & 9 & 7.5 & 5.86 & \\
\hline E1 & & & & 0.498 & 26.45 & 9 & 7.5 & 5.90 & $-21.31 \%$ \\
\hline & & & & 0.500 & 26.75 & 9 & 7.5 & 5.94 & \\
\hline MEL 228 & 2.7182 & 2.8929 & $6.43 \%$ & 0.484 & 85.30 & 11 & 21.7 & 16.02 & \\
\hline $\mathrm{F} 1$ & & & & 0.522 & 85.85 & 11 & 21.7 & 14.95 & $-28.92 \%$ \\
\hline & & & & 0.440 & 74.05 & 11 & 21.7 & 15.30 & \\
\hline & & $500 \mathrm{HRS}$ & $S$ IN R-22 & @194F. & $\rightarrow 24$ HRS & S @ 302 F & & & $R-22 \cdots>3$ \\
\hline NO/MY/NO & 5.0578 & 5.0538 & $-0.08 \%$ & 0.443 & 160.00 & 22 & 17.4 & 16.42 & \\
\hline A2 & & & & 0.413 & 148.10 & 22 & 17.4 & 16.30 & $-4.67 \%$ \\
\hline & & & & 0.428 & 160.50 & 22 & 17.4 & 17.05 & \\
\hline$D A / M Y / D A$ & 5.3272 & 5.2660 & $-1.15 \%$ & 0.370 & 105.90 & 22 & 13.7 & 13.01 & \\
\hline$B 2$ & & & & 0.380 & 108.50 & 22 & 13.7 & 12.98 & $-6.85 \%$ \\
\hline & & & & 0.343 & 92.80 & 22 & 13.7 & 12.30 & \\
\hline MYLAR MO & 2.6565 & 2.6395 & $-0.64 \%$ & 0.425 & 74.10 & 9 & 21.7 & 19.37 & \\
\hline $\mathrm{C} 2$ & & & & 0.478 & 95.15 & 9 & 21.7 & 22.12 & $-1.71 \%$ \\
\hline & & & & 0.471 & 95.35 & 9 & 21.7 & 22.49 & \\
\hline NOMEX 410 & 2.8327 & 2.8511 & $0.65 \%$ & 0.518 & 99.80 & 11 & 18.7 & 17.51 & \\
\hline 02 & & & & 0.528 & 111.60 & 11 & 18.7 & 19.21 & $-1.85 \%$ \\
\hline & & & & 0.483 & 97.40 & 11 & 18.7 & 18.33 & \\
\hline NO/MI 418 & 2.7239 & 2.7331 & $0.34 \%$ & 0.490 & 27.20 & 10 & 7.5 & 5.55 & \\
\hline E2 & & & & 0.516 & 27.40 & 10 & 7.5 & 5.31 & $-27.30 \%$ \\
\hline & & & & 0.493 & 27.10 & 10 & 7.5 & 5.50 & \\
\hline MEL 228 & 2.9914 & 2.9870 & $-0.15 \%$ & 0.424 & 80.80 & 11 & 21.7 & 17.32 & \\
\hline $\mathrm{F} 2$ & & & & 0.483 & 88.55 & 11 & 21.7 & 16.67 & $-20.68 \%$ \\
\hline & & & & 0.510 & 99.00 & 11 & 21.7 & 17.65 & \\
\hline
\end{tabular}




\begin{tabular}{|c|c|c|c|c|c|c|c|c|}
\hline \multicolumn{8}{|c|}{ After 500 hour exposure@194 $\left.@ 190^{\circ} \mathrm{C}\right)$} & \multirow[b]{2}{*}{$\begin{array}{c}\text { VISUAL } \\
\text { EXP }\end{array}$} \\
\hline ID & $\begin{array}{c}\text { STRETCH } \\
\text { (inch) }\end{array}$ & $\begin{array}{c}\% \\
\text { ELONG }\end{array}$ & $\begin{array}{l}\text { BASE } \\
\text { ELONG } \\
\text { (AVE) }\end{array}$ & $\begin{array}{c}\text { ELONG } \\
\% \\
\text { CHANGE }\end{array}$ & $\begin{array}{c}\text { BASE } \\
\text { DIE } \\
(\mathrm{AVE})\end{array}$ & EXP DIE & $\begin{array}{c}\text { DIE \% } \\
\text { CHANGE }\end{array}$ & \\
\hline \multirow{3}{*}{$\begin{array}{l}\mathrm{NO} / \mathrm{MY} / \mathrm{NO} \\
\mathrm{A} 1\end{array}$} & 0.98 & $24.5 \%$ & $20.0 \%$ & & $>18.97$ & $>16.72$ & & \multirow{3}{*}{$\begin{array}{l}\text { bubbles } \\
\text { pulling } \\
\text { away }\end{array}$} \\
\hline & 0.89 & $22.3 \%$ & $20.0 \%$ & $20.8 \%$ & $>18.97$ & $>16.80 \mathrm{f}$ & flash & \\
\hline & 1.03 & $25.8 \%$ & $20.0 \%$ & & $>18.97=$ & $>16.53$ & & \\
\hline \multirow{3}{*}{$\begin{array}{l}\mathrm{DA} / \mathrm{MY} / \mathrm{DA} \\
\mathrm{B} 1\end{array}$} & 0.71 & $35.5 \%$ & $46.0 \%$ & & $>15.27$ & $>13.03$ & & \multirow{3}{*}{$\begin{array}{l}\text { slighlty } \\
\text { warped }\end{array}$} \\
\hline & 0.68 & $34.0 \%$ & $46.0 \%$ & $.3 .6 \%$ & $>15.27$ & $>13.87 f$ & flash & \\
\hline & 0.58 & $29.0 \%$ & $46.0 \%$ & & $>15.27=$ & $>12.64$ & & \\
\hline \multirow{3}{*}{$\begin{array}{l}\text { MYLARMO } \\
\text { C1 }\end{array}$} & 2.16 & $108.0 \%$ & $131.0 \%$ & & $>14.91$ & $>12.81$ & & \multirow[t]{3}{*}{$\mathrm{N} / \mathrm{C}$} \\
\hline & 2.94 & $147.0 \%$ & $131.0 \%$ & $0.8 \%$ & $>14.91$ & $>12.63 \mathrm{f}$ & flash & \\
\hline & 2.82 & $141.0 \%$ & $131.0 \%$ & & $>14.91$ & $>12.08$ & & \\
\hline \multirow{3}{*}{$\begin{array}{l}\text { NO } 410 \\
\text { D1 }\end{array}$} & 0.51 & $12.8 \%$ & $17.0 \%$ & & 10.67 & 11.26 & & \multirow[t]{3}{*}{$\sqrt{N / C}$} \\
\hline & 0.61 & $15.3 \%$ & $17.0 \%$ & $-19.1 \%$ & $i 0.67$ & 9.85 & $-1.6 \%$ & \\
\hline & 0.53 & $13.3 \%$ & $17.0 \%$ & & 10.67 & 10.40 & & \\
\hline \multirow{3}{*}{$\begin{array}{l}\text { NO Ml } 418 \\
\text { E1 }\end{array}$} & 0.08 & $2.0 \%$ & $4.0 \%$ & & 10.23 & 9.65 & & \multirow[t]{3}{*}{$\mathrm{N} / \mathrm{C}$} \\
\hline & 0.08 & $2.0 \%$ & $4.0 \%$ & $.50 .0 \%$ & 10.23 & 10.61 & $-4.0 \%$ & \\
\hline & 0.08 & $2.0 \%$ & $4.0 \%$ & & 10.23 & 9.20 & & \\
\hline \multirow{4}{*}{$\begin{array}{l}\text { MEL } 228 \\
\text { F1 }\end{array}$} & 3.11 & $155.5 \%$ & $160.0 \%$ & & $>14.22$ & $>12.85$ & & \multirow[t]{3}{*}{$\overline{\mathrm{N} / \mathrm{C}}$} \\
\hline & 2.46 & $123.0 \%$ & $160.0 \%$ & $.16 .4 \%$ & $\geq 14.22$ & $>12.60 \mathrm{f}$ & flash & \\
\hline & 2.46 & $123.0 \%$ & $160.0 \%$ & & $>14.22$ & $>13.10$ & & \\
\hline & \multicolumn{7}{|c|}{ After 500 hour exposure plus a 24 hour airbake @ 302 $\mathrm{F}\left(150^{\circ}\right.$} & $\left.{ }^{\circ} \mathrm{C}\right)$. \\
\hline \multirow{3}{*}{$\begin{array}{l}\text { NO/MY/NO } \\
\text { A2 }\end{array}$} & 0.82 & $20.5 \%$ & $20.0 \%$ & & $>18.97$ & $>17.10$ & & \multirow{3}{*}{$\begin{array}{l}\text { bubbles } \\
\text { pulling } \\
\text { away }\end{array}$} \\
\hline & 0.73 & $18.3 \%$ & $20.0 \%$ & $9.6 \%$ & $>18.97$ & $>15.83$ & flash & \\
\hline & 1.08 & $27.0 \%$ & $20.0 \%$ & & $>18.97$ & $>16.17$ & & \\
\hline \multirow{3}{*}{$\begin{array}{l}\mathrm{DA} / \mathrm{MY} / \mathrm{DA} \\
\mathrm{B} 2\end{array}$} & 0.58 & $29.0 \%$ & $46.0 \%$ & & $>15.27$ & $>13.76$ & & \multirow{3}{*}{$\begin{array}{l}\text { slighlty } \\
\text { warped }\end{array}$} \\
\hline & 1.24 & $62.0 \%$ & $46.0 \%$ & $-17.4 \%$ & $\geq 15.27$ & $>13.79$ & flash & \\
\hline & 0.46 & $23.0 \%$ & $46.0 \%$ & & $>15.27$ & $>14.21$ & & \\
\hline \multirow{3}{*}{$\begin{array}{l}\text { MYLARMO } \\
\mathrm{C} 2\end{array}$} & 1.84 & $92.0 \%$ & $131.0 \%$ & & $>14.91$ & $>13.47$ & & \multirow[t]{3}{*}{$\mathrm{N} / \mathrm{C}$} \\
\hline & 2.96 & $148.0 \%$ & $131.0 \%$ & $.0 .6 \%$ & $>14.91$ & $>13.42$ & flash & \\
\hline & 3.01 & $150.5 \%$ & $131.0 \%$ & & $>14.91$ & $>13.82$ & & \\
\hline \multirow{3}{*}{$\begin{array}{l}\text { NOMEX 410 } \\
\text { D2 }\end{array}$} & 0.53 & $13.3 \%$ & $17.0 \%$ & & 10.67 & 10.77 & & \multirow{3}{*}{$\mathrm{N} / \mathrm{C}$} \\
\hline & 0.76 & $19.0 \%$ & $17.0 \%$ & $.4 .4 \%$ & 10.67 & 11.70 & $-0.1 \%$ & \\
\hline & 0.66 & $16.5 \%$ & $17.0 \%$ & & 10.67 & 9.52 & & \\
\hline \multirow{3}{*}{$\begin{array}{l}\text { NO/MI } 418 \\
\text { E2 }\end{array}$} & 0.10 & $2.5 \%$ & $4.0 \%$ & & 10.23 & 8.32 & & \multirow[t]{3}{*}{$\mathrm{N} / \mathrm{C}$} \\
\hline & 0.08 & $2.0 \%$ & $4.0 \%$ & $-43.8 \%$ & 10.23 & 8.60 & $-16.8 \%$ & \\
\hline & 0.09 & $2.3 \%$ & $4.0 \%$ & & 10.23 & 8.60 & & \\
\hline & 3.11 & $155.5 \%$ & $160.0 \%$ & & $>14.22$ & $>14.64$ & & $N / C$ \\
\hline $\mathrm{F} 2$ & 2.92 & $146.0 \%$ & $160.0 \%$ & $-5.6 \%$ & $>14.22$ & $>14.24$ & flash & \\
\hline & 3.03 & $151.5 \%$ & $160.0 \%$ & & $>14.22$ & $>14.12$ & & \\
\hline
\end{tabular}




\section{SLEEVING}

\begin{tabular}{|c|c|c|c|c|}
\hline & \multicolumn{4}{|c|}{500 HRS IN R-22 @ 194F } \\
\hline & WT & EXPWT & $\begin{array}{c}\% \\
\text { CHANGE }\end{array}$ & EXP VIS \\
\hline $\begin{array}{l}\text { A1 } \\
\text { NOMEX }\end{array}$ & 0.5558 & 0.6409 & $15.31 \%$ & $\mathrm{~N} / \mathrm{C}$ \\
\hline $\begin{array}{l}\text { B1 } \\
\text { MYAR }\end{array}$ & 0.4789 & 0.5195 & $8.48 \%$ & Bubbles \\
\hline & 0.3944 & 0.4228 & $7.20 \%$ & $\mathrm{~N} / \mathrm{C}$ \\
\hline & R-22@ @ 194 & $\cdots>24 \mathrm{hrs}$ & @ 302F & \\
\hline $\begin{array}{l}\text { A2 } \\
\text { NOMEX }\end{array}$ & 0.5569 & 0.5619 & $0.90 \%$ & $\mathrm{~N} / \mathrm{C}$ \\
\hline $\begin{array}{l}\text { B2 } \\
\text { MYLAR }\end{array}$ & 0.4564 & 0.4524 & $-0.88 \%$ & $\begin{array}{c}\text { Bubbles } \\
\text { pulled away }\end{array}$ \\
\hline $\begin{array}{l}\mathrm{C} 2 \\
\mathrm{NO} / \mathrm{MY}\end{array}$ & 0.3920 & 0.3871 & $-1.25 \%$ & \begin{tabular}{|c|} 
Pockets where \\
mylar pulled away \\
\end{tabular} \\
\hline
\end{tabular}




\begin{tabular}{|c|c|c|c|c|c|c|c|c|c|}
\hline & \multicolumn{9}{|c|}{500 HRS IN R-22@194 F } \\
\hline $\mathrm{D}$ & WT & EXPWT & $\begin{array}{c}\text { WT \% } \\
\text { CHANGE }\end{array}$ & $\begin{array}{l}\text { BREAK } \\
\text { LOAD } \\
\text { (AVE) }\end{array}$ & $\begin{array}{c}\text { BREAK } \\
\text { LOAD } \\
\text { EPP }\end{array}$ & $\begin{array}{c}\% \\
\text { CHANGE } \\
\text { BPK } \\
\text { LOAD }\end{array}$ & $\begin{array}{c}\text { STRETCH } \\
(\text { INCH })\end{array}$ & $\%$ ELONG & EXPVIS \\
\hline \multirow{3}{*}{$\begin{array}{l}\text { A } 1 \\
\text { Glass }\end{array}$} & \multirow[t]{3}{*}{1.6516} & \multirow[t]{3}{*}{1.6425} & \multirow[t]{3}{*}{$-0.55 \%$} & 39.02 & 46.45 & & 0.03 & $1.50 \%$ & $\mathrm{~N} / \mathrm{C}$ \\
\hline & & & & 39.02 & 60.10 & $35.32 \%$ & 0.05 & $2.50 \%$ & \\
\hline & & & & 39.02 & 51.85 & & 0.04 & $2.00 \%$ & \\
\hline \multirow{3}{*}{$\begin{array}{l}\mathrm{B} 1 \\
\text { Polyester }\end{array}$} & \multirow[t]{3}{*}{0.6986} & \multirow[t]{3}{*}{0.7449} & \multirow[t]{3}{*}{$6.63 \%$} & 56.12 & 59.60 & & 0.73 & $36.50 \%$ & $\mathrm{~N} / \mathrm{C}$ \\
\hline & & & & 56.12 & 64.25 & $6.20 \%$ & 0.82 & $41.00 \%$ & \\
\hline & & & & 56.12 & 54.95 & & 0.54 & $27.00 \%$ & \\
\hline \multirow{4}{*}{$\begin{array}{l}\text { C1 } \\
\text { Permacel }\end{array}$} & \multirow[t]{3}{*}{1.6012} & \multirow[t]{3}{*}{1.6280} & \multirow[t]{3}{*}{$1.67 \%$} & 88.50 & 98.70 & & 0.10 & $5.00 \%$ & Rolled up \\
\hline & & & & 88.50 & 97.55 & $15.12 \%$ & 0.08 & $4.00 \%$ & but unrolled \\
\hline & & & & 88.50 & 109.40 & & 0.09 & $4.50 \%$ & in minutes \\
\hline & \multicolumn{7}{|c|}{500 HRS in R-22 $>302$ F 24 HRS } & & \\
\hline \multirow{3}{*}{$\begin{array}{l}\text { A2 } \\
\text { Glass }\end{array}$} & \multirow[t]{3}{*}{1.5674} & \multirow[t]{3}{*}{1.5812} & \multirow[t]{3}{*}{$0.88 \%$} & 39.02 & 60.15 & & 0.05 & $2.50 \%$ & $\mathrm{~N} / \mathrm{C}$ \\
\hline & & & & 39.02 & 44.80 & $42.15 \%$ & 0.04 & $2.00 \%$ & \\
\hline & & & & 39.02 & 61.45 & & 0.06 & $3.00 \%$ & \\
\hline \multirow{3}{*}{$\begin{array}{l}\mathrm{B} 2 \\
\text { Polyester }\end{array}$} & \multirow[t]{3}{*}{0.6622} & \multirow[t]{3}{*}{0.6598} & \multirow[t]{3}{*}{$-0.36 \%$} & 56.12 & 60.75 & & 0.72 & $36.00 \%$ & $\mathrm{~N} / \mathrm{C}$ \\
\hline & & & & 56.12 & 50.70 & $1.48 \%$ & 0.51 & $25.50 \%$ & \\
\hline & & & & 56.12 & 59.40 & & 0.66 & $33.00 \%$ & \\
\hline \multirow{3}{*}{$\begin{array}{l}\mathrm{C} 2 \\
\text { Permacel }\end{array}$} & \multirow[t]{3}{*}{1.5453} & \multirow[t]{3}{*}{1.4775} & \multirow[t]{3}{*}{$-4.39 \%$} & 88.50 & 96.55 & & 0.08 & $4.00 \%$ & $\mathrm{~N} / \mathrm{C}$ \\
\hline & & & & 88.50 & 124.90 & $25.29 \%$ & 0.10 & $5.00 \%$ & \\
\hline & & & & 88.50 & 111.20 & & 0.09 & $4.50 \%$ & \\
\hline
\end{tabular}


TIE CORDS

\begin{tabular}{|c|c|c|c|c|c|c|c|c|c|}
\hline \multicolumn{10}{|c|}{500 HRS IN R-22@194 F } \\
\hline ID & WT & EXPWT & $\begin{array}{c}\text { WT \% } \\
\text { CHANGE }\end{array}$ & $\begin{array}{l}\text { EXP } \\
\text { VIS }\end{array}$ & $\begin{array}{l}\text { BREAK } \\
\text { LOAD } \\
\text { (AVE) }\end{array}$ & $\begin{array}{c}\text { BREAK } \\
\text { LOAD } \\
\text { EXP } \\
(\text { ave })\end{array}$ & $\begin{array}{c}\% \\
\text { CHANGE } \\
\text { BPK } \\
\text { LOAD }\end{array}$ & $\begin{array}{c}\text { STRETCH } \\
\text { (Inch) }\end{array}$ & $\begin{array}{c}\% \\
\text { ELONG }\end{array}$ \\
\hline \multirow[t]{4}{*}{ A1 } & \multirow[t]{3}{*}{0.3021} & \multirow{3}{*}{0.3225} & \multirow[t]{3}{*}{$6.75 \%$} & $\mathrm{~N} / \mathrm{C}$ & 28.36 & 29.90 & & 0.43 & $21.5 \%$ \\
\hline & & & & & 28.36 & 31.70 & \multirow{2}{*}{$14.77 \%$} & 0.45 & $22.5 \%$ \\
\hline & & & & & 28.36 & 36.05 & & 0.49 & $24.5 \%$ \\
\hline & \multicolumn{9}{|c|}{500 HRS IN R-22 -> 24 HRS @ 302F } \\
\hline \multirow[t]{3}{*}{ A2 } & \multirow[t]{3}{*}{0.2948} & \multirow[t]{3}{*}{0.2939} & \multirow[t]{3}{*}{$-0.305 \%$} & $\mathrm{~N} / \mathrm{C}$ & 28.36 & 24.35 & & 0.53 & $26.5 \%$ \\
\hline & & & & & 28.36 & 28.40 & \multirow[t]{2}{*}{$-1.80 \%$} & 0.4 & $20.0 \%$ \\
\hline & & & & & 28.36 & 30.80 & & 0.45 & $22.5 \%$ \\
\hline
\end{tabular}




\begin{tabular}{|c|c|c|c|c|c|c|c|}
\hline & 500 HRS IN & $1-22 @ 194$ & & & & & \\
\hline ID & WT & EXPWT & $\begin{array}{c}\text { WT \% } \\
\text { CHANGE }\end{array}$ & EXP VIS & $\begin{array}{c}\text { BASE DIE } \\
\text { (AVE) }\end{array}$ & EXPDIE & $\begin{array}{c}\text { DIE\% } \\
\text { CHANGE }\end{array}$ \\
\hline DMD & 4.4054 & 4.4423 & $0.84 \%$ & $\mathrm{~N} / \mathrm{C}$ & 9.61 & 9.81 & \\
\hline A1 1 & & & & & 9.61 & 8.41 & $-4.51 \%$ \\
\hline & & & & & 9.61 & 9.31 & \\
\hline DTMD & 4.1084 & 4.1436 & $0.86 \%$ & $\mathrm{~N} / \mathrm{C}$ & 9.95 & 7.63 & \\
\hline $\mathrm{B1}$ & & & & & 9.95 & 8.91 & $-16.18 \%$ \\
\hline & & & & & 9.95 & 8.48 & \\
\hline & R-22@194 & $\mathrm{F} \rightarrow 24 \mathrm{HRS}$ & @ 302F & & & & \\
\hline DMD & 4.4279 & 4.4188 & $-0.21 \%$ & $N / C$ & 9.61 & 8.32 & \\
\hline A2 & & & & & 9.61 & 10.28 & $1.77 \%$ \\
\hline & & & & & 9.61 & 10.74 & \\
\hline DTMD & 4.1332 & 4.1239 & $-0.23 \%$ & $\mathrm{~N} / \mathrm{C}$ & 9.95 & 8.83 & \\
\hline B2 & & & & & 9.95 & 7.94 & $-15.28 \%$ \\
\hline & & & & & 9.95 & 8.52 & \\
\hline
\end{tabular}




\section{Appendix D}

Experimental Data for HCFC-123 Exposure at $90^{\circ} \mathrm{C}\left(194^{\circ} \mathrm{F}\right)$ 


\begin{tabular}{|c|c|c|c|c|c|c|c|c|c|c|}
\hline & 500 HRS IN & R-123@1 & $94 \mathrm{~F}$ & & & & & & & \\
\hline ID & WT & EXPWT & $\begin{array}{c}\text { WT \% } \\
\text { CHANGE }\end{array}$ & EXP VIS & $\begin{array}{c}\text { BASE BRN } \\
\text { OLT } \\
(A \vee E)\end{array}$ & $\begin{array}{c}\text { EXP BRN } \\
\text { OUT }\end{array}$ & $\begin{array}{c}\text { BRN OUTT } \\
\% \\
\text { CHANGE }\end{array}$ & $\begin{array}{c}\text { BASE DIE } \\
(\text { A } V E)\end{array}$ & EXP DIE & $\begin{array}{c}\text { DIE \% } \\
\text { CHANGE }\end{array}$ \\
\hline A 1 & 23.7354 & 23.7655 & $0.127 \%$ & $\mathrm{~N} / \mathrm{C}$ & 576 & 404 & & 15.80 & 12.60 & \\
\hline & & & & & 576 & 397 & $-28.9 \%$ & 15.80 & 14.19 & $-17.7 \%$ \\
\hline & & & & & 576 & 427 & & 15.80 & 12.23 & \\
\hline B1 & 22.5491 & 22.6785 & $0.574 \%$ & $\mathrm{~N} / \mathrm{C}$ & 736 & 723 & & 11.62 & 11.98 & \\
\hline & & & & & 736 & 724 & $-1.6 \%$ & 11.62 & 12.99 & $7.4 \%$ \\
\hline & & & & & 736 & 726 & & 11.62 & 12.48 & \\
\hline$c 1$ & 22.1161 & 22.1216 & $0.025 \%$ & $\mathrm{~N} / \mathrm{C}$ & 579 & 417 & & 16.58 & 12.76 & \\
\hline & & & & & 579 & 406 & $-29.5 \%$ & 16.58 & 13.57 & $-17.0 \%$ \\
\hline & & & & & 579 & 402 & & 16.58 & 14.96 & \\
\hline & $R-123-->24$ & HRS@30 & $2 F$ & & & & & & & \\
\hline$A 2$ & 23.4865 & 23.4900 & $0.015 \%$ & $\mathrm{~N} / \mathrm{C}$ & 576 & 415 & & 15.80 & 13.83 & \\
\hline & & & & & 576 & 413 & $-28.2 \%$ & 15.80 & 14.40 & $-15.0 \%$ \\
\hline & & & & & 576 & 413 & & 15.80 & 12.07 & \\
\hline$B 2$ & 22.6786 & 22.6687 & $-0.044 \%$ & $\mathrm{~N} / \mathrm{C}$ & 736 & 731 & & 11.62 & 11.90 & \\
\hline & & & & & 736 & 724 & $-0.9 \%$ & 11.62 & 12.62 & $4.6 \%$ \\
\hline & & & & & 736 & 734 & & 11.62 & 11.95 & \\
\hline$\overline{c 2}$ & 21.8389 & 21.8421 & $0.015 \%$ & $\mathrm{~N} / \mathrm{C}$ & 579 & 501 & & 16.58 & 13.92 & \\
\hline & & & & & 579 & 421 & $-22.7 \%$ & 16.58 & 11.42 & $-28.3 \%$ \\
\hline & & & & & 579 & 421 & & 16.58 & 10.33 & \\
\hline
\end{tabular}


500 HOURS IN R-123@194 F

\begin{tabular}{|c|c|c|c|c|c|c|c|c|c|c|c|c|}
\hline & VARN & $\begin{array}{l}\text { TWISTED } \\
\text { PAIR WT }\end{array}$ & $\begin{array}{l}\text { VARN } \\
W T\end{array}$ & $\begin{array}{c}\text { EXP T } \\
\text { PAIR WT }\end{array}$ & $\begin{array}{c}\text { WT \% } \\
\text { CHANGE }\end{array}$ & $\begin{array}{l}\text { EXP } \\
\text { VIS }\end{array}$ & BASE DIE & EXP DIE & $\begin{array}{c}\text { DIE \% } \\
\text { CHANGE }\end{array}$ & $\begin{array}{c}\text { BASE } \\
\text { BURN } \\
\text { OUT }\end{array}$ & $\begin{array}{c}\text { EXP } \\
\text { BURN } \\
\text { OUT }\end{array}$ & $\begin{array}{c}\text { BRNOUT } \\
\% \\
\text { CHANGE }\end{array}$ \\
\hline \multirow{3}{*}{$\begin{array}{l}P \\
O \\
L\end{array}$} & \multirow{3}{*}{$\begin{array}{l}\text { U.475 } \\
\text { A } 1\end{array}$} & \multirow[t]{3}{*}{22.7137} & & \multirow[t]{3}{*}{22.8386} & \multirow[t]{3}{*}{$0.550 \%$} & $\mathrm{~N} / \mathrm{C}$ & 16.24 & 15.19 & & 430 & 249 & \\
\hline & & & & & & & 16.24 & 15.95 & $-1.15 \%$ & 430 & 249 & $-41.40 \%$ \\
\hline & & & & & & & 16.24 & 17.02 & & 430 & 258 & \\
\hline \multirow{3}{*}{$\begin{array}{l}Y \\
E \\
S\end{array}$} & \multirow{3}{*}{$\begin{array}{l}Y-390 \\
B 1\end{array}$} & \multirow[t]{3}{*}{22.6176} & & \multirow[t]{3}{*}{22.8601} & \multirow[t]{3}{*}{$1.072 \%$} & $\mathrm{~N} / \mathrm{C}$ & 18.77 & 13.29 & & 510 & 306 & \\
\hline & & & & & & & 18.77 & 14.60 & $-14.95 \%$ & 510 & 339 & $-36.99 \%$ \\
\hline & & & & & & & 18.77 & 20.00 & & 510 & 319 & \\
\hline \multirow{4}{*}{$\begin{array}{l}T \\
E \\
R\end{array}$} & ER-610 & \multirow[t]{3}{*}{22.2736} & & \multirow[t]{3}{*}{22.3135} & \multirow[t]{3}{*}{$0.179 \%$} & $\mathrm{~N} / \mathrm{C}$ & 15.57 & 15.18 & & 442 & 399 & \\
\hline & \multirow{2}{*}{ C1 } & & & & & & 15.57 & 14.99 & $.8 .95 \%$ & 442 & 403 & $-10.11 \%$ \\
\hline & & & & & & & 15.57 & 12.36 & & 442 & 390 & \\
\hline & $Y-833$ & \multirow[t]{3}{*}{22.6802} & & \multirow[t]{3}{*}{22.6763} & \multirow[t]{3}{*}{$-0.017 \%$} & $\mathrm{~N} / \mathrm{C}$ & 12.04 & 10.54 & & 578 & 453 & \\
\hline \multirow{2}{*}{$\begin{array}{l}P \\
O\end{array}$} & \multirow{2}{*}{ D1 } & & & & & & 12.04 & 15.44 & $13.18 \%$ & 578 & 412 & $-24.91 \%$ \\
\hline & & & & & & & 12.04 & 14.90 & & 578 & 437 & \\
\hline \multirow{3}{*}{$\begin{array}{l}\mathrm{L} \\
Y \\
A\end{array}$} & 923 & \multirow[t]{3}{*}{23.7597} & & \multirow[t]{3}{*}{24.0263} & \multirow[t]{3}{*}{$1.122 \%$} & $N / C$ & 16.76 & 6.22 & & 606 & 289 & \\
\hline & E1 & & & & & & 16.76 & 11.01 & $-44.33 \%$ & 606 & 262 & $-54.29 \%$ \\
\hline & & & & & & & 16.76 & 10.76 & & 606 & 280 & \\
\hline$M$ & $150-800$ & 22.3989 & & 22.4717 & $0.32 .5 \%$ & $\mathrm{~N} / \mathrm{C}$ & 19.08 & 19.77 & & 580 & 277 & \\
\hline 1 & $\mathrm{~F} 1$ & & & & & & 19.08 & 15.92 & $-18.41 \%$ & 580 & 250 & $.56 .55 \%$ \\
\hline$D$ & & & & & & & 19.08 & 11.01 & & 580 & 229 & \\
\hline$F$ & & $R-123 \cdots$ & 24 hour & (a) 302 F & & & & & & & & \\
\hline & U.475 & 22.8372 & & 22.8086 & $-0.125 \%$ & $N / C$ & 16.24 & 13.87 & & 430 & 273 & \\
\hline 1 & $A 2$ & & & & & & 16.24 & 20.00 & $-3.04 \%$ & 430 & 291 & $-35.12 \%$ \\
\hline$M$ & & & & & & & 16.24 & 13.37 & & 430 & 273 & \\
\hline 1 & $Y-390$ & 22.9613 & & 22.9870 & $0.112 \%$ & $\mathrm{~N} / \mathrm{C}$ & 18.77 & 16.85 & & 510 & 395 & \\
\hline$D$ & B2 & & & & & & 18.77 & 12.91 & $-19.91 \%$ & 510 & 281 & $-35.56 \%$ \\
\hline$E$ & & & & & & & 18.77 & 15.34 & & 510 & 310 & \\
\hline & ER-610 & 22.0776 & & 22.0751 & $-0.011 \%$ & $\mathrm{~N} / \mathrm{C}$ & 15.57 & 15.95 & & 442 & 394 & \\
\hline & $\mathrm{C} 2$ & & & & & & 15.57 & 16.43 & $-2.06 \%$ & 442 & 405 & $-9.65 \%$ \\
\hline & & & & & & & 15.57 & 13.37 & & 442 & 399 & \\
\hline & $Y-833$ & 22.6822 & & 22.6676 & $-0.064 \%$ & $\mathrm{~N} / \mathrm{C}$ & 12.04 & 14.27 & & 578 & 470 & \\
\hline & D2 & & & & & & 12.04 & 12.70 & $9.14 \%$ & 578 & 440 & $-24.11 \%$ \\
\hline & & & & & & & 12.04 & 12.45 & & 578 & 406 & \\
\hline & 923 & 23.8838 & & 23.9120 & $0.118 \%$ & $\mathrm{~N} / \mathrm{C}$ & 16.76 & 17.63 & & 606 & 273 & \\
\hline & E2 & & & & & & 16.76 & 14.35 & $-2.96 \%$ & 606 & 269 & $-55.01 \%$ \\
\hline & & & & & & & 16.76 & 16.81 & & 606 & 276 & \\
\hline & $150-800$ & 22.2352 & & 22.2337 & $-0.007 \%$ & $\mathrm{~N} / \mathrm{C}$ & 19.08 & 13.82 & & 580 & 258 & \\
\hline & & & & & & & 19.08 & 13.47 & $-26.92 \%$ & 580 & 294 & $.47 .70 \%$ \\
\hline & & & & & & & 19.08 & 14.54 & & 580 & 358 & \\
\hline
\end{tabular}




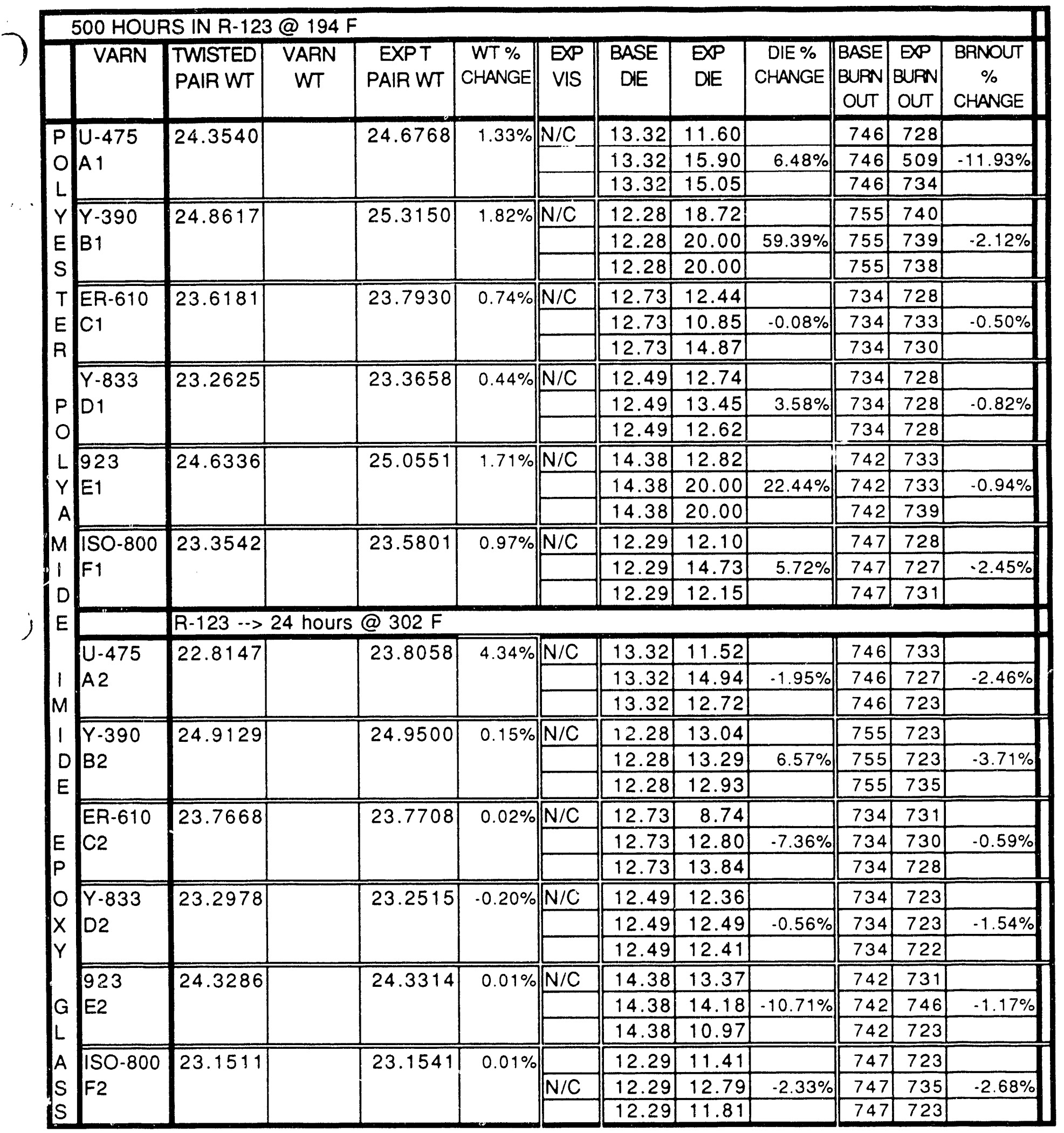


500 HOURS IN R-123@194 F

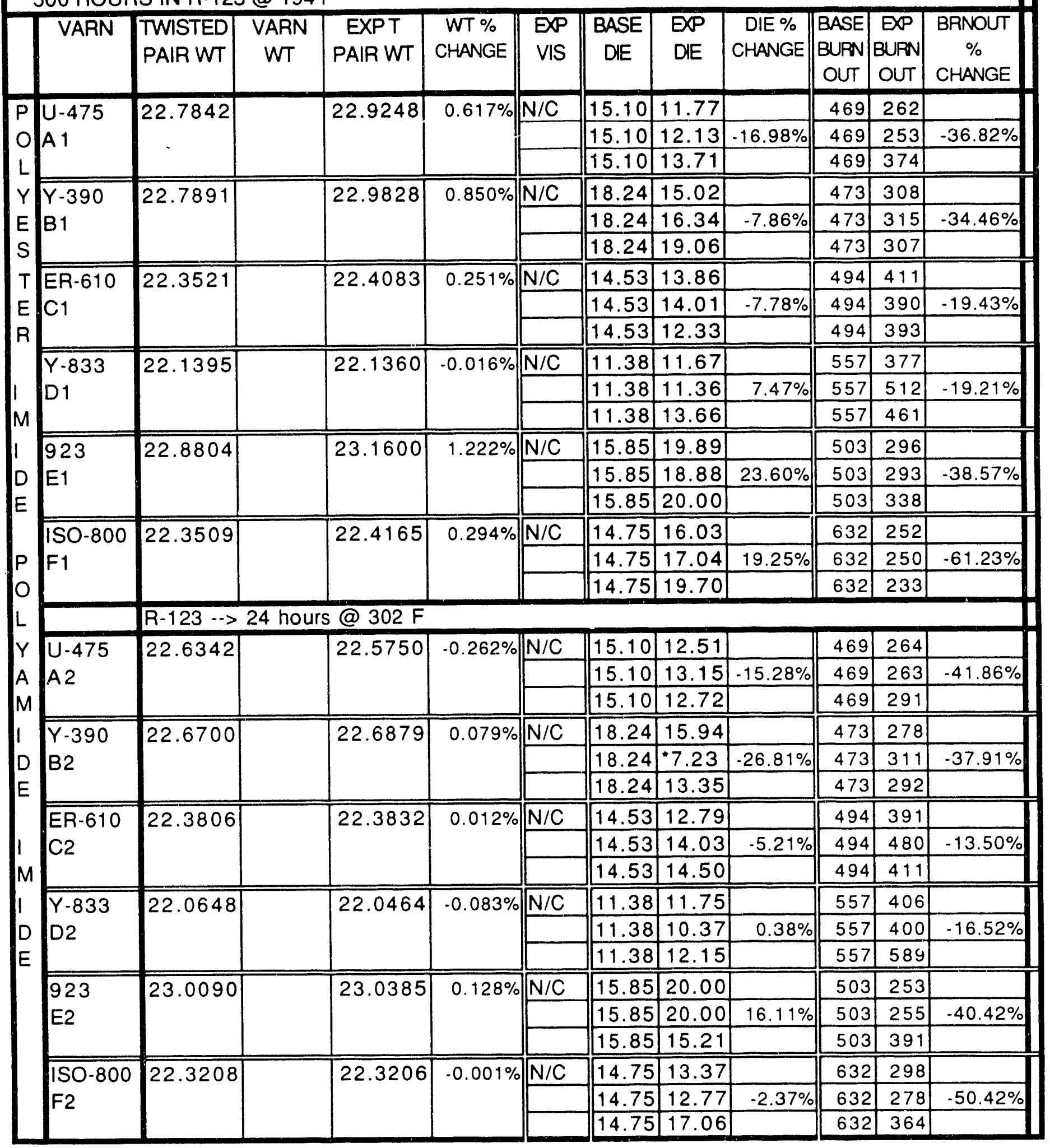

D-4

"Crack in varnish. Didnot use data. 


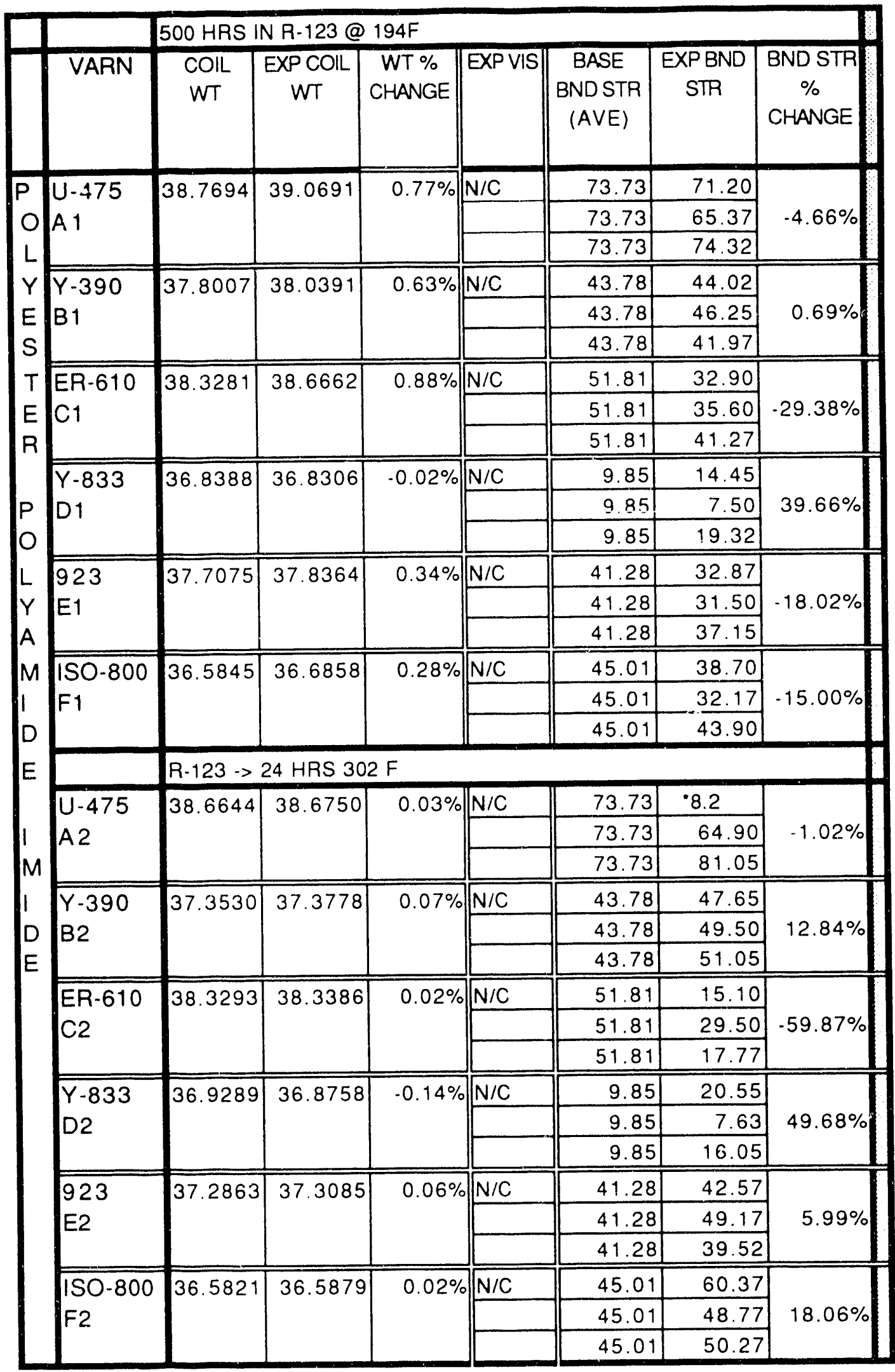

D) -5

-This number was not included in the average 


\begin{tabular}{|c|c|c|c|c|c|c|c|c|}
\hline & & $500 \mathrm{HRS}$ & IN R-123 & $194 \mathrm{~F}$ & & & & \\
\hline & VARN & $\begin{array}{l}\text { COIL } \\
\text { WT }\end{array}$ & $\begin{array}{c}\text { EXP COIL } \\
W T\end{array}$ & $\begin{array}{c}\text { WT \% } \\
\text { CHANGE }\end{array}$ & EXP VIS & $\begin{array}{c}\text { BASE } \\
\text { BND STR } \\
(\text { AVE })\end{array}$ & $\begin{array}{c}\text { EXPBND } \\
\text { STR }\end{array}$ & $\begin{array}{c}\text { BND STR } \\
\% \\
\text { CHANGE }\end{array}$ \\
\hline$P$ & $U-475$ & 36.4002 & 36.8578 & $1.26 \%$ & $\mathrm{~N} / \mathrm{C}$ & 40.14 & 52.77 & \\
\hline 0 & A 1 & & & & & 40.14 & 60.22 & $34.85 \%$ \\
\hline$L$ & & & & & & 40.14 & 49.40 & \\
\hline$Y$ & $Y-390$ & 35.6135 & 36.1703 & $1.56 \%$ & $\mathrm{~N} / \mathrm{C}$ & 36.12 & 48.00 & \\
\hline$E$ & B1 & & & & & 36.12 & 44.35 & $25.51 \%$ \\
\hline$s$ & & & & & & 36.12 & 43.65 & \\
\hline $\mathrm{TT}$ & ER-610 & 36.6823 & 36.9602 & $0.76 \%$ & $\mathrm{~N} / \mathrm{C}$ & 35.96 & 37.85 & \\
\hline E & $\mathrm{C}_{1}$ & & & & & 35.96 & 32.07 & $-4.64 \%$ \\
\hline$R$ & & & & & & 35.96 & 32.95 & \\
\hline & $Y-833$ & 36.3794 & 36.9719 & $1.63 \%$ & $\mathrm{~N} / \mathrm{C}$ & 33.14 & 6.58 & \\
\hline$P$ & D1 & & & & & 33.14 & 9.36 & $.75 .50 \%$ \\
\hline 0 & & & & & & 33.14 & 8.42 & \\
\hline L & 923 & 35.8908 & 36.5263 & $1.77 \%$ & $\mathrm{~N} / \mathrm{C}$ & 40.52 & 43.92 & \\
\hline$Y$ & E1 & & & & & 40.52 & 42.20 & $5.45 \%$ \\
\hline A) & & & & & & 40.52 & 42.07 & \\
\hline MI & 1SO-800 & 34.8244 & 34.9667 & $0.41 \%$ & $\mathrm{~N} / \mathrm{C}$ & 20.20 & 24.75 & \\
\hline 1 & $\mathrm{~F} 1$ & & & & & 20.20 & 23.35 & $5.15 \%$ \\
\hline$D$ & & & & & & 20.20 & 15.62 & \\
\hline$E$ & & R-123 -> & 24 HRS 3 & $2 \mathrm{~F}$ & & & & \\
\hline & $U-475$ & 36.4661 & 36.4817 & $0.04 \%$ & $N / C$ & 40.14 & 15.25 & \\
\hline 1 & $A 2$ & & & & & 40.14 & 22.32 & $-53.02 \%$ \\
\hline$M$ & & & & & & 40.14 & 19.00 & \\
\hline 1 & $Y-390$ & 36.1981 & 36.2055 & $0.02 \%$ & $\mathrm{~N} / \mathrm{C}$ & 36.12 & 9.78 & \\
\hline$D$ & B2 & & & & & 36.12 & 6.50 & $-78.84 \%$ \\
\hline$E$ & & & & & & 36.12 & 6.65 & \\
\hline & ER-610 & 36.0871 & 36.1171 & $0.08 \%$ & $\mathrm{~N} / \mathrm{C}$ & 35.96 & 31.17 & \\
\hline$E$ & $\mathrm{C} 2$ & & & & & 35.96 & 25.27 & $-16.98 \%$ \\
\hline$P$ & & & & & & 35.96 & 33.12 & \\
\hline 0 & $Y-833$ & 36.4580 & 36.4588 & $0.00 \%$ & $\mathrm{~N} / \mathrm{C}$ & 33.14 & 15.57 & \\
\hline$x$ & D2 & & & & & 33.14 & 5.60 & $-69.75 \%$ \\
\hline$Y$ & & & & & & 33.14 & 8.90 & \\
\hline & 923 & 36.4986 & 36.5091 & $0.03 \%$ & $\mathrm{~N} / \mathrm{C}$ & 40.52 & 9.00 & \\
\hline $\mathrm{G}$ & E2 & & & & & 40.52 & 10.00 & $-80.42 \%$ \\
\hline$L$ & & & & & & 40.52 & 4.80 & \\
\hline$A$ & $150-800$ & 34.9478 & 34.9635 & $0.04 \%$ & $\mathrm{~N} / \mathrm{C}$ & 20.20 & 21.60 & \\
\hline$S \mid$ & F2 & & & & & 20.20 & 18.57 & $1.22 \%$ \\
\hline$S$ & & & & & & 20.20 & 21.17 & \\
\hline
\end{tabular}




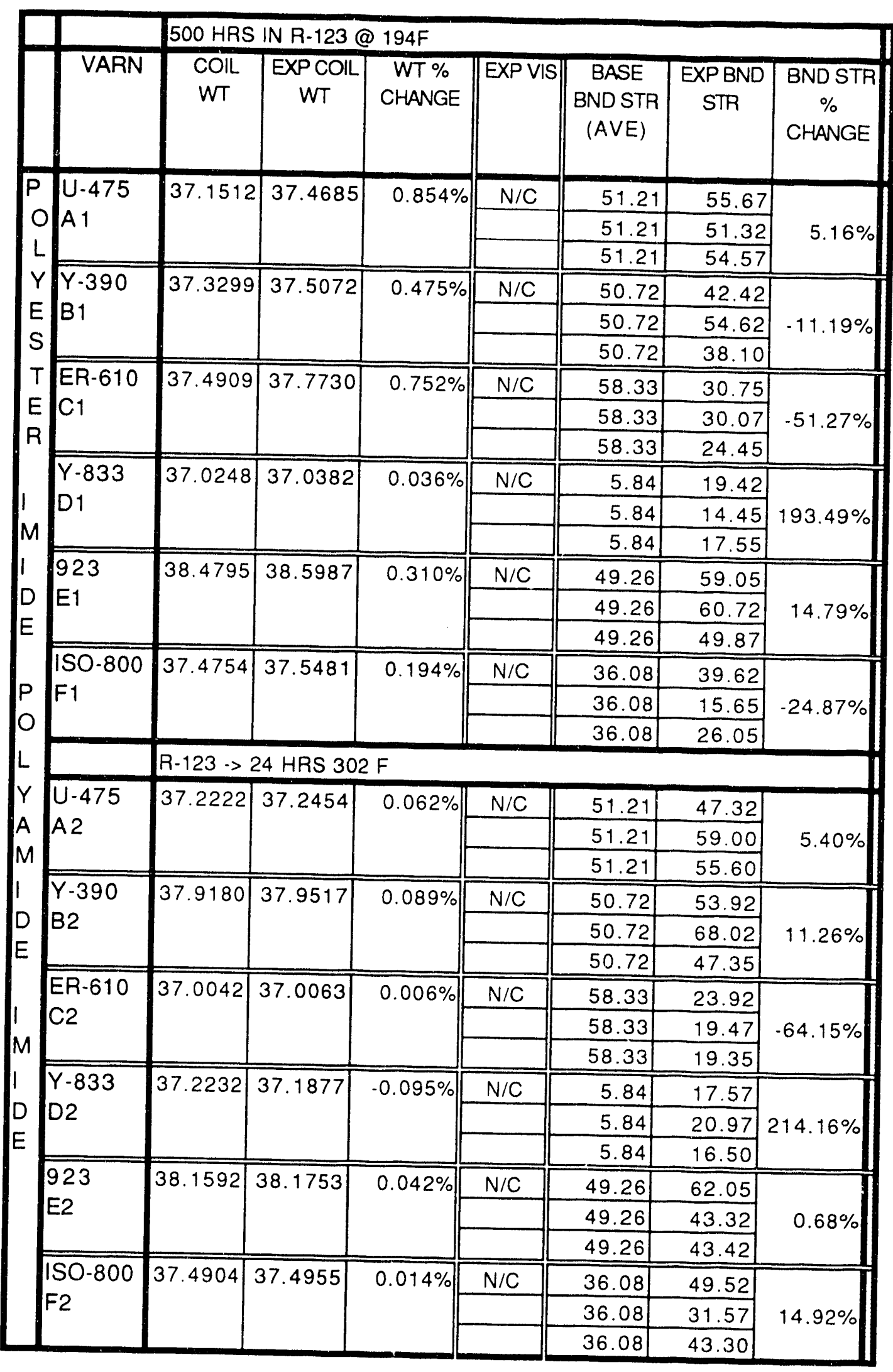




\begin{tabular}{|c|c|c|c|c|c|c|}
\hline & $500 \mathrm{HRS}$ IN & $\mathrm{R}-123 @ 1$ & & & & \\
\hline ID & WT & EXPWT & $\begin{array}{l}\text { WT \% } \\
\text { CHANGE }\end{array}$ & EXP VIS & EXPFLEX & \\
\hline$A 1$ & 5.6564 & 5.6574 & $0.018 \%$ & $\mathrm{~N} / \mathrm{C}$ & & \\
\hline & & & & & YES & \\
\hline $\mathrm{B1}$ & 5.4009 & 54356 & $0.642 \%$ & $N / C$ & & \\
\hline & & & & & YES & \\
\hline & & & & & & \\
\hline $\mathrm{C} 1$ & 5.5316 & 5.5351 & $0.063 \%$ & $\mathrm{~N} / \mathrm{C}$ & & \\
\hline & & & & & YES & \\
\hline & $\mathrm{R}-123-3$ & $20 \mathrm{~F} 24 \mathrm{HR}$ & & & & \\
\hline A? ? & 56678 & $\frac{0<24 \pi}{56659}$ & & Nec & & \\
\hline & 0.0070 & 0.0059 & $-0.034 \%$ & N/L & YES & \\
\hline & & & & & & \\
\hline B2 & 5.4456 & 5.4407 & $-0.090 \%$ & $\mathrm{~N} / \mathrm{C}$ & & \\
\hline & & & & & YES & \\
\hline C2 & 5.4676 & 5.4679 & $0.005 \%$ & $\mathrm{~N} / \mathrm{C}$ & & \\
\hline & & & & & YES & \\
\hline & & & & & & \\
\hline
\end{tabular}




\begin{tabular}{|c|c|c|c|c|c|c|c|c|}
\hline & & & 500 HRS IN & R-123@1 & $194 \mathrm{~F}$ & & & \\
\hline & VARN & WIREWT & VARN WT & $\begin{array}{c}\text { EXPWRE } \\
W T\end{array}$ & $\begin{array}{c}\text { WT \% } \\
\text { CHANGE }\end{array}$ & $\begin{array}{l}\text { EXP } \\
V I S\end{array}$ & $\begin{array}{l}\text { EXP } \\
\text { FLEX }\end{array}$ & \\
\hline $\mathrm{P}$ & $U-475$ & 5.8590 & & 5.9103 & $0.876 \%$ & $\mathrm{~N} / \mathrm{C}$ & & \\
\hline 이 & A 1 & & & & & & YES & \\
\hline L & & & & & & & & \\
\hline $\mathrm{Y}$ & $Y-390$ & 5.9629 & & 6.0131 & $0.842 \%$ & $\mathrm{~N} / \mathrm{C}$ & & \\
\hline $\mathrm{E}$ & & & & & & & NO & \\
\hline s & & & & & & & & \\
\hline $\mathrm{T}$ & ER-610 & 5.7751 & & 5.7845 & $0.163 \%$ & $\mathrm{~N} / \mathrm{C}$ & & \\
\hline$E$ & & & & & & & YES & \\
\hline $\mathrm{R}$ & & & & & & & & \\
\hline & $Y-833$ & 5.6990 & & 5.7065 & $0.132 \%$ & $\mathrm{~N} / \mathrm{C}$ & & \\
\hline$P$ & & & & & & & YES & \\
\hline 0 & & & & & & & & \\
\hline$L$ & $\overline{923}$ & 6.0113 & & 6.0755 & $1.068 \%$ & $\mathrm{~N} / \mathrm{C}$ & & \\
\hline Y & E1 & & & & & & NO & \\
\hline A & & & & & & & & \\
\hline M & ISO-800 & 5.7057 & & 5.7242 & $0.324 \%$ & $\mathrm{~N} / \mathrm{C}$ & & \\
\hline 1 & & & & & & & YES & \\
\hline$D$ & & & & & & & & \\
\hline$E$ & & $R-123>2$ & 24 HRS @ 3 & $302 \mathrm{~F}$ & & & & \\
\hline & U-475 & 5.7712 & & 5.7626 & $-0.149 \%$ & $\mathrm{~N} / \mathrm{C}$ & & \\
\hline 1 & A2 & & & & & & NO & \\
\hline$M$ & & & & & & & & \\
\hline 1 & $Y-390$ & 5.9251 & & 5.9361 & $0.186 \%$ & $\mathrm{~N} / \mathrm{C}$ & & \\
\hline$D$ & & & & & & & NO & \\
\hline$E$ & & & & & & & & \\
\hline & $\mid \begin{array}{l}\text { ER-610 } \\
\mathrm{C} 2\end{array}$ & 5.8035 & & 5.8011 & $-0.041 \%$ & $\mathrm{~N} / \mathrm{C}$ & YYES & \\
\hline & & & & & & & & \\
\hline & $Y-833$ & 5.6618 & & 5.6586 & $-0.057 \%$ & $\mathrm{~N} / \mathrm{C}$ & & \\
\hline & D2 & & & & & & NO & \\
\hline & 923 & 5.9486 & & 5.9600 & $0.192 \%$ & $\mathrm{~N} / \mathrm{C}$ & & \\
\hline & E2 & & & & & & NO & \\
\hline & & & & & & & & \\
\hline & $150-800$ & 5.7947 & & 5.7930 & $-0.029 \%$ & $\mathrm{~N} / \mathrm{C}$ & & \\
\hline & & & & & & & YES & \\
\hline
\end{tabular}

) 


\begin{tabular}{|c|c|c|c|c|c|c|c|}
\hline & & & $00 \mathrm{HRS}$ & VR-123@ & $94 \mathrm{~F}$ & & \\
\hline & VARN & WIREWT & $\begin{array}{l}\text { VARN } \\
\text { WT }\end{array}$ & $\begin{array}{c}\text { EXPWRE } \\
W T\end{array}$ & $\begin{array}{l}\text { WT \% } \\
\text { CHANGE }\end{array}$ & EXP VIS| & $\begin{array}{l}\text { EXP } \\
\text { FLEX }\end{array}$ \\
\hline$P$ & U-475 & 5.7099 & & 5.8056 & $1.676 \%$ & $\mathrm{~N} / \mathrm{C}$ & \\
\hline 이 & A 1 & & & & & & YES \\
\hline & & & & & & & \\
\hline $\mathrm{Y}$ & $Y-390$ & 5.9136 & & 6.0223 & $1.837 \%$ & $\mathrm{~N} / \mathrm{C}$ & \\
\hline $\mathrm{E}$ & B1 & & & & & & NO \\
\hline & & & & & & & \\
\hline$T$ & ER-610 & 5.5336 & & $5.5781 \mid$ & $0.804 \%$ & $\mathrm{~N} / \mathrm{C}$ & \\
\hline$E$ & & & & & & & YES \\
\hline$R$ & & & & & & & \\
\hline & $Y-833$ & 5.5418 & & 5.5840 & $0.761 \%$ & $\mathrm{~N} / \mathrm{C}$ & \\
\hline $\mathrm{P}$ & & & & & & & YES \\
\hline 이 & & & & & & & \\
\hline L & 923 & 5.9358 & & 6.0555 & $2.017 \%$ & $\mathrm{~N} / \mathrm{C}$ & \\
\hline $\mathrm{Y}$ & E1 & & & & & & $\mathrm{NO}$ \\
\hline A & & & & & & & \\
\hline$M$ & $150-800$ & 5.6518 & & 5.7057 & $0.954 \%$ & $\mathrm{~N} / \mathrm{C}$ & \\
\hline 1 & & & & & & & YES \\
\hline D & & & & & & & \\
\hline$E$ & & $R-123>2$ & 4 HRS & $302 \mathrm{~F}$ & & & \\
\hline & U-475 & 5.6653 & & 5.6563 & $-0.159 \%$ & $\mathrm{~N} / \mathrm{C}$ & \\
\hline 1 & A2 2 & & & & & & NO \\
\hline$M$ & & & & & & & \\
\hline 1 & $Y-390$ & 5.7697 & & 5.7865 & $0.291 \%$ & $\mathrm{~N} / \mathrm{C}$ & \\
\hline D & B2 & & & & & & NO \\
\hline E & & & & & & & \\
\hline & ER-610 & 5.6574 & & 5.6540 & $-0.060 \%$ & $\mathrm{~N} / \mathrm{C}$ & \\
\hline E & & & & & & & YES \\
\hline $\mathrm{P}$ & & & & & & & \\
\hline 0 & Y-833 & 5.6218 & & 5.6215 & $-0.005 \%$ & $\mathrm{~N} / \mathrm{C}$ & \\
\hline $\mid \begin{array}{l}x \\
2\end{array}$ & D2 & & & & & & YES \\
\hline$Y$ & & & & & & & \\
\hline & 923 & 5.8970 & & 5.9089 & $0.202 \%$ & $\mathrm{~N} / \mathrm{C}$ & \\
\hline$G$ & E2 & & & & & & NO \\
\hline L & & & & & & & \\
\hline$A$ & ISO-800 & 5.5673 & & 5.5686 & $0.023 \%$ & $\mathrm{~N} / \mathrm{C}$ & \\
\hline$S$ & $\mathrm{~F} 2$ & & & & & & NO \\
\hline S & & & & & & & \\
\hline
\end{tabular}




\begin{tabular}{|c|c|c|c|c|c|c|c|c|}
\hline & & & 500 HRS IN & 3-123@194 & & & & \\
\hline & VARN & WIRE WT & VARN WT & $\begin{array}{c}\text { EXPWRE } \\
W T\end{array}$ & $\begin{array}{l}\text { WT \% } \\
\text { CHANGE }\end{array}$ & EXPVIS & $\begin{array}{l}\text { EXP } \\
\text { FLEX }\end{array}$ & \\
\hline$P$ & U-475 & 5.6813 & & 5.7321 & $0.894 \%$ & $N / C$ & & \\
\hline 이 & A 1 & & & & & & YES & \\
\hline $\mathrm{L}$ & & & & & & & & \\
\hline Y & $Y-390$ & 5.8165 & & 5.8778 & $1.054 \%$ & $N / C$ & & \\
\hline$E$ & & & & & & & YES & \\
\hline$s$ & & & & & & & & \\
\hline$T$ & ER-610 & 5.5091 & & 5.5147 & $0.102 \%$ & $N / C$ & & \\
\hline$E$ & C1 & & & & & & YES & \\
\hline 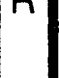 & $Y-833$ & 5.5816 & & 5.5900 & $0.150 \%$ & $\| N / C$ & & \\
\hline 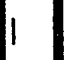 & & & & & & & NO & \\
\hline M & & & & & & & & \\
\hline 1 & 923 & 5.7696 & & 5.8405 & $1.229 \%$ & $\mathrm{~N} / \mathrm{C}$ & & \\
\hline D & E1 & & & & & & NO & \\
\hline & ISO-800 & 5.5894 & & 5.6052 & $0.283 \%$ & $N / C$ & & \\
\hline $\mathrm{P}$ & & & & & & & NO & \\
\hline 0 & & & & & & & & \\
\hline L & & $R-123 \rightarrow 24$ & HRS @ 30 & & & & & \\
\hline$Y$ & U-475 & 5.6784 & & 5.6746 & $-0.067 \%$ & $N / C$ & & \\
\hline A & & & & & & & NO & \\
\hline M & & & & & & & & \\
\hline 10 & $Y-390$ & 5.6971 & & 5.7057 & $0.151 \%$ & $N / C$ & & \\
\hline$D$ & & & & & & & NO & \\
\hline ᄃ & & 55242 & & 5.5213 & $-0.052 \%$ & $\| N / C$ & & \\
\hline 1 & $\begin{array}{ll}\mathrm{E} 2 \mathrm{~B} \\
\mathrm{C} 2\end{array}$ & $0.5<4<1$ & & $0.5<101$ & & & YES & \\
\hline$M$ & & & & & & & & \\
\hline 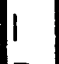 & $\begin{array}{l}-833 \\
\end{array}$ & 5.5245 & & 5.5244 & $-0.002 \%$ & $\mathrm{~N} / \mathrm{C}$ & & \\
\hline$D$ & & & & & & & NNO & \\
\hline & 923 & 5.7992 & & 5.8093 & $0.174 \%$ & $N / C$ & & \\
\hline & E2 & & & & & & NO & \\
\hline & ISO-800 & 55757 & & 5.5760 & $0.005 \%$ & $N / C$ & & \\
\hline & & & & & & & NO & \\
\hline & & & & & & & & \\
\hline
\end{tabular}

) 


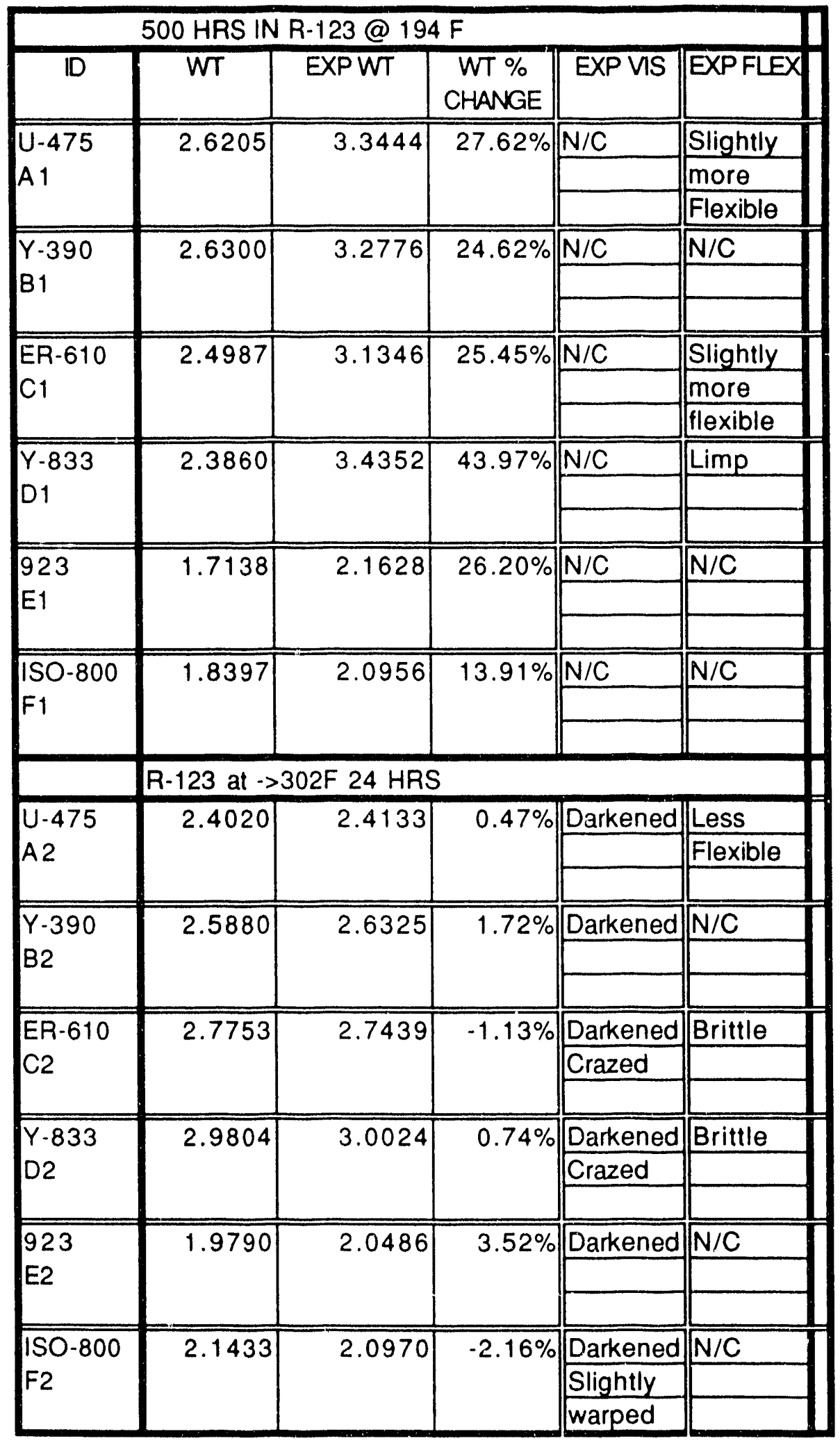




\begin{tabular}{|c|c|c|c|c|c|c|c|c|c|}
\hline & & 500 & IN R-1 & $33 @ 194$ & & & & & \\
\hline ID & WT & EXPWT & $\begin{array}{c}\text { WT \% } \\
\text { CHANGE }\end{array}$ & $\begin{array}{c}\text { SAMPLE } \\
\text { WIDTH }\end{array}$ & $\begin{array}{l}\text { BREAK } \\
\text { LOAD }\end{array}$ & $\begin{array}{c}\text { SAMPLE } \\
\text { THICKNESS } \\
\text { Mils }\end{array}$ & $\begin{array}{c}\text { TENSILE } \\
\text { STR } \\
\text { BASE }\end{array}$ & $\begin{array}{l}\text { TENSILE } \\
\text { STREXP }\end{array}$ & $\begin{array}{c}\% \\
\text { CHANGE } \\
\text { TENSILE }\end{array}$ \\
\hline NO/MY/NO & 4.9226 & 5.9564 & $21.00 \%$ & 0.394 & 123.00 & 22 & 17.4 & 14.19 & \\
\hline$A 1$ & & & & 0.425 & 130.60 & 22 & 17.4 & 13.96 & $-18.98 \%$ \\
\hline & & & & 0.470 & 146.20 & 22 & 17.4 & 14.14 & \\
\hline$D A / M Y / D A$ & 4.4844 & 4.8280 & $7.66 \%$ & 0.374 & 85.95 & 23 & 13.7 & 9.99 & \\
\hline B1 & & & & 0.460 & 108.70 & 23 & 13.7 & 10.27 & $-25.07 \%$ \\
\hline & & & & 0.460 & 111.40 & 23 & 13.7 & 10.53 & \\
\hline MYLAR MO & 2.3394 & 2.5315 & $8.21 \%$ & 0.410 & 91.75 & 10 & 21.7 & 22.38 & \\
\hline $\mathrm{C} 1$ & & & & 0.494 & 89.10 & 10 & 21.7 & 18.04 & $-7.66 \%$ \\
\hline & & & & 0.486 & 95.75 & 10 & 21.7 & 19.70 & \\
\hline NO 410 & 2.3193 & 2.6688 & $15.07 \%$ & 0.484 & 86.25 & 11 & 18.7 & 16.20 & \\
\hline D1 & & & & 0.529 & 97.20 & 11 & 18.7 & 16.70 & $-12.50 \%$ \\
\hline & & & & 0.507 & 90.25 & 11 & 18.7 & 16.18 & \\
\hline NO Ml 418 & 2.3454 & 2.3625 & $0.73 \%$ & 0.488 & 27.40 & 10 & 7.5 & 5.61 & \\
\hline E1 & & & & 0.512 & 29.80 & 10 & 7.5 & 5.82 & $-23.85 \%$ \\
\hline & & & & 0.473 & 26.95 & 10 & 7.5 & 5.70 & \\
\hline MEL 228 & 2.2604 & 2.4439 & $8.12 \%$ & 0.394 & 75.25 & 11 & 21.7 & 17.36 & \\
\hline $\mathrm{F} 1$ & & & & 0.486 & 95.25 & 11 & 21.7 & 17.82 & $-20.15 \%$ \\
\hline & & & & 0.487 & 90.00 & 11 & 21.7 & 16.80 & \\
\hline & & $500 \mathrm{HR}$ & IN R-12: & $3 @ 194$ & $=->24 \mathrm{HP}$ & 35@302 F & & & \\
\hline NO/MY/NO & 5.0617 & 5.0416 & $-0.40 \%$ & 0.376 & 135.50 & 22 & 17.4 & 16.38 & \\
\hline A2 & & & & 0.465 & 170.00 & 22 & 17.4 & 16.62 & $-7.08 \%$ \\
\hline & & & & 0.501 & 170.90 & 22 & 17.4 & 15.51 & \\
\hline$D A / M Y / D A$ & 4.5162 & 4.4576 & $-1.30 \%$ & 0.370 & 102.70 & 22 & 13.7 & 12.62 & \\
\hline$B 2$ & & & & 0.411 & 113.10 & 22 & 13.7 & 12.51 & $-7.23 \%$ \\
\hline & & & & 0.446 & 127.60 & 22 & 13.7 & 13.00 & \\
\hline MYLAR MO & 2.2684 & 2.2583 & $-0.45 \%$ & 0.418 & 78.30 & 10 & 21.7 & 18.73 & \\
\hline $\mathrm{C} 2$ & & & & 0.515 & 100.50 & 10 & 21.7 & 19.51 & $-11.52 \%$ \\
\hline & & & & 0.497 & 96.20 & 10 & 21.7 & 19.36 & \\
\hline NOMEX 410 & 2.4916 & 2.4942 & $0.10 \%$ & 0.500 & 97.45 & 10 & 18.7 & 19.49 & \\
\hline D2 & & & & 0.510 & 103.10 & 10 & 18.7 & 20.22 & $4.18 \%$ \\
\hline & & & & 0.480 & 89.95 & 10 & 18.7 & 18.74 & \\
\hline $\mathrm{NO} / \mathrm{MI} 418$ & 2.3845 & 2.3851 & $0.03 \%$ & 0.485 & 28.45 & 10 & 7.5 & 5.87 & \\
\hline E2 & & & & 0.521 & 28.80 & 10 & 7.5 & 5.53 & $-22.67 \%$ \\
\hline & & & & 0.492 & 29.55 & 10 & 7.5 & 6.01 & \\
\hline MEL 228 & 2.4424 & 2.4380 & $-0.18 \%$ & 0.482 & 93.00 & 10 & 21.7 & 19.29 & \\
\hline $\mathrm{F} 2$ & & & & 0.485 & 92.15 & 10 & 21.7 & 19.00 & $-15.36 \%$ \\
\hline & & & & 0.481 & 80.85 & 10 & 21.7 & 16.81 & \\
\hline
\end{tabular}




\begin{tabular}{|c|c|c|c|c|c|c|c|c|}
\hline & After 500 & hour ex & sure to & HCFC- 1 & 23@19 & ${ }^{\circ} \mathrm{F}\left(90^{\circ} \mathrm{C}\right.$ & & \\
\hline 10 & $\begin{array}{c}\text { STRETCH } \\
\text { (inch) }\end{array}$ & $\begin{array}{c}\% \\
\text { ELONG }\end{array}$ & $\begin{array}{l}\text { BASE } \\
\text { ELONG } \\
\text { (AVE) }\end{array}$ & $\begin{array}{c}\text { ELONG } \\
\% \\
\text { CHANGE }\end{array}$ & $\begin{array}{c}\text { BASE } \\
\text { DIE } \\
(A \vee E)\end{array}$ & EXPDIE & $\begin{array}{c}\text { DIE \% } \\
\text { CHANGE }\end{array}$ & $\begin{array}{l}\text { VISUAL } \\
\text { EXP }\end{array}$ \\
\hline $\mathrm{NO} / \mathrm{MY} / \mathrm{NO}$ & 0.56 & $14.0 \%$ & $20.0 \%$ & & $>18.97$ & $>18.21$ & & $\mathrm{~N} / \mathrm{C}$ \\
\hline A1 & 0.79 & $19.8 \%$ & $20.0 \%$ & $-12.9 \%$ & $>18.97$ & $>18.69$ & flash & \\
\hline & 0.74 & $18.5 \%$ & $20.0 \%$ & & $>18.97$ & $>18.94$ & & \\
\hline$D A / M Y / D A$ & 0.50 & $25.0 \%$ & $46.0 \%$ & & $\geq 15.27$ & $>15.14$ & & $\mathrm{~N} / \mathrm{C}$ \\
\hline B1 & 0.53 & $26.5 \%$ & $46.0 \%$ & $-40.6 \%$ & $>15.27$ & $>15.64$ & flash & \\
\hline & 0.61 & $30.5 \%$ & $46.0 \%$ & & $>15.27$ & $>14.97$ & & \\
\hline MYLAR MO & 2.19 & $109.5 \%$ & $131.0 \%$ & & $>14.91$ & $>14.19$ & & $\mathrm{~N} / \mathrm{C}$ \\
\hline $\mathrm{C} 1$ & 2.22 & $111.0 \%$ & $131.0 \%$ & $-8.5 \%$ & $>14.91$ & $>14.3$ & flash & \\
\hline & 2.78 & $139.0 \%$ & $131.0 \%$ & & $>14.91$ & $>14.97$ & & \\
\hline NO 410 & 0.47 & $11.8 \%$ & $17.0 \%$ & & 10.67 & 12.30 & & $\mathrm{~N} / \mathrm{C}$ \\
\hline D1 & 0.52 & $13.0 \%$ & $17.0 \%$ & $-30.9 \%$ & 10.67 & 11.27 & $7.8 \%$ & \\
\hline & 0.42 & $10.5 \%$ & $17.0 \%$ & & 10.67 & 10.94 & & \\
\hline NO Ml 418 & 0.05 & $1.3 \%$ & $4.0 \%$ & & 10.23 & 10.79 & & $\mathrm{~N} / \mathrm{C}$ \\
\hline E1 & 0.06 & $1.5 \%$ & $4.0 \%$ & $-60.4 \%$ & 10.23 & 9.65 & $2.9 \%$ & \\
\hline & 0.08 & $2.0 \%$ & $4.0 \%$ & & 10.23 & 11.15 & & \\
\hline MEL 228 & 3.24 & $162.0 \%$ & $160.0 \%$ & & $\geq 14.22$ & $>13.94$ & & $\mathrm{~N} / \mathrm{C}$ \\
\hline $\mathrm{F} 1$ & 3.54 & $177.0 \%$ & $160.0 \%$ & $3.9 \%$ & $>14.22$ & $>13.36$ & flash & \\
\hline & 3.19 & $159.5 \%$ & $160.0 \%$ & & $>14.22$ & $>13.64$ & & \\
\hline & After 500 & hour ex & josure plu & us 24 ho & ur airbak & re@19 & $4^{\circ} \mathrm{F}\left(90^{\circ} \mathrm{C}\right)$ & \\
\hline NO/MY/NO & 0.64 & $16.0 \%$ & $20.0 \%$ & & $>18.97$ & $>17.93$ & & Bubbles \\
\hline $\mathrm{A} 2$ & 0.78 & $19.5 \%$ & $20.0 \%$ & $-20.4 \%$ & $\geq 18.97$ & $>17.24$ & flash & pulled a \\
\hline & 0.49 & $12.3 \%$ & $20.0 \%$ & & $>18.97$ & $>16.51$ & & away" \\
\hline$D A / M Y / D A$ & 0.49 & $24.5 \%$ & $46.0 \%$ & & $\geq 15.27$ & $>13.70$ & & warped \\
\hline $\mathrm{B2}$ & 0.49 & $24.5 \%$ & $46.0 \%$ & $-43.1 \%$ & $>15.27$ & $>13.94$ & flash & \\
\hline & 0.59 & $29.5 \%$ & $46.0 \%$ & & $>15.27$ & $>14.94$ & & \\
\hline MYLAR MO & 2.79 & $139.5 \%$ & $131.0 \%$ & & $>14.91$ & $>14.74$ & & $\mathrm{~N} / \mathrm{C}$ \\
\hline C2 & 3.09 & $154.5 \%$ & $131.0 \%$ & $13.5 \%$ & $\geq 14.91$ & $>14.14$ & flash & \\
\hline & 3.04 & $152.0 \%$ & $131.0 \%$ & & $>14.91$ & $>14.94$ & & \\
\hline NOMEX 410 & 0.49 & $12.3 \%$ & $17.0 \%$ & & 10.67 & 10.10 & & $\mathrm{~N} / \mathrm{C}$ \\
\hline D2 & 0.49 & $12.3 \%$ & $17.0 \%$ & $.32 .4 \%$ & 10.67 & 11.31 & $1.0 \%$ & \\
\hline & 0.40 & $10.0 \%$ & $17.0 \%$ & & 10.67 & 10.91 & & \\
\hline $\mathrm{NO} / \mathrm{MI} 418$ & 0.06 & $1.5 \%$ & $4.0 \%$ & & 10.23 & 10.19 & & $\mathrm{~N} / \mathrm{C}$ \\
\hline E2 & 0.04 & $1.0 \%$ & $4.0 \%$ & $-66.7 \%$ & 10.23 & 9.80 & $-4.9 \%$ & \\
\hline & 0.06 & $1.5 \%$ & $4.0 \%$ & & 10.23 & 9.19 & & \\
\hline MEL 228 & 3.42 & $171.0 \%$ & $160.0 \%$ & & $\geq 14.22$ & $>13.94$ & & $\mathrm{~N} / \mathrm{C}$ \\
\hline $\mathrm{F} 2$ & 3.37 & $168.5 \%$ & $160.0 \%$ & $-7.8 \%$ & $>14.22$ & $>14.54$ & flash & \\
\hline & 2.06 & $103.0 \%$ & $160.0 \%$ & & $>14.22$ & $>14.64$ & & \\
\hline
\end{tabular}




\begin{tabular}{|c|c|c|c|c|}
\hline & \multicolumn{4}{|c|}{500 HRS IN R-123@194F } \\
\hline & WT & EXPWT & $\begin{array}{c}\% \\
\text { CHANGE }\end{array}$ & EXP VIS \\
\hline $\begin{array}{l}\text { A1 } \\
\text { NOMEX }\end{array}$ & 0.5521 & 0.8661 & $56.87 \%$ & $\mathrm{~N} / \mathrm{C}$ \\
\hline $\begin{array}{l}\text { B1 } \\
\text { MYLAR }\end{array}$ & 0.5001 & 0.6193 & $23.84 \%$ & $\mathrm{~N} / \mathrm{C}$ \\
\hline C1 & 0.3953 & 0.4845 & $22.57 \%$ & $\mathrm{~N} / \mathrm{C}$ \\
\hline & R-123@19 & $\mathrm{F} \cdots \cdots>24 \mathrm{hrs}$ & s@302F & \\
\hline $\begin{array}{l}\text { A2 } \\
\text { NOMEX }\end{array}$ & 0.5453 & 0.5264 & $-3.47 \%$ & Darkened \\
\hline $\begin{array}{l}\text { B2 } \\
\text { MYLAR }\end{array}$ & 0.4608 & 0.4533 & $-1.63 \%$ & \begin{tabular}{|c|} 
Darkened \\
Warped \\
\end{tabular} \\
\hline $\begin{array}{l}\mathrm{C} 2 \\
\mathrm{NO} / \mathrm{MY}\end{array}$ & 0.3935 & 0.3832 & $-2.62 \%$ & \begin{tabular}{|c|} 
Darkened \\
Warped \\
\end{tabular} \\
\hline
\end{tabular}




\begin{tabular}{|c|c|c|c|c|c|c|c|c|c|}
\hline & \multicolumn{9}{|c|}{500 HRS INR-123@194F } \\
\hline $\mathrm{D}$ & WT & EXPWT & $\begin{array}{c}\text { WT \% } \\
\text { CHANGE }\end{array}$ & $\begin{array}{l}\text { BREAK } \\
\text { LOAD } \\
\text { (AVE) }\end{array}$ & $\begin{array}{l}\text { BREAK } \\
\text { LOAD } \\
\text { EPP }\end{array}$ & $\begin{array}{c}\% \\
\text { CHANGE } \\
\text { BPK } \\
\text { LOAD }\end{array}$ & $\begin{array}{c}\text { STRETCH } \\
(\mathrm{INCH})\end{array}$ & $\%$ ELONG & EXPVIS \\
\hline \multirow{3}{*}{$\begin{array}{l}\text { A1 } \\
\text { Glass }\end{array}$} & \multirow[t]{3}{*}{1.5632} & \multirow[t]{3}{*}{1.5969} & \multirow[t]{3}{*}{$2.16 \%$} & 39.02 & 36.85 & & 0.07 & $3.50 \%$ & $\mathrm{~N} / \mathrm{C}$ \\
\hline & & & & 39.02 & 47.35 & $0.69 \%$ & 0.05 & $2.50 \%$ & \\
\hline & & & & 39.02 & 32.05 & & 0.04 & $2.00 \%$ & \\
\hline \multirow{3}{*}{$\begin{array}{l}\text { B1 } \\
\text { Polyester }\end{array}$} & \multirow[t]{3}{*}{0.6933} & \multirow[t]{3}{*}{0.7383} & \multirow[t]{3}{*}{$6.49 \%$} & 56.12 & 60.15 & & 0.73 & $36.50 \%$ & $\mathrm{~N} / \mathrm{C}$ \\
\hline & & & & 56.12 & 58.40 & $5.81 \%$ & 0.66 & $33.00 \%$ & \\
\hline & & & & 56.12 & 59.60 & & 0.77 & $38.50 \%$ & \\
\hline \multirow{4}{*}{$\begin{array}{l}\text { P1 } \\
\text { Permacel }\end{array}$} & \multirow[t]{3}{*}{1.8506} & \multirow[t]{3}{*}{1.7466} & \multirow[t]{3}{*}{$-5.62 \%$} & 88.50 & 78.85 & & 0.07 & $3.50 \%$ & Rolled \\
\hline & & & & 88.50 & 81.05 & $-12.34 \%$ & 0.09 & $4.50 \%$ & up,turn \\
\hline & & & & 88.50 & 72.85 & & 0.08 & $4.00 \%$ & green \\
\hline & \multicolumn{7}{|c|}{$500 \mathrm{HRS}$ in R-123 $>302 \mathrm{~F} 24 \mathrm{HRS}$} & & \\
\hline \multirow{3}{*}{$\begin{array}{l}\text { A2 } \\
\text { Glass }\end{array}$} & \multirow[t]{3}{*}{1.5994} & \multirow[t]{3}{*}{1.5976} & \multirow[t]{3}{*}{$-0.11 \%$} & 39.02 & 59.05 & & 0.05 & $2.50 \%$ & $\mathrm{~N} / \mathrm{C}$ \\
\hline & & & & 39.02 & 49.95 & $18.83 \%$ & 0.05 & $2.50 \%$ & \\
\hline & & & & 39.02 & 30.10 & & 0.04 & $2.00 \%$ & \\
\hline \multirow{3}{*}{$\begin{array}{l}\text { B2 } \\
\text { Polyester }\end{array}$} & \multirow[t]{3}{*}{0.6907} & \multirow[t]{3}{*}{0.6801} & \multirow[t]{3}{*}{$.1 .53 \%$} & 56.12 & 52.80 & & 0.50 & $25.00 \%$ & $\mathrm{~N} / \mathrm{C}$ \\
\hline & & & & 56.12 & 60.70 & $2.90 \%$ & 0.70 & $35.00 \%$ & \\
\hline & & & & 56.12 & 59.75 & & 0.60 & $30.00 \%$ & \\
\hline \multirow{3}{*}{$\begin{array}{l}\mathrm{C} 2 \\
\text { Permacel }\end{array}$} & \multirow[t]{3}{*}{1.7841} & \multirow[t]{3}{*}{1.5248} & \multirow[t]{3}{*}{$-14.53 \%$} & 88.50 & 96.90 & & 0.09 & $4.50 \%$ & Darkeneó \\
\hline & & & & 88.50 & 98.49 & $20.49 \%$ & 0.10 & $5.00 \%$ & \\
\hline & & & & 88.50 & 124.50 & & 0.11 & $5.50 \%$ & \\
\hline
\end{tabular}




\begin{tabular}{|c|c|c|c|c|c|c|c|c|c|}
\hline \multicolumn{10}{|c|}{500 HRS IN R-123@194 F } \\
\hline ID & WT & EXPWT & $\begin{array}{c}\text { WT \% } \\
\text { CHANGE }\end{array}$ & $\begin{array}{l}\text { EXP } \\
\text { VIS }\end{array}$ & $\begin{array}{l}\text { BREAK } \\
\text { LOAD } \\
\text { (AVE) }\end{array}$ & $\begin{array}{l}\text { BREAK } \\
\text { LOAD } \\
\text { EXP } \\
(\operatorname{av} \theta)\end{array}$ & $\begin{array}{c}\% \\
\text { CHANGE } \\
\text { BPK } \\
\text { LOAD }\end{array}$ & $\begin{array}{c}\text { STRETCH } \\
\text { (Inch) }\end{array}$ & $\begin{array}{c}\% \\
\text { ELONG }\end{array}$ \\
\hline \multirow[t]{4}{*}{ A1 } & \multirow[t]{3}{*}{0.2827} & \multirow[t]{3}{*}{0.2996} & \multirow[t]{3}{*}{$5.98 \%$} & $\mathrm{~N} / \mathrm{C}$ & 28.36 & 33.55 & & 0.23 & $11.5 \%$ \\
\hline & & & & & 28.36 & 28.55 & $10.13 \%$ & 0.20 & $10.0 \%$ \\
\hline & & & & & 28.36 & 31.60 & & 0.19 & $9.5 \%$ \\
\hline & \multicolumn{9}{|c|}{500 HRS IN R-123 -> 24 HRS @ 302F } \\
\hline \multirow[t]{3}{*}{$A 2$} & \multirow[t]{3}{*}{0.2824} & \multirow[t]{3}{*}{0.2868} & \multirow[t]{3}{*}{$1.558 \%$} & $\mathrm{~N} / \mathrm{C}$ & 28.36 & 30.80 & & 0.29 & $14.5 \%$ \\
\hline & & & & & 28.36 & 30.45 & $4.96 \%$ & 0.29 & $14.5 \%$ \\
\hline & & & & & 28.36 & 28.05 & & 0.29 & $14.5 \%$ \\
\hline
\end{tabular}


LEAD WIRE INSULATION

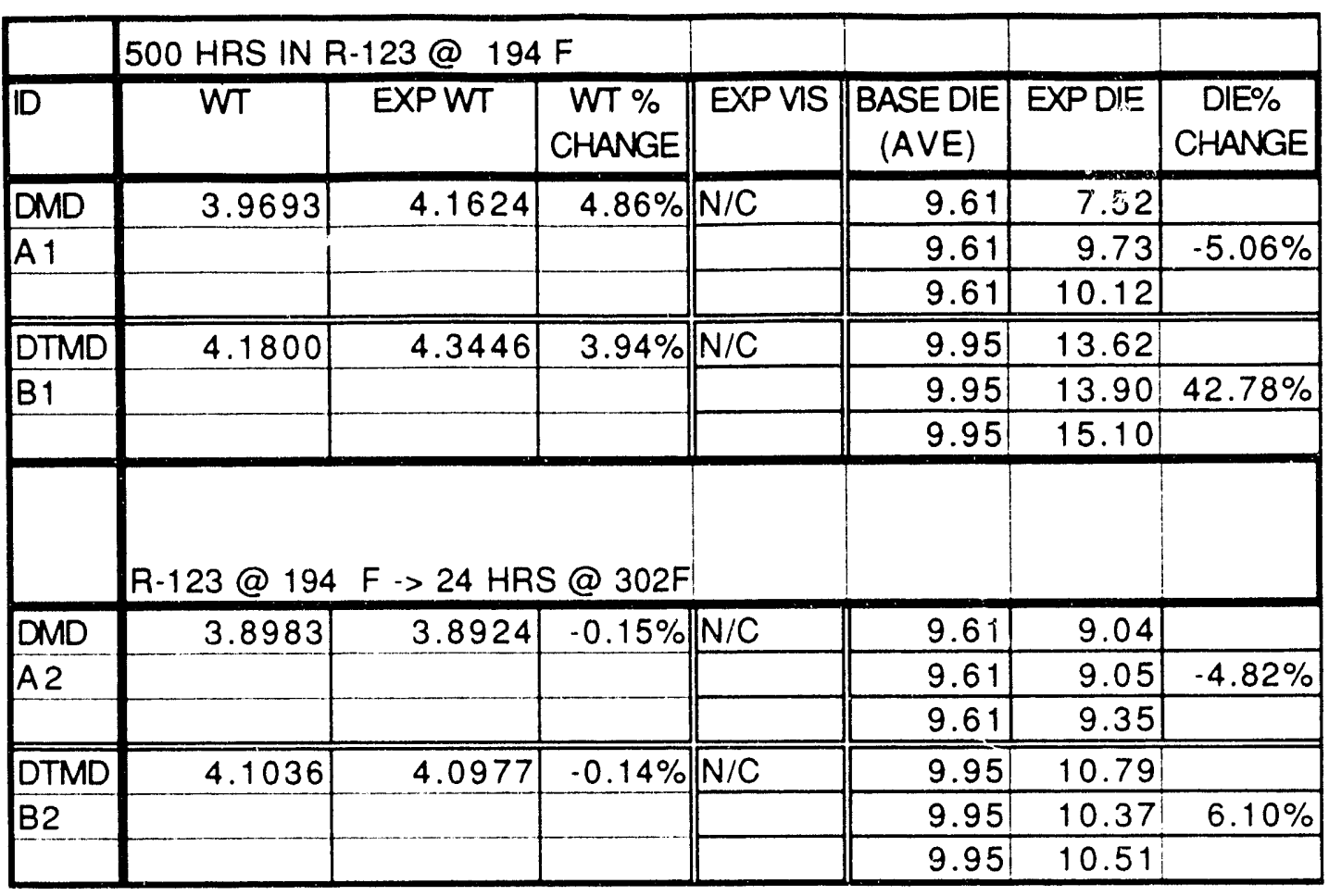




\section{Appendix E}

Experimental Data for HCFC-124 Exposure at $90^{\circ} \mathrm{C}\left(194^{\circ} \mathrm{F}\right)$ 


\begin{tabular}{|c|c|c|c|c|c|c|c|c|c|c|}
\hline & 500 HRS IN & R-124@1 & $94 \mathrm{~F}$ & & & & & & & \\
\hline ID & $W T$ & EXP WT & $\begin{array}{c}\text { WT \% } \\
\text { CHANGE }\end{array}$ & EXP VIS & $\begin{array}{c}\text { BASE BRN } \\
\text { OUT } \\
(A \vee E)\end{array}$ & $\begin{array}{c}\text { EXPBRN } \\
\text { OUT }\end{array}$ & $\begin{array}{c}\text { BRN OUT } \\
\% \\
\text { CHANGE }\end{array}$ & $\begin{array}{c}\text { BASE DIE } \\
\text { (AVE) }\end{array}$ & EXPDIE & $\begin{array}{c}\text { DIE \% } \\
\text { CHANGE }\end{array}$ \\
\hline A 1 & 26.8128 & 26.8163 & $0.013 \%$ & $N / C$ & 576 & 415 & & 15.80 & 15.43 & \\
\hline & & & & & 576 & 454 & $-20.9 \%$ & 15.80 & 16.12 & $3.1 \%$ \\
\hline & & & & & 576 & 497 & & 15.80 & 17.30 & \\
\hline $\mathrm{B} 1$ & 24.6689 & 24.7140 & $0.183 \%$ & $\mathrm{~N} / \mathrm{C}$ & 736 & 733 & & 11.62 & 11.15 & \\
\hline & & & & & 736 & 730 & $-0.7 \%$ & 11.62 & 12.21 & $1.5 \%$ \\
\hline & & & & & 736 & 730 & & 11.62 & 12.01 & \\
\hline$\overline{C 1}$ & 25.2283 & 25.2294 & $0.004 \%$ & $\mathrm{~N} / \mathrm{C}$ & 579 & 395 & & 16.58 & 11.32 & \\
\hline & & & & & 579 & 561 & $-13.6 \%$ & 16.58 & 13.63 & $-17.0 \%$ \\
\hline & & & & & 579 & 544 & & 16.58 & 16.35 & \\
\hline & $R-124-->24$ & HRS@30 & $2 F$ & & & & & & & \\
\hline A2 & 23.1202 & 23.1164 & $-0.016 \%$ & $\mathrm{~N} / \mathrm{C}$ & 576 & 479 & & 15.80 & 15.06 & \\
\hline & & & & & 576 & 423 & $-19.7 \%$ & 15.80 & 14.98 & $-2.8 \%$ \\
\hline & & & & & 576 & 486 & & 15.80 & 16.02 & \\
\hline$\overline{\mathrm{B} 2}$ & 23.9815 & 23.9635 & $-0.075 \%$ & $\mathrm{~N} / \mathrm{C}$ & 736 & 658 & & 11.62 & 12.00 & \\
\hline & & & & & 736 & 655 & $-12.4 \%$ & 11.62 & 11.62 & $0.3 \%$ \\
\hline & & & & & 736 & 621 & & 11.62 & 11.34 & \\
\hline$\overline{\mathrm{C} 2}$ & 23.5253 & 23.5238 & $-0.006 \%$ & $\mathrm{~N} / \mathrm{C}$ & 579 & 521 & & 16.58 & 14.22 & \\
\hline & & & & & 579 & 570 & $-9.9 \%$ & 16.58 & 9.75 & $-16.6 \%$ \\
\hline & & & & & 579 & 474 & & 16.58 & 17.49 & \\
\hline
\end{tabular}




\begin{tabular}{|c|c|c|c|c|c|c|c|c|c|c|}
\hline \multicolumn{11}{|c|}{500 HOURS IN R-124@194 F } \\
\hline VARN & $\begin{array}{l}\text { TWISTED } \\
\text { PAIR WT }\end{array}$ & $\begin{array}{c}\text { EXPT } \\
\text { PAIR WT }\end{array}$ & $\begin{array}{c}\text { WT \% } \\
\text { CHANGE }\end{array}$ & \begin{tabular}{|l|} 
EXP \\
VIS
\end{tabular} & BASEDIE & EXP DIE & $\begin{array}{c}\text { DIE \% } \\
\text { CHANGE }\end{array}$ & $\begin{array}{c}\text { BASE } \\
\text { BURN } \\
\text { OUT }\end{array}$ & $\begin{array}{c}\text { EXP } \\
\text { BUPN } \\
\text { OUT }\end{array}$ & $\begin{array}{c}\text { BRNOUT } \\
\% \\
\text { CHANGE }\end{array}$ \\
\hline \multirow{3}{*}{$\begin{array}{l}\text { U-475 } \\
\text { A } 1 \\
\end{array}$} & \multirow[t]{3}{*}{21.4225} & \multirow[t]{3}{*}{21.4566} & \multirow[t]{3}{*}{$0.159 \%$} & $\mathrm{~N} / \mathrm{C}$ & 16.24 & 16.30 & & 430 & 332 & \\
\hline & & & & & 16.24 & 12.77 & $-5.25 \%$ & 430 & 318 & $-27.13 \%$ \\
\hline & & & & & 16.24 & 17.09 & & 430 & 290 & \\
\hline \multirow{3}{*}{$\begin{array}{l}Y-390 \\
B 1\end{array}$} & \multirow[t]{3}{*}{21.8935} & \multirow[t]{3}{*}{21.9259} & \multirow[t]{3}{*}{$0.148 \%$} & $\mathrm{~N} / \mathrm{C}$ & 18.77 & 19.66 & & 510 & 423 & \\
\hline & & & & & 18.77 & 20.00 & $4.23 \%$ & 510 & 416 & $-18.82 \%$ \\
\hline & & & & & 18.77 & 19.03 & & 510 & 403 & \\
\hline$T \longdiv { \text { ER-610 } }$ & \multirow[t]{3}{*}{21.8850} & \multirow[t]{3}{*}{21.9070} & \multirow[t]{3}{*}{$0.101 \%$} & $\mathrm{~N} / \mathrm{C}$ & 15.57 & 13.93 & & 442 & 411 & \\
\hline \multirow{2}{*}{ C1 } & & & & & 15.57 & 13.29 & $-15.24 \%$ & 442 & 407 & $-7.16 \%$ \\
\hline & & & & & 15.57 & 12.37 & & 442 & 413 & \\
\hline \multirow{3}{*}{$\begin{array}{l}Y-833 \\
D 1\end{array}$} & \multirow[t]{3}{*}{21.6046} & \multirow[t]{3}{*}{21.6004} & \multirow[t]{3}{*}{$-0.019 \%$} & $\mathrm{~N} / \mathrm{C}$ & 12.04 & 9.73 & & 578 & 510 & \\
\hline & & & & & 12.04 & 12.53 & $-6.15 \%$ & 578 & 392 & $-21.22 \%$ \\
\hline & & & & & 12.04 & 11.64 & & 578 & 464 & \\
\hline \multirow{3}{*}{$\begin{array}{l}923 \\
E 1\end{array}$} & \multirow[t]{3}{*}{22.5209} & 22.5604 & $0.175 \%$ & $\mathrm{~N} / \mathrm{C}$ & 16.76 & broke & & 606 & 421 & \\
\hline & & & & & 16.76 & 20.00 & $-5.58 \%$ & 606 & 418 & $-32.45 \%$ \\
\hline & & & & & 16.76 & 11.65 & & 606 & 389 & \\
\hline$M \longdiv { 1 S O - 8 0 0 }$ & 22.0486 & 22.0563 & $0.035 \%$ & $\mathrm{~N} / \mathrm{C}$ & 19.08 & 14.30 & & 580 & 558 & \\
\hline I F1 & & & & & 19.08 & 12.47 & $-25.40 \%$ & 580 & 580 & $-1.90 \%$ \\
\hline$D$ & & & & & 19.08 & 15.93 & & 580 & 569 & \\
\hline$E$ & 24 HOURS & SAT $302 \mathrm{~F}$ & & & & & & & & \\
\hline$U-475$ & 21.6149 & 21.6137 & $-0.006 \%$ & $\mathrm{~N} / \mathrm{C}$ & 16.24 & 13.36 & & 430 & 274 & \\
\hline 1 A2 & & & & & 16.24 & 13.99 & $-5.48 \%$ & 430 & 271 & $-36.74 \%$ \\
\hline$M$ & & & & & 16.24 & 18.70 & & 430 & 271 & \\
\hline$1 \longdiv { Y - 3 9 0 }$ & 21.7951 & 21.7538 & $-0.189 \%$ & $\mathrm{~N} / \mathrm{C}$ & 18.77 & 12.99 & & 510 & 477 & \\
\hline $\mathrm{D} / \mathrm{B} 2$ & & & & & 18.77 & 20.00 & $-7.57 \%$ & 510 & 410 & $-10.46 \%$ \\
\hline$E$ & & & & & 18.77 & 19.06 & & 510 & 483 & \\
\hline ER-610 & 21.5499 & 21.5448 & $-0.024 \%$ & $\mathrm{~N} / \mathrm{C}$ & 15.57 & 12.27 & & 442 & 414 & \\
\hline $\mathrm{C} 2$ & & & & & 15.57 & 12.17 & $-21.52 \%$ & 442 & -127 & $-2.87 \%$ \\
\hline & & & & & 15.57 & BROKE & & 442 & 447 & \\
\hline$Y-833$ & 21.5756 & 21.5590 & $-0.077 \%$ & $\mathrm{~N} / \mathrm{C}$ & 12.04 & 11.06 & & 578 & 567 & \\
\hline D2 & & & & & 12.04 & 9.17 & $-12.21 \%$ & 578 & 555 & $-7.73 \%$ \\
\hline & & & & & 12.04 & 11.48 & & 578 & 478 & \\
\hline 923 & 22.2525 & 22.2537 & $0.005 \%$ & $\mathrm{~N} / \mathrm{C}$ & 16.76 & 20.00 & & 606 & 370 & \\
\hline E2 & & & & & $i 6.76$ & 20.00 & $19.33 \%$ & 606 & 395 & $-37.02 \%$ \\
\hline & & & & & 16.76 & 20.00 & & 606 & 380 & \\
\hline ISO-800 & 22.0184 & 22.0156 & $-0.013 \%$ & $N / C$ & 19.08 & 18.79 & & 580 & 589 & \\
\hline $\mathrm{F} 2$ & & & & & 19.08 & 13.13 & $-13.75 \%$ & 580 & 564 & $.1 .09 \%$ \\
\hline & & & & & 19.08 & 17.45 & & 580 & 568 & \\
\hline
\end{tabular}


500 HOURS IN R-124@194F

\begin{tabular}{|c|c|c|c|c|c|c|c|c|c|c|c|}
\hline & VARN & $\begin{array}{l}\text { TWISTED } \\
\text { PAIR WT }\end{array}$ & $\begin{array}{c}\text { EXPT } \\
\text { PAIR WT }\end{array}$ & $\begin{array}{c}\text { WT \% } \\
\text { CHANGE }\end{array}$ & $\begin{array}{l}\text { EXP } \\
\text { VIS }\end{array}$ & $\begin{array}{c}\text { BASE } \\
\text { DIE }\end{array}$ & $\begin{array}{l}\text { EXP } \\
\text { DIE }\end{array}$ & $\begin{array}{c}\text { DIE \% } \\
\text { CHANGE }\end{array}$ & $\begin{array}{c}\text { BASE } \\
\text { BURN } \\
\text { OUT }\end{array}$ & $\begin{array}{c}\text { EXP } \\
\text { BURN } \\
\text { OUT }\end{array}$ & $\begin{array}{c}\text { BRNOUT } \\
\% \\
\text { CHANGE }\end{array}$ \\
\hline \multirow{3}{*}{$\begin{array}{l}P \\
O \\
L\end{array}$} & \multirow{3}{*}{$\begin{array}{l}\text { U-475 } \\
\mathrm{A}_{1}\end{array}$} & \multirow[t]{3}{*}{22.9533} & \multirow[t]{3}{*}{23.2238} & \multirow[t]{3}{*}{$1.18 \%$} & $\mathrm{~N} / \mathrm{C}$ & 13.32 & 13.78 & & 746 & 734 & \\
\hline & & & & & & 13.32 & 15.14 & $8.13 \%$ & 746 & 724 & $-3.57 \%$ \\
\hline & & & & & & 13.32 & 14.29 & & 746 & 700 & \\
\hline \multirow{3}{*}{$\begin{array}{l}Y \\
E \\
S\end{array}$} & \multirow{3}{*}{$\begin{array}{l}Y-390 \\
B 1\end{array}$} & \multirow[t]{3}{*}{23.2879} & \multirow[t]{3}{*}{23.3028} & \multirow[t]{3}{*}{$0.06 \%$} & $\mathrm{~N} / \mathrm{C}$ & 12.28 & 12.31 & & 755 & 735 & \\
\hline & & & & & & 12.28 & 10.13 & $-8.93 \%$ & 755 & 743 & $-2.38 \%$ \\
\hline & & & & & & 12.28 & 11.11 & & 755 & 733 & \\
\hline \multirow{4}{*}{$\begin{array}{l}T \\
E \\
R\end{array}$} & \multirow{3}{*}{$\begin{array}{l}\text { ER-610 } \\
\text { C1 }\end{array}$} & \multirow[t]{3}{*}{22.5862} & \multirow[t]{3}{*}{22.6263} & \multirow[t]{3}{*}{$0.18 \%$} & $\mathrm{~N} / \mathrm{C}$ & 12.73 & 13.85 & & 734 & 728 & \\
\hline & & & & & & 12.73 & 14.57 & $9.45 \%$ & 734 & 728 & $-0.82 \%$ \\
\hline & & & & & & 12.73 & 13.38 & & 734 & 728 & \\
\hline & $Y-833$ & \multirow[t]{3}{*}{21.1237} & \multirow[t]{3}{*}{21.1519} & \multirow[t]{3}{*}{$0.13 \%$} & $\mathrm{~N} / \mathrm{C}$ & 12.49 & 12.69 & & 734 & 726 & \\
\hline \multirow{2}{*}{$\begin{array}{l}P \\
O\end{array}$} & \multirow{2}{*}{ D1 } & & & & & 12.49 & 9.95 & $-6.73 \%$ & 734 & 725 & $-1.36 \%$ \\
\hline & & & & & & 12.49 & 12.31 & & 734 & 721 & \\
\hline \multirow{3}{*}{$\begin{array}{l}\mathrm{L} \\
\mathrm{Y} \\
\mathrm{A}\end{array}$} & \multirow{3}{*}{$\begin{array}{l}\overline{923} \\
E 1\end{array}$} & \multirow[t]{3}{*}{23.5952} & \multirow[t]{3}{*}{23.6168} & $0.09 \%$ & $\mathrm{~N} / \mathrm{C}$ & 14.38 & 14.59 & & 742 & 734 & \\
\hline & & & & & & 14.38 & 13.98 & $-7.79 \%$ & 742 & 728 & $-1.48 \%$ \\
\hline & & & & & & 14.38 & 11.21 & & 742 & 731 & \\
\hline$M$ & ISO-800 & 22.9195 & 23.1347 & $0.94 \%$ & $\mathrm{~N} / \mathrm{C}$ & 12.29 & 12.67 & & 747 & 696 & \\
\hline 1 & $\mathrm{~F} 1$ & & & & & 12.29 & 12.30 & $-3.06 \%$ & 747 & 728 & $-4.42 \%$ \\
\hline$D$ & & & & & & 12.29 & 10.77 & & 747 & 718 & \\
\hline$E$ & & $R-124 \quad->$ & 24 hours & @ $302 \mathrm{~F}$ & & & & & & & \\
\hline & U-475 & 22.8840 & 22.8661 & $-0.08 \%$ & $\mathrm{~N} / \mathrm{C}$ & 13.32 & 11.69 & & 746 & 727 & \\
\hline 1 & $A 2$ & & & & & 13.32 & 13.45 & $-6.38 \%$ & 746 & 737 & $-2.06 \%$ \\
\hline$M$ & & & & & & 13.32 & 12.27 & & 746 & 728 & \\
\hline 1 & $Y-390$ & 23.2460 & 23.2468 & $0.00 \%$ & $\mathrm{~N} / \mathrm{C}$ & 12.28 & 11.98 & & 755 & 737 & \\
\hline D & B2 & & & & & 12.28 & 12.90 & $6.32 \%$ & 755 & 726 & $-3.11 \%$ \\
\hline$E$ & & & & & & 12.28 & 14.29 & & 755 & 741 & \\
\hline & ER-610 & 22.1358 & 22.1309 & $-0.02 \%$ & $\mathrm{~N} / \mathrm{C}$ & 12.73 & 13.17 & & 734 & 674 & \\
\hline$E$ & $\mathrm{C} 2$ & & & & & 12.73 & 11.32 & $-0.76 \%$ & 734 & 726 & $-5.09 \%$ \\
\hline$P$ & & & & & & 12.73 & 13.41 & & 734 & 690 & \\
\hline 0 & $Y-833$ & 21.2747 & 21.2619 & $-0.06 \%$ & $\mathrm{~N} / \mathrm{C}$ & 12.49 & 12.69 & & 734 & 727 & \\
\hline$x$ & D2 & & & & & 12.49 & 12.07 & $-1.33 \%$ & 734 & 726 & $-1.00 \%$ \\
\hline$Y$ & & & & & & 12.49 & 12.21 & & 734 & $72:$ & \\
\hline & 923 & 23.4878 & 23.4504 & $-0.16 \%$ & $\mathrm{~N} / \mathrm{C}$ & 14.38 & 12.04 & & 742 & $72 i^{\prime}$ & \\
\hline$G$ & E2 & & & & & 14.38 & 17.30 & $-5.10 \%$ & 742 & 733 & $-2.02 \%$ \\
\hline$L$ & & & & & & 14.38 & 11.60 & & 742 & 736 & \\
\hline A & ISO-800 & 23.4103 & 23.4085 & $-0.01 \%$ & & 12.29 & 11.42 & & 747 & 727 & \\
\hline$S$ & & & & & $\mathrm{~N} / \mathrm{C}$ & 12.29 & 12.18 & $-10.31 \%$ & 747 & 735 & $-2.14 \%$ \\
\hline$S$ & & & & & & 12.29 & 9.47 & & 747 & 725 & \\
\hline
\end{tabular}




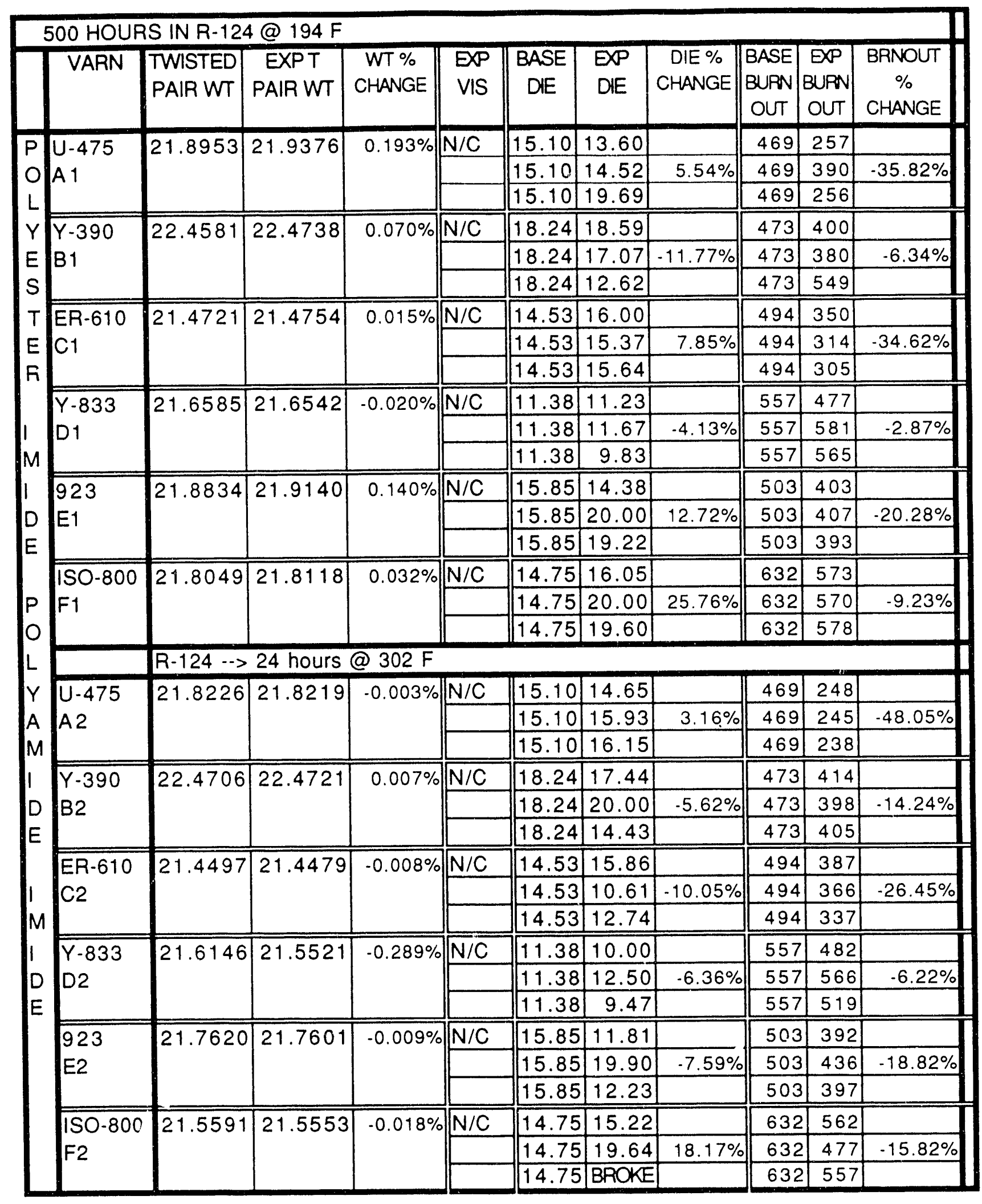




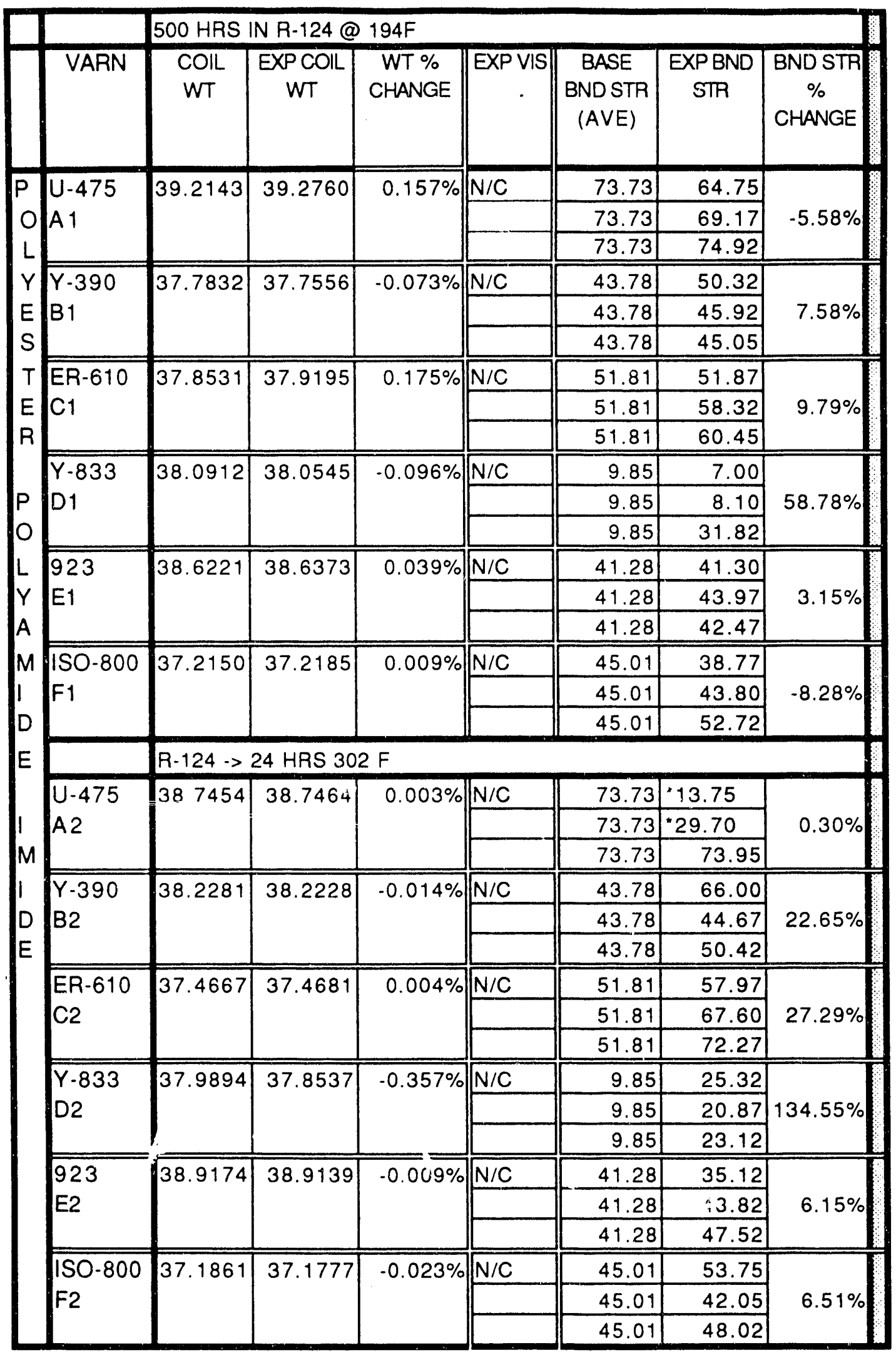

E-5

-These values were obtained with speed set at $20 \mathrm{in} / \mathrm{min}$. 


\begin{tabular}{|c|c|c|c|c|c|c|c|c|}
\hline \multirow[t]{2}{*}{$\square$} & \multirow{2}{*}{ VARN } & \multicolumn{7}{|c|}{500 HRS IN R-124@194F } \\
\hline & & $\begin{array}{l}\text { COIL } \\
\text { WT }\end{array}$ & $\begin{array}{c}\text { EXP COIL } \\
W T\end{array}$ & $\begin{array}{c}\text { WT \% } \\
\text { CHANGE }\end{array}$ & EXP VIS & $\begin{array}{c}\text { BASE } \\
\text { BND STR } \\
(\text { AVE })\end{array}$ & $\begin{array}{c}\text { EXPBND } \\
\text { STR }\end{array}$ & $\begin{array}{c}\text { BND STR } \\
\% \\
\text { CHANGE }\end{array}$ \\
\hline \multirow{3}{*}{$\begin{array}{l}P \\
O \\
L\end{array}$} & \multirow{3}{*}{$\begin{array}{l}U-475 \\
A_{1} \\
\end{array}$} & \multirow{3}{*}{36.9795} & \multirow{3}{*}{37.0610} & \multirow{3}{*}{$0.220 \%$} & $\mathrm{~N} / \mathrm{C}$ & 40.14 & 32.52 & \multirow{3}{*}{$-16.84 \%$} \\
\hline & & & & & & 40.14 & 32.27 & \\
\hline & & & & & & 40.14 & 35.35 & \\
\hline \multirow{3}{*}{\multicolumn{2}{|c|}{$\begin{array}{l}Y-390 \\
B 1\end{array}$}} & \multirow[t]{3}{*}{36.9108} & \multirow[t]{3}{*}{36.9729} & \multirow[t]{3}{*}{$0.168 \%$} & $\mathrm{~N} / \mathrm{C}$ & 36.12 & 37.35 & \multirow{3}{*}{$0.67 \%$} \\
\hline & & & & & & 36.12 & 39.22 & \\
\hline & & & & & & 36.12 & 32.52 & \\
\hline \multirow{4}{*}{$\begin{array}{l}T \\
E \\
R\end{array}$} & ER-610 & \multirow[t]{3}{*}{37.1115} & \multirow[t]{3}{*}{37.1695} & \multirow[t]{3}{*}{$0.156 \%$} & $\mathrm{~N} / \mathrm{C}$ & 35.96 & 32.72 & \\
\hline & $C_{1}$ & & & & & 35.96 & 35.87 & $-5.86 \%$ \\
\hline & & & & & & 35.96 & 32.97 & \\
\hline & $\overline{Y-833}$ & 36.3222 & 36.3535 & $0.086 \%$ & $\mathrm{~N} / \mathrm{C}$ & 33.14 & 32.47 & \\
\hline$P$ & D1 & & & & & 33.14 & 28.95 & $-8.40 \%$ \\
\hline 0 & & & & & & 33.14 & 29.65 & \\
\hline $\mathrm{L}$ & 923 & 35.9855 & 36.1269 & $0.393 \%$ & $\mathrm{~N} / \mathrm{C}$ & 40.52 & 44.72 & \\
\hline$Y$ & E1 & & & & & 40.52 & 56.90 & $22.85 \%$ \\
\hline A & & & & & & 40.52 & 47.72 & \\
\hline$M$ & $150-800$ & 35.6655 & 35.6779 & $0.035 \%$ & $\mathrm{~N} / \mathrm{C}$ & 20.20 & 19.95 & \\
\hline 1 & $\mathrm{~F} 1$ & & & & & 20.20 & 19.60 & $-7.15 \%$ \\
\hline$D$ & & & & & & 20.20 & 16.72 & \\
\hline$E$ & & $R-124 \rightarrow$ & 24 HRS 30 & $2 F$ & & & & \\
\hline & $U-475$ & 37.1098 & 37.1129 & $0.008 \%$ & $\mathrm{~N} / \mathrm{C}$ & 40.14 & 34.42 & \\
\hline 1 & A2 & & & & & 40.14 & 39.17 & $-9.48 \%$ \\
\hline$M$ & & & & & & 40.14 & 35.42 & \\
\hline 1 & $Y-390$ & 36.3441 & 36.3526 & $0.023 \%$ & Varnish & 36.12 & 35.10 & \\
\hline$D$ & $B 2$ & & & & Pockets & 36.12 & 35.87 & $0.17 \%$ \\
\hline$E$ & & & & & & 36.12 & 37.57 & \\
\hline & ER-610 & 37.2841 & 37.2795 & $-0.012 \%$ & $\mathrm{~N} / \mathrm{C}$ & 35.96 & 42.45 & \\
\hline$E$ & & & & & & 35.96 & 35.52 & $6.73 \%$ \\
\hline$P$ & & & & & & 35.96 & 37.17 & \\
\hline 0 & $Y-833$ & 36.9409 & 36.9460 & $0.014 \%$ & $\mathrm{~N} / \mathrm{C}$ & 33.14 & 34.87 & \\
\hline$x$ & D2 & & & & & 33.14 & 30.15 & $8.92 \%$ \\
\hline Y & & & & & & 33.14 & 43.27 & \\
\hline & 923 & 37.0416 & 37.0523 & $0.029 \%$ & $\mathrm{~N} / \mathrm{C}$ & 40.52 & 49.67 & \\
\hline $\mathrm{G}$ & E2 & & & & & 40.52 & 48.00 & $17.61 \%$ \\
\hline$L$ & & & & & & 40.52 & 45.30 & \\
\hline A & $150-800$ & 35.7067 & 35.7010 & $-0.016 \%$ & $\mathrm{~N} / \mathrm{C}$ & 20.20 & 18.20 & \\
\hline$S$ & $\mathrm{~F} 2$ & & & & & 20.20 & 17.82 & $-5.58 \%$ \\
\hline$S$ & & & & & & 20.20 & 21.20 & \\
\hline
\end{tabular}




\begin{tabular}{|c|c|c|c|c|c|c|c|c|c|}
\hline \multirow{2}{*}{7} & & \multirow{2}{*}{ VARN } & \multicolumn{7}{|c|}{500 HRS IN R-124@194F } \\
\hline & & & $\begin{array}{l}\text { COIL } \\
\text { WT }\end{array}$ & $\begin{array}{c}\text { EXP COIL } \\
W T\end{array}$ & $\begin{array}{c}\text { WT \% } \\
\text { CHANGE }\end{array}$ & EXP VIS & $\begin{array}{c}\text { BASE } \\
\text { BND STR } \\
(A V E)\end{array}$ & $\begin{array}{c}\text { EXP BND } \\
\text { STR }\end{array}$ & $\begin{array}{c}\text { BND STR } \\
\% \\
\text { CHANGE }\end{array}$ \\
\hline & \multirow{3}{*}{$\begin{array}{l}P \\
O \\
L\end{array}$} & \multirow{3}{*}{$\begin{array}{l}U-475 \\
\text { A } 1\end{array}$} & \multirow[t]{3}{*}{38.7159} & \multirow[t]{3}{*}{38.7754} & \multirow[t]{3}{*}{$0.154 \%$} & $\mathrm{~N} / \mathrm{C}$ & 51.21 & 55.00 & \multirow{3}{*}{$5.48 \%$} \\
\hline & & & & & & & 51.21 & 46.90 & \\
\hline & & & & & & & 51.21 & 60.15 & \\
\hline & Y & \multirow{3}{*}{$\begin{array}{l}Y-390 \\
B 1\end{array}$} & \multirow[t]{3}{*}{37.8168} & \multirow[t]{3}{*}{37.8353} & \multirow[t]{3}{*}{$0.049 \%$} & $\mathrm{~N} / \mathrm{C}$ & 50.72 & 66.60 & \multirow{3}{*}{$20.86 \%$} \\
\hline & \multirow{2}{*}{$\begin{array}{l}E \\
S\end{array}$} & & & & & & 50.72 & 58.60 & \\
\hline & & & & & & & 50.72 & 58.70 & \\
\hline & \multirow{3}{*}{$\begin{array}{l}T \\
E \\
R\end{array}$} & \multirow{3}{*}{$\begin{array}{l}\text { ER-610 } \\
\text { C1 }\end{array}$} & 36.2751 & 36.3299 & $0.151 \%$ & $\mathrm{~N} / \mathrm{C}$ & 58.33 & 49.77 & \\
\hline & & & & & & & 58.33 & 54.12 & $-5.91 \%$ \\
\hline & & & & & & & 58.33 & 60.75 & \\
\hline & & $Y-833$ & 37.5073 & 37.5010 & $-0.017 \%$ & $\mathrm{~N} / \mathrm{C}$ & 5.84 & 18.10 & \\
\hline & | & D1 & & & & & 5.84 & 8.10 & $170.26 \%$ \\
\hline & $M$ & & & & & & 5.84 & 21.15 & \\
\hline & 1 & 923 & 38.8092 & 38.8148 & $0.014 \%$ & $\mathrm{~N} / \mathrm{C}$ & 49.26 & 40.75 & \\
\hline & D & E1 & & & & & 49.26 & 41.32 & $-20.02 \%$ \\
\hline & $E$ & & & & & & 49.26 & 36.12 & \\
\hline & & ISO-800 & 37.6076 & 37.6148 & $0.019 \%$ & $\mathrm{~N} / \mathrm{C}$ & 36.08 & 47.22 & \\
\hline & $P$ & $\mathrm{~F} 1$ & & & & & 36.08 & 39.07 & $25.50 \%$ \\
\hline ) & 0 & & & & & & 36.08 & 49.55 & \\
\hline & $L$ & & $R-124->2$ & 4 HRS 302 & & & & & \\
\hline & Y & $U-475$ & 38.4566 & 38.4570 & $0.001 \%$ & $\mathrm{~N} / \mathrm{C}$ & 51.21 & 53.67 & \\
\hline & A & $A 2$ & & & & & 51.21 & 53.80 & $11.17 \%$ \\
\hline & M & & & & & & 51.21 & 63.32 & \\
\hline & 1 & $Y-390$ & 38.0765 & 38.0746 & $-0.005 \%$ & $\mathrm{~N} / \mathrm{C}$ & 50.72 & 67.10 & \\
\hline & D & B2 & & & & & 50.72 & 54.77 & $22.99 \%$ \\
\hline & $E$ & & & & & & 50.72 & 65.27 & \\
\hline & & ER-610 & 36.3845 & 36.3613 & $-0.064 \%$ & $\mathrm{~N} / \mathrm{C}$ & 58.33 & 65.80 & \\
\hline & 1 & $\mathrm{C} 2$ & & & & & 58.33 & 56.97 & $6.33 \%$ \\
\hline & M & & & & & & 58.33 & 63.30 & \\
\hline & 1 & $Y-833$ & 36.7843 & 36.7485 & $-0.097 \%$ & $\mathrm{~N} / \mathrm{C}$ & 5.84 & 2.00 & \\
\hline & $D$ & D2 & & & & & 5.84 & 29.87 & $160.22 \%$ \\
\hline & E & & & & & & 5.84 & 13.72 & \\
\hline & & 923 & 38.3985 & 38.3535 & $-0.117 \%$ & $\mathrm{~N} / \mathrm{C}$ & 49.26 & 59.07 & \\
\hline & & E2 & & & & & 49.26 & 35.77 & $-4.55 \%$ \\
\hline & & & & & & & 49.26 & 46.22 & \\
\hline & & $150-800$ & 37.6736 & 37.6665 & $-0.019 \%$ & $\mathrm{~N} / \mathrm{C}$ & 36.08 & 32.97 & \\
\hline & & F2 & & & & & 36.08 & 32.77 & $2.42 \%$ \\
\hline & & & & & & & 36.08 & 45.12 & \\
\hline
\end{tabular}




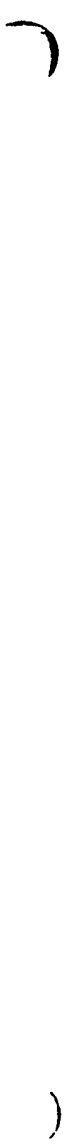

\begin{tabular}{|c|c|c|c|c|c|}
\hline & 500 HRS IN & R-124@1 & & & \\
\hline ID & WT & EXPWT & $\begin{array}{l}\text { WT \% } \\
\text { CHANGE }\end{array}$ & EXP VIS & EXPFLEX \\
\hline$A 1$ & 5.4458 & 5.4482 & $0.044 \%$ & $\mathrm{~N} / \mathrm{C}$ & \\
\hline & & & & & YES \\
\hline B1 & 54206 & 54437 & $0260 \%$ & $N / C$ & \\
\hline & & & & & YES \\
\hline & & & & & \\
\hline $\mathrm{C1}$ & 5.2747 & 5.2769 & $0.042 \%$ & $N / C$ & \\
\hline & & & & & YES \\
\hline & & & & & \\
\hline & $R-124 \rightarrow 3$ & $02 F 24 \mathrm{HR}$ & & & \\
\hline A2 & 5.4788 & 5.4764 & $-0.044 \%$ & $\mathrm{NiC}$ & \\
\hline & & & & & YES \\
\hline B2 & 5.4733 & 5.4726 & $-0.013 \%$ & $\mathrm{~N} / \mathrm{C}$ & \\
\hline & & & & & YES \\
\hline $\mathrm{C} 2$ & 5.483 & 5.4779 & $-0.093 \%$ & $\mathrm{~N} / \mathrm{C}$ & \\
\hline & & & & & YES \\
\hline & & & & & \\
\hline
\end{tabular}




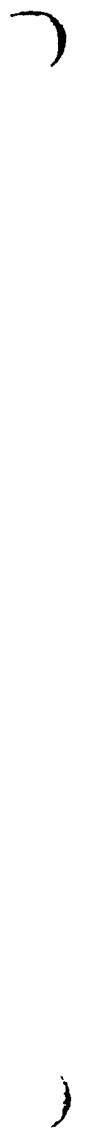

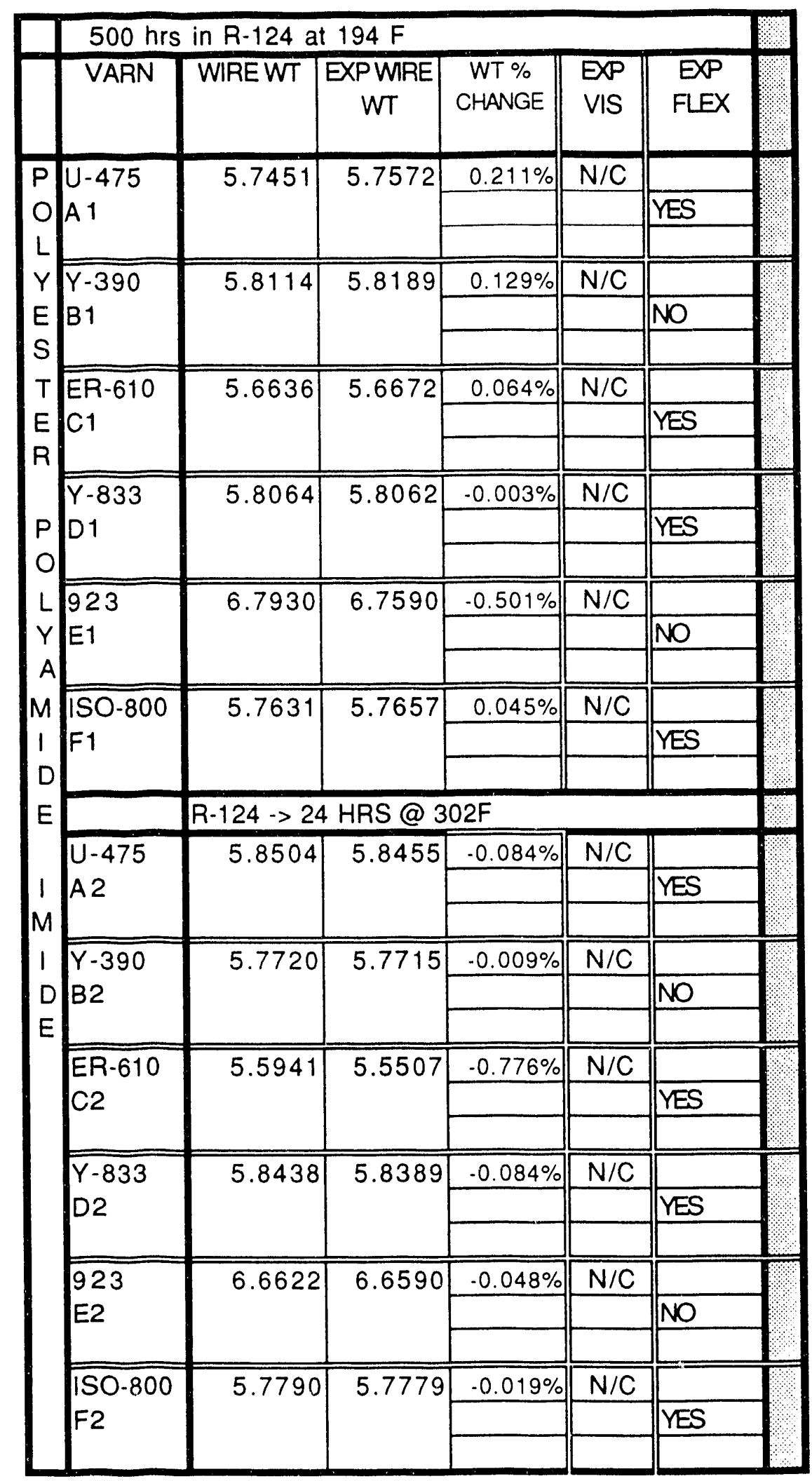




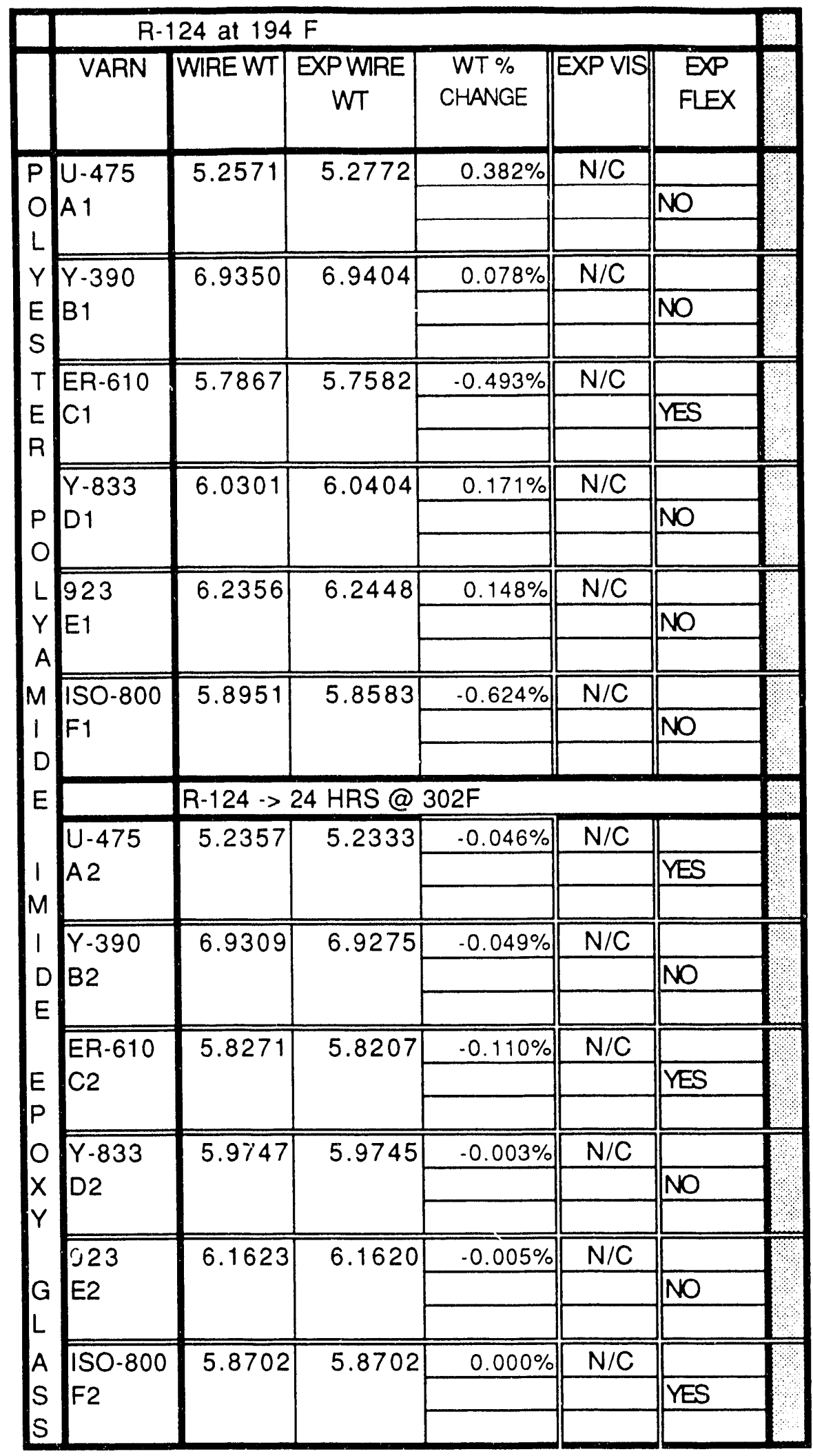




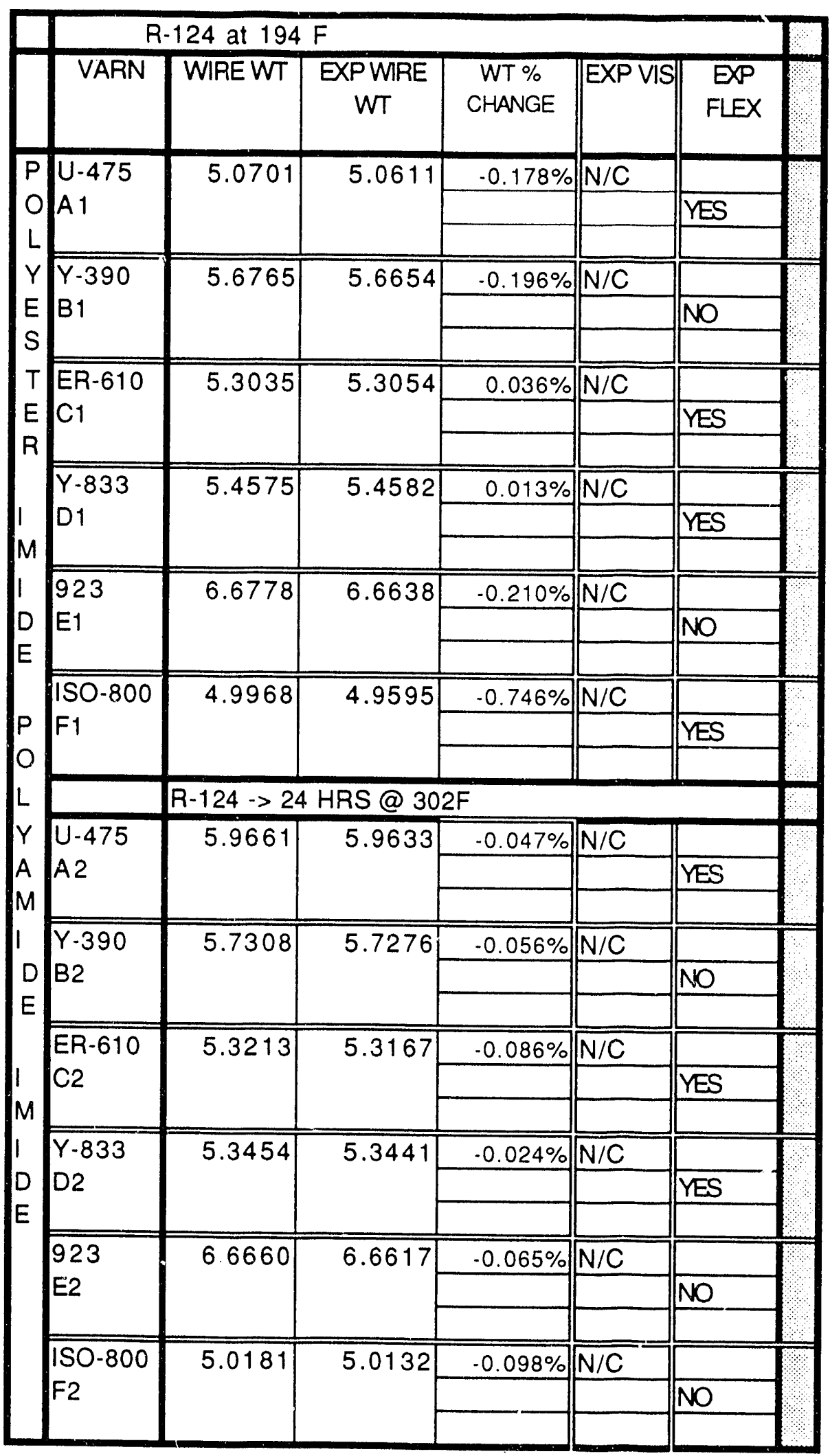




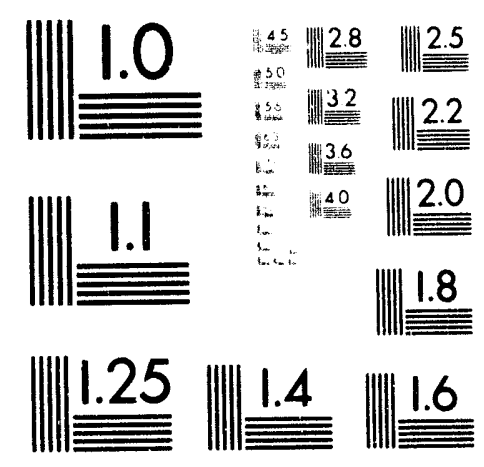



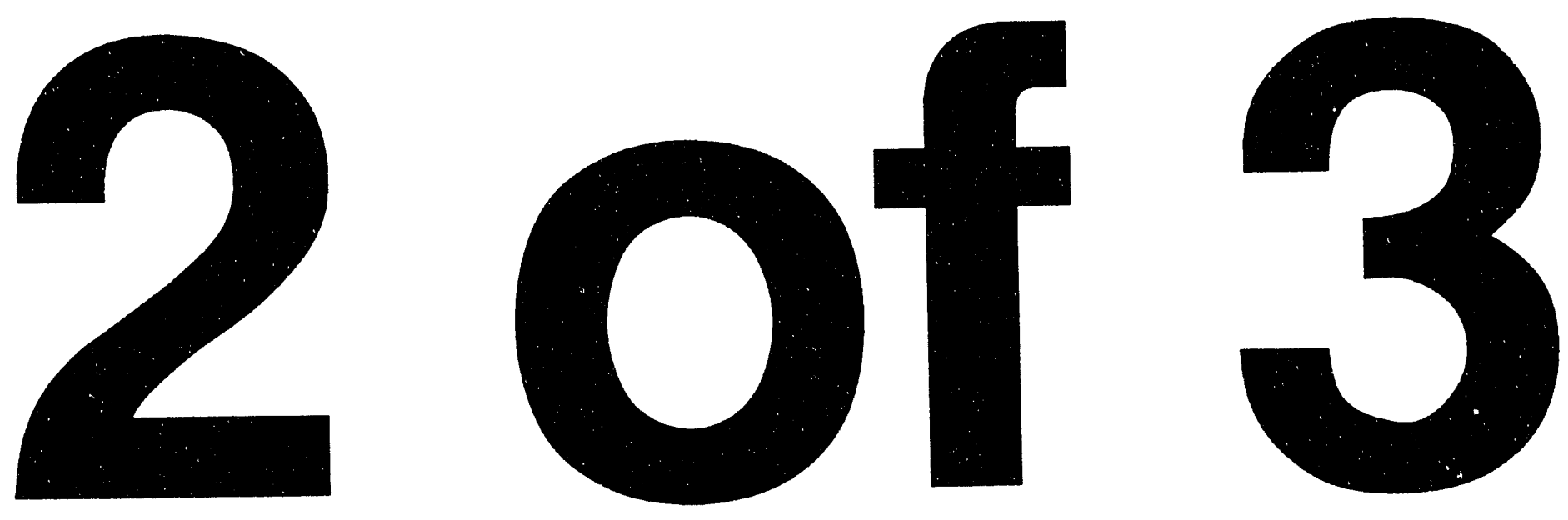


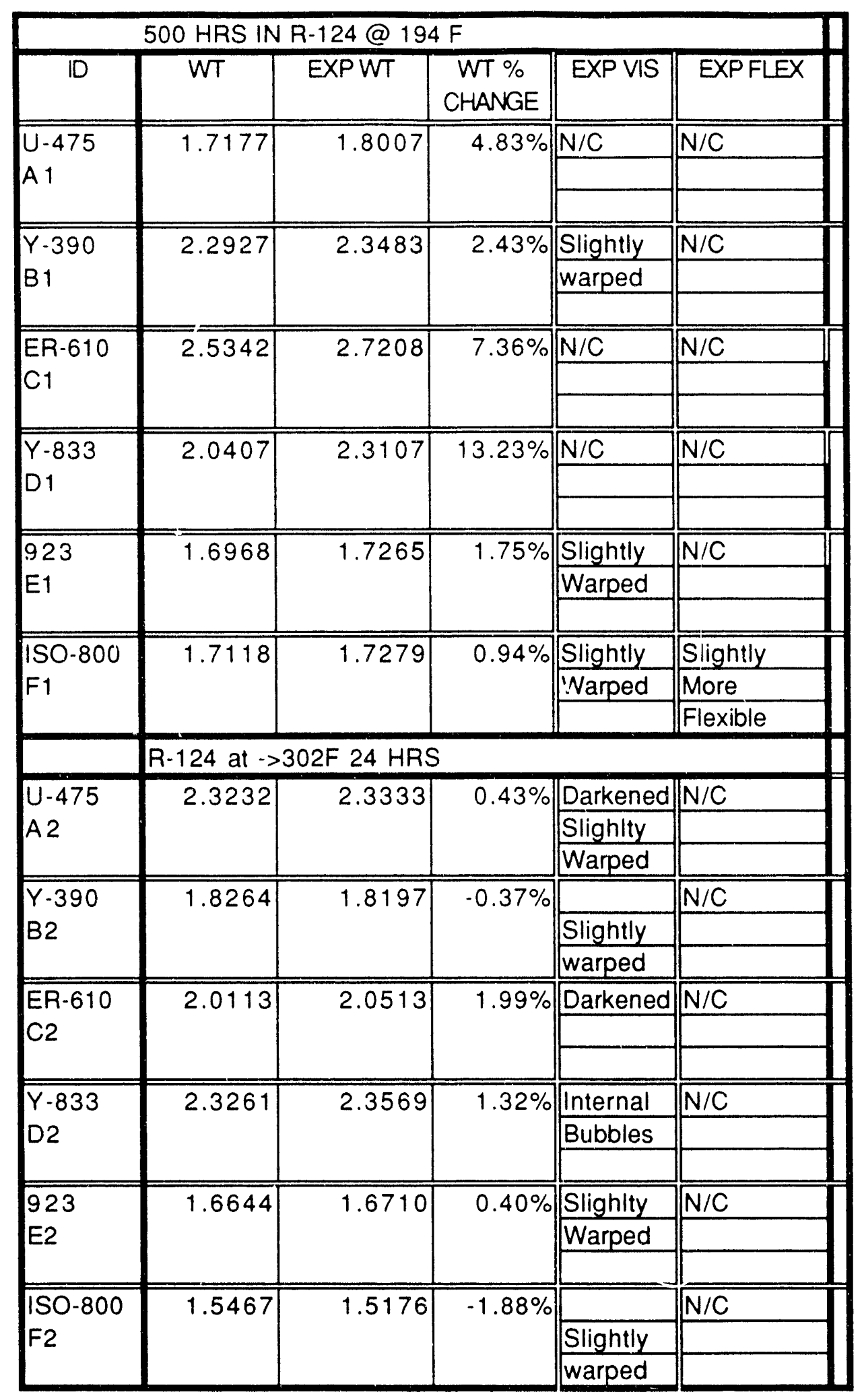




\begin{tabular}{|c|c|c|c|c|c|c|c|c|c|}
\hline \multicolumn{10}{|c|}{500 HR IN R-124@194F } \\
\hline 10 & WT & EXPWT & \begin{tabular}{c|} 
WT \% \\
CHANGE
\end{tabular} & $\begin{array}{l}\text { SAMPLE } \\
\text { WIDTH }\end{array}$ & $\begin{array}{l}\text { BREAK } \\
\text { LOAD }\end{array}$ & $\begin{array}{c}\text { SAMPLE } \\
\text { THICKNESS } \\
\text { MILS }\end{array}$ & $\begin{array}{c}\text { TENSILE } \\
\text { STR } \\
\text { BASE }\end{array}$ & $\begin{array}{l}\text { TENSILE } \\
\text { STREXP }\end{array}$ & $\begin{array}{c}\% \\
\text { CHANGE: } \\
\text { TENSILE }\end{array}$ \\
\hline \multirow{3}{*}{$\begin{array}{l}\text { NO/MY/NO } \\
\text { A1 }\end{array}$} & \multirow[t]{3}{*}{4.3892} & \multirow[t]{3}{*}{4.6516} & \multirow[t]{3}{*}{$5.98 \%$} & 0.408 & 143.80 & 22 & 17.4 & 16.02 & \\
\hline & & & & 0.348 & 123.80 & 22 & 17.4 & 16.17 & $.7 .19 \%$ \\
\hline & & & & 0.392 & 140.20 & 22 & 17.4 & 16.26 & \\
\hline \multirow{3}{*}{$\begin{array}{l}\text { DA/MY/DA } \\
\text { B1 }\end{array}$} & \multirow{3}{*}{4.4190} & \multirow{3}{*}{4.6139} & \multirow{3}{*}{$4.41 \%$} & 0.390 & 107.40 & 22 & 13.7 & 12.52 & \\
\hline & & & & 0.475 & 125.70 & 22 & 13.7 & 12.03 & $-9.69 \%$ \\
\hline & & & & 0.410 & 113.40 & 22 & 13.7 & 12.57 & \\
\hline \multirow{3}{*}{$\begin{array}{l}\text { MYLAR MO } \\
\text { C1 }\end{array}$} & \multirow[t]{3}{*}{2.3154} & \multirow[t]{3}{*}{2.4045} & \multirow[t]{3}{*}{$3.85 \%$} & 0.461 & 84.15 & 10 & 21.7 & 18.25 & \\
\hline & & & & 0.440 & 84.30 & 10 & 21.7 & 19.16 & $-13.87 \%$ \\
\hline & & & & 0.398 & 74.25 & 10 & 21.7 & 18.66 & \\
\hline \multirow{3}{*}{$\begin{array}{l}\text { NO } 410 \\
\text { D1 }\end{array}$} & \multirow[t]{3}{*}{2.1084} & \multirow{3}{*}{2.1714} & \multirow{3}{*}{$2.99 \%$} & 0.505 & 97.75 & 10 & 18.7 & 19.36 & \\
\hline & & & & 0.435 & 92.25 & 10 & 18.7 & 21.21 & $6.47 \%$ \\
\hline & & & & 0.510 & 97.75 & 10 & 18.7 & 19.17 & \\
\hline \multirow{3}{*}{$\begin{array}{l}\text { NO Ml } 418 \\
\text { E1 }\end{array}$} & \multirow[t]{3}{*}{2.4478} & \multirow{3}{*}{2.4525} & \multirow[t]{3}{*}{$0.19 \%$} & 0.507 & 28.70 & 9 & 7.5 & 6.29 & \\
\hline & & & & 0.478 & 26.20 & 9 & 7.5 & 6.09 & $-17.00 \%$ \\
\hline & & & & 0.489 & 27.70 & 9 & 7.5 & 6.29 & \\
\hline \multirow{4}{*}{$\begin{array}{l}\text { MEL } 228 \\
\text { F1 }\end{array}$} & \multirow[t]{3}{*}{2.3964} & \multirow[t]{3}{*}{2.4502} & \multirow[t]{3}{*}{$2.25 \%$} & 0.530 & 105.30 & 11 & 21.7 & 18.06 & \\
\hline & & & & 0.522 & 101.80 & 11 & 21.7 & 17.73 & $.18 .43 \%$ \\
\hline & & & & 0.485 & 92.35 & 11 & 21.7 & 17.31 & \\
\hline & & 500 HRS & 5 IN R-124 & $4 @ 194$ & $\mathrm{~F} \rightarrow 24 \mathrm{HF}$ & 7S@302 F & & & $R-124$ \\
\hline NO/MYINO & 4.5503 & 4.5526 & $0.05 \%$ & 0.495 & 187.50 & 22 & 17.4 & 17.22 & \\
\hline A2 & & & & 0.375 & 131.20 & 22 & 17.4 & 15.90 & $.4 .01 \%$ \\
\hline & & & & 0.350 & 130.80 & 22 & 17.4 & 16.99 & \\
\hline$D A / M Y / D A$ & 4.2276 & 4.2519 & $0.57 \%$ & 0.342 & 102.20 & 22 & 13.7 & 13.58 & \\
\hline$B 2$ & & & & 0.387 & 107.90 & 22 & 13.7 & 12.67 & $-4.63 \%$ \\
\hline & & & & 0.438 & 124.70 & 22 & 13.7 & 12.94 & \\
\hline MYLAR MO & 2.2746 & 2.2745 & $0.00 \%$ & 0.502 & 89.35 & 10 & 21.7 & 17.80 & \\
\hline $\mathrm{C} 2$ & & & & 0.469 & 96.00 & 10 & 21.7 & 20.47 & $.13 .91 \%$ \\
\hline & & & & 0.414 & 73.60 & 10 & 21.7 & 17.78 & \\
\hline NOMEX 410 & 2.0827 & 2.0535 & $-1.40 \%$ & 0.506 & 100.30 & 10 & 18.7 & 19.82 & \\
\hline D2 & & & & 0.487 & 97.00 & 10 & 18.7 & 19.92 & $7.67 \%$ \\
\hline & & & & 0.511 & 105.60 & 10 & 18.7 & 20.67 & \\
\hline NO/MI 418 & 2.1911 & 2.2088 & $0.81 \%$ & 0.510 & 26.15 & 9 & 7.5 & 5.70 & \\
\hline E2 & & & & 0.462 & 24.55 & 9 & 7.5 & 5.90 & $-23.00 \%$ \\
\hline & & & & 0.526 & 27.10 & 9 & 7.5 & 5.72 & \\
\hline MEL 228 & 2.4317 & 2.4355 & $0.16 \%$ & 0.412 & 77.70 & 10 & 21.7 & 18.86 & \\
\hline $\mathrm{F} 2$ & & & & 0.413 & 72.30 & 10 & 21.7 & 17.51 & $-14.69 \%$ \\
\hline & & & & 0.399 & 76.50 & 10 & 21.7 & 19.17 & \\
\hline
\end{tabular}




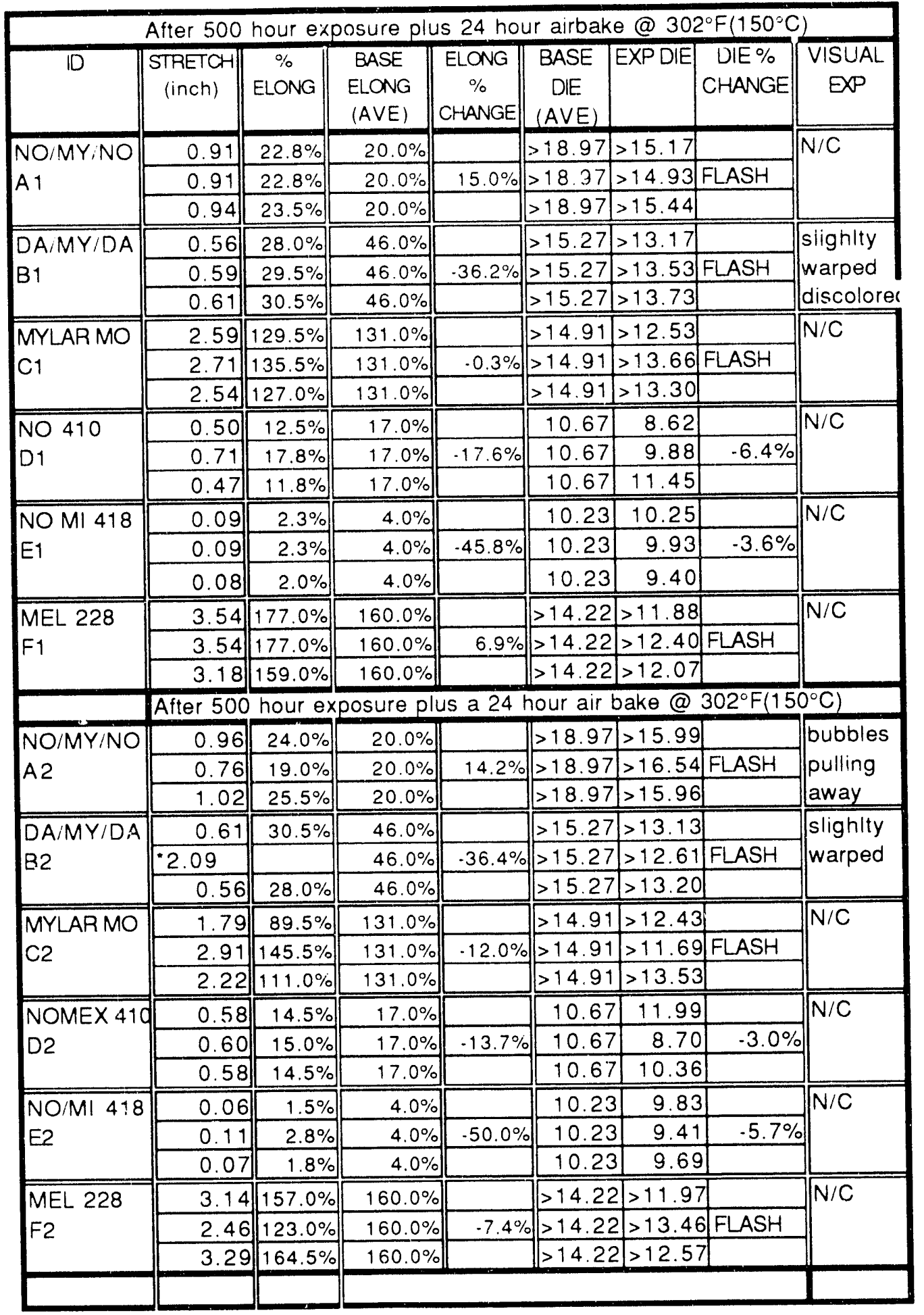


)

\begin{tabular}{|c|c|c|c|c|}
\hline & \multicolumn{4}{|c|}{500 HRS IN R-124@194F } \\
\hline & $\overline{W T}$ & EXPWT & $\begin{array}{c}\% \\
\text { CHANGE }\end{array}$ & EXPVIS \\
\hline $\begin{array}{l}\mathrm{A} 1 \\
\text { NOMEX }\end{array}$ & 0.5374 & 0.6092 & $13.36 \%$ & $\mathrm{~N} / \mathrm{C}$ \\
\hline$\overline{B 1}$ & 0.4755 & 0.5050 & $6.20 \%$ & $\mathrm{~N} / \mathrm{C}$ \\
\hline$\overline{C 1}$ & 0.3947 & 0.4155 & $5.27 \%$ & $\mathrm{~N} / \mathrm{C}$ \\
\hline & $\mathrm{R}-124 @ 19$ & $F \cdots>24 \mathrm{hr}$ & $@ 302 F$ & \\
\hline $\begin{array}{l}\mathrm{A} 2 \\
\text { NOMEX }\end{array}$ & 0.5779 & 0.5726 & $-0.92 \%$ & $\mathrm{~N} / \mathrm{C}$ \\
\hline \begin{tabular}{|l} 
B2 \\
MYLAR
\end{tabular} & 0.4659 & 0.4637 & $-0.47 \%$ & Some delamination \\
\hline $\begin{array}{l}\mathrm{C} 2 \\
\text { NO/MY }\end{array}$ & 0.4026 & $\overline{0.4012}$ & $-0.35 \%$ & $\begin{array}{l}\text { Pockects where } \\
\text { mylar pulled away }\end{array}$ \\
\hline
\end{tabular}




\begin{tabular}{|c|c|c|c|c|c|c|c|c|c|}
\hline & \multicolumn{9}{|c|}{500 HRS IN R-124@194F } \\
\hline $\mathrm{DD}$ & WT & EXPWT & $\begin{array}{c}\text { WT \% } \\
\text { CHANGE }\end{array}$ & $\begin{array}{l}\text { BREAK } \\
\text { LOAD } \\
\text { (AVE) }\end{array}$ & $\begin{array}{l}\text { BREAK } \\
\text { LOAD } \\
\text { EXP }\end{array}$ & $\begin{array}{c}\% \\
\text { CHANGE } \\
\text { BAK } \\
\text { LOAD }\end{array}$ & $\begin{array}{c}\text { STRETCH } \\
(\text { INCH) }\end{array}$ & $\%$ ELONG & EXP VIS \\
\hline \multirow{3}{*}{$\begin{array}{l}\text { A } 1 \\
\text { Glass }\end{array}$} & \multirow[t]{3}{*}{1.4492} & \multirow[t]{3}{*}{1.4525} & \multirow[t]{3}{*}{$0.23 \%$} & 39.02 & 31.00 & & 0.07 & $3.50 \%$ & $\mathrm{~N} / \mathrm{C}$ \\
\hline & & & & 39.02 & 54.85 & $12.16 \%$ & 0.05 & $2.50 \%$ & \\
\hline & & & & 39.02 & 45.45 & & 0.04 & $2.00 \%$ & \\
\hline \multirow{3}{*}{$\begin{array}{l}\text { B1 } \\
\text { Polyester }\end{array}$} & \multirow[t]{3}{*}{0.7147} & \multirow[t]{3}{*}{0.7420} & \multirow[t]{3}{*}{$3.82 \%$} & 56.12 & 47.00 & & 0.36 & $18.00 \%$ & $\mathrm{~N} / \mathrm{C}$ \\
\hline & & & & 56.12 & 63.05 & $5.55 \%$ & 0.67 & $33.50 \%$ & \\
\hline & & & & 56.12 & 67.65 & & 0.89 & $44.50 \%$ & \\
\hline \multirow{4}{*}{$\begin{array}{l}\text { C1 } \\
\text { Permacel }\end{array}$} & \multirow[t]{3}{*}{1.6045} & \multirow{3}{*}{1.6477} & \multirow{3}{*}{$2.69 \%$} & 88.50 & 116.40 & & 0.10 & $5.00 \%$ & $\mathrm{~N} / \mathrm{C}$ \\
\hline & & & & 88.50 & 85.90 & $10.64 \%$ & 0.08 & $4.00 \%$ & Ends Slightly \\
\hline & & & & 88.50 & 91.45 & & 0.08 & $4.00 \%$ & rolled up \\
\hline & \multicolumn{7}{|c|}{500 HRS in R-124 $>302 F \quad 24$ HRS } & & \\
\hline \multirow{3}{*}{$\begin{array}{l}\text { A2 } \\
\text { Glass }\end{array}$} & \multirow[t]{3}{*}{1.5190} & \multirow[t]{3}{*}{1.5150} & \multirow[t]{3}{*}{$-0.26 \%$} & 39.02 & 40.40 & & 0.05 & $2.50 \%$ & $\mathrm{~N} / \mathrm{C}$ \\
\hline & & & & 39.02 & 46.75 & $11.61 \%$ & 0.05 & $2.50 \%$ & \\
\hline & & & & 39.02 & 43.50 & & 0.04 & $2.00 \%$ & \\
\hline \multirow{3}{*}{$\begin{array}{l}\text { B2 } \\
\text { Polyester }\end{array}$} & \multirow[t]{3}{*}{0.6668} & \multirow[t]{3}{*}{0.6656} & \multirow[t]{3}{*}{$-0.18 \%$} & 56.12 & 64.65 & & 0.81 & $40.50 \%$ & $\mathrm{~N} / \mathrm{C}$ \\
\hline & & & & 56.12 & 52.50 & $4.39 \%$ & 0.53 & $26.50 \%$ & \\
\hline & & & & 56.12 & 58.60 & & 0.69 & $34.50 \%$ & \\
\hline \multirow{3}{*}{$\begin{array}{l}\text { C2 } \\
\text { Permacel }\end{array}$} & \multirow[t]{3}{*}{1.5211} & \multirow[t]{3}{*}{1.4117} & \multirow[t]{3}{*}{$-7.19 \%$} & 88.50 & 139.30 & & 0.09 & $4.50 \%$ & Slightly \\
\hline & & & & 88.50 & 129.50 & $43.47 \%$ & 0.08 & $4.00 \%$ & Darkened \\
\hline & & & & 88.50 & 112.10 & & 0.08 & $4.00 \%$ & \\
\hline
\end{tabular}


TIE CORDS

\begin{tabular}{|c|c|c|c|c|c|c|c|c|c|}
\hline \multicolumn{10}{|c|}{500 HRS IN R-124@194F } \\
\hline ID & WT & EXPWT & $\begin{array}{c}\text { WT \% } \\
\text { CHANGE }\end{array}$ & $\begin{array}{l}\text { EXP } \\
\text { VIS }\end{array}$ & $\begin{array}{l}\text { BREAK } \\
\text { LOAD } \\
\text { (AVE) }\end{array}$ & $\begin{array}{c}\text { BREAK } \\
\text { LOAD } \\
\text { EXP } \\
(\text { ave })\end{array}$ & $\begin{array}{c}\% \\
\text { CHANGE } \\
\text { BAK } \\
\text { LOAD }\end{array}$ & $\begin{array}{c}\text { STRETCH } \\
\text { (Inch) }\end{array}$ & $\begin{array}{c}\% \\
\text { ELONG }\end{array}$ \\
\hline \multirow[t]{3}{*}{ A1 } & \multirow[t]{3}{*}{0.2834} & \multirow[t]{3}{*}{0.2916} & \multirow[t]{3}{*}{$2.89 \%$} & $\mathrm{~N} / \mathrm{C}$ & 28.36 & 39.95 & & 0.30 & $15.0 \%$ \\
\hline & & & & & 28.36 & 36.55 & \multirow{2}{*}{$35.75 \%$} & 0.33 & $16.5 \%$ \\
\hline & & & & & 28.36 & 39.00 & & 0.41 & $20.5 \%$ \\
\hline & \multicolumn{9}{|c|}{500 HRS IN R-124 $\because 24$ HRS@ @302F } \\
\hline \multirow[t]{3}{*}{$A 2$} & \multirow[t]{3}{*}{0.2812} & \multirow[t]{3}{*}{0.2802} & \multirow[t]{3}{*}{$-0.356 \%$} & $\mathrm{~N} / \mathrm{C}$ & 28.36 & 31.55 & & 0.38 & $19.0 \%$ \\
\hline & & & & & 28.36 & 33.15 & $12.72 \%$ & 0.41 & $20.5 \%$ \\
\hline & & & & & 28.36 & 31.20 & & 0.40 & $20.0 \%$ \\
\hline
\end{tabular}




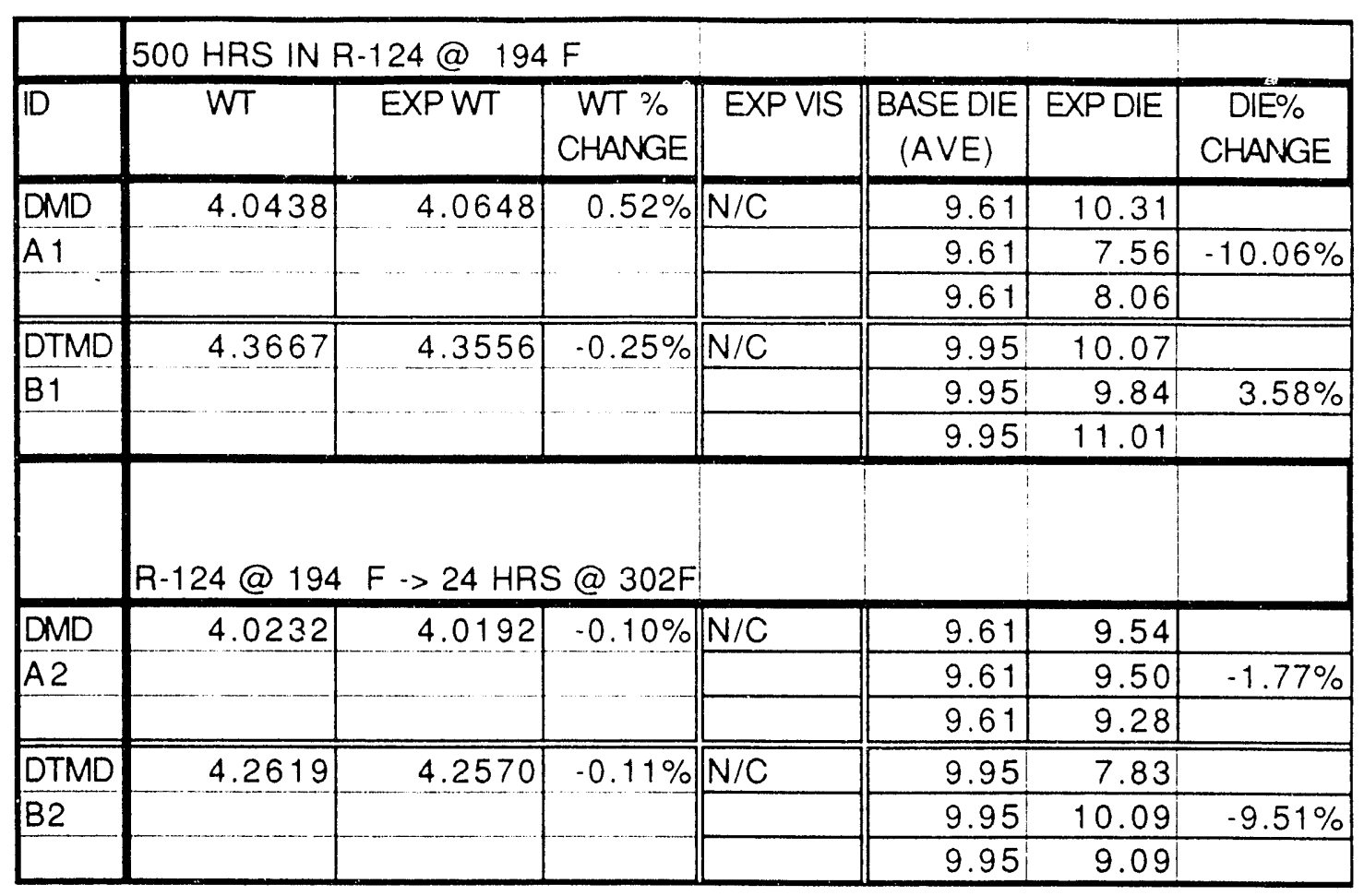




\section{Appendix F}

Experimental Data for HCFC-142b Exposure at $90^{\circ} \mathrm{C}\left(194^{\circ} \mathrm{F}\right)$ 


\begin{tabular}{|c|c|c|c|c|c|c|c|c|c|c|}
\hline & 500 HRS IN & R-142b@ & $194 \mathrm{~F}$ & & & & & & & \\
\hline ID & WT & EXPWT & $\begin{array}{c}\text { WT \% } \\
\text { CHANGE }\end{array}$ & EXP VIS & $\begin{array}{c}\text { BASE BRN } \\
\text { OUT } \\
\text { (AVE) }\end{array}$ & $\begin{array}{c}\text { EXP BRN } \\
\text { OUT }\end{array}$ & $\begin{array}{c}\text { BRN OUT } \\
\% \\
\text { CHANGE }\end{array}$ & $\begin{array}{c}\text { BASE DIE } \\
(A \vee E)\end{array}$ & EXP DIE & $\begin{array}{c}\text { DIE \% } \\
\text { CHANGE }\end{array}$ \\
\hline A1 & 25.6511 & 25.7016 & $0.197 \%$ & $N / C$ & 576 & 230 & & 15.80 & 16.26 & \\
\hline & & & & & 576 & 272 & $-54.9 \%$ & 15.80 & 17.32 & $7.5 \%$ \\
\hline & & & & & 576 & 277 & & 15.80 & 17.39 & \\
\hline $\mathrm{B1}$ & 24.3511 & 24.4163 & $0.268 \%$ & $N / C$ & 736 & 735 & & 11.62 & 12.03 & \\
\hline & & & & & 736 & 730 & $-0.5 \%$ & 11.62 & 12.82 & $5.1 \%$ \\
\hline & & & & & 736 & 732 & & 11.62 & 11.78 & \\
\hline $\mathrm{C1}$ & 25.0366 & 25.0444 & $0.031 \%$ & $\mathrm{~N} / \mathrm{C}$ & 579 & 284 & & 16.58 & 16.19 & \\
\hline & & & & & 579 & 287 & $-54.9 \%$ & 16.58 & 15.30 & $-10.5 \%$ \\
\hline & & & & & 579 & 213 & & 16.58 & 13.04 & \\
\hline & $R-142 b$ & & & & & & & & & \\
\hline A2 & 25.2514 & 25.2543 & $0.011 \%$ & $\mathrm{~N} / \mathrm{C}$ & 576 & 524 & & 15.80 & 14.09 & \\
\hline & & & & & 576 & 519 & $-12.7 \%$ & 15.80 & 16.90 & $-9.8 \%$ \\
\hline & & & & & 576 & 466 & & 15.80 & 11.78 & \\
\hline $\mathrm{B2}$ & 24.4042 & 24.4077 & $0.014 \%$ & $\mathrm{~N} / \mathrm{C}$ & 736 & 650 & & 11.62 & 12.42 & \\
\hline & & & & & 736 & 651 & $-8.0 \%$ & 11.62 & 12.69 & $6.0 \%$ \\
\hline & & & & & 736 & 730 & & 11.62 & 11.83 & \\
\hline $\mathrm{C2}$ & 25.3238 & 25.3244 & $0.002 \%$ & $\mathrm{~N} / \mathrm{C}$ & 579 & 450 & & 16.58 & 18.00 & \\
\hline & & & & & 579 & 337 & $-28.4 \%$ & 16.58 & 18.86 & $10.4 \%$ \\
\hline & & & & & 579 & 457 & & 16.58 & 18.04 & \\
\hline
\end{tabular}




\begin{tabular}{|c|c|c|c|c|c|c|c|c|c|c|c|}
\hline \multicolumn{12}{|c|}{500 HOURS IN R-142b @ 194 F } \\
\hline & VARN & $\begin{array}{l}\text { TWISTED } \\
\text { PAIR WT }\end{array}$ & $\begin{array}{l}\text { Exp Pair } \\
\text { weight }\end{array}$ & $\begin{array}{c}\text { WT \% } \\
\text { CHANGE }\end{array}$ & $\begin{array}{l}\text { EXP } \\
\text { VIS }\end{array}$ & |BASE DIE & EXPDIE & $\begin{array}{c}\text { DII } \% \\
\text { CHANGE }\end{array}$ & $\begin{array}{c}\text { BASE } \\
\text { BURN } \\
\text { OUT }\end{array}$ & $\begin{array}{l}\text { EXP } \\
\text { BUPN } \\
\text { OUT }\end{array}$ & $\begin{array}{c}\text { BRNOUT } \\
\% \\
\text { CHANGE }\end{array}$ \\
\hline \multirow{3}{*}{$\begin{array}{l}P \\
O \\
L\end{array}$} & \multirow{3}{*}{$\begin{array}{l}\text { U-475 } \\
\text { A } 1\end{array}$} & \multirow[t]{3}{*}{22.2514} & \multirow[t]{3}{*}{22.3185} & \multirow[t]{3}{*}{$0.302 \%$} & $N / C$ & 16.24 & 17.70 & & 430 & 212 & \\
\hline & & & & & & 16.24 & 15.87 & $2.81 \%$ & 430 & 162 & $-54.65 \%$ \\
\hline & & & & & & 16.24 & 16.52 & & 430 & 211 & \\
\hline \multirow{3}{*}{$\begin{array}{l}Y \\
E \\
S\end{array}$} & \multirow{3}{*}{$\begin{array}{l}Y-390 \\
B 1\end{array}$} & \multirow[t]{3}{*}{22.8135} & \multirow[t]{3}{*}{22.9002} & \multirow[t]{3}{*}{$0.380 \%$} & $\mathrm{~N} / \mathrm{C}$ & 18.77 & 20.00 & & 510 & 447 & \\
\hline & & & & & & 18.77 & 17.20 & $1.58 \%$ & 510 & 320 & $-24.31 \%$ \\
\hline & & & & & & 18.77 & 20.00 & & 510 & 391 & \\
\hline \multirow{4}{*}{$\begin{array}{l}T \\
E \\
R\end{array}$} & ER-610 & \multirow[t]{3}{*}{21.4572} & \multirow[t]{3}{*}{21.5180} & \multirow[t]{3}{*}{$0.283 \%$} & $\mathrm{~N} / \mathrm{C}$ & 15.57 & 13.03 & & 442 & 330 & \\
\hline & \multirow{2}{*}{$c_{1}$} & & & & & 15.57 & 15.73 & $-13.12 \%$ & 442 & 318 & $-30.17 \%$ \\
\hline & & & & & & 15.57 & 11.82 & & 442 & 278 & \\
\hline & $\overline{Y-833}$ & \multirow{3}{*}{23.2688} & \multirow[t]{3}{*}{23.2737} & \multirow[t]{3}{*}{$0.021 \%$} & $N / C$ & 12.04 & 11.48 & & 578 & 349 & \\
\hline \multirow{2}{*}{$\begin{array}{l}P \\
O\end{array}$} & \multirow{2}{*}{ D1 } & & & & & 12.04 & 11.03 & $-4.35 \%$ & 578 & 472 & $-34.83 \%$ \\
\hline & & & & & & 12.04 & 12.04 & & 578 & 309 & \\
\hline \multirow{3}{*}{$\begin{array}{l}\mathrm{L} \\
\mathrm{Y} \\
\mathrm{A}\end{array}$} & 923 & \multirow[t]{3}{*}{22.8538} & \multirow[t]{3}{*}{22.9475} & $0.410 \%$ & $\mathrm{~N} / \mathrm{C}$ & 16.76 & 19.66 & & 606 & 337 & \\
\hline & E1 & & & & & 16.76 & 18.30 & $3.88 \%$ & 606 & 336 & $.41 .09 \%$ \\
\hline & & & & & & 16.76 & 14.27 & & 606 & 398 & \\
\hline$M$ & ISO-800 & 21.6544 & 21.6819 & $0.127 \%$ & $\mathrm{~N} / \mathrm{C}$ & 19.08 & 16.09 & & 580 & 285 & \\
\hline 1 & F1 & & & & & 19.08 & 20.00 & $-2.01 \%$ & 580 & 313 & $-47.93 \%$ \\
\hline$D$ & & & & & & 19.08 & 20.00 & & 580 & 308 & \\
\hline$E$ & & 24 HOUR & S AT 302 & & & & & & & & \\
\hline & U-475 & 22.1512 & 22.1542 & $0.014 \%$ & $\mathrm{~N} / \mathrm{C}$ & 16.24 & 16.59 & & 430 & 217 & \\
\hline 1 & A2 & & & & & 16.24 & 15.89 & $0.02 \%$ & 430 & 217 & $-47.60 \%$ \\
\hline$M$ & & & & & & 16.24 & 16.25 & & 430 & 242 & \\
\hline 1 & $Y-390$ & 22.9632 & 22.9882 & $0.109 \%$ & $\mathrm{~N} / \mathrm{C}$ & 18.77 & 16.91 & & 510 & 394 & \\
\hline$D$ & B2 & & & & & 18.77 & 16.29 & $-8.03 \%$ & 510 & 423 & $-23.53 \%$ \\
\hline$E$ & & & & & & 18.77 & 18.59 & & 510 & 353 & \\
\hline & ER-610 & 21.7431 & 21.7448 & $0.008 \%$ & $\mathrm{~N} / \mathrm{C}$ & 15.57 & 14.04 & & 442 & 372 & \\
\hline & C2 & & & & & 15.57 & 14.65 & $-9.18 \%$ & 442 & 366 & $-16.52 \%$ \\
\hline & & & & & & 15.57 & 13.73 & & 442 & $36 \overline{9}$ & \\
\hline & $Y-833$ & 23.6078 & 23.6080 & $0.001 \%$ & $N / C$ & 12.04 & 11.10 & & 578 & 513 & \\
\hline & D2 & & & & & 12.04 & 9.89 & $-11.68 \%$ & 578 & 504 & $-12.00 \%$ \\
\hline & & & & & & 12.04 & 10.91 & & 578 & 509 & \\
\hline & 923 & 22.6395 & 22.6474 & $0.035 \%$ & $\mathrm{~N} / \mathrm{C}$ & 16.76 & 20.00 & & 606 & 303 & \\
\hline & E2 & & & & & 16.76 & 10.83 & $-9.49 \%$ & 606 & 377 & $-45.76 \%$ \\
\hline & & & & & & 16.76 & 14.68 & & 606 & 306 & \\
\hline & ISO-800 & 21.7078 & 21.7090 & $0.006 \%$ & $\mathrm{~N} / \mathrm{C}$ & 19.08 & 17.09 & & 580 & 507 & \\
\hline & $\mathrm{F} 2$ & & & & & 19.08 & 20.00 & $-2.90 \%$ & 580 & 401 & $-22.87 \%$ \\
\hline & & & & & & 19.08 & 18.49 & & 580 & 434 & \\
\hline
\end{tabular}


500 HOURS IN R-142b @ 194 $\mathrm{F}$

\begin{tabular}{|c|c|c|c|c|c|c|c|c|c|c|c|}
\hline & VARN & $\begin{array}{l}\text { TWSTED } \\
\text { PAIR WT }\end{array}$ & $\begin{array}{c}\text { EXPT } \\
\text { PAIR WT }\end{array}$ & $\begin{array}{c}\text { WT \% } \\
\text { CHANGE }\end{array}$ & $\begin{array}{l}\text { EXP } \\
\text { VIS }\end{array}$ & $\begin{array}{c}\text { BASE } \\
\text { DIE }\end{array}$ & $\begin{array}{l}\text { EXP } \\
\text { DIE }\end{array}$ & $\begin{array}{c}\text { DIE \% } \\
\text { CHANGE }\end{array}$ & $\begin{array}{l}\text { BASE } \\
\text { BURN } \\
\text { OUT }\end{array}$ & \begin{tabular}{|c|} 
EXP \\
BURN \\
OUT
\end{tabular} & $\begin{array}{c}\text { BRNOUT } \\
\% \\
\text { CHANGE }\end{array}$ \\
\hline \multirow{3}{*}{$\begin{array}{l}P \\
O \\
L\end{array}$} & \multirow{3}{*}{$\begin{array}{l}\text { U-475 } \\
\text { A } 1\end{array}$} & \multirow[t]{3}{*}{26.3169} & \multirow[t]{3}{*}{26.4343} & \multirow[t]{3}{*}{$0.45 \%$} & $N / C$ & 13.32 & 18.70 & & 746 & 683 & \\
\hline & & & & & & 13.32 & 16.63 & $18.54 \%$ & 746 & 648 & $-10.05 \%$ \\
\hline & & & & & & 13.32 & 12.04 & & 746 & 682 & \\
\hline \multirow{3}{*}{$\begin{array}{l}Y \\
E \\
S\end{array}$} & $Y-390$ & \multirow[t]{3}{*}{23.3508} & \multirow[t]{3}{*}{23.4053} & \multirow[t]{3}{*}{$0.23 \%$} & $\mathrm{~N} / \mathrm{C}$ & 12.28 & 11.11 & & 755 & 691 & \\
\hline & \multirow{2}{*}{ B1 } & & & & & 12.28 & 12.70 & $-5.65 \%$ & 755 & 687 & $-9.36 \%$ \\
\hline & & & & & & 12.28 & 10.95 & & 755 & 675 & \\
\hline \multirow{4}{*}{$\begin{array}{l}T \\
E \\
R\end{array}$} & ER-610 & \multirow[t]{3}{*}{25.1144} & \multirow[t]{3}{*}{25.1901} & \multirow[t]{3}{*}{$0.30 \%$} & $\mathrm{~N} / \mathrm{C}$ & 12.73 & 15.30 & & 734 & 615 & \\
\hline & \multirow[t]{2}{*}{ C1 } & & & & & 12.73 & 13.22 & $9.66 \%$ & 734 & 655 & $-12.67 \%$ \\
\hline & & & & & & 12.73 & 13.36 & & 734 & 653 & \\
\hline & $Y-833$ & \multirow[t]{3}{*}{22.0235} & \multirow[t]{3}{*}{22.0715} & \multirow[t]{3}{*}{$0.22 \%$} & $\mathrm{~N} / \mathrm{C}$ & 12.49 & 10.92 & & 734 & 652 & \\
\hline \multirow{2}{*}{$\begin{array}{l}\mathrm{P} \\
\mathrm{O}\end{array}$} & \multirow[t]{2}{*}{ D1 } & & & & & 12.49 & 13.43 & $0.69 \%$ & 734 & 633 & $-11.40 \%$ \\
\hline & & & & & & 12.49 & 13.38 & & 734 & 666 & \\
\hline \multirow{3}{*}{$\begin{array}{l}\mathrm{L} \\
\mathrm{Y} \\
\mathrm{A}\end{array}$} & \multirow{3}{*}{$\begin{array}{l}923 \\
E 1\end{array}$} & \multirow[t]{3}{*}{26.8758} & \multirow[t]{3}{*}{26.9648} & $0.33 \%$ & $\mathrm{~N} / \mathrm{C}$ & 14.38 & 12.23 & & 742 & 698 & \\
\hline & & & & & & 14.38 & 12.23 & $-17.50 \%$ & 742 & 669 & $-8.54 \%$ \\
\hline & & & & & & 14.38 & 11.13 & & 742 & 669 & \\
\hline$M$ & $150-800$ & 29.2546 & 29.3207 & $0.23 \%$ & $\mathrm{~N} / \mathrm{C}$ & 12.29 & 12.82 & & 747 & 725 & \\
\hline 1 & $F 1$ & & & & & 12.29 & 13.09 & $1.27 \%$ & 747 & 724 & $-2.81 \%$ \\
\hline$D$ & & & & & & 12.29 & 11.43 & & 747 & 729 & \\
\hline$E$ & & $R-142 b$ & 24 hours & @ $302 \mathrm{~F}$ & & & & & & & \\
\hline & U-475 & 26.6580 & 26.6723 & $0.05 \%$ & $\mathrm{~N} / \mathrm{C}$ & 13.32 & 13.48 & & 746 & 685 & \\
\hline 1 & $A 2$ & & & & & 13.32 & 12.27 & $-1.93 \%$ & 746 & 683 & $-8.04 \%$ \\
\hline M & & & & & & 13.32 & 13.44 & & 746 & 690 & \\
\hline 1 & $Y-390$ & 23.1102 & 23.1155 & $0.02 \%$ & $\mathrm{~N} / \mathrm{C}$ & 12.28 & 11.33 & & 755 & 691 & \\
\hline$D$ & B2 & & & & & 12.28 & 12.20 & $-3.58 \%$ & 755 & 688 & $-8.68 \%$ \\
\hline$E$ & & & & & & 12.28 & 11.99 & & 755 & 708 & \\
\hline & ER-610 & 25.1733 & 25.1717 & $-0.01 \%$ & $\mathrm{~N} / \mathrm{C}$ & 12.73 & 12.17 & & 734 & 654 & \\
\hline$E$ & C2 & & & & & 12.73 & 11.47 & $-6.42 \%$ & 734 & 667 & $-10.58 \%$ \\
\hline$P$ & & & & & & 12.73 & 12.10 & & 734 & 648 & \\
\hline 0 & $Y-833$ & 21.8724 & 21.8676 & $-0.02 \%$ & $\mathrm{~N} / \mathrm{C}$ & 12.49 & 12.63 & & 734 & 649 & \\
\hline$x$ & D2 & & & & & 12.49 & 12.60 & $0.29 \%$ & 734 & 650 & $-12.67 \%$ \\
\hline Y & & & & & & 12.49 & 12.35 & & 734 & 624 & \\
\hline & 923 & 26.2261 & 26.2384 & $0.05 \%$ & $\mathrm{~N} / \mathrm{C}$ & 14.38 & 11.56 & & 742 & 687 & \\
\hline$G$ & E2 & & & & & 14.38 & 15.16 & $-17.55 \%$ & 742 & 716 & $-7.41 \%$ \\
\hline$L$ & & & & & & 14.38 & 8.85 & & 742 & 667 & \\
\hline$A$ & ISO-800 & 29.4112 & 29.4122 & $0.00 \%$ & & 12.29 & 12.85 & & 747 & 726 & \\
\hline S & $\mathrm{F} 2$ & & & & $\mathrm{~N} / \mathrm{C}$ & 12.29 & 11.97 & $1.84 \%$ & 747 & 725 & $-2.88 \%$ \\
\hline$S$ & & & & & & 12.29 & 12.73 & & 747 & 725 & \\
\hline
\end{tabular}


500 HOURS IN R-142b @ 194 F

\begin{tabular}{|c|c|c|c|c|c|c|c|c|c|c|c|}
\hline & VARN & $\begin{array}{l}\text { TWSTED } \\
\text { PAIR WT }\end{array}$ & $\begin{array}{c}\text { EXP T } \\
\text { PAIR WT }\end{array}$ & $\begin{array}{c}\text { WT \% } \\
\text { CHANGE }\end{array}$ & $\begin{array}{l}\text { EXP } \\
\text { VIS }\end{array}$ & $\begin{array}{c}\text { BASE } \\
\text { DIE }\end{array}$ & $\begin{array}{l}\text { EXP } \\
\text { DIE }\end{array}$ & $\begin{array}{c}\text { DIE \% } \\
\text { CHANGE }\end{array}$ & $\begin{array}{l}\text { BASE } \\
\text { BURN } \\
\text { OUT }\end{array}$ & $\begin{array}{c}\text { EXP } \\
\text { BUPN } \\
\text { OUT }\end{array}$ & $\begin{array}{c}\text { BRNOUT } \\
\% \\
\text { CHANGE }\end{array}$ \\
\hline \multirow{3}{*}{$\begin{array}{l}P \\
O \\
L\end{array}$} & \multirow{3}{*}{$\begin{array}{l}\text { U-475 } \\
\text { A } 1\end{array}$} & 23.5589 & 23.6081 & $0.209 \%$ & $N / C$ & 15.10 & 11.51 & & 469 & 322 & \\
\hline & & & & & & 15.10 & 11.70 & $.25 .70 \%$ & 469 & 293 & $-31.91 \%$ \\
\hline & & & & & & 15.10 & 10.45 & & 469 & 343 & \\
\hline \multirow{3}{*}{$\begin{array}{l}Y \\
E \\
S\end{array}$} & \multirow{3}{*}{$\begin{array}{l}Y-390 \\
B 1\end{array}$} & 21.9781 & 22.0902 & $0.510 \%$ & $\mathrm{~N} / \mathrm{C}$ & 18.24 & 12.36 & & 473 & 333 & \\
\hline & & & & & & 18.24 & 14.39 & $-29.84 \%$ & 473 & 380 & $-24.03 \%$ \\
\hline & & & & & & \begin{tabular}{|l|}
18.24 \\
\end{tabular} & 11.64 & & 473 & 365 & \\
\hline \multirow{4}{*}{$\begin{array}{l}T \\
E \\
R\end{array}$} & \multirow{3}{*}{$\begin{array}{l}\text { ER-610 } \\
\text { C1 }\end{array}$} & 23.3581 & 23.4222 & $0.275 \%$ & $\mathrm{~N} / \mathrm{C}$ & \begin{tabular}{|l|l|}
14.53 \\
\end{tabular} & 18.20 & & 494 & 240 & \\
\hline & & & & & & \begin{tabular}{|l|}
14.53 \\
\end{tabular} & 16.94 & $14.75 \%$ & 494 & 252 & $-48.99 \%$ \\
\hline & & & & & & 14.53 & 14.88 & & 494 & 264 & \\
\hline \multirow{3}{*}{\multicolumn{2}{|c|}{$\begin{array}{l}Y-833 \\
D 1\end{array}$}} & 24.9671 & 25.0433 & $0.305 \%$ & $\mathrm{~N} / \mathrm{C}$ & 11.38 & 13.04 & & 557 & 243 & \\
\hline & & & & & & 11.38 & 14.78 & $19.16 \%$ & 557 & 223 & $-59.61 \%$ \\
\hline & & & & & & 11.38 & 12.86 & & 557 & 209 & \\
\hline \multirow{4}{*}{$\begin{array}{l}I \\
D \\
E\end{array}$} & \multirow{3}{*}{$\begin{array}{l}923 \\
E 1\end{array}$} & 23.1154 & 23.1816 & $0.286 \%$ & $\mathrm{~N} / \mathrm{C}$ & 15.85 & 20.00 & & 503 & 297 & \\
\hline & & & & & & 15.85 & 18.13 & $20.15 \%$ & 503 & 363 & $-30.68 \%$ \\
\hline & & & & & & \begin{tabular}{|l|}
15.85 \\
\end{tabular} & 19.00 & & 503 & 386 & \\
\hline & \multirow{4}{*}{$\begin{array}{l}\mathrm{ISO}-800 \\
\mathrm{~F} 1\end{array}$} & 22.1179 & 22.1443 & $0.119 \%$ & $\mathrm{~N} / \mathrm{C}$ & 14.75 & 17.59 & & 632 & 389 & \\
\hline$P$ & & & & & & \begin{tabular}{|l|}
14.75 \\
\end{tabular} & 15.88 & $20.84 \%$ & 632 & 308 & $.45 .68 \%$ \\
\hline 0 & & & & & & 14.75 & 20.00 & & 632 & 333 & \\
\hline$L$ & & R-142b --> & $>24$ hours & @ $302 F$ & & & & & & & \\
\hline \multirow{3}{*}{$\begin{array}{l}Y \\
A \\
M\end{array}$} & \multirow{3}{*}{$\begin{array}{l}U-475 \\
A 2\end{array}$} & 24.1347 & 24.1367 & $0.008 \%$ & $\mathrm{~N} / \mathrm{C}$ & \begin{tabular}{|l|}
15.10 \\
\end{tabular} & 10.96 & & 469 & 340 & \\
\hline & & & & & & 15.10 & 11.56 & $-26.05 \%$ & 469 & 435 & $-9.81 \%$ \\
\hline & & & & & & 15.10 & 10.98 & & 469 & 494 & \\
\hline 1 & \multirow{3}{*}{$\begin{array}{l}\bar{Y}-\overline{390} \\
B 2\end{array}$} & 22.0216 & 22.0238 & $0.010 \%$ & $\mathrm{~N} / \mathrm{C}$ & 18.24 & 17.26 & & 473 & 385 & \\
\hline$D$ & & & & & & 18.24 & 12.51 & $-22.94 \%$ & 473 & 397 & $-17.41 \%$ \\
\hline$E$ & & & & & & \begin{tabular}{|l|}
18.24 \\
\end{tabular} & 15.60 & & 473 & 390 & \\
\hline & \multirow{3}{*}{$\begin{array}{l}\text { ER-610 } \\
\text { C2 }\end{array}$} & 22.4370 & 22.4406 & $0.016 \%$ & $\mathrm{~N} / \mathrm{C}$ & 14.53 & 20.00 & & 494 & 296 & \\
\hline 1 & & & & & & 14.53 & 13.82 & $5.18 \%$ & 494 & 277 & $-41.84 \%$ \\
\hline$M$ & & & & & & 14.53 & 12.03 & & 494 & 289 & \\
\hline \multirow{9}{*}{ D } & \multirow{3}{*}{$\begin{array}{l}Y-833 \\
D 2\end{array}$} & 24.7470 & 24.7455 & $0.010 \%$ & $\mathrm{~N} / \mathrm{C}$ & 11.38 & 15.09 & & 557 & 216 & \\
\hline & & & & & & \begin{tabular}{|l|}
11.38 \\
\end{tabular} & 16.06 & $46.95 \%$ & 557 & 222 & $-59.31 \%$ \\
\hline & & & & & & \begin{tabular}{|l|}
11.38 \\
\end{tabular} & 19.02 & & 557 & 242 & \\
\hline & \multirow{3}{*}{$\begin{array}{l}923 \\
E 2\end{array}$} & 23.0308 & 23.0354 & $0.020 \%$ & $\mathrm{~N} / \mathrm{C}$ & 15.85 & 17.00 & & 503 & 335 & \\
\hline & & & & & & 15.85 & 11.21 & $1.39 \%$ & 503 & 309 & $-36.12 \%$ \\
\hline & & & & & & \begin{tabular}{|l|}
15.85 \\
\end{tabular} & 20.00 & & 503 & 320 & \\
\hline & \multirow{3}{*}{$\begin{array}{l}\text { ISO-800 } \\
\mathrm{F} 2\end{array}$} & 22.2205 & 22.2222 & $0.008 \%$ & $\mathrm{~N} / \mathrm{C}$ & \begin{tabular}{|l|l|}
14.75 \\
\end{tabular} & 14.72 & & 632 & 354 & \\
\hline & & & & & & \begin{tabular}{|l|}
14.75 \\
\end{tabular} & 13.69 & $1.54 \%$ & 632 & 514 & $-33.76 \%$ \\
\hline & & & & & & 14.75 & 16.52 & & 632 & 388 & \\
\hline
\end{tabular}




\begin{tabular}{|c|c|c|c|c|c|c|c|c|}
\hline & & 0 HRS & IN R-142b & $194^{\circ} \mathrm{F}$ & & & & \\
\hline & VARN & $\begin{array}{l}\text { COIL } \\
\text { WT }\end{array}$ & $\begin{array}{c}\text { EXP COIL } \\
W T\end{array}$ & $\begin{array}{c}\text { WT \% } \\
\text { CHANGE }\end{array}$ & EXP VIS & \begin{tabular}{|c|} 
BASE \\
BND STR \\
(AVE)
\end{tabular} & $\begin{array}{c}\text { EXPBND } \\
\text { STR }\end{array}$ & $\begin{array}{c}\text { BND STR } \\
\% \\
\text { CHANGE }\end{array}$ \\
\hline$P$ & U.475 & 39.6774 & 39.7926 & $0.290 \%$ & $\mathrm{~N} / \mathrm{C}$ & 73.73 & 51.45 & \\
\hline 0 & A 1 & & & & & 73.73 & 66.25 & $-23.12 \%$ \\
\hline L & & & & & & 73.73 & 52.35 & \\
\hline$Y$ & $Y-390$ & 38.9263 & 38.9807 & $0.140 \%$ & $\mathrm{~N} / \mathrm{C}$ & 43.78 & 53.65 & \\
\hline$E$ & B1 & & & & & 43.78 & 55.15 & $16.26 \%$ \\
\hline$S$ & & & & & & 43.78 & 43.90 & \\
\hline$T$ & ER-610 & 37.9514 & 38.0910 & $0.368 \%$ & $\mathrm{~N} / \mathrm{C}$ & 51.81 & 47.85 & \\
\hline$E$ & $C_{1}$ & & & & & 51.81 & 50.75 & $-2.62 \%$ \\
\hline$R$ & & & & & & 51.81 & 52.75 & \\
\hline & $Y-833$ & 37.8674 & 37.8941 & $0.071 \%$ & $\mathrm{~N} / \mathrm{C}$ & 9.85 & 48.65 & \\
\hline$P$ & D1 & & & & & 9.85 & 9.65 & $167.34 \%$ \\
\hline 0 & & & & & & 9.85 & 20.70 & \\
\hline $\mathrm{L}$ & 923 & 39.2632 & 39.3330 & $0.178 \%$ & $\mathrm{~N} / \mathrm{C}$ & 41.28 & 42.45 & \\
\hline Y & E1 & & & . & & 41.28 & 48.90 & $5.22 \%$ \\
\hline$A$ & & & & & & 41.28 & 38.95 & \\
\hline$M$ & ISO-800 & 38.3631 & 38.3900 & $0.070 \%$ & $\mathrm{~N} / \mathrm{C}$ & 45.01 & 45.80 & \\
\hline & $F_{1}$ & & & & & 45.01 & 36.40 & $-8.69 \%$ \\
\hline D & & & & & & 45.01 & 38.85 & \\
\hline$E$ & & $R-142 b->$ & 24 HRS 3 & $22^{\circ} \mathrm{F}$ & & & & \\
\hline & $U-475$ & 41.1729 & 41.1730 & $0.000 \%$ & $\mathrm{~N} / \mathrm{C}$ & 73.73 & 58.80 & \\
\hline & A2 & & & & & 73.73 & 62.00 & $-9.70 \%$ \\
\hline$M$ & & & & & & 73.73 & 71.15 & \\
\hline 1 & Y.390 & 39.1542 & 39.1519 & $-0.006 \%$ & $\mathrm{~N} / \mathrm{C}$ & 43.78 & 57.15 & \\
\hline$D$ & B2 & & & & & 43.78 & 58.75 & $30.46 \%$ \\
\hline$E$ & & & & & & 43.78 & 55.45 & \\
\hline & ER-610 & 38.9205 & 38.9228 & $0.006 \%$ & $\mathrm{~N} / \mathrm{C}$ & 51.81 & 61.15 & \\
\hline & $\mathrm{C} 2$ & & & & & 51.81 & 62.90 & $22.31 \%$ \\
\hline & & & & & & 51.81 & 66.05 & \\
\hline & $Y-833$ & 38.6547 & 38.6543 & $-0.001 \%$ & $\mathrm{~N} / \mathrm{C}$ & 9.85 & 57.65 & \\
\hline & D2 & & & & & 9.85 & 52.05 & $361.93 \%$ \\
\hline & & & & & & 9.85 & 26.80 & \\
\hline & 923 & 38.9026 & 38.8995 & $-0.008 \%$ & $\mathrm{~N} / \mathrm{C}$ & 41.28 & 66.20 & \\
\hline & E2 & & & & & 41.28 & 47.25 & $25.48 \%$ \\
\hline & & & & & & 41.28 & 41.95 & \\
\hline & ISO-800 & 37.3546 & 37.3863 & $0.085 \%$ & $N / C$ & 45.01 & 54.15 & \\
\hline & $\mathrm{F} 2$ & & & & & 45.01 & 39.90 & $13.38 \%$ \\
\hline & & & & & & 45.01 & 59.05 & \\
\hline
\end{tabular}




\begin{tabular}{|c|c|c|c|c|c|c|c|c|}
\hline & & 500 HRS & IN R-142b & @ 194으 & & & & \\
\hline & VARN & $\begin{array}{l}\text { COIL } \\
\text { WT }\end{array}$ & $\begin{array}{c}\text { EXP COIL } \\
W T\end{array}$ & $\begin{array}{c}\text { WT \% } \\
\text { CHANGE }\end{array}$ & EXPVIS| & \begin{tabular}{|c|} 
BASE \\
BND STR \\
(AVE)
\end{tabular} & $\begin{array}{c}\text { EXP BND } \\
\text { STR }\end{array}$ & $\begin{array}{c}\text { BND STR } \\
\% \\
\text { CHANGE }\end{array}$ \\
\hline$P$ & $U-475$ & 37.1752 & 37.3126 & $0.370 \%$ & $\mathrm{~N} / \mathrm{C}$ & 40.14 & 48.75 & \\
\hline 이 & A 1 & & & & & 40.14 & 36.05 & $6.71 \%$ \\
\hline LL & & & & & & 40.14 & 43.70 & \\
\hline Y & Y-390 & 38.4599 & 38.5376 & $0.202 \%$ & $\mathrm{~N} / \mathrm{C}$ & 36.12 & 39.15 & \\
\hline$E$ & B1 & & & & & 36.12 & 39.15 & $9.54 \%$ \\
\hline $\mathrm{s}$ & & & & & & 36.12 & 40.40 & \\
\hline$T$ & ER-610 & 39.2710 & 39.4002 & $0.329 \%$ & $\mathrm{~N} / \mathrm{C}$ & 35.96 & 32.90 & \\
\hline$E$ & C1 & & & & & 35.96 & 37.90 & $.1 .00 \%$ \\
\hline$R$ & & & & & & 35.96 & 36.00 & \\
\hline & $Y-833$ & 36.2675 & 36.3489 & $0.224 \%$ & $\mathrm{~N} / \mathrm{C}$ & 33.14 & 27.25 & \\
\hline$P$ & D1 & & & & & 33.14 & 18.60 & $-38.09 \%$ \\
\hline 0 & & & & & & 33.14 & 15.70 & \\
\hline$L$ & 923 & 38.4320 & 38.4873 & $0.144 \%$ & $\mathrm{~N} / \mathrm{C}$ & 40.52 & 39.20 & \\
\hline$Y$ & E1 & & & & & 40.52 & 34.50 & $.8 .28 \%$ \\
\hline$A$ & & & & & & 40.52 & 37.80 & \\
\hline$M$ & $150-800$ & 36.8168 & 36.8582 & $0.112 \%$ & $\mathrm{~N} / \mathrm{C}$ & 20.20 & 24.55 & \\
\hline 1 & $F_{1}$ & & & & & 20.20 & 6.50 & $-11.80 \%$ \\
\hline$D$ & & & & & & 20.20 & 22.40 & \\
\hline$E$ & & $R-142 b->$ & 24 HRS & $02^{\circ} \mathrm{F}$ & & & & \\
\hline & U-475 & 37.6223 & 37.6403 & $0.048 \%$ & $\mathrm{~N} / \mathrm{C}$ & 40.14 & 38.70 & \\
\hline 1 & A2 & & & & & 40.14 & 35.50 & $-8.07 \%$ \\
\hline$M$ & & & & & & 40.14 & 36.50 & \\
\hline 1 & Y-390 & 38.0742 & 38.0844 & $0.027 \%$ & $\mathrm{~N} / \mathrm{C}$ & 36.12 & 31.55 & \\
\hline$D$ & B2 & & & & & 36.12 & 37.20 & $-1.81 \%$ \\
\hline$E$ & & & & & & 36.12 & 37.65 & \\
\hline & ER-610 & 37.7659 & 37.7702 & $0.011 \%$ & $\mathrm{~N} / \mathrm{C}$ & 35.96 & 34.10 & \\
\hline$E$ & & & & & & 35.96 & 35.50 & $-0.54 \%$ \\
\hline$P$ & & & & & & 35.96 & 37.70 & \\
\hline 0 & Y-833 & 36.5670 & 36.5665 & $-0.001 \%$ & $\mathrm{~N} / \mathrm{C}$ & 33.14 & 32.35 & \\
\hline$x$ & D2 & & & & & 33.14 & 33.25 & $-16.11 \%$ \\
\hline$Y$ & & & & & & 33.14 & 17.80 & \\
\hline & 923 & 38.2670 & 38.2661 & $-0.002 \%$ & $\mathrm{~N} / \mathrm{C}$ & 40.52 & 34.45 & \\
\hline$G$ & E2 & & & & & 40.52 & 29.95 & $-19.38 \%$ \\
\hline L & & & & & & 40.52 & 33.60 & \\
\hline$A$ & ISO-800 & 37.1301 & 37.1209 & $-0.025 \%$ & $\mathrm{~N} / \mathrm{C}$ & 20.20 & 24.95 & \\
\hline S & $\mathrm{F} 2$ & & & & & 20.20 & 16.85 & $-2.39 \%$ \\
\hline$S$ & & & & & & 20.20 & 17.35 & \\
\hline
\end{tabular}




\begin{tabular}{|c|c|c|c|c|c|c|c|c|}
\hline & & مחו & $-142 b$ & $34^{\circ} \mathrm{F}$ & & & & \\
\hline & VARN & $\begin{array}{l}\text { COIL } \\
\text { WT }\end{array}$ & $\begin{array}{c}\text { EXP COIL } \\
W T\end{array}$ & $\begin{array}{c}\text { WT \% } \\
\text { CHANGE }\end{array}$ & EXP VIS & $\begin{array}{c}\text { BASE } \\
\text { BND STR } \\
(A \vee E)\end{array}$ & $\begin{array}{c}\text { EXP BND } \\
\text { STR }\end{array}$ & $\begin{array}{c}\text { BND STR } \\
\% \\
\text { CHANGE }\end{array}$ \\
\hline$P$ & U.475 & 40.4592 & 40.5632 & $0.257 \%$ & $\mathrm{~N} / \mathrm{C}$ & 51.21 & 60.95 & \\
\hline 0 & A 1 & & & & & 51.21 & 64.60 & $26.24 \%$ \\
\hline$L$ & & & & & & 51.21 & 68.40 & \\
\hline$Y$ & $\mathrm{Y}-390$ & 38.8349 & 38.8761 & $0.106 \%$ & $\mathrm{~N} / \mathrm{C}$ & 50.72 & 43.35 & \\
\hline$E$ & B1 & & & & & 50.72 & 43.05 & $-12.59 \%$ \\
\hline$S$ & & & & & & 50.72 & 46.60 & \\
\hline$T$ & ER-610 & 40.0091 & 40.1035 & $0.236 \%$ & $\mathrm{~N} / \mathrm{C}$ & 58.33 & 41.05 & \\
\hline$E$ & C1 & & & & & 58.33 & 67.20 & $-11.45 \%$ \\
\hline$R$ & & & & & & 58.33 & 46.70 & \\
\hline & $Y-833$ & 38.2793 & 38.2726 & $-0.018 \%$ & $\mathrm{~N} / \mathrm{C}$ & 5.84 & 34.75 & \\
\hline 1 & D1 & & & & & 5.84 & 8.00 & $306.96 \%$ \\
\hline$M$ & & & & & & 5.84 & 28.55 & \\
\hline 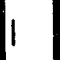 & 923 & 40.8484 & 40.8950 & $0.114 \%$ & $\mathrm{~N} / \mathrm{C}$ & 49.26 & 45.70 & \\
\hline$D$ & E1 & & & & & 49.26 & 35.20 & $-17.07 \%$ \\
\hline$E$ & & & & & & 49.26 & 41.65 & \\
\hline & $150-800$ & 38.3174 & 38.3424 & $0.065 \%$ & $\mathrm{~N} / \mathrm{C}$ & 36.08 & 44.95 & \\
\hline$P$ & $\mathrm{~F} 1$ & & & & & 36.08 & 56.55 & $41.86 \%$ \\
\hline 0 & & & & & & 36.08 & 52.05 & \\
\hline L & & R-142b-> & 24 HAS 30 & $2^{\circ} \mathrm{F}$ & & & & \\
\hline Y & U-475 & 38.8175 & 38.8068 & $.0 .028 \%$ & $\mathrm{~N} / \mathrm{C}$ & 51.21 & 54.85 & \\
\hline$A$ & $A 2$ & & & & & 51.21 & 57.90 & $10.18 \%$ \\
\hline$M$ & & & & & & 51.21 & 56.52 & \\
\hline 1 & $Y-390$ & 38.7690 & 38.7516 & $-0.045 \%$ & $\mathrm{~N} / \mathrm{C}$ & 50.72 & 44.77 & \\
\hline$D$ & B2 & & & & & 50.72 & 46.52 & $-11.55 \%$ \\
\hline$E$ & & & & & & 50.72 & 43.30 & \\
\hline & ER-610 & 39.2222 & 39.2052 & $-0.043 \%$ & $\mathrm{~N} / \mathrm{C}$ & 58.33 & 80.80 & \\
\hline I & $\mathrm{C} 2$ & & & & & 58.33 & 58.30 & $10.21 \%$ \\
\hline$M$ & & & & & & 58.33 & 53.75 & \\
\hline 1 & $Y-833$ & 40.1391 & 40.0481 & $-0.227 \%$ & $\mathrm{~N} / \mathrm{C}$ & 5.84 & 29.92 & \\
\hline$D$ & D2 & & & & & 5.84 & 29.97 & $528.65 \%$ \\
\hline$E$ & & & & & & 5.84 & 50.25 & \\
\hline & 923 & 40.4078 & 40.3954 & $-0.031 \%$ & $N / C$ & 49.26 & 45.42 & \\
\hline & E2 & & & & & 49.26 & 55.25 & $1.72 \%$ \\
\hline & & & & & & 49.26 & 49.65 & \\
\hline & ISO-800 & 38.2570 & 38.2463 & $-0.028 \%$ & $\mathrm{~N} / \mathrm{C}$ & 36.08 & 60.90 & \\
\hline & $\mathrm{F} 2$ & & & & & 36.08 & 36.40 & $38.37 \%$ \\
\hline & & & & & & 36.08 & 52.47 & \\
\hline
\end{tabular}




\begin{tabular}{|c|c|c|c|c|c|}
\hline & 500 HRS IN & 8-142b@ & $94^{\circ} \mathrm{F}$ & & \\
\hline ID & WT & EXPWT & $\begin{array}{l}\text { WT \% } \\
\text { CHANGE }\end{array}$ & EXP VIS & EXP FLEX \\
\hline A 1 & 4.2682 & 4.2904 & $0.520 \%$ & $\mathrm{~N} / \mathrm{C}$ & \\
\hline & & & & & YES \\
\hline$\overline{B 1}$ & 4.2503 & 4.3073 & $1.341 \%$ & $\bar{N} / \mathrm{C}$ & \\
\hline & & & & & YES \\
\hline C1 & 3.9537 & 3.9962 & $1.075 \%$ & $\mathrm{~N} / \mathrm{C}$ & \\
\hline & & & & & YES \\
\hline & $R-142 b \rightarrow$ & $302^{\circ} \mathrm{F}$ for 2 & HRS & & \\
\hline A2 & 4.0574 & 4.0567 & $-0.017 \%$ & $\mathrm{~N} / \mathrm{C}$ & \\
\hline & & & & & YES \\
\hline $\mathrm{B2}$ & 4.5649 & 4.5627 & $-0.048 \%$ & $\overline{N / C}$ & \\
\hline & & & & & YES \\
\hline $\mathrm{C} 2$ & 3.9148 & 3.913 & $-0.046 \%$ & $\mathrm{~N} / \mathrm{C}$ & \\
\hline & & & & & YES \\
\hline & & & & & \\
\hline
\end{tabular}




\begin{tabular}{|c|c|c|c|c|c|c|}
\hline & $500 \mathrm{hrs}$ & $R-142 b a$ & It $194^{\circ} \mathrm{F}$ & & & \\
\hline & VARN & WIRE WT & $\begin{array}{c}\text { EXPWIRE } \\
W T\end{array}$ & $\begin{array}{c}\text { WT \% } \\
\text { CHANGE }\end{array}$ & $\begin{array}{l}\text { EXP } \\
\text { VIS }\end{array}$ & $\begin{array}{l}\text { EXP } \\
\text { FLEX }\end{array}$ \\
\hline$P$ & $U-475$ & 5.3285 & 5.3624 & $0.636 \%$ & $\mathrm{~N} / \mathrm{C}$ & \\
\hline o & A 1 & & & & & YES \\
\hline L & & & & & & \\
\hline$Y$ & $Y-390$ & 5.0661 & 5.0845 & $0.363 \%$ & $\mathrm{~N} / \mathrm{C}$ & \\
\hline$E$ & $B 1$ & & & & & $\mathrm{NO}$ \\
\hline$S$ & & & & & & \\
\hline$T$ & ER-610 & 4.7466 & 4.7491 & $0.053 \%$ & $\mathrm{~N} / \mathrm{C}$ & \\
\hline$E$ & C1 & & & & & YES \\
\hline$n$ & $Y-833$ & 5.1636 & 51655 & $0.037 \%$ & $\mathrm{~N} / \mathrm{C}$ & \\
\hline$P$ & D1 & & & & & YES \\
\hline 0 & & & & & & \\
\hline$L$ & 923 & 5.2390 & 5.2592 & $0.386 \%$ & $\mathrm{~N} / \mathrm{C}$ & \\
\hline$Y$ & E1 & & & & & NO \\
\hline A & & & & & & \\
\hline$M$ & ISO-800 & 5.0703 & 5.0756 & $0.105 \%$ & $\mathrm{~N} / \mathrm{C}$ & \\
\hline 1 & $F 1$ & & & & & $\mathrm{NO}$ \\
\hline$D$ & & & & & & \\
\hline$E$ & & $R-142 b->2$ & 24 HRS @ & $302^{\circ} \mathrm{F}$ & & \\
\hline & U-475 & 5.3445 & 5.3450 & $0.009 \%$ & $\mathrm{~N} / \mathrm{C}$ & \\
\hline 1 & $A 2$ & & & & & YES \\
\hline$M$ & & & & & & \\
\hline 1 & $Y-390$ & 5.0482 & 5.0458 & $-0.048 \%$ & $\mathrm{~N} / \mathrm{C}$ & \\
\hline$D$ & B2 & & & & & $\mathrm{NO}$ \\
\hline$E$ & & & & & & \\
\hline & ER-610 & 4.7660 & 4.7651 & $-0.019 \%$ & $\mathrm{~N} / \mathrm{C}$ & \\
\hline & $\mathrm{C} 2$ & & & & & YES \\
\hline & & & & & $N / C$ & \\
\hline & $\begin{array}{l}y-833 \\
D 2\end{array}$ & 5.4104 & 5.4129 & $-0.065 \%$ & & YES \\
\hline & 923 & 5.2444 & 5.2454 & $0.019 \%$ & $\mathrm{~N} / \mathrm{C}$ & \\
\hline & E2 & & & & & $\mathrm{NO}$ \\
\hline & & 5.1130 & 5.1110 & $-0.039 \%$ & $\mathrm{~N} / \mathrm{C}$ & \\
\hline & F2 & & & & & NO \\
\hline & & & & & & \\
\hline
\end{tabular}




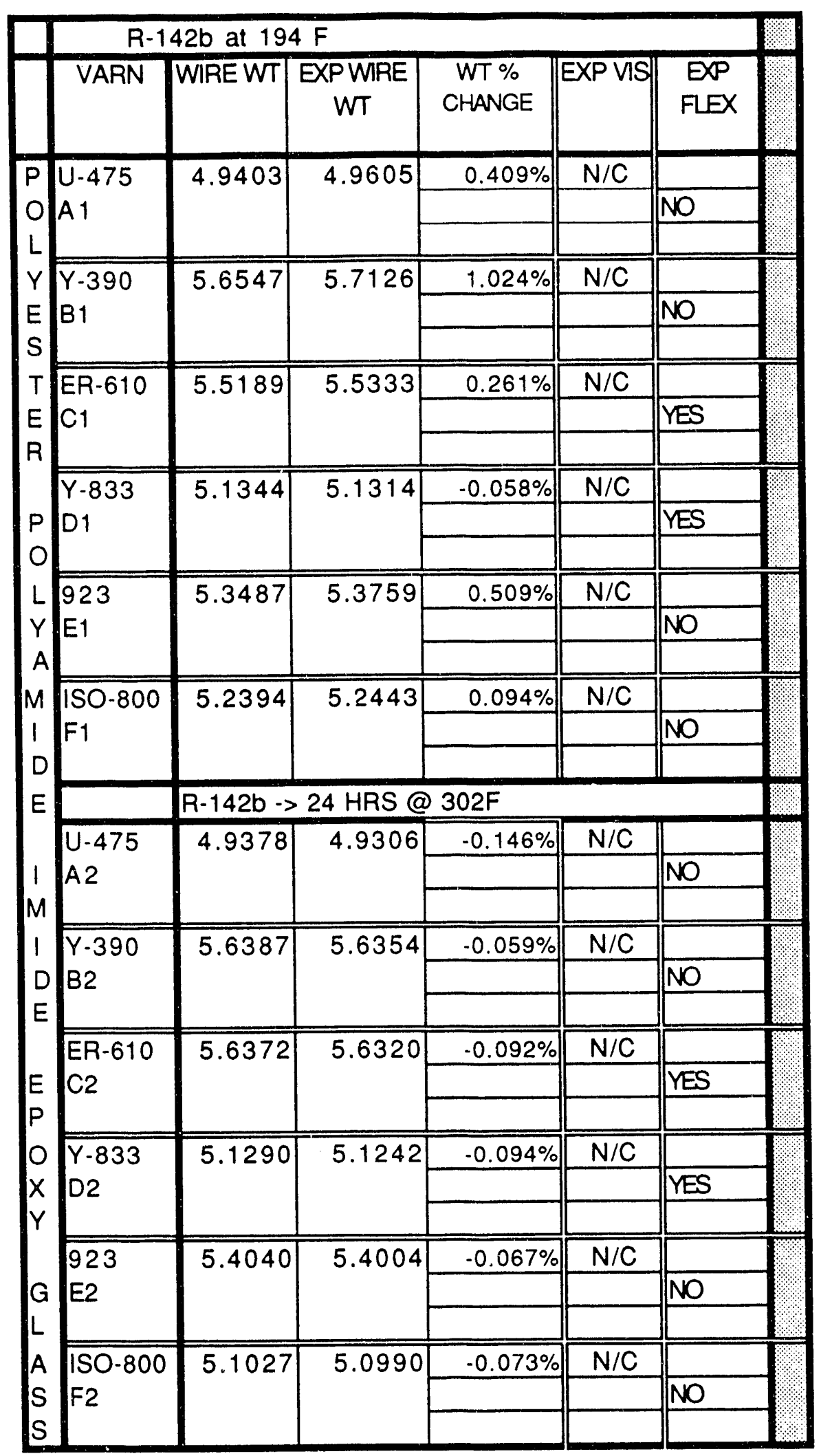




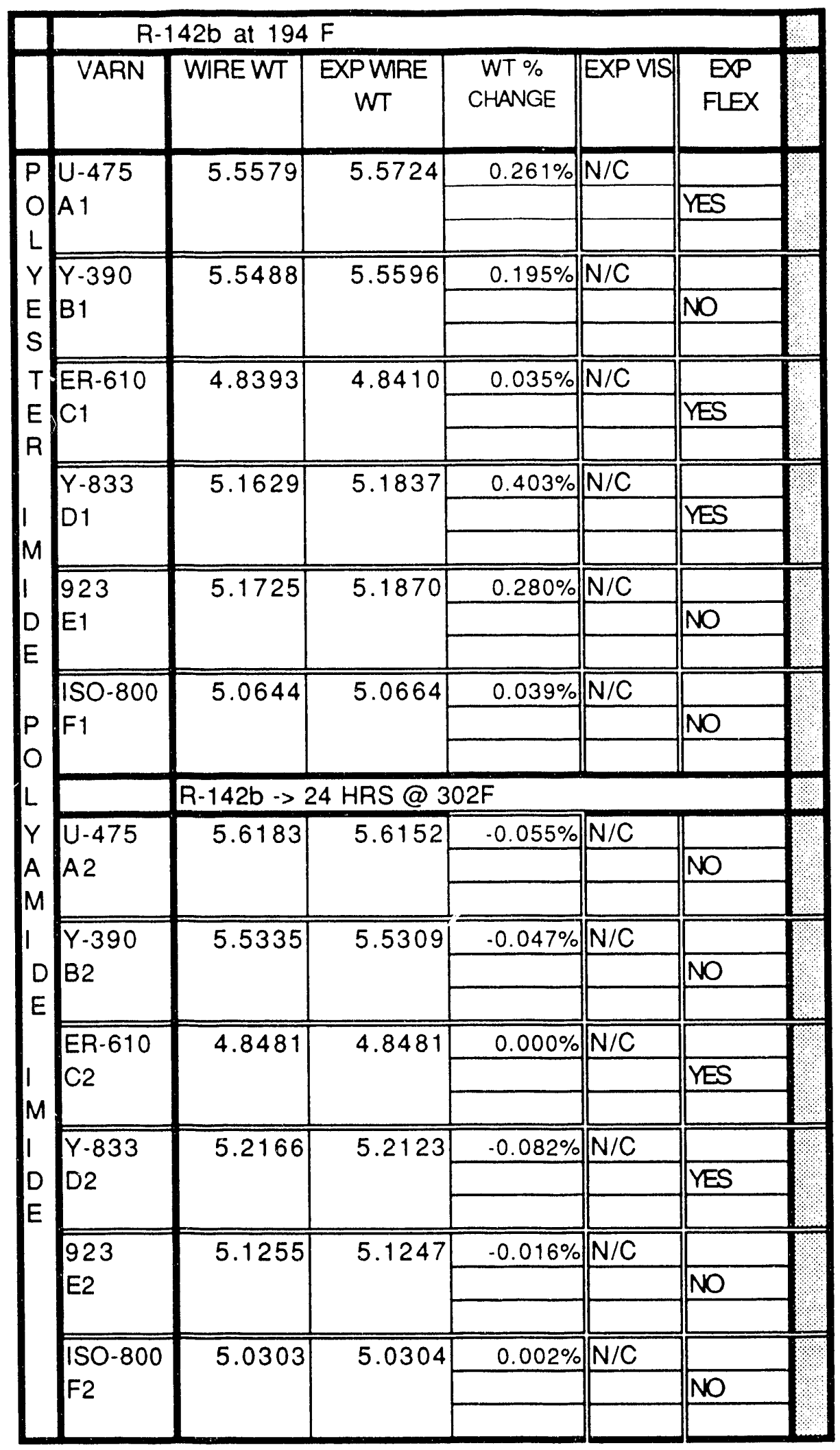

) 


\begin{tabular}{|c|c|c|c|c|c|}
\hline \multicolumn{6}{|c|}{500 HRS IN R-142b @ 194F } \\
\hline ID & WT & EXPWT & $\begin{array}{c}\text { WT \% } \\
\text { CHANGE }\end{array}$ & EXPVIS & EXPFLEX \\
\hline $\begin{array}{l}-475 \\
\text { A } 1\end{array}$ & 2.3328 & 2.4757 & $6.13 \%$ & $\mathrm{~N} / \mathrm{C}$ & $\mathrm{N} / \mathrm{C}$ \\
\hline $\begin{array}{l}Y-390 \\
B 1\end{array}$ & 1.8525 & 1.9728 & $6.49 \%$ & $\mathrm{~N} / \mathrm{C}$ & $\mathrm{N} / \mathrm{C}$ \\
\hline $\begin{array}{l}\text { ER-610 } \\
\text { C1 }\end{array}$ & 2.0392 & 2.2438 & $10.03 \%$ & $\mathrm{~N} / \mathrm{C}$ & $\mathrm{N} / \mathrm{C}$ \\
\hline $\begin{array}{l}\mathrm{Y}-833 \\
\mathrm{D} 1\end{array}$ & 2.1642 & 2.3002 & $6.28 \%$ & $\mathrm{~N} / \mathrm{C}$ & $N / C$ \\
\hline 923 & 1.9760 & 2.0977 & $6.16 \%$ & $\mathrm{~N} / \mathrm{C}$ & $N / C$ \\
\hline & & & & & \\
\hline $\begin{array}{l}\mathrm{ISO}-800 \\
\mathrm{~F} 1\end{array}$ & 1.8749 & 1.8753 & $0.02 \%$ & $\mathrm{~N} / \mathrm{C}$ & $\mathrm{N} / \mathrm{C}$ \\
\hline & $R-142 b$ at & $->24$ hour & at $302^{\circ} \mathrm{F}$. & & \\
\hline $\begin{array}{l}\text { U-475 } \\
\text { A2 }\end{array}$ & 2.1650 & 2.1325 & $-1.50 \%$ & \begin{tabular}{|l|} 
Darkened \\
slightly \\
We:.ped \\
\end{tabular} & $N / C$ \\
\hline $\begin{array}{l}Y-390 \\
B 2\end{array}$ & 2.2528 & 2.1869 & $-2.93 \%$ & \begin{tabular}{|l|} 
Few \\
Internal \\
Bubbles \\
\end{tabular} & $\mathrm{N} / \mathrm{C}$ \\
\hline $\begin{array}{l}\text { ER-610 } \\
\text { C2 }\end{array}$ & 2.3422 & 2.3367 & $-0.23 \%$ & \begin{tabular}{|l|} 
Few \\
Internal \\
Bubbles \\
\end{tabular} & \begin{tabular}{|l|} 
darkened \\
\end{tabular} \\
\hline $\begin{array}{l}\mathrm{Y}-833 \\
\mathrm{D} 2\end{array}$ & 2.5179 & 2.3634 & $-6.14 \%$ & \begin{tabular}{|l|} 
Many \\
Internal \\
bubbles
\end{tabular} & brittle \\
\hline \begin{tabular}{|l}
923 \\
E2
\end{tabular} & 2.0426 & 2.0362 & $-0.31 \%$ & \begin{tabular}{|l|} 
few \\
Internal \\
bubbles \\
\end{tabular} & $N / C$ \\
\hline $\begin{array}{l}150-800 \\
\text { F2 }\end{array}$ & 1.9216 & 1.8191 & $-5.33 \%$ & \begin{tabular}{|l|} 
Few \\
Internal \\
bubbles \\
\end{tabular} & \begin{tabular}{|l} 
very \\
warped
\end{tabular} \\
\hline
\end{tabular}




\begin{tabular}{|c|c|c|c|c|c|c|c|c|c|}
\hline \multicolumn{10}{|c|}{500 HR IN R-142b @ 194º } \\
\hline ID & WT & EXPWT & $\begin{array}{c}\text { WT \% } \\
\text { CHANGE }\end{array}$ & $\begin{array}{l}\text { SAMPLE } \\
\text { WIDTH }\end{array}$ & $\begin{array}{c}\text { BREAK } \\
\text { LOAD }\end{array}$ & \begin{tabular}{|c|} 
SAMPLE \\
THICKNESS \\
Mils
\end{tabular} & $\begin{array}{c}\text { TENSILE } \\
\text { STR } \\
\text { BASE }\end{array}$ & $\begin{array}{l}\text { TENSILE } \\
\text { STREXP }\end{array}$ & $\begin{array}{c}\% \\
\text { CHANGE } \\
\text { TENSILE }\end{array}$ \\
\hline \multirow{3}{*}{$\begin{array}{l}\text { NO/MY/NO } \\
\text { A1 }\end{array}$} & \multirow[t]{3}{*}{4.5092} & \multirow[t]{3}{*}{4.6627} & \multirow[t]{3}{*}{$3.40 \%$} & 0.392 & 132.70 & 21 & 17.4 & 16.12 & \\
\hline & & & & 0.446 & 157.10 & 21 & 17.4 & 16.77 & $-5.16 \%$ \\
\hline & & & & 0.452 & 157.70 & 21 & 17.4 & 16.61 & \\
\hline \multirow{3}{*}{$\begin{array}{l}\text { DA/MY/DA } \\
\text { B1 }\end{array}$} & \multirow[t]{3}{*}{4.3303} & \multirow[t]{3}{*}{4.5003} & \multirow[t]{3}{*}{$3.93 \%$} & 0.418 & 102.30 & 21 & 13.7 & 11.65 & \\
\hline & & & & 0.488 & 136.30 & 21 & 13.7 & 13.30 & $-7.72 \%$ \\
\hline & & & & 0.395 & 107.60 & 21 & 13.7 & 12.97 & \\
\hline \multirow{3}{*}{$\begin{array}{l}\text { MYLAR MO } \\
\text { C1 }\end{array}$} & \multirow[t]{3}{*}{2.4419} & \multirow[t]{3}{*}{2.5224} & \multirow[t]{3}{*}{$3.30 \%$} & 0.498 & 102.40 & 10 & 21.7 & 20.56 & \\
\hline & & & & 0.475 & 96.20 & 10 & 21.7 & 20.25 & $-8.68 \%$ \\
\hline & & & & 0.417 & 77.70 & 10 & 21.7 & 18.63 & \\
\hline \multirow{3}{*}{$\begin{array}{l}\text { NO } 410 \\
\text { D1 }\end{array}$} & \multirow{3}{*}{2.3591} & \multirow{3}{*}{2.3717} & \multirow[t]{3}{*}{$0.53 \%$} & 0.505 & 99.40 & 10 & 18.7 & 19.68 & \\
\hline & & & & 0.440 & 84.70 & 10 & 18.7 & 19.25 & $2.80 \%$ \\
\hline & & & & 0.495 & 92.75 & 10 & 18.7 & 18.74 & \\
\hline \multirow{3}{*}{$\begin{array}{l}\text { NO MI } 418 \\
\text { E1 }\end{array}$} & \multirow[t]{3}{*}{2.2739} & \multirow[t]{3}{*}{2.2827} & \multirow[t]{3}{*}{$0.39 \%$} & 0.489 & 26.50 & 9 & 7.5 & 6.02 & \\
\hline & & & & 0.477 & 25.30 & 9 & 7.5 & 5.89 & $-19.99 \%$ \\
\hline & & & & 0.490 & 26.85 & 9 & 7.5 & 6.09 & \\
\hline \multirow{3}{*}{$\begin{array}{l}\text { MEL } 228 \\
\text { F1 }\end{array}$} & \multirow[t]{3}{*}{2.4609} & \multirow{3}{*}{2.5631} & \multirow[t]{3}{*}{$4.15 \%$} & 0.452 & 82.60 & 10 & 21.7 & 18.27 & \\
\hline & & & & 0.514 & 98.90 & 10 & 21.7 & 19.24 & $-12.41 \%$ \\
\hline & & & & 0.483 & 94.20 & 10 & 21.7 & 19.50 & \\
\hline & & $500 \mathrm{HR}$ & IN R-142 & 2b@194 & ${ }^{\circ} \mathrm{F} \rightarrow 24 \mathrm{H}$ & tRS@302० & & & \\
\hline NO/MY/NO & 4.3502 & 4.3645 & $0.33 \%$ & 0.476 & 173.50 & 21 & 17.4 & 17.36 & \\
\hline$A 2$ & & & & 0.502 & 164.60 & 21 & 17.4 & 15.61 & $-4.80 \%$ \\
\hline & & & $\theta^{\circ}$ & 0.404 & 141.90 & 21 & 17.4 & 16.73 & \\
\hline$D A / M Y / D A$ & 4.1032 & 4.0864 & $-0.41 \%$ & 0.378 & 107.30 & 21 & 13.7 & 13.52 & \\
\hline$B 2$ & & & & 0.424 & 118.90 & 21 & 13.7 & 13.35 & $-3.89 \%$ \\
\hline & & & & 0.411 & 109.00 & 21 & 13.7 & 12.63 & \\
\hline MYLAR MO & 2.2106 & 2.2071 & $-0.16 \%$ & 0.405 & 74.85 & 10 & 21.7 & 18.48 & \\
\hline $\mathrm{C} 2$ & & & & 0.429 & 84.35 & 10 & 21.7 & 19.66 & $-13.36 \%$ \\
\hline & & & & 0.414 & 75.60 & 10 & 21.7 & 18.26 & \\
\hline NOMEX 410 & 2.2600 & 2.2456 & $-0.64 \%$ & 0.505 & 92.60 & 10 & 18.7 & 18.34 & \\
\hline $\mathrm{D} 2$ & & & & 0.488 & 96.85 & 10 & 18.7 & 19.85 & $5.31 \%$ \\
\hline & & & & 0.447 & 93.40 & 10 & 18.7 & 20.89 & \\
\hline $\mathrm{NO} / \mathrm{MI} 4: 8$ & 2.3795 & 2.3579 & $-0.91 \%$ & 0.499 & 25.65 & 9 & 7.5 & 5.71 & \\
\hline E? & & & & 0.508 & 27.80 & 9 & 7.5 & 6.08 & $-21.48 \%$ \\
\hline & & & & 0.503 & 26.60 & 9 & 7.5 & 5.88 & \\
\hline MEL 228 & 2.5156 & 2.5105 & $-0.20 \%$ & 0.492 & 92.10 & 10 & 21.7 & 18.72 & \\
\hline $\mathrm{F} 2$ & & & & 0.431 & 80.50 & 10 & 21.7 & 18.68 & $-12.77 \%$ \\
\hline & & & & 0.481 & 93.25 & 10 & 21.7 & 19.39 & \\
\hline
\end{tabular}




\begin{tabular}{|c|c|c|c|c|c|c|c|c|}
\hline \multicolumn{8}{|c|}{ After 500 hour exposure @ $194^{\circ} \mathrm{F}\left(90^{\circ} \mathrm{C}\right)$} & \multirow[b]{2}{*}{ VISUAL EXP } \\
\hline ID & $\begin{array}{c}\text { STRETCH } \\
\text { (inch) }\end{array}$ & $\begin{array}{c}\% \\
\text { ELONG }\end{array}$ & $\begin{array}{l}\text { BASE } \\
\text { ELONG } \\
\text { (AVE) }\end{array}$ & $\begin{array}{c}\text { ELONG } \\
\% \\
\text { CHANGE }\end{array}$ & $\begin{array}{c}\text { BASE } \\
\text { DIE } \\
\text { (AVE) }\end{array}$ & EXP DIE & $\begin{array}{c}\text { DIE \% } \\
\text { CHANGE }\end{array}$ & \\
\hline \multirow{3}{*}{$\begin{array}{l}\mathrm{NO} / \mathrm{MY} / \mathrm{NO} \\
\mathrm{A} 1\end{array}$} & 0.85 & $21.3 \%$ & $20.0 \%$ & & $>18.97$ & $>15.60$ & & \multirow[t]{3}{*}{$\mathrm{N} / \mathrm{C}$} \\
\hline & 1.02 & $25.5 \%$ & $20.0 \%$ & $21.7 \%$ & $>18.97$ & $>16.31$ & flash & \\
\hline & 1.05[ & $26.3 \%$ & $20.0 \%$ & & $>18.97$ & $>17.14$ & & \\
\hline \multirow{3}{*}{$\begin{array}{l}\mathrm{CA} / \mathrm{MY} / \mathrm{DA} \\
\mathrm{B} 1\end{array}$} & 0.69 & $34.5 \%$ & $46.0 \%$ & & $>15.27$ & $>15.10$ & & \multirow{3}{*}{$\begin{array}{l}\text { slighlty } \\
\text { warped }\end{array}$} \\
\hline & 0.62 & $31.0 \%$ & $46.0 \%$ & $-30.8 \%$ & $>15.27$ & $>14.42$ & flash & \\
\hline & $0.60]$ & $30.0 \%$ & $46.0 \%$ & & $>15.27$ & $>14.56$ & & \\
\hline \multirow{3}{*}{$\begin{array}{l}\text { MYLAR MO } \\
\text { C1 }\end{array}$} & 3.26 & $163.0 \%$ & $131.0 \%$ & & $>14.91$ & $>13.91$ & & \multirow[t]{3}{*}{$\mathrm{N} / \mathrm{C}$} \\
\hline & 3.18 & $159.0 \%$ & $131.0 \%$ & $17.2 \%$ & $>14.91$ & $>13.89$ & flash & \\
\hline & 2.77 & $138.5 \%$ & $131.0 \%$ & & $>14.91$ & $>14.08$ & & \\
\hline \multirow{3}{*}{$\begin{array}{l}\text { NO } 410 \\
\text { D1 }\end{array}$} & 0.55 & $13.8 \%$ & $17.0 \%$ & & 10.67 & 10.39 & & \multirow[t]{3}{*}{$\mathrm{N} / \mathrm{C}$} \\
\hline & 0.53 & $13.3 \%$ & $17.0 \%$ & $.26 .0 \%$ & 10.67 & 9.60 & $-0.8 \%$ & \\
\hline & 0.43 & $10.8 \%$ & $17.0 \%$ & & 10.67 & 11.76 & & \\
\hline \multirow{3}{*}{$\begin{array}{l}\text { NO MI } 418 \\
\text { E1 }\end{array}$} & 0.07 & $1.8 \%$ & $4.0 \%$ & & 10.23 & 9.45 & & \multirow[t]{3}{*}{$\mathrm{N} / \mathrm{C}$} \\
\hline & 0.08 & $2.0 \%$ & $4.0 \%$ & $-54.2 \%$ & 10.23 & 10.28 & $-2.7 \%$ & \\
\hline & 0.07 & $1.8 \%$ & $4.0 \%$ & & 10.23 & 10.13 & & \\
\hline \multirow{3}{*}{$\begin{array}{l}\text { MEL } 228 \\
\text { F1 }\end{array}$} & 3.15 & $157.5 \%$ & $160.0 \%$ & & $>14.22$ & $>12.37$ & & \multirow[t]{3}{*}{$\mathrm{N} / \mathrm{C}$} \\
\hline & 3.47 & $173.5 \%$ & $160.0 \%$ & $5.3 \%$ & $>14.22$ & $>14.21$ & flash & \\
\hline & 3.49 & $174.5 \%$ & $160.0 \%$ & & $>14.22$ & $>13.84$ & & \\
\hline & \multicolumn{7}{|c|}{ After 500 hour exposure plus a 24 h } & $\left.0^{\circ} \mathrm{C}\right)$ \\
\hline \multirow{3}{*}{$\begin{array}{l}\mathrm{NO} / \mathrm{MY} / \mathrm{NO} \\
\mathrm{A} 2\end{array}$} & 0.81 & $20.3 \%$ & $20.0 \%$ & & $>18.97$ & $>15.72$ & & \multirow{3}{*}{$\begin{array}{l}\text { Bubbles } \\
\text { delamination }\end{array}$} \\
\hline & 0.39 & $9.8 \%$ & $20.0 \%$ & $.16 .7 \%$ & $>18.97$ & $>15.90$ & flash & \\
\hline & 0.80 & $20.0 \%$ & $20.0 \%$ & & $>18.97$ & $>16.27$ & & \\
\hline \multirow{3}{*}{$\begin{array}{l}\mathrm{DA} / \mathrm{MY} / \mathrm{DA} \\
\mathrm{B} 2\end{array}$} & 0.52 & $26.0 \%$ & $46.0 \%$ & & $>15.27$ & $>12.73$ & & \multirow{3}{*}{$\begin{array}{l}\text { slighlty } \\
\text { warped }\end{array}$} \\
\hline & 0.56 & $28.0 \%$ & $46.0 \%$ & $-46.0 \%$ & $>15.27$ & $>12.70$ & flash & \\
\hline & 0.41 & $20.5 \%$ & $46.0 \%$ & & $>15.27$ & $>12.89$ & & \\
\hline \multirow{3}{*}{$\begin{array}{l}\text { MYLAR MO } \\
\text { C2 }\end{array}$} & 2.64 & $132.0 \%$ & $131.0 \%$ & & $>14.91$ & $>13.22$ & & \multirow[t]{3}{*}{$\mathrm{N} / \mathrm{C}$} \\
\hline & 2.99 & $149.5 \%$ & $131.0 \%$ & $6.7 \%$ & $>14.91$ & $>11.66$ & flash & \\
\hline & 2.76 & $138.0 \%$ & $131.0 \%$ & & $>14.91$ & $>13.19$ & & \\
\hline \multirow{3}{*}{$\begin{array}{l}\text { NOMEX 410 } \\
\text { D2 }\end{array}$} & 0.42 & $10.5 \%$ & $17.0 \%$ & & 10.67 & 9.81 & & \multirow[t]{3}{*}{$\mathrm{N} / \mathrm{C}$} \\
\hline & 0.55 & $13.8 \%$ & $17.0 \%$ & $-23.0 \%$ & 10.67 & 10.22 & $-8.4 \%$ & \\
\hline & 0.60 & $15.0 \%$ & $17.0 \%$ & & 10.67 & 9.30 & & \\
\hline \multirow{3}{*}{$\begin{array}{l}\text { NO/MI } 418 \\
\text { E2 }\end{array}$} & 0.08 & $2.0 \%$ & $4.0 \%$ & & 10.23 & 9.90 & & \multirow[t]{3}{*}{$\mathrm{N} / \mathrm{C}$} \\
\hline & 0.09 & $2.3 \%$ & $4.0 \%$ & $-47.9 \%$ & 10.23 & 9.42 & $-8.0 \%$ & \\
\hline & 0.08 & $2.0 \%$ & $4.0 \%$ & & 10.23 & 8.91 & & \\
\hline MEL 228 & 3.24 & $162.0 \%$ & $160.0 \%$ & & $>14.22$ & $>14.25$ & & $\mathrm{~N} / \mathrm{C}$ \\
\hline $\mathrm{F} 2$ & 3.32 & $166.0 \%$ & $160.0 \%$ & $5.6 \%$ & $>14.22$ & $>14.21$ & flash & \\
\hline & 3.58 & $179.0 \%$ & $160.0 \%$ & & $>14.22$ & $>12.16$ & & \\
\hline
\end{tabular}




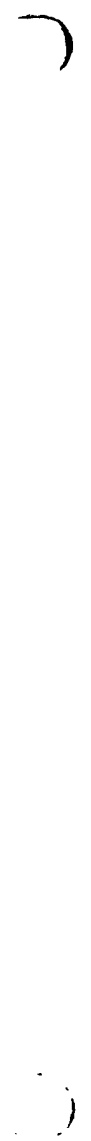

\begin{tabular}{|c|c|c|c|c|}
\hline & \multicolumn{4}{|c|}{500 HRS IN R-142b @194 F } \\
\hline & WT & EXPWT & $\begin{array}{c}\% \\
\text { CHANGE } \\
\end{array}$ & EXP VIS \\
\hline $\begin{array}{l}\text { A } 1 \\
\text { NOMEX }\end{array}$ & 0.5407 & 0.5735 & $6.07 \%$ & $\mathrm{~N} / \mathrm{C}$ \\
\hline $\begin{array}{l}\text { B1 } \\
\text { MYLAR }\end{array}$ & 0.4647 & 0.5048 & $8.63 \%$ & $\begin{array}{c}\frac{\text { Bubble }}{\text { between }} \\
\text { layers }\end{array}$ \\
\hline $\begin{array}{l}\mathrm{C} 1 \\
\mathrm{NO} / \mathrm{MY}\end{array}$ & 0.4025 & 0.4164 & $3.45 \%$ & $\mathrm{~N} / \mathrm{C}$ \\
\hline & R-142b@19 & $4^{\circ} \mathrm{F}-\cdots>24$ & irs@302 & \\
\hline $\begin{array}{l}\text { A2 } \\
\text { NOMEX }\end{array}$ & 0.5363 & 0.5242 & $-2.26 \%$ & $\mathrm{~N} / \mathrm{C}$ \\
\hline $\begin{array}{l}\text { B2 } \\
\text { MYLAR }\end{array}$ & 0.4614 & 0.4602 & $-0.26 \%$ & \begin{tabular}{|c|} 
ome delamination \\
see photo \\
\end{tabular} \\
\hline $\begin{array}{l}\mathrm{C} 2 \\
\mathrm{NO} / \mathrm{MY}\end{array}$ & 0.4037 & 0.4005 & $-0.79 \%$ & $\begin{array}{l}\text { Pockects where } \\
\text { mylar pulled away } \\
\text { "see photo }\end{array}$ \\
\hline
\end{tabular}

) 


\begin{tabular}{|c|c|c|c|c|c|c|c|c|c|}
\hline & \multicolumn{9}{|c|}{500 HRS IN R-142b @ 194 F } \\
\hline D & WT & EXPWT & $\begin{array}{c}\text { WT \% } \\
\text { CHANGE }\end{array}$ & $\begin{array}{l}\text { BREAK } \\
\text { LOAD } \\
\text { (AVE) }\end{array}$ & $\begin{array}{c}\text { BREAK } \\
\text { LOAD } \\
\text { EXP }\end{array}$ & $\begin{array}{c}\% \\
\text { CHANGE } \\
\text { BRK } \\
\text { LOAD }\end{array}$ & $\begin{array}{c}\text { STRETCH } \\
(\mathrm{INCH})\end{array}$ & $\%$ ELONG & EXP VIS \\
\hline \multirow{3}{*}{$\begin{array}{l}\text { A } 1 \\
\text { Glass }\end{array}$} & \multirow[t]{3}{*}{1.5101} & \multirow[t]{3}{*}{1.5089} & \multirow[t]{3}{*}{$-0.08 \%$} & 39.02 & 45.30 & & 0.07 & $3.50 \%$ & $\mathrm{~N} / \mathrm{C}$ \\
\hline & & & & 39.02 & 46.50 & $19.77 \%$ & 0.05 & $2.50 \%$ & \\
\hline & & & & 39.02 & 48.40 & & 0.05 & $2.50 \%$ & \\
\hline \multirow{3}{*}{$\begin{array}{l}\text { B1 } \\
\text { Polyester }\end{array}$} & \multirow[t]{3}{*}{0.6358} & \multirow[t]{3}{*}{0.6595} & \multirow[t]{3}{*}{$3.73 \%$} & 56.12 & 60.90 & & 0.67 & $33.50 \%$ & $\mathrm{~N} / \mathrm{C}$ \\
\hline & & & & 56.12 & 58.75 & $5.07 \%$ & 0.64 & $32.00 \%$ & \\
\hline & & & & 56.12 & 57.25 & & 0.65 & $32.50 \%$ & \\
\hline \multirow{4}{*}{$\begin{array}{l}\text { C1 } \\
\text { Permacel }\end{array}$} & \multirow[t]{3}{*}{1.4760} & \multirow[t]{3}{*}{1.5803} & \multirow[t]{3}{*}{$7.07 \%$} & 88.50 & 111.10 & & 0.09 & $4.50 \%$ & Darkened \\
\hline & & & & 88.50 & 88.35 & $1.66 \%$ & 0.09 & $4.70 \%$ & \\
\hline & & & & 88.50 & 70.45 & & 0.10 & $5.00 \%$ & \\
\hline & \multicolumn{7}{|c|}{$500 \mathrm{HRS}$ in R-142b $>302^{\circ} \mathrm{F} 24 \mathrm{HRS}$} & & \\
\hline \multirow{3}{*}{$\begin{array}{l}\text { A2 } \\
\text { Glass }\end{array}$} & \multirow[t]{3}{*}{1.3670} & \multirow[t]{3}{*}{1.3669} & \multirow[t]{3}{*}{$.0 .01 \%$} & 39.02 & 46.65 & & 0.04 & $2.00 \%$ & $\mathrm{~N} / \mathrm{C}$ \\
\hline & & & & 39.02 & 53.45 & $17.97 \%$ & 0.06 & $3.00 \%$ & \\
\hline & & & & 39.02 & 38.00 & & 0.05 & $2.50 \%$ & \\
\hline \multirow{3}{*}{$\begin{array}{l}\text { B2 } \\
\text { Polyester }\end{array}$} & \multirow[t]{3}{*}{0.5821} & \multirow[t]{3}{*}{0.5845} & \multirow[t]{3}{*}{$0.41 \%$} & 56.12 & 57.45 & & 0.62 & $31.00 \%$ & $\mathrm{~N} / \mathrm{C}$ \\
\hline & & & & 56.12 & 52.65 & $-2.95 \%$ & 0.51 & $25.50 \%$ & \\
\hline & & & & 56.12 & 53.30 & & 0.53 & $26.50 \%$ & \\
\hline \multirow{3}{*}{$\begin{array}{l}\mathrm{C} 2 \\
\text { Permacel }\end{array}$} & \multirow[t]{3}{*}{1.2669} & \multirow[t]{3}{*}{1.1915} & \multirow[t]{3}{*}{$-5.95 \%$} & 88.50 & 97.25 & & 0.10 & $5.00 \%$ & \\
\hline & & & & 88.50 & 99.65 & $6.78 \%$ & 0.14 & $7.00 \%$ & Darkenad \\
\hline & & & & 88.50 & 86.60 & & 0.14 & $7.00 \%$ & \\
\hline
\end{tabular}




\begin{tabular}{|c|c|c|c|c|c|c|c|c|c|}
\hline \multicolumn{10}{|c|}{500 HRS IN R-142b @ 194. F } \\
\hline ID & WT & EXPWT & $\begin{array}{c}\text { WT \% } \\
\text { CHANGE }\end{array}$ & $\begin{array}{l}\text { EXP } \\
\text { VIS }\end{array}$ & $\begin{array}{l}\text { BREAK } \\
\text { LOAD } \\
\text { (AVE) }\end{array}$ & $\begin{array}{c}\text { BFEAK } \\
\text { LOAD } \\
\text { EXP } \\
(\text { ave })\end{array}$ & $\begin{array}{c}\% \\
\text { CHANGE } \\
\text { BRK } \\
\text { LOAD }\end{array}$ & $\begin{array}{c}\text { STRETCH } \\
\text { (Inch) }\end{array}$ & $\begin{array}{c}\% \\
\text { ELONG }\end{array}$ \\
\hline \multirow[t]{4}{*}{ A1 } & \multirow[t]{3}{*}{0.2500} & \multirow[t]{3}{*}{0.2565} & \multirow[t]{3}{*}{$2.60 \%$} & $\mathrm{~N} / \mathrm{C}$ & 28.36 & 35.65 & & 0.37 & $18.5 \%$ \\
\hline & & & & & 28.36 & 33.25 & $14.36 \%$ & 0.36 & $18.0 \%$ \\
\hline & & & & & 28.36 & 28.40 & & 0.32 & $16.0 \%$ \\
\hline & \multicolumn{9}{|c|}{500 HRS IN R-142b $>24$ HRS @ $302^{\circ} \mathrm{F}$} \\
\hline \multirow[t]{3}{*}{ A2 } & \multirow[t]{3}{*}{0.2464} & \multirow[t]{3}{*}{0.2462} & \multirow[t]{3}{*}{$-0.081 \%$} & $\mathrm{~N} / \mathrm{C}$ & 28.36 & 28.05 & & 0.47 & $23.5 \%$ \\
\hline & & & & & 28.36 & 26.55 & $-2.15 \%$ & 0.42 & $21.0 \%$ \\
\hline & & & & & 28.36 & 28.65 & & 0.45 & $22.5 \%$ \\
\hline
\end{tabular}




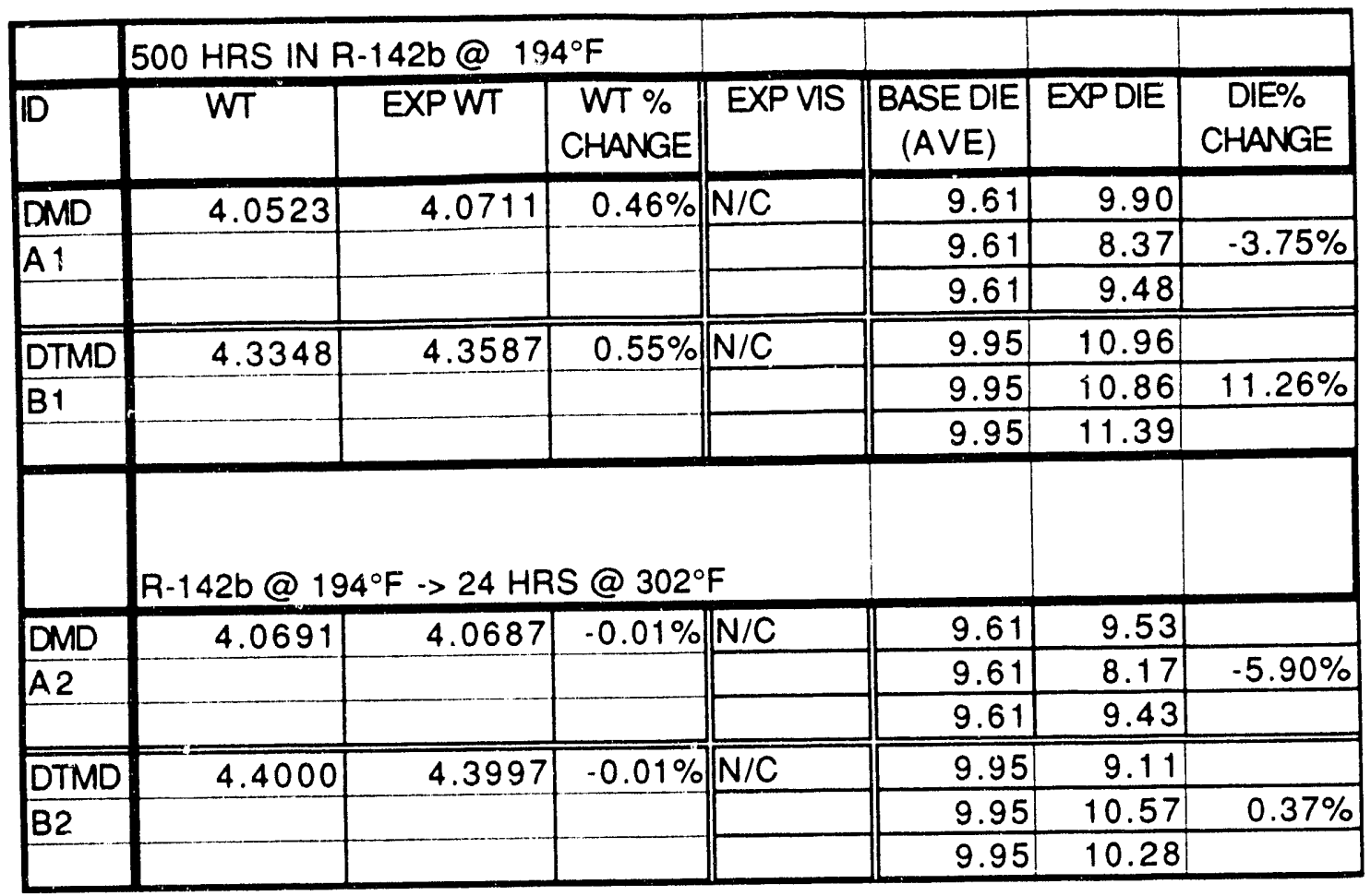




\section{Appendix G}

Experimental Data for HFC-152a Exposure at $90^{\circ} \mathrm{C}\left(194^{\circ} \mathrm{F}\right)$ 


\begin{tabular}{|c|c|c|c|c|c|c|c|c|c|c|}
\hline & 500 HRS IN & R-152a@ & $194 \mathrm{~F}$ & & & & & & & \\
\hline ID & WT & EXPWT & $\begin{array}{c}\text { WT\% } \\
\text { CHANGE }\end{array}$ & $\begin{array}{l}\text { EXP } \\
\text { VIS }\end{array}$ & $\begin{array}{c}\text { BASE } \\
\text { BRNOUT } \\
(A \vee E)\end{array}$ & $\begin{array}{c}\text { EXP BRN } \\
\text { OUT }\end{array}$ & $\begin{array}{c}\text { BRN OUT } \\
\% \\
\text { CHANGE }\end{array}$ & $\begin{array}{l}\text { BASE } \\
\text { DIE } \\
\text { (AVE) }\end{array}$ & EXP DIE & $\begin{array}{c}\text { DIE \% } \\
\text { CHANGE }\end{array}$ \\
\hline A 1 & 24.8314 & 24.8562 & $0.100 \%$ & $\mathrm{~N} / \mathrm{C}$ & 576 & 429 & & 15.80 & 20.00 & \\
\hline & & & & & 576 & 402 & $-28.4 \%$ & 15.80 & 13.01 & $7.5 \%$ \\
\hline & & & & & 576 & 407 & & 15.80 & 17.95 & \\
\hline B1 & 24.7799 & 24.8528 & $0.294 \%$ & $\mathrm{~N} / \mathrm{C}$ & 736 & 731 & & 11.62 & 12.87 & \\
\hline & & & & & 736 & 733 & $-3.5 \%$ & 11.62 & 12.09 & $7.8 \%$ \\
\hline & & & & & 736 & 667 & & 11.62 & 12.62 & \\
\hline C1 & 22.6199 & 22.6448 & $0.110 \%$ & $\mathrm{~N} / \mathrm{C}$ & 579 & 454 & & 16.58 & 12.95 & \\
\hline & & & & & 579 & 452 & $-18.9 \%$ & 16.58 & 9.95 & $22.7 \%$ \\
\hline & & & & & 579 & 503 & & 16.58 & 15.57 & \\
\hline & $R-152 a-->2$ & 4 HRS@ & $302 F$ & & & & & & & \\
\hline A2 & 24.7114 & 24.7101 & $-0.005 \%$ & $\mathrm{~N} / \mathrm{C}$ & 576 & 491 & & 15.80 & 15.88 & \\
\hline & & & & & 576 & 467 & $-11.2 \%$ & 15.80 & 16.16 & $-1.0 \%$ \\
\hline & & & & & 576 & 576 & & 15.80 & 14.87 & \\
\hline B2 & 24.2052 & 24.2038 & $-0.006 \%$ & $\mathrm{~N} / \mathrm{C}$ & 736 & 712 & & 11.62 & 11.03 & \\
\hline & & & & & 736 & 671 & $-5.8 \%$ & 11.62 & 10.83 & $-5.1 \%$ \\
\hline & & & & & 736 & 697 & & 11.62 & 11.23 & \\
\hline $\mathrm{C2}$ & 24.0461 & 24.0452 & $-0.004 \%$ & $\mathrm{~N} / \mathrm{C}$ & 579 & 454 & & 16.58 & 14.89 & \\
\hline & & & & & 579 & 520 & $-15.8 \%$ & 16.58 & 18.21 & $-3.3 \%$ \\
\hline & & & & & 579 & 488 & & 16.58 & 14.99 & \\
\hline
\end{tabular}


500 HOURS IN R-152a @ 194 F

\begin{tabular}{|c|c|c|c|c|c|c|c|c|c|c|c|}
\hline & VARN & $\begin{array}{l}\text { TWISTED } \\
\text { PAIR WT }\end{array}$ & $\begin{array}{c}\text { EXPT } \\
\text { PAIR WT }\end{array}$ & $\begin{array}{c}\text { WT \% } \\
\text { CHANGE }\end{array}$ & $\begin{array}{l}\text { EXP } \\
\text { VIS }\end{array}$ & BASE DIE & EXP DIE & $\begin{array}{c}\text { DIE \% } \\
\text { CHANGE }\end{array}$ & $\begin{array}{l}\text { BASE } \\
\text { BUPN } \\
\text { OIT }\end{array}$ & $\begin{array}{c}\text { EXP } \\
\text { BUPN } \\
\text { OUT }\end{array}$ & $\begin{array}{c}\text { BRNOUT } \\
\% \\
\text { CHANGE }\end{array}$ \\
\hline \multirow{3}{*}{$\begin{array}{l}P \\
O \\
L\end{array}$} & \multirow{3}{*}{$\begin{array}{l}\text { U-475 } \\
\text { A } 1\end{array}$} & \multirow[t]{3}{*}{21.0623} & \multirow[t]{3}{*}{21.1329} & \multirow[t]{3}{*}{$0.335 \%$} & $\mathrm{~N} / \mathrm{C}$ & 16.24 & 12.44 & & 430 & 363 & \\
\hline & & & & & & 16.24 & 12.02 & $-18.19 \%$ & 430 & 438 & $.12 .17 \%$ \\
\hline & & & & & & 16.24 & 15.40 & & 430 & 332 & \\
\hline \multirow{3}{*}{$\begin{array}{l}Y \\
E \\
S\end{array}$} & \multirow{3}{*}{$\begin{array}{l}Y-390 \\
B 1\end{array}$} & \multirow[t]{3}{*}{21.1133} & \multirow[t]{3}{*}{21.1509} & \multirow[t]{3}{*}{$0.178 \%$} & $\mathrm{~N} / \mathrm{C}$ & 18.77 & 15.97 & & 510 & 312 & \\
\hline & & & & & & 18.77 & 15.51 & $-15.17 \%$ & 510 & 295 & $-41.24 \%$ \\
\hline & & & & & & 18.77 & 16.29 & & 510 & 292 & \\
\hline \multirow{4}{*}{$\begin{array}{l}T \\
E \\
R\end{array}$} & ER-610 & \multirow[t]{3}{*}{21.6699} & \multirow[t]{3}{*}{21.7622} & \multirow[t]{3}{*}{$0.426 \%$} & $\mathrm{~N} / \mathrm{C}$ & 15.57 & 11.51 & & 442 & 418 & \\
\hline & \multirow[t]{2}{*}{$C_{1}$} & & & & & 15.57 & 14.53 & $-9.08 \%$ & 442 & 434 & $2.04 \%$ \\
\hline & & & & & & 15.57 & 16.43 & & 442 & 501 & \\
\hline & $Y-833$ & \multirow{3}{*}{21.0502} & \multirow[t]{3}{*}{21.0690} & \multirow[t]{3}{*}{$0.089 \%$} & $\mathrm{~N} / \mathrm{C}$ & 12.04 & 8.34 & & 578 & 440 & \\
\hline \multirow{2}{*}{$\begin{array}{l}P \\
O\end{array}$} & & & & & & 12.04 & 9.69 & $-5.18 \%$ & 578 & 425 & $-26.41 \%$ \\
\hline & & & & & & 12.04 & 16.22 & & 578 & 411 & \\
\hline \multirow{3}{*}{$\begin{array}{l}\mathrm{L} \\
\mathrm{Y} \\
\mathrm{A}\end{array}$} & 923 & \multirow[t]{3}{*}{22.4011} & \multirow[t]{3}{*}{22.5076} & \multirow[t]{3}{*}{$0.475 \%$} & $\mathrm{~N} / \mathrm{C}$ & 16.76 & 15.90 & & 606 & 552 & \\
\hline & E1 & & & & & 16.76 & 9.16 & $-23.33 \%$ & 606 & 553 & $-12.05 \%$ \\
\hline & & & & & & 16.76 & 13.49 & & 606 & 494 & \\
\hline$M$ & $150-800$ & 21.4539 & 21.5023 & $0.226 \%$ & $\mathrm{~N} / \mathrm{C}$ & 19.08 & 17.88 & & 580 & 500 & \\
\hline 1 & F1 & & & & & 19.08 & 12.72 & $-21.35 \%$ & 580 & 479 & $-14.20 \%$ \\
\hline$D$ & & & & & & 19.08 & 14.42 & & 580 & 514 & \\
\hline$E$ & & 24 HOUR & S AT $302 F$ & & & & & & & & \\
\hline & U-475 & 21.3650 & 21.3896 & $0.115 \%$ & $\mathrm{~N} / \mathrm{C}$ & 16.24 & 14.47 & & 430 & 267 & \\
\hline 1 & A 2 & & & & & 16.24 & 16.34 & $-8.37 \%$ & 430 & 390 & $-22.25 \%$ \\
\hline$M$ & & & & & & 16.24 & 13.83 & & 430 & 346 & \\
\hline 1 & $Y-390$ & 21.1121 & 21.1148 & $0.013 \%$ & $\mathrm{~N} / \mathrm{C}$ & 18.77 & 9.81 & & 510 & 314 & \\
\hline$D$ & B2 & & & & & 18.77 & 10.37 & $-40.45 \%$ & 510 & 358 & $-30.92 \%$ \\
\hline$E$ & & & & & & 18.77 & 13.35 & & 510 & 385 & \\
\hline & ER-610 & 21.9094 & 21.9133 & $0.018 \%$ & $\mathrm{~N} / \mathrm{C}$ & 15.57 & 11.08 & & 442 & 418 & \\
\hline & $\mathrm{C} 2$ & & & & & 15.57 & 10.10 & $-26.05 \%$ & 442 & 425 & $-1.06 \%$ \\
\hline & & & & & & 15.57 & 13.36 & & 442 & 469 & \\
\hline & $Y-833$ & 21.6282 & 21.6186 & $-0.044 \%$ & $\mathrm{~N} / \mathrm{C}$ & 12.04 & 10.58 & & 578 & 535 & \\
\hline & D2 & & & & & 12.04 & 11.74 & $-6.40 \%$ & 578 & 490 & $-12.05 \%$ \\
\hline & & & & & & 12.04 & 11.49 & & 578 & 500 & \\
\hline & 923 & 22.3357 & 22.3421 & $0.029 \%$ & $\mathrm{~N} / \mathrm{C}$ & 16.76 & 6.43 & & 606 & 558 & \\
\hline & E2 & & & & & 16.76 & 9.61 & $-50.14 \%$ & 606 & 564 & $-12.21 \%$ \\
\hline & & & & & & 16.76 & 9.03 & & 606 & 474 & \\
\hline & $150-800$ & 21.4186 & 21.4165 & $-0.010 \%$ & $\mathrm{~N} / \mathrm{C}$ & 19.08 & 14.15 & & 580 & 474 & \\
\hline & $\mathrm{F} 2$ & & & & & 19.08 & 14.59 & $-24.63 \%$ & 580 & 430 & $-16.55 \%$ \\
\hline & & & & & & 19.08 & 14.40 & & 580 & 548 & \\
\hline
\end{tabular}


500 HOURS IN R-152a @ $194^{\circ} \mathrm{F}$

\begin{tabular}{|c|c|c|c|c|c|c|c|c|c|c|c|}
\hline & VARN & $\begin{array}{l}\text { TWISTED } \\
\text { PAIR WT }\end{array}$ & $\begin{array}{c}\text { EXPT } \\
\text { PAIR WT }\end{array}$ & \begin{tabular}{|c|} 
WT \% \\
CHANGE
\end{tabular} & $\begin{array}{l}\text { EXP } \\
\text { VIS }\end{array}$ & $\begin{array}{c}\text { BASE } \\
\text { DIE }\end{array}$ & $\begin{array}{l}\text { EXP } \\
\text { DIE }\end{array}$ & \begin{tabular}{|c|} 
DIE \% \\
CHANGE
\end{tabular} & $\begin{array}{c}\text { BASE } \\
\text { BURN } \\
\text { OUT }\end{array}$ & $\begin{array}{c}\text { EXP } \\
\text { BURN } \\
\text { OUT }\end{array}$ & $\begin{array}{c}\text { BRNOUT } \\
\% \\
\text { CHANGE }\end{array}$ \\
\hline \multirow{6}{*}{$\begin{array}{l}P \\
O \\
L \\
Y \\
E \\
S \\
-\end{array}$} & \multirow{3}{*}{$\begin{array}{l}U-475 \\
\text { A } 1\end{array}$} & 22.3329 & 22.4749 & $0.64 \%$ & $\mathrm{~N} / \mathrm{C}$ & 13.32 & 10.13 & & 746 & 669 & \\
\hline & & & & & & 13.32 & 12.87 & $-6.91 \%$ & 746 & 665 & $-9.34 \%$ \\
\hline & & & & & & 13.32 & 14.20 & & 746 & 695 & \\
\hline & \multirow{3}{*}{$\begin{array}{l}Y-390 \\
B 1\end{array}$} & \multirow[t]{3}{*}{23.4162} & \multirow{3}{*}{23.5983} & \multirow[t]{3}{*}{$0.78 \%$} & $\mathrm{~N} / \mathrm{C}$ & 12.28 & 12.86 & & 755 & 709 & \\
\hline & & & & & & 12.28 & 13.28 & $2.42 \%$ & 755 & 730 & $-3.93 \%$ \\
\hline & & & & & & 12.28 & 11.59 & & 755 & 737 & \\
\hline & \multirow{3}{*}{$\begin{array}{l}\text { ER-610 } \\
\text { C1 }\end{array}$} & \multirow[t]{3}{*}{22.2562} & \multirow[t]{3}{*}{22.3454} & \multirow[t]{3}{*}{$0.40 \%$} & $\mathrm{~N} / \mathrm{C}$ & 12.73 & 12.63 & & 734 & 657 & \\
\hline \multirow{2}{*}{$\begin{array}{l}T \\
E \\
R\end{array}$} & & & & & & 12.73 & 13.74 & $1.31 \%$ & 734 & 733 & $-3.91 \%$ \\
\hline & & & & & & 12.73 & 12.32 & & 734 & 726 & \\
\hline & \multirow{3}{*}{$\begin{array}{l}Y-833 \\
D 1\end{array}$} & \multirow[t]{3}{*}{22.5501} & \multirow[t]{3}{*}{22.6615} & \multirow[t]{3}{*}{$0.49 \%$} & $\mathrm{~N} / \mathrm{C}$ & 12.49 & 11.98 & & 734 & 729 & \\
\hline & & & & & & 12.49 & 11.17 & $-6.11 \%$ & 734 & 656 & $-8.36 \%$ \\
\hline & & & & & & 12.49 & 12.03 & & 734 & 633 & \\
\hline \multirow{3}{*}{$\begin{array}{l}\mathrm{L} \\
\mathrm{Y} \\
\mathrm{A}\end{array}$} & \multirow{3}{*}{$\begin{array}{l}923 \\
E 1\end{array}$} & \multirow[t]{3}{*}{22.7557} & \multirow[t]{3}{*}{22.9268} & \multirow[t]{3}{*}{$0.75 \%$} & $N / C$ & 14.38 & 10.13 & & 742 & 689 & \\
\hline & & & & & & 14.38 & 9.83 & $-20.96 \%$ & 742 & 728 & $-3.68 \%$ \\
\hline & & & & & & 14.38 & 14.14 & & 742 & 727 & \\
\hline \multirow{3}{*}{$\begin{array}{l}M \\
1 \\
D\end{array}$} & \multirow{3}{*}{$\begin{array}{l}\text { ISO-800 } \\
\mathrm{F} 1\end{array}$} & \multirow[t]{3}{*}{26.1670} & 26.3362 & $0.65 \%$ & $\mathrm{~N} / \mathrm{C}$ & 12.29 & 12.93 & & 747 & 730 & \\
\hline & & & & & & 12.29 & 12.65 & $3.55 \%$ & 747 & 726 & $-2.59 \%$ \\
\hline & & & & & & 12.29 & 12.60 & & 747 & 727 & \\
\hline$F$ & & $R-152 a \cdots$ & 24 hours & $302 \mathrm{~F}$ & & & & & & & \\
\hline & Uি-475 & 22.2596 & 22.2730 & $0.06 \%$ & $N / C$ & 13.32 & 12.47 & & 746 & 703 & \\
\hline & $A 2$ & & & & & 13.32 & 12.64 & $-6.83 \%$ & 746 & 675 & $-6.03 \%$ \\
\hline V & & & & & & 13.32 & 12.12 & & 746 & 725 & \\
\hline & $Y-390$ & 23.2541 & 23.2654 & $0.05 \%$ & $\mathrm{~N} / \mathrm{C}$ & 12.28 & 11.79 & & 755 & 728 & \\
\hline D & B2 & & & & & 12.28 & 12.46 & $2.36 \%$ & 755 & 728 & $-3.58 \%$ \\
\hline & & & & & & 12.28 & 13.46 & & 755 & 728 & \\
\hline & ER-610 & 23.0588 & 23.0541 & $-0.02 \%$ & $\mathrm{~N} / \mathrm{C}$ & 12.73 & 11.70 & & 734 & 729 & \\
\hline & C2 & & & & & 12.73 & 11.65 & $-10.53 \%$ & 734 & 647 & $-6.86 \%$ \\
\hline & & & & & & 12.73 & 10.82 & & 734 & 675 & \\
\hline & $Y-833$ & 22.0554 & 22.0849 & $0.13 \%$ & $\mathrm{~N} / \mathrm{C}$ & 12.49 & 12.12 & & 734 & 664 & \\
\hline & 52 & & & & & 12.49 & 11.51 & $-7.05 \%$ & 734 & 679 & $-8.13 \%$ \\
\hline & & & & & & 12.49 & 11.20 & & 734 & 680 & \\
\hline & 923 & 22.8751 & 22.8728 & $-0.01 \%$ & $\mathrm{~N} / \mathrm{C}$ & 14.38 & 10.79 & & 742 & 668 & \\
\hline 7 & E2 & & & & & 14.38 & 10.82 & $-24.92 \%$ & 742 & 728 & $-9.97 \%$ \\
\hline & & & & & & 14.38 & 10.78 & & 742 & 715 & \\
\hline & ISO-800 & 23.3631 & 23.3646 & $0.01 \%$ & & 12.29 & 10.92 & & 747 & 730 & \\
\hline & F2 & & & & $\mathrm{N} / \mathrm{C}$ & 12.29 & 11.90 & $-6.83 \%$ & 747 & 732 & $-2.14 \%$ \\
\hline & & & & & & 12.29 & 11.53 & & 747 & 729 & \\
\hline
\end{tabular}




\begin{tabular}{|c|c|c|c|c|c|c|c|c|c|c|c|}
\hline \multicolumn{12}{|c|}{500 HOURS IN R-152a@ 194 F } \\
\hline & VARN & $\begin{array}{l}\text { TWISTED } \\
\text { PAIR WT }\end{array}$ & $\begin{array}{c}\text { EXPT } \\
\text { PAIR WT }\end{array}$ & $\begin{array}{c}\text { WT \% } \\
\text { CHANGE }\end{array}$ & $\begin{array}{l}\text { EXP } \\
\text { VIS }\end{array}$ & $\begin{array}{c}\text { BASE } \\
\text { DIE }\end{array}$ & $\begin{array}{l}\text { EXP } \\
\text { DIE }\end{array}$ & $\begin{array}{c}\text { DIE \% } \\
\text { CHANGE }\end{array}$ & $\begin{array}{c}\text { BASE } \\
\text { BUPN } \\
\text { OUT }\end{array}$ & $\begin{array}{c}\text { EXP } \\
\text { BURN } \\
\text { OUT }\end{array}$ & $\begin{array}{c}\text { BRNOUT } \\
\% \\
\text { CHANGE }\end{array}$ \\
\hline \multirow{3}{*}{$\begin{array}{l}P \\
O \\
L\end{array}$} & \multirow{3}{*}{$\begin{array}{l}\text { U-475 } \\
\text { A } 1 \\
\end{array}$} & \multirow[t]{3}{*}{21.5151} & \multirow[t]{3}{*}{21.5316} & \multirow[t]{3}{*}{$0.077 \%$} & $\mathrm{~N} / \mathrm{C}$ & 15.10 & 9.51 & & 469 & 449 & \\
\hline & & & & & & 15.10 & 10.10 & $-34.61 \%$ & 469 & 417 & $-6.11 \%$ \\
\hline & & & & & & 15.10 & 10.01 & & 469 & 455 & \\
\hline \multirow{3}{*}{$\begin{array}{l}Y \\
E \\
S\end{array}$} & \multirow{3}{*}{$\begin{array}{l}Y-390 \\
B 1\end{array}$} & \multirow[t]{3}{*}{22.3769} & \multirow[t]{3}{*}{22.4665} & \multirow[t]{3}{*}{$0.400 \%$} & $\mathrm{~N} / \mathrm{C}$ & 18.24 & 20.00 & & 473 & 404 & \\
\hline & & & & & & 18.24 & 20.00 & $9.65 \%$ & 473 & 433 & $-8.32 \%$ \\
\hline & & & & & & 18.24 & 20.00 & & 473 & 464 & \\
\hline \multirow{4}{*}{$\begin{array}{l}T \\
E \\
R\end{array}$} & ER-610 & \multirow[t]{3}{*}{21.1644} & \multirow[t]{3}{*}{21.2074} & \multirow[t]{3}{*}{$0.203 \%$} & $\mathrm{~N} / \mathrm{C}$ & 14.53 & 12.17 & & 494 & 318 & \\
\hline & \multirow{2}{*}{ C1 } & & & & & 14.53 & 14.85 & $-8.33 \%$ & 494 & 379 & $-36.77 \%$ \\
\hline & & & & & & 14.53 & 12.94 & & 494 & 240 & \\
\hline & \multirow{3}{*}{$\begin{array}{l}-833 \\
D 1\end{array}$} & \multirow[t]{3}{*}{22.1708} & \multirow[t]{3}{*}{22.2422} & \multirow[t]{3}{*}{$0.322 \%$} & $\mathrm{~N} / \mathrm{C}$ & 11.38 & 13.13 & & 557 & 547 & \\
\hline 1 & & & & & & 11.38 & 20.00 & $42.77 \%$ & 557 & 529 & $-4.31 \%$ \\
\hline$M$ & & & & & & 11.38 & 15.61 & & 557 & 523 & \\
\hline & \multirow{3}{*}{$\begin{array}{l}923 \\
E_{1}\end{array}$} & \multirow[t]{3}{*}{22.9794} & \multirow[t]{3}{*}{23.0795} & $0.436 \%$ & $\mathrm{~N} / \mathrm{C}$ & 15.85 & 8.30 & & 503 & 582 & \\
\hline$D$ & & & & & & 15.85 & 13.50 & $-12.09 \%$ & 503 & 592 & $11.46 \%$ \\
\hline$E$ & & & & & & 15.85 & 20.00 & & 503 & 508 & \\
\hline & $150-800$ & 18.4390 & 18.4777 & $0.210 \%$ & $\mathrm{~N} / \mathrm{C}$ & 14.75 & 12.25 & & 632 & 562 & \\
\hline$P$ & $\mathrm{~F} 1$ & & & & & 14.75 & \begin{tabular}{|l|}
14.57 \\
\end{tabular} & $-8.75 \%$ & 632 & 561 & $-11.13 \%$ \\
\hline 0 & & & & & & 14.75 & 13.56 & & 632 & 562 & \\
\hline$L$ & & R-152a -- & $>24$ hours & @ $302 F$ & & & & & & & \\
\hline Y & $U-475$ & 21.4513 & 21.4253 & $-0.121 \%$ & $\mathrm{~N} / \mathrm{C}$ & 15.10 & 10.17 & & 469 & 559 & \\
\hline$A$ & $A 2$ & & & & & 15.10 & 11.24 & $-31.43 \%$ & 469 & 436 & $3.62 \%$ \\
\hline$M$ & & & & & & 15.10 & 9.65 & & 469 & 463 & \\
\hline 1 & $Y-390$ & 22.3525 & 22.3606 & $0.036 \%$ & $\mathrm{~N} / \mathrm{C}$ & 18.24 & 7.30 & & 473 & 450 & \\
\hline$D$ & B2 & & & & & 18.24 & 10.70 & $-36.29 \%$ & 473 & 427 & $-2.89 \%$ \\
\hline$E$ & & & & & & 18.24 & 12.54 & & 473 & 501 & \\
\hline & ER-610 & 21.2577 & 21.3018 & $0.207 \%$ & $\mathrm{~N} / \mathrm{C}$ & 14.53 & 11.38 & & 494 & 351 & \\
\hline 1 & $\mathrm{C} 2$ & & & & & 14.53 & 11.13 & $-9.64 \%$ & 494 & 373 & $-29.76 \%$ \\
\hline$M$ & & & & & & 14.53 & 16.88 & & 494 & 317 & \\
\hline 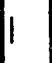 & $Y-833$ & 21.9670 & 21.9633 & $-0.017 \%$ & $\mathrm{~N} / \mathrm{C}$ & 11.38 & 11.03 & & 557 & 350 & \\
\hline$D$ & D2 & & & & & 11.38 & 14.16 & \begin{tabular}{|l|}
$12.39 \%$ \\
\end{tabular} & 557 & 350 & $-37.52 \%$ \\
\hline$E$ & & & & & & 11.38 & 13.18 & & 557 & 344 & \\
\hline & 923 & 23.0549 & 23.0573 & $0.010 \%$ & $\mathrm{~N} / \mathrm{C}$ & 15.85 & 11.84 & & 503 & 551 & \\
\hline & E2 & & & & & 15.85 & 18.76 & $-3.01 \%$ & 503 & 512 & $2.72 \%$ \\
\hline & & & & & & 15.85 & 15.52 & & 503 & 487 & \\
\hline & $150-800$ & 21.7205 & 21.7203 & $-0.001 \%$ & $\mathrm{~N} / \mathrm{C}$ & 14.75 & 9.20 & & 632 & 558 & \\
\hline & $\mathrm{F} 2$ & & & & & 14.75 & 9.07 & $-32.61 \%$ & 632 & 475 & $.16 .24 \%$ \\
\hline & & & & & & 14.75 & 11.55 & & 632 & 555 & \\
\hline
\end{tabular}




\begin{tabular}{|c|c|c|c|c|c|c|c|c|}
\hline & & $\mathrm{OH}$ & $152 a$ & D $194^{\circ} \mathrm{F}$ & & & & \\
\hline & VARN & $\begin{array}{l}\text { COIL } \\
\text { WT }\end{array}$ & $\begin{array}{c}\text { EXP COIL } \\
W T\end{array}$ & $\begin{array}{c}\text { WT \% } \\
\text { CHANGE }\end{array}$ & EXP VIS & $\begin{array}{c}\text { BASE } \\
\text { BND STR } \\
(A \vee E)\end{array}$ & $\begin{array}{c}\text { EXPBND } \\
\text { STR }\end{array}$ & $\begin{array}{c}\text { BND STR } \\
\% \\
\text { CHANGE }\end{array}$ \\
\hline$P$ & $U-475$ & 39.4503 & 39.6157 & $0.419 \%$ & $\mathrm{~N} / \mathrm{C}$ & 73.73 & 63.25 & \\
\hline 0 & A 1 & & & & & 73.73 & 48.75 & $-23.30 \%$ \\
\hline L & & & & & & 73.73 & 57.65 & \\
\hline$Y$ & $Y-390$ & 39.6170 & 39.7676 & $0.380 \%$ & $\mathrm{~N} / \mathrm{C}$ & 43.78 & 38.75 & \\
\hline$E$ & B1 & & & & & 43.78 & 38.30 & $-5.66 \%$ \\
\hline$S$ & & & & & & 43.78 & 46.85 & \\
\hline$T$ & ER-610 & 39.4370 & 39.5862 & $0.378 \%$ & $\mathrm{~N} / \mathrm{C}$ & 51.81 & 61.25 & \\
\hline$E$ & C1 & & & & & 51.81 & 55.65 & $9.31 \%$ \\
\hline$R$ & & & & & & 51.81 & 53.00 & \\
\hline & $Y-833$ & 39.3500 & 39.4063 & $0.143 \%$ & $\mathrm{~N} / \mathrm{C}$ & 9.85 & 32.80 & \\
\hline$P$ & D1 & & & & & 9.85 & 9.40 & $181.22 \%$ \\
\hline $\mathrm{O}$ & & & & & & 9.85 & 40.90 & \\
\hline L & 923 & 37.9121 & 38.0459 & $0.353 \%$ & $\mathrm{~N} / \mathrm{C}$ & 41.28 & 30.40 & \\
\hline$Y$ & E1 & & & & & 41.28 & 27.75 & $-27.61 \%$ \\
\hline A & & & & & & 41.28 & 31.50 & \\
\hline$M$ & ISO-800 & 38.9161 & 39.0020 & $0.221 \%$ & $\mathrm{~N} / \mathrm{C}$ & 45.01 & 49.00 & \\
\hline | & $F 1$ & & & & & 45.01 & 39.75 & $.1 .41 \%$ \\
\hline D & & & & & & 45.01 & 48.30 & \\
\hline$E$ & & R-152a $>$ & $24 \mathrm{HRS} 3$ & $2^{\circ} \mathrm{F}$ & & & & \\
\hline & $U-475$ & 40.0779 & 40.0836 & $0.014 \%$ & $\mathrm{~N} / \mathrm{C}$ & 73.73 & 6.00 & \\
\hline 1 & A2 & & & & & 73.73 & 9.00 & $-91.18 \%$ \\
\hline$M$ & & & & & & 73.73 & 4.00 & \\
\hline I & $Y-390$ & 38.8683 & 38.8711 & $0.007 \%$ & $\mathrm{~N} / \mathrm{C}$ & 43.78 & 9.50 & \\
\hline D & B2 & & & & & 43.78 & 6.50 & $-83.10 \%$ \\
\hline$E$ & & & & & & 43.78 & 6.20 & \\
\hline & ER-610 & 39.3700 & 39.3680 & $-0.005 \%$ & $\mathrm{~N} / \mathrm{C}$ & 51.81 & 8.60 & \\
\hline & C2 & & & & & 51.81 & 8.80 & $-84.82 \%$ \\
\hline & & & & & & 51.81 & 6.20 & \\
\hline & $Y-833$ & 39.3364 & 39.2506 & $-0.218 \%$ & $\mathrm{~N} / \mathrm{C}$ & 9.85 & 18.60 & \\
\hline & D2 & & & & & 9.85 & 25.12 & $152.18 \%$ \\
\hline & & & & & & 9.85 & 30.80 & \\
\hline & 923 & 38.3294 & 38.3301 & $0.002 \%$ & $\mathrm{~N} / \mathrm{C}$ & 41.28 & 30.92 & \\
\hline & E2 & & & & & 41.28 & 27.90 & $-19.36 \%$ \\
\hline & & & & & & 41.28 & 41.05 & \\
\hline & ISO-800 & 37.7502 & 37.7388 & $-0.030 \%$ & $\mathrm{~N} / \mathrm{C}$ & 45.01 & 26.60 & \\
\hline & F2 & & & & & 45.01 & 31.62 & $-36.70 \%$ \\
\hline & & & & & & 45.01 & 27.25 & \\
\hline
\end{tabular}




\begin{tabular}{|c|c|c|c|c|c|c|c|c|}
\hline & & 500 HRS I & IN R-152a & D $194^{\circ} \mathrm{F}$ & & & & \\
\hline & VARN & $\begin{array}{l}\text { COIL } \\
\text { WT }\end{array}$ & $\begin{array}{c}\text { EXP COIL } \\
W T\end{array}$ & $\begin{array}{c}\text { WT \% } \\
\text { CHANGE }\end{array}$ & EXPVIS|| & $\begin{array}{c}\text { BASE } \\
\text { BND STR } \\
\text { (AVE) }\end{array}$ & $\begin{array}{c}\text { EXPBND } \\
\text { STR }\end{array}$ & $\begin{array}{c}\text { BND STR } \\
\% \\
\text { CHANGE }\end{array}$ \\
\hline$P$ & U-475 & 37.9781 & 37.7047 & $-0.720 \%$ & $\mathrm{~N} / \mathrm{C}$ & 40.14 & 46.40 & \\
\hline O & A 1 & & & & & 40.14 & 45.90 & $23.57 \%$ \\
\hline $\mathrm{L}$ & & & & & & 40.14 & 56.50 & \\
\hline $\mathrm{Y}$ & $Y-390$ & $37.5730 \mid$ & 37.7732 & $0.533 \%$ & $\mathrm{~N} / \mathrm{C}$ & 36.12 & 53.15 & \\
\hline E & B1 & & & & & 36.12 & 56.15 & $43.27 \%$ \\
\hline $\mathrm{S}$ & & & & & & 36.12 & 45.95 & \\
\hline$T$ & ER-610 & 36.9680 & 37.1863 & $0.591 \%$ N & $\mathrm{N} / \mathrm{C}$ & 35.96 & 42.30 & \\
\hline$E$ & C1 & & & & & 35.96 & 38.95 & $24.72 \%$ \\
\hline$R$ & & & & & & 35.96 & 53.30 & \\
\hline & $Y-833$ & 36.4794 & 36.5855 & $0.291 \%$ & $\mathrm{~N} / \mathrm{C}$ & 33.14 & 30.50 & \\
\hline$P$ & D1 & & & & & 33.14 & 3.50 & $-59.26 \%$ \\
\hline 0 & & & & & & 33.14 & 6.50 & \\
\hline$L$ & 923 & 38.4704 & 38.6675 & $0.512 \%$ & $\mathrm{~N} / \mathrm{C}$ & 40.52 & 50.65 & \\
\hline $\mathrm{Y}$ & E1 & & & & & 40.52 & 36.30 & $10.36 \%$ \\
\hline A & & & & & & 40.52 & 47.20 & \\
\hline$M$ & $150-800$ & 37.6078 & 37.7456 & $0.366 \%$ & $\mathrm{~N} / \mathrm{C}$ & 20.20 & 28.25 & \\
\hline 1 & $F 1$ & & & & & 20.20 & 27.25 & $40.43 \%$ \\
\hline$D$ & & & & & & 20.20 & 29.60 & \\
\hline$E$ & & R-152a -> & - 24 HRS 3 & $02^{\circ} \mathrm{F}$ & & & & \\
\hline & $U-475$ & 37.2778 & 37.2701 & $.0 .021 \%$ & $\mathrm{~N} / \mathrm{C}$ & 40.14 & *4.03 & \\
\hline 1 & A 2 & & & & & 40.14 & 21.80 & $-38.63 \%$ \\
\hline$M$ & & & & & & 40.14 & 27.47 & \\
\hline 1 & $Y-390$ & 37.8149 & 37.7934 & $-0.057 \%$ & $N / C$ & 36.12 & 2.65 & \\
\hline$D$ & B2 & & & & & 36.12 & 17.82 & $-47.62 \%$ \\
\hline$E$ & & & & & & 36.12 & 20.02 & \\
\hline & ER-610 & 37.3670 & 37.3662 & $-0.002 \%$ & $\mathrm{~N} / \mathrm{C}$ & 35.96 & 26.52 & \\
\hline$E$ & C2 & & & & & 35.96 & 30.52 & $-22.84 \%$ \\
\hline$P$ & & & & & & 35.96 & 26.20 & \\
\hline 0 & $Y-833$ & 36.7123 & 36.6887 & $-0.064 \%$ & $\mathrm{~N} / \mathrm{C}$ & 33.14 & 21.92 & \\
\hline$x$ & D2 & & & & & 33.14 & 22.27 & $-48.51 \%$ \\
\hline Y & & & & & & 33.14 & 7.00 & \\
\hline & 923 & 38.4742 & 38.4756 & $0.004 \%$ & $\mathrm{~N} / \mathrm{C}$ & 40.52 & 22.92 & \\
\hline $\mathrm{G}$ & E2 & & & & & 40.52 & 17.75 & $-52.09 \%$ \\
\hline L & & & & & & 40.52 & 17.57 & \\
\hline A & ISO-800 & 37.5588 & 37.5866 & $0.074 \%$ & $\mathrm{~N} / \mathrm{C}$ & 20.20 & 18.15 & \\
\hline $\mathrm{s}$ & F2 & & & & & 20.20 & 17.15 & $-6.85 \%$ \\
\hline $\mathrm{s}$ & & & & & & 20.20 & 21.15 & \\
\hline
\end{tabular}




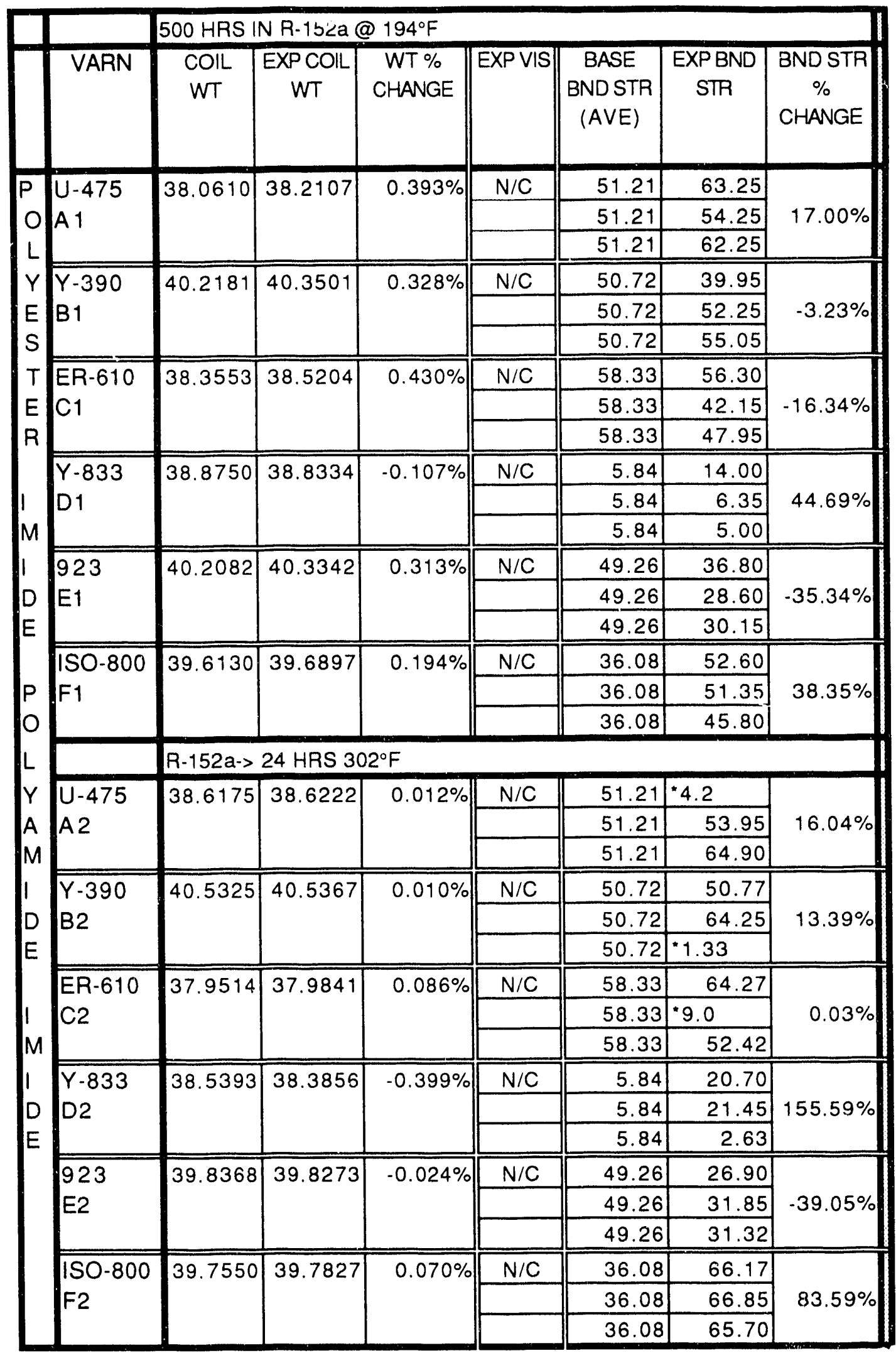

"Experimental Data was not used in the average due to unusually low result. 


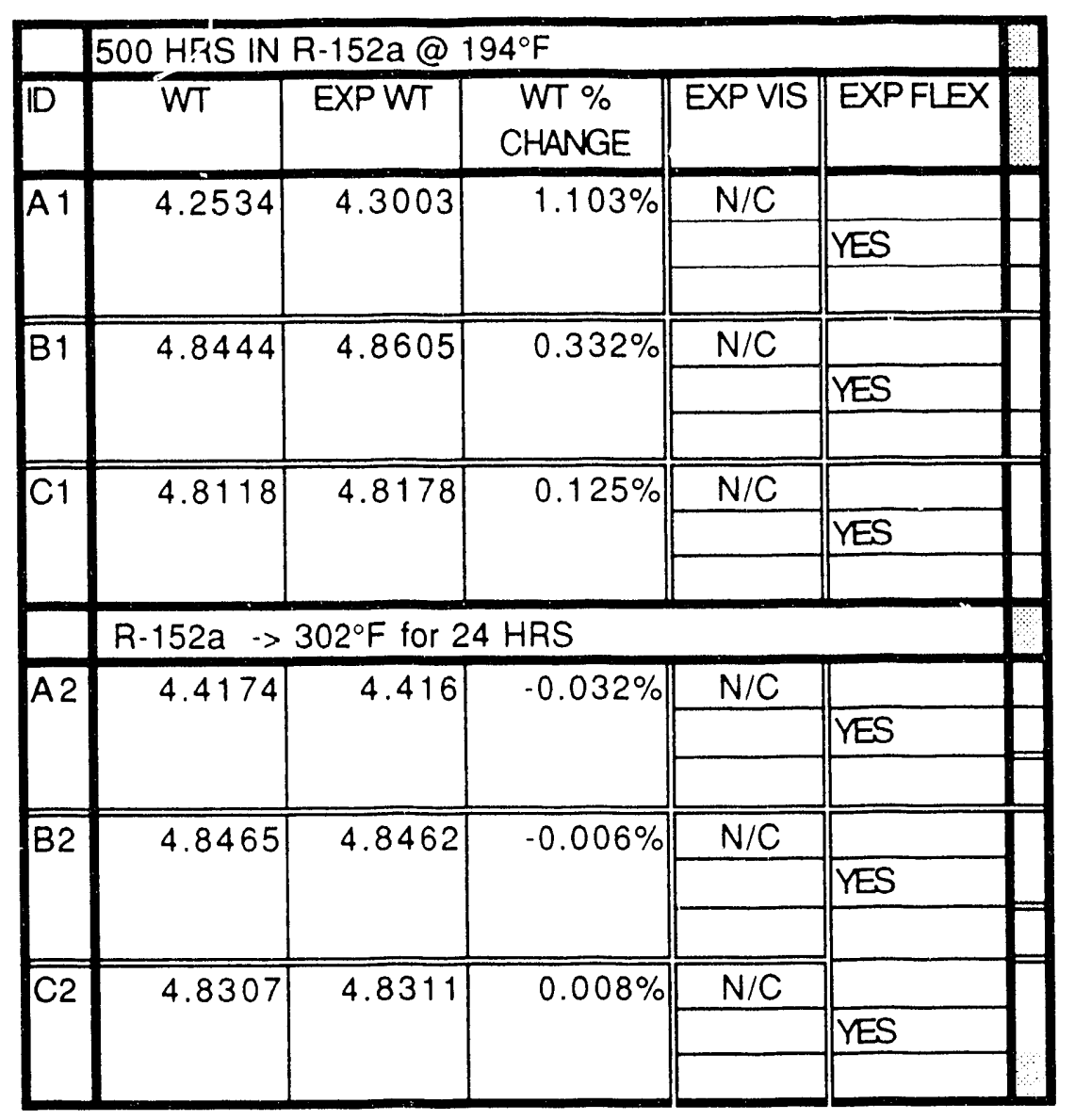




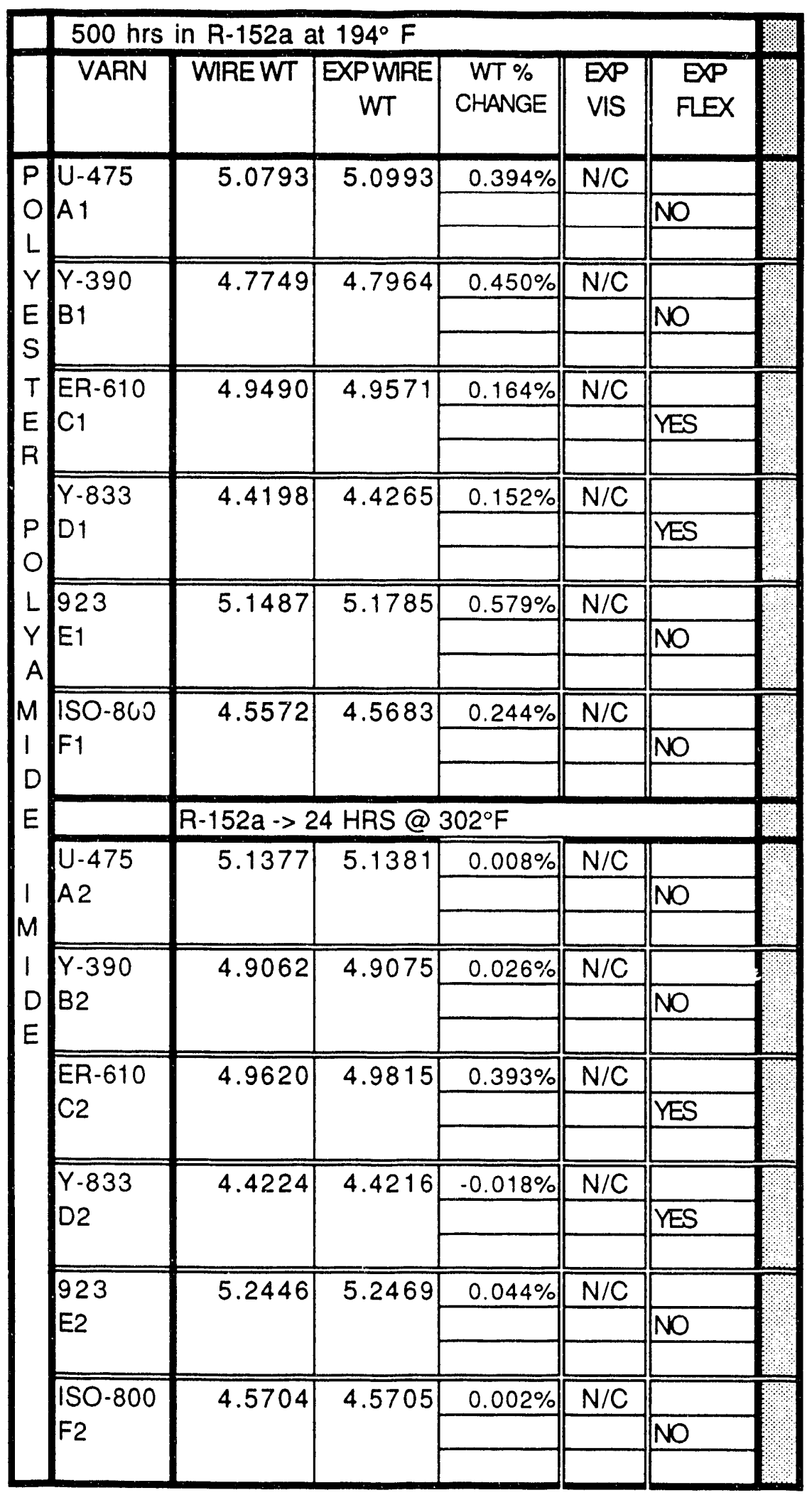




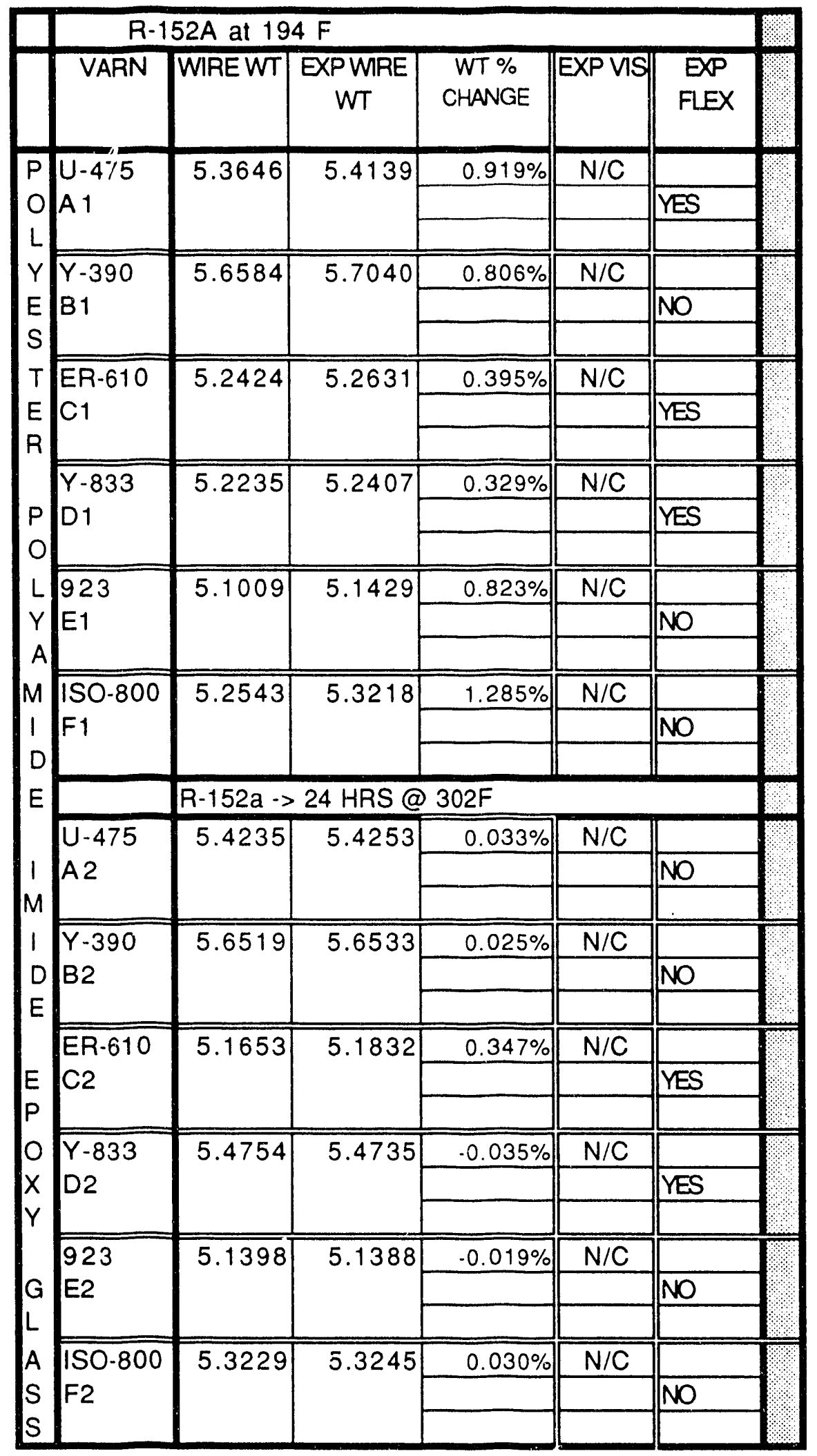




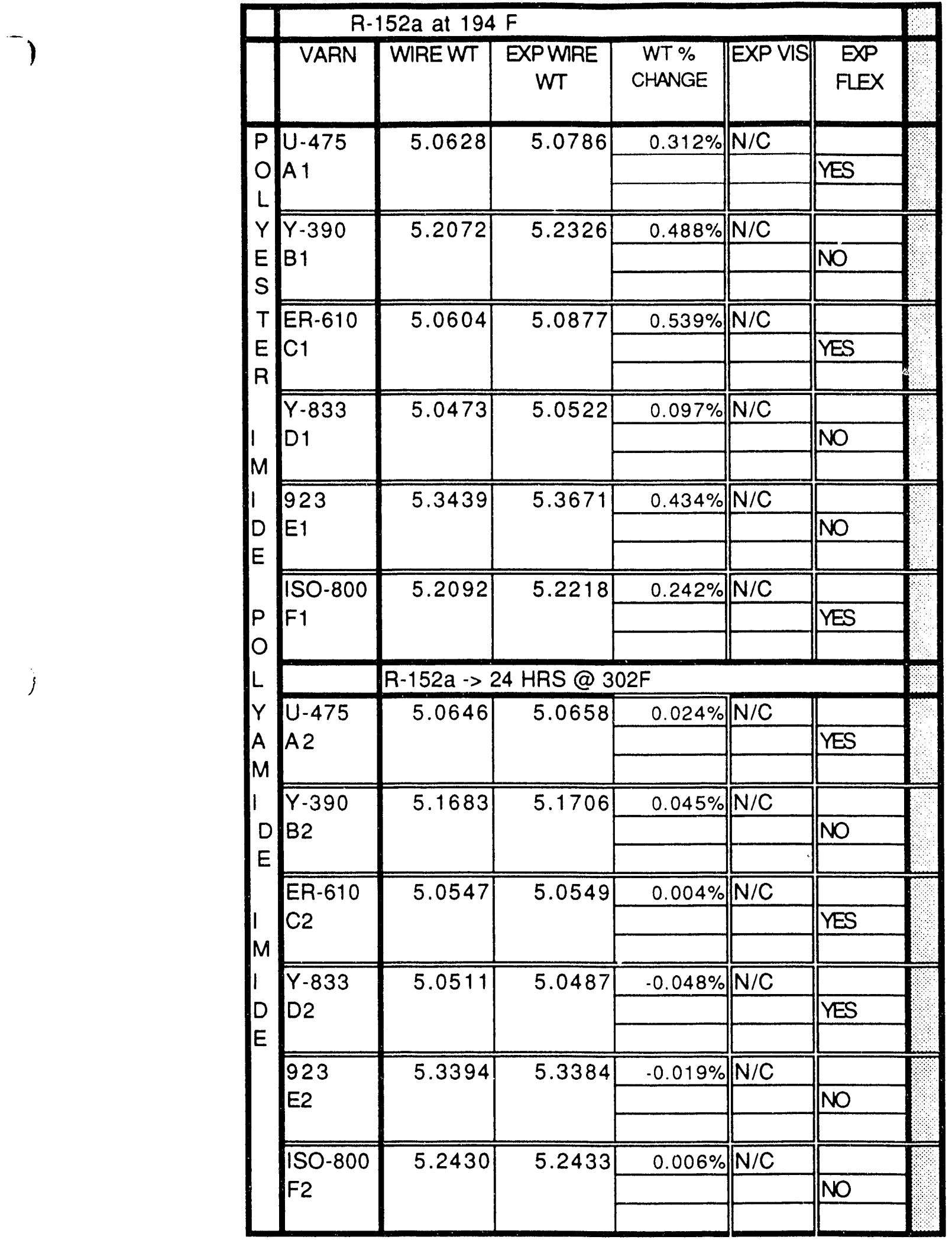


500 HRS IN R-152a @ 194 F

\begin{tabular}{|c|c|c|c|c|c|}
\hline ID & WT & EXPWT & $\begin{array}{c}\text { WT \% } \\
\text { CHANGE }\end{array}$ & EXP VIS & EXPFLEX \\
\hline$U-475$ & 2.7075 & 2.8816 & $6.43 \%$ & $\mathrm{~N} / \mathrm{C}$ & $\mathrm{N} / \mathrm{C}$ \\
\hline$Y-390$ & 2.1165 & 2.2205 & $4.91 \% \|$ & $\mathrm{N} / \mathrm{C}$ & $\mathrm{N} / \mathrm{C}$ \\
\hline ER-610 & 3.0231 & 3.2992 & $9.13 \%$ & $\mathrm{~N} / \mathrm{C}$ & $\mathrm{N} / \mathrm{C}$ \\
\hline \multirow{3}{*}{$\begin{array}{l}Y-833 \\
D 1\end{array}$} & \multirow[t]{3}{*}{2.2243} & \multirow[t]{3}{*}{2.4034} & \multirow[t]{3}{*}{$8.05 \%$} & $\mathrm{~N} / \mathrm{C}$ & Slightly \\
\hline & & & & & More \\
\hline & & & & & Flexible \\
\hline 923 & 1.9237 & 2.0239 & $5.21 \%$ & $N / C$ & $\mathrm{~N} / \mathrm{C}$ \\
\hline \multirow{2}{*}{$\begin{array}{l}\mathrm{ISO}-800 \\
\mathrm{~F} 1\end{array}$} & 1.4577 & 1.4593 & $0.11 \%$ & $\mathrm{~N} / \mathrm{C}$ & $\mathrm{N} / \mathrm{C}$ \\
\hline & \multicolumn{5}{|c|}{$\mathrm{R}-152 \mathrm{a}$ at $\cdots>24$ hour at $302^{\circ} \mathrm{F}$. } \\
\hline \multirow{3}{*}{$\begin{array}{l}U-475 \\
A 2\end{array}$} & \multirow[t]{2}{*}{2.4002} & \multirow[t]{2}{*}{2.3680} & \multirow[t]{2}{*}{$-1.34 \%$} & Few & $\mathrm{N} / \mathrm{C}$ \\
\hline & & & & Internal & \\
\hline & \multirow{4}{*}{2.1426} & \multirow{4}{*}{2.0738} & \multirow{4}{*}{$-3.21 \%$} & & \\
\hline \multirow{3}{*}{$\begin{array}{l}Y-390 \\
B 2\end{array}$} & & & & Few & $\mathrm{N} / \mathrm{C}$ \\
\hline & & & & Internal & \\
\hline & & & & Bubbles & \\
\hline \multirow{3}{*}{$\begin{array}{l}\text { ER-610 } \\
\text { C2 }\end{array}$} & \multirow[t]{3}{*}{2.2565} & \multirow[t]{3}{*}{2.2679} & \multirow[t]{3}{*}{$0.51 \%$} & Two & $N / C$ \\
\hline & & & & Internal & \\
\hline & & & & Bubbles & \\
\hline \multirow{3}{*}{$\begin{array}{l}Y-833 \\
D 2\end{array}$} & \multirow[t]{3}{*}{2.5410} & \multirow[t]{3}{*}{2.3516} & \multirow[t]{3}{*}{$-7.45 \%$} & Many & brittle \\
\hline & & & & Internal & slightly \\
\hline & & & & bubbles & Iwarped \\
\hline \multirow{2}{*}{$\begin{array}{l}923 \\
\text { E2 }\end{array}$} & \multirow[t]{2}{*}{1.7713} & \multirow[t]{2}{*}{1.7432} & \multirow[t]{2}{*}{$-1.59 \%$} & few & Brittle \\
\hline & & & & $\frac{\text { Internal }}{\text { bubbles }}$ & \\
\hline \multirow{3}{*}{$\begin{array}{l}\mathrm{ISO}-800 \\
\mathrm{~F} 2\end{array}$} & \multirow[t]{3}{*}{1.7211} & \multirow[t]{3}{*}{1.6226} & \multirow[t]{3}{*}{$-5.72 \%$} & Many & brittle \\
\hline & & & & Internal & slightly \\
\hline & & & & bubbles & warped \\
\hline
\end{tabular}




\begin{tabular}{|c|c|c|c|c|c|c|c|c|c|}
\hline \multicolumn{10}{|c|}{ 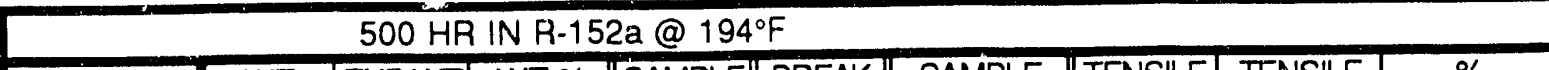 } \\
\hline ID & WT & EXPWT & \begin{tabular}{c|} 
WT \% \\
CHANGE
\end{tabular} & $\begin{array}{l}\text { SAMPLE } \\
\text { WIDTH }\end{array}$ & $\begin{array}{l}\text { BREAK } \\
\text { LOAD }\end{array}$ & $\begin{array}{c}\text { SAMPLE } \\
\text { THICKNESS } \\
M, S\end{array}$ & \begin{tabular}{|c|} 
TENSILE \\
STR \\
BASE \\
\end{tabular} & $\begin{array}{l}\text { TENSILE } \\
\text { STREXP }\end{array}$ & $\begin{array}{c}\% \\
\text { CHANGE } \\
\text { TENSILE }\end{array}$ \\
\hline \multirow{3}{*}{$\begin{array}{l}\text { NO/MY/NO } \\
\text { A1 }\end{array}$} & \multirow[t]{3}{*}{4.7048} & \multirow[t]{3}{*}{4.8982} & \multirow[t]{3}{*}{$4.11 \%$} & 0.473 & 165.90 & 21 & 17.4 & 16.70 & \\
\hline & & & & 0.403 & 142.90 & 21 & 17.4 & 16.89 & $-4.22 \%$ \\
\hline & & & & 0.401 & 138.20 & 21 & 17.4 & 16.41 & \\
\hline \multirow{3}{*}{$\begin{array}{l}\text { DA/MYiDA } \\
\text { B1 }\end{array}$} & \multirow[t]{3}{*}{4.3255} & \multirow[t]{3}{*}{4.4528} & \multirow[t]{3}{*}{$2.94 \%$} & 0.464 & 120.30 & 21 & 13.7 & 12.35 & \\
\hline & & & & 0.366 & 101.20 & 21 & 13.7 & 13.17 & $-6.35 \%$ \\
\hline & & & & 0.415 & 113.10 & 21 & 13.7 & 12.98 & \\
\hline \multirow{3}{*}{$\begin{array}{l}\text { MYLAR MO } \\
\text { C1 }\end{array}$} & \multirow[t]{3}{*}{2.3334} & \multirow{3}{*}{$2 . \therefore 3$} & \multirow[t]{3}{*}{$2.87 \%$} & 0.459 & 94.40 & 10 & 21.7 & 20.57 & \\
\hline & & & & 0.371 & 72.70 & 10 & 21.7 & 19.60 & $-9.24 \%$ \\
\hline & & & & 0.423 & 80.05 [ & 10 & 21.7 & 18.92 & \\
\hline \multirow{3}{*}{$\begin{array}{l}\text { NO } 410 \\
\text { D1 }\end{array}$} & \multirow[t]{3}{*}{2.3604} & \multirow[t]{3}{*}{2.4802} & \multirow{3}{*}{$5.08 \%$} & 0.496 & 94.95 & 10 & 18.7 & 19.14 & \\
\hline & & & & 0.521 & 106.80 & 10 & 18.7 & 20.50 & $6.73 \%$ \\
\hline & & & & 0.494 & 99.95 & 10 & 18.7 & 20.23 & \\
\hline \multirow{3}{*}{$\begin{array}{l}\text { NO MI } 418 \\
\text { E1 }\end{array}$} & \multirow{3}{*}{2.2491} & \multirow{3}{*}{2.2942} & \multirow[t]{3}{*}{$2.01 \%$} & 0.481 & 25.85 & 10 & 7.5 & 5.37 & \\
\hline & & & & 0.505 & 26.90 & 10 & 7.5 & 5.33 & $-28.35 \%$ \\
\hline & & & & 0.499 & 27.05 & 10 & 7.5 & 5.42 & \\
\hline \multirow{3}{*}{$\begin{array}{l}\text { MEL } 228 \\
\text { F1 }\end{array}$} & \multirow[t]{3}{*}{2.3339} & \multirow[t]{3}{*}{2.4041} & \multirow[t]{3}{*}{$3.01 \%$} & 0.373 & 72.15 & 10 & 21.7 & 19.34 & \\
\hline & & & & 0.480 & 80.10 & 10 & 21.7 & 16.69 & $-15.22 \%$ \\
\hline & & & & 0.470 & 90.05 & 10 & 21.7 & 19.16 & \\
\hline & & $500 \mathrm{HRS}$ & IN R-152 & ?a@194 & ${ }^{\circ} \mathrm{F}->24 \mathrm{H}$ & $5 @ 302^{\circ}$ & & & \\
\hline $\mathrm{NC/MY/NO}$ & 4.4061 & 4.4033 & $-0.06 \%$ & 0.418 & 149.70 & 21 & 1.7 & 17.05 & \\
\hline & & & & 0.333 & $i 18.20$ & 21 & 17.4 & 16.90 & $-1.78 \%$ \\
\hline & & & & 0.539 & 196.00 & 21 & 17.4 & 17.32 & \\
\hline $\mathrm{DA} / \mathrm{MY} / \mathrm{DA}$ & 4.9090 & 4.8904 & $-0.38 \%$ & 0.398 & 116.50 & 21 & 13.7 & 13.94 & \\
\hline B2 & & & & 0.392 & 110.00 & 21 & 13.7 & 13.36 & $0.06 \%$ \\
\hline & & & & 0.422 & 122.50 & 21 & 13.7 & 13.82 & \\
\hline MYLARMO & 2.2682 & 2.2863 & $0.80 \%$ & 0.402 & 75.20 & 10 & 21.7 & 18.71 & \\
\hline $\mathrm{C} 2$ & & & & 0.481 & 95.05 & 10 & 21.7 & 19.76 & $-10.28 \%$ \\
\hline & & & & 0.407 & 81.15 & 10 & 21.7 & 19.94 & \\
\hline$\overline{\text { NOMEX } 410}$ & 2.2259 & 2.2213 & $.0 .21 \%$ & 0.500 & 98.60 & 10 & 18.7 & 19.72 & \\
\hline D2 & & & & 0.508 & 92.75 & 10 & 18.7 & 18.26 & $3.20 \%$ \\
\hline & & & & 0.498 & 99.20 & 10 & 18.7 & 19.92 & \\
\hline NO/MI 418 & 2.1262 & 2.1171 & $-0.43 \%$ & 0.503 & 26.65 & 10 & 7.5 & 5.30 & \\
\hline & & & & 0.473 & 27.60 & 10 & 7.5 & 5.84 & $-26.22 \%$ \\
\hline & & & & 0.492 & 26.90 & 10 & 7.5 & 5.47 & \\
\hline MEL 228 & 2.3115 & 2.3109 & $-0.03 \%$ & 0.458 & 88.90 & 10 & 21.7 & 19.41 & \\
\hline & & & & 0.490 & 95.65 & 10 & 21.7 & 19.52 & $-9.35 \%$ \\
\hline & & & & 0.433 & 86.95 & 10 & 21.7 & 20.08 & \\
\hline
\end{tabular}




\begin{tabular}{|c|c|c|c|c|c|c|c|c|}
\hline \multicolumn{8}{|c|}{ After 500 hour exposure @ $194^{\circ} \mathrm{F}\left(90^{\circ} \mathrm{C}\right)$} & \multirow[b]{2}{*}{$\begin{array}{c}\text { VISUAL } \\
\text { EXP }\end{array}$} \\
\hline ID & $\begin{array}{c}\text { STRETCH } \\
\text { (inch) }\end{array}$ & $\begin{array}{c}\% \\
\text { ELONG }\end{array}$ & $\begin{array}{l}\text { BASE } \\
\text { ELONG } \\
\text { (AV:) }\end{array}$ & $\begin{array}{c}\text { ELONG } \\
\% \\
\text { CHANGE }\end{array}$ & $\begin{array}{c}\text { BASE } \\
\text { DIE } \\
\text { (AVE) }\end{array}$ & EXPDIE & $\begin{array}{c}\text { DIE \% } \\
\text { CHANGE }\end{array}$ & \\
\hline \multirow{3}{*}{$\begin{array}{l}\mathrm{NO} / \mathrm{MY} / \mathrm{NO} \\
\mathrm{A} 1\end{array}$} & 1.24 & $31.0 \%$ & $20.0 \%$ & & $>18.97$ & $>16.23$ & & \multirow[t]{3}{*}{$\mathrm{N} / \mathrm{C}$} \\
\hline & 1.27 & $31.8 \%$ & $20.0 \%$ & $55.8 \%$ & $>18.97$ & $>15.93$ & flash & \\
\hline & 1.23 & $30.8 \%$ & $20.0 \%$ & & $>18.97$ & $>16.85$ & & \\
\hline \multirow{3}{*}{$\begin{array}{l}\mathrm{DA} / \mathrm{MY} / \mathrm{DA} \\
\mathrm{B} 1\end{array}$} & 0.60 & $30.0 \%$ & $46.0 \%$ & & $\geq 15.27$ & $>14.10$ & & \multirow{3}{*}{$\begin{array}{l}\text { slighlty } \\
\text { warped }\end{array}$} \\
\hline & 0.64 & $32.0 \%$ & $46.0 \%$ & $-31.2 \%$ & $>15.27$ & $>12.18$ & flash & \\
\hline & 0.66 & $33.0 \%$ & $46.0 \%$ & & $>15.27$ & $>14.07$ & & \\
\hline \multirow{3}{*}{$\begin{array}{l}\text { MYLARMO } \\
\text { C1 }\end{array}$} & 3.21 & $160.5 \%$ & $131.0 \%$ & & $>14.91$ & $>13.07$ & & \multirow[t]{3}{*}{$\mathrm{N} / \mathrm{C}$} \\
\hline & 3.07 & $153.5 \%$ & $131.0 \%$ & $17.0 \%$ & $>14.91$ & $>13.31$ & flash & \\
\hline & 2.92 & $146.0 \%$ & $131.0 \%$ & & $>14.91$ & $>13.39$ & & \\
\hline \multirow{3}{*}{$\begin{array}{l}\text { NO } 410 \\
\text { D1 }\end{array}$} & 0.62 & $15.5 \%$ & $17.0 \%$ & & 10.67 & 10.34 & & \multirow[t]{3}{*}{$N / C$} \\
\hline & 0.85 & $21.3 \%$ & $17.0 \%$ & $11.3 \%$ & 10.67 & 10.35 & $-0.1 \%$ & \\
\hline & 0.80 & $20.0 \%$ & $17.0 \%$ & & 10.07 & 11.28 & & \\
\hline \multirow{3}{*}{$\begin{array}{l}\text { NO MI } 418 \\
: 1\end{array}$} & 0.11 & $2.8 \%$ & $4.0 \%$ & & 10.23 & 9.73 & & \multirow[t]{3}{*}{$\mathrm{N} / \mathrm{C}$} \\
\hline & 0.12 & $3.0 \%$ & $4.0 \%$ & $-27.1 \%$ & 10.23 & 9.30 & $-9.0 \%$ & \\
\hline & 0.12 & $3.0 \%$ & $4.0 \%$ & & 10.23 & 8.91 & & \\
\hline \multirow{4}{*}{$\begin{array}{l}\text { MEL } 228 \\
\text { F1 }\end{array}$} & 3.46 & $173.0 \%$ & $160.0 \%$ & & $\geq 14.22$ & $>12.63$ & & \multirow[t]{3}{*}{$N / C$} \\
\hline & 2.19 & $109.5 \%$ & $160.0 \%$ & $-5.2 \%$ & $>14.22$ & $>13.46$ & flash & \\
\hline & 3.45 & $172.5 \%$ & $160.0 \%$ & & $>14.22$ & $>13.66$ & & \\
\hline & After 500 & hour ex & oosure plu & us 24 ho & ur@30 & $2^{\circ} \mathrm{F}\left(150^{\circ}\right.$ & $\left.{ }^{\circ} \mathrm{C}\right)$ & \\
\hline \multirow{3}{*}{$\begin{array}{l}\mathrm{NO} / \mathrm{MY} / \mathrm{NO} \\
\mathrm{A} 2\end{array}$} & 0.65 & $16.3 \%$ & $20.0 \%$ & & $>18.97$ & $>16.23$ & & \multirow[t]{3}{*}{$\mathrm{N} / \mathrm{C}$} \\
\hline & 0.75 & $18.8 \%$ & $20.0 \%$ & $-9.6 \%$ & $>18.97$ & $>16.96$ & flash & \\
\hline & 0.77 & $19.3 \%$ & $20.0 \%$ & & $>18.97$ & $>17.76$ & & \\
\hline \multirow{3}{*}{$\begin{array}{l}\mathrm{DA} / \mathrm{MY} / \mathrm{DA} \\
\mathrm{B} 2\end{array}$} & 0.56 & $28.0 \%$ & $46.0 \%$ & & $\geq 15.27$ & $>15.39$ & & \multirow{3}{*}{$\begin{array}{l}\text { slighity } \\
\text { warped }\end{array}$} \\
\hline & 0.56 & $28.0 \%$ & $46.0 \%$ & $-38.0 \%$ & $\geq 15.27$ & $>14.64$ & flash & \\
\hline & 0.59 & $29.5 \%$ & $46.0 \%$ & & $>15.27$ & $>13.41$ & & \\
\hline \multirow{3}{*}{$\begin{array}{l}\text { MYLAR MO } \\
\text { C2 }\end{array}$} & 2.69 & $134.5 \%$ & $131.0 \%$ & & $\geq 14.91$ & $>13.46$ & & \multirow[t]{3}{*}{$N / C$} \\
\hline & 2.84 & $142.0 \%$ & $131.0 \%$ & $8.9 \%$ & $\because 14.91$ & $>13.26$ & flash & \\
\hline & 3.03 & $151.5 \%$ & $131.0 \%$ & & $>14.91$ & $>13.25$ & & \\
\hline \multirow{3}{*}{$\begin{array}{l}\text { NOMEX 410 } \\
\text { D2 }\end{array}$} & 0.53 & $13.3 \%$ & $17.0 \%$ & & 10.67 & 10.00 & & \multirow[t]{3}{*}{$\sqrt{N / C}$} \\
\hline & 0.36 & $9.0 \%$ & $17.0 \%$ & $-30.4 \%$ & 10.67 & 10.52 & $-2.9 \%$ & \\
\hline & 0.53 & $13.3 \%$ & $17.0 \%$ & & 10.67 & 10.57 & & \\
\hline \multirow{3}{*}{$\begin{array}{l}\mathrm{NO} / \mathrm{MI} 418 \\
\mathrm{E2}\end{array}$} & 0.06 & $1.5 \%$ & $4.0 \%$ & & 10.23 & 7.94 & & \multirow[t]{3}{*}{$\mathrm{N} / \mathrm{C}$} \\
\hline & 0.07 & $1.8 \%$ & $4.0 \%$ & $-56.3 \%$ & 10.23 & 9.93 & $-12.1 \%$ & \\
\hline & 0.08 & $2.0 \%$ & $4.0 \%$ & & 10.23 & 9.10 & & \\
\hline \multirow{3}{*}{$\begin{array}{l}\text { MEL } 228 \\
\text { F2 }\end{array}$} & 3.27 & $163.5 \%$ & $160.0 \%$ & & $>14.22$ & $>13.57$ & & $N / C$ \\
\hline & 3.34 & $167.0 \%$ & $160.0 \%$ & $4.9 \%$ & $>14.22$ & $>13.30$ & flash & \\
\hline & 3.46 & $173.0 \%$ & $160.0 \%$ & & $>14.22$ & $>13.98$ & & \\
\hline
\end{tabular}




\begin{tabular}{|c|c|c|c|c|}
\hline & \multicolumn{4}{|c|}{500 HRS IN R-152a @ 194ㄷ F } \\
\hline & WT & EXPWT & $\begin{array}{c}\% \\
\text { CHANGE }\end{array}$ & EXP VIS \\
\hline & 0.5371 & 0.6003 & $11.77 \%$ & $\mathrm{~N} / \mathrm{C}$ \\
\hline \multirow{3}{*}{$\begin{array}{l}\text { B1 } \\
\text { MYLAR }\end{array}$} & \multirow[t]{3}{*}{0.4612} & \multirow[t]{3}{*}{0.5023} & \multirow[t]{3}{*}{$8.91 \%$} & Bubble \\
\hline & & & & between \\
\hline & & & & layers \\
\hline \multirow{2}{*}{$\begin{array}{l}\mathrm{C} 1 \\
\mathrm{NO} / \mathrm{MY}\end{array}$} & 0.3583 & 0.4200 & $17.22 \%$ & $\mathrm{~N} / \mathrm{C}$ \\
\hline & \multicolumn{4}{|c|}{ R-152a@ $194^{\circ} \mathrm{F}-. .->24$ hrs @ 302 $\mathrm{F}$} \\
\hline A2 & 0.5433 & 0.5435 & $0.04 \%$ & $\mathrm{~N} / \mathrm{C}$ \\
\hline \multirow{2}{*}{$\begin{array}{l}\text { B2 } \\
\text { MYAR }\end{array}$} & \multirow[t]{2}{*}{0.4789} & \multirow[t]{2}{*}{0.4779} & \multirow[t]{2}{*}{$-0.21 \%$} & Some delamination \\
\hline & & & & see photo \\
\hline \multirow{2}{*}{$\begin{array}{l}\mathrm{C} 2 \\
\mathrm{NO} / \mathrm{MY}\end{array}$} & \multirow[t]{2}{*}{0.4085} & \multirow[t]{2}{*}{0.4053} & \multirow[t]{2}{*}{$-0.78 \%$} & Pockects where \\
\hline & & & & \begin{tabular}{|c|} 
mylar pulled away \\
"see photo
\end{tabular} \\
\hline
\end{tabular}




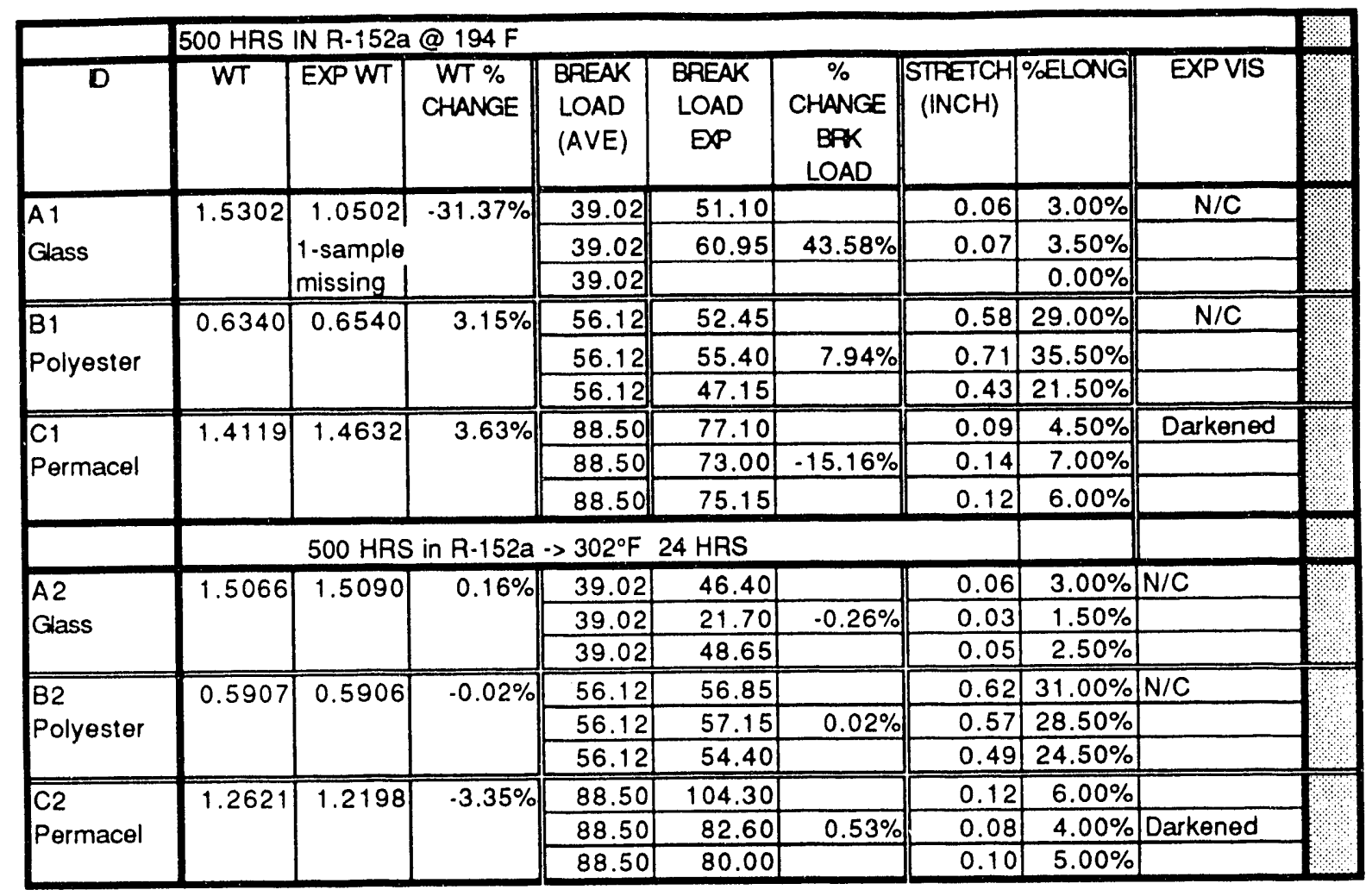




\begin{tabular}{|c|c|c|c|c|c|c|c|c|c|}
\hline \multicolumn{10}{|c|}{500 HRS IN R-152a@194F } \\
\hline iD & WT & EXPWT & $\begin{array}{c}\text { WT \% } \\
\text { CHANGE }\end{array}$ & $\begin{array}{l}\text { EXP } \\
\text { VIS }\end{array}$ & $\begin{array}{l}\text { BREAK } \\
\text { LOAD } \\
\text { (AVE) }\end{array}$ & $\begin{array}{c}\text { BREAK } \\
\text { LOAD } \\
\text { EXP } \\
\text { (ave) }\end{array}$ & $\begin{array}{c}\% \\
\text { CHANGE } \\
\text { BRK } \\
\text { LOAD }\end{array}$ & $\begin{array}{c}\text { STRETCH } \\
\text { (lnch) }\end{array}$ & $\begin{array}{c}\% \\
\text { ELONG }\end{array}$ \\
\hline \multirow[t]{4}{*}{ A1 } & \multirow[t]{3}{*}{0.2602} & \multirow[t]{3}{*}{0.2705} & \multirow[t]{3}{*}{$3.96 \%$} & $\mathrm{~N} / \mathrm{C}$ & 28.36 & 33.05 & & 0.40 & $20.0 \%$ \\
\hline & & & & & 28.36 & 31.00 & \multirow[t]{2}{*}{$13.42 \%$} & 037 & $18.5 \%$ \\
\hline & & & & & 28.36 & 32.45 & & 0.38 & $19.0 \%$ \\
\hline & \multicolumn{9}{|c|}{500 HRS IN R-152a -> 24 HRS @ 302 ${ }^{\circ} \mathrm{F}$} \\
\hline \multirow[t]{3}{*}{$A 2$} & \multirow[t]{3}{*}{0.2493} & \multirow[t]{3}{*}{0.2509} & \multirow[t]{3}{*}{$0.642 \%$} & $\mathrm{~N} / \mathrm{C}$ & 28.36 & 25.90 & & 0.43 & $21.5 \%$ \\
\hline & & & & & 28.36 & 38.85 & $14.36 \%$ & 0.45 & $22.5 \%$ \\
\hline & & & & & 28.36 & 32.55 & & 0.45 & $22.5 \%$ \\
\hline
\end{tabular}




\begin{tabular}{|c|c|c|c|c|c|c|c|}
\hline & 500 HRS IN & 152a@19 & $4^{\circ} \mathrm{F}$ & & & & \\
\hline ID & WT & EXPWT & $\begin{array}{c}\text { WT \% } \\
\text { CHANGE }\end{array}$ & EXP VIS & $\begin{array}{c}\text { BASE DIE } \\
\text { (AVE) }\end{array}$ & EXP DIE & $\begin{array}{c}\text { DIE\% } \\
\text { CHANGE }\end{array}$ \\
\hline DMD & 4.0361 & 4.0565 & $0.51 \%$ & $\mathrm{~N} / \mathrm{C}$ & 9.61 & 9.49 & \\
\hline$A 1$ & & & & & 9.61 & 8.64 & $-11.48 \%$ \\
\hline & & & & & 9.61 & 7.39 & \\
\hline DTMD & 4.3611 & 4.3812 & $0.46 \%$ & $\mathrm{~N} / \mathrm{C}$ & 9.95 & 10.28 & \\
\hline B1 & & & & & 9.95 & 9.16 & $-9.11 \%$ \\
\hline & & & & & 9.95 & 7.69 & \\
\hline & R-152a@19 & $\mathrm{F}->24 \mathrm{HR}$ & S@302ㄷ & & & & \\
\hline DMD & 4.0487 & 4.0456 & $-0.08 \%$ & $\mathrm{~N} / \mathrm{C}$ & 9.61 & 9.78 & \\
\hline A2 2 & & & & & 9.61 & 9.94 & $1.98 \%$ \\
\hline & & & & & 9.61 & 9.68 & \\
\hline DTMD & 4.4312 & 4.4288 & $-0.05 \%$ & $\mathrm{~N} / \mathrm{C}$ & 9.95 & 9.18 & \\
\hline $\mathrm{B} 2$ & & & & & 9.95 & 9.77 & $-0.40 \%$ \\
\hline & & & & & 9.95 & 10.78 & \\
\hline
\end{tabular}




\section{Appendix $\mathbf{H}$}

Experimental Data for HFC-134a Exposure at $90^{\circ} \mathrm{C}\left(194^{\circ} \mathrm{F}\right)$ 


\begin{tabular}{|c|c|c|c|c|c|c|c|c|c|c|}
\hline & 500 HRS IN & R-134a@ & $194 \mathrm{~F}$ & & & & & & & \\
\hline ID & WT & EXPWT & $\begin{array}{c}\text { WT \% } \\
\text { CHANGE }\end{array}$ & EXP VIS & $\begin{array}{c}\text { BASE BRN } \\
\text { OUT } \\
\text { (AVE) }\end{array}$ & $\begin{array}{c}\text { EXP BRN } \\
\text { OUT }\end{array}$ & $\begin{array}{c}\text { BRN OUT } \\
\% \\
\text { CHANGE }\end{array}$ & 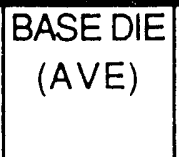 & EXP DIE & $\begin{array}{c}\text { DIE \% } \\
\text { CHANGE }\end{array}$ \\
\hline A 1 & 21.7392 & 21.7518 & $0.058 \%$ & $\mathrm{~N} / \mathrm{C}$ & 576 & 451 & & 15.80 & 17.82 & \\
\hline & & & & & 576 & 459 & $-23.0 \%$ & 15.80 & 17.51 & $6.9 \%$ \\
\hline & & & & & 576 & 421 & & 15.80 & 15.32 & \\
\hline$\overline{\mathrm{B1}}$ & 23.9183 & 23.9778 & $0.249 \%$ & $\mathrm{~N} / \mathrm{C}$ & 736 & 720 & & 11.62 & 11.06 & \\
\hline & & & & & 736 & 721 & $-2.1 \%$ & 11.62 & 12.26 & $3.0 \%$ \\
\hline & & & & & 736 & 721 & & 11.62 & 12.59 & \\
\hline C1 & 21.5932 & 21.6071 & $0.064 \%$ & $\mathrm{~N} / \mathrm{C}$ & 579 & 411 & & 16.58 & 13.42 & \\
\hline & & & & & 579 & 443 & $-24.8 \%$ & 16.58 & 17.40 & $-1.2 \%$ \\
\hline & & & & & 579 & 453 & & 16.58 & 18.34 & \\
\hline & $R-134 a-->2$ & tHRS@ & $02 \mathrm{~F}$ & & & & & & & \\
\hline$A 2$ & 21.7854 & 21.7880 & $0.012 \%$ & $\mathrm{~N} / \mathrm{C}$ & 576 & 549 & & 15.80 & 11.65 & \\
\hline & & & & & 576 & 611 & $-1.5 \%$ & 15.80 & 11.93 & $-20.4 \%$ \\
\hline & & & & & 576 & 542 & & 15.80 & 14.13 & \\
\hline$B 2$ & 23.0417 & 23.0430 & $0.006 \%$ & $\mathrm{~N} / \mathrm{C}$ & 736 & 720 & & 11.62 & 10.94 & \\
\hline & & & & & 736 & 720 & $-6.3 \%$ & 11.62 & 10.38 & $-8.5 \%$ \\
\hline & & & & & 736 & 630 & & 11.62 & 10.58 & \\
\hline $\mathrm{C} 2$ & 21.2863 & 21.2890 & $0.013 \%$ & $\mathrm{~N} / \mathrm{C}$ & 579 & 549 & & 16.58 & 13.29 & \\
\hline & & & & & 579 & 571 & $-2.9 \%$ & 16.58 & 17.03 & $-12.6 \%$ \\
\hline & & & & & 579 & 567 & & 16.58 & 13.13 & \\
\hline
\end{tabular}


500 HOURS IN R-134a @ 194 F

\begin{tabular}{|c|c|c|c|c|c|c|c|c|c|c|}
\hline VARN & $\begin{array}{l}\text { TWISTED } \\
\text { PAIR WT }\end{array}$ & $\begin{array}{c}\text { EXP T } \\
\text { PAIR WT }\end{array}$ & $\begin{array}{c}\text { WT \% } \\
\text { CHANGE }\end{array}$ & $\begin{array}{l}\text { EXP } \\
\text { VIS }\end{array}$ & BASE DIE & EXPDIE & $\begin{array}{c}\text { DIE \% } \\
\text { CHANGE }\end{array}$ & $\begin{array}{c}\text { BASE } \\
\text { BUPN } \\
\text { OUT }\end{array}$ & $\begin{array}{c}\text { EXP } \\
\text { BURN } \\
\text { OUT }\end{array}$ & $\begin{array}{c}\text { BRNOUT } \\
\% \\
\text { CHANGE }\end{array}$ \\
\hline \multirow{3}{*}{$\begin{array}{l}\text { U-475 } \\
\text { A } 1\end{array}$} & \multirow[t]{3}{*}{22.4783} & \multirow[t]{3}{*}{22.5036} & \multirow[t]{3}{*}{$0.113 \%$} & $\mathrm{~N} / \mathrm{C}$ & 16.24 & 19.75 & & 430 & 546 & \\
\hline & & & & & 16.24 & 15.16 & $6.44 \%$ & 430 & 414 & $4.88 \%$ \\
\hline & & & & & 16.24 & 16.95 & & 430 & 393 & \\
\hline \multirow{3}{*}{$\begin{array}{l}Y-390 \\
B 1\end{array}$} & \multirow[t]{3}{*}{23.1672} & \multirow[t]{3}{*}{23.1955} & \multirow[t]{3}{*}{$0.122 \%$} & $\mathrm{~N} / \mathrm{C}$ & 18.77 & 20.00 & & 510 & 452 & \\
\hline & & & & & 18.77 & 15.73 & $-4.05 \%$ & 510 & 446 & $-13.99 \%$ \\
\hline & & & & & 18.77 & 18.30 & & 510 & 418 & \\
\hline$T$ ER-610 & \multirow[t]{3}{*}{22.3418} & \multirow[t]{3}{*}{22.3397} & \multirow[t]{3}{*}{$-0.009 \%$} & $\mathrm{~N} / \mathrm{C}$ & 15.57 & 15.33 & & 442 & 385 & \\
\hline \multirow{2}{*}{ C1 } & & & & & 15.57 & 16.37 & $3.79 \%$ & 442 & 367 & $-14.78 \%$ \\
\hline & & & & & 15.57 & 16.78 & & 442 & 37.8 & \\
\hline$Y-833$ & \multirow{3}{*}{22.4853} & \multirow{3}{*}{22.4881} & \multirow[t]{3}{*}{$0.012 \%$} & $\mathrm{~N} / \mathrm{C}$ & 12.04 & 14.76 & & 578 & 552 & \\
\hline \multirow[t]{2}{*}{ D1 } & & & & & 12.04 & 11.17 & $0.97 \%$ & 578 & 552 & $-4.27 \%$ \\
\hline & & & & & 12.04 & 10.54 & & 578 & 556 & \\
\hline \multirow{3}{*}{$\begin{array}{l}923 \\
E 1\end{array}$} & \multirow[t]{3}{*}{23.8426} & \multirow[t]{3}{*}{23.8723} & \multirow[t]{3}{*}{$0.125 \%$} & $\mathrm{~N} / \mathrm{C}$ & 16.76 & 18.56 & & 606 & 566 & \\
\hline & & & & & 16.76 & 20.00 & $16.47 \%$ & 606 & 440 & $-12.49 \%$ \\
\hline & & & & & 16.76 & 20.00 & & 606 & 585 & \\
\hline$M \mid 1 S O-800$ & 22.3021 & 22.3146 & $0.056 \%$ & $\mathrm{~N} / \mathrm{C}$ & 19.08 & 16.38 & & 580 & 553 & \\
\hline $1 F 1$ & & & & & 19.08 & 14.94 & $-18.45 \%$ & 580 & 462 & $9.66 \%$ \\
\hline & & & & & 19.08 & 15.36 & & 580 & 557 & \\
\hline$E$ & & & & & & & & & & \\
\hline$U-475$ & 22.6408 & 22.6460 & $0.023 \%$ & $\mathrm{~N} / \mathrm{C}$ & 16.24 & 13.83 & & 430 & 302 & \\
\hline $1 \mathrm{~A} 2$ & & & & & 16.24 & 13.39 & $-18.10 \%$ & 430 & 276 & $-32.48 \%$ \\
\hline$M$ & & & & & 16.24 & 12.68 & & 430 & 293 & \\
\hline $11 Y-390$ & 23.1389 & 23.1410 & $0.009 \%$ & $\mathrm{~N} / \mathrm{C}$ & 18.77 & 20.00 & & 510 & 408 & \\
\hline$D B 2$ & & & & & 18.77 & 15.30 & $-17.05 \%$ & 510 & 422 & $-18.43 \%$ \\
\hline$E$ & & & & & 18.77 & 11.41 & & 510 & 418 & \\
\hline ER-610 & 22.2601 & 22.2604 & $0.001 \%$ & $\mathrm{~N} / \mathrm{C}$ & 15.57 & 12.75 & & 442 & 407 & \\
\hline $\mathrm{C} 2$ & & & & & 15.57 & 13.98 & $-14.22 \%$ & 442 & 414 & $-6.94 \%$ \\
\hline & & & & & 15.57 & 13.34 & & 442 & 413 & \\
\hline$\overline{Y-833}$ & 22.4472 & 22.4356 & $-0.052 \%$ & $\mathrm{~N} / \mathrm{C}$ & 12.04 & 10.86 & & 578 & 573 & \\
\hline D2 & & & & & 12.04 & 9.67 & $.18 .30 \%$ & 578 & 493 & $-6.46 \%$ \\
\hline & & & & & 12.04 & 8.98 & & 578 & 556 & \\
\hline 923 & 23.4721 & 23.4640 & $-0.035 \%$ & $\mathrm{~N} / \mathrm{C}$ & 16.76 & 19.30 & & 606 & 577 & \\
\hline E2 & & & & & 16.76 & 20.00 & $8.41 \%$ & 606 & 555 & $-8.69 \%$ \\
\hline & & & & & 16.76 & 15.21 & & 606 & 528 & \\
\hline ISO-800 & 22.5023 & 22.5007 & $-0.007 \%$ & $\mathrm{~N} / \mathrm{C}$ & 19.08 & 13.45 & & 580 & 429 & \\
\hline $\mathrm{F} 2$ & & & & & 19.08 & 14.64 & $-20.48 \%$ & 580 & 585 & $-8.05 \%$ \\
\hline & & & & & 19.08 & 17.43 & & 580 & 586 & \\
\hline
\end{tabular}




\begin{tabular}{|c|c|c|c|c|c|c|c|c|c|c|c|}
\hline \multicolumn{12}{|c|}{500 HOURS IN R-134a @ 194 F } \\
\hline & VARN & $\begin{array}{l}\text { TWISTED } \\
\text { PAIR WT }\end{array}$ & $\begin{array}{c}\text { EXPT } \\
\text { PAIR WT }\end{array}$ & $\begin{array}{c}\text { WT \% } \\
\text { CHANGE }\end{array}$ & $\begin{array}{l}\text { EXP } \\
\text { VIS }\end{array}$ & $\begin{array}{c}\text { BASE } \\
\text { DIE }\end{array}$ & $\begin{array}{l}\text { EXP } \\
\text { DIE }\end{array}$ & $\begin{array}{c}\text { DIE \% } \\
\text { CHANGE }\end{array}$ & $\begin{array}{l}\text { BASE } \\
\text { BURN } \\
\text { OUT }\end{array}$ & \begin{tabular}{|c|} 
EXP \\
BURN \\
OUT
\end{tabular} & $\begin{array}{c}\text { BRNOUT } \\
\% \\
\text { CHANGE }\end{array}$ \\
\hline \multirow{6}{*}{$\begin{array}{l}P \\
O \\
L \\
Y \\
E \\
S\end{array}$} & \multirow{3}{*}{$\begin{array}{l}\text { U-475 } \\
\text { A } 1\end{array}$} & \multirow[t]{3}{*}{24.4352} & \multirow[t]{3}{*}{24.4862} & \multirow[t]{3}{*}{$0.21 \%$} & $\mathrm{~N} / \mathrm{C}$ & 13.32 & 8.88 & & 746 & 721 & \\
\hline & & & & & & 13.32 & 12.92 & $-13.11 \%$ & 746 & 720 & $-3.35 \%$ \\
\hline & & & & & & 13.32 & 12.92 & & 746 & 722 & \\
\hline & \multirow{3}{*}{$\begin{array}{l}Y-390 \\
B 1\end{array}$} & \multirow[t]{3}{*}{24.9362} & \multirow[t]{3}{*}{24.9814} & \multirow[t]{3}{*}{$0.18 \%$} & $\mathrm{~N} / \mathrm{C}$ & 12.28 & 14.19 & & 755 & 720 & \\
\hline & & & & & & 12.28 & 14.39 & $9.69 \%$ & 755 & 722 & $-4.42 \%$ \\
\hline & & & & & & 12.28 & 11.83 & & 755 & 723 & \\
\hline \multirow{4}{*}{$\begin{array}{l}T \\
E \\
R\end{array}$} & ER-610 & \multirow[t]{3}{*}{23.4987} & \multirow[t]{3}{*}{23.5430} & \multirow[t]{3}{*}{$0.19 \%$} & $\mathrm{~N} / \mathrm{C}$ & 12.73 & 13.47 & & 734 & 722 & \\
\hline & \multirow{2}{*}{ C1 } & & & & & 12.73 & 13.72 & $9.66 \%$ & 734 & 721 & $-1.73 \%$ \\
\hline & & & & & & 12.73 & 14.69 & & 734 & 721 & \\
\hline & \multirow{3}{*}{$\begin{array}{l}Y-833 \\
D 1\end{array}$} & \multirow[t]{3}{*}{23.3314} & \multirow[t]{3}{*}{23.3758} & \multirow[t]{3}{*}{$0.19 \%$} & $\mathrm{~N} / \mathrm{C}$ & 12.49 & 12.73 & & 734 & 721 & \\
\hline$P$ & & & & & & 12.49 & 13.41 & $3.60 \%$ & 734 & 722 & $-1.73 \%$ \\
\hline 0 & & & & & & 12.49 & 12.68 & & 734 & 721 & \\
\hline$L$ & \multirow{3}{*}{$\begin{array}{l}923 \\
E 1 \\
\end{array}$} & \multirow[t]{3}{*}{24.9493} & \multirow[t]{3}{*}{24.9923} & \multirow[t]{3}{*}{$0.17 \%$} & $\mathrm{~N} / \mathrm{C}$ & 14.38 & 13.77 & & 742 & 723 & \\
\hline$Y$ & & & & & & 14.38 & 18.02 & $8.00 \%$ & 742 & 722 & $-2.61 \%$ \\
\hline A & & & & & & 14.38 & 14.80 & & 742 & 723 & \\
\hline \multirow{3}{*}{$\begin{array}{l}M \\
1 \\
D\end{array}$} & ISO-800 & 24.5264 & 24.5529 & $0.11 \%$ & $N / C$ & 12.29 & 13.49 & & 747 & 720 & \\
\hline & F1 & & & & & 12.29 & 13.71 & $10.60 \%$ & 747 & 722 & $-3.44 \%$ \\
\hline & & & & & & 12.29 & 13.58 & & 747 & 722 & \\
\hline$E$ & & R-134a -. & $>24$ hours & @ 302 F & & & & & & & \\
\hline & U-475 & 24.5684 & 24.5723 & $0.02 \%$ & $N / C$ & 13.32 & 12.09 & & 746 & 721 & \\
\hline 1 & A2 & & & & & 13.32 & 12.43 & $-8.31 \%$ & 746 & 722 & $-3.31 \%$ \\
\hline$M$ & & & & & & 13.32 & 12.12 & & 746 & 721 & \\
\hline 1 & $Y-390$ & 24.9671 & 24.9671 & $0.00 \%$ & $\mathrm{~N} / \mathrm{C}$ & 12.28 & 13.92 & & 755 & 721 & \\
\hline$D$ & B2 & & & & & 12.28 & 13.25 & $11.13 \%$ & 755 & 722 & $-4.44 \%$ \\
\hline$E$ & & & & & & 12.28 & 13.77 & & 755 & 585 & \\
\hline & ER-610 & 23.6134 & 23.6134 & $0.00 \%$ & $\mathrm{~N} / \mathrm{C}$ & 12.73 & 11.07 & & 734 & 715 & \\
\hline$E$ & $\mathrm{C} 2$ & & & & & 12.73 & 12.08 & $.10 .45 \%$ & 734 & 670 & $-4.41 \%$ \\
\hline$P$ & & & & & & 12.73 & 11.05 & & 734 & 720 & \\
\hline 0 & $Y-833$ & 23.1894 & 23.1754 & $-0.06 \%$ & $\mathrm{~N} / \mathrm{C}$ & 12.49 & 11.38 & & 734 & 721 & \\
\hline$x$ & D2 & & & & & 12.49 & 12.95 & $-0.67 \%$ & 734 & 720 & $-1.84 \%$ \\
\hline$Y$ & & & & & & 12.49 & 12.89 & & 734 & $n / a$ & \\
\hline & 923 & 25.6773 & 25.6786 & $0.01 \%$ & $\mathrm{~N} / \mathrm{C}$ & 14.38 & 11.63 & & 742 & 720 & \\
\hline$G$ & E2 & & & & & 14.38 & 14.39 & $-8.37 \%$ & 742 & 543 & $-2.96 \%$ \\
\hline$L$ & & & & & & 14.38 & 13.51 & & 742 & 621 & \\
\hline$A$ & $150-800$ & 24.4514 & 24.4501 & $-0.01 \%$ & & 12.29 & 11.56 & & 747 & 720 & \\
\hline$S$ & F2 & & & & $\mathrm{N} / \mathrm{C}$ & 12.29 & 12.03 & $-4.37 \%$ & 747 & 721 & $-3.55 \%$ \\
\hline S & & & & & & 12.29 & 11.67 & & 747 & 542 & \\
\hline
\end{tabular}




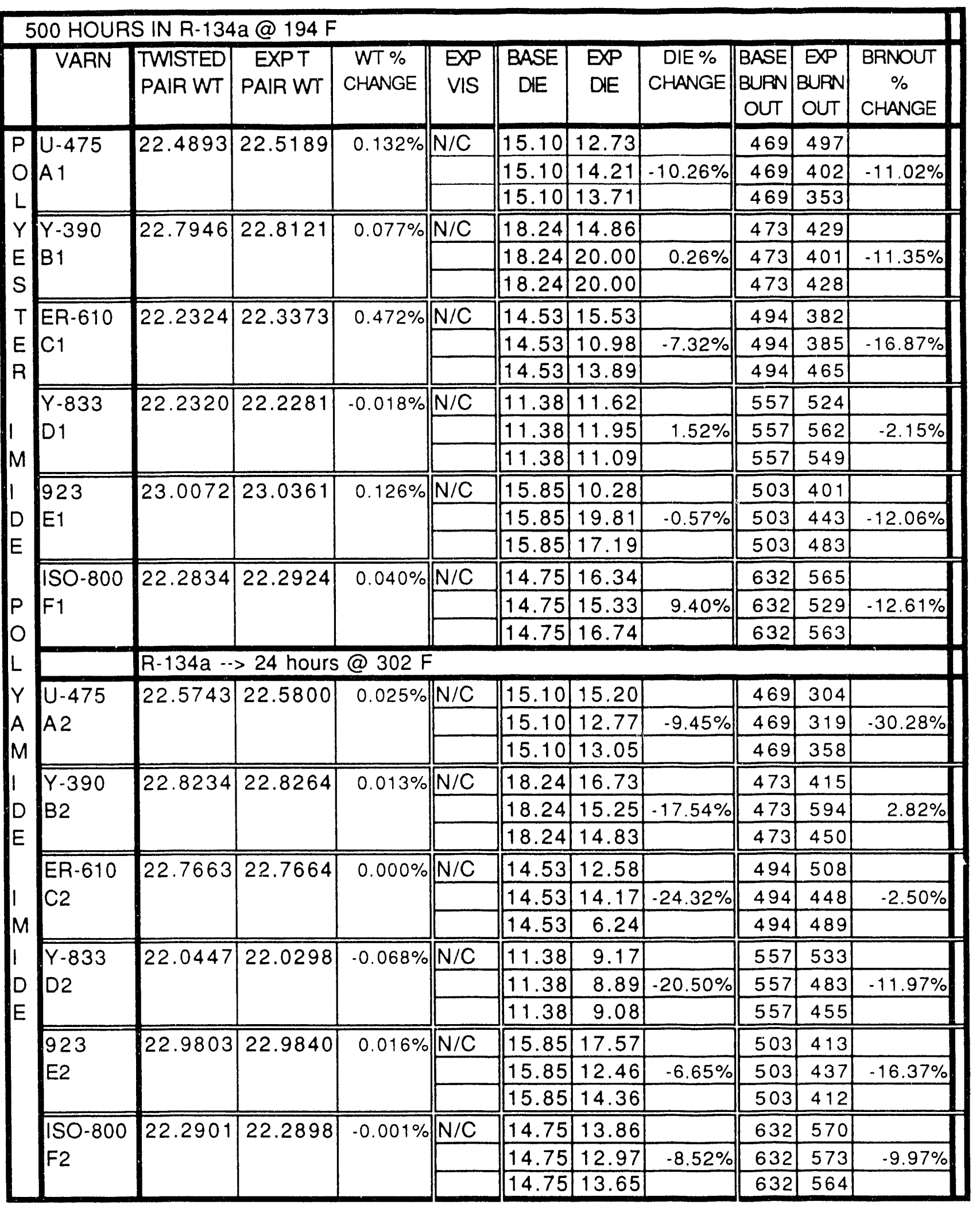




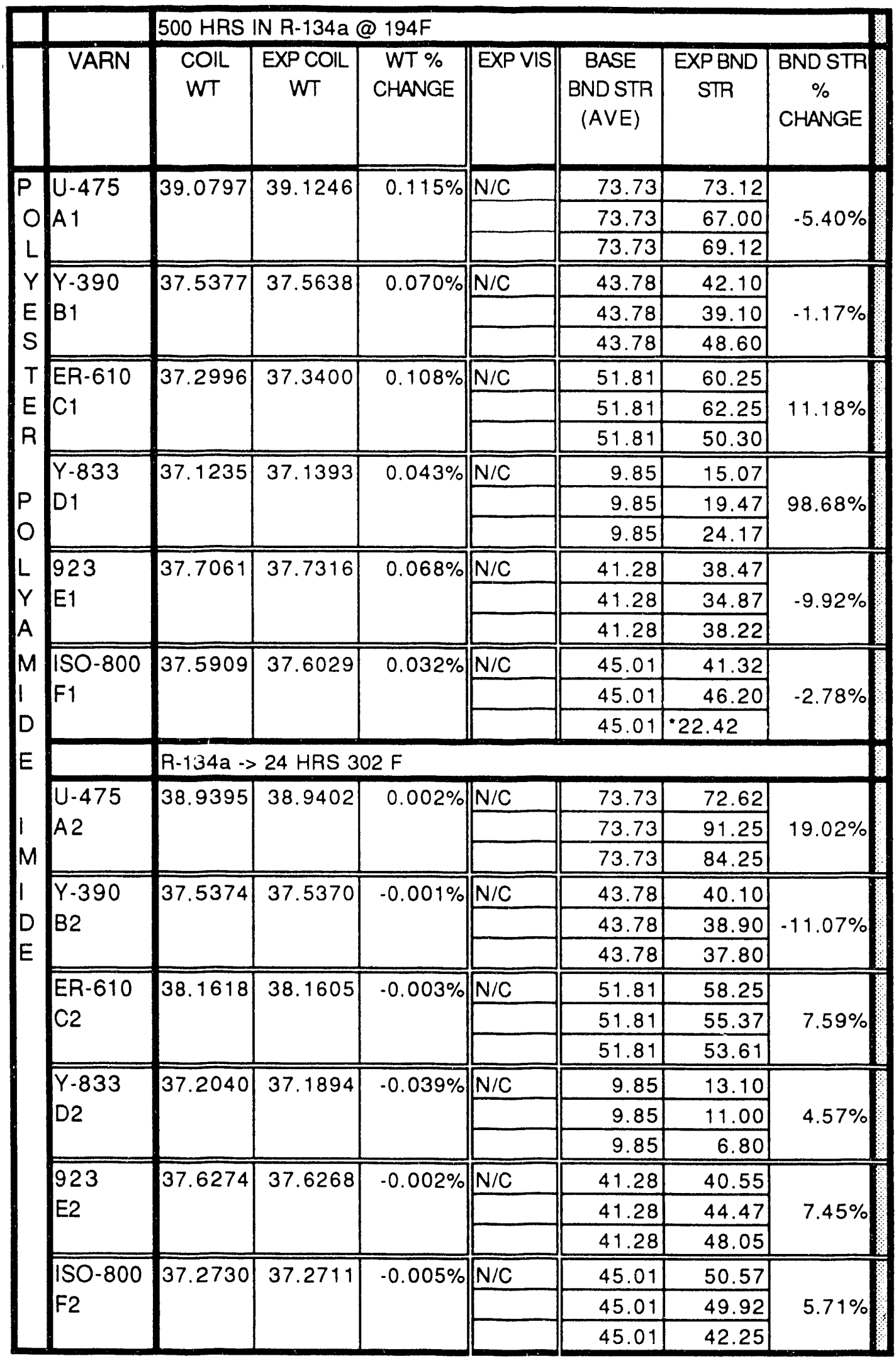

$\mathrm{H}-5$

*This number was not included in the average 


\begin{tabular}{|c|c|c|c|c|c|c|c|c|}
\hline & & 500 & IN R-134a & @ 194F & & & & \\
\hline & VARN & $\begin{array}{l}\text { COIL } \\
\text { WT }\end{array}$ & $\begin{array}{c}\text { EXP COIL } \\
W T\end{array}$ & $\begin{array}{c}\text { WT \% } \\
\text { CHANGE }\end{array}$ & EXP VIS & $\begin{array}{c}\text { BASE } \\
\text { BND STR } \\
(\text { AVE })\end{array}$ & $\begin{array}{c}\text { EXPBND } \\
\text { STR }\end{array}$ & $\begin{array}{c}\text { BND STR } \\
\% \\
\text { CHANGE }\end{array}$ \\
\hline$P$ & U-475 & 37.1147 & 37.1729 & $0.157 \%$ & $\mathrm{~N} / \mathrm{C}$ & 40.14 & 37.42 & \\
\hline 0 & $A 1$ & & & & & 40.14 & 33.40 & $-9.47 \%$ \\
\hline L & & & & & & 40.14 & 38.20 & \\
\hline$Y$ & $Y-390$ & 36.3902 & 36.4363 & $0.127 \%$ & $\mathrm{~N} / \mathrm{C}$ & 36.12 & 41.32 & \\
\hline$E$ & B1 & & & & & 36.12 & 35.47 & $0.63 \%$ \\
\hline S & & & & & & 36.12 & 32.25 & \\
\hline$T$ & ER-610 & 37.1452 & 37.1909 & $0.123 \%$ & $\mathrm{~N} / \mathrm{C}$ & 35.96 & 28.32 & \\
\hline$E$ & & & & & & 35.96 & 34.40 & $-14.73 \%$ \\
\hline$R$ & & & & & & 35.96 & 29.27 & \\
\hline & $\overline{Y-833}$ & 36.5883 & 36.6357 & $0.130 \%$ & $\mathrm{~N} / \mathrm{C}$ & 33.14 & 35.30 & \\
\hline$P$ & $D 1$ & & & & & 33.14 & 34.70 & $2.65 \%$ \\
\hline 0 & & & & & & 33.14 & 32.05 & \\
\hline$L$ & 923 & 36.3319 & 36.3932 & $0.169 \%$ & $\mathrm{~N} / \mathrm{C}$ & 40.52 & 42.35 & \\
\hline Y & & & & & & 40.52 & 46.02 & $11.83 \%$ \\
\hline A & & & & & & 40.52 & 47.57 & \\
\hline$M$ & $150-800$ & 35.2993 & 35.3187 & $0.055 \%$ & $\mathrm{~N} / \mathrm{C}$ & 20.20 & 21.70 & \\
\hline 1 & $F_{1}$ & & & & & 20.20 & 19.00 & $-6.93 \%$ \\
\hline$D$ & & & & & & 20.20 & 15.70 & \\
\hline$E$ & & R-134a $\rightarrow$ & 24 HRS 3 & $02 \mathrm{~F}$ & & & & \\
\hline & U-475 & 37.1800 & 37.1817 & $0.005 \%$ & $\mathrm{~N} / \mathrm{C}$ & 40.14 & 45.20 & \\
\hline 1 & $A 2$ & & & & & 40.14 & 37.82 & $0.00 \%$ \\
\hline$M$ & & & & & & 40.14 & 37.40 & \\
\hline 1 & $Y-390$ & 36.2323 & 36.2332 & $0.002 \%$ & $\mathrm{~N} / \mathrm{C}$ & 36.12 & 34.82 & \\
\hline$D$ & B2 & & & & & 36.12 & 35.72 & $-2.19 \%$ \\
\hline$E$ & & & & & & 36.12 & 35.45 & \\
\hline & ER-610 & 36.2481 & 36.2462 & $-0.005 \%$ & $N / C$ & 35.96 & 31.75 & \\
\hline$E$ & C2 & & & & & 35.96 & 31.72 & $-5.46 \%$ \\
\hline$P$ & & & & & & 35.96 & 38.52 & \\
\hline 0 & $Y-833$ & 36.5078 & 36.5057 & $-0.006 \%$ & $\mathrm{~N} / \mathrm{C}$ & 33.14 & 31.22 & \\
\hline$x$ & $D 2$ & & & & & 33.14 & 31.17 & $-11.53 \%$ \\
\hline$Y$ & & & & & & 33.14 & 25.57 & \\
\hline & 923 & 36.3960 & 36.3862 & $-0.027 \%$ & $\mathrm{~N} / \mathrm{C}$ & 40.52 & 40.70 & \\
\hline$G$ & E2 & & & & & 40.52 & 36.95 & $0.44 \%$ \\
\hline$L$ & & & & & & 40.52 & 44.45 & \\
\hline A & ISO-800 & 35.0732 & 35.0733 & $0.000 \%$ & $\mathrm{~N} / \mathrm{C}$ & 20.20 & 18.77 & \\
\hline$S$ & $\mathrm{~F} 2$ & & & & & 20.20 & 19.47 & $-6.83 \%$ \\
\hline$S$ & & & & & & 20.20 & 18.22 & \\
\hline
\end{tabular}




\begin{tabular}{|c|c|c|c|c|c|c|c|c|}
\hline & & $500 \mathrm{HRS}$ & IN R-134a & @ 194F & & & & \\
\hline & VARN & $\begin{array}{l}\text { COIL } \\
\text { WT }\end{array}$ & $\begin{array}{c}\text { EXP COIL } \\
W T\end{array}$ & $\begin{array}{c}\text { WT \% } \\
\text { CHANGE }\end{array}$ & EXP VIS & $\begin{array}{c}\text { BASE } \\
\text { BND STR } \\
\text { (AVE) }\end{array}$ & $\begin{array}{c}\text { EXPBND } \\
\text { STR }\end{array}$ & $\begin{array}{c}\text { BND STR } \\
\% \\
\text { CHANGE }\end{array}$ \\
\hline $\mathrm{P}$ & U-475 & 37.4206 & 37.4664 & $0.122 \%$ & $\mathrm{NOC}$ & 51.21 & 55.60 & \\
\hline 0 & A 1 & & & & & 51.21 & 68.42 & $18.38 \%$ \\
\hline$L$ & & & & & & 51.21 & 57.85 & \\
\hline Y & $Y-390$ & 38.5014 & 38.5438 & $0.110 \%$ & $\mathrm{~N} / \mathrm{C}$ & 50.72 & 48.77 & \\
\hline$E$ & B1 & & & & & 50.72 & 46.60 & $-4.43 \%$ \\
\hline$S$ & & & & & & 50.72 & 50.05 & \\
\hline $\mathrm{T}$ & ER-610 & 37.6576 & 37.6928 & $0.093 \%$ & $\mathrm{~N} / \mathrm{C}$ & 58.33 & 65.80 & \\
\hline$E$ & C1 & & & & & 58.33 & 58.55 & $5.09 \%$ \\
\hline$R$ & & & & & & 58.33 & 59.55 & \\
\hline & $Y-833$ & 37.0345 & 37.0488 & $0.039 \%$ & $\mathrm{~N} / \mathrm{C}$ & 5.84 & 27.15 & \\
\hline 1 & D1 & & & & & 5.84 & 21.02 & $327.63 \%$ \\
\hline M & & & & & & 5.84 & 26.75 & \\
\hline I & 923 & 38.2019 & 38.2190 & $0.045 \%$ & $\mathrm{~N} / \mathrm{C}$ & 49.26 & 32.35 & \\
\hline$D$ & $E 1$ & & & & & 49.26 & 49.72 & $.14 .66 \%$ \\
\hline$E$ & & & & & & 49.26 & 44.05 & \\
\hline & ISO-800 & 37.5214 & 37.5375 & $0.043 \%$ & $\mathrm{~N} / \mathrm{C}$ & 36.08 & 43.87 & \\
\hline $\mathrm{P}$ & & & & & & 36.08 & 40.15 & $19.98 \%$ \\
\hline O & & & & & & 36.08 & 45.85 & \\
\hline L & & R-134a -> & 24 HRS 3 & $2 \mathrm{~F}$ & & & & \\
\hline Y & $U-475$ & 37.6974 & 37.6956 & $-0.005 \%$ & $\mathrm{~N} / \mathrm{C}$ & 51.21 & 64.72 & \\
\hline A & $\mathrm{A} 2$ & & & & & 51.21 & 60.55 & $22.87 \%$ \\
\hline M & & & & & & 51.21 & 63.50 & \\
\hline 1 & $Y-390$ & 38.2755 & 38.2774 & $0.005 \%$ & $\mathrm{~N} / \mathrm{C}$ & 50.72 & 47.80 & \\
\hline$D$ & B2 & & & & & 50.72 & 37.42 & $-14.98 \%$ \\
\hline$E$ & & & & & & 50.72 & 44.15 & \\
\hline & ER-610 & 37.4381 & 37.4361 & $-0.005 \%$ & $\mathrm{~N} / \mathrm{C}$ & 58.33 & 60.95 & \\
\hline 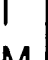 & $\mathrm{C} 2$ & & & & & 58.33 & 62.12 & $10.97 \%$ \\
\hline M & & & & & & 58.33 & 71.12 & \\
\hline 1 & $Y-833$ & 36.6439 & 36.6266 & $-0.047 \%$ & $\mathrm{~N} / \mathrm{C}$ & 5.84 & 9.00 & \\
\hline$D$ & D2 & & & & & 5.84 & 32.85 & $395.55 \%$ \\
\hline$E$ & & & & & & 5.84 & 44.97 & \\
\hline & 923 & 38.1007 & 38.0994 & $-0.003 \%$ & $\mathrm{~N} / \mathrm{C}$ & 49.26 & 40.95 & \\
\hline & E2 & & & & & 49.26 & 53.20 & $9.77 \%$ \\
\hline & & & & & & 49.26 & 68.07 & \\
\hline & ISO-800 & 37.3299 & 37.3291 & $-0.002 \%$ & $\mathrm{~N} / \mathrm{C}$ & 36.08 & 35.95 & \\
\hline & $F 2$ & & & & & 36.08 & 36.90 & $-12.32 \%$ \\
\hline & & & & & & 36.08 & 22.05 & \\
\hline
\end{tabular}


SINGLE MAG WIRE WITHOUT VARNISH

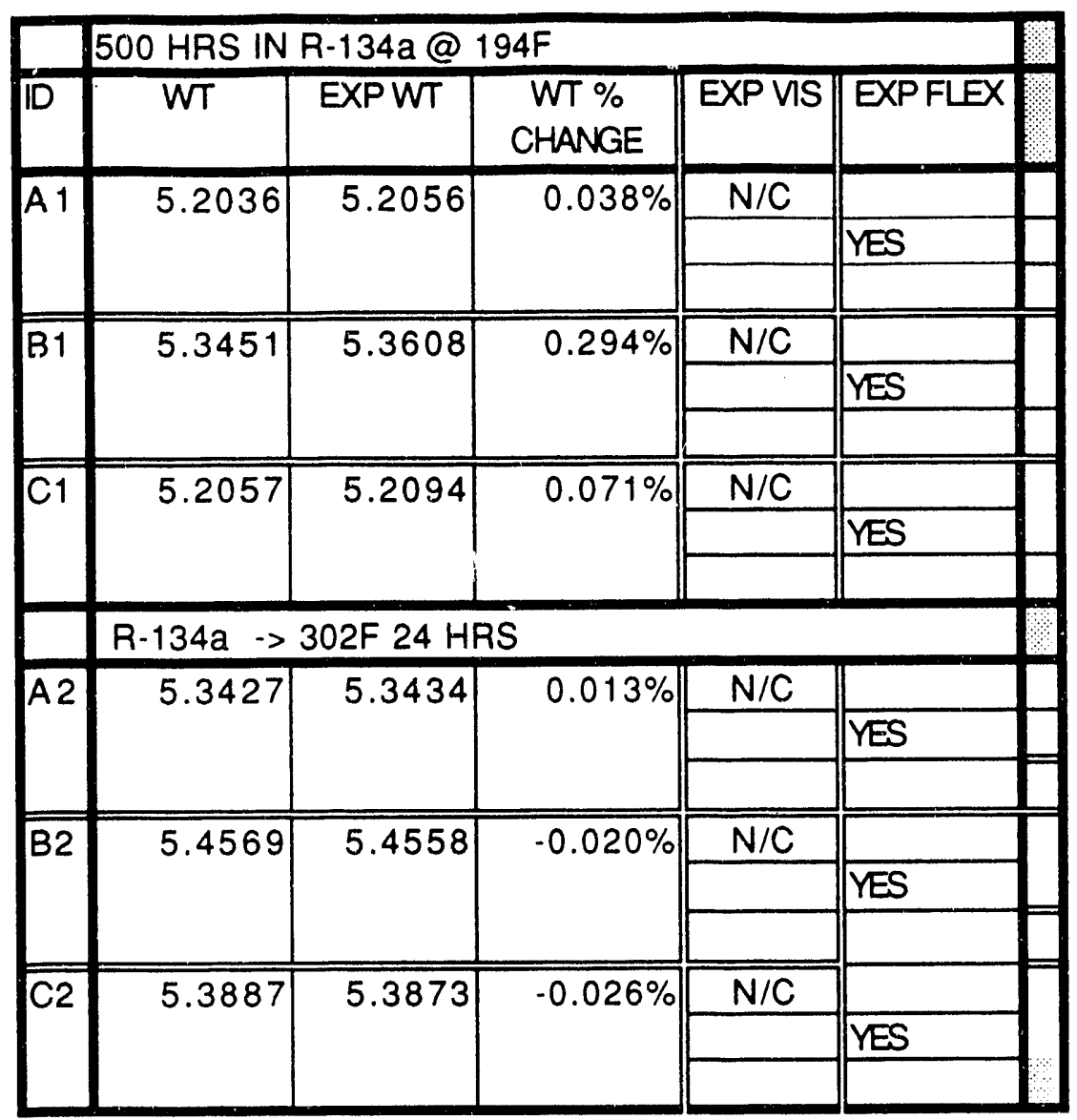




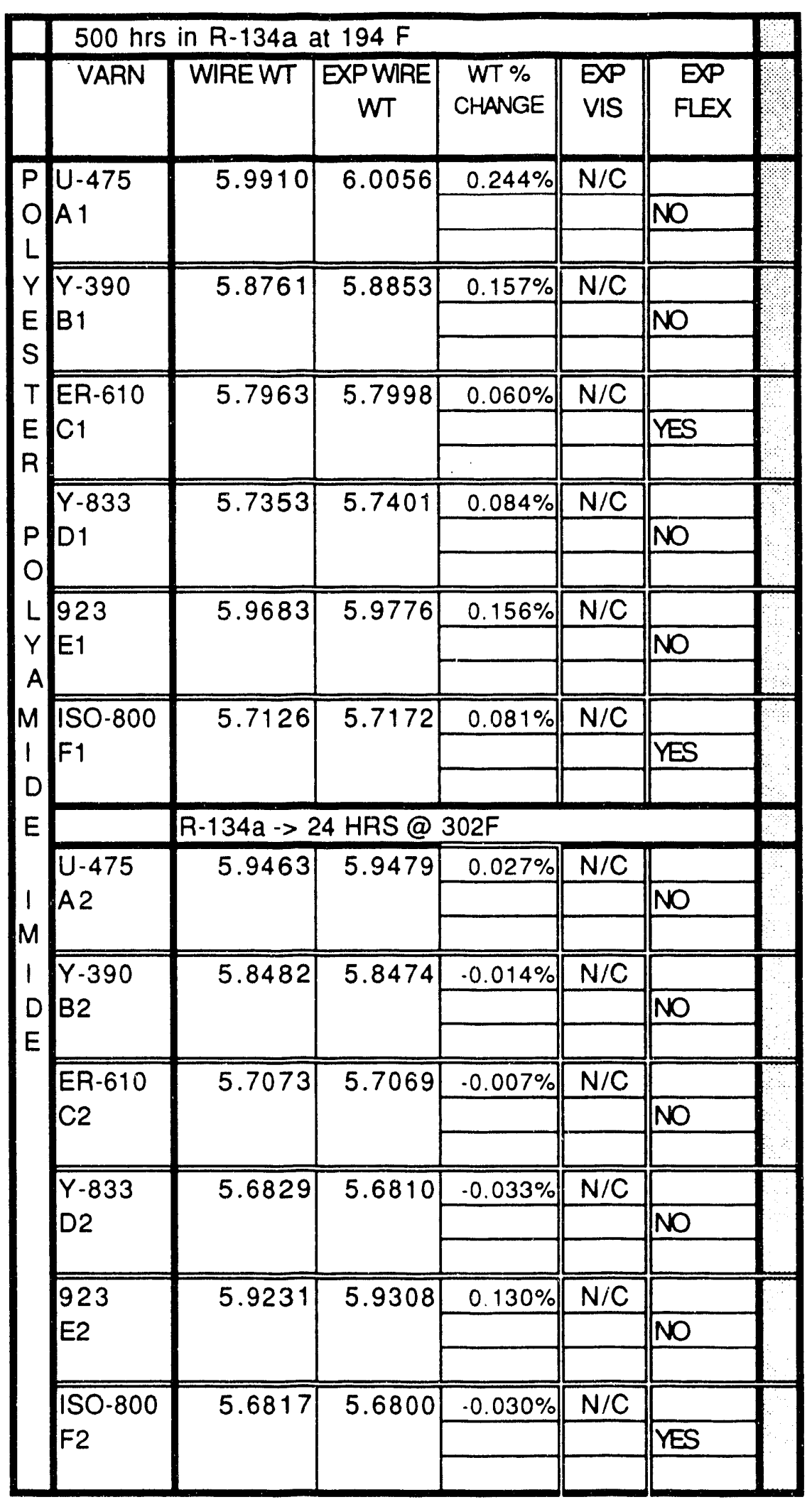









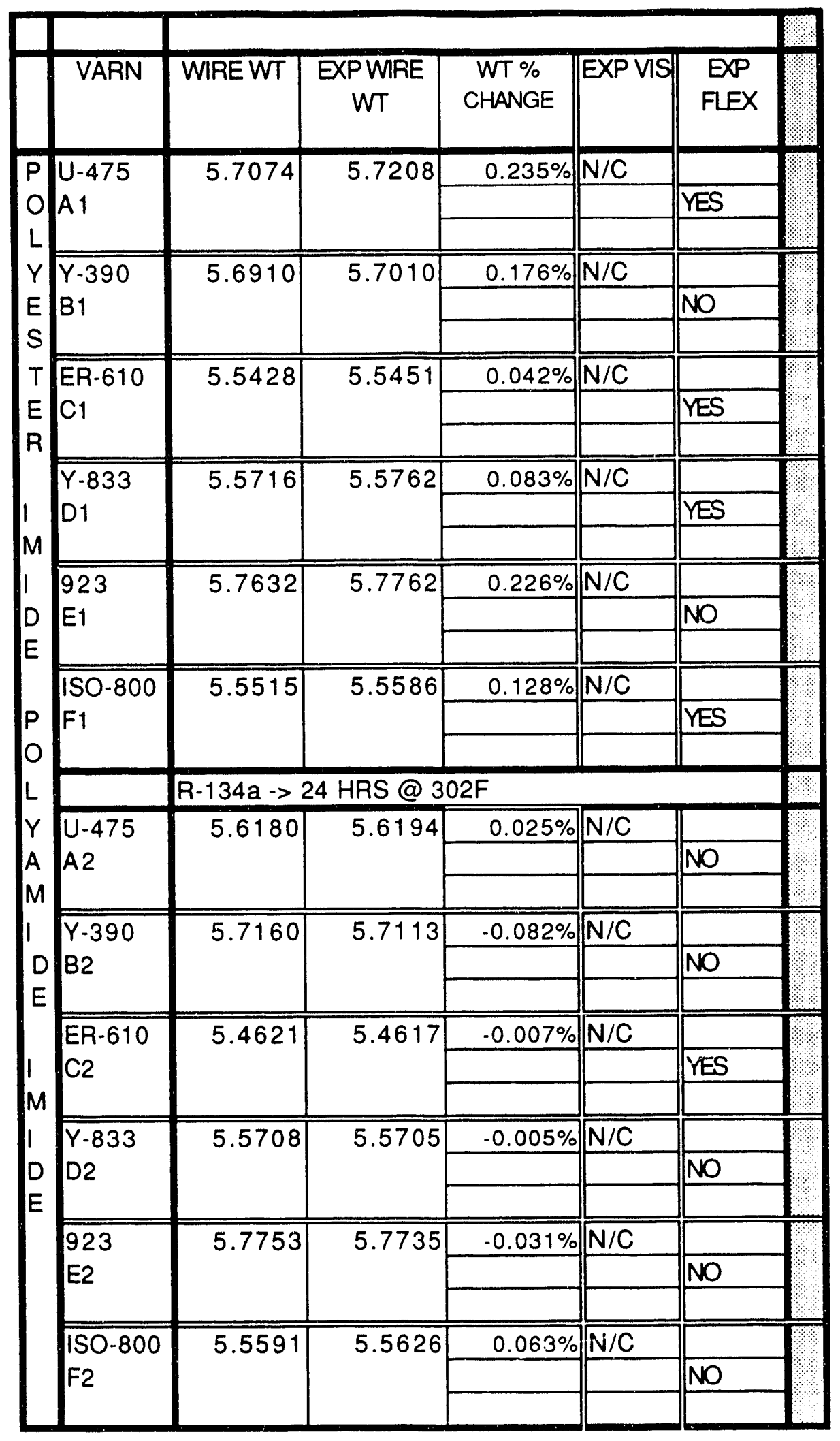

) 


\begin{tabular}{|c|c|c|c|c|c|}
\hline & $500 \mathrm{HRS}$ IN & 2-134a@19 & & & \\
\hline$\overline{I D}$ & $\overline{W T}$ & EXPWT & $\begin{array}{l}\text { WT \% } \\
\text { CHANGE }\end{array}$ & EXPVIS & EXPFLEX \\
\hline$U-475$ & 2.5242 & 2.5664 & $1.67 \%$ & $N / C$ & $\mathrm{~N} / \mathrm{C}$ \\
\hline $\begin{array}{l}Y-390 \\
B 1\end{array}$ & 2.7829 & 2.8159 & $1.19 \%$ & \begin{tabular}{|l} 
Slightly \\
Warped
\end{tabular} & $\mathrm{N} / \mathrm{C}$ \\
\hline & & & & & \\
\hline $\begin{array}{l}\text { ER-610 } \\
\mathrm{C} 1\end{array}$ & 2.4045 & 2.4713 & $2.78 \%$ & $N / C$ & $\mathrm{~N} / \mathrm{C}$ \\
\hline$\overline{Y-833}$ & 2.6305 & 2.7540 & $4.69 \%$ & $\mathrm{~N} / \mathrm{C}$ & $\mathrm{N} / \mathrm{C}$ \\
\hline D1 & & & & & \\
\hline 923 & 2.1588 & 2.1904 & $1.46 \%$ & $\mathrm{~N} / \mathrm{C}$ & $\mathrm{N} / \mathrm{C}$ \\
\hline E1 & & & & & \\
\hline ISO-800 & 1.3455 & 1.3578 & $0.91 \%$ & Slightly & $\mathrm{N} / \mathrm{C}$ \\
\hline & & & & Warped & \\
\hline & $\mathrm{R}-134 \mathrm{a}$ at - & $302 \mathrm{~F} 24 \mathrm{HF}$ & & & \\
\hline $\mathrm{U}-475$ & 2.4729 & 2.4316 & $-1.67 \%$ & |Darkened & $N / C$ \\
\hline A2 & & & & Slighlty & \\
\hline & & & & Warped & \\
\hline$Y-390$ & 2.1316 & 2.0918 & $-1.87 \%$ & Darkened & $\mathrm{N} / \mathrm{C}$ \\
\hline & & & & Slightly & \\
\hline & & & & warped & \\
\hline ER-610 & 2.7314 & 2.7129 & $-0.68 \%$ & Darkened & $N / C$ \\
\hline & & & & & \\
\hline$\overline{Y-833}$ & 2.2510 & 2.2529 & $0.08 \%$ & $N / C$ & $N / C$ \\
\hline D2 & & & & & \\
\hline 923 & 1.8864 & 1.8827 & $-0.20 \%$ & $\mathrm{~N} / \mathrm{C}$ & $\mathrm{N} / \mathrm{C}$ \\
\hline E2 & & & & & \\
\hline $1 \mathrm{SO}-800$ & 2.0169 & 1.9608 & $-2.78 \%$ & Darkened & $N / C$ \\
\hline & & & & Slightly & \\
\hline & & & & warped & \\
\hline
\end{tabular}


500 HR IN R-123@194F

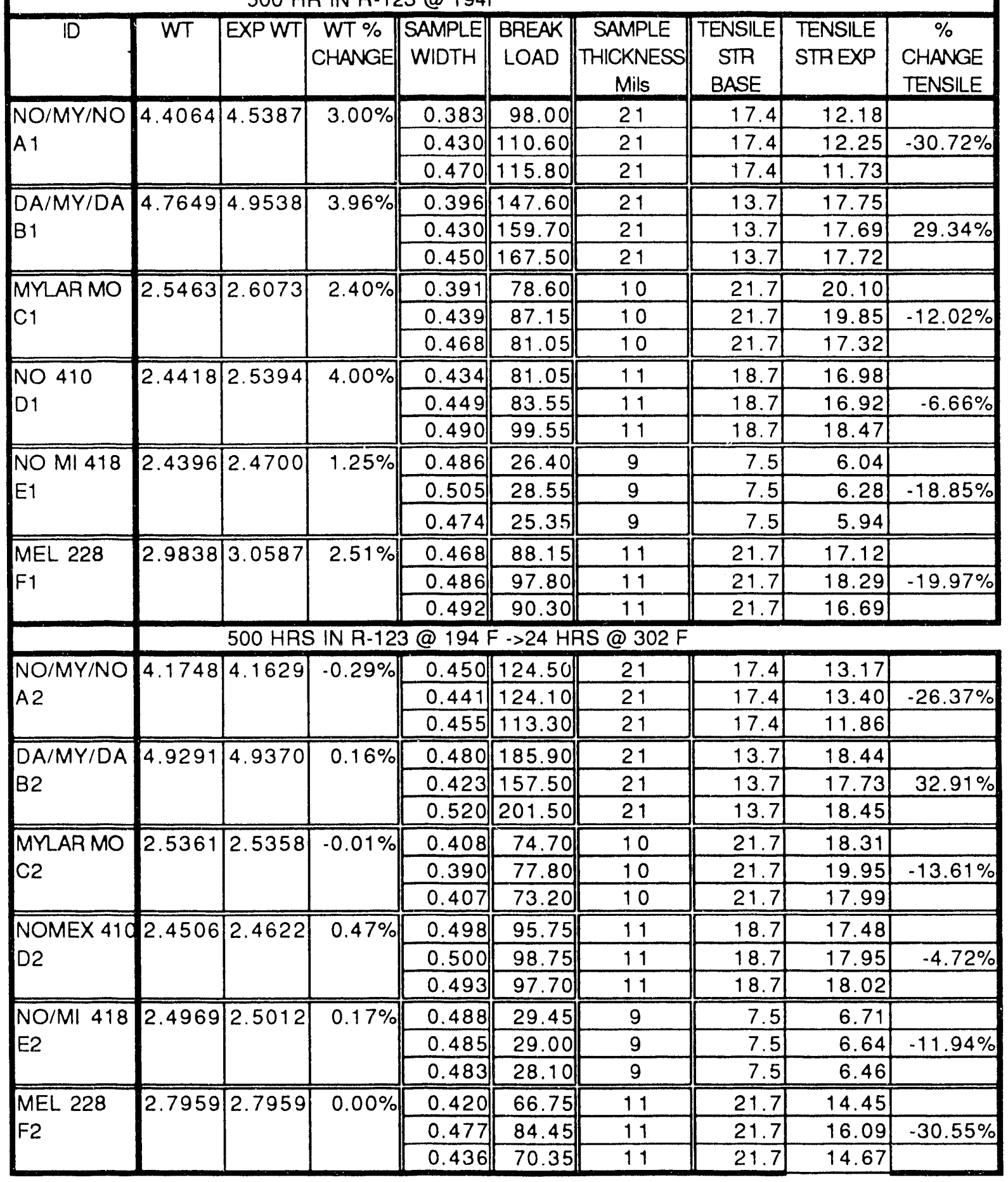




\begin{tabular}{|c|c|c|c|c|c|c|c|c|}
\hline \multicolumn{8}{|c|}{ After 500 hour exposure to R-134a@ $194^{\circ} \mathrm{F}\left(90^{\circ} \mathrm{C}\right)$} & \multirow[b]{2}{*}{$\begin{array}{c}\text { VISUAL } \\
\text { EXP }\end{array}$} \\
\hline ID & $\begin{array}{c}\text { STRETCH } \\
\text { (inch) }\end{array}$ & $\begin{array}{c}\% \\
\text { ELONG }\end{array}$ & $\begin{array}{l}\text { BASE } \\
\text { ELONG } \\
\text { (AVE) }\end{array}$ & $\begin{array}{c}\text { ELONG } \\
\% \\
\text { CHANGE }\end{array}$ & $\begin{array}{c}\text { BASE } \\
\text { DIE } \\
(A \vee E)\end{array}$ & EXPDIE & $\begin{array}{c}\text { DIE \% } \\
\text { CHANGE }\end{array}$ & \\
\hline \multirow{3}{*}{$\begin{array}{l}\mathrm{NO} / \mathrm{MY} / \mathrm{NO} \\
\mathrm{A} 1\end{array}$} & 1.17 & $29.3 \%$ & $20.0 \%$ & & $>18.97$ & $>14.54$ & & \multirow{3}{*}{$\begin{array}{l}\text { Slightly } \\
\text { warped } \\
\text { bubbles }\end{array}$} \\
\hline & 1.11 & $27.8 \%$ & $20.0 \%$ & $38.3 \%$ & $>18.97$ & $>14.40$ & flash & \\
\hline & 1.04 & $26.0 \%$ & $20.0 \%$ & & $>18.97$ & $>14.96$ & & \\
\hline \multirow{3}{*}{$\begin{array}{l}D A / M Y / D A \\
B 1\end{array}$} & 0.49 & $24.5 \%$ & $46.0 \%$ & & $\geq 15.27$ & $>15.86$ & & \multirow[t]{3}{*}{$\mathrm{N} / \mathrm{C}$} \\
\hline & 0.44 & $22.0 \%$ & $46.0 \%$ & $-46.7 \%$ & $\geq 15.27$ & $>15.94$ & flash & \\
\hline & 0.54 & $27.0 \%$ & $46.0 \%$ & & $>15.27$ & $>17.29$ & & \\
\hline \multirow{3}{*}{$\begin{array}{l}\text { MYLARMO } \\
\text { C1 }\end{array}$} & 2.76 & $138.0 \%$ & $131.0 \%$ & & $>14.91$ & $>12.86$ & & \multirow[t]{3}{*}{$\| N / C$} \\
\hline & 2.88 & $144.0 \%$ & $131.0 \%$ & $.7 .4 \%$ & $>14.91$ & $>14.94$ & flash & \\
\hline & 1.64 & $82.0 \%$ & $131.0 \%$ & & $>14.91$ & $>13.62$ & & \\
\hline \multirow{3}{*}{$\begin{array}{l}\text { NO } 410 \\
\text { D1 }\end{array}$} & 0.52 & $13.0 \%$ & $17.0 \%$ & & 10.67 & 10.01 & & \multirow[t]{3}{*}{$\sqrt{N / C}$} \\
\hline & 0.49 & $12.3 \%$ & $17.0 \%$ & $-19.6 \%$ & 10.67 & 11.94 & $6.0 \%$ & \\
\hline & 0.63 & $15.8 \%$ & $17.0 \%$ & & 10.67 & 11.99 & & \\
\hline \multirow{3}{*}{$\begin{array}{l}\text { NO MI } 418 \\
\text { E1 }\end{array}$} & 0.09 & $2.3 \%$ & $4.0 \%$ & & 10.23 & 9.24 & & \multirow[t]{3}{*}{$N / C$} \\
\hline & 0.09 & $2.3 \%$ & $4.0 \%$ & $-43.8 \%$ & 10.23 & 10.61 & $-2.7 \%$ & \\
\hline & 0.09 & $2.3 \%$ & $4.0 \%$ & & 10.23 & 10.00 & & \\
\hline \multirow{4}{*}{$\begin{array}{l}\text { MEL } 228 \\
\text { F1 }\end{array}$} & 3.09 & $154.5 \%$ & $160.0 \%$ & & $>14.22$ & $>14.24$ & & \multirow[t]{3}{*}{$\sqrt{\mathrm{N} / \mathrm{C}}$} \\
\hline & 3.33 & $166.5 \%$ & $160.0 \%$ & $-5.1 \%$ & $\geq 14.22$ & $>13.78$ & flash & \\
\hline & 2.69 & $134.5 \%$ & $160.0 \%$ & & $>14.22$ & $>14.10$ & & \\
\hline & \multicolumn{7}{|c|}{ After 500 hour exposure plus 24 hour airbake at $150^{\circ} \mathrm{C}\left(302^{\circ}\right.$} & F) \\
\hline \multirow{3}{*}{$\begin{array}{l}\mathrm{NO} / \mathrm{MY} / \mathrm{NO} \\
\mathrm{A2}\end{array}$} & 1.12 & $28.0 \%$ & $20.0 \%$ & & $>18.97$ & $>13.83$ & & \multirow[t]{3}{*}{ Bubbles } \\
\hline & 1.19 & $29.8 \%$ & $20.0 \%$ & $31.3 \%$ & $>18.97$ & $>12.10$ & flash & \\
\hline & 0.84 & $21.0 \%$ & $20.0 \%$ & & $>18.97$ & $>12.11$ & & \\
\hline \multirow{3}{*}{$\begin{array}{l}\mathrm{DA} / \mathrm{MY} / \mathrm{DA} \\
\mathrm{B} 2\end{array}$} & 0.47 & $23.5 \%$ & $46.0 \%$ & & $>15.27$ & $>16.64$ & & \multirow{3}{*}{$\begin{array}{l}\text { slightly } \\
\text { warped }\end{array}$} \\
\hline & 0.47 & $23.5 \%$ & $46.0 \%$ & $-48.2 \%$ & $\geq 15.27$ & $>16.76$ & flash & \\
\hline & 0.49 & $24.5 \%$ & $46.0 \%$ & & $>15.27$ & $>15.96$ & & \\
\hline \multirow{3}{*}{$\begin{array}{l}\text { MYLAR MO } \\
\text { C2 }\end{array}$} & 2.08 & $104.0 \%$ & $131.0 \%$ & & $>14.91$ & $>13.49$ & & \multirow[t]{3}{*}{ bubbles } \\
\hline & 2.79 & $139.5 \%$ & $131.0 \%$ & $-11.2 \%$ & $\geq 14.91$ & $>13.54$ & flash & \\
\hline & 2.11 & $105.5 \%$ & $131.0 \%$ & & $>14.91$ & $>13.86$ & & \\
\hline \multirow{3}{*}{$\begin{array}{l}\text { NOMEX 410 } \\
\text { D2 }\end{array}$} & 0.46 & $11.5 \%$ & $17.0 \%$ & & 10.67 & 10.50 & & \multirow[t]{3}{*}{$\sqrt{N / C}$} \\
\hline & 0.52 & $13.0 \%$ & $17.0 \%$ & $-28.4 \%$ & 10.67 & 11.77 & $3.9 \%$ & \\
\hline & 0.48 & $12.0 \%$ & $17.0 \%$ & & 10.67 & 11.00 & & \\
\hline \multirow{3}{*}{$\begin{array}{l}\mathrm{NO} / \mathrm{MI} 418 \\
\mathrm{E} 2\end{array}$} & 0.07 & $1.8 \%$ & $4.0 \%$ & & 10.23 & 11.19 & & \multirow[t]{3}{*}{$\sqrt{N / C}$} \\
\hline & 0.09 & $2.3 \%$ & $4.0 \%$ & $.56 .3 \%$ & 10.23 & 310.10 & $2.4 \%$ & \\
\hline & 0.05 & $1.3 \%$ & $4.0 \%$ & & 10.23 & 10.13 & & \\
\hline MEL 228 & 0.57 & $28.5 \%$ & $160.0 \%$ & & $>14.22$ & $>14.73$ & & bubbles \\
\hline $\mathrm{F} 2$ & 3.81 & $190.5 \%$ & $160.0 \%$ & $-42.5 \%$ & $>14.22$ & $>14.49$ & flash & \\
\hline & 1.14 & $57.0 \%$ & $160.0 \%$ & & $>14.22$ & $>14.14$ & & \\
\hline
\end{tabular}


SLEEVING

\begin{tabular}{|c|c|c|c|c|}
\hline & \multicolumn{4}{|c|}{500 HRS IN R-134a @ 194F } \\
\hline & WT & EXPWT & $\begin{array}{c}\% \\
\text { CHANGE }\end{array}$ & EXP VIS \\
\hline $\begin{array}{l}\text { A1 } \\
\text { NOMEX }\end{array}$ & 0.5209 & 0.5496 & $5.51 \%$ & $\mathrm{~N} / \mathrm{C}$ \\
\hline \begin{tabular}{|l} 
B1 \\
MYLAR
\end{tabular} & 0.4804 & 0.4956 & $3.16 \%$ & Bubbles \\
\hline $\begin{array}{l}\text { N1 } \\
\text { NO/MY }\end{array}$ & 0.3927 & 0.4045 & $3.00 \%$ & $\mathrm{~N} / \mathrm{C}$ \\
\hline & R-134a@1 & $4 F-\ldots>24 h$ & s@302f & \\
\hline $\begin{array}{l}\text { A2 } \\
\text { NOMEX }\end{array}$ & 0.5296 & 0.5319 & $0.43 \%$ & $\mathrm{~N} / \mathrm{C}$ \\
\hline $\begin{array}{l}\text { B2 } \\
\text { MYLAR }\end{array}$ & 0.4211 & 0.4213 & $0.05 \%$ & $\begin{array}{c}\text { Delamination } \\
\text { Pockets } \\
\end{array}$ \\
\hline $\begin{array}{l}\mathrm{C} 2 \\
\mathrm{NO} / \mathrm{MY}\end{array}$ & 0.3944 & 0.3937 & $-0.18 \%$ & $\begin{array}{c}\text { Delamination } \\
\text { Pockets } \\
\end{array}$ \\
\hline
\end{tabular}




\begin{tabular}{|c|c|c|c|c|c|c|c|c|c|}
\hline & 500 HRS & IN R-134a & @ 194F & & & & & & \\
\hline$D$ & WT & EXP WT & $\begin{array}{c}\text { WT \% } \\
\text { CHANGE }\end{array}$ & $\begin{array}{l}\text { BREAK } \\
\text { LOAD } \\
\text { (AVE) }\end{array}$ & $\begin{array}{c}\text { BREAK } \\
\text { LOAD } \\
\text { EPP }\end{array}$ & $\begin{array}{c}\% \\
\text { CHANGE } \\
\text { BFK } \\
\text { LOAD }\end{array}$ & $\begin{array}{c}\text { STRETCH } \\
(\text { INCH) }\end{array}$ & $\%$ ELONG & EXP VIS \\
\hline A1 & 1.7086 & 1.7075 & $-0.06 \%$ & 39.02 & 48.05 & & 0.06 & $3.00 \%$ & $\mathrm{~N} / \mathrm{C}$ \\
\hline Glass & & & & 39.02 & 57.15 & $25.11 \%$ & 0.05 & $2.50 \%$ & \\
\hline & & & & 39.02 & 41.25 & & 0.05 & $2.50 \%$ & \\
\hline$B 1$ & 0.7054 & 0.7226 & $2.44 \%$ & 56.12 & 67.47 & & 0.72 & $36.00 \%$ & $\mathrm{~N} / \mathrm{C}$ \\
\hline Polyester & & & & 56.12 & 63.77 & $12.56 \%$ & 0.58 & $29.00 \%$ & \\
\hline & & & & 56.12 & 58.27 & & 0.55 & $27.50 \%$ & \\
\hline & 1.6459 & 1.7019 & $3.40 \%$ & 88.50 & 102.30 & & 0.08 & $4.00 \%$ & \\
\hline Permacel & & & & 88.50 & 105.90 & $27.34 \%$ & 0.08 & $4.00 \%$ & \\
\hline & & & & 88.50 & 129.90 & & 0.09 & $4.50 \%$ & \\
\hline & & $500 \mathrm{HRS}$ & in $\mathrm{R}-134 \mathrm{a}$ & $>302 \mathrm{~F}$ & 24 HRS & & & & \\
\hline$\widehat{A 2}$ & 1.6612 & 1.6517 & $.0 .57 \%$ & 39.02 & 48.80 & & 0.05 & $2.50 \%$ & $\mathrm{~N} / \mathrm{C}$ \\
\hline Glass & & & & 39.02 & 49.60 & $16.44 \%$ & 0.05 & $2.50 \%$ & \\
\hline & & & & 39.02 & 37.90 & & 0.05 & $2.50 \%$ & \\
\hline & 0.6874 & 0.6888 & $0.20 \%$ & 56.12 & 49.32 & & 0.41 & $20.50 \%$ & $\mathrm{~N} / \mathrm{C}$ \\
\hline Polyester & & & & 56.12 & 49.37 & $-8.17 \%$ & 0.42 & $21.00 \%$ & \\
\hline & & & & 56.12 & 55.92 & & 0.52 & $26.00 \%$ & \\
\hline & 1.6204 & 1.5174 & $-6.36 \%$ & 88.50 & 117.10 & & 0.08 & $4.00 \%$ & Darkened \\
\hline Permacel & & & & 88.50 & 124.30 & $39.74 \%$ & 0.10 & $5.00 \%$ & \\
\hline & & & & 88.50 & 129.60 & & 0.11 & $5.50 \%$ & \\
\hline
\end{tabular}




\begin{tabular}{|c|c|c|c|c|c|c|c|c|c|}
\hline \multicolumn{10}{|c|}{500 HRS IN R-134a @ 194 F } \\
\hline ID & WT & EXP WT & $\begin{array}{c}\text { WT \% } \\
\text { CHANGE }\end{array}$ & $\begin{array}{l}E X P \\
V I S\end{array}$ & $\begin{array}{l}\text { BREAK } \\
\text { LOAD } \\
\text { (AVE) }\end{array}$ & $\begin{array}{l}\text { BREAK } \\
\text { LOAD } \\
\text { EXP } \\
(\text { ave })\end{array}$ & $\begin{array}{c}\% \\
\text { CHANGE } \\
\text { BPK } \\
\text { LOAD }\end{array}$ & $\begin{array}{c}\text { STRETCH } \\
\text { (Inch) }\end{array}$ & $\begin{array}{c}\% \\
\text { ELONG }\end{array}$ \\
\hline \multirow[t]{4}{*}{ A1 } & \multirow[t]{3}{*}{0.3059} & \multirow[t]{3}{*}{0.3116} & \multirow[t]{3}{*}{$1.86 \%$} & $\mathrm{~N} / \mathrm{C}$ & 28.36 & 35.00 & & 0.32 & $16.0 \%$ \\
\hline & & & & & 28.36 & 29.45 & \multirow[t]{2}{*}{$13.95 \%$} & 0.28 & $14.0 \%$ \\
\hline & & & & & 28.36 & 32.50 & & 0.29 & $14.5 \%$ \\
\hline & \multicolumn{9}{|c|}{500 HRS IN R-134a $>24$ HRS @ 302F } \\
\hline \multirow[t]{3}{*}{$A 2$} & \multirow[t]{3}{*}{0.2889} & \multirow[t]{3}{*}{0.2875} & \multirow[t]{3}{*}{$-0.485 \%$} & $\mathrm{~N} / \mathrm{C}$ & 28.36 & 23.70 & & 0.37 & $18.5 \%$ \\
\hline & & & & & 28.36 & 29.77 & $0.93 \%$ & 0.5 & $25.0 \%$ \\
\hline & & & & & 28.36 & 30.82 & & 0.42 & $21.0 \%$ \\
\hline
\end{tabular}


LEAD WIRE INSULATION

\begin{tabular}{|c|c|c|c|c|c|c|c|}
\hline & 500 HRS IN F & 134a@19 & $4 \mathrm{~F}$ & & & & \\
\hline ID & WT & EXPWT & $\begin{array}{c}\text { WT \% } \\
\text { CHANGE }\end{array}$ & EXP VIS & $\begin{array}{c}\text { BASE DIE } \\
\text { (AVE) }\end{array}$ & EXP DIE & $\begin{array}{c}\text { DIE\% } \\
\text { CHANGE }\end{array}$ \\
\hline DMD & 4.0043 & 4.0190 & $0.37 \%$ & $\mathrm{~N} / \mathrm{C}$ & 9.61 & 9.91 & \\
\hline A1 & & & & & 9.61 & 9.82 & $1.63 \%$ \\
\hline & & & & & 9.61 & 9.57 & \\
\hline DTMD & 4.3321 & 4.3455 & $0.31 \%$ & $\mathrm{~N} / \mathrm{C}$ & 9.95 & 7.64 & \\
\hline$B 1$ & & & & & 9.95 & 8.49 & $-10.02 \%$ \\
\hline & & & & & 9.95 & 10.73 & \\
\hline & R-134a@19 & $F \rightarrow 24 \mathrm{HF}$ & 3S@302 & & & & \\
\hline DMD & 3.9967 & 3.9951 & $-0.04 \%$ & See Photd & 9.61 & 8.60 & \\
\hline$A 2$ & & & & & 9.61 & 8.61 & $-9.43 \%$ \\
\hline & & & & & 9.61 & 8.90 & \\
\hline DTMD & 4.3818 & 4.3766 & $-0.12 \%$ & See Phot & 9.95 & 10.58 & \\
\hline $\mathrm{B2}$ & & & & & 9.95 & 11.29 & $8.54 \%$ \\
\hline & & & & & 9.95 & 10.53 & \\
\hline
\end{tabular}




\section{Appendix I}

Experimental Data for HFC-134 Exposure at $90^{\circ} \mathrm{C}\left(194^{\circ} \mathrm{F}\right)$ 


\begin{tabular}{|c|c|c|c|c|c|c|c|c|c|c|}
\hline & 500 HRS IN & R-134@1 & $94 \mathrm{~F}$ & & & & & & & \\
\hline ID & WT & EXPWT & $\begin{array}{c}\text { WT \% } \\
\text { CHANGE }\end{array}$ & EXP VIS & $\begin{array}{c}\text { BASE BRN } \\
\text { OUT } \\
\text { (AVE) }\end{array}$ & $\begin{array}{c}\text { EXP BRN } \\
\text { OUT }\end{array}$ & $\begin{array}{c}\text { BRN OUTT } \\
\% \\
\text { CHANGE }\end{array}$ & $\begin{array}{c}\text { BASE DIE } \\
\text { (AVE) }\end{array}$ & EXP DIE & $\begin{array}{c}\text { DIE \% } \\
\text { CHANGE }\end{array}$ \\
\hline A 1 & 25.52 & 25.5571 & $0.145 \%$ & $\mathrm{~N} / \mathrm{C}$ & 576 & 433 & & 15.80 & 12.57 & \\
\hline & & & & & 576 & 461 & $-23.1 \%$ & 15.80 & 12.76 & $-27.0 \%$ \\
\hline & & & & & 576 & 434 & & 15.80 & 9.26 & \\
\hline B1 & 24.1469 & 24.1747 & $0.115 \%$ & $\mathrm{~N} / \mathrm{C}$ & 736 & 732 & & 11.62 & 12.30 & \\
\hline & & & & & 736 & 732 & $-1.7 \%$ & 11.62 & 13.15 & $7.0 \%$ \\
\hline & & & & & 736 & 707 & & 11.62 & 11.86 & \\
\hline $\mathrm{C} 1$ & 24.3298 & 24.3586 & $0.118 \%$ & $\mathrm{~N} / \mathrm{C}$ & 579 & 400 & & 16.58 & 16.85 & \\
\hline & & & & & 579 & 405 & $-30.1 \%$ & 16.58 & 18.73 & $10.2 \%$ \\
\hline & & & & & 579 & 409 & & 16.58 & 19.23 & \\
\hline & $500 \mathrm{hr}$ in $\mathrm{P}$ & 134 plus & $4 \mathrm{hr}$ at 30 & & & & & & & \\
\hline A2 & 25.6523 & 25.6559 & $0.014 \%$ & $\mathrm{~N} / \mathrm{C}$ & 576 & 480 & & 15.80 & 11.70 & \\
\hline & & & & & 576 & 466 & $-19.5 \%$ & 15.80 & 10.79 & $-22.7 \%$ \\
\hline & & & & & 576 & 445 & & 15.80 & 14.14 & \\
\hline $\mathrm{B2}$ & 25.1286 & 25.1326 & $0.016 \%$ & $\mathrm{~N} / \mathrm{C}$ & 736 & 730 & & 11.62 & 12.78 & \\
\hline & & & & & 736 & 733 & $-0.6 \%$ & 11.62 & 11.55 & $3.0 \%$ \\
\hline & & & & & 736 & 732 & & 11.62 & 11.57 & \\
\hline $\mathrm{C} 2$ & 22.8864 & 22.8908 & $0.019 \%$ & $N / C$ & 579 & 571 & & 16.58 & 13.41 & \\
\hline & & & & & 579 & 559 & $-1.8 \%$ & 16.58 & 9.37 & $-30.1 \%$ \\
\hline & & & & & 579 & 576 & & 16.58 & 11.97 & \\
\hline
\end{tabular}


500 HOURS IN R-134@ 194 F

7

\begin{tabular}{|c|c|c|c|c|c|c|c|c|c|c|}
\hline VARN & $\begin{array}{l}\text { TWISTED } \\
\text { PAIR WT }\end{array}$ & $\begin{array}{l}\text { Exp Pair } \\
\text { weight }\end{array}$ & $\begin{array}{c}\text { WT \% } \\
\text { CHANGE }\end{array}$ & $\begin{array}{l}\text { EXP } \\
\text { VIS }\end{array}$ & BASE DIE & EXPDIE & $\begin{array}{c}\text { DIE \% } \\
\text { CHANGE }\end{array}$ & $\begin{array}{c}\text { BASE } \\
\text { BURN } \\
\text { OUT }\end{array}$ & $\begin{array}{c}\text { EXP } \\
\text { BURN } \\
\text { OUT }\end{array}$ & $\begin{array}{c}\text { BRNOUT } \\
\% \\
\text { CHANGE }\end{array}$ \\
\hline \multirow{3}{*}{$\begin{array}{l}\text { U-475 } \\
\text { A1 }\end{array}$} & \multirow[t]{3}{*}{22.3767} & \multirow[t]{3}{*}{22.4549} & \multirow[t]{3}{*}{$0.349 \%$} & $\mathrm{~N} / \mathrm{C}$ & 16.24 & 12.28 & & 430 & 364 & \\
\hline & & & & & 16.24 & 16.36 & $-9.42 \%$ & 430 & 428 & $-8.84 \%$ \\
\hline & & & & & 16.24 & 15.49 & & 430 & 384 & \\
\hline \multirow{3}{*}{$\begin{array}{l}\mathrm{Y}-390 \\
\mathrm{~B} 1\end{array}$} & \multirow{3}{*}{22.5770} & \multirow{3}{*}{22.6392} & \multirow[t]{3}{*}{$0.276 \%$} & $\mathrm{~N} / \mathrm{C}$ & 18.77 & 13.72 & & 510 & 532 & \\
\hline & & & & & 18.77 & 19.39 & $-5.68 \%$ & 510 & 565 & $-0.07 \%$ \\
\hline & & & & & 18.77 & 20.00 & & 510 & 432 & \\
\hline $\mathrm{T}$ ER-610 & \multirow[t]{3}{*}{25.3129} & \multirow[t]{3}{*}{25.3540} & \multirow[t]{3}{*}{$0.162 \%$} & $\mathrm{~N} / \mathrm{C}$ & 15.57 & 18.55 & & 442 & 392 & \\
\hline \multirow[t]{2}{*}{ C1 } & & & & & 15.57 & 18.95 & $14.13 \%$ & 442 & 326 & $-21.87 \%$ \\
\hline & & & & & 15.57 & 15.81 & & 442 & 318 & \\
\hline \multirow{3}{*}{$\begin{array}{l}Y-833 \\
D 1\end{array}$} & \multirow[t]{3}{*}{21.5035} & \multirow[t]{3}{*}{21.5201} & \multirow[t]{3}{*}{$0.077 \%$} & $\mathrm{~N} / \mathrm{C}$ & 12.04 & 12.31 & & 578 & 460 & \\
\hline & & & & & 12.04 & 11.38 & $-2.80 \%$ & 578 & 450 & $-16.44 \%$ \\
\hline & & & & & 12.04 & 11.42 & & 578 & 539 & \\
\hline \multirow{3}{*}{$\begin{array}{l}923 \\
E 1\end{array}$} & \multirow[t]{3}{*}{23.2030} & \multirow[t]{3}{*}{23.2606} & \multirow[t]{3}{*}{$0.248 \%$} & $\mathrm{~N} / \mathrm{C}$ & 16.76 & 20.00 & & 606 & 591 & \\
\hline & & & & & 16.76 & 11.74 & $2.90 \%$ & 606 & 562 & $-7.32 \%$ \\
\hline & & & & & 16.76 & 20.00 & & 606 & 532 & \\
\hline$M \longdiv { I S O - 8 0 0 }$ & 21.5581 & 21.5910 & $0.153 \%$ & $\mathrm{~N} / \mathrm{C}$ & 19.08 & broke & & 580 & 478 & \\
\hline $1 F_{1}$ & & & & & 19.08 & 18.77 & $1.60 \%$ & 580 & 446 & $-17.93 \%$ \\
\hline$D$ & & & & & 19.08 & 20.00 & & 580 & 504 & \\
\hline$E$ & 24 HOUR & S AT $302 \mathrm{~F}$ & & & & & & & & \\
\hline$U-475$ & 23.1981 & 23.1979 & $-0.001 \%$ & $\mathrm{~N} / \mathrm{C}$ & 16.24 & 13.10 & & 430 & 417 & \\
\hline $1 \mid A 2$ & & & & & 16.24 & 10.87 & $-25.92 \%$ & 430 & 328 & $-18.22 \%$ \\
\hline$M$ & & & & & 16.24 & 12.12 & & 430 & 310 & \\
\hline$\overline{Y-390}$ & 24.0372 & 24.0446 & $0.031 \%$ & $\mathrm{~N} / \mathrm{C}$ & 18.77 & 14.05 & & 510 & 471 & \\
\hline$D \mid B 2$ & & & & & 18.77 & 9.10 & $-38.22 \%$ & 510 & 579 & $-0.20 \%$ \\
\hline$E$ & & & & & 18.77 & 11.64 & & 510 & 477 & \\
\hline ER-610 & 22.7487 & 22.7509 & $0.010 \%$ & $\mathrm{~N} / \mathrm{C}$ & 15.57 & 13.28 & & 442 & 347 & \\
\hline $\mathrm{C} 2$ & & & & & 15.57 & 12.88 & $-21.90 \%$ & 442 & 431 & $-17.72 \%$ \\
\hline & & & & & 15.57 & 10.32 & & 442 & 313 & \\
\hline$Y-833$ & 21.3948 & 21.3904 & $-0.021 \%$ & $\mathrm{~N} / \mathrm{C}$ & 12.04 & 9.34 & & 578 & 428 & \\
\hline D2 & & & & & 12.04 & 10.10 & $-18.83 \%$ & 578 & 504 & $-15.92 \%$ \\
\hline & & & & & 12.04 & 9.88 & & 578 & 526 & \\
\hline 923 & 23.7700 & 23.7792 & $0.039 \%$ & $\mathrm{~N} / \mathrm{C}$ & 16.76 & 10.99 & & 606 & 521 & \\
\hline E2 & & & & & 16.76 & 10.50 & $-30.81 \%$ & 606 & 471 & $-20.41 \%$ \\
\hline & & & & & 16.76 & 13.30 & & 606 & 455 & \\
\hline ISO-800 & 24.7974 & 24.8003 & $0.012 \%$ & $\mathrm{~N} / \mathrm{C}$ & 19.08 & 14.43 & & 580 & 544 & \\
\hline $\mathrm{F} 2$ & & & & & 19.08 & 19.87 & $-7.93 \%$ & 580 & 567 & $-3.74 \%$ \\
\hline & & & & & 19.08 & 18.40 & & 580 & 564 & \\
\hline
\end{tabular}




\begin{tabular}{|c|c|c|c|c|c|c|c|c|c|c|c|}
\hline \multicolumn{12}{|c|}{500 HOURS IN R-134@194F } \\
\hline & VARN & $\begin{array}{l}\text { TWISTED } \\
\text { PAIR WT }\end{array}$ & $\begin{array}{c}\text { EXPT } \\
\text { PAIR WT }\end{array}$ & \begin{tabular}{c|} 
WT \% \\
CHANGE
\end{tabular} & $\begin{array}{l}\text { EXP } \\
\text { VIS }\end{array}$ & $\begin{array}{l}\text { BASE } \\
\text { DIE }\end{array}$ & $\begin{array}{l}\text { EXP } \\
\text { DIE }\end{array}$ & $\begin{array}{c}\text { DIE \% } \\
\text { CHANGE }\end{array}$ & $\begin{array}{c}\text { BASE } \\
\text { BURN } \\
\text { OUT }\end{array}$ & $\begin{array}{c}\text { EXP } \\
\text { BURN } \\
\text { OUT }\end{array}$ & $\begin{array}{c}\text { BRNOUT } \\
\% \\
\text { CHANGE }\end{array}$ \\
\hline \multirow{3}{*}{$\begin{array}{l}P \\
O \\
L\end{array}$} & \multirow{3}{*}{$\begin{array}{l}\text { U-475 } \\
\text { A } 1\end{array}$} & \multirow[t]{3}{*}{27.0522} & \multirow[t]{3}{*}{27.0610} & \multirow[t]{3}{*}{$0.03 \%$} & $\mathrm{~N} / \mathrm{C}$ & 13.32 & 12.36 & & 746 & 736 & \\
\hline & & & & & & 13.32 & 13.25 & $-4.60 \%$ & 746 & 735 & $-0.98 \%$ \\
\hline & & & & & & 13.32 & 12.51 & & 746 & 745 & \\
\hline \multirow{3}{*}{$\begin{array}{l}Y \\
E \\
S\end{array}$} & \multirow{3}{*}{$\begin{array}{l}\text { Y-390 } \\
B 1\end{array}$} & \multirow[t]{3}{*}{26.0751} & \multirow[t]{3}{*}{26.0745} & \multirow[t]{3}{*}{$0.00 \%$} & $\mathrm{~N} / \mathrm{C}$ & 12.28 & 10.89 & & 755 & 743 & \\
\hline & & & & & & 12.28 & 11.93 & $-4.02 \%$ & 755 & 736 & $-2.16 \%$ \\
\hline & & & & & & 12.28 & 12.54 & & 755 & 737 & \\
\hline \multirow{4}{*}{$\begin{array}{l}T \\
E \\
R\end{array}$} & \multirow{3}{*}{$\begin{array}{l}\text { ER-610 } \\
\text { C1 }\end{array}$} & \multirow[t]{3}{*}{26.2937} & \multirow[t]{3}{*}{26.2898} & \multirow[t]{3}{*}{$-0.01 \%$} & $\mathrm{~N} / \mathrm{C}$ & 12.73 & 13.17 & & 734 & 730 & \\
\hline & & & & & & 12.73 & 11.94 & $-5.47 \%$ & 734 & 727 & $-0.86 \%$ \\
\hline & & & & & & 12.73 & 10.99 & & 734 & 726 & \\
\hline & $Y-833$ & \multirow[t]{3}{*}{25.4006} & \multirow[t]{3}{*}{25.3880} & \multirow[t]{3}{*}{$-0.05 \%$} & $\mathrm{~N} / \mathrm{C}$ & 12.49 & 12.71 & & 734 & 734 & \\
\hline \multirow{2}{*}{$\begin{array}{l}P \\
0\end{array}$} & \multirow[t]{2}{*}{ D1 } & & & & & 12.49 & 12.83 & $1.63 \%$ & 734 & 735 & $-0.14 \%$ \\
\hline & & & & & & 12.49 & 12.54 & & 734 & 730 & \\
\hline \multirow{3}{*}{$\begin{array}{l}\mathrm{L} \\
\mathrm{Y} \\
\mathrm{A}\end{array}$} & \multirow{3}{*}{$\begin{array}{l}923 \\
E 1\end{array}$} & \multirow[t]{3}{*}{26.0053} & 26.0103 & $0.02 \%$ & $\mathrm{~N} / \mathrm{C}$ & 14.38 & 11.24 & & 742 & 732 & \\
\hline & & & & & & 14.38 & 11.27 & $-21.14 \%$ & 742 & 759 & $0.67 \%$ \\
\hline & & & & & & 14.38 & 11.51 & & 742 & 750 & \\
\hline$M$ & ISO-800 & 26.3863 & 26.3853 & $0.00 \%$ & $\mathrm{~N} / \mathrm{C}$ & 12.29 & 12.24 & & 747 & 753 & \\
\hline 1 & $\mathrm{~F} 1$ & & & & & 12.29 & 11.66 & $-5.78 \%$ & 747 & 738 & $0.04 \%$ \\
\hline$D$ & & & & & & 12.29 & 10.84 & & 747 & 751 & \\
\hline$E$ & & $R-134 \cdots$ & 24 hours & @ $302 \mathrm{~F}$ & & & & & & & \\
\hline & $U-475$ & 26.6020 & 26.6042 & $0.01 \%$ & $\mathrm{~N} / \mathrm{C}$ & 13.32 & 12.01 & & 746 & 741 & \\
\hline 1 & $A 2$ & & & & & 13.32 & 12.86 & $-5.63 \%$ & 746 & 742 & $-1.03 \%$ \\
\hline$M$ & & & & & & 13.32 & 12.84 & & 746 & 732 & \\
\hline 1 & $Y-390$ & 26.8160 & 26.8160 & $0.00 \%$ & $\mathrm{~N} / \mathrm{C}$ & 12.28 & 9.74 & & 755 & 737 & \\
\hline$D$ & $B 2$ & & & & & 12.28 & 11.86 & $-8.55 \%$ & 755 & 748 & $-1.66 \%$ \\
\hline$E$ & & & & & & 12.28 & 12.09 & & 755 & 743 & \\
\hline & ER-610 & 26.9333 & 26.9027 & $-0.11 \%$ & $\mathrm{~N} / \mathrm{C}$ & 12.73 & 11.22 & & 734 & 730 & \\
\hline$E$ & $C 2$ & & & & & \begin{tabular}{|l|}
12.73 \\
\end{tabular} & 13.10 & $-6.47 \%$ & 734 & 726 & $-0.73 \%$ \\
\hline$P$ & & & & & & 12.73 & 11.40 & & 734 & 730 & \\
\hline 0 & $Y-833$ & 25.3814 & 25.3594 & $-0.09 \%$ & $\mathrm{~N} / \mathrm{C}$ & 12.49 & 12.49 & & 734 & 727 & \\
\hline$x$ & D2 & & & & & 12.49 & 12.01 & $-2.86 \%$ & 734 & 730 & $-0.68 \%$ \\
\hline$Y$ & & & & & & 12.49 & 11.90 & & 734 & 730 & \\
\hline & $\overline{923}$ & 25.2467 & 25.2424 & $-0.02 \%$ & $\mathrm{~N} / \mathrm{C}$ & 14.38 & 12.10 & & 742 & 748 & \\
\hline$G$ & E2 & & & & & 14.38 & 10.73 & $.20 .91 \%$ & 742 & 751 & $0.81 \%$ \\
\hline$L$ & & & & & & 14.38 & 11.29 & & 742 & 753 & \\
\hline A & ISO-800 & 26.1409 & 26.1437 & $0.01 \%$ & & 12.29 & 12.08 & & 747 & 738 & \\
\hline$S$ & $\mathrm{~F} 2$ & & & & $\mathrm{~N} / \mathrm{C}$ & 12.29 & 11.01 & $-5.61 \%$ & 747 & 736 & $-1.34 \%$ \\
\hline$S$ & & & & & & 12.29 & 11.71 & & 747 & 738 & \\
\hline
\end{tabular}




\begin{tabular}{|c|c|c|c|c|c|c|c|c|c|c|c|}
\hline \multicolumn{12}{|c|}{500 HOURS IN R-134@194 F } \\
\hline & VARN & $\begin{array}{l}\text { TWISTED } \\
\text { PAIR WT }\end{array}$ & $\begin{array}{c}\text { EXPT } \\
\text { PAIR WT }\end{array}$ & $\begin{array}{c}\text { WT \% } \\
\text { CHANGE }\end{array}$ & $\begin{array}{l}\text { EXP } \\
\text { VIS }\end{array}$ & $\begin{array}{c}\text { BASE } \\
\text { DIE }\end{array}$ & $\begin{array}{l}\text { EXP } \\
\text { DIE }\end{array}$ & $\begin{array}{c}\text { DIE } \% \\
\text { CHANGE }\end{array}$ & $\begin{array}{l}\text { BASE } \\
\text { BUPN } \\
\text { OUT }\end{array}$ & \begin{tabular}{|c|} 
EXP \\
BUPN \\
OUT
\end{tabular} & $\begin{array}{c}\text { BRNOUT } \\
\% \\
\text { CHANGE }\end{array}$ \\
\hline \multirow{4}{*}{$\begin{array}{l}P \\
0 \\
L\end{array}$} & \multirow{3}{*}{$\begin{array}{l}\text { U-475 } \\
\text { A } 1\end{array}$} & 22.9348 & 22.9409 & $0.027 \%$ & $N / C$ & 15.10 & 12.55 & & 469 & 569 & \\
\hline & & & & & & 15.10 & 11.18 & $.20 .49 \%$ & 469 & 454 & $10.66 \%$ \\
\hline & & & & & & 15.10 & 12.29 & & 469 & 534 & \\
\hline & \multirow{3}{*}{$\begin{array}{l}\mathrm{Y}-390 \\
\mathrm{~B} 1\end{array}$} & 24.1008 & 24.1626 & $0.256 \%$ & $\mathrm{~N} / \mathrm{C}$ & 18.24 & 20.00 & & 473 & 467 & \\
\hline \multirow{2}{*}{$\begin{array}{l}Y \\
E \\
S\end{array}$} & & & & & & 18.24 & 20.00 & $9.65 \%$ & 473 & 468 & $-1.90 \%$ \\
\hline & & & & & & 18.24 & 20.00 & & 473 & 457 & \\
\hline \multirow{3}{*}{\multicolumn{2}{|c|}{$\begin{array}{l}T \\
E \\
E\end{array}$}} & 21.6848 & 21.7132 & $0.131 \%$ & $\mathrm{~N} / \mathrm{C}$ & 14.53 & 11.00 & & 494 & 392 & \\
\hline & & & & & & 14.53 & 15.40 & $.0 .44 \%$ & 494 & 391 & $.21 .05 \%$ \\
\hline & & & & & & 14.53 & 17.00 & & 494 & 387 & \\
\hline \multirow{3}{*}{\multicolumn{2}{|c|}{$\begin{array}{l}Y-833 \\
D 1 \\
\end{array}$}} & 25.1992 & 25.2828 & $0.332 \%$ & $\mathrm{~N} / \mathrm{C}$ & 11.38 & 17.64 & & 557 & 438 & \\
\hline & & & & & & 11.38 & 18.40 & $61.54 \%$ & 557 & 397 & $.26 .45 \%$ \\
\hline & & & & & & 11.38 & 19.11 & & 557 & 394 & \\
\hline \multirow{4}{*}{$\begin{array}{l}\text { D } \\
E\end{array}$} & \multirow{3}{*}{$\begin{array}{l}923 \\
E 1\end{array}$} & 23.3072 & 23.3705 & $0.272 \%$ & $\mathrm{~N} / \mathrm{C}$ & 15.85 & 11.96 & & 503 & 589 & \\
\hline & & & & & & 15.85 & 15.71 & $-17.67 \%$ & 503 & 445 & $-3.78 \%$ \\
\hline & & & & & & 15.85 & 11.48 & & 503 & 418 & \\
\hline \multirow{4}{*}{\multicolumn{2}{|c|}{$\begin{array}{l}\text { ISO-800 } \\
\mathrm{F} 1\end{array}$}} & 22.1375 & 22.1650 & $0.124 \%$ & $\mathrm{~N} / \mathrm{C}$ & 14.75 & 13.50 & & 632 & 551 & \\
\hline & & & & & & 14.75 & 19.40 & $19.39 \%$ & 632 & 537 & $.13 .29 \%$ \\
\hline & & & & & & 14.75 & 19.93 & & 632 & 556 & \\
\hline $\mathrm{L}$ & & $R-134 \cdots$ & 24 hours & @ $302 F$ & & & & & & & \\
\hline \multirow{3}{*}{$\begin{array}{l}Y \\
A \\
M\end{array}$} & \multirow{3}{*}{$\begin{array}{l}\text { U-475 } \\
\text { A2 }\end{array}$} & 23.8288 & 23.8331 & $0.018 \%$ & $\mathrm{~N} / \mathrm{C}$ & 15.10 & 17.36 & & 469 & 253 & \\
\hline & & & & & & 15.10 & 18.95 & $16.56 \%$ & 469 & 291 & $-37.95 \%$ \\
\hline & & & & & & 15.10 & 16.49 & & 469 & 329 & \\
\hline 1 & \multirow{3}{*}{$\begin{array}{l}\mathrm{Y}-390 \\
B 2\end{array}$} & 24.0029 & 24.0048 & $0.008 \%$ & $\mathrm{~N} / \mathrm{C}$ & 18.24 & 15.56 & & 473 & 568 & \\
\hline$D$ & & & & & & 18.24 & 18.41 & $-11.90 \%$ & 473 & 503 & $15.15 \%$ \\
\hline E & & & & & & 18.24 & 13.73 & & 473 & 563 & \\
\hline \multirow{3}{*}{\multicolumn{2}{|c|}{$\begin{array}{l}\text { ER-610 } \\
\text { C2 }\end{array}$}} & 23.6103 & 23.6109 & $0.003 \%$ & $\mathrm{~N} / \mathrm{C}$ & 14.53 & 16.84 & & 494 & 379 & \\
\hline & & & & & & 14.53 & 13.62 & $4.08 \%$ & 494 & 353 & $-27.13 \%$ \\
\hline$M$ & & & & & & 14.53 & 14.91 & & 494 & 348 & \\
\hline \multirow{3}{*}{\multicolumn{2}{|c|}{$\begin{array}{l}Y \\
D\end{array}$}} & 23.8421 & 23.8328 & $-0.039 \%$ & $\mathrm{~N} / \mathrm{C}$ & 11.38 & 10.68 & & 557 & 428 & \\
\hline & & & & & & 11.38 & 11.08 & $-5.83 \%$ & 557 & 578 & $-5.86 \%$ \\
\hline & & & & & & 11.38 & 10.39 & & 557 & 567 & \\
\hline \multirow{3}{*}{\multicolumn{2}{|c|}{$\begin{array}{l}923 \\
E 2\end{array}$}} & 23.6598 & 23.6589 & $-0.004 \%$ & $N / C$ & 15.85 & 12.62 & & 503 & 479 & \\
\hline & & & & & & 15.85 & 12.46 & $-17.43 \%$ & 503 & 491 & $0.86 \%$ \\
\hline & & & & & & 15.85 & 14.18 & & 503 & 552 & \\
\hline \multirow{3}{*}{\multicolumn{2}{|c|}{$\begin{array}{l}\text { ISO-800 } \\
\text { F2 }\end{array}$}} & 24.0365 & 24.0368 & $0.001 \%$ & $\mathrm{~N} / \mathrm{C}$ & 14.75 & 19.79 & & 632 & 484 & \\
\hline & & & & & & 14.75 & 13.69 & $8.52 \%$ & 632 & 568 & $-18.04 \%$ \\
\hline & & & & & & 14.75 & 14.54 & & 632 & 502 & \\
\hline
\end{tabular}




\begin{tabular}{|c|c|c|c|c|c|c|c|c|}
\hline & & 500 HRS & IN R-134@ & $194^{\circ} \mathrm{F}$ & & & & \\
\hline & VARN & $\begin{array}{l}\text { COIL } \\
\text { WT }\end{array}$ & $\begin{array}{c}\text { EXP COIL } \\
W T\end{array}$ & $\begin{array}{c}\text { WT \% } \\
\text { CHANGE }\end{array}$ & EXPVIS & $\begin{array}{c}\text { BASE } \\
\text { BND STR } \\
\text { (AVE) }\end{array}$ & $\begin{array}{c}\text { EXPBND } \\
\text { STR }\end{array}$ & $\begin{array}{c}\text { BND STR } \\
\% \\
\text { CHANGE }\end{array}$ \\
\hline $\mathrm{P}$ & U-475 & 40.1112 & 40.2182 & $0.267 \%$ & $\mathrm{~N} / \mathrm{C}$ & 73.73 & 54.30 & \\
\hline 0 & A1 & & & & & 73.73 & 48.82 & $-31.01 \%$ \\
\hline$L$ & & & & & & 73.73 & 49.47 & \\
\hline$Y$ & $Y-390$ & 39.5473 & 39.6004 & $0.134 \%$ & $\mathrm{~N} / \mathrm{C}$ & 43.78 & 71.47 & \\
\hline$E$ & B1 & & & & & 43.78 & 52.70 & $28.46 \%$ \\
\hline$S$ & & & & & & 43.78 & 44.55 & \\
\hline $\mathrm{T}$ & ER-610 & 38.9483 & 39.0510 & $0.264 \%$ & $\mathrm{~N} / \mathrm{C}$ & 51.81 & 43.40 & \\
\hline$E$ & C1 & & & & & 51.81 & 48.50 & $-10.18 \%$ \\
\hline$R$ & & & & & & 51.81 & 47.70 & \\
\hline & $Y-833$ & 37.8394 & 37.8747 & $0.093 \%$ & $\mathrm{~N} / \mathrm{C}$ & 9.85 & 35.67 & \\
\hline$P$ & D1 & & & & & 9.85 & 5.85 & $66.23 \%$ \\
\hline 0 & & & & & & 9.85 & 7.60 & \\
\hline$L$ & 923 & 39.3268 & 39.3827 & $0.142 \%$ & $\mathrm{~N} / \mathrm{C}$ & 41.28 & 56.27 & \\
\hline Y & E1 & & & & & 41.28 & 43.65 & $10.78 \%$ \\
\hline A & & & & & & 41.28 & 37.27 & \\
\hline$M$ & |1SO-800 & 38.8790 & 38.9129 & $0.087 \%$ & $\mathrm{~N} / \mathrm{C}$ & 45.01 & 50.27 & \\
\hline & F1 & & & & & 45.01 & 34.55 & $-5.78 \%$ \\
\hline$D$ & & & & & & 45.01 & 35.10 & \\
\hline$E$ & & R-134 -> & 24 HRS 30 & & & & & \\
\hline & U-475 & 39.4902 & 39.4891 & $-0.003 \%$ & $N / C$ & 73.73 & 48.77 & \\
\hline I & A2 2 & & & & & 73.73 & 35.52 & $-31.05 \%$ \\
\hline$M$ & & & & & & 73.73 & 66.15 & \\
\hline I & Y & 39.7613 & 39.7723 & $0.028 \%$ & $\mathrm{~N} / \mathrm{C}$ & 43.78 & 69.35 & \\
\hline D & B2 & & & & & 43.78 & 74.52 & $45.52 \%$ \\
\hline$E$ & & & & & & 43.78 & 47.26 & \\
\hline & ER-610 & 39.7521 & 39.7531 & $0.003 \%$ & $N / C$ & 51.81 & 47.50 & \\
\hline & C2 & & & & & 51.81 & 17.70 & $-23.28 \%$ \\
\hline & & & & & & 51.81 & 54.05 & \\
\hline & $Y-833$ & 38.7982 & 38.7912 & $-0.018 \%$ & $N / C$ & 9.85 & 18.40 & \\
\hline & D2 & & & & & 9.85 & 49.90 & $201.76 \%$ \\
\hline & & & & & & 9.85 & 20.87 & \\
\hline & 923 & 39.7495 & 39.7468 & $-0.007 \%$ & $N / C$ & 41.28 & 40.90 & \\
\hline & E2 & & & & & 41.28 & 43.70 & $2.12 \%$ \\
\hline & & & & & & 41.28 & 41.87 & \\
\hline & ISO-800 & 38.2454 & 38.2462 & $0.002 \%$ & $\mathrm{~N} / \mathrm{C}$ & 45.01 & 53.42 & \\
\hline & F2 & & & & & 45.01 & 48.40 & $0.16 \%$ \\
\hline & & & & & & 45.01 & 33.42 & \\
\hline
\end{tabular}




\begin{tabular}{|c|c|c|c|c|c|c|c|c|}
\hline & & $500 \mathrm{HRS}$ & IN R-134 & $194^{\circ} \mathrm{F}$ & & & & \\
\hline & VARN & $\begin{array}{l}\text { COIL } \\
W T\end{array}$ & $\begin{array}{c}\text { EXP COIL } \\
W T\end{array}$ & $\begin{array}{c}\text { WT \% } \\
\text { CHANGE }\end{array}$ & EXP VIS & $\begin{array}{c}\text { BASE } \\
\text { BND STR } \\
\text { (AVE) }\end{array}$ & $\begin{array}{c}\text { EXPBND } \\
\text { STR }\end{array}$ & $\begin{array}{c}\text { BND STR } \\
\% \\
\text { CHANGE }\end{array}$ \\
\hline $\mathrm{P}$ & U-475 & 37.3566 & 37.4843 & $0.342 \%$ & $\mathrm{~N} / \mathrm{C}$ & 40.14 & 37.20 & \\
\hline 0 & A 1 & & & & & 40.14 & 44.97 & $5.36 \%$ \\
\hline L & & & & & & 40.14 & 44.70 & \\
\hline$Y$ & $Y-390$ & 37.5060 & 37.6283 & $0.326 \%$ & $N / C$ & 36.12 & 52.02 & \\
\hline$E$ & B1 & & & & & 36.12 & 52.27 & $40.54 \%$ \\
\hline$S$ & & & & & & 36.12 & 48.00 & \\
\hline$T$ & ER-610 & 37.4990 & 37.6254 & $0.337 \%$ & $\mathrm{~N} / \mathrm{C}$ & 35.96 & 38.95 & \\
\hline$E$ & & & & & & 35.96 & 39.47 & $2.56 \%$ \\
\hline$R$ & & & & & & 35.96 & 32.22 & \\
\hline & $Y-833$ & 36.5017 & 36.6252 & $0.338 \%$ & $\mathrm{~N} / \mathrm{C}$ & 33.14 & 29.75 & \\
\hline$P$ & D1 & & & & & 33.14 & 35.37 & $-19.49 \%$ \\
\hline 0 & & & & & & 33.14 & 14.92 & \\
\hline$L$ & 923 & 38.3531 & 38.4203 & $0.175 \%$ & $\mathrm{~N} / \mathrm{C}$ & 40.52 & 39.02 & \\
\hline$Y$ & E1 & & & & & 40.52 & 38.65 & $2.04 \%$ \\
\hline A & & & & & & 40.52 & 46.37 & \\
\hline$M$ & $150-800$ & 36.3362 & 36.3712 & $0.096 \%$ & $\mathrm{~N} / \mathrm{C}$ & 20.20 & 23.40 & \\
\hline 1 & F1 & & & & & 20.20 & 24.32 & $11.91 \%$ \\
\hline$D$ & & & & & & 20.20 & 20.10 & \\
\hline$E$ & & $R-134 \rightarrow$ & 24 HRS 30 & $2^{\circ} \mathrm{F}$ & & & & \\
\hline & U-475 & 38.3979 & 38.3980 & $0.000 \%$ & $\mathrm{~N} / \mathrm{C}$ & 40.14 & 38.27 & \\
\hline 1 & $A 2$ & & & & & 40.14 & 36.65 & $-5.88 \%$ \\
\hline$M$ & & & & & & 40.14 & 38.42 & \\
\hline 1 & $Y-390$ & 36.7192 & 36.7171 & $-0.006 \%$ & $\mathrm{~N} / \mathrm{C}$ & 36.12 & 38.75 & \\
\hline$D$ & B2 & & & & & 36.12 & 40.15 & $13.48 \%$ \\
\hline$E$ & & & & & & 36.12 & 44.07 & \\
\hline & ER-610 & 37.8417 & 37.8425 & $0.002 \%$ & $\mathrm{~N} / \mathrm{C}$ & 35.96 & 30.70 & \\
\hline$E$ & C2 & & & & & 35.96 & broken & $-8.20 \%$ \\
\hline$P$ & & & & & & 35.96 & 35.32 & \\
\hline 0 & $Y-833$ & 36.5801 & 36.5821 & $0.005 \%$ & $\mathrm{~N} / \mathrm{C}$ & 33.14 & 2.05 & \\
\hline$x$ & D2 & & & & & 33.14 & 30.65 & $-47.27 \%$ \\
\hline$Y$ & & & & & & 33.14 & 19.72 & \\
\hline & 923 & 39.6921 & 39.6901 & $-0.005 \%$ & $\mathrm{~N} / \mathrm{C}$ & 40.52 & 34.30 & \\
\hline$G$ & E2 & & & & & 40.52 & 36.87 & $-11.92 \%$ \\
\hline$L$ & & & & & & 40.52 & 35.90 & \\
\hline$A$ & ISO-800 & 36.4985 & 36.4921 & $-0.018 \%$ & $\mathrm{~N} / \mathrm{C}$ & 20.20 & 24.35 & \\
\hline$S$ & F2 & & & & & 20.20 & 19.97 & $6.83 \%$ \\
\hline$S$ & & & & & & 20.20 & 20.42 & \\
\hline
\end{tabular}


HELICAL COILS/WIRE C...HFC-134@ 90 C $\left(194^{\circ} \mathrm{F}\right)$






\begin{tabular}{|c|c|c|c|c|c|c|}
\hline \multicolumn{7}{|c|}{500 HRS IN R-134@ 194으 } \\
\hline DD & & EXPWT & $\begin{array}{c}\text { WT \% } \\
\text { CHANGE }\end{array}$ & EXPVIS & EXPFLEX & \\
\hline \multirow[t]{2}{*}{$\mathrm{A} 1$} & \multirow[t]{2}{*}{5.0972} & \multirow[t]{2}{*}{5.1077} & \multirow[t]{2}{*}{$0.206 \%$} & $\mathrm{~N} / \mathrm{C}$ & & \\
\hline & & & & & YES & \\
\hline \multirow{2}{*}{$B 1$} & \multirow{2}{*}{6.077} & \multirow{2}{*}{6.095} & \multirow{2}{*}{$0.296 \%$} & $\mathrm{~N} / \mathrm{C}$ & & \\
\hline & & & & & YES & \\
\hline \multirow{3}{*}{ C1 } & \multirow{2}{*}{3.9145} & \multirow{2}{*}{3.9223} & \multirow{2}{*}{$0.199 \%$} & $\mathrm{~N} / \mathrm{C}$ & & \\
\hline & & & & & YES & \\
\hline & \multicolumn{6}{|c|}{$\mathrm{R}-134>302^{\circ} \mathrm{F}$ for $24 \mathrm{HRS}$} \\
\hline \multirow[t]{2}{*}{ A2 } & \multirow[t]{2}{*}{5.0449} & \multirow[t]{2}{*}{5.0487} & \multirow[t]{2}{*}{$0.075 \%$} & $\mathrm{~N} / \mathrm{C}$ & & \\
\hline & & & & & YES & \\
\hline \multirow{2}{*}{$\overline{B 2}$} & \multirow{2}{*}{6.0727} & \multirow{2}{*}{6.0789} & \multirow{2}{*}{$0.102 \%$} & $\mathrm{~N} / \mathrm{C}$ & & \\
\hline & & & & & YES & \\
\hline \multirow{3}{*}{$\mathrm{C} 2$} & \multirow{3}{*}{5.6072} & \multirow{3}{*}{5.6132} & \multirow[t]{3}{*}{$0.107 \%$} & $\mathrm{~N} / \mathrm{C}$ & & \\
\hline & & & & & YES & \\
\hline & & & & & & \\
\hline
\end{tabular}

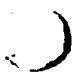




\begin{tabular}{|c|c|c|c|c|c|c|c|}
\hline & $500 \mathrm{hrs}$ & in $\mathrm{R}-134 \mathrm{a}$ & $t$ & & & & \\
\hline & VARN & WIRE WT & \begin{tabular}{|c|} 
EXPWIRE \\
$W T$
\end{tabular} & \begin{tabular}{l||} 
WT \% \\
CHANGE
\end{tabular} & $\begin{array}{l}\text { EXP } \\
\text { VIS }\end{array}$ & $\begin{array}{l}\text { EXP } \\
\text { FLEX }\end{array}$ & \\
\hline $\mathrm{P}$ & U-475 & 5.2879 & 5.3057 & $0.337 \%$ & $\mathrm{~N} / \mathrm{C}$ & & \\
\hline 이 & A 1 & & & & & NO & \\
\hline L & & & & & & & \\
\hline$Y \mid$ & $Y-390$ & 5.5591 & 5.5787 & $0.353 \%$ & $\mathrm{~N} / \mathrm{C}$ & & \\
\hline$E$ & & & & & & NO & \\
\hline$s$ & & & & & & & \\
\hline$T$ & ER-610 & 5.2970 & 5.3067 & $0.18 .3 \%$ & $\mathrm{~N} / \mathrm{C}$ & & \\
\hline $\mathrm{E}$ & & & & & & YES & \\
\hline & & & & & & & \\
\hline$|p|$ & $Y-833$ & 5.8143 & 5.8253 & $0.189 \%$ & $\mathrm{~N} / \mathrm{C}$ & YES & \\
\hline o & & & & & & & \\
\hline 니 & $\overline{923}$ & 5.5400 & 5.5627 & $0.410 \%$ & $\mathrm{~N} / \mathrm{C}$ & & \\
\hline $\mathrm{Y}$ & E1 & & & & & NO & \\
\hline A & & & & & & & \\
\hline M & $150-800$ & 5.4424 & 5.4524 & $0.184 \%$ & $\mathrm{~N} / \mathrm{C}$ & & \\
\hline 1 & & & & & & NO & \\
\hline D & & & & & & & \\
\hline$E$ & & $R-134>24$ & 4 HRS @ 30 & $02^{\circ} \mathrm{F}$ & & & \\
\hline & U-475 & 5.3553 & 5.3647 & $0.176 \%$ & $\mathrm{~N} / \mathrm{C}$ & & \\
\hline 1 & A2 & & & & & NO & \\
\hline M & & & & & & & \\
\hline 1 & $Y-390$ & 5.4998 & 5.5085 & $0.158 \%$ & $\mathrm{~N} / \mathrm{C}$ & & \\
\hline$D \mid$ & B2 & & & & & NO & \\
\hline $\mathrm{E}$ & & & & & & & \\
\hline & ER-610 & 5.3108 & 5.3137 & $0.055 \%$ & $\mathrm{~N} / \mathrm{C}$ & & \\
\hline & & & & & & YES & \\
\hline & $Y-833$ & 5.9941 & 5.9942 & $0.002 \%$ & $\mathrm{~N} / \mathrm{C}$ & & \\
\hline & D2 & & & & & YES & \\
\hline & & 54951 & 55016 & $0118 \%$ & $N / C$ & & \\
\hline & $E 2$ & | 19.4 & & & & NO & \\
\hline & & & & & & & \\
\hline & ISO-800 & 5.4805 & 5.4853 & $0.088 \%$ & $\mathrm{~N} / \mathrm{C}$ & & \\
\hline & F2 & & & & & NO & \\
\hline
\end{tabular}




\begin{tabular}{|l|l|l|l|l||l|l|}
\hline & \multicolumn{3}{|c|}{ R-134 at 194 F } & WT \\
\hline & VARN & WIRE WT & EXPWRE & $\begin{array}{c}\text { WT } \\
\text { CHANGE }\end{array}$ & EXPVIS & EXP \\
FLEX
\end{tabular}




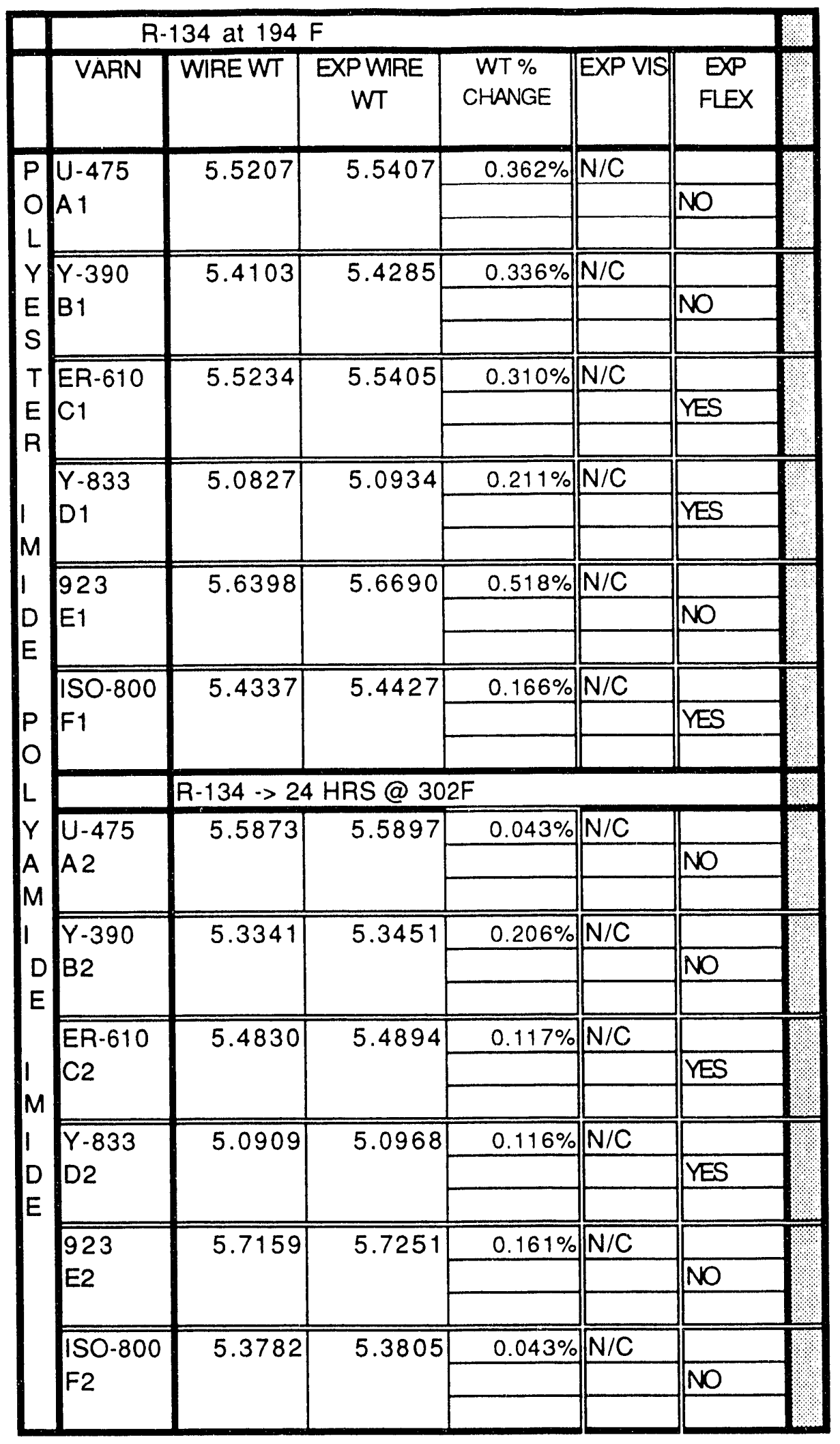




\begin{tabular}{|c|c|c|c|c|c|}
\hline & 00 HRS IN & $2-134$ & & & \\
\hline$\overline{I D}$ & $\overline{W T}$ & EXPWT & \begin{tabular}{c|} 
WT \% \\
CHANGE
\end{tabular} & EXPVIS & EXPFLEX \\
\hline U-475 & 2.1529 & 2.3152 & $7.54 \%$ & $\mathrm{~N} / \mathrm{C}$ & slightly \\
\hline A 1 & & & & & more \\
\hline & & & & & flexible \\
\hline$Y-390$ & 2.1604 & 2.2580 & $4.52 \%$ & slightly & slightly \\
\hline B1 & & & & warped & more \\
\hline & & & & & flexible \\
\hline ER-610 & 2.4726 & 2.6976 & $9.10 \%$ & darkened & slightly \\
\hline & & & & & more \\
\hline & & & & & flexible \\
\hline$Y-833$ & 2.7859 & 2.9962 & $7.55 \%$ & $\mathrm{~N} / \mathrm{C}$ & slightly \\
\hline D1 & & & & & more \\
\hline & & & & & flexible \\
\hline 923 & 1.6399 & 1.7599 & $7.32 \%$ & $\mathrm{~N} / \mathrm{C}$ & $N / C$ \\
\hline$E 1$ & & & & & \\
\hline $150-800$ & 1.8409 & 1.8202 & $-1.12 \%$ & warped & slightly \\
\hline & & & & & more \\
\hline & & & & & flexible \\
\hline & $2-134$ at - & 24 hour at & $302^{\circ} \mathrm{F}$. & & \\
\hline$U-475$ & 2.0050 & 2.0228 & $0.89 \%$ & Darkened & $N / C$ \\
\hline A2 & & & & slightly & few \\
\hline & & & & warped & pockets \\
\hline$Y-390$ & 2.2055 & 2.1807 & $-1.12 \%$ & Few & $N / C$ \\
\hline & & & & Internal & \\
\hline & & & & Bubbles & \\
\hline ER-610 & 1.9302 & 1.9588 & $1.48 \%$ & & $\mathrm{~N} / \mathrm{C}$ \\
\hline & & & & darkened & darkened \\
\hline$\overline{Y-833}$ & 2.3043 & 2.2420 & $-2.70 \%$ & Many & brittle \\
\hline D2 & & & & Internal & \\
\hline & & & & bubbles & \\
\hline 923 & 1.8422 & 1.8620 & $1.07 \%$ & many & \\
\hline E2 & & & & Internal & $N / C$ \\
\hline & & & & bubbles & \\
\hline $150-800$ & 1.3540 & 1.2483 & $-7.81 \%$ & many & \\
\hline & & & & Internal & brittle \\
\hline & & & & bubbles & warped \\
\hline
\end{tabular}




\begin{tabular}{|c|c|c|c|c|c|c|c|c|c|}
\hline & & $500 \mathrm{HR}$ & 8 & $12400^{2}$ & & & & & \\
\hline ID & WT & EXPWT & $\begin{array}{c}\text { WT \% } \\
\text { CHANGE }\end{array}$ & $\begin{array}{l}\text { SAMPLE } \\
\text { WIDTH }\end{array}$ & $\begin{array}{l}\text { BREAK } \\
\text { LOAD }\end{array}$ & $\begin{array}{c}\text { SAMPLE } \\
\text { THICKNESS } \\
\text { Mils }\end{array}$ & $\begin{array}{c}\text { TENSILE } \\
\text { STR } \\
\text { BASE }\end{array}$ & $\begin{array}{l}\text { TENSILE } \\
\text { STR EXP }\end{array}$ & $\begin{array}{c}\% \\
\text { CHANGE } \\
\text { TENSILE }\end{array}$ \\
\hline NO/MY/NO & 4.4740 & 4.7677 & $6.56 \%$ & 0.384 & 128.40 & 21 & 17.4 & 15.92 & \\
\hline$A i$ & & & & 0.448 & 152.10 & 21 & 17.4 & 16.17 & $-7.66 \%$ \\
\hline & & & & 0.475 & 160.70 & 21 & 17.4 & 16.11 & \\
\hline DA/MY/DA & 4.3937 & 4.6407 & $5.62 \%$ & 0.396 & 101.20 & 21 & 13.7 & 12.17 & \\
\hline B1 & & & & 0.398 & 105.90 & 21 & 13.7 & 12.67 & $-11.20 \%$ \\
\hline & & & & 0.482 & 118.00 & 21 & 13.7 & 11.66 & \\
\hline MYLAR MO & 2.3007 & 2.4161 & $5.02 \%$ & 0.417 & 79.25 & 10 & 21.7 & 19.00 & \\
\hline $\mathrm{C} 1$ & & & & 0.515 & 102.50 & 10 & 21.7 & 19.90 & $-13.83 \%$ \\
\hline & & & & 0.461 & 79.25 & 10 & 21.7 & 17.19 & \\
\hline NO 410 & 2.1866 & 2.2467 & $2.75 \%$ & 0.525 & 102.50 & 10 & 18.7 & 19.52 & \\
\hline D1 & & & & 0.442 & 84.70 & 10 & 18.7 & 19.16 & $3.92 \%$ \\
\hline & & & & 0.505 & 99.05 & 10 & 18.7 & 19.61 & \\
\hline NO MI 418 & 2.4751 & 2.5373 & $2.51 \%$ & 0.468 & 23.35 & 10 & 7.5 & 4.99 & \\
\hline E1 & & & & 0.495 & 25.90 & 10 & 7.5 & 5.23 & $-31.28 \%$ \\
\hline & & & & 0.478 & 25.05 & 10 & 7.5 & 5.24 & \\
\hline MEL 228 & 2.3316 & 2.4496 & $5.06 \%$ & 0.454 & 88.80 & 11 & 21.7 & 17.78 & \\
\hline$F 1$ & & & & 0.496 & 101.40 & 11 & 21.7 & 18.59 & $-18.03 \%$ \\
\hline & & & & 0.468 & 87.50 & 11 & 21.7 & 17.00 & \\
\hline & & $500 \mathrm{HRS}$ & IN R-134 & $4 @ 194^{\circ} \mathrm{F}$ & $=->24 \mathrm{HR}$ & 3S@302\% F & & & \\
\hline $\mathrm{NO} / \mathrm{MY} / \mathrm{NO}$ & 5.2862 & 5.2948 & $0.16 \%$ & 0.400 & 137.60 & 21 & 17.4 & 16.38 & \\
\hline $\mathrm{A} 2$ & & & & 0.455 & 146.40 & 21 & 17.4 & 15.32 & $-6.53 \%$ \\
\hline & & & & 0.460 & 165.10 & 21 & 17.4 & 17.09 & \\
\hline$D A / M Y / D A$ & 4.3847 & 4.3693 & $-0.35 \%$ & 0.418 & 117.40 & 21 & 13.7 & 13.37 & \\
\hline$B 2$ & & & & 0.458 & 130.70 & 21 & 13.7 & 13.59 & $-1.42 \%$ \\
\hline & & & & 0.488 & 138.90 & 21 & 13.7 & 13.55 & \\
\hline MYLAR MO & 2.2237 & 2.2229 & $-0.04 \%$ & 0.350 & 70.35 & 10 & 21.7 & 20.10 & \\
\hline $\mathrm{C} 2$ & & & & 0.433 & 85.05 & 10 & 21.7 & 19.64 & $-8.65 \%$ \\
\hline & & & & 0.406 & 80.10 & 10 & 21.7 & 19.73 & \\
\hline NOMEX 410 & 2.2360 & 2.2461 & $0.45 \%$ & 0.479 & 102.10 & 10 & 18.7 & 21.32 & \\
\hline $\mathrm{D} 2$ & & & & 0.412 & 83.70 & 10 & 18.7 & 20.32 & $11.24 \%$ \\
\hline & & & & 0.503 & 104.50 & 10 & 18.7 & 20.78 & \\
\hline NO/MI 418 & 2.4630 & 2.4665 & $0.14 \%$ & 0.485 & 27.50 & 10 & 7.5 & 5.67 & \\
\hline E2 & & & & 0.510 & 29.10 & 10 & 7.5 & 5.71 & $-24.10 \%$ \\
\hline & & & & 0.490 & 27.95 & 10 & 7.5 & 5.70 & \\
\hline MEL 228 & 2.3939 & 2.3957 & $0.08 \%$ & 0.463 & 92.25 & 10 & 21.7 & 19.92 & \\
\hline $\mathrm{F} 2$ & & & & 0.508 & 97.75 & 10 & 21.7 & 19.24 & $-10.17 \%$ \\
\hline & & & & 0.480 & 92.70 & 10 & 21.7 & 19.31 & \\
\hline
\end{tabular}




\begin{tabular}{|c|c|c|c|c|c|c|c|c|}
\hline \multicolumn{8}{|c|}{ After 500 hour exposure to R-134@194. $\mathrm{F}\left(90^{\circ} \mathrm{C}\right)$} & \multirow[b]{2}{*}{ VISUAL EXP } \\
\hline 10 & $\begin{array}{c}\text { STRETCH } \\
\text { (inch) }\end{array}$ & $\begin{array}{c}\% \\
\text { ELONG }\end{array}$ & $\begin{array}{l}\text { BASE } \\
\text { ELONG } \\
\text { (AVE) }\end{array}$ & $\begin{array}{c}\text { ELONG } \\
\% \\
\text { CHANGE }\end{array}$ & $\begin{array}{c}\text { BASE } \\
\text { DIE } \\
(A \vee E)\end{array}$ & EXPDIE & $\begin{array}{c}\text { DIE \% } \\
\text { CHANGE }\end{array}$ & \\
\hline \multirow{3}{*}{$\begin{array}{l}\text { NO/MY/NO } \\
\text { A1 }\end{array}$} & 1.16 & $29.0 \%$ & $20.0 \%$ & & $>18.97$ & $>17.17$ & & \multirow[t]{3}{*}{$\overline{N / C}$} \\
\hline & 1.08 & $27.0 \%$ & $20.0 \%$ & $37.9 \%$ & $>18.97$ & $>17.04$ & FLASH & \\
\hline & 1.07 & $26.8 \%$ & $20.0 \%$ & & $>18.97$ & $>16.18$ & & \\
\hline \multirow{3}{*}{$\begin{array}{l}\mathrm{DA} / \mathrm{MY} / \mathrm{DA} \\
\mathrm{B} 1\end{array}$} & 0.60 & $30.0 \%$ & $46.0 \%$ & & $>15.27$ & $>14.55$ & & \multirow{3}{*}{$\begin{array}{l}\text { slighlty } \\
\text { warped }\end{array}$} \\
\hline & 0.62 & $31.0 \%$ & $46.0 \%$ & $-35.9 \%$ & $>15.27$ & $>14.16$ & FLASH & \\
\hline & 0.55 & $27.5 \%$ & $46.0 \%$ & & $>15.27$ & $>14.46$ & & \\
\hline \multirow{3}{*}{$\begin{array}{l}\text { MYLARMO } \\
\text { C1 }\end{array}$} & 2.75 & $137.5 \%$ & $131.0 \%$ & & $>14.91$ & $>13.93$ & & \multirow[t]{3}{*}{$\mathrm{N} / \mathrm{C}$} \\
\hline & 3.11 & $155.5 \%$ & $131.0 \%$ & $-1.9 \%$ & $>14.91$ & $>13.61$ & FLASH & \\
\hline & 1.85 & $92.5 \%$ & $131.0 \%$ & & $>14.91$ & $>12.77$ & & \\
\hline \multirow{3}{*}{$\begin{array}{l}\text { NO } 410 \\
\text { D1 }\end{array}$} & 0.57 & $14.3 \%$ & $17.0 \%$ & & 10.67 & 12.17 & & \multirow[t]{3}{*}{$N / C$} \\
\hline & 0.61 & $15.3 \%$ & $17.0 \%$ & $-13.7 \%$ & 10.67 & 9.71 & $2.0 \%$ & \\
\hline & 0.58 & $14.5 \%$ & $17.0 \%$ & & 10.67 & 10.78 & & \\
\hline \multirow{3}{*}{$\begin{array}{l}\text { NO MI } 418 \\
\text { E1 }\end{array}$} & 0.10 & $2.5 \%$ & $4.0 \%$ & & 10.23 & 8.60 & & \multirow[t]{3}{*}{$N / C$} \\
\hline & 0.11 & $2.8 \%$ & $4.0 \%$ & $-29.2 \%$ & 10.23 & 10.66 & FLASH & \\
\hline & 0.13 & $3.3 \%$ & $4.0 \%$ & & 10.23 & 9.04 & & \\
\hline \multirow{3}{*}{$\begin{array}{l}\text { MEL } 228 \\
\text { F1 }\end{array}$} & 3.44 & $172.0 \%$ & $160.0 \%$ & & $>14.22$ & $>14.11$ & & \multirow[t]{3}{*}{$N / C$} \\
\hline & 3.71 & $185.5 \%$ & $160.0 \%$ & $7.9 \%$ & $>14.22$ & $>13.35$ & FLASH & \\
\hline & 3.21 & $160.5 \%$ & $160.0 \%$ & & $>14.22$ & $>14.27$ & & \\
\hline & \multicolumn{8}{|c|}{ After 500 hour exposure plus a 24 hour airbake @ $302^{\circ} \mathrm{F}\left(150^{\circ}\right.$} \\
\hline \multirow{3}{*}{$\begin{array}{l}\mathrm{NO} / \mathrm{MY} / \mathrm{NO} \\
\mathrm{A} 2\end{array}$} & 0.55 & $13.8 \%$ & $20.0 \%$ & & $>18.97$ & $>16.01$ & & \multirow{3}{*}{$\begin{array}{l}\text { Bubbles } \\
\text { delamination } \\
\text { pulled away }\end{array}$} \\
\hline & 0.30 & $7.5 \%$ & $20.0 \%$ & $-37.1 \%$ & $>18.97$ & $>16.53$ & FLASH & \\
\hline & 0.66 & $16.5 \%$ & $20.0 \%$ & & $>18.97$ & $>18.48$ & & \\
\hline \multirow{3}{*}{$\begin{array}{l}\mathrm{DA} / \mathrm{MY} / \mathrm{DA} \\
\mathrm{B} 2\end{array}$} & 0.52 & $26.0 \%$ & $46.0 \%$ & & $>15.27$ & $>13.04$ & & \multirow{3}{*}{$\begin{array}{l}\text { slighity } \\
\text { warped }\end{array}$} \\
\hline & 0.54 & $27.0 \%$ & $46.0 \%$ & $-40.2 \%$ & $>15.27$ & $>14.74$ & FLASH & \\
\hline & 0.59 & $29.5 \%$ & $46.0 \%$ & & $>15.27$ & $>15.93$ & & \\
\hline \multirow{3}{*}{$\begin{array}{l}\text { MYLARMO } \\
\text { C2 }\end{array}$} & 2.86 & $143.0 \%$ & $131.0 \%$ & & $>14.91$ & $>13.17$ & & \multirow[t]{3}{*}{$\mathrm{N} / \mathrm{C}$} \\
\hline & 2.62 & $131.0 \%$ & $131.0 \%$ & $6.0 \%$ & $>14.91$ & $>13.71$ & FLASH & \\
\hline & 2.85 & $142.5 \%$ & $131.0 \%$ & & $>14.91$ & $>13.23$ & & \\
\hline \multirow{3}{*}{$\begin{array}{l}\text { NOMEX 410 } \\
\text { D2 }\end{array}$} & 0.64 & $16.0 \%$ & $17.0 \%$ & & 10.67 & 10.70 & & \multirow[t]{3}{*}{$N / C$} \\
\hline & 0.53 & $13.3 \%$ & $17.0 \%$ & $-16.2 \%$ & 10.67 & 9.98 & $-1.3 \%$ & \\
\hline & 0.54 & $13.5 \%$ & $17.0 \%$ & & 10.67 & 10.90 & & \\
\hline \multirow{3}{*}{$\begin{array}{l}\text { NOIMI } 418 \\
\text { E2 }\end{array}$} & 0.07 & $1.8 \%$ & $4.0 \%$ & & 10.23 & 9.28 & & \multirow[t]{3}{*}{$\mathrm{N} / \mathrm{C}$} \\
\hline & 0.07 & $1.8 \%$ & $4.0 \%$ & $-58.3 \%$ & 10.23 & 7.88 & $-17.3 \%$ & \\
\hline & 0.06 & $1.5 \%$ & $4.0 \%$ & & 10.23 & 8.23 & & \\
\hline MEL. 228 & 3.49 & $174.5 \%$ & $160.0 \%$ & & $>14.22$ & $>14.71$ & & $\mathrm{~N} / \mathrm{C}$ \\
\hline $\mathrm{F} 2$ & 3.16 & $158.0 \%$ & $160.0 \%$ & $3.2 \%$ & $>14.22$ & $>15.21$ & FLASH & \\
\hline & 3.26 & $163.0 \%$ & $160.0 \%$ & & $>14.22$ & $>13.91$ & & \\
\hline
\end{tabular}




\begin{tabular}{|c|c|c|c|c|}
\hline & 500 HRS IN & -134@194 & & \\
\hline & WT & EXPWT & $\begin{array}{c}\% \\
\text { CHANGE }\end{array}$ & EXP VIS \\
\hline & 0.5363 & 0.5862 & $9.30 \%$ & $\mathrm{~N} / \mathrm{C}$ \\
\hline & 0.4464 & 0.4756 & $6.54 \%$ & Bubbles \\
\hline & & & & between \\
\hline & & & & layers \\
\hline C1 & 0.3923 & 0.4133 & $5.35 \%$ & $\mathrm{~N} / \mathrm{C}$ \\
\hline & R-134@19 & $\mathrm{F} \ldots . .->24 \mathrm{hr}$ & $\mathrm{s} @ 302^{\circ} \mathrm{F}$ & \\
\hline A2 & 0.5512 & 0.5531 & $0.34 \%$ & $\mathrm{~N} / \mathrm{C}$ \\
\hline & 0.4971 & 0.4974 & $0.06 \%$ & Some delamination \\
\hline MYLAR & & & & Pockets \\
\hline $\mathrm{IC2}$ & 03968 & 03082 & $038 \%$ & Pockentc whare \\
\hline NO/MY & & & & mylar pulled away \\
\hline & & & & "see photo \\
\hline
\end{tabular}




\begin{tabular}{|c|c|c|c|c|c|c|c|c|c|}
\hline & 500 HRS & IN R-134 & $@ 194 \mathrm{~F}$ & & & & & & \\
\hline D & WT & EXPWT & $\begin{array}{c}\text { WT \% } \\
\text { CHANGE }\end{array}$ & $\begin{array}{l}\text { BREAK } \\
\text { LOAD } \\
\text { (AVE) }\end{array}$ & $\begin{array}{l}\text { BREAK } \\
\text { LOAD } \\
\text { EXP }\end{array}$ & $\begin{array}{c}\% \\
\text { CHANGE } \\
\text { BRK } \\
\text { LOAD }\end{array}$ & $\begin{array}{c}\text { STRETCH } \\
(\text { INCH) }\end{array}$ & \%ELONG & EXF'VIS \\
\hline$A 1$ & 1.4875 & 1.4935 & $0.40 \%$ & 39.02 & 55.90 & & 0.06 & $3.00 \%$ & $\mathrm{~N} / \mathrm{C}$ \\
\hline Glass & & & & 39.02 & 56.60 & $36.13 \%$ & 0.05 & $2.50 \%$ & \\
\hline & & & & 39.02 & 46.85 & & 0.06 & $3.00 \%$ & \\
\hline$\overline{\mathrm{B1}}$ & 0.7018 & 0.7407 & $5.54 \%$ & 56.12 & 51.90 & & 0.69 & $34.50 \%$ & $\mathrm{~N} / \mathrm{C}$ \\
\hline Polyester & & & & 56.12 & 59.05 & $0.50 \%$ & 0.76 & $38.00 \%$ & \\
\hline & & & & 56.12 & 58.25 & & 0.48 & $24.00 \%$ & \\
\hline C1 & 1.3719 & 1.5332 & $11.76 \%$ & 88.50 & 104.40 & & 0.13 & $6.50 \%$ & Darkened \\
\hline Permacel & & & & 88.50 & 83.30 & $0.92 \%$ & 0.11 & $5.50 \%$ & \\
\hline & & & & 88.50 & 80.25 & & 0.13 & $6.50 \%$ & \\
\hline & & $500 \mathrm{HRS}$ & in R-134. & $302^{\circ} \mathrm{F}$ & $4 \mathrm{HRS}$ & & & & \\
\hline A2 & 1.4283 & 1.4308 & $0.18 \%$ & 39.02 & 50.35 & & 0.77 & $38.50 \%$ & $\mathrm{~N} / \mathrm{C}$ \\
\hline Glass & & & & 39.02 & 50.15 & $28.87 \%$ & 0.53 & $26.50 \%$ & \\
\hline & & & & 39.02 & 50.35 & & 0.57 & $28.50 \%$ & \\
\hline & 0.6780 & 0.6799 & $0.28 \%$ & 56.12 & 62.65 & & 0.05 & $2.50 \%$ & $\mathrm{~N} / \mathrm{C}$ \\
\hline Polyester & & & & 56.12 & 54.60 & $3.47 \%$ & 0.05 & $2.50 \%$ & \\
\hline & & & & 56.12 & 56.95 & & 0.06 & $3.00 \%$ & \\
\hline & 1.3053 & 1.2703 & $-2.68 \%$ & 88.50 & 120.90 & & 0.13 & $6.50 \%$ & $\mathrm{~N} / \mathrm{C}$ \\
\hline Permacel & & & & 88.50 & 81.60 & $-3.69 \%$ & 0.09 & $4.50 \%$ & \\
\hline & & & & 88.50 & 53.20 & & 0.08 & $4.00 \%$ & \\
\hline
\end{tabular}


TIE CORD----HFC-134

\begin{tabular}{|c|c|c|c|c|c|c|c|c|c|}
\hline \multicolumn{10}{|c|}{500 HRS IN R-134@194. F } \\
\hline ID & WT & EXPWT & $\begin{array}{c}\text { WT \% } \\
\text { CHANGE }\end{array}$ & $\begin{array}{l}\text { EXP } \\
\text { VIS }\end{array}$ & $\begin{array}{l}\text { BREAK } \\
\text { LOAD } \\
\text { (AVE) }\end{array}$ & $\begin{array}{c}\text { BREAK } \\
\text { LOAD } \\
\text { EXP } \\
(\operatorname{av} \theta)\end{array}$ & $\begin{array}{c}\% \\
\text { CHANGE } \\
\text { BPK } \\
\text { LOAD }\end{array}$ & $\begin{array}{c}\text { STRETCH } \\
\text { (Inch) }\end{array}$ & $\begin{array}{c}\% \\
\text { ELONG }\end{array}$ \\
\hline \multirow[t]{3}{*}{$A 1}$. & \multirow[t]{3}{*}{0.2273} & \multirow[t]{3}{*}{0.2381} & \multirow[t]{3}{*}{$4.75 \%$} & $\mathrm{~N} / \mathrm{C}$ & 28.36 & 28.50 & & 0.43 & $21.5 \%$ \\
\hline & & & & & 28.36 & 33.00 & \multirow[t]{2}{*}{$8.25 \%$} & 0.42 & $21.0 \%$ \\
\hline & & & & & 28.36 & 30.60 & & 0.37 & $18.5 \%$ \\
\hline & \multicolumn{9}{|c|}{500 HRS IN R-134 -> 24 HRS @ $302^{\circ} \mathrm{F}$} \\
\hline \multirow[t]{3}{*}{ A2 } & \multirow[t]{3}{*}{$0.262 \%$} & \multirow[t]{3}{*}{0.2632} & \multirow[t]{3}{*}{$0.381 \%$} & $\mathrm{~N} / \mathrm{C}$ & 28.36 & 28.70 & & 0.36 & $18.0 \%$ \\
\hline & & & & & 28.36 & 29.95 & \multirow[t]{2}{*}{$4.84 \%$} & 0.40 & $20.0 \%$ \\
\hline & & & & & 28.36 & 30.55 & & 0.39 & $19.5 \%$ \\
\hline
\end{tabular}




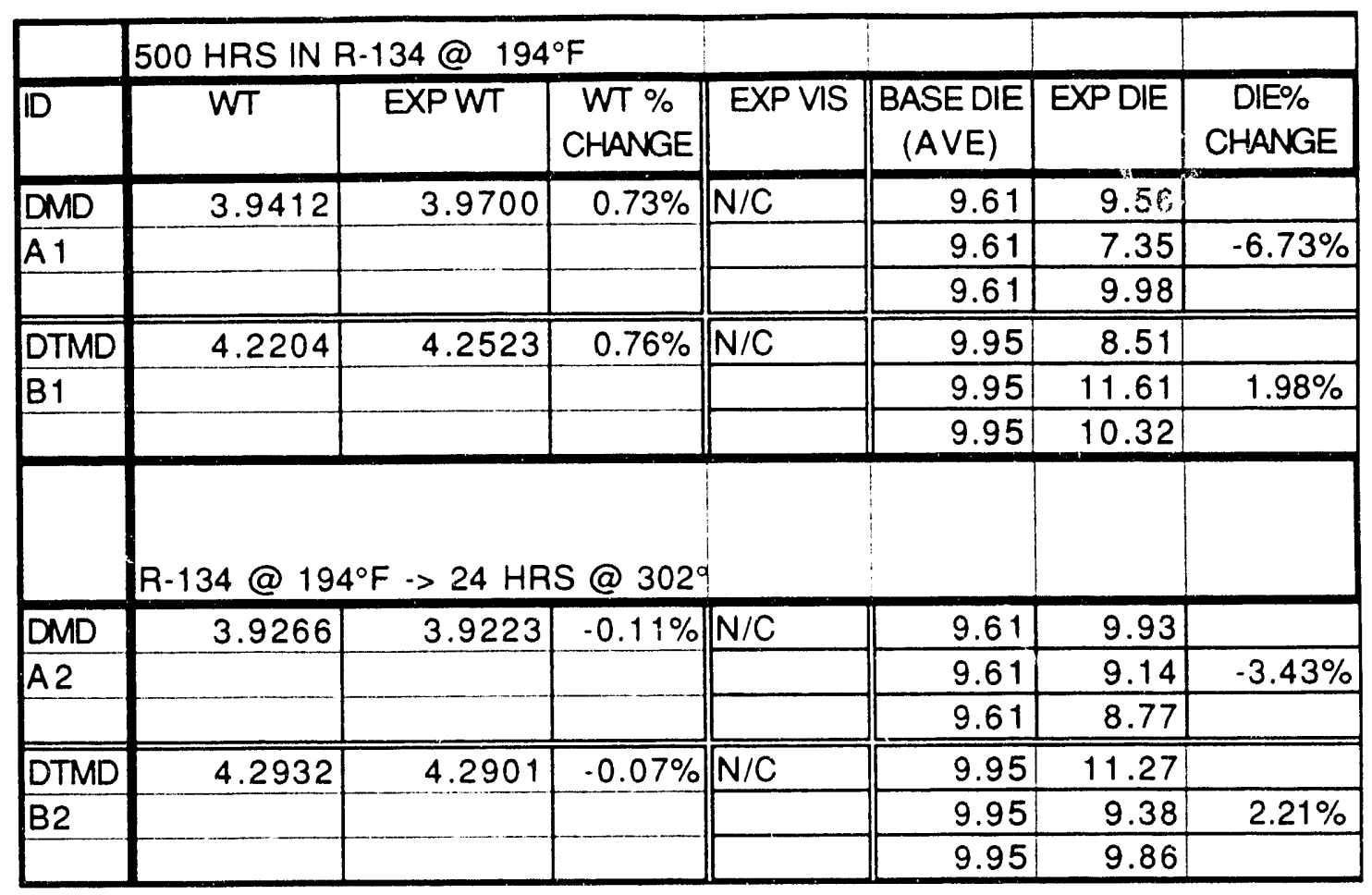




\section{Appendix $\mathbf{J}$}

Experimental Data for HFC-125 Exposure at $60^{\circ} \mathrm{C}\left(140^{\circ} \mathrm{F}\right)$ 


\begin{tabular}{|c|c|c|c|c|c|c|c|c|c|c|}
\hline & 500 HRS IN & R-125@1 & $40 \mathrm{~F}$ & & & & & & & \\
\hline ID & WT & EXPWT & $\begin{array}{c}\text { WT \% } \\
\text { CHANGE }\end{array}$ & EXP VIS & $\begin{array}{c}\text { BASE BRN } \\
\text { OUT } \\
(A \vee E)\end{array}$ & $\begin{array}{c}\text { EXP BRN } \\
\text { OUT }\end{array}$ & $\begin{array}{c}\text { BRN OUTT } \\
\% \\
\text { CHANGE }\end{array}$ & $\begin{array}{c}\text { BASE DIE } \\
\text { (AVE) }\end{array}$ & EXPDIE & $\begin{array}{c}\text { DIE \% } \\
\text { CHANGE }\end{array}$ \\
\hline A 1 & 24.6950 & 24.6671 & $-0.113 \%$ & $\mathrm{~N} / \mathrm{C}$ & 576 & 536 & & 15.80 & 17.54 & \\
\hline & & & & & 576 & 506 & $-11.2 \%$ & 15.80 & 14.31 & $0.0 \%$ \\
\hline & & & & & 576 & 493 & & 15.80 & 15.53 & \\
\hline B1 & 26.8289 & 26.8308 & $0.007 \%$ & $\mathrm{~N} / \mathrm{C}$ & 736 & 693 & & 11.62 & 12.40 & \\
\hline & & & & & 736 & 727 & $-5.4 \%$ & 11.62 & 10.17 & $0.1 \%$ \\
\hline & & & & & 736 & 668 & & 11.62 & 12.31 & \\
\hline C1 & 25.0019 & 25.0042 & $0.009 \%$ & $\mathrm{~N} / \mathrm{C}$ & 579 & 573 & & 16.58 & 16.70 & \\
\hline & & & & & 579 & 587 & $0.4 \%$ & 16.58 & 16.63 & $4.3 \%$ \\
\hline & & & & & 579 & 584 & & 16.58 & 18.53 & \\
\hline & $R-125 \cdots>24$ & HRS@30 & $2 \mathrm{~F}$ & & & & & & & \\
\hline A2 & 24.1706 & 24.1727 & $0.009 \%$ & $\mathrm{~N} / \mathrm{C}$ & 576 & 576 & & 15.80 & 20.00 & \\
\hline & & & & & 576 & 552 & $-2.4 \%$ & 15.80 & 20.00 & $13.2 \%$ \\
\hline & & & & & 576 & 558 & & 15.80 & 13.66 & \\
\hline $\mathrm{B2}$ & 27.0670 & 27.0673 & $0.001 \%$ & $\mathrm{~N} / \mathrm{C}$ & 736 & 652 & & 11.62 & 12.27 & \\
\hline & & & & & 736 & 729 & $-4.4 \%$ & 11.62 & 11.65 & $2.0 \%$ \\
\hline & & & & & 736 & 730 & & 11.62 & 11.65 & \\
\hline $\mathrm{C} 2$ & 24.5087 & 24.5103 & $0.007 \%$ & $\mathrm{~N} / \mathrm{C}$ & 579 & 499 & & 16.58 & 16.70 & \\
\hline & & & & & 579 & 576 & $-5.0 \%$ & 16.58 & 13.42 & $-8.4 \%$ \\
\hline & & & & & 579 & 576 & & 16.58 & 15.42 & \\
\hline
\end{tabular}




\begin{tabular}{|c|c|c|c|c|c|c|c|c|c|c|c|}
\hline \multicolumn{12}{|c|}{500 HOURS IN R-1.5 @140F } \\
\hline & VARN & $\begin{array}{l}\text { TWISTED } \\
\text { PAIR WT }\end{array}$ & $\begin{array}{c}\text { EXP T } \\
\text { PAIR WT }\end{array}$ & $\begin{array}{c}\text { WT \% } \\
\text { CHANGE }\end{array}$ & $\begin{array}{l}\text { EXP } \\
\text { VIS }\end{array}$ & BASE DIE & EXP DIE & $\begin{array}{c}\text { DIE \% } \\
\text { CHANGE }\end{array}$ & $\begin{array}{c}\text { BASE } \\
\text { BURN } \\
\text { OUT }\end{array}$ & $\begin{array}{l}\text { EXP } \\
\text { BURN } \\
\text { OUT }\end{array}$ & $\begin{array}{c}\text { BRNOUT } \\
\% \\
\text { CHANGE }\end{array}$ \\
\hline \multirow{3}{*}{$\begin{array}{l}P \\
O \\
L \\
V\end{array}$} & \multirow{3}{*}{$\begin{array}{l}U-475 \\
\text { A } 1\end{array}$} & \multirow[t]{3}{*}{23.9222} & \multirow[t]{3}{*}{23.9170} & \multirow[t]{3}{*}{$-0.022 \%$} & $\mathrm{~N} / \mathrm{C}$ & 16.24 & 9.24 & & 430 & 269 & \\
\hline & & & & & & 16.24 & 12.17 & $-24.86 \%$ & 430 & 379 & $-21.09 \%$ \\
\hline & & & & & & 16.24 & 15.20 & & 430 & 370 & \\
\hline \multirow{3}{*}{$\begin{array}{l}Y \\
E \\
S\end{array}$} & \multirow{3}{*}{$\begin{array}{l}Y-390 \\
B 1\end{array}$} & \multirow[t]{3}{*}{21.8923} & \multirow[t]{3}{*}{21.9054} & \multirow[t]{3}{*}{$0.060 \%$} & $\mathrm{~N} / \mathrm{C}$ & 18.77 & 13.09 & & 510 & 402 & \\
\hline & & & & & & 18.77 & 11.51 & $-21.65 \%$ & 510 & 398 & $-18.82 \%$ \\
\hline & & & & & & 18.77 & 19.52 & & 510 & 442 & \\
\hline \multirow{4}{*}{$\begin{array}{l}T \\
E \\
R\end{array}$} & \multirow{3}{*}{$\begin{array}{l}\text { ER-610 } \\
\text { C1 }\end{array}$} & \multirow[t]{3}{*}{23.5401} & \multirow[t]{3}{*}{23.5332} & \multirow[t]{3}{*}{$-0.029 \%$} & $\mathrm{~N} / \mathrm{C}$ & 15.57 & 14.69 & & 442 & 399 & \\
\hline & & & & & & 15.57 & 17.08 & $10.83 \%$ & 442 & 405 & $-9.35 \%$ \\
\hline & & & & & & 15.57 & 20.00 & & 442 & 398 & \\
\hline & \multirow{3}{*}{$\begin{array}{l}Y-833 \\
D 1\end{array}$} & \multirow[t]{3}{*}{24.4172} & \multirow[t]{3}{*}{24.4103} & \multirow[t]{3}{*}{$-0.028 \%$} & $\mathrm{~N} / \mathrm{C}$ & 12.04 & 12.01 & & 578 & 570 & \\
\hline \multirow{2}{*}{$\begin{array}{l}P \\
0\end{array}$} & & & & & & 12.04 & 10.15 & $-4.43 \%$ & 578 & 546 & $-3.11 \%$ \\
\hline & & & & & & 12.04 & 12.36 & & 578 & 564 & \\
\hline \multirow{3}{*}{$\begin{array}{l}L \\
Y \\
A\end{array}$} & \multirow{3}{*}{$\begin{array}{l}923 \\
E 1\end{array}$} & \multirow[t]{3}{*}{22.1638} & 22.1559 & $-0.036 \%$ & $\mathrm{~N} / \mathrm{C}$ & 16.76 & 10.76 & & 606 & 403 & \\
\hline & & & & & & 16.76 & 12.68 & $-29.22 \%$ & 606 & 406 & $-30.64 \%$ \\
\hline & & & & & & 16.76 & 12.15 & & 606 & 452 & \\
\hline$M$ & $150-800$ & 21.7035 & 21.6579 & $-0.210 \%$ & $\mathrm{~N} / \mathrm{C}$ & 19.08 & 18.60 & & 580 & 545 & \\
\hline 1 & $\mathrm{~F} 1$ & & & & & 19.08 & 19.72 & $-2.69 \%$ & 580 & 493 & $-8.16 \%$ \\
\hline$D$ & & & & & & 19.08 & 17.38 & & 580 & 560 & \\
\hline$E$ & & 24 HOURS & S AT $302 \mathrm{~F}$ & & & & & & & & \\
\hline & U.475 & 24.1100 & 24.1036 & $-0.027 \%$ & $\mathrm{~N} / \mathrm{C}$ & 16.24 & 14.58 & & 430 & 436 & \\
\hline 1 & A2 & & & & & 16.24 & 15.18 & $-7.29 \%$ & 430 & 346 & $-15.97 \%$ \\
\hline$M$ & & & & & & 16.24 & 15.41 & & 430 & 302 & \\
\hline 1 & $\overline{Y-390}$ & 22.0861 & 22.0791 & $.0 .032 \%$ & $\mathrm{~N} / \mathrm{C}$ & 18.77 & 13.62 & & 510 & 422 & \\
\hline$D$ & B2 & & & & & 18.77 & 20.00 & $-21.35 \%$ & 510 & 469 & $-4.44 \%$ \\
\hline$E$ & & & & & & 18.77 & 10.67 & & 510 & 571 & \\
\hline & ER-610 & 23.4269 & 23.4270 & $0.000 \%$ & $\mathrm{~N} / \mathrm{C}$ & 15.57 & 14.33 & & 442 & 413 & \\
\hline & $\mathrm{C} 2$ & & & & & 15.57 & 11.91 & $-18.28 \%$ & 442 & 33 & $-6.03 \%$ \\
\hline & & & & & & 15.57 & 11.93 & & 442 & 400 & \\
\hline & $\overline{Y-833}$ & 24.0632 & 24.0619 & $-0.005 \%$ & $\mathrm{~N} / \mathrm{C}$ & 12.04 & 11.97 & & 578 & 441 & \\
\hline & D2 & & & & & 12.04 & 10.96 & $-3.43 \%$ & 578 & 546 & $-10.61 \%$ \\
\hline & & & & & & 12.04 & 11.95 & & 578 & 563 & \\
\hline & 923 & 26.2051 & 26.1994 & $-0.022 \%$ & $\mathrm{~N} / \mathrm{C}$ & 6.76 & 17.67 & & 606 & 441 & \\
\hline & E2 & & & & & 16.76 & 20.00 & $7.52 \%$ & 606 & 430 & $.29 .59 \%$ \\
\hline & & & & & & 16.76 & 16.39 & & 606 & 409 & \\
\hline & $150-800$ & 21.7807 & 21.7782 & $.0 .011 \%$ & $\mathrm{~N} / \mathrm{C}$ & 19.08 & 19.74 & & 580 & 461 & \\
\hline & $\mathrm{F} 2$ & & & & & 19.08 & 17.79 & $-2.32 \%$ & 580 & 583 & $-8.51 \%$ \\
\hline & & & & & & 13.08 & 18.38 & & 580 & 548 & \\
\hline
\end{tabular}




\begin{tabular}{|c|c|c|c|c|c|c|c|c|c|c|c|}
\hline \multicolumn{12}{|c|}{500 HOURS IN R-125@140F } \\
\hline & VARN & $\begin{array}{l}\text { TWISTED } \\
\text { PAIR WT }\end{array}$ & $\begin{array}{c}\text { EXPT } \\
\text { PAIR WT }\end{array}$ & $\begin{array}{c}\text { WT \% } \\
\text { CHANGE }\end{array}$ & $\begin{array}{l}\text { EXP } \\
\text { VIS }\end{array}$ & $\begin{array}{c}\text { BASE } \\
\text { DIE }\end{array}$ & $\begin{array}{l}\text { EXP } \\
\text { DIE }\end{array}$ & $\begin{array}{c}\text { DIE \% } \\
\text { CHANGE }\end{array}$ & \begin{tabular}{|c|} 
BASE \\
BURN \\
OUT \\
\end{tabular} & \begin{tabular}{|c|} 
EXP \\
BUPN \\
OUT
\end{tabular} & $\begin{array}{c}\text { BRNOUT } \\
\% \\
\text { CHANGE }\end{array}$ \\
\hline \multirow{4}{*}{$\begin{array}{l}P \\
O \\
L \\
Y\end{array}$} & \multirow{3}{*}{$\begin{array}{l}\text { U-475 } \\
\text { A } 1\end{array}$} & 26.6872 & 26.6797 & $-0.03 \%$ & $\mathrm{~N} / \mathrm{C}$ & 13.32 & 13.45 & & 746 & 707 & \\
\hline & & & & & & 13.32 & 14.39 & $1.20 \%$ & 746 & 708 & $-5.41 \%$ \\
\hline & & & & & & 13.32 & 12.60 & & 746 & 702 & \\
\hline \multirow{3}{*}{\multicolumn{2}{|c|}{$\begin{array}{r}Y-390 \\
B 1\end{array}$}} & 27.2615 & 27.2601 & $-0.01 \%$ & $\mathrm{~N} / \mathrm{C}$ & 12.28 & 12.92 & & 755 & 741 & \\
\hline & & & & & & 12.28 & 11.96 & $2.17 \%$ & 755 & 738 & $-1.99 \%$ \\
\hline$S$ & & & & & & 12.28 & 12.76 & & 755 & 741 & \\
\hline \multirow{4}{*}{$\begin{array}{l}T \\
E \\
R\end{array}$} & \multirow{3}{*}{$\begin{array}{l}\text { ER-610 } \\
\text { C1 }\end{array}$} & 26.7332 & 26.7288 & $-0.02 \%$ & $\mathrm{~N} / \mathrm{C}$ & 12.73 & 13.40 & & 734 & 729 & \\
\hline & & & & & & 12.73 & 14.03 & $7.02 \%$ & 734 & 730 & $-4.59 \%$ \\
\hline & & & & & & 12.73 & 13.44 & & 734 & 642 & \\
\hline & \multirow{3}{*}{$\begin{array}{l}Y-833 \\
D 1\end{array}$} & 29.7619 & 29.7548 & $-0.02 \%$ & $\mathrm{~N} / \mathrm{C}$ & 12.49 & 13.00 & & 734 & 730 & \\
\hline \multirow{2}{*}{$\begin{array}{l}P \\
O\end{array}$} & & & & & & 12.49 & 11.91 & $-1.01 \%$ & 734 & 728 & $-0.68 \%$ \\
\hline & & & & & & 12.49 & 12.18 & & 734 & 729 & \\
\hline \multirow{3}{*}{$\begin{array}{l}L \\
Y \\
A\end{array}$} & \multirow{3}{*}{$\begin{array}{l}923 \\
E 1\end{array}$} & 28.1158 & 28.1046 & $-0.04 \%$ & $\mathrm{~N} / \mathrm{C}$ & 14.38 & 12.36 & & 742 & 731 & \\
\hline & & & & & & 14.38 & 12.69 & $-7.88 \%$ & 742 & 735 & $-1.44 \%$ \\
\hline & & & & & & 14.38 & 14.69 & & 742 & 728 & \\
\hline \multirow{3}{*}{$\begin{array}{c}M \\
I \\
D \\
F\end{array}$} & \multirow{3}{*}{$\begin{array}{l}\text { ISO-800 } \\
\mathrm{F} 1\end{array}$} & \multirow{3}{*}{24.1429} & \multirow[t]{3}{*}{24.1358} & \multirow[t]{3}{*}{$-0.03 \%$} & $\mathrm{~N} / \mathrm{C}$ & \begin{tabular}{|l|}
12.29 \\
\end{tabular} & 13.47 & & 747 & 720 & \\
\hline & & & & & & 12.29 & 12.20 & $0.84 \%$ & 747 & 731 & $-3.12 \%$ \\
\hline & & & & & & 12.29 & 11.51 & & 747 & 720 & \\
\hline \multirow{4}{*}{ 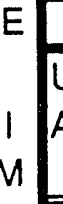 } & & \multicolumn{10}{|c|}{ R-125 $\rightarrow 24$ hours@ @ 302 F } \\
\hline & \multirow{3}{*}{$\begin{array}{l}U-475 \\
A 2\end{array}$} & 26.7216 & 26.7084 & $-0.05 \%$ & $N / C$ & 13.32 & 13.25 & & 746 & 698 & \\
\hline & & & & & & 13.32 & 12.56 & $-8.68 \%$ & 746 & 729 & $-3.71 \%$ \\
\hline & & & & & & 13.32 & 10.68 & & 746 & 728 & \\
\hline \multirow{3}{*}{$\begin{array}{l}\text { I } \\
D \\
E\end{array}$} & \multirow{3}{*}{$\begin{array}{l}Y-390 \\
B 2\end{array}$} & 27.4061 & 27.3552 & $-0.19 \%$ & $\mathrm{~N} / \mathrm{C}$ & 12.28 & 11.47 & & 755 & 743 & \\
\hline & & & & & & 12.28 & 12.72 & $2.50 \%$ & 755 & 747 & $-1.32 \%$ \\
\hline & & & & & & 12.28 & 13.57 & & 755 & 747 & \\
\hline & \multirow{3}{*}{$\begin{array}{l}\text { ER-610 } \\
\text { C2 }\end{array}$} & 26.1216 & 26.1115 & $-0.04 \%$ & $\mathrm{~N} / \mathrm{C}$ & \begin{tabular}{|l|}
12.73 \\
\end{tabular} & 13.59 & & 734 & 648 & \\
\hline$E$ & & & & & & 12.73 & 12.55 & $2.78 \%$ & 734 & 659 & $-9.85 \%$ \\
\hline$P$ & & & & & & 12.73 & 13.11 & & 734 & 678 & \\
\hline \multirow{3}{*}{$\begin{array}{l}O \\
X \\
Y\end{array}$} & \multirow{3}{*}{$\begin{array}{l}Y-833 \\
D 2\end{array}$} & 25.6699 & 25.6588 & $.0 .04 \%$ & $\mathrm{~N} / \mathrm{C}$ & \begin{tabular}{|l|}
12.49 \\
\end{tabular} & 12.31 & & 734 & 728 & \\
\hline & & & & & & \begin{tabular}{|l|}
12.49 \\
\end{tabular} & 12.31 & $0.51 \%$ & 734 & 727 & $-1.95 \%$ \\
\hline & & & & & & \begin{tabular}{|l|}
12.49 \\
\end{tabular} & 13.04 & & 734 & 704 & \\
\hline & \multirow{3}{*}{$\begin{array}{l}923 \\
E 2\end{array}$} & 27.5259 & 27.5167 & $-0.03 \%$ & $\mathrm{~N} / \mathrm{C}$ & 14.38 & 12.55 & & 742 & 734 & \\
\hline$G$ & & & & & & 14.38 & 11.60 & $-8.4, \%$ & 742 & 735 & $-1.08 \%$ \\
\hline L & & & & & & 14.38 & 15.36 & & 742 & 729 & \\
\hline A & ISO-800 & 24.6862 & 24.6650 & $-0.09 \%$ & & 12.29 & 11.37 & & 747 & 733 & \\
\hline$S$ & F2 & & & & $\mathrm{N} / \mathrm{C}$ & 12.29 & 12.10 & $-0.81 \%$ & 747 & 732 & $-1.94 \%$ \\
\hline$S$ & & & & & & 12.29 & 13.10 & & 747 & 731 & \\
\hline
\end{tabular}




\begin{tabular}{|c|c|c|c|c|c|c|c|c|c|c|c|}
\hline \multicolumn{12}{|c|}{500 HOURS INR-125@140 F } \\
\hline & VARN & $\begin{array}{l}\text { TWISTED } \\
\text { PAIR WT }\end{array}$ & $\begin{array}{c}\text { EXP T } \\
\text { PAIR WT }\end{array}$ & $\begin{array}{c}\text { WT \% } \\
\text { CHANGE }\end{array}$ & $\begin{array}{l}\text { EXP } \\
\text { VIS }\end{array}$ & \begin{tabular}{|c|} 
BASE \\
DIE
\end{tabular} & $\begin{array}{l}\text { EXP } \\
\text { DIE }\end{array}$ & $\begin{array}{c}\text { DIE \% } \\
\text { CHANGE }\end{array}$ & $\begin{array}{l}\text { BASE } \\
\text { BURN } \\
\text { OUT }\end{array}$ & $\begin{array}{c}\text { EXP } \\
\text { BURN } \\
\text { OUT }\end{array}$ & $\begin{array}{c}\text { BRNOUT } \\
\% \\
\text { CHANGE }\end{array}$ \\
\hline \multirow{3}{*}{$\begin{array}{l}P \\
O \\
L \\
Y\end{array}$} & \multirow{3}{*}{$\begin{array}{l}U-475 \\
\text { A } 1\end{array}$} & \multirow[t]{3}{*}{24.5921} & \multirow[t]{3}{*}{24.5643} & \multirow[t]{3}{*}{$-0.113 \%$} & $\mathrm{~N} / \mathrm{C}$ & 15.10 & 8.22 & & 469 & 364 & \\
\hline & & & & & & 15.10 & broke & $-43.18 \%$ & 469 & 251 & $-36.11 \%$ \\
\hline & & & & & & 15.10 & 8.94 & & 469 & 284 & \\
\hline \multirow{3}{*}{\multicolumn{2}{|c|}{$\begin{array}{l}Y-390 \\
B 1\end{array}$}} & \multirow[t]{3}{*}{21.8227} & \multirow[t]{3}{*}{21.8228} & \multirow[t]{3}{*}{$0.000 \%$} & $\mathrm{~N} / \mathrm{C}$ & 18.24 & 20.00 & & 473 & 456 & \\
\hline & & & & & & 18.24 & 13.41 & $-2.39 \%$ & 473 & 422 & $-3.49 \%$ \\
\hline s & & & & & & 18.24 & 20.00 & & 473 & 491 & \\
\hline \multirow{4}{*}{$\begin{array}{l}T \\
E \\
R\end{array}$} & \multirow{3}{*}{$\begin{array}{l}\text { ER-610 } \\
\text { C1 }\end{array}$} & \multirow[t]{3}{*}{22.6876} & \multirow[t]{3}{*}{22.6602} & \multirow[t]{3}{*}{$-0.121 \%$} & $\mathrm{~N} / \mathrm{C}$ & 14.53 & 16.04 & & 494 & 388 & \\
\hline & & & & & & 14.53 & 15.89 & $8.63 \%$ & 494 & 370 & $-22.06 \%$ \\
\hline & & & & & & 14.53 & 15.42 & & 494 & 397 & \\
\hline \multirow{3}{*}{\multicolumn{2}{|c|}{$\begin{array}{l}Y-833 \\
D 1\end{array}$}} & \multirow[t]{3}{*}{23.9051} & \multirow[t]{3}{*}{23.8516} & \multirow[t]{3}{*}{$-0.224 \%$} & $\mathrm{~N} / \mathrm{C}$ & 11.38 & 11.51 & & 557 & 592 & \\
\hline & & & & & & 11.38 & 11.70 & $2.58 \%$ & 557 & 502 & $-0.90 \%$ \\
\hline & & & & & & 11.38 & 11.81 & & 557 & 562 & \\
\hline \multirow{4}{*}{$\begin{array}{l}I \\
D \\
E\end{array}$} & \multirow{3}{*}{$\begin{array}{l}923 \\
E 1\end{array}$} & \multirow[t]{3}{*}{23.2545} & \multirow[t]{3}{*}{23.2469} & $-0.033 \%$ & $\mathrm{~N} / \mathrm{C}$ & 15.85 & 20.00 & & 503 & 506 & \\
\hline & & & & & & 15.85 & 15.29 & $16.28 \%$ & 503 & 403 & $-5.70 \%$ \\
\hline & & & & & & 15.85 & 20.00 & & 503 & 514 & \\
\hline & $150-800$ & 22.1990 & 22.1527 & $-0.209 \%$ & $\mathrm{~N} / \mathrm{C}$ & 14.75 & 15.71 & & 632 & 501 & \\
\hline$P$ & $\mathrm{~F} 1$ & & & & & 14.75 & 13.33 & $5.88 \%$ & 632 & 599 & $-14.66 \%$ \\
\hline 0 & & & & & & 14.75 & 17.81 & & 632 & 518 & \\
\hline$L$ & & $R-125 \cdots$ & 24 hours & @ 302 F & & & & & & & \\
\hline$Y$ & U-475 & 24.4209 & 24.4132 & $-0.032 \%$ & $\mathrm{~N} / \mathrm{C}$ & 15.10 & broke & & 469 & 289 & \\
\hline A & $A 2$ & & & & & 15.10 & 20.00 & $10.79 \%$ & 469 & 258 & $-43.00 \%$ \\
\hline$M$ & & & & & & 15.10 & 13.46 & & 469 & 255 & \\
\hline 1 & $Y-390$ & 21.5328 & 21.5267 & $-0.028 \%$ & $\mathrm{~N} / \mathrm{C}$ & 18.24 & 20.00 & & 473 & 395 & \\
\hline$D$ & $B 2$ & & & & & 18.24 & 11.02 & \begin{tabular}{|l|}
$.14 .97 \%$ \\
\end{tabular} & 473 & 394 & $-17.20 \%$ \\
\hline$E$ & & & & & & 18.24 & 20.00 & & 473 & 386 & \\
\hline & ER-610 & 22.6428 & 22.6436 & $0.004 \%$ & $\mathrm{~N} / \mathrm{C}$ & 14.53 & 16.88 & & 494 & 483 & \\
\hline 1 & $\mathrm{C} 2$ & & & & & 14.53 & 18.17 & $14.77 \%$ & 494 & 391 & $-10.46 \%$ \\
\hline$M$ & & & & & & 14.53 & 14.98 & & 494 & 453 & \\
\hline 1 & $Y-833$ & 23.9028 & 23.8549 & $-0.200 \%$ & $\mathrm{~N} / \mathrm{C}$ & 11.38 & 11.96 & & 557 & 588 & \\
\hline$D$ & D2 & & & & & 11.38 & 11.10 & $3.28 \%$ & 557 & 586 & $5.80 \%$ \\
\hline$E$ & & & & & & 11.38 & 12.20 & & 557 & 587 & \\
\hline & 923 & 23.1540 & 23.1502 & $-0.016 \%$ & $\mathrm{~N} / \mathrm{C}$ & 15.85 & 20.00 & & 503 & 461 & \\
\hline & E2 & & & & & 15.85 & 20.00 & $26.18 \%$ & 503 & 428 & $-5.70 \%$ \\
\hline & & & & & & 15.85 & 20.00 & & 503 & 534 & \\
\hline & $150-800$ & 21.9142 & 21.9090 & $-0.024 \%$ & $\mathrm{~N} / \mathrm{C}$ & 14.75 & 15.77 & & 632 & 577 & \\
\hline & $\mathrm{F} 2$ & & & & & 14.75 & 12.02 & $-9.99 \%$ & 632 & 571 & $-9.34 \%$ \\
\hline & & & & & & 14.75 & 12.04 & & 632 & 571 & \\
\hline
\end{tabular}




\begin{tabular}{|c|c|c|c|c|c|c|c|c|c|}
\hline & & \multirow[b]{2}{*}{ VARN } & \multicolumn{7}{|c|}{500 HRS IN R-125@140F } \\
\hline & & & $\begin{array}{l}\text { COIL } \\
\text { WT }\end{array}$ & $\begin{array}{c}\text { EXP COIL } \\
W T\end{array}$ & $\begin{array}{c}\text { WT \% } \\
\text { CHANGE }\end{array}$ & EXP VIS & $\begin{array}{c}\text { BASE } \\
\text { BND STR } \\
\text { (AVE) }\end{array}$ & $\begin{array}{c}\text { EXPBND } \\
\text { STR }\end{array}$ & $\begin{array}{c}\text { BND STR } \\
\% \\
\text { CHANGE }\end{array}$ \\
\hline & $P$ & $U-475$ & 39.3142 & 39.3074 & $-0.017 \%$ & $\mathrm{~N} / \mathrm{C}$ & 73.73 & 56.55 & \\
\hline & 0 & A 1 & & & & & 73.73 & 49.80 & $-24.31 \%$ \\
\hline & $L$ & & & & & & 73.73 & 61.07 & \\
\hline & Y & $Y-390$ & 38.1491 & 38.1542 & $0.013 \%$ & $\mathrm{~N} / \mathrm{C}$ & 43.78 & 48.30 & \\
\hline & $E$ & B1 & & & & & 43.78 & 41.82 & $-0.85 \%$ \\
\hline & S & & & & & & 43.78 & 40.10 & \\
\hline & $T$ & ER-610 & 38.4070 & 38.3580 & $-0.128 \%$ & $\mathrm{~N} / \mathrm{C}$ & 51.81 & 51.55 & \\
\hline & $E$ & C1 & & & & & 51.81 & 49.92 & $2.84 \%$ \\
\hline & $R$ & & & & & & 51.81 & 58.37 & \\
\hline & & $Y-833$ & 37.3510 & 37.3497 & $-0.003 \%$ & $\mathrm{~N} / \mathrm{C}$ & 9.85 & 5.00 & \\
\hline & $\mathrm{P}$ & D1 & & & & & 9.85 & 5.40 & $-27.58 \%$ \\
\hline & 0 & & & & & & 9.85 & 11.00 & \\
\hline & L & 923 & 38.2370 & 38.2395 & $0.007 \%$ & $\mathrm{~N} / \mathrm{C}$ & 41.28 & 30.80 & \\
\hline & Y & E1 & & & & & 41.28 & 42.15 & $-9.95 \%$ \\
\hline & A & & & & & & 41.28 & 38.57 & \\
\hline & M & ISO-800 & 37.1214 & 37.1160 & $-0.015 \%$ & $\mathrm{~N} / \mathrm{C}$ & 45.01 & 50.05 & \\
\hline & | & F1 & & & & & 45.01 & 42.80 & $3.14 \%$ \\
\hline$i$ & $D$ & & & & & & 45.01 & 42.62 & \\
\hline & $E$ & & $R-125->$ & 24 HRS 30 & $2 F$ & & & & \\
\hline & & U-475 & 39.3577 & $39.353 C$ & $.0 .012 \%$ & $\mathrm{~N} / \mathrm{C}$ & 73.73 & 54.75 & \\
\hline & 1 & A2 & & & & & 73.73 & 75.00 & $-10.69 \%$ \\
\hline & $M$ & & & & & & 73.73 & 56.70 & \\
\hline & | & $Y-390$ & 39.5198 & 39.5019 & $-0.045 \%$ & $\mathrm{~N} / \mathrm{C}$ & 43.78 & 60.05 & \\
\hline & D & B2 & & & & & 43.78 & 78.16 & $42.04 \%$ \\
\hline & $E$ & & & & & & 43.78 & 48.35 & \\
\hline & & ER-610 & 38.4961 & 38.4704 & $-0.067 \%$ & $\mathrm{~N} / \mathrm{C}$ & 51.81 & 57.05 & \\
\hline & & C2 & & & & & 51.81 & 56.50 & $13.52 \%$ \\
\hline & & & & & & & 51.81 & 62.90 & \\
\hline & & $Y-833$ & 38.0796 & 38.0468 & $-0.086 \%$ & $\mathrm{~N} / \mathrm{C}$ & 9.85 & 11.95 & \\
\hline & & D2 & & & & & 9.85 & 5.00 & $-29.10 \%$ \\
\hline & & & & & & & 9.85 & 4.00 & \\
\hline & & 923 & 38.9747 & 38.9567 & $-0.046 \%$ & $\mathrm{~N} / \mathrm{C}$ & 41.28 & 36.20 & \\
\hline & & E2 & & & & & 41.28 & 10.20 & $-11.46 \%$ \\
\hline & & & & & & & 41.28 & 33.25 & \\
\hline & & ISO-800 & 36.9124 & 36.8551 & $-0.155 \%$ & $\mathrm{~N} / \mathrm{C}$ & 45.01 & 44.15 & \\
\hline & & F2 & & & & & 45.01 & 49.40 & $2.05 \%$ \\
\hline & & & & & & & 45.01 & 44.25 & \\
\hline
\end{tabular}




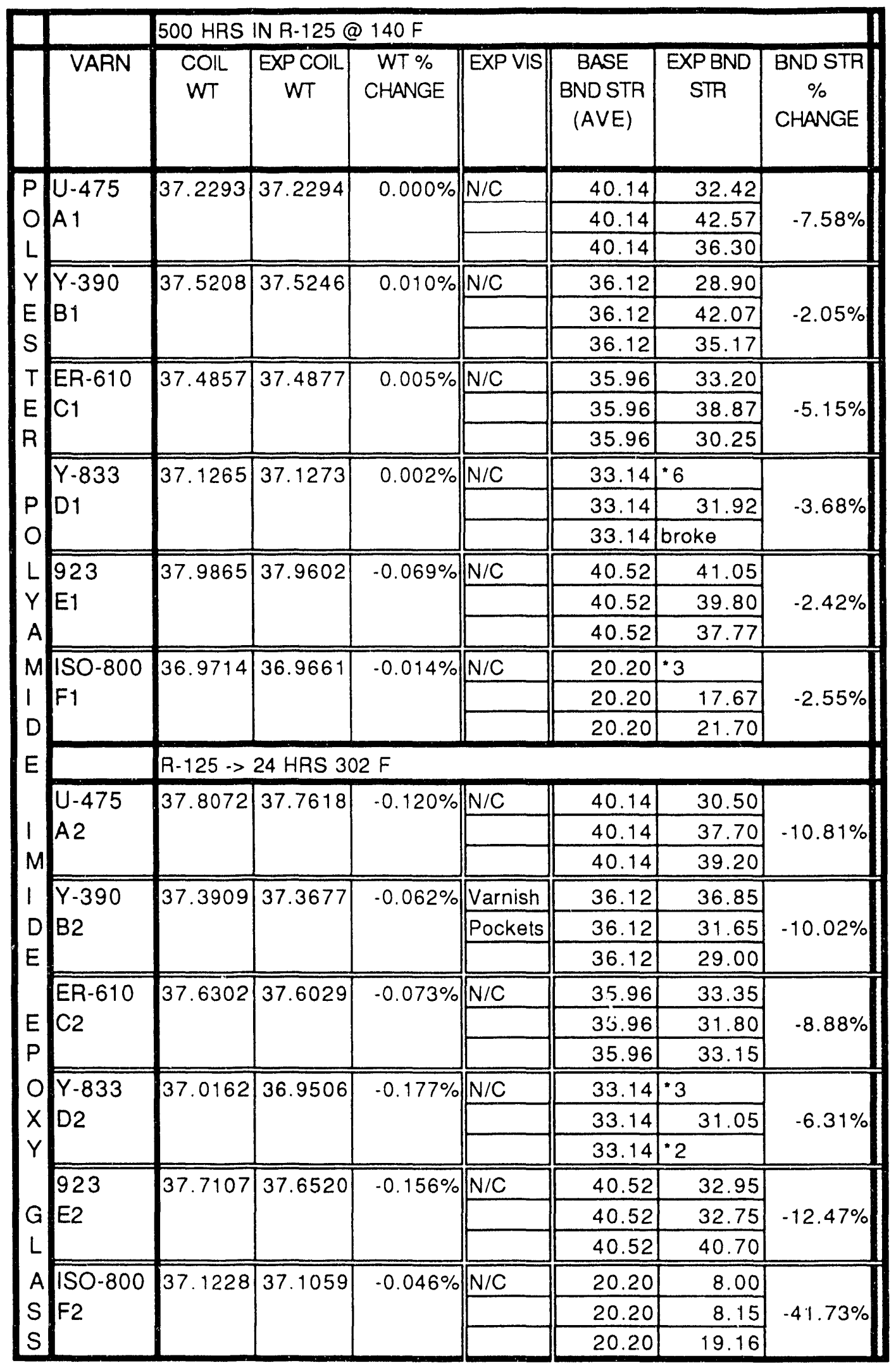

J-6

"Bond strength not included in average 
HELICAL COILSNWIRE C

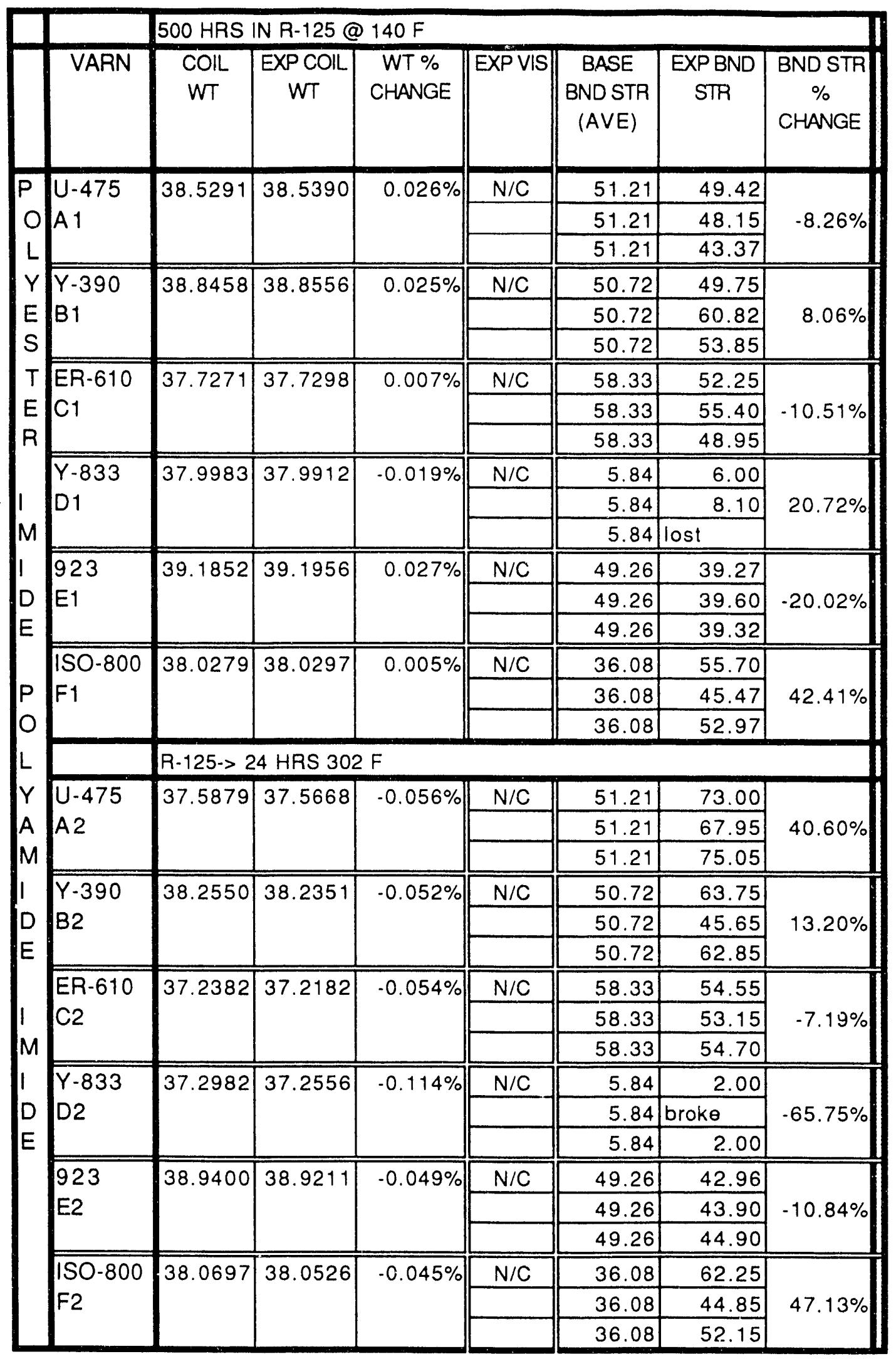


SINGLE MAG WIRE WITHOUT VARNISH

\begin{tabular}{|c|c|c|c|c|c|}
\hline & 500 HRS IN & R-125@1 & & & \\
\hline ID & WT & EXPWT & $\begin{array}{l}\text { WT \% } \\
\text { CHANGE }\end{array}$ & EXPVS & EXPFLEX \\
\hline$A 1$ & 5.7870 & 5.7673 & $-0.340 \%$ & $\mathrm{~N} / \mathrm{C}$ & \\
\hline & & & & & YES \\
\hline$\overline{\mathrm{B} 1}$ & 6.2820 & 6.2627 & $-0.307 \%$ & $\mathrm{~N} / \mathrm{C}$ & \\
\hline & & & & & YES \\
\hline C1 & 5.5910 & 5.5512 & $-0.712 \%$ & $\bar{N} / \mathrm{C}$ & \\
\hline & & & & & YES \\
\hline & $R-125 \rightarrow 3$ & $2 \mathrm{~F} 24 \mathrm{HR}$ & & & \\
\hline A2 & 5.8551 & 5.8545 & $-0.010 \%$ & $\mathrm{~N} / \mathrm{C}$ & \\
\hline & & & & & YES \\
\hline$\overline{B 2}$ & 6.2047 & 6.2019 & $-0.045 \%$ & $\mathrm{~N} / \mathrm{C}$ & \\
\hline & & & & & YES \\
\hline$\overline{\mathrm{C} 2}$ & 5.6289 & 5.6327 & $0.068 \%$ & $\mathrm{~N} / \mathrm{C}$ & \\
\hline & & & & & YES \\
\hline & & & & & \\
\hline
\end{tabular}




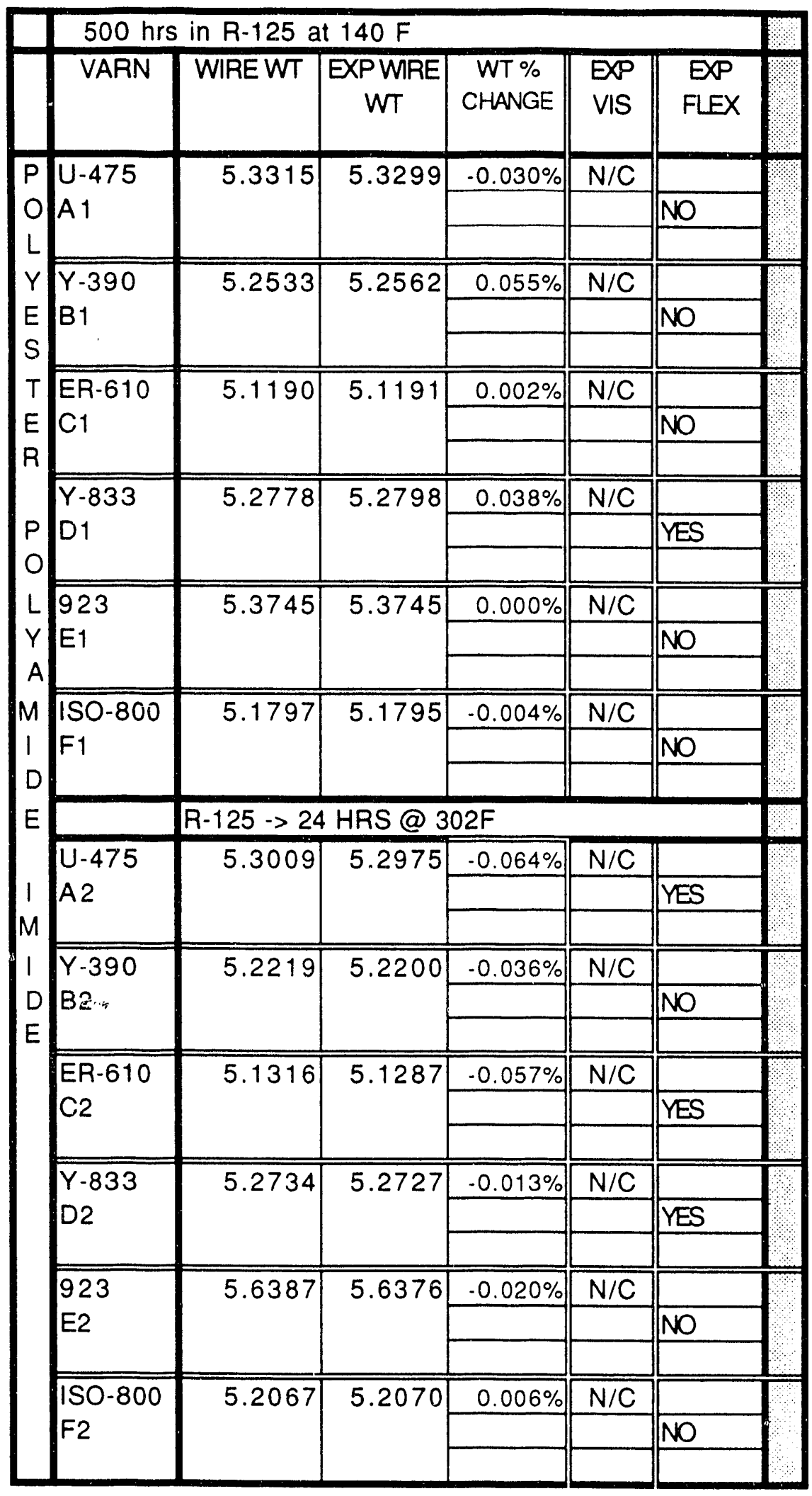




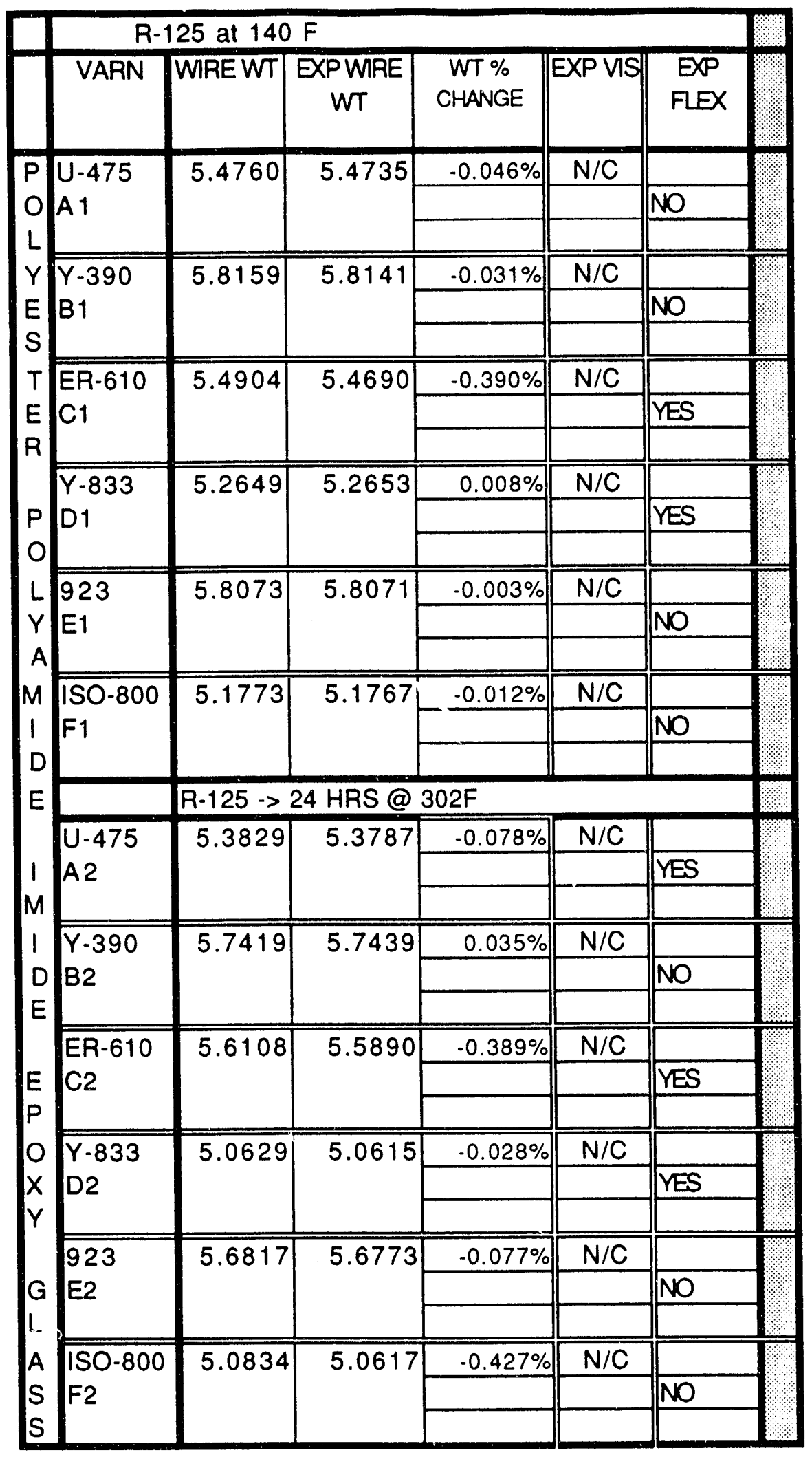




\begin{tabular}{|l|l|l|l|l||l|l|}
\hline & \multicolumn{3}{|c|}{ R-125 at 140 F } & \\
\hline & VARN & WIRE WT & EXPWRE & WT $\%$ \\
CHT & CHANGE & & EXPVIS \\
FLEX
\end{tabular}




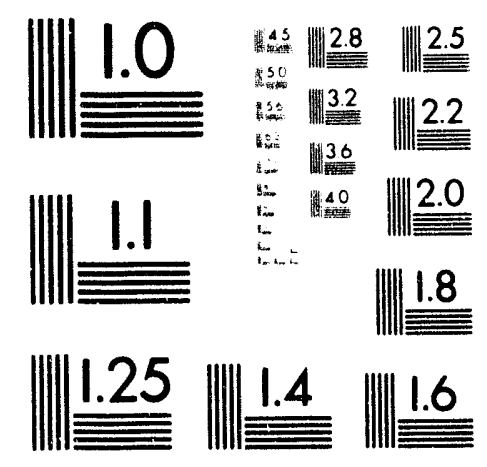



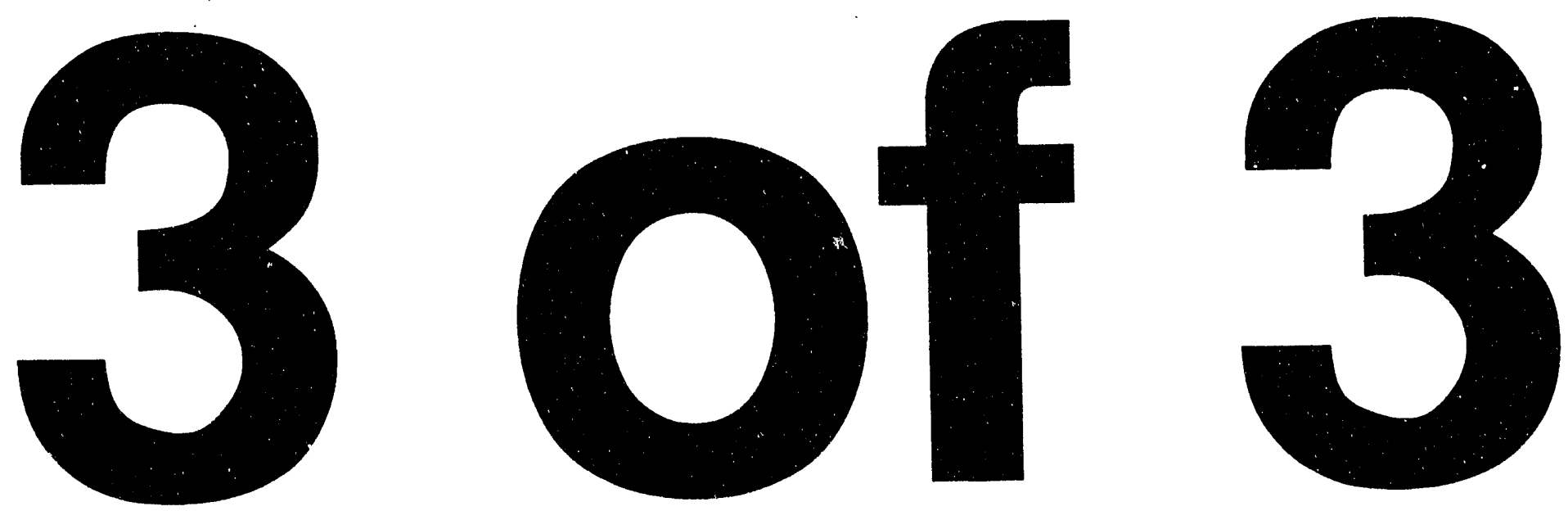


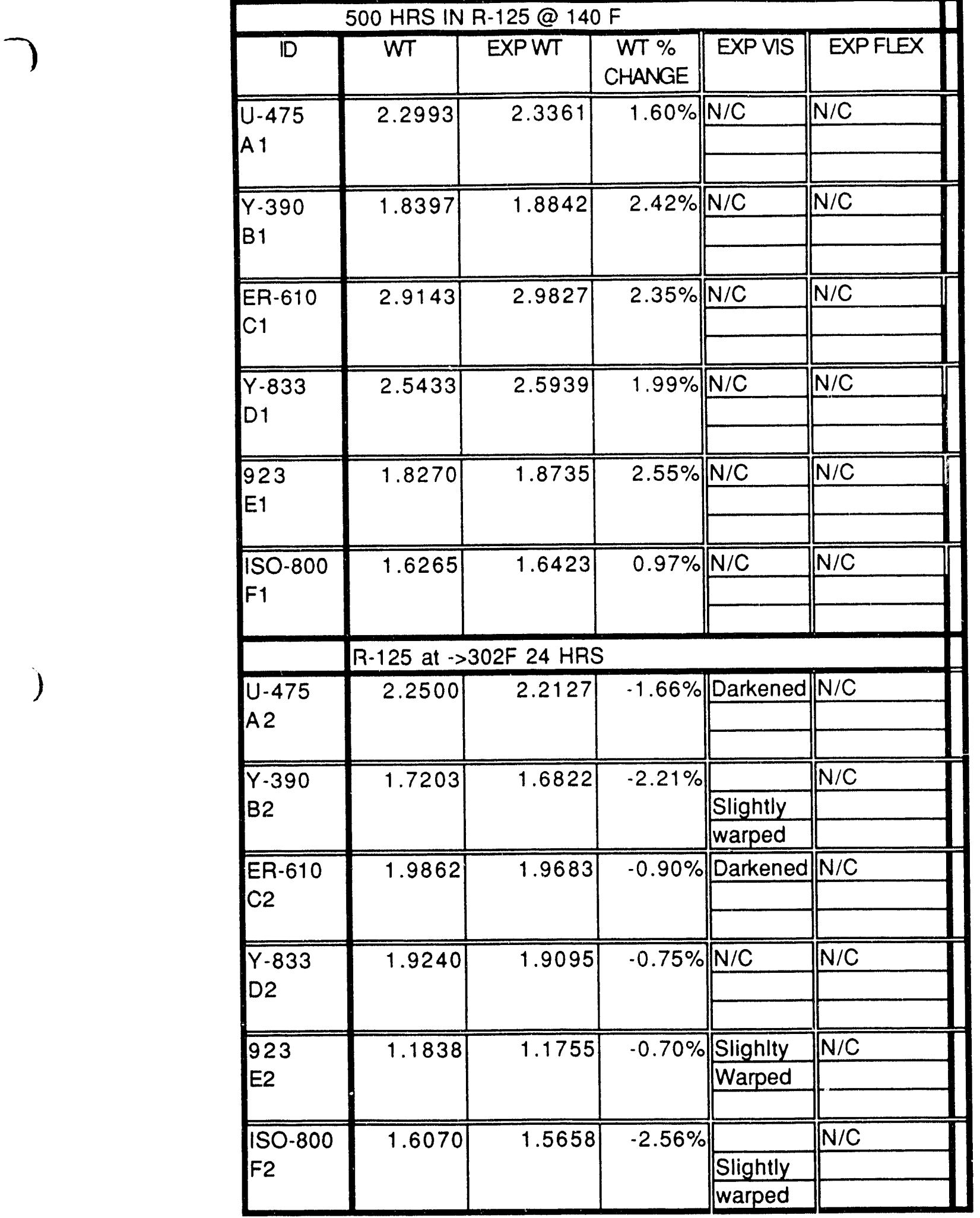




\begin{tabular}{|c|c|c|c|c|c|c|c|c|c|}
\hline & & $500 \mathrm{H}$ & Z IN R & @ 140 & & & & & \\
\hline ID & WT & EXPWT & $\begin{array}{c}\text { WT \% } \\
\text { CHANGE }\end{array}$ & $\begin{array}{l}\text { SAMPLE } \\
\text { WIDTH }\end{array}$ & $\begin{array}{c}\text { BREAK } \\
\text { LOAD }\end{array}$ & $\begin{array}{c}\text { SAMPLE } \\
\text { THICKNESS } \\
\text { Mils }\end{array}$ & $\begin{array}{c}\text { TENSILE } \\
\text { STR } \\
\text { BASE }\end{array}$ & $\begin{array}{l}\text { TENSILE } \\
\text { STREXP }\end{array}$ & $\begin{array}{c}\% \\
\text { CHANGE } \\
\text { TENSILE }\end{array}$ \\
\hline NO/MY/NO & 4.5625 & 4.5628 & $0.01 \%$ & 0.412 & 145.30 & 21 & 17.4 & 16.79 & \\
\hline$A 1$ & & & & 0.494 & 159.20 & 21 & 17.4 & 15.35 & $-5.14 \%$ \\
\hline & & & & 0.410 & 149.60 & 21 & 17.4 & 17.38 & \\
\hline$D A / M Y / D A$ & 4.0705 & 4.1196 & $1.21 \%$ & 0.407 & 116.40 & 21 & 13.7 & 13.62 & \\
\hline B1 & & & & 0.397 & 109.10 & 21 & 13.7 & 13.09 & $-3.84 \%$ \\
\hline & & & & 0.379 & 102.00 & 21 & 13.7 & 12.82 & \\
\hline MYLAR MO & 2.1565 & 2.1584 & $0.09 \%$ & 0.495 & 87.50 & 10 & 21.7 & 17.68 & \\
\hline C1 & & & & 0.438 & 78.45 & 10 & 21.7 & 17.91 & $-12.13 \%$ \\
\hline & & & & 0.418 & 90.35 & 10 & 21.7 & 21.61 & \\
\hline NO 410 & 2.9521 & 2.9432 & $-0.64 \%$ & 0.458 & 84.65 & 11 & 18.7 & 16.80 & \\
\hline D1 & & & & 0.526 & 102.00 & 11 & 18.7 & 17.63 & $-6.50 \%$ \\
\hline & & & & 0.432 & 85.65 & 11 & 18.7 & 18.02 & \\
\hline NO MI 418 & 2.3753 & 2.3642 & $-0.47 \%$ & 0.500 & 27.75 & 9 & 7.5 & 6.17 & \\
\hline E1 & & & & 0.509 & 29.00 & 9 & 7.5 & 6.33 & $-16.61 \%$ \\
\hline & & & & 0.485 & 27.35 & 9 & 7.5 & 6.27 & \\
\hline MEL 228 & 2.2965 & 2.2983 & $0.08 \%$ & 0.416 & 82.25 & 10 & 21.7 & 19.77 & \\
\hline$F 1$ & & & & $0.44 \mathrm{G}$ & 76.55 & 10 & 21.7 & 17.40 & $-13.27 \%$ \\
\hline & & & & 0.467 & 90.10 & 10 & 21.7 & 19.29 & \\
\hline & & 500 HRS & IN R-12 & $5->24 \mathrm{HR}$ & is@ @ 302 & & & & $R \cdot 125 \cdots$ \\
\hline $\mathrm{NO} / \mathrm{MY} / \mathrm{NO}$ & 4.4613 & 4.4584 & $-0.07 \%$ & 0.521 & 153.00 & 21 & 17.4 & 13.98 & \\
\hline$A 2$ & & & & 0.417 & 154.30 & 21 & 17.4 & 17.62 & $-17.02 \%$ \\
\hline & & & & 0.442 & 108.70 & 21 & 17.4 & 11.71 & \\
\hline $\mathrm{DA} / \mathrm{MY} / \mathrm{DA}$ & 4.5090 & 4.4627 & $-1.03 \%$ & 0.393 & 114.70 & 21 & 13.7 & 13.90 & \\
\hline 82 & & & & 0.337 & 93.75 & 21 & 13.7 & 13.25 & $-2.43 \%$ \\
\hline & & & & 0.444 & 120.80 & 21 & 13.7 & 12.96 & \\
\hline MYLAR MO & 2.3352 & 2.3320 & $-0.14 \%$ & 0.402 & 75.70 & 10 & 21.7 & 18.83 & \\
\hline $\mathrm{C} 2$ & & & & 0.420 & 84.00 & 10 & 21.7 & 20.00 & $-12.21 \%$ \\
\hline & & & & 0.425 & 77.85 & 10 & 21.7 & 18.32 & \\
\hline NOMEX 410 & 2.2651 & 2.2001 & $-2.87 \%$ & 0.439 & 82.95 & 11 & 18.7 & 17.18 & \\
\hline 02 & & & & 0.513 & 108.60 & 11 & 18.7 & 19.25 & $-3.60 \%$ \\
\hline & & & & 0.485 & 94.20 & 11 & 18.7 & 17.66 & \\
\hline NO/MI 418 & 2.2896 & 2.2532 & $-1.59 \%$ & 0.454 & 26.20 & 9 & 7.5 & 6.41 & \\
\hline E2 & & & & 0.489 & 26.70 & 9 & 7.5 & 6.07 & $-14.26 \%$ \\
\hline & & & & 0.477 & 29.25 & 9 & 7.5 & 6.81 & \\
\hline MEL 228 & 2.3828 & 2.3784 & $-0.18 \%$ & 0.427 & 82.95 & 10 & 21.7 & 19.43 & \\
\hline $\mathrm{F} 2$ & & & & 0.493 & 95.05 & 10 & 21.7 & 19.28 & $-11.78 \%$ \\
\hline & & & & 0.448 & 83.90 & 10 & 21.7 & 18.73 & \\
\hline
\end{tabular}




\begin{tabular}{|c|c|c|c|c|c|c|c|c|}
\hline & After 500 & hour ex & ssure@ & $140^{\circ} \mathrm{F}(6$ & $\left.0^{\circ} \mathrm{C}\right)$ & & & \\
\hline ID & $\begin{array}{c}\text { STRETCH } \\
\text { (inch) }\end{array}$ & $\begin{array}{c}\% \\
\text { ELONG }\end{array}$ & $\begin{array}{c}\text { BASE } \\
\text { ELONG } \\
\text { (AVE) }\end{array}$ & $\begin{array}{c}\text { ELONG } \\
\% \\
\text { CHANGE }\end{array}$ & $\begin{array}{c}\text { BASE } \\
\text { DIE } \\
\text { (AVE) }\end{array}$ & EXPDIE & $\begin{array}{c}\text { DIE \% } \\
\text { CHANGE }\end{array}$ & $\begin{array}{c}\text { VISUAL } \\
\text { EXP }\end{array}$ \\
\hline NO/MY/NO & 0.94 & $23.5 \%$ & $20.0 \%$ & & $>18.97$ & $>14.67$ & & $\mathrm{~N} / \mathrm{C}$ \\
\hline & 0.48 & $12.0 \%$ & $20.0 \%$ & $2.5 \%$ & $>18.97$ & $>15.01$ & flash & \\
\hline & 1.04 & $26.0 \%$ & $20.0 \%$ & & $>18.97$ & $>14.11$ & & \\
\hline$D A / M Y / D A$ & 0.64 & $32.0 \%$ & $46.0 \%$ & & $>15.27$ & $>12.75$ & & $\mathrm{~N} / \mathrm{C}$ \\
\hline B1 & 0.59 & $29.5 \%$ & $46.0 \%$ & $-33.2 \%$ & $>15.27$ & $>12.99$ & flash & \\
\hline & lost & \#\#\#\#\# & $46.0 \%$ & & $>15.27$ & $>13.34$ & & \\
\hline MYLAR MO & 1.84 & $92.0 \%$ & $131.0 \%$ & & $>14.91$ & $>13.62$ & & $N / C$ \\
\hline C1 & 1.94 & $97.0 \%$ & $131.0 \%$ & $-10.2 \%$ & $\geq 14.91$ & $>14.05$ & flash & \\
\hline & 3.28 & $164.0 \%$ & $131.0 \%$ & & $>14.91$ & $>12.57$ & & \\
\hline NO 410 & 0.55 & $13.8 \%$ & $17.0 \%$ & & 10.67 & 15.04 & & $\mathrm{~N} / \mathrm{C}$ \\
\hline D1 & 0.63 & $15.8 \%$ & $17.0 \%$ & $-11.3 \%$ & 10.67 & 11.16 & $16.4 \%$ & \\
\hline & 0.63 & $15.8 \%$ & $17.0 \%$ & & 10.67 & 11.07 & & \\
\hline MI 418 & 0.08 & $2.0 \%$ & $4.0 \%$ & & 10.23 & 9.50 & & $\mathrm{~N} / \mathrm{C}$ \\
\hline E1 & 0.09 & $2.3 \%$ & $4.0 \%$ & $-45.8 \%$ & 10.23 & 10.04 & $-5.9 \%$ & \\
\hline & 0.09 & $2.3 \%$ & $4.0 \%$ & & 10.23 & 9.34 & & \\
\hline MEL 228 & 3.34 & $167.0 \%$ & $160.0 \%$ & & $>14.22$ & $>12.29$ & & $\mathrm{~N} / \mathrm{C}$ \\
\hline$F 1$ & 2.46 & $123.0 \%$ & $160.0 \%$ & $-5.3 \%$ & $>14.22$ & $>13.29$ & flash & \\
\hline & 3.29 & $164.5 \%$ & $160.0 \%$ & & $>14.22$ & $>12.17$ & & \\
\hline & After 500 & hour $\theta$ ) & osure pl & $24 r$ & hour air & bake at & & $\left.0^{\circ} \mathrm{C}\right)$ \\
\hline NO/MY/NO & .13 & $3.2 \%$ & $20.0 \%$ & & $>18.97$ & $>14.25$ & & bubbles \\
\hline$A 2$ & 0.72 & $18.0 \%$ & $20.0 \%$ & $-16.9 \%$ & $>18.97$ & $>14.78$ & flash & pulling \\
\hline & 0.61 & $15.3 \%$ & $20.0 \%$ & & $>18.97$ & $>14.67$ & & away \\
\hline$D A / M Y / D A$ & 0.49 & $24.5 \%$ & $46.0 \%$ & & $>15.27$ & $>13.40$ & & $\mathrm{~N} / \mathrm{C}$ \\
\hline$B 2$ & 0.55 & $27.5 \%$ & $46.0 \%$ & $-47.5 \%$ & $>15.27$ & $>13.36$ & flash & \\
\hline & 0.41 & $20.5 \%$ & $46.0 \%$ & & $>15.27$ & $>14.23$ & & \\
\hline MYLAR MO & 2.45 & $122.5 \%$ & $131.0 \%$ & & $>14.91$ & $>13.63$ & & $\mathrm{~N} / \mathrm{C}$ \\
\hline $\mathrm{C} 2$ & 2.89 & $144.5 \%$ & $131.0 \%$ & $-1.4 \%$ & $>14.91$ & $>14.20$ & flash & \\
\hline & 2.41 & $120.5 \%$ & $131.0 \%$ & & $>14.91$ & $>12.10$ & & \\
\hline NOMEX 410 & C. 61 & $15.3 \%$ & $17.0 \%$ & & 10.67 & 10.35 & & $\mathrm{~N} / \mathrm{C}$ \\
\hline D2 & 0.56 & $14.0 \%$ & $17.0 \%$ & $-20.6 \%$ & 10.67 & 9.46 & $-7.9 \%$ & \\
\hline & 0.45 & $11.3 \%$ & $17.0 \%$ & & 10.67 & 9.66 & & \\
\hline $\mathrm{NO} / \mathrm{MI} 418$ & 0.06 & $1.5 \%$ & $4.0 \%$ & & 10.23 & 10.90 & & $\mathrm{~N} / \mathrm{C}$ \\
\hline E2 & 0.07 & $1.8 \%$ & $4.0 \%$ & $-56.3 \%$ & 10.23 & 8.68 & $-4.3 \%$ & \\
\hline & 0.08 & $2.0 \%$ & $4.0 \%$ & & 10.23 & 9.80 & & \\
\hline MEL 228 & 3.22 & $161.0 \%$ & $160.0 \%$ & & $\geq 14.22$ & $>13.14$ & & $\mathrm{~N} / \mathrm{C}$ \\
\hline $\mathrm{F} 2$ & 3.16 & $158.0 \%$ & $160.0 \%$ & $-2.5 \%$ & $\geq 14.22$ & $>14.34$ & flash & \\
\hline & 2.98 & $149.0 \%$ & $160.0 \%$ & & $>14.22$ & $>13.48$ & & \\
\hline
\end{tabular}




\begin{tabular}{|c|c|c|c|c|}
\hline & \multicolumn{4}{|c|}{500 HRS IN R-125@140F } \\
\hline & WT & EXPWT & $\begin{array}{c}\% \\
\text { CHANGE }\end{array}$ & EXP VIS \\
\hline & 0.5570 & 0.5620 & $0.90 \%$ & $\mathrm{~N} / \mathrm{C}$ \\
\hline & 0.5210 & 0.5262 & $1.00 \%$ & $\mathrm{~N} / \mathrm{C}$ \\
\hline $\mathrm{C1}$ & 0.4036 & 0.4042 & $0.15 \%$ & $\mathrm{~N} / \mathrm{C}$ \\
\hline & $R-125 \quad \cdots>2$ & hrs@30? & & \\
\hline & 0.5653 & 0.5438 & $-3.80 \%$ & $\mathrm{~N} / \mathrm{C}$ \\
\hline$B 2$ & 0.4806 & 0.4772 & $-0.71 \%$ & Some delamination \\
\hline & & & & "see photo \\
\hline $\mathrm{C} 2$ & 0.3983 & 0.3860 & $-3.09 \%$ & Pockects where \\
\hline NO/MY & & & & mylar pulled away \\
\hline & & & & "see photo \\
\hline
\end{tabular}




\begin{tabular}{|c|c|c|c|c|c|c|c|c|c|}
\hline & 0 HRS & IN R-125 & @ $140 \mathrm{~F}$ & & & & & & \\
\hline$\overline{1 D}$ & WT & EXPWT & $\begin{array}{c}\text { WT \% } \\
\text { CHANGE }\end{array}$ & $\begin{array}{l}\text { BREAK } \\
\text { LOAD } \\
\text { (AVE) }\end{array}$ & $\begin{array}{l}\text { BREAK } \\
\text { LOAD } \\
\text { EXP }\end{array}$ & $\begin{array}{c}\% \\
\text { CHANGE } \\
\text { BPK } \\
\text { LOAD }\end{array}$ & $\begin{array}{l}\text { STRETCH } \\
\text { (INCH) }\end{array}$ & $\%$ \%LONG & EXP VS \\
\hline A1 & 1.5188 & 1.5119 & $-0.45 \%$ & 39.02 & 34.20 & & 0.03 & $1.50 \%$ & $\mathrm{~N} / \mathrm{C}$ \\
\hline Glass & & & & 39.02 & 34.30 & $-3.13 \%$ & 0.04 & $2.00 \%$ & \\
\hline & & & & 39.02 & 44.90 & & 0.03 & $1.50 \%$ & \\
\hline$\overline{\mathrm{B} 1}$ & 0.6223 & 0.6309 & $1.38 \%$ & 56.12 & 63.00 & & 0.64 & $32.00 \%$ & $\mathrm{~N} / \mathrm{C}$ \\
\hline Polyester & & & & 56.12 & 59.45 & $5.73 \%$ & 0.54 & $27.00 \%$ & \\
\hline & & & & 56.12 & 55.55 & & 0.62 & $31.00 \%$ & \\
\hline C1 & 1.7227 & 1.8374 & $6.66 \%$ & 88.50 & 146.30 & & 0.12 & $6.00 \%$ & $\mathrm{~N} / \mathrm{C}$ \\
\hline Permacel & & & & 88.50 & 89.30 & $27.65 \%$ & 0.10 & $5.00 \%$ & \\
\hline & & & & 88.50 & 103.30 & & 0.09 & $4.50 \%$ & \\
\hline & & $500 \mathrm{HRS}$ & in $\mathrm{R}-125$ & $302 \mathrm{~F} 2$ & 4 HRS & & & & \\
\hline A2 & 1.3779 & 1.3735 & $-0.32 \%$ & 39.02 & 42.90 & & 0.04 & $2.00 \%$ & $\mathrm{~N} / \mathrm{C}$ \\
\hline Glass & & & & 39.02 & 49.25 & $30.70 \%$ & 0.04 & $2.00 \%$ & \\
\hline & & & & 39.02 & 60.85 & & 0.05 & $2.50 \%$ & \\
\hline & 0.6050 & 0.6027 & $-0.38 \%$ & 56.12 & 55.90 & & 0.52 & $26.00 \%$ & $\mathrm{~N} / \mathrm{C}$ \\
\hline Polyester & & & & 56.12 & 50.70 & $.0 .60 \%$ & 0.43 & $21.50 \%$ & \\
\hline & & & & 56.12 & 60.75 & & 0.70 & $35.00 \%$ & \\
\hline $\mathrm{C2}$ & 1.4518 & 1.3762 & $-5.21 \%$ & 88.50 & 117.00 & & 0.08 & $4.00 \%$ & Slightly \\
\hline Permacel & & & & 88.50 & 124.70 & $37.85 \%$ & 0.09 & $4.50 \%$ & Darkened \\
\hline & & & & 88.50 & 124.30 & & 0.09 & $4.50 \%$ & \\
\hline
\end{tabular}


TIE CORDS

\begin{tabular}{|c|c|c|c|c|c|c|c|c|c|}
\hline \multicolumn{10}{|c|}{500 HRS IN R-125@140F } \\
\hline ID & WT & EXPWT & $\begin{array}{c}\text { WT \% } \\
\text { CHANGE }\end{array}$ & $\begin{array}{l}\text { EXP } \\
\text { VIS }\end{array}$ & $\begin{array}{l}\text { BREAK } \\
\text { LOAD } \\
\text { (AVE) }\end{array}$ & $\begin{array}{l}\text { BREAK } \\
\text { LOAD } \\
\text { EPP } \\
\text { (ave) }\end{array}$ & $\begin{array}{c}\% \\
\text { CHANGE } \\
\text { BRK } \\
\text { LOAD }\end{array}$ & $\begin{array}{c}\text { STRETCH } \\
\text { (Inch) }\end{array}$ & $\begin{array}{c}\% \\
\text { ELONG }\end{array}$ \\
\hline \multirow[t]{4}{*}{$A 1$} & \multirow[t]{3}{*}{0.2789} & \multirow[t]{3}{*}{0.2801} & \multirow[t]{3}{*}{$0.43 \%$} & $\mathrm{~N} / \mathrm{C}$ & 28.36 & 42.20 & & 0.20 & $10.0 \%$ \\
\hline & & & & & 28.36 & 38.95 & $38.52 \%$ & 0.23 & $11.5 \%$ \\
\hline & & & & & 28.36 & 36.70 & & 0.23 & $11.5 \%$ \\
\hline & \multicolumn{9}{|c|}{500 HRS IN R-125 -> 24 HRS @ 302F } \\
\hline \multirow[t]{3}{*}{ A2 } & \multirow[t]{3}{*}{0.2601} & \multirow[t]{3}{*}{0.2576} & \multirow[t]{3}{*}{$-0.961 \%$} & $\mathrm{~N} / \mathrm{C}$ & 28.36 & 30.00 & & 0.44 & $22.0 \%$ \\
\hline & & & & & 28.36 & 30.25 & $9.02 \%$ & 0.42 & $21.0 \%$ \\
\hline & & & & & 28.36 & 32.50 & & 0.39 & $19.5 \%$ \\
\hline
\end{tabular}




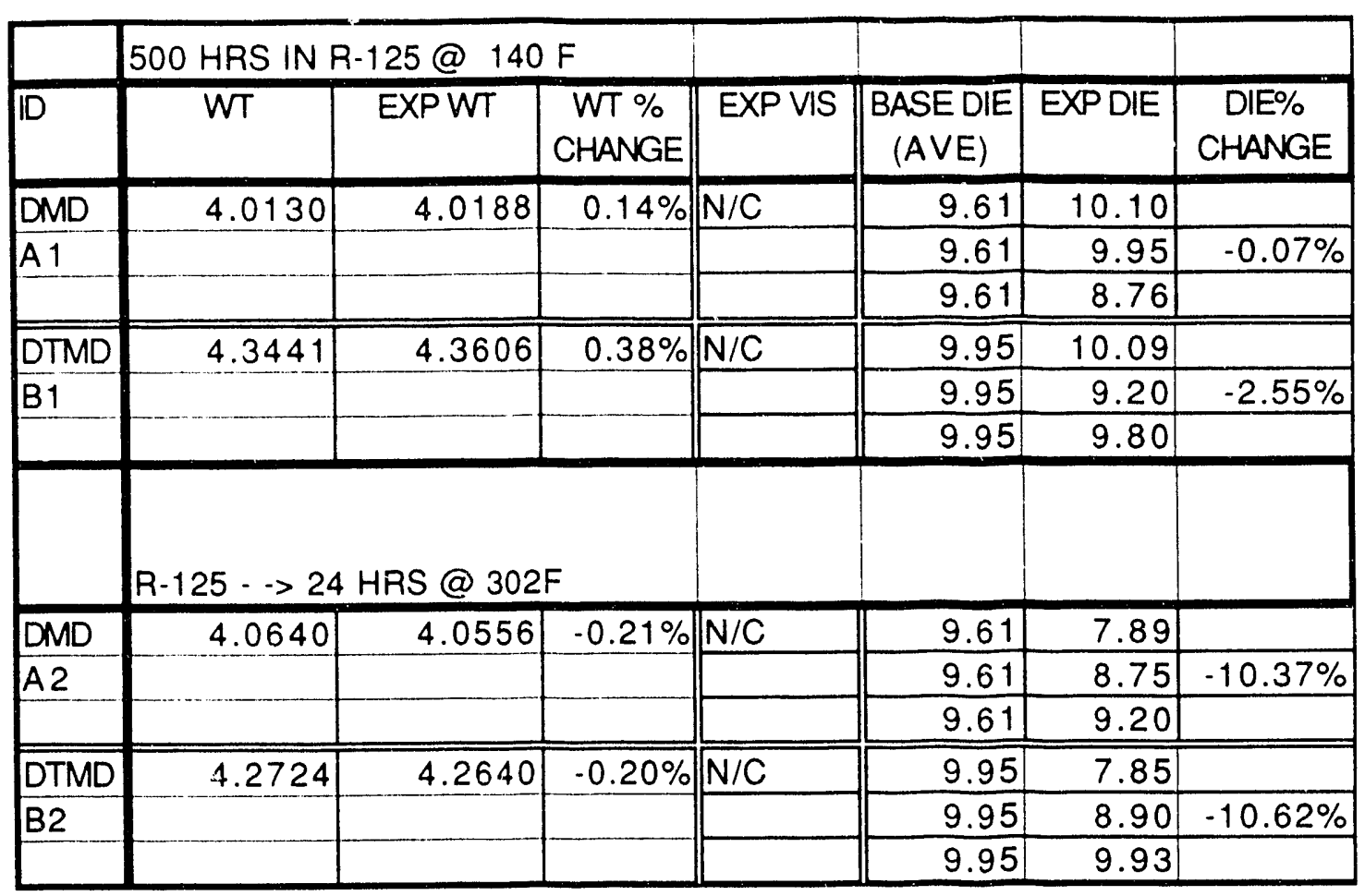




\section{Appendix K}

Experimenta! Data for HFC-32 Exposure at $60^{\circ} \mathrm{C}\left(140^{\circ} \mathrm{F}\right)$ 


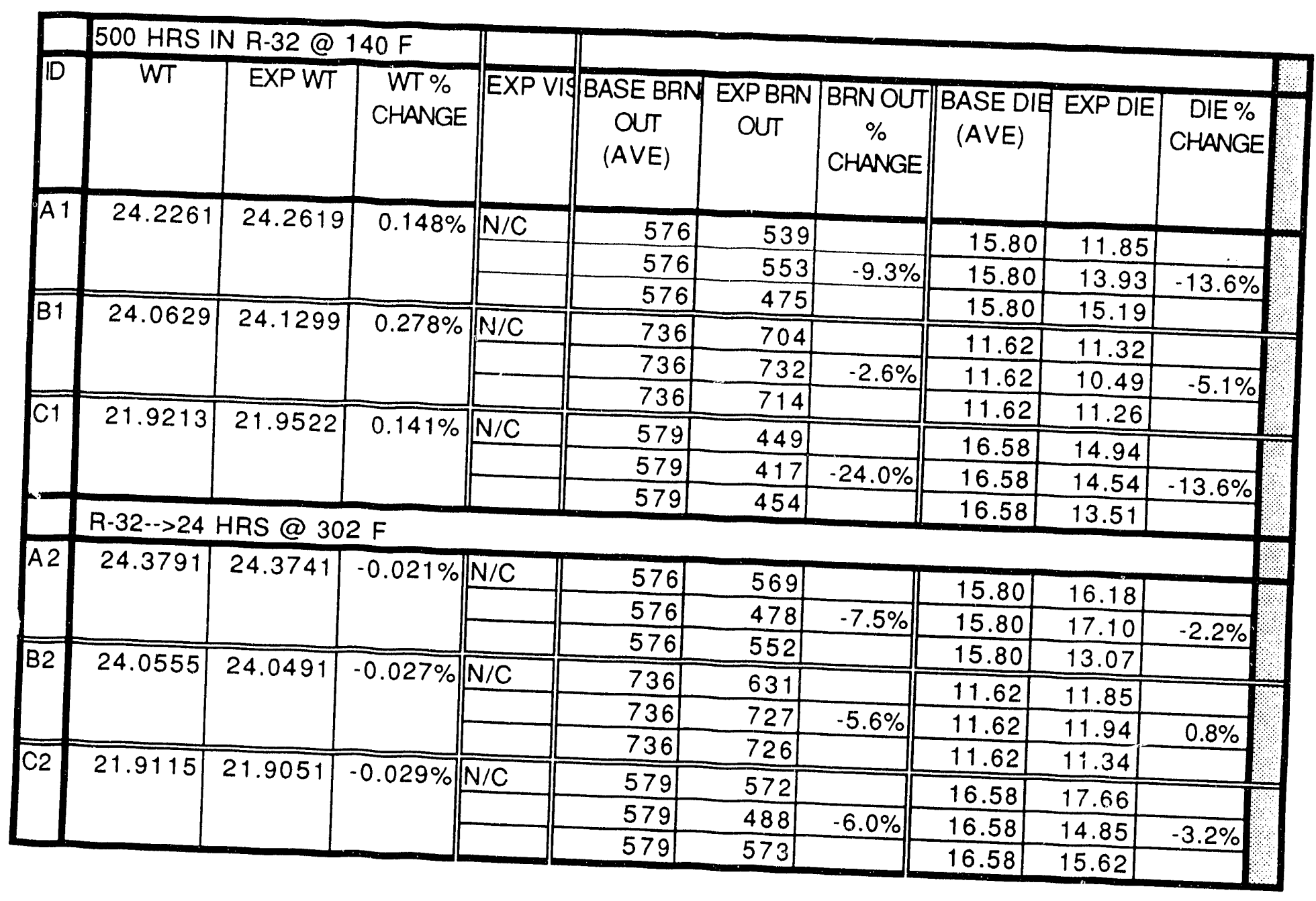




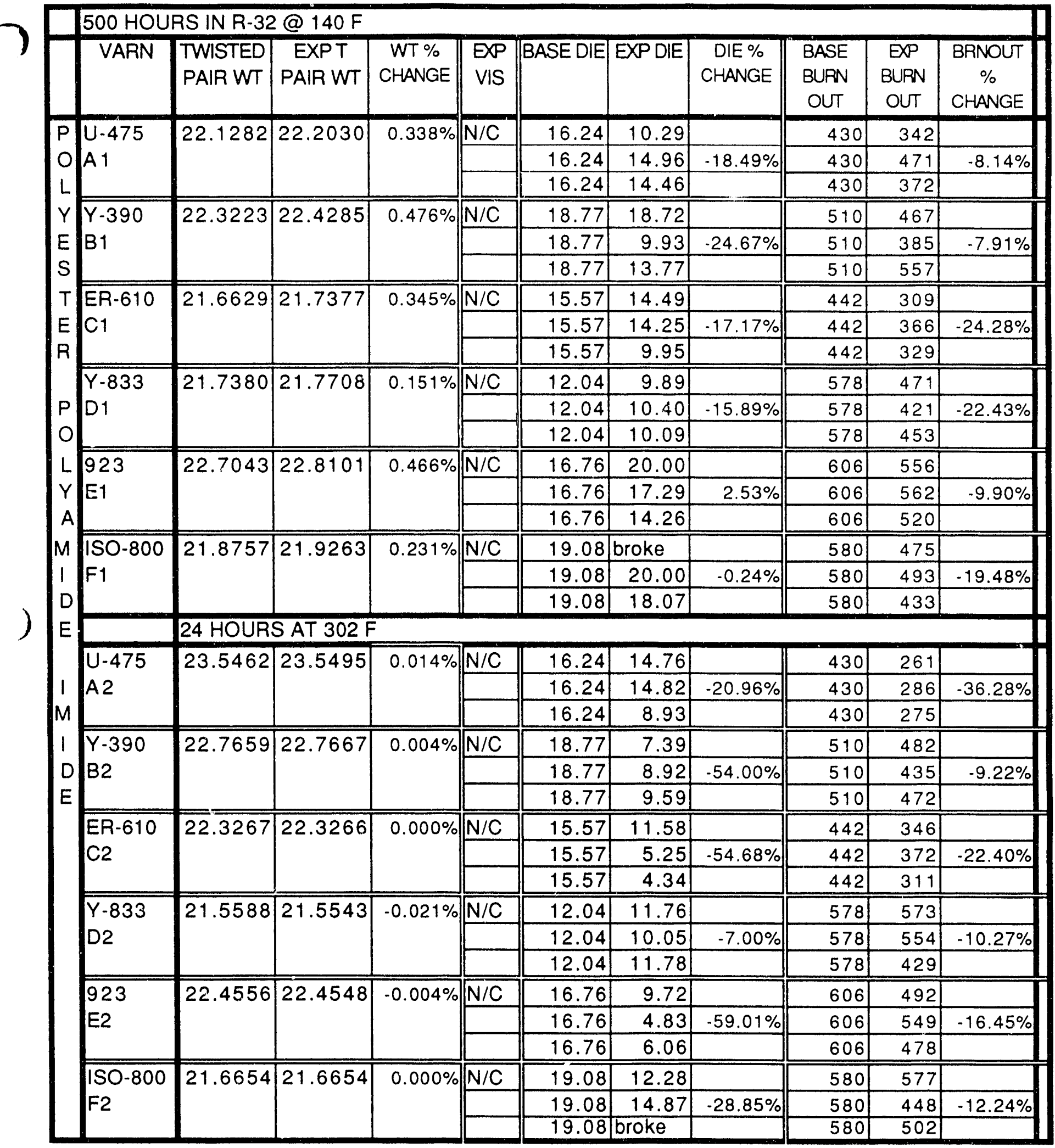

) 


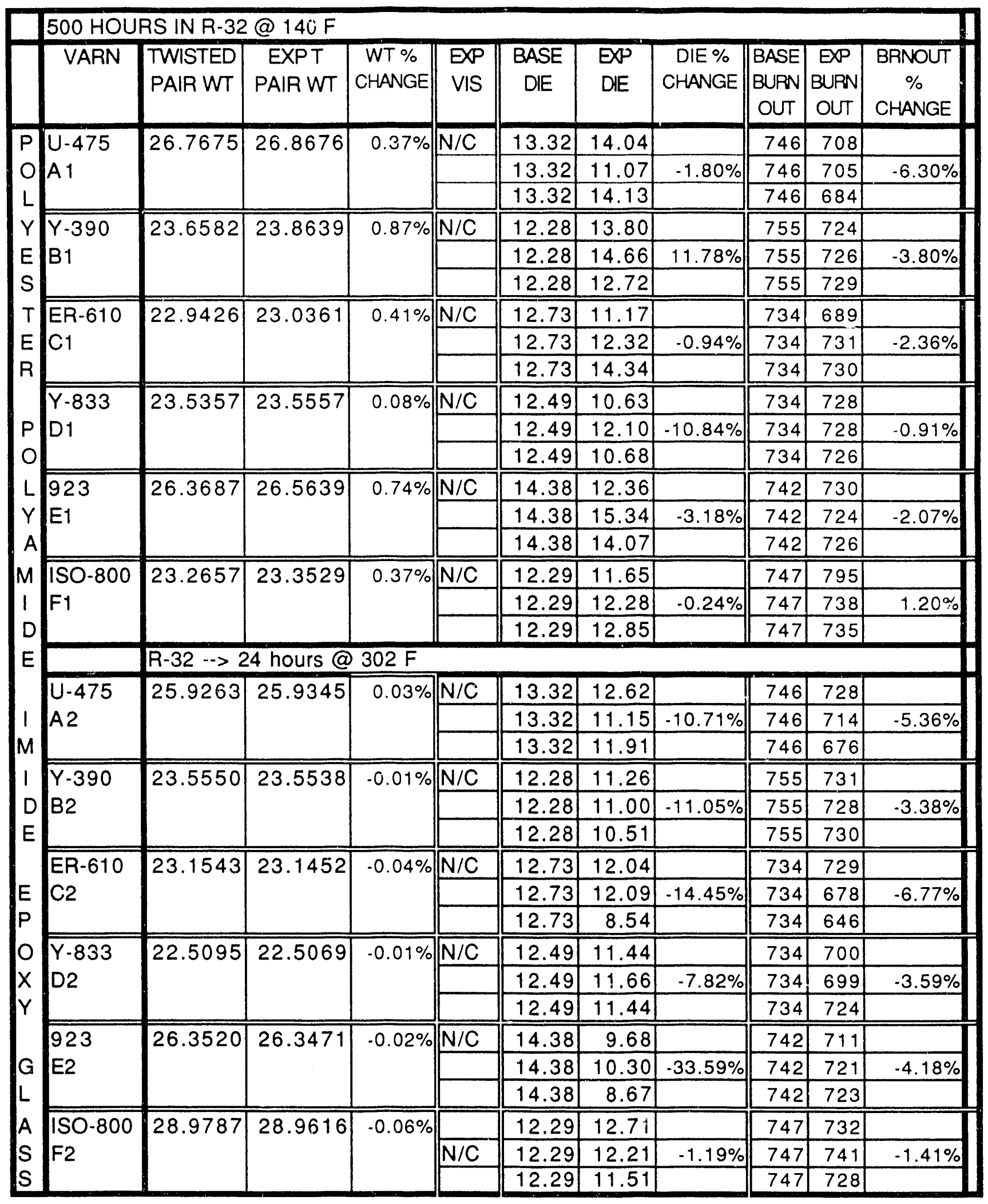




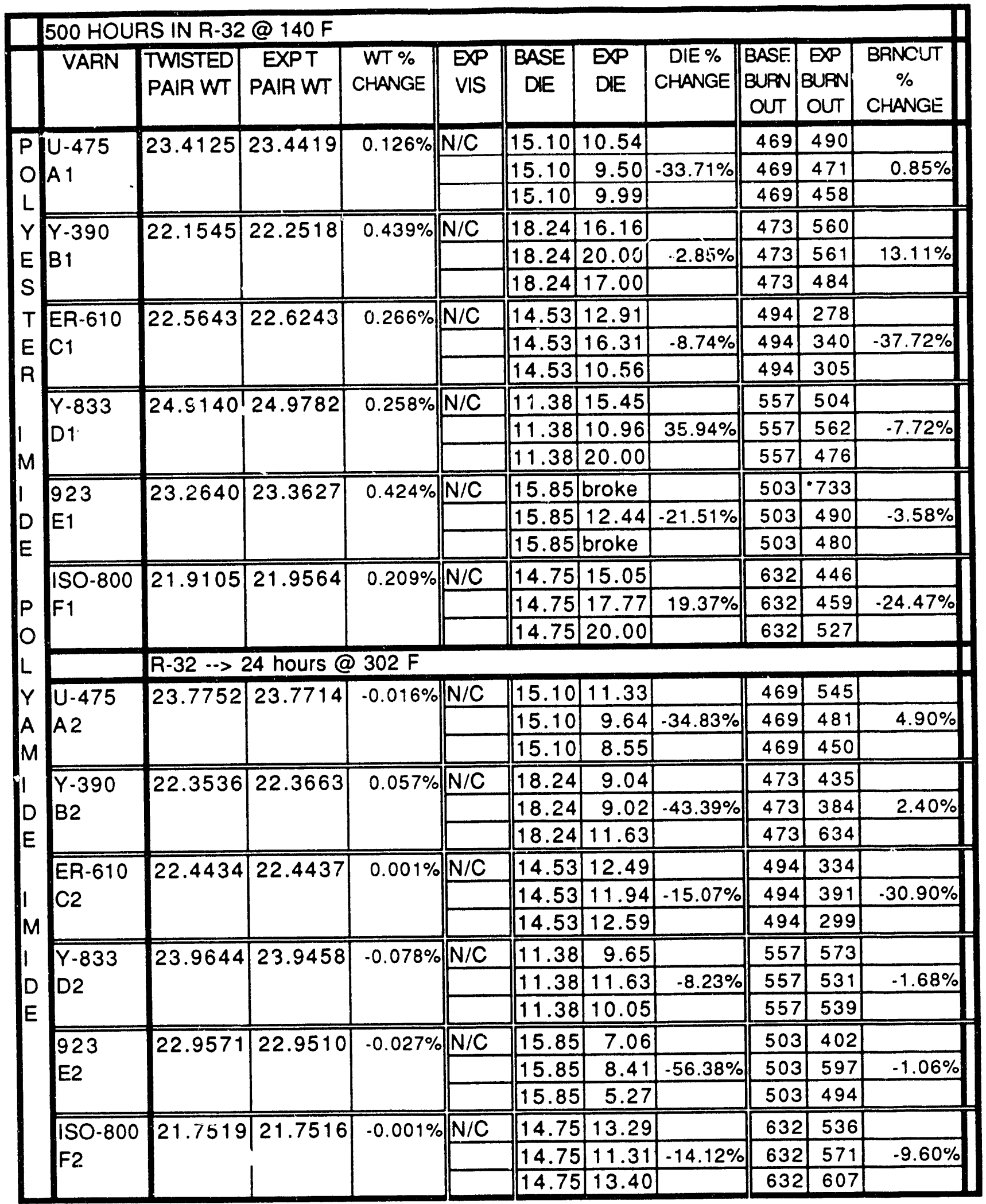

$\mathrm{K}-4$

"Left lid of Burnout Tester open to observe sample. 


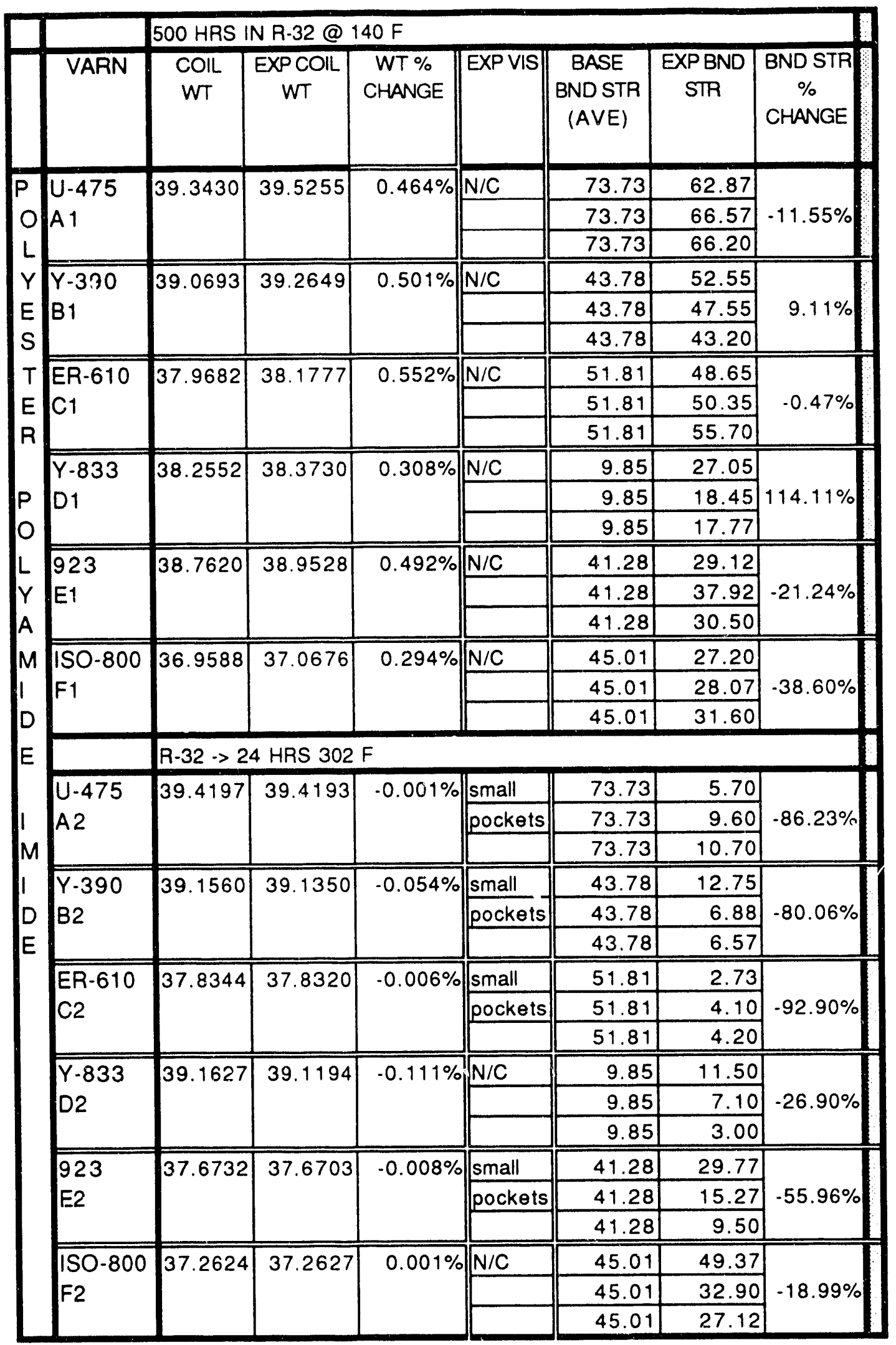




\begin{tabular}{|c|c|c|c|c|c|c|c|c|}
\hline & & 500 HRS I & IN R.32 @ & $140 \mathrm{~F}$ & & & & \\
\hline & VARN & $\begin{array}{l}\text { COIL } \\
\text { WT }\end{array}$ & $\mid \begin{array}{c}\text { EXP COIL } \\
W T\end{array}$ & $\begin{array}{c}\text { WT \% } \\
\text { CHANGE }\end{array}$ & EXP VIS & $\begin{array}{c}\text { BASE } \\
\text { BND STR } \\
(A \vee E)\end{array}$ & $\begin{array}{c}\text { EXPBND } \\
\text { STR }\end{array}$ & $\begin{array}{c}\text { BND STR } \\
\% \\
\text { C.HANGE }\end{array}$ \\
\hline $\mathrm{P}$ & $U-475$ & 37.1538 & 37.4124 & $0.696 \%$ & $\mathrm{~N} / \mathrm{C}$ & 40.14 & 59.80 & \\
\hline 0 & A 1 & & & & & 40.14 & 43.27 & $38.12 \%$ \\
\hline L & & & & & & 40.14 & 63.25 & \\
\hline $\mathrm{Y}$ & $Y-390$ & 37.3189 & 37.6404 & $0.861 \%$ & $\mathrm{~N} / \mathrm{C}$ & 36.12 & 60.20 & \\
\hline$E$ & B1 & & & & & 36.12 & 57.82 & $75.18 \%$ \\
\hline $\mathrm{s}$ & & & & & & 36.12 & 71.80 & \\
\hline$T$ & ER-610 & 38.0592 & 38.2561 & $0.517 \%$ & $\mathrm{~N} / \mathrm{C}$ & 35.96 & 49.37 & \\
\hline$E$ & C1 & & & & & 35.96 & 48.35 & $34.63 \%$ \\
\hline$R$ & & & & & & 35.96 & 47.52 & \\
\hline & $Y-833$ & 36.9525 & 37.1375 & $0.501 \%$ & $\mathrm{~N} / \mathrm{C}$ & 33.14 & broke & \\
\hline $\mathrm{P}$ & D1 & & & & & 33.14 & 58.55 & $83.77 \%$ \\
\hline 0 & & & & & & 33.14 & 63.25 & \\
\hline L & 923 & 38.6222 & 38.8701 & $0.642 \%$ & $\mathrm{~N} / \mathrm{C}$ & 40.52 & 54.50 & \\
\hline $\mathrm{Y}$ & E1 & & & & & 40.52 & 46.92 & $21.82 \%$ \\
\hline A & & & & & & 40.52 & 46.67 & \\
\hline$M$ & ISO-800 & 37.6491 & 37.7581 & $0.290 \%$ & $\mathrm{~N} / \mathrm{C}$ & 20.20 & 26.57 & \\
\hline 1 & F1 & & & & & 20.20 & 24.87 & $28.61 \%$ \\
\hline$D$ & & & & & & 20.20 & 26.50 & \\
\hline$E$ & & $R-32>2$ & 4 HRS 302 & & & & & \\
\hline & $U-475$ & 38.1236 & 38.1176 & $-0.016 \%$ & small & 40.14 & 26.82 & \\
\hline 1 & A2 & & & & pockets & 40.14 & 27.05 & $-34.86 \%$ \\
\hline$M$ & & & & & in Varn & 40.14 & 24.57 & \\
\hline 1 & $Y-390$ & 37.3841 & 37.7274 & $0.918 \%$ & small & 36.12 & 25.90 & \\
\hline$D$ & $B 2$ & & & & pocket & 36.12 & 17.92 & $-36.81 \%$ \\
\hline$E$ & & & & & in Varn & 36.12 & 24.65 & \\
\hline & ER-610 & 37.2288 & 37.3500 & $0.326 \%$ & small & 35.96 & 30.27 & \\
\hline$E$ & & & & & pocket & 35.96 & 21.40 & $-31.60 \%$ \\
\hline$P$ & & & & & in Varn & 35.96 & 22.12 & \\
\hline 0 & Y-833 & 36.2789 & 36.2671 & $-0.033 \%$ & small & 33.14 & 30.80 & \\
\hline$x$ & D2 & & & & pocket & 33.14 & 25.57 & $-17.85 \%$ \\
\hline$Y$ & & & & & in Varn & 33.14 & 25.30 & \\
\hline & 923 & 38.6152 & 38.6101 & $-0.013 \%$ & small & 40.52 & 11.00 & \\
\hline $\mathrm{G}$ & E2 & & & & pocket & 40.52 & 10.30 & $-74.58 \%$ \\
\hline$L$ & & & & & in Varn & 40.52 & 9.60 & \\
\hline A & ISO-800 & 37.7990 & 37.7507 & $-0.128 \%$ & $\mathrm{~N} / \mathrm{C}$ & 20.20 & 19.15 & \\
\hline$S$ & F2 & & & & & 20.20 & 17.52 & $-5.71 \%$ \\
\hline$S$ & & & & & & 20.20 & 20.47 & \\
\hline
\end{tabular}




\begin{tabular}{|c|c|c|c|c|c|c|c|c|}
\hline & & 500 HRS I & IN R-32 @ & $140 \mathrm{~F}$ & & & & \\
\hline & VARN & $\begin{array}{l}\text { COIL } \\
W T\end{array}$ & $\begin{array}{c}\text { EXP COIL } \\
W T\end{array}$ & $\begin{array}{c}\text { WT \% } \\
\text { CHANGE }\end{array}$ & EXP VIS & \begin{tabular}{|c|} 
BASE \\
BND STR \\
(AVE)
\end{tabular} & $\begin{array}{c}\text { EXPBND } \\
\text { STR }\end{array}$ & $\begin{array}{c}\text { BND STR } \\
\% \\
\text { CHANGE }\end{array}$ \\
\hline$P$ & U-475 & 38.3767 & 38.5541 & $0.462 \%$ & $\mathrm{~N} / \mathrm{C}$ & 51.21 & 40.20 & \\
\hline 0 & A 1 & & & & & 51.21 & 68.27 & $4.37 \%$ \\
\hline $\mathrm{L}$ & & & & & & 51.21 & 51.87 & \\
\hline$Y$ & $Y-390$ & 39.2515 & 39.4782 & $0.578 \%$ & $\mathrm{~N} / \mathrm{C}$ & 50.72 & 48.82 & \\
\hline$E$ & B1 & & & & & 50.72 & 45.95 & $-8.43 \%$ \\
\hline$S$ & & & & & & 50.72 & 44.57 & \\
\hline$T$ & ER-610 & 37.2554 & 37.4610 & $0.552 \%$ & $\mathrm{~N} / \mathrm{C}$ & 58.33 & 44.87 & \\
\hline$E$ & C1 & & & & & 58.33 & 58.85 & $-15.26 \%$ \\
\hline$R$ & & & & & & 58.33 & 44.57 & \\
\hline & $Y-833$ & 37.5577 & 37.6008 & $0.115 \%$ & $\mathrm{~N} / \mathrm{C}$ & 5.84 & 10.16 & \\
\hline | & D1 & & & & & 5.84 & 26.70 & $215.58 \%$ \\
\hline M & & & & & & 5.84 & broke & \\
\hline | & 923 & 38.1450 & 38.6672 & $1.369 \%$ & $\mathrm{~N} / \mathrm{C}$ & 49.26 & 33.75 & \\
\hline D & E1 & & & & & 49.26 & 37.45 & $-27.63 \%$ \\
\hline$E$ & & & & & & 49.26 & 35.75 & \\
\hline & $150-800$ & 37.5587 & 37.6634 & $0.279 \%$ & $\mathrm{~N} / \mathrm{C}$ & 36.08 & 9.70 & \\
\hline$P$ & F1 & & & & & 36.08 & 32.17 & $-19.82 \%$ \\
\hline 0 & & & & & & 36.08 & 44.92 & \\
\hline L & & R-32-> 24 & HRS 302 & & & & & \\
\hline$Y$ & U-475 & 38.1246 & 38.1192 & $-0.014 \%$ & small & 51.21 & 60.00 & \\
\hline A & $A 2$ & & & & pockets & 51.21 & 53.92 & $-20.97 \%$ \\
\hline$M$ & & & & & in Varn & 51.21 & 7.50 & \\
\hline I & $Y-390$ & 38.8664 & 38.8773 & $0.028 \%$ & small & 50.72 & 14.25 & \\
\hline D & B2 & & & & pockets & 50.72 & 33.62 & $-41.76 \%$ \\
\hline$E$ & & & & & in Varn & 50.72 & 40.75 & \\
\hline & ER-610 & 37.3591 & 37.2226 & $-0.365 \%$ & small & 58.33 & 3.50 & \\
\hline 1 & C2 & & & & pockets & 58.33 & 3.00 & $-94.51 \%$ \\
\hline M & & & & & in Varn & 58.33 & 3.10 & \\
\hline 1 & Y-833 & 37.7553 & 37.6547 & $-0.266 \%$ & $\mathrm{~N} / \mathrm{C}$ & 5.84 & 7.10 & \\
\hline D & D2 & & & & & 5.84 & 3.50 & $-9.25 \%$ \\
\hline$E$ & & & & & & 5.84 & broke & \\
\hline & 923 & 39.2109 & 39.2013 & $-0.024 \%$ & small & 49.26 & 8.50 & \\
\hline & E2 & & & & pockets & 49.26 & 9.00 & $-82.88 \%$ \\
\hline & & & & & in Varn & 49.26 & 7.80 & \\
\hline & ISO-800 & 38.5920 & 38.5496 & $-0.110 \%$ & $\mathrm{~N} / \mathrm{C}$ & 36.08 & 52.97 & \\
\hline & F2 & & & & & 36.08 & 47.17 & $13.40 \%$ \\
\hline & & & & & & 36.08 & 22.60 & \\
\hline
\end{tabular}




\begin{tabular}{|c|c|c|c|c|c|c|}
\hline & $300 \mathrm{HRS} \mathrm{IN}$ & $3-32 @ 140$ & & & & \\
\hline ID & $\overline{W T}$ & EXPWT & $\begin{array}{l}\text { WT \% } \\
\text { CHANGE }\end{array}$ & EXPVIS & EXPFLEX & \\
\hline A1 & 4.7835 & 4.7904 & $0.144 \%$ & $\mathrm{~N} / \mathrm{C}$ & & \\
\hline & & & & & YYES & \\
\hline$\overline{\mathrm{B1}}$ & $\overline{4.8552}$ & 4.8697 & $0.299 \%$ & $\mathrm{~N} / \mathrm{C}$ & & \\
\hline & & & & & YES & \\
\hline & & & 01730 & N/C & & \\
\hline C1 & 5.5652 & 5.5748 & $0.173 \%$ & & YYES & \\
\hline & & & & & & \\
\hline & $R-32>3$ & F $24 \mathrm{HRS}$ & & & & \\
\hline A2 & 4.6566 & 4.6557 & $-0.019 \%$ & $\mathrm{~N} / \mathrm{C}$ & & \\
\hline & & & & & YES & \\
\hline$\overline{B 2}$ & 4.8191 & 4.8198 & $0.015 \%$ & $\mathrm{~N} / \mathrm{C}$ & & \\
\hline & & & & & YES & \\
\hline C2 & 5.5709 & 5.5698 & $-0.020 \%$ & $N / C$ & & \\
\hline & & & & & YES & \\
\hline & & & & & & \\
\hline
\end{tabular}




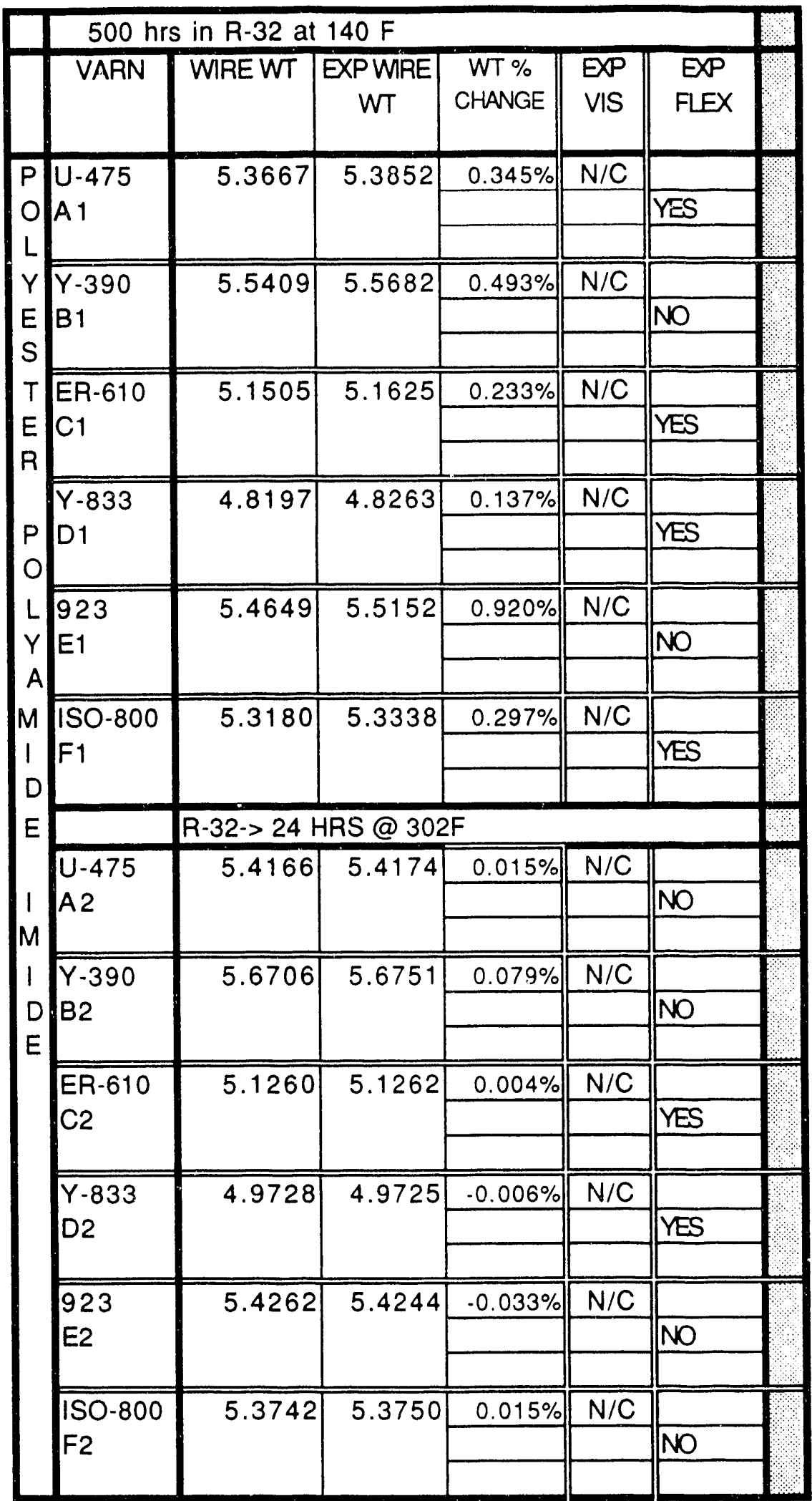

K-9 


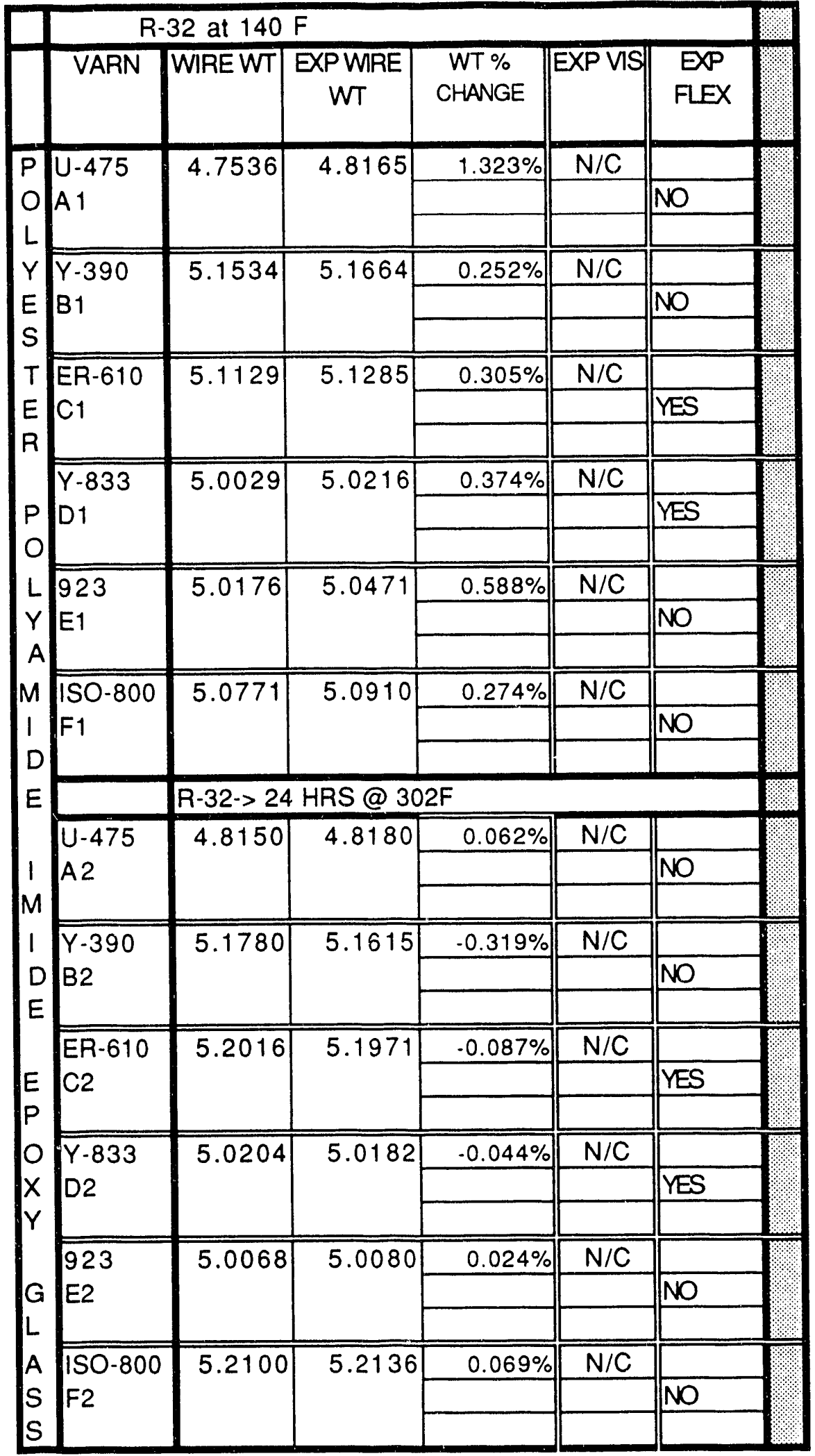




\begin{tabular}{|c|c|c|c|c|c|c|c|}
\hline & \multicolumn{6}{|c|}{ R.32 at $140 \mathrm{~F}$} & \\
\hline & VARN & WIREWT & \begin{tabular}{c|} 
EXPWIRE \\
WT
\end{tabular} & $\begin{array}{l}\text { WT \% } \\
\text { CHANGE }\end{array}$ & EXPVIS & $\begin{array}{l}\text { EXP } \\
\text { FLEX }\end{array}$ & \\
\hline \multirow{4}{*}{$\begin{array}{l}\mathrm{P} \\
\mathrm{O} \\
\mathrm{L} \\
\mathrm{y}\end{array}$} & \multirow{3}{*}{\begin{tabular}{|l|} 
U -475 \\
A 1 \\
\end{tabular}} & \multirow[t]{3}{*}{5.4099} & \multirow[t]{3}{*}{5.4291} & $0.355 \%$ & $N / C$ & & \\
\hline & & & & & & YES & \\
\hline & & & & & & & \\
\hline & \multirow{3}{*}{$\begin{array}{l}Y-390 \\
B 1\end{array}$} & \multirow[t]{3}{*}{5.2117} & \multirow[t]{3}{*}{5.2370} & $0.485 \%$ & $N / C$ & & \\
\hline E & & & & & & NO & \\
\hline s! & & & & & & & \\
\hline \multirow{3}{*}{$\begin{array}{l}T \\
E \\
R\end{array}$} & \multirow{2}{*}{$\begin{array}{l}\text { ER-610 } \\
\text { C1 }\end{array}$} & \multirow[t]{2}{*}{5.5031} & \multirow[t]{2}{*}{5.5118} & $0.158 \%$ & $N / C$ & & \\
\hline & & & & & & YES & \\
\hline & \multirow{3}{*}{$\begin{array}{l}Y-833 \\
D 1\end{array}$} & \multirow[t]{3}{*}{4.8109} & \multirow[t]{3}{*}{4.8196} & $0.181 \%$ & $\mathrm{~N} / \mathrm{C}$ & & \\
\hline \multirow{2}{*}{ M } & & & & & & YES & \\
\hline & & & & & & & \\
\hline \multirow{3}{*}{ D } & \multirow{3}{*}{$\begin{array}{l}923 \\
E 1\end{array}$} & \multirow[t]{3}{*}{5.5256} & \multirow[t]{3}{*}{5.5486} & $0.416 \%$ & $N / C$ & & \\
\hline & & & & & & NO & \\
\hline & & & & & & & \\
\hline & \multirow{3}{*}{$\begin{array}{l}50-800 \\
F 1\end{array}$} & \multirow[t]{3}{*}{ (5.3274 } & \multirow[t]{3}{*}{5.3384} & $0.206 \%$ & $\mathrm{~N} / \mathrm{C}$ & & \\
\hline $\mathrm{P}$ & & & & & & NO & \\
\hline 0 & & & & & & & \\
\hline$L$ & & \multicolumn{5}{|c|}{ R-32 >> 24 HRS @ 302F } & \\
\hline \multirow{3}{*}{$\begin{array}{l}Y \\
A \\
M\end{array}$} & \multirow{3}{*}{$\begin{array}{l}\text { U-475 } \\
\text { A2 }\end{array}$} & 5.3004 & 5.3028 & $0.045 \%$ & $\mathrm{~N} / \mathrm{C}$ & & \\
\hline & & & & & & NO & \\
\hline & & & & & & & \\
\hline 1 & $\overline{Y-390}$ & 5.3558 & $\overline{5.3562}$ & $0.007 \%$ & $\mathrm{~N} / \mathrm{C}$ & & \\
\hline$D$ & & & & & & NO & \\
\hline E & & & & & & & \\
\hline & ER-610 & 5.6200 & 5.6206 & $0.011 \%$ & $N / C$ & & \\
\hline$|M|$ & & & & & & YES & \\
\hline 1 & & 10105 & & & & & \\
\hline D & $\mathrm{D} 2$ & 4.8190 & 4.8196 & $0.002 \%$ & N/C & IVCo & \\
\hline I- & & & & & & & \\
\hline & 923 & 5.5091 & 5.5148 & $0.103 \%$ & $\overline{N / C}$ & & \\
\hline & E2 & & & & & NO & \\
\hline & & & & & & & \\
\hline & $150-800$ & 5.4299 & 5.4301 & $0.004 \%$ & $\mathrm{~N} / \mathrm{C}$ & & \\
\hline & & & & & & NO & \\
\hline
\end{tabular}




\begin{tabular}{|c|c|c|c|c|c|}
\hline & 500 HRS IN & 7-32@140 & & & \\
\hline$\overline{I D}$ & WT & EXPWT & $\begin{array}{c}\text { WT \% } \\
\text { CHANGE }\end{array}$ & EXPVIS & EXPFLEX \\
\hline$U-475$ & 2.0610 & 2.2277 & $8.09 \%$ & $\mathrm{~N} / \mathrm{C}$ & Slightly \\
\hline A 1 & & & & & more \\
\hline & & & & & Flexible \\
\hline$Y-390$ & 2.2609 & 2.4151 & $6.82 \%$ & $\mathrm{~N} / \mathrm{C}$ & slightly \\
\hline B1 & & & & & more \\
\hline & & & & & flexible \\
\hline ER-610 & 2.1411 & 2.3385 & $9.22 \%$ & $\mathrm{~N} / \mathrm{C}$ & more \\
\hline & & & & & flexible \\
\hline Y-833 & 1.9698 & 2.1565 & $9.48 \%$ & $\mathrm{~N} / \mathrm{C}$ & flexible \\
\hline D1 & & & & & \\
\hline & & & & & \\
\hline 923 & 1.6573 & 1.7579 & $6.07 \%$ & slightly & more \\
\hline E1 & & & & warped & flexible \\
\hline & 16188 & 16967 & $481 \%$ & warped & sliahtlv \\
\hline $\begin{array}{l}\text { ISO-800 } \\
\text { F1 }\end{array}$ & 1.6188 & 1.6960 & & & |limginiy \\
\hline & & & & & flexible \\
\hline & $R-32--->302$ & for $24 \mathrm{HR}$ & & & \\
\hline U-475 & 2.7049 & 2.6479 & $-2.11 \%$ & many & brittle \\
\hline A2 & & & & pockets & \\
\hline & & & & darkened & \\
\hline$Y-390$ & 2.4693 & 2.3974 & $-2.91 \%$ & many & warped \\
\hline & & & & pockets & brittle \\
\hline ER-610 & 23109 & 2,3080 & $-0.13 \%$ & few & darkened \\
\hline $\mathrm{C} 2$ & & & & big & $\mathrm{N} / \mathrm{C}$ \\
\hline & & & & pockets & \\
\hline$\overline{Y-833}$ & 2.6208 & 2.5472 & $-2.81 \%$ & many & \\
\hline D2 & & & & pockets & brittle \\
\hline & & & & lightened & \\
\hline$\overline{923}$ & 1.9706 & 1.8615 & $-5.54 \%$ & many & \\
\hline E2 & & & & pockets & brittle \\
\hline |ISO-800 & 1.4728 & 1.3592 & $-7.71 \%$ & many & 7 warped \\
\hline & & & & pockets & brittle \\
\hline & & & & lightened & \\
\hline
\end{tabular}




\begin{tabular}{|c|c|c|c|c|c|c|c|c|c|}
\hline \multicolumn{10}{|c|}{500 HR IN R-32@140 F } \\
\hline 10 & WT & EXPWT & $\begin{array}{c}\text { WT \% } \\
\text { CHANGE }\end{array}$ & $\begin{array}{l}\text { SAMPLE } \\
\text { WIDTH }\end{array}$ & $\begin{array}{l}\text { BREAK } \\
\text { LOAD }\end{array}$ & $\begin{array}{c}\text { SAMPLE } \\
\text { THICKNESS } \\
\text { Mils }\end{array}$ & $\begin{array}{c}\text { TENSILE } \\
\text { STR } \\
\text { BASE }\end{array}$ & $\begin{array}{l}\text { TENSILE } \\
\text { STREXP }\end{array}$ & $\begin{array}{c}\% \\
\text { CHANGE } \\
\text { TENSILE }\end{array}$ \\
\hline \multirow{3}{*}{$\begin{array}{l}\mathrm{NO} / \mathrm{MY} / \mathrm{NO} \\
\mathrm{A} 1\end{array}$} & \multirow[t]{3}{*}{4.6528} & \multirow[t]{3}{*}{4.9171} & \multirow[t]{3}{*}{$5.68 \%$} & 0.381 & 122.30 & 21 & 17.4 & 15.29 & \\
\hline & & & & 0.364 & 114.60 & 21 & 17.4 & 14.99 & $-12.13 \%$ \\
\hline & & & & 0.384 & 125.70 & 21 & 17.4 & 15.59 & \\
\hline \multirow{3}{*}{$\begin{array}{l}\mathrm{DA} / \mathrm{MY} / \mathrm{DA} \\
\mathrm{B} 1\end{array}$} & \multirow[t]{3}{*}{4.5349} & \multirow{3}{*}{4.7017} & \multirow{3}{*}{$3.68 \%$} & 0.527 & 131.90 & 21 & 13.7 & 11.92 & \\
\hline & & & & 0.431 & 111.10 & 21 & 13.7 & 12.27 & $-12.19 \%$ \\
\hline & & & & 0.476 & 118.90 & 21 & 13.7 & 11.89 & \\
\hline \multirow{3}{*}{$\begin{array}{l}\text { MYAR MO } \\
\text { C1 }\end{array}$} & \multirow[t]{3}{*}{2.1239} & \multirow[t]{3}{*}{2.2094} & \multirow[t]{3}{*}{$4.03 \%$} & 0.432 & 89.90 & 10 & 21.7 & 20.81 & \\
\hline & & & & 0.493 & 97.65 & 10 & 21.7 & 19.81 & $-6.71 \%$ \\
\hline & & & & 0.477 & 95.95 & 10 & 21.7 & 20.12 & \\
\hline \multirow{3}{*}{$\begin{array}{l}\text { NO } 410 \\
\text { D1 }\end{array}$} & \multirow[t]{3}{*}{2.0229} & \multirow[t]{3}{*}{2.1510} & \multirow[t]{3}{*}{$6.33 \%$} & 0.531 & 98.05 & 11 & 18.7 & 16.79 & \\
\hline & & & & 0.422 & 81.55 & 11 & 18.7 & 17.57 & $-7.71 \%$ \\
\hline & & & & 0.501 & 96.00 & 11 & 18.7 & 17.42 & \\
\hline \multirow{3}{*}{$\begin{array}{l}\text { NO MI } 418 \\
\text { E1 }\end{array}$} & \multirow[t]{3}{*}{2.5480} & \multirow[t]{3}{*}{2.6046} & \multirow[t]{3}{*}{$2.22 \%$} & 0.475 & 23.45 & 9 & 7.5 & 5.49 & \\
\hline & & & & 0.480 & 24.30 & 9 & 7.5 & 5.63 & $-24.87 \%$ \\
\hline & & & & 0.490 & 25.55 & 9 & 7.5 & 5.79 & \\
\hline \multirow{4}{*}{$\begin{array}{l}\text { MEL } 228 \\
\text { F1 }\end{array}$} & \multirow[t]{3}{*}{2.3562} & \multirow[t]{3}{*}{2.4685} & \multirow[t]{3}{*}{$4.77 \%$} & 0.470 & 86.80 & 10 & 21.7 & 18.47 & \\
\hline & & & & 0.395 & 76.95 & 10 & 21.7 & 19.48 & $-11.43 \%$ \\
\hline & & & & 0.495 & 97.55 & 10 & 21.7 & 19.71 & \\
\hline & & $->24 \mathrm{HF}$ & 35@ 302 & & & & & & $\because>302 F$ \\
\hline NO/MY/NO & 5.0134 & 5.0066 & $-0.14 \%$ & 0.381 & 144.10 & 21 & 17.4 & 18.01 & \\
\hline A2 & & & & 0.361 & 131.10 & 21 & 17.4 & 17.29 & $1.48 \%$ \\
\hline & & & & 0.505 & 187.40 & 21 & 17.4 & 17.67 & \\
\hline$D A / M Y / D A$ & 4.4380 & 4.4317 & $-0.14 \%$ & 0.340 & 95.45 & 21 & 13.7 & 13.37 & \\
\hline $\mathrm{B} 2$ & & & & 0.365 & 107.40 & 21 & 13.7 & 14.01 & $-0.53 \%$ \\
\hline & & & & 0.420 & 119.10 & 21 & 13.7 & 13.50 & \\
\hline MYLAR MO & 2.3016 & 2.3074 & $0.25 \%$ & 0.392 & 79.60 & 10 & 21.7 & 20.31 & \\
\hline $\mathrm{C} 2$ & & & & 0.530 & 107.50 & 10 & 21.7 & 20.28 & $.7 .63 \%$ \\
\hline & & & & 0.385 & 75.25 & 10 & 21.7 & 19.55 & \\
\hline NOMEX 410 & 2.5629 & 2.5573 & $-0.22 \%$ & 0.438 & 91.60 & 10 & 18.7 & 20.91 & \\
\hline D2 & & & & 0.492 & 101.80 & 10 & 18.7 & 20.69 & $10.98 \%$ \\
\hline & & & & 0.502 & 103.70 & 10 & 18.7 & 20.66 & \\
\hline NO/MI 418 & 2.2461 & 2.2466 & $0.02 \%$ & 0.527 & 29.50 & 9 & 7.5 & 6.22 & \\
\hline E2 & & & & 0.480 & 25.45 & 9 & 7.5 & 5.89 & $-19.35 \%$ \\
\hline & & & & 0.487 & 26.45 & 9 & 7.5 & 6.03 & \\
\hline MEL 228 & 2.2656 & 2.2710 & $0.24 \%$ & 0.474 & 78.55 & 10 & 21.7 & 16.57 & \\
\hline $\mathrm{F} 2$ & & & & 0.436 & 83.40 & 10 & 21.7 & 19.13 & $-15.78 \%$ \\
\hline & & & & 0.522 & 99.85 & 10 & 21.7 & 19.13 & \\
\hline
\end{tabular}




\begin{tabular}{|c|c|c|c|c|c|c|c|c|}
\hline \multicolumn{8}{|c|}{ After 500 hour exposure@140 $@\left(60^{\circ} \mathrm{C}\right)$} & \multirow[b]{2}{*}{ VISUAL EXP } \\
\hline ID & $\begin{array}{c}\text { STRETCH } \\
\text { (inch) }\end{array}$ & $\begin{array}{c}\% \\
\text { ELONG }\end{array}$ & $\begin{array}{l}\text { BASE } \\
\text { ELONG } \\
\text { (AVE) }\end{array}$ & $\begin{array}{c}\text { ELONG } \\
\% \\
\text { CHANGE }\end{array}$ & $\begin{array}{c}\text { BASE } \\
\text { DIE } \\
(A \vee E)\end{array}$ & EXPDIE & $\begin{array}{c}\text { DIE \% } \\
\text { CHANGE }\end{array}$ & \\
\hline \multirow{3}{*}{$\begin{array}{l}\mathrm{NO} / \mathrm{MY} / \mathrm{NO} \\
\mathrm{A} 1\end{array}$} & 1.23 & $30.8 \%$ & $20.0 \%$ & & $>18.97$ & $>14.84$ & & \multirow[t]{3}{*}{$\mathrm{N} / \mathrm{C}$} \\
\hline & 0.96 & $24.0 \%$ & $20.0 \%$ & $38.8 \%$ & $>18.97$ & $>14.14$ & flash & \\
\hline & 1.26 [ & $31.5 \%$ & $20.0 \%$ & & $>18.97$ & $>14.40$ & & \\
\hline \multirow{3}{*}{$\begin{array}{l}\mathrm{DA} / \mathrm{MY} / \mathrm{DA} \\
\mathrm{B} 1\end{array}$} & 0.62 & $31.0 \%$ & $46.0 \%$ & & $>15.27$ & $>12.47$ & & \multirow[t]{3}{*}{$\mathrm{N} / \mathrm{C}$} \\
\hline & 0.61 & $30.5 \%$ & $46.0 \%$ & $-33.3 \%$ & $\geq 15.27$ & $>13.36$ & flash & \\
\hline & 0.61 & $30.5 \%$ & $46.0 \%$ & & $>15.27$ & $>12.93$ & & \\
\hline \multirow{3}{*}{$\begin{array}{l}\text { MYLAR MO } \\
\text { C1 }\end{array}$} & 3.21 & $160.5 \%$ & $131.0 \%$ & & $>14.91$ & $>13.17$ & & \multirow[t]{3}{*}{$N / C$} \\
\hline & 3.09 & $154.5 \%$ & $131.0 \%$ & $21.9 \%$ & $>14.91$ & $>13.24$ & flash & \\
\hline & 3.28 & $164.0 \%$ & $131.0 \%$ & & $>14.91$ & $>13.34$ & & \\
\hline \multirow{3}{*}{$\begin{array}{l}\text { NO } 410 \\
\text { D1 }\end{array}$} & 0.74 & $18.5 \%$ & $17.0 \%$ & & 10.67 & 11.17 & & \multirow[t]{3}{*}{$\sqrt{N / C}$} \\
\hline & 0.79 & $19.8 \%$ & $17.0 \%$ & $13.2 \%$ & 10.67 & 11.20 & $3.3 \%$ & \\
\hline & 0.78 & $19.5 \%$ & $17.0 \%$ & & 10.67 & 10.71 & & \\
\hline \multirow{3}{*}{$\begin{array}{l}\text { NO Ml } 418 \\
\text { E1 }\end{array}$} & 0.07 & $1.8 \%$ & $4.0 \%$ & & 10.23 & 10.25 & & \multirow{3}{*}{$N / C$} \\
\hline & 0.07 & $1.8 \%$ & $4.0 \%$ & $-56.3 \%$ & 10.23 & 8.50 & $-6.1 \%$ & \\
\hline & 0.07 & $1.8 \%$ & $4.0 \%$ & & 10.23 & 10.07 & & \\
\hline \multirow{4}{*}{$\begin{array}{l}\text { MEL } 228 \\
\text { F1 }\end{array}$} & 3.37 & $168.5 \%$ & $160.0 \%$ & & $>14.22$ & $>13.26$ & & \multirow[t]{3}{*}{$\sqrt{N / C}$} \\
\hline & 3.49 & $174.5 \%$ & $160.0 \%$ & $9.7 \%$ & $\geq 14.22$ & $>13.81$ & flash & \\
\hline & 3.67 & $183.5 \%$ & $160.0 \%$ & & $>14.22$ & $>12.20$ & & \\
\hline & & \multicolumn{6}{|c|}{ After 500 hour exposure plus a 24 hour airbake at $302^{\circ} \mathrm{F}$} & $\left(150^{\circ} \mathrm{C}\right)$ \\
\hline \multirow{3}{*}{$\begin{array}{l}\mathrm{NO} / \mathrm{MY} / \mathrm{NO} \\
\mathrm{A} 2\end{array}$} & 1.00 & $25.0 \%$ & $20.0 \%$ & & $>18.97$ & $>14.96$ & & \multirow{3}{*}{$\begin{array}{l}\text { few bubbles } \\
\text { between } \\
\text { layer }\end{array}$} \\
\hline & 0.93 & $23.3 \%$ & $20.0 \%$ & $20.4 \%$ & $>18.97$ & $>13.71$ & flash & \\
\hline & 0.96 & $24.0 \%$ & $20.0 \%$ & & $>18.97$ & $>14.21$ & & \\
\hline \multirow{3}{*}{$\begin{array}{l}\mathrm{DA} / \mathrm{MY} / \mathrm{DA} \\
\mathrm{B} 2\end{array}$} & 0.47 & $23.5 \%$ & $46.0 \%$ & & $>15.27$ & $>13.94$ & & \multirow{3}{*}{$\begin{array}{l}\text { few bubbles } \\
\text { between } \\
\text { layer }\end{array}$} \\
\hline & 0.56 & $28.0 \%$ & $46.0 \%$ & $-43.8 \%$ & $\geq 15.27$ & $>13.21$ & flash & \\
\hline & 0.52 & $26.0 \%$ & $46.0 \%$ & & $>15.27$ & $>14.24$ & & \\
\hline \multirow{3}{*}{$\begin{array}{l}\text { MYLARMO } \\
\text { C2 }\end{array}$} & 2.94 & $147.0 \%$ & $131.0 \%$ & & $>14.91$ & $>12.82$ & & \multirow[t]{3}{*}{$N / C$} \\
\hline & 3.17 & $158.5 \%$ & $131.0 \%$ & $10.1 \%$ & $>14.91$ & $>13.61$ & flash & \\
\hline & 2.54 & $127.0 \%$ & $131.0 \%$ & & $>14.91$ & $>12.42$ & & \\
\hline \multirow{3}{*}{$\begin{array}{l}\text { NOMEX 410 } \\
\text { D2 }\end{array}$} & 0.66 & $16.5 \%$ & $17.0 \%$ & & 10.67 & 10.60 & & \multirow[t]{3}{*}{$N / C$} \\
\hline & 0.61 & $15.3 \%$ & $17.0 \%$ & $-3.4 \%$ & 10.67 & 11.07 & $6.0 \%$ & \\
\hline & 0.70 & $17.5 \%$ & $17.0 \%$ & & 10.67 & 12.27 & & \\
\hline \multirow{3}{*}{$\begin{array}{l}\mathrm{NO} / \mathrm{MI} 418 \\
\mathrm{E2}\end{array}$} & 0.08 & $2.0 \%$ & $4.0 \%$ & & 10.23 & 10.95 & & \multirow{3}{*}{$N / C$} \\
\hline & 0.06 & $1.5 \%$ & $4.0 \%$ & $.58 .3 \%$ & 10.23 & 9.94 & $-4.1 \%$ & \\
\hline & 0.06 & $1.5 \%$ & $4.0 \%$ & & 10.23 & 8.55 & & \\
\hline MEL 228 & 3.14 & $157.0 \%$ & $160.0 \%$ & & $>14.22$ & $>14.23$ & & Few \\
\hline F2 & 3.09 & $154.5 \%$ & $160.0 \%$ & $-0.8 \%$ & $>14.22$ & $>12.39$ & flash & bubbles \\
\hline & 3.29 & $164.5 \%$ & $160.0 \%$ & & $\| 14.22$ & $>13.46$ & & \\
\hline
\end{tabular}




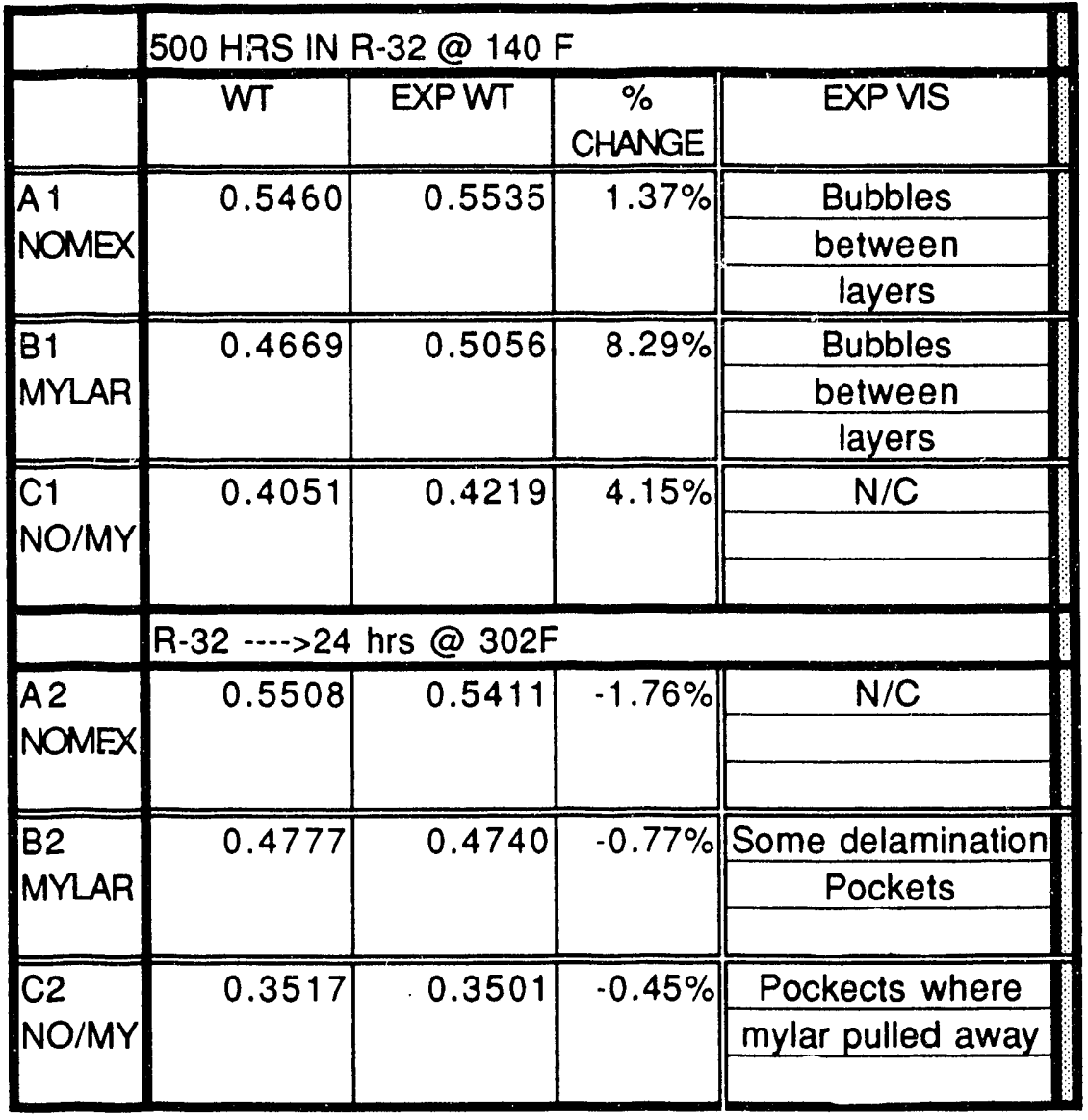




\begin{tabular}{|c|c|c|c|c|c|c|c|c|c|}
\hline & \multicolumn{9}{|c|}{500 HRS IN R-32@140F } \\
\hline$D$ & WT & EXPWT & $\begin{array}{c}\text { WT \% } \\
\text { CHANGE }\end{array}$ & $\begin{array}{l}\text { BREAK } \\
\text { LOAD } \\
\text { (AVE) }\end{array}$ & $\begin{array}{l}\text { BREAK } \\
\text { LOAD } \\
\text { EXP }\end{array}$ & $\begin{array}{c}\% \\
\text { CHANGE } \\
\text { BPK } \\
\text { LOAD }\end{array}$ & $\begin{array}{c}\text { STRETCH } \\
\text { (INCH) }\end{array}$ & $\%$ ELONG & EXP VIS \\
\hline \multirow{3}{*}{$\begin{array}{l}\text { A } 1 \\
\text { Glass }\end{array}$} & \multirow[t]{3}{*}{1.4662} & \multirow[t]{3}{*}{1.4682} & \multirow[t]{3}{*}{$0.14 \%$} & 39.02 & 46.20 & & 0.06 & $3.00 \%$ & $\mathrm{~N} / \mathrm{C}$ \\
\hline & & & & 39.02 & 41.95 & $15.75 \%$ & 0.06 & $3.00 \%$ & \\
\hline & & & & 39.02 & 47.35 & & 0.06 & $3.00 \%$ & \\
\hline \multirow{3}{*}{$\begin{array}{l}\text { B1 } \\
\text { Polyester }\end{array}$} & \multirow[t]{3}{*}{0.6329} & \multirow[t]{3}{*}{0.6543} & \multirow[t]{3}{*}{$3.38 \%$} & 56.12 & 59.55 & & 0.63 & $31.50 \%$ & $\mathrm{~N} / \mathrm{C}$ \\
\hline & & & & 56.12 & 51.15 & $2.52 \%$ & 0.45 & $22.50 \%$ & \\
\hline & & & & 56.12 & 61.90 & & 0.75 & $37.50 \%$ & \\
\hline \multirow{4}{*}{$\begin{array}{l}\text { C1 } \\
\text { Permacel }\end{array}$} & \multirow{3}{*}{1.3595} & \multirow[t]{3}{*}{1.3650} & \multirow[t]{3}{*}{$0.40 \%$} & 88.50[ & 96.60 & & 0.14 & $7.00 \%$ & $\mathrm{~N} / \mathrm{C}$ \\
\hline & & & & 88.50 & 83.70 & $-7.68 \%$ & 0.14 & $7.00 \%$ & \\
\hline & & & & 88.50 & 64.80 & & 0.13 & $6.50 \%$ & \\
\hline & \multicolumn{7}{|c|}{$500 \mathrm{HRS}$ in R-32 $>302 \mathrm{~F} 24 \mathrm{HRS}$} & & \\
\hline \multirow{3}{*}{$\begin{array}{l}\text { A2 } \\
\text { Glass }\end{array}$} & \multirow[t]{3}{*}{1.3560} & \multirow[t]{3}{*}{1.3593} & \multirow[t]{3}{*}{$0.24 \%$} & 39.02 & 41.20 & & 0.05 & $2.50 \%$ & $\mathrm{~N} / \mathrm{C}$ \\
\hline & & & & 39.02 & 44.92 & $23.56 \%$ & 0.07 & $3.50 \%$ & \\
\hline & & & & 39.02 & 58.52 & & 0.05 & $2.50 \%$ & \\
\hline \multirow{3}{*}{$\begin{array}{l}\text { B2 } \\
\text { Polyester }\end{array}$} & \multirow[t]{3}{*}{0.5705} & \multirow[t]{3}{*}{0.5708} & \multirow[t]{3}{*}{$0.05 \%$} & 56.12 & 59.10 & & 0.71 & $35.50 \%$ & $\mathrm{~N} / \mathrm{C}$ \\
\hline & & & & 56.12 & $53 \cdot 2$ & $0.69 \%$ & 0.51 & $25.50 \%$ & \\
\hline & & & & 56.12 & 57.30 & & 0.63 & $31.50 \%$ & \\
\hline \multirow{3}{*}{$\begin{array}{l}\text { C2 } \\
\text { Permacel }\end{array}$} & \multirow[t]{3}{*}{1.4109} & \multirow[t]{3}{*}{1.3579} & \multirow[t]{3}{*}{$-3.76 \%$} & 88.50 & 74.47 & & 0.10 & $5.00 \%$ & $\mathrm{~N} / \mathrm{C}$ \\
\hline & & & & 88.50 & 58.77 & $-11.28 \%$ & 0.06 & $3.00 \%$ & \\
\hline & & & & 88.50 & 102.30 & & 0.10 & $5.00 \%$ & \\
\hline
\end{tabular}


TIE CORDS

\begin{tabular}{|c|c|c|c|c|c|c|c|c|c|}
\hline \multicolumn{10}{|c|}{500 HRS IN R.32@140F } \\
\hline ID & WT & EXPWT & $\begin{array}{c}\text { WT \% } \\
\text { CHANGE }\end{array}$ & $\begin{array}{l}\text { EXP } \\
\text { VIS }\end{array}$ & $\begin{array}{l}\text { BREAK } \\
\text { LOAD } \\
\text { (AVE) }\end{array}$ & $\begin{array}{c}\text { BREAK } \\
\text { LOAD } \\
\text { EXP } \\
(\text { ave })\end{array}$ & $\begin{array}{c}\% \\
\text { CHANGE } \\
\text { BRK } \\
\text { LOAD }\end{array}$ & $\begin{array}{c}\text { STRETCH } \\
\text { (Inch) }\end{array}$ & $\begin{array}{c}\% \\
\text { ELONG }\end{array}$ \\
\hline \multirow[t]{4}{*}{$A 1$} & \multirow[t]{3}{*}{0.2670} & \multirow[t]{3}{*}{0.2721} & \multirow[t]{3}{*}{$1.91 \%$} & $\mathrm{~N} / \mathrm{C}$ & 28.36 & 36.65 & & 0.37 & $18.5 \%$ \\
\hline & & & & & 28.36 & 33.95 & \multirow{2}{*}{$25.94 \%$} & 0.34 & $17.0 \%$ \\
\hline & & & & & 28.36 & 36.55 & & 0.34 & $17.0 \%$ \\
\hline & \multicolumn{9}{|c|}{500 HRS IN R-32 -> 24 HRS @ 302F } \\
\hline \multirow[t]{3}{*}{ A2 } & \multirow[t]{3}{*}{0.2680} & \multirow[t]{3}{*}{0.2624} & \multirow[t]{3}{*}{$-2.09 \%$} & $\mathrm{~N} / \mathrm{C}$ & 28.36 & 33.27 & & 0.39 & $19.5 \%$ \\
\hline & & & & & 28.36 & 31.67 & $8.12 \%$ & 0.38 & $19.0 \%$ \\
\hline & & & & & 28.36 & 27.05 & & 0.35 & $17.5 \%$ \\
\hline
\end{tabular}




\begin{tabular}{|c|c|c|c|c|c|c|c|}
\hline & 500 HRS IN & $32 @ 140$ & & & & & \\
\hline ID & WT & EXP WT & $\begin{array}{c}\text { WT \% } \\
\text { CHANGE }\end{array}$ & EXP VS & $\begin{array}{c}\text { BASE DIE } \\
\text { (AVE) }\end{array}$ & EXPDIE & $\begin{array}{c}\text { DIE\% } \\
\text { CHANGE }\end{array}$ \\
\hline DMD & 4.0420 & 4.0576 & $0.39 \%$ & $N / C$ & 9.61 & 8.64 & \\
\hline A1 & & & & & 9.61 & 9.37 & $-4.13 \%$ \\
\hline & & & & & 9.61 & 9.63 & \\
\hline$\overline{\text { DTMD }}$ & 4.3599 & 4.4126 & $1.21 \%$ & $\mathrm{~N} / \mathrm{C}$ & 9.95 & 8.42 & \\
\hline$B 1$ & & & & & 9.95 & 9.07 & $-9.41 \%$ \\
\hline & & & & & 9.95 & 9.55 & \\
\hline & $R-32-\rightarrow 24$ & RS @ 302F & & & & & \\
\hline DMD & 4.1134 & 4.1122 & $-0.03 \%$ & $\mathrm{~N} / \mathrm{C}$ & 9.61 & 8.90 & \\
\hline$A 2$ & & & & & 9.61 & 9.14 & $-4.13 \%$ \\
\hline & & & & & 9.61 & 9.60 & \\
\hline DTMD & 4.3652 & 4.3633 & $-0.04 \%$ & $\mathrm{~N} / \mathrm{C}$ & 9.95 & 9.94 & \\
\hline B2 & & & & & 9.95 & 7.06 & $-4.25 \%$ \\
\hline & & & & & 9.95 & 11.58 & \\
\hline
\end{tabular}




\section{Appendix L}

\section{Experimental Data for HFC-143a Exposure at $60^{\circ} \mathrm{C}\left(140^{\circ} \mathrm{F}\right)$}




\begin{tabular}{|c|c|c|c|c|c|c|c|c|c|c|}
\hline & 500 HRS IN & R-143a@ & $140 \mathrm{~F}$ & & & & & & & \\
\hline ID & WT & EXPWT & $\begin{array}{c}\text { WT \% } \\
\text { CHANGE }\end{array}$ & EXP VIS & $\begin{array}{c}\text { BASE BRN } \\
\text { OUT } \\
\text { (AVE) }\end{array}$ & $\begin{array}{c}\text { EXPBRN } \\
\text { OUT }\end{array}$ & $\begin{array}{c}\text { BRN OUT } \\
\% \\
\text { CHANGE }\end{array}$ & $\mid \begin{array}{c}\text { BASE DIE } \\
\text { (AVE) }\end{array}$ & EXPDIE & $\begin{array}{c}\text { DIE \% } \\
\text { CHANGE }\end{array}$ \\
\hline$A 1$ & 24.6950 & 24.6989 & $0.016 \%$ & $\mathrm{~N} / \mathrm{C}$ & 576 & 546 & & 15.80 & 19.03 & \\
\hline & & & & & 576 & 561 & $-6.5 \%$ & 15.80 & 18.70 & $18.4 \%$ \\
\hline & & & & & 576 & 509 & & 15.80 & 18.37 & \\
\hline B1 & 22.8557 & 22.8670 & $0.049 \%$ & $\mathrm{~N} / \mathrm{C}$ & 736 & 735 & & 11.62 & 12.02 & \\
\hline & & & & & 736 & 733 & $-0.3 \%$ & 11.62 & 12.31 & $1.5 \%$ \\
\hline & & & & & 736 & 733 & & 11.62 & 11.04 & \\
\hline$\overline{C 1}$ & 24.7617 & 24.7638 & $0.008 \%$ & $\mathrm{~N} / \mathrm{C}$ & 579 & 494 & & 16.58 & 11.87 & \\
\hline & & & & & 579 & 553 & $-10.1 \%$ & 16.58 & 17.15 & $-12.8 \%$ \\
\hline & & & & & 579 & 514 & & 16.58 & 14.36 & \\
\hline & $R-143 a-->2$ & 4 HRS@3 & $02 \mathrm{~F}$ & & & & & & & \\
\hline A2 & 24.5883 & 24.5695 & $-0.076 \%$ & $\mathrm{~N} / \mathrm{C}$ & 576 & 599 & & 15.80 & 12.16 & \\
\hline & & & & & 576 & 581 & $0.6 \%$ & 15.80 & 17.79 & $-0.1 \%$ \\
\hline & & & & & 576 & 558 & & 15.80 & 17.42 & \\
\hline $\mathrm{B2}$ & 22.5195 & 22.5155 & $-0.018 \%$ & $\mathrm{~N} / \mathrm{C}$ & 736 & 730 & & 11.62 & 10.83 & \\
\hline & & & & & 736 & 730 & $-0.8 \%$ & 11.62 & 10.09 & $-8.0 \%$ \\
\hline & & & & & 736 & 730 & & 11.62 & 11.14 & \\
\hline $\mathrm{C2}$ & 24.3511 & 24.3490 & $-0.009 \%$ & $\mathrm{~N} / \mathrm{C}$ & 579 & 501 & & 16.58 & 17.00 & \\
\hline & & & & & 579 & 528 & $-16.5 \%$ & 16.58 & 10.52 & $-12.4 \%$ \\
\hline & & & & & 579 & 422 & & 16.58 & 16.06 & \\
\hline
\end{tabular}


500 HOURS IN R-143a@ $140 \mathrm{~F}$

\begin{tabular}{|c|c|c|c|c|c|c|c|c|c|c|}
\hline VARN & $\begin{array}{l}\text { TWISTED } \\
\text { PAIR WT }\end{array}$ & $\begin{array}{c}\text { EXPT } \\
\text { PAIR WT }\end{array}$ & $\begin{array}{c}\text { WT \% } \\
\text { CHANGE }\end{array}$ & $\begin{array}{l}\text { EXP } \\
\text { VIS }\end{array}$ & BASE DIE & EXPDIE & $\begin{array}{c}\text { DIE \% } \\
\text { CHANGE }\end{array}$ & $\begin{array}{c}\text { BASE } \\
\text { BURN } \\
\text { OUT }\end{array}$ & $\begin{array}{l}\text { EXP } \\
\text { BURN } \\
\text { OUT }\end{array}$ & $\begin{array}{c}\text { BRNOUT } \\
\% \\
\text { CHANGE }\end{array}$ \\
\hline \multirow{3}{*}{$\begin{array}{l}\text { U-475 } \\
\text { A } 1\end{array}$} & \multirow[t]{3}{*}{23.3173} & \multirow[t]{3}{*}{23.3265} & \multirow[t]{3}{*}{$0.039 \%$} & $\mathrm{~N} / \mathrm{C}$ & 16.24 & 20.00 & & 430 & 371 & \\
\hline & & & & & 16.24 & 20.00 & $12.58 \%$ & 430 & 368 & $-22.25 \%$ \\
\hline & & & & & 16.24 & 14.85 & & 430 & 264 & \\
\hline \multirow{3}{*}{$\begin{array}{l}Y-390 \\
B 1\end{array}$} & \multirow[t]{3}{*}{22.6923} & \multirow[t]{3}{*}{22.6999} & \multirow[t]{3}{*}{$0.033 \%$} & $\mathrm{~N} / \mathrm{C}$ & 18.77 & 20.00 & & 510 & 460 & \\
\hline & & & & & 18.77 & 14.09 & $-23.92 \%$ & 510 & 421 & $-7.78 \%$ \\
\hline & & & & & 18.77 & 8.75 & & 510 & 530 & \\
\hline \multirow{3}{*}{$\begin{array}{l}\text { ER-610 } \\
\text { C1 }\end{array}$} & \multirow[t]{3}{*}{22.5762} & \multirow[t]{3}{*}{22.5798} & \multirow[t]{3}{*}{$0.016 \%$} & $\mathrm{~N} / \mathrm{C}$ & 15.57 & 13.04 & & 442 & 366 & \\
\hline & & & & & 15.57 & 13.09 & $-9.51 \%$ & 442 & 391 & $-14.48 \%$ \\
\hline & & & & & 15.57 & 16.14 & & 442 & 377 & \\
\hline \multirow{3}{*}{$\begin{array}{l}Y-833 \\
D 1\end{array}$} & \multirow[t]{3}{*}{21.2975} & \multirow[t]{3}{*}{21.3001} & \multirow[t]{3}{*}{$0.012 \%$} & $\mathrm{~N} / \mathrm{C}$ & 12.04 & 13.29 & & 578 & 555 & \\
\hline & & & & & 12.04 & 10.09 & $-7.34 \%$ & 578 & 567 & $-4.27 \%$ \\
\hline & & & & & 12.04 & 10.09 & & 578 & 538 & \\
\hline \multirow{3}{*}{$\begin{array}{l}923 \\
\text { E1 }\end{array}$} & \multirow[t]{3}{*}{22.6822} & \multirow[t]{3}{*}{22.6943} & \multirow[t]{3}{*}{$0.053 \%$} & $\mathrm{~N} / \mathrm{C}$ & 16.76 & broke & & 606 & 452 & \\
\hline & & & & & 16.76 & 20.00 & $19.33 \%$ & 606 & 405 & $-25.91 \%$ \\
\hline & & & & & 16.76 & 20.00 & & 606 & 490 & \\
\hline$M \longdiv { 1 S O - 8 0 0 }$ & 21.5037 & 21.5047 & $0.005 \%$ & $\mathrm{~N} / \mathrm{C}$ & 19.08 & 20.00 & & 580 & 481 & \\
\hline I $\mid F 1$ & & & & & 19.08 & 20.00 & $4.82 \%$ & 580 & 511 & $-10.69 \%$ \\
\hline D & & & & & 19.08 & 20.00 & & 580 & 562 & \\
\hline$E$ & 24 HOUR & SAT $302 \mathrm{~F}$ & & & & & & & & \\
\hline$U-475$ & 23.4289 & 23.4304 & $0.006 \%$ & $\mathrm{~N} / \mathrm{C}$ & 16.24 & 20.00 & & 430 & 369 & \\
\hline $1 \mathrm{~A} 2$ & & & & & 16.24 & 20.00 & $23.15 \%$ & 430 & 378 & $.19 .84 \%$ \\
\hline$M$ & & & & & 16.24 & 20.00 & & 430 & 287 & \\
\hline$1 \longdiv { Y - 3 9 0 }$ & 21.7695 & 21.7706 & $0.005 \%$ & $\mathrm{~N} / \mathrm{C}$ & 18.77 & 20.00 & & 510 & 485 & \\
\hline$D B 2$ & & & & & 18.77 & 20.00 & $6.55 \%$ & 510 & 449 & $-4.44 \%$ \\
\hline$E$ & & & & & 18.77 & 20.00 & & 510 & 528 & \\
\hline ER-610 & 22.1502 & 22.1520 & $0.008 \%$ & $\mathrm{~N} / \mathrm{C}$ & 15.57 & 15.94 & & 442 & 384 & \\
\hline $\mathrm{C} 2$ & & & & & 15.57 & 15.50 & $-4.73 \%$ & 442 & 380 & $-12.97 \%$ \\
\hline & & & & & 15.57 & 13.06 & & 442 & 390 & \\
\hline$Y .833$ & 21.1923 & 21.1946 & $0.011 \%$ & $\mathrm{~N} / \mathrm{C}$ & 12.04 & 11.01 & & 578 & 528 & \\
\hline D2 & & & & & 12.04 & 11.89 & $-0.14 \%$ & 578 & 494 & $-14.07 \%$ \\
\hline & & & & & 12.04 & 13.17 & & 578 & 468 & \\
\hline 923 & 22.5625 & 22.5659 & $0.015 \%$ & $N / C$ & 16.76 & broke & & 606 & 472 & \\
\hline E2 & & & & & 16.76 & 20.00 & $19.33 \%$ & 606 & 502 & $-19.80 \%$ \\
\hline & & & & & 16.76 & 20.00 & & 606 & 484 & \\
\hline $150-800$ & 21.9676 & 21.9696 & $0.009 \%$ & $\mathrm{~N} / \mathrm{C}$ & 19.08 & 14.45 & & 580 & 534 & \\
\hline F2 & & & & & 19.08 & 16.01 & $-19.16 \%$ & 580 & 460 & $-10.63 \%$ \\
\hline & & & & & 19.08 & 15.81 & & 580 & 561 & \\
\hline
\end{tabular}




\begin{tabular}{|c|c|c|c|c|c|c|c|c|c|c|c|}
\hline \multicolumn{12}{|c|}{500 HOURS IN R-143a@140F } \\
\hline & VARN & $\begin{array}{l}\text { TWISTED } \\
\text { PAIR WT }\end{array}$ & $\begin{array}{c}\text { EXPT } \\
\text { PAIR WT }\end{array}$ & $\begin{array}{c}\text { WT \% } \\
\text { CHANGE }\end{array}$ & $\begin{array}{l}\text { EPP } \\
\text { VIS }\end{array}$ & $\begin{array}{c}\text { BASE } \\
\text { DIE }\end{array}$ & $\begin{array}{l}\text { EXP } \\
\text { DIE }\end{array}$ & $\begin{array}{c}\text { DIE \% } \\
\text { CHANGE }\end{array}$ & $\begin{array}{c}\text { BASE } \\
\text { BUPN } \\
\text { OUT }\end{array}$ & $\begin{array}{c}\text { EXP } \\
\text { BURN } \\
\text { OUT }\end{array}$ & $\begin{array}{c}\text { BRNOUT } \\
\% \\
\text { CHANGE }\end{array}$ \\
\hline \multirow{3}{*}{\begin{tabular}{l|l}
$P$ \\
0
\end{tabular}} & \multirow{3}{*}{$\begin{array}{l}\text { U-475 } \\
\text { A1 }\end{array}$} & \multirow[t]{3}{*}{22.4180} & \multirow[t]{3}{*}{22.4280} & \multirow[t]{3}{*}{$0.04 \%$} & $\mathrm{~N} / \mathrm{C}$ & 13.32 & 12.58 & & 746 & 719 & \\
\hline & & & & & & 13.32 & 10.81 & $-13.11 \%$ & 746 & 730 & $-4.83 \%$ \\
\hline & & & & & & 13.32 & 11.33 & & 746 & 681 & \\
\hline \multirow{3}{*}{$\begin{array}{l}Y \\
E \\
S\end{array}$} & \multirow{3}{*}{$\begin{array}{l}Y-390 \\
B 1\end{array}$} & \multirow[t]{3}{*}{24.4351} & \multirow[t]{3}{*}{24.4480} & \multirow[t]{3}{*}{$0.05 \%$} & $\mathrm{~N} / \mathrm{C}$ & 12.28 & 10.69 & & 755 & 740 & \\
\hline & & & & & & 12.28 & 12.55 & $-5.37 \%$ & 755 & 736 & $-2.56 \%$ \\
\hline & & & & & & 12.28 & broke & & 755 & 731 & \\
\hline \multirow{3}{*}{\multicolumn{2}{|c|}{$\begin{array}{ll}T & \text { ER-610 } \\
\text { E } & C 1 \\
R & \\
\end{array}$}} & \multirow{3}{*}{22.8381} & \multirow[t]{3}{*}{22.8474} & \multirow[t]{3}{*}{$0.04 \%$} & $\mathrm{~N} / \mathrm{C}$ & 12.73 & 10.80 & & 734 & 642 & \\
\hline & & & & & & 12.73 & 13.04 & $-4.95 \%$ & 734 & 728 & $-4.77 \%$ \\
\hline & & & & & & 12.73 & 12.46 & & 734 & 727 & \\
\hline \multirow{3}{*}{$\begin{array}{l}P \\
O\end{array}$} & $\overline{Y-833}$ & \multirow[t]{3}{*}{21.4211} & \multirow[t]{3}{*}{21.4328} & \multirow[t]{3}{*}{$0.05 \%$} & $\mathrm{~N} / \mathrm{C}$ & 12.49 & 11.95 & & 734 & 706 & \\
\hline & \multirow{2}{*}{ D1 } & & & & & 12.49 & 11.38 & $-7.21 \%$ & 734 & 698 & $-7.08 \%$ \\
\hline & & & & & & 12.49 & 11.44 & & 734 & 642 & \\
\hline \multirow{3}{*}{$\begin{array}{l}L \\
Y \\
A\end{array}$} & \multirow{3}{*}{$\begin{array}{l}923 \\
E 1\end{array}$} & \multirow[t]{3}{*}{22.3550} & \multirow[t]{3}{*}{22.3653} & $0.05 \%$ & $\mathrm{~N} / \mathrm{C}$ & 14.38 & 11.65 & & 742 & 726 & \\
\hline & & & & & & 14.38 & 11.40 & $-19.82 \%$ & 742 & 726 & $-2.25 \%$ \\
\hline & & & & & & 14.38 & 11.54 & & 742 & 724 & \\
\hline$M$ & $150-800$ & 23.3855 & 23.3542 & $-0.13 \%$ & $\mathrm{~N} / \mathrm{C}$ & 12.29 & 12.02 & & 747 & 726 & \\
\hline 1 & $\mathrm{~F} 1$ & & & & & 12.29 & 12.47 & $1.95 \%$ & 747 & 730 & $-2.28 \%$ \\
\hline$D$ & & & & & & 12.29 & 13.10 & & 747 & 734 & \\
\hline$E$ & & R-143a --> & 24 hours & @ $302 F$ & & & & & & & \\
\hline & U-475 & 22.4680 & 22.4703 & $0.01 \%$ & $\mathrm{~N} / \mathrm{C}$ & 13.32 & 13.62 & & 746 & 683 & \\
\hline 1 & A2 & & & & & 13.32 & 13.46 & $-0.38 \%$ & 746 & 712 & $-5.54 \%$ \\
\hline$M$ & & & & & & 13.32 & \begin{tabular}{|l|}
12.73 \\
\end{tabular} & & 746 & 719 & \\
\hline 1 & $Y-390$ & 22.7931 & 22.7973 & $0.02 \%$ & $\mathrm{~N} / \mathrm{C}$ & 12.28 & 12.60 & & 755 & 734 & \\
\hline$D$ & $B ?$ & & & & & 12.28 & 13.11 & $1.19 \%$ & 755 & 738 & $-2.52 \%$ \\
\hline$E$ & & & & & & 12.28 & 11.57 & & 755 & 741 & \\
\hline & ER-610 & 22.7974 & 22.8029 & $0.02 \%$ & $\mathrm{~N} / \mathrm{C}$ & 12.73 & 11.32 & & 734 & 732 & \\
\hline$E$ & $\mathrm{C} 2$ & & & & & 12.73 & 10.47 & $-14.64 \%$ & 734 & 730 & $-0.59 \%$ \\
\hline$P$ & & & & & & 12.73 & 10.81 & & 734 & 727 & \\
\hline 0 & $Y-833$ & 21.9966 & 21.9925 & $-0.02 \%$ & $\mathrm{~N} / \mathrm{C}$ & 12.49 & 10.91 & & 734 & 683 & \\
\hline$x$ & D2 & & & & & 12.49 & 10.65 & $-10.73 \%$ & 734 & 718 & $-5.59 \%$ \\
\hline$Y$ & & & & & & 12.49 & 11.89 & & 734 & 678 & \\
\hline & 923 & 22.5370 & 22.5405 & $0.02 \%$ & $\mathrm{~N} / \mathrm{C}$ & 14.38 & 11.92 & & 742 & 725 & \\
\hline$G$ & E2 & & & & & 14.38 & 12.18 & $-16.09 \%$ & 742 & 730 & $-2.29 \%$ \\
\hline$L$ & & & & & & 14.38 & \begin{tabular}{|l|}
12.10 \\
\end{tabular} & & 742 & 727 & \\
\hline$A$ & $150-800$ & 23.6019 & 23.6012 & $0.00 \%$ & & 12.29 & \begin{tabular}{|l|}
12.43 \\
\end{tabular} & & 747 & 662 & \\
\hline$s$ & & & & & $\mathrm{~N} / \mathrm{C}$ & 12.29 & \begin{tabular}{|l|}
12.57 \\
\end{tabular} & $-1.44 \%$ & 747 & 664 & $-11.24 \%$ \\
\hline$S$ & & & & & & 12.29 & 11.34 & & 747 & 651 & \\
\hline
\end{tabular}




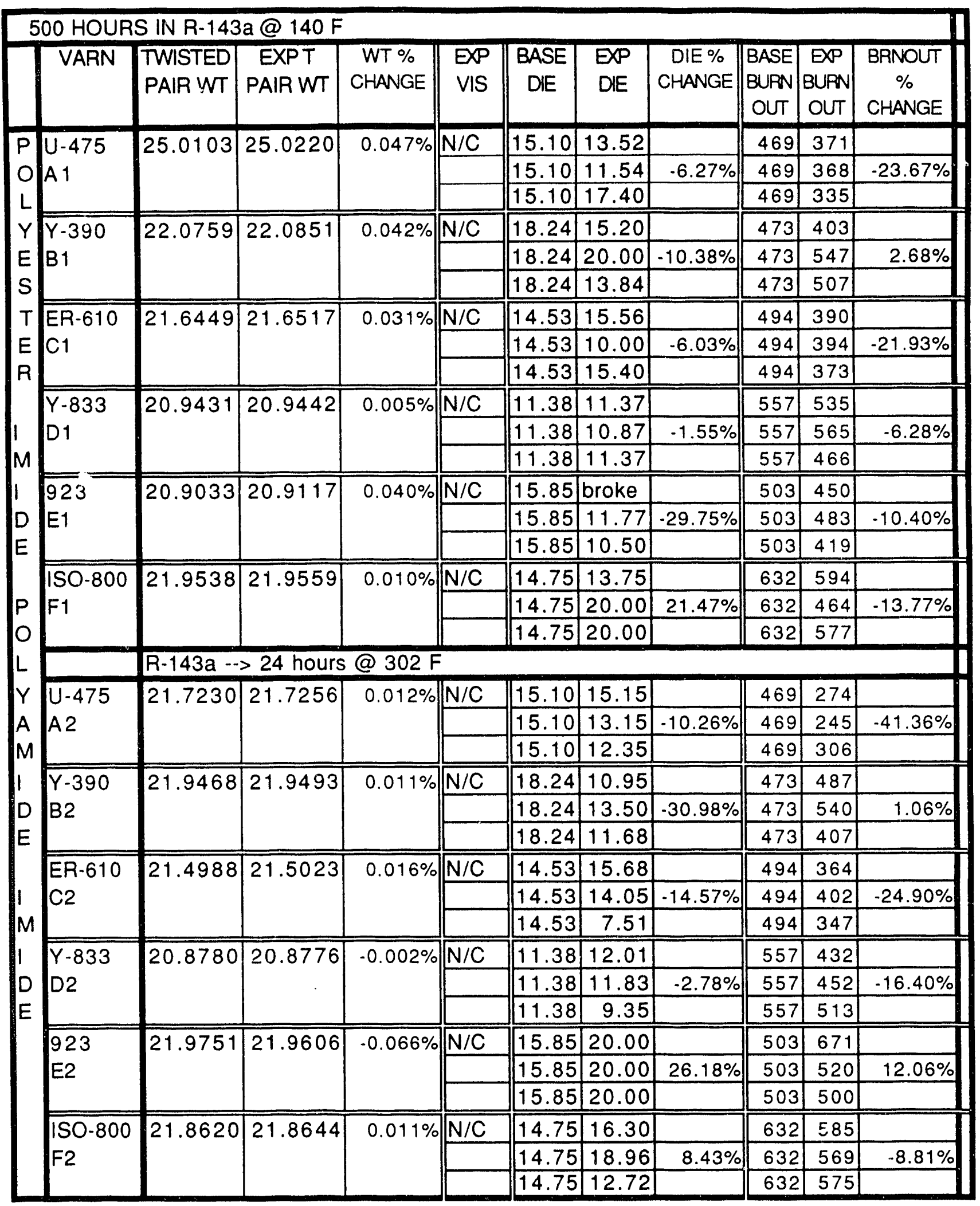




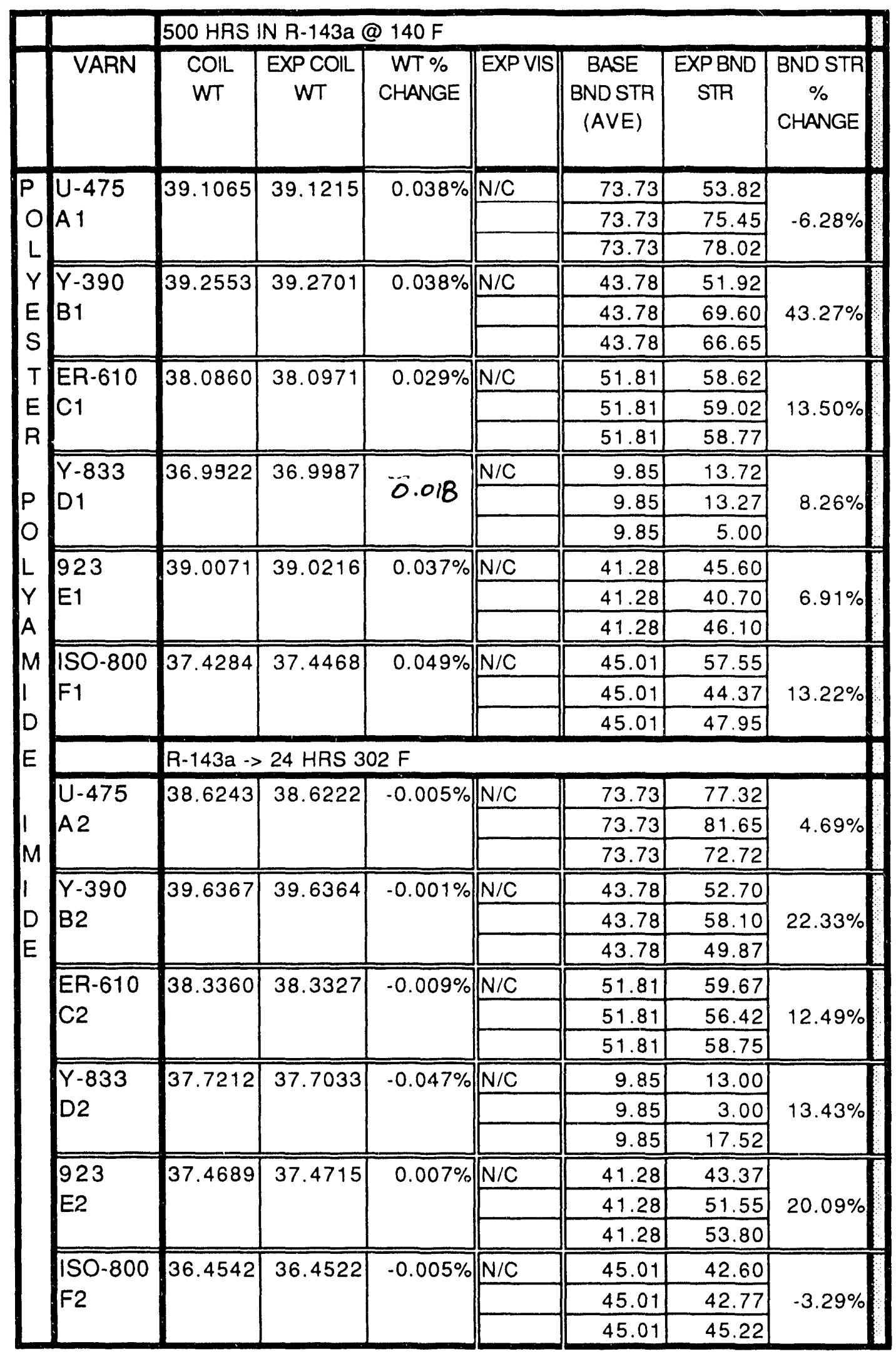


500 HRS IN R-143a @ 140 F

\begin{tabular}{|c|c|c|c|c|c|c|c|c|}
\hline & VARN & $\begin{array}{l}\text { COIL } \\
\text { WT }\end{array}$ & $\begin{array}{c}\text { EXP COIL } \\
W T\end{array}$ & $\begin{array}{c}\text { WT \% } \\
\text { CHANGE }\end{array}$ & EXP VIS & $\begin{array}{c}\text { BASE } \\
\text { BND STR } \\
\text { (AVE) }\end{array}$ & $\begin{array}{c}\text { EXPBND } \\
\text { STR }\end{array}$ & $\begin{array}{c}\text { BND STR } \\
\% \\
\text { CHANGE }\end{array}$ \\
\hline \multirow{3}{*}{$\begin{array}{l}P \\
O \\
L\end{array}$} & \multirow{3}{*}{$\begin{array}{l}\text { U-475 } \\
\text { A } 1\end{array}$} & \multirow[t]{3}{*}{36.9782} & \multirow[t]{3}{*}{36.9946} & \multirow[t]{3}{*}{$0.044 \%$} & $\mathrm{~N} / \mathrm{C}$ & 40.14 & 30.42 & \multirow{3}{*}{$-15.35 \%$} \\
\hline & & & & & & 40.14 & 35.95 & \\
\hline & & & & & & 40.14 & 35.57 & \\
\hline \multirow{3}{*}{\multicolumn{2}{|c|}{$\begin{array}{l}Y-390 \\
B 1\end{array}$}} & \multirow[t]{3}{*}{37.8674} & \multirow[t]{3}{*}{37.9066} & \multirow[t]{3}{*}{$0.104 \%$} & $\mathrm{~N} / \mathrm{C}$ & 36.12 & 31.75 & \multirow{3}{*}{$-2.28 \%$} \\
\hline & & & & & & 36.12 & 36.47 & \\
\hline$S$ & & & & & & 36.12 & 37.67 & \\
\hline \multirow{3}{*}{$\begin{array}{l}T \\
E \\
R\end{array}$} & \multirow{3}{*}{$\mathrm{C} 1$} & \multirow[t]{3}{*}{36.6769} & \multirow[t]{3}{*}{36.6698} & \multirow[t]{3}{*}{$-0.019 \%$} & $\mathrm{~N} / \mathrm{C}$ & 35.96 & 35.87 & \multirow{3}{*}{$-0.94 \%$} \\
\hline & & & & & & 35.96 & 33.85 & \\
\hline & & & & & & 35.96 & 37.15 & \\
\hline \multirow{3}{*}{$\begin{array}{l}P \\
O\end{array}$} & $Y-833$ & \multirow[t]{3}{*}{36.8205} & \multirow[t]{3}{*}{36.8364} & \multirow[t]{3}{*}{$0.043 \%$} & $\mathrm{~N} / \mathrm{C}$ & 33.14 & 3 & \multirow{3}{*}{$2.50 \%$} \\
\hline & \multirow{2}{*}{ D1 } & & & & & 33.14 & 33.97 & \\
\hline & & & & & & 33.14 & .2 & \\
\hline \multirow{3}{*}{$\begin{array}{l}L \\
Y \\
A\end{array}$} & 923 & \multirow[t]{3}{*}{37.2599} & \multirow[t]{3}{*}{37.2741} & $0.038 \%$ & $\mathrm{~N} / \mathrm{C}$ & 40.52 & 33.25 & \\
\hline & E1 & & & & & 40.52 & 33.45 & $-15.99 \%$ \\
\hline & & & & & & 40.52 & 35.42 & \\
\hline$M$ & ISO-800 & 35.9889 & 36.0120 & $0.064 \%$ & $\mathrm{~N} / \mathrm{C}$ & 20.20 & 17.25 & \\
\hline 1 & $\mathrm{~F} 1$ & & & & & 20.20 & 19.85 & $-7.06 \%$ \\
\hline$D$ & & & & & & 20.20 & 19.22 & \\
\hline E & & R-143a -> & 24 HRS 3 & $02 \mathrm{~F}$ & & & & \\
\hline & $U-475$ & 37.4278 & 37.4299 & $0.006 \%$ & $\mathrm{~N} / \mathrm{C}$ & 40.14 & 34.67 & \\
\hline 1 & A2 & & & & & 40.14 & 34.42 & $-12.69 \%$ \\
\hline$M$ & & & & & & 40.14 & 36.05 & \\
\hline 1 & $Y-390$ & 37.0193 & 37.0215 & $0.006 \%$ & Varnish & 36.12 & 33.80 & \\
\hline$D$ & $B 2$ & & & & Pockets & 36.12 & 31.62 & $.11 .18 \%$ \\
\hline$E$ & & & & & & 36.12 & 30.82 & \\
\hline & ER-610 & 37.8022 & 37.8032 & $0.003 \%$ & $\mathrm{~N} / \mathrm{C}$ & 35.96 & 37.07 & \\
\hline$E$ & C2 & & & & & 35.96 & 34.67 & $-1.69 \%$ \\
\hline$P$ & & & & & & 35.96 & 34.32 & \\
\hline 0 & $Y-833$ & 36.9060 & 36.9001 & $-0.016 \%$ & $\mathrm{~N} / \mathrm{C}$ & 33.14 & 34.55 & \\
\hline$x$ & D2 & & & & & 33.14 & 40.25 & $12.85 \%$ \\
\hline$Y$ & & & & & & 33.14 & $* 2$ & \\
\hline & 923 & 37.2280 & 37.2297 & $0.005 \%$ & $\mathrm{~N} / \mathrm{C}$ & 40.52 & 33.12 & \\
\hline$G$ & E2 & & & & & 40.52 & 34.10 & $-15.91 \%$ \\
\hline $\mathrm{L}$ & & & & & & 40.52 & 35.00 & \\
\hline A & ISO-800 & 36.8279 & 36.8258 & $-0.006 \%$ & $\mathrm{~N} / \mathrm{C}$ & 20.20 & 18.42 & \\
\hline 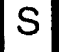 & & & & & & 20.20 & 13.95 & $-25.30 \%$ \\
\hline$S$ & & & & & & 20.20 & 12.90 & \\
\hline
\end{tabular}

L-6

*Bond strength not included in average 


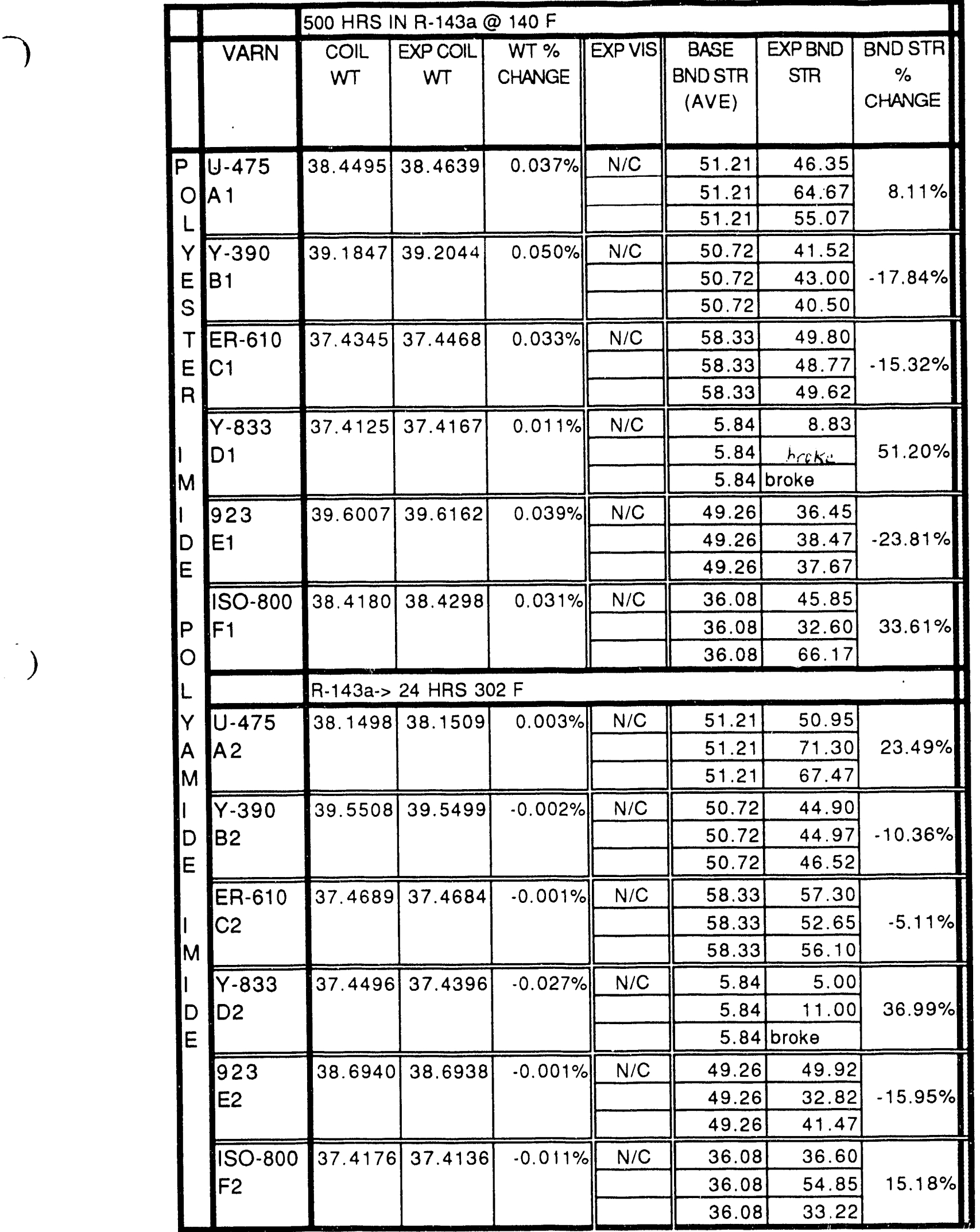




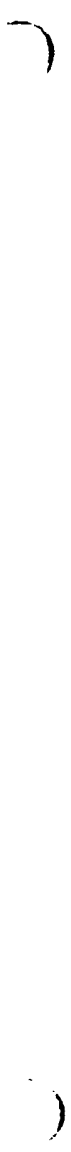

\begin{tabular}{|c|c|c|c|c|c|c|}
\hline & 500 HRS IN & $\overline{43}$ & & & & \\
\hline ID & $\overline{W T}$ & EXPWT & $\begin{array}{c}\text { WT \% } \\
\text { CHANGE }\end{array}$ & EXPVIS & EXPFLEX & \\
\hline A1 & 4.5234 & 4.5264 & $0.066 \%$ & $\mathrm{~N} / \mathrm{C}$ & & \\
\hline & & & & & YES & \\
\hline & & & & & & \\
\hline B1 & 5.3852 & 5.3919 & $0.124 \%$ & $\mathrm{~N} / \mathrm{C}$ & & \\
\hline & & & & & YES & \\
\hline lc1 & 5050 & 5020 & $0728 \%$ & Nic & & \\
\hline & 0.0300 & 0.0546 & & & YYES & \\
\hline & & & & & & \\
\hline & $R-143 a \rightarrow$ & $302 \mathrm{~F} 24 \mathrm{HI}$ & & & & \\
\hline A2 & 4.5243 & 4.5259 & $0.035 \%$ & $\mathrm{~N} / \mathrm{C}$ & & \\
\hline & & & & & YES & \\
\hline & & & & & & \\
\hline$\overline{B 2}$ & 5.3884 & 5.3905 & $0.039 \%$ & $\mathrm{~N} / \mathrm{C}$ & & \\
\hline & & & & & YES & \\
\hline $\mathrm{C} 2$ & 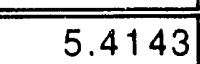 & 5.4141 & $-0.004 \%$ & $\mathrm{~N} / \mathrm{C}$ & & \\
\hline & & & & & YES & \\
\hline & & & & & & \\
\hline
\end{tabular}

) 


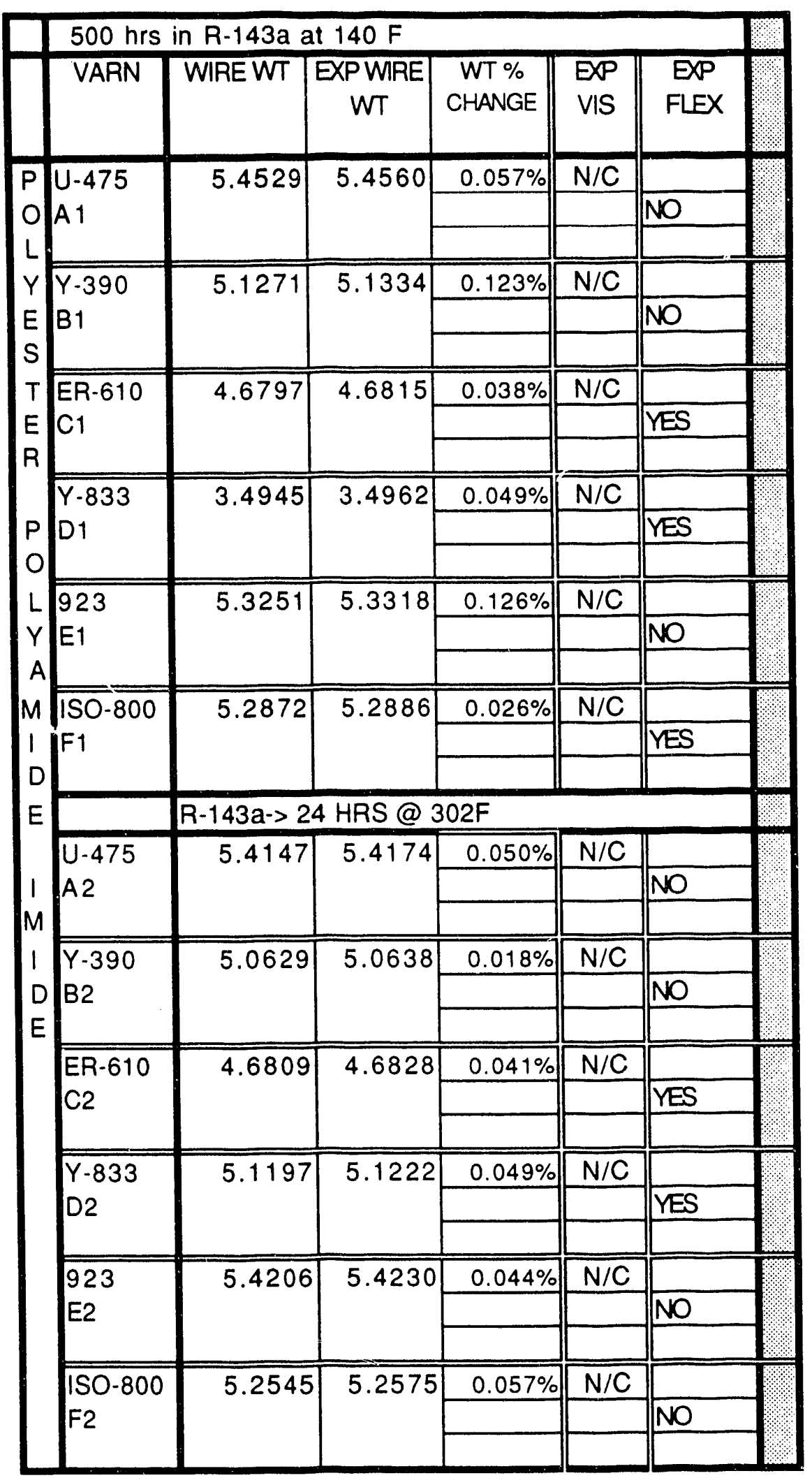




\begin{tabular}{|c|c|c|c|c|c|c|c|}
\hline & \multicolumn{6}{|c|}{$\mathrm{R}-143 \mathrm{a}$ at $140 \mathrm{~F}$} & \\
\hline & VARN & WIREWT & $\begin{array}{c}\text { EXPWRE } \\
W T\end{array}$ & $\begin{array}{l}\text { WT \% } \\
\text { CHANGE }\end{array}$ & EXPVIS| & $\begin{array}{l}\text { EXP } \\
\text { FLEX }\end{array}$ & \\
\hline & \multirow{3}{*}{$\begin{array}{l}\text { U-475 } \\
\text { A } 1\end{array}$} & \multirow[t]{3}{*}{5.2250} & \multirow[t]{3}{*}{5.2289} & $0.075 \%$ & $\mathrm{~N} / \mathrm{C}$ & & \\
\hline & & & & & & NO & \\
\hline & & & & & & & \\
\hline \multirow{3}{*}{$\begin{array}{l}Y \\
E \\
S\end{array}$} & \multirow{2}{*}{$\begin{array}{l}Y-390 \\
B 1\end{array}$} & \multirow[t]{2}{*}{5.4727} & \multirow[t]{2}{*}{5.4768} & $0.075 \%$ & $\mathrm{~N} / \mathrm{C}$ & & \\
\hline & & & & & & NO & \\
\hline & \multirow{3}{*}{$\begin{array}{l}\text { ER-610 } \\
\text { C1 }\end{array}$} & \multirow{3}{*}{5.2696} & \multirow{3}{*}{5.2726} & & & & \\
\hline \multirow{2}{*}{$\begin{array}{lll}T & E \\
E & C\end{array}$} & & & & $0.057 \%$ & $\mathrm{~N} / \mathrm{C}$ & & \\
\hline & & & & & & YES & \\
\hline \multirow{3}{*}{$\begin{array}{l}P \\
0\end{array}$} & \multirow{3}{*}{$\begin{array}{l}Y-833 \\
D 1\end{array}$} & \multirow[t]{3}{*}{5.1963} & \multirow[t]{3}{*}{5.1985} & $0.042 \%$ & $\mathrm{~N} / \mathrm{C}$ & & \\
\hline & & & & & & YES & \\
\hline & & & & & & & \\
\hline \multirow{3}{*}{\multicolumn{2}{|c|}{$\begin{array}{lll} & 923 \\
& & \\
& & \\
A & \\
\end{array}$}} & \multirow[t]{3}{*}{5.5336} & \multirow[t]{3}{*}{5.5378} & $0.076 \%$ & $\mathrm{~N} / \mathrm{C}$ & & \\
\hline & & & & & & NO & \\
\hline & & & & & & & \\
\hline \multirow{2}{*}{$\begin{array}{l}M \\
1 \\
D\end{array}$} & \multirow{2}{*}{$\begin{array}{l}\text { ISO-800 } \\
\mathrm{F} 1\end{array}$} & \multirow[t]{2}{*}{5.7690} & \multirow[t]{2}{*}{5.7717} & $0.047 \%$ & $\mathrm{~N} / \mathrm{C}$ & & \\
\hline & & & & & & NO & \\
\hline \multirow[t]{2}{*}{ E } & & \multicolumn{5}{|c|}{$R-143 a->24 H R S @ 302 F$} & \\
\hline & & 5.1490 & 5.1502 & $0.024 \%$ & $\mathrm{~N} / \mathrm{C}$ & & \\
\hline 1 & A2 & & & & & NO & \\
\hline M & 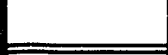 & & & & & & \\
\hline 1 & $Y-390$ & 5.5616 & 5.56 .33 & $0.031 \%$ & $\mathrm{~N} / \mathrm{C}$ & & \\
\hline$D$ & B2 & & & & & NO & \\
\hline 다 & FR-610 & 52221 & 52254 & & & & \\
\hline $\mathrm{E}$ & & & & & & YES & \\
\hline$P$ & & & & & & & \\
\hline 0 & $Y-833$ & 5.1738 & 5.1726 & $-0.023 \%$ & $\mathrm{~N} / \mathrm{C}$ & & \\
\hline$x \mid$ & D2 & & & & & YES & \\
\hline$Y$ & & & & & & & \\
\hline & 923 & 5.4839 & 5.4874 & $0.064 \%$ & $\mathrm{~N} / \mathrm{C}$ & & \\
\hline G & E2 & & & & & NO & \\
\hline 1 & & & & & & & \\
\hline A & ISO-800 & 5.8020 & 5.8043 & $0.040 \%$ & $\mathrm{~N} / \mathrm{C}$ & & \\
\hline S & & & & & & NNo & \\
\hline
\end{tabular}

) 


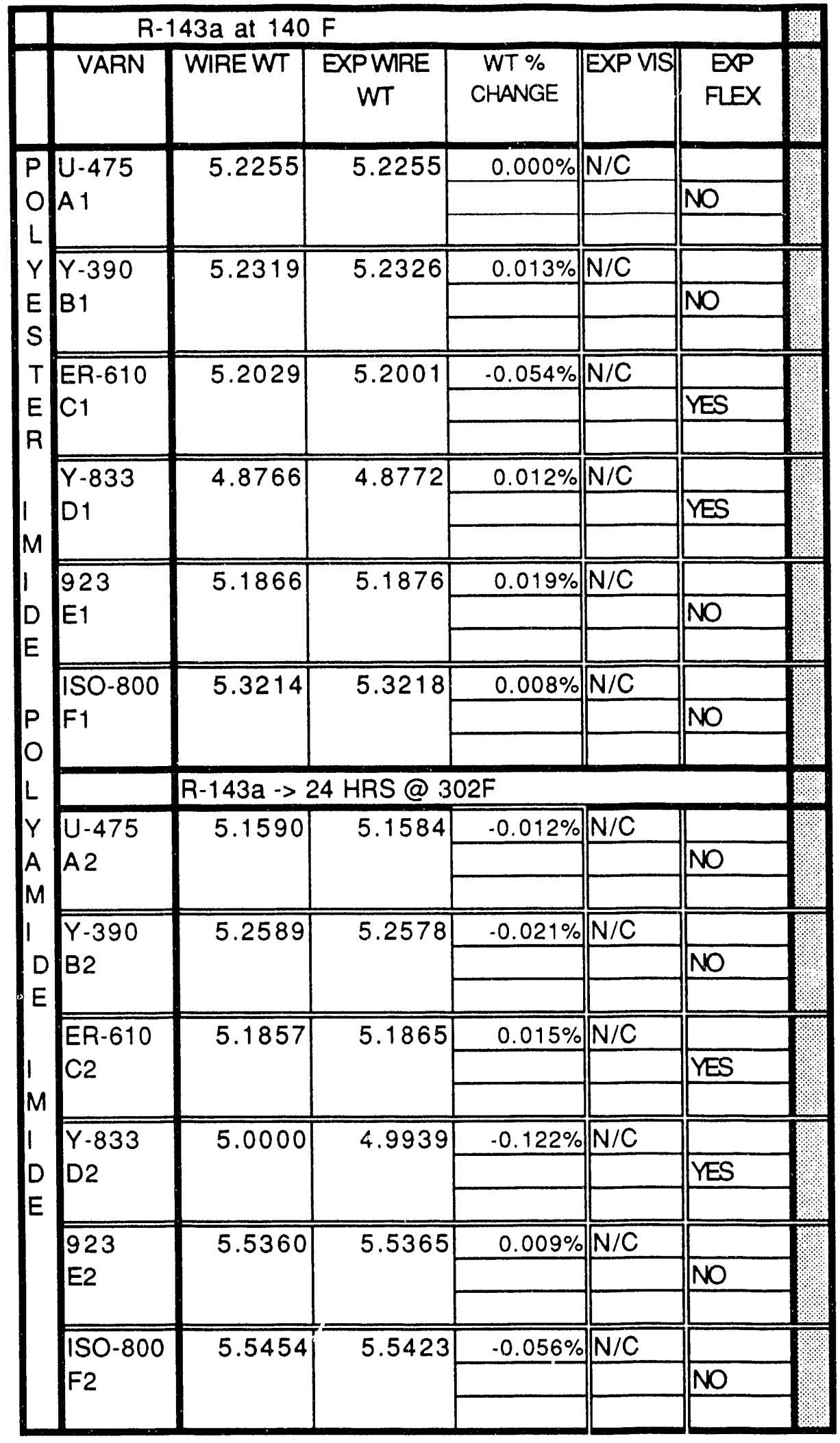




\begin{tabular}{|c|c|c|c|c|c|}
\hline & 500 HRS IN & R-143a@1 & & & \\
\hline ID & WT & EXPWT & $\begin{array}{l}\text { WT \% } \\
\text { CHANGE }\end{array}$ & EXPVIS & EXPFLEX \\
\hline $\begin{array}{l}\text { U-475 } \\
\text { A } 1\end{array}$ & 1.8987 & 1.9215 & $1.20 \%$ & $\frac{\text { Slightly }}{\text { Warped }}$ & $\mathrm{N} / \mathrm{C}$ \\
\hline$\overline{Y-390}$ & 1.7599 & 1.7748 & $0.85 \%$ & Warped & $\mathrm{N} / \mathrm{C}$ \\
\hline B1 & & & & & \\
\hline ER-610 & 2.3821 & 2.3922 & $0.42 \%$ & $\overline{i v / C}$ & $\mathrm{~N} / \mathrm{C}$ \\
\hline & & & & & \\
\hline$\overline{Y-833}$ & 2.0257 & 2.1295 & $5.12 \%$ & $\overline{\mathrm{N} / \mathrm{C}}$ & $\mathrm{N} / \mathrm{C}$ \\
\hline D1 & & & & & \\
\hline 923 & 1.5000 & 1.5250 & $1.67 \%$ & $\mathrm{~N} / \mathrm{C}$ & $\mathrm{N} / \mathrm{C}$ \\
\hline E1 & & & & & \\
\hline & & & & & \\
\hline $150-800$ & 1.7347 & 1.7368 & $0.12 \%$ & Slightly & $\mathrm{N} / \mathrm{C}$ \\
\hline & & & & warped & \\
\hline & $\mathrm{R}-143 a$ at & $302 \mathrm{~F} 24 \mathrm{HF}$ & & & \\
\hline$U-475$ & 1.9587 & 1.9500 & $-0.44 \%$ & Darkened & $N / C$ \\
\hline A2 & & & & Slightly & \\
\hline & & & & Wamped & \\
\hline$\overline{Y-390}$ & 1.6945 & 1.6811 & $-0.79 \%$ & Warped & $\mathrm{N} / \mathrm{C}$ \\
\hline & & & & & \\
\hline ER-610 & 2.6742 & 2.6679 & $\mid-0.24 \%$ & Darkened & $\mathrm{N} / \mathrm{C}$ \\
\hline & & & & & \\
\hline$\overline{Y-833}$ & 2.4564 & 2.4614 & $0.20 \%$ & $\mathrm{~N} / \mathrm{C}$ & $\mathrm{N} / \mathrm{C}$ \\
\hline D2 & & & & & \\
\hline 923 & 1.8952 & 1.8950 & $-0.01 \%$ & $\mathrm{~N} / \mathrm{C}$ & $\mathrm{N} / \mathrm{C}$ \\
\hline E2 & & & & & \\
\hline & 15616 & 15324 & $-187 \%$ & Warped & $1 / \mathrm{N} / \mathrm{C}$ \\
\hline $\begin{array}{l}\text { SO-800 } \\
\text { F2 }\end{array}$ & 1.5616 & 1.5324 & $-1.81 \%$ & |Warped & \\
\hline & & & & & \\
\hline
\end{tabular}




\begin{tabular}{|c|c|c|c|c|c|c|c|c|c|}
\hline \multicolumn{10}{|c|}{500 HR IN R-143a@140 F } \\
\hline ID & WT & EXPWT & $\begin{array}{c}\text { WT \% } \\
\text { CHANGE }\end{array}$ & $\begin{array}{l}\text { SAMPLE } \\
\text { WIDTH }\end{array}$ & $\begin{array}{l}\text { BREAK } \\
\text { LOAD }\end{array}$ & $\begin{array}{c}\text { SAMPLE } \\
\text { THICKNESS } \\
\text { Mils }\end{array}$ & $\begin{array}{c}\text { TENSILE } \\
\text { STR } \\
\text { BASE }\end{array}$ & $\begin{array}{l}\text { TENSILE } \\
\text { STREXP }\end{array}$ & $\begin{array}{c}\% \\
\text { CHANGE } \\
\text { TENSILE }\end{array}$ \\
\hline \multirow{3}{*}{$\begin{array}{l}\text { NO/MY/NO } \\
\text { A1 }\end{array}$} & \multirow[t]{3}{*}{4.7438} & \multirow[t]{3}{*}{4.8079} & \multirow[t]{3}{*}{$1.35 \%$} & 0.490 & 150.50 & 21 & 17.4 & 14.63 & \\
\hline & & & & 0.387 & 141.50 & 21 & 17.4 & 17.41 & $-5.91 \%$ \\
\hline & & & & 0.375 & 134.50 & 21 & 17.4 & 17.08 & \\
\hline \multirow{3}{*}{$\begin{array}{l}\mathrm{DA} / \mathrm{MY} / \mathrm{DA} \\
\mathrm{B} 1\end{array}$} & \multirow[t]{3}{*}{4.2047} & \multirow[t]{3}{*}{4.2951} & \multirow[t]{3}{*}{$2.15 \%$} & 0.356 & 101.20 & 21 & 13.7 & 13.54 & \\
\hline & & & & 0.429 & 118.30 & 21 & 13.7 & 13.13 & $-2.67 \%$ \\
\hline & & & & 0.415 & 116.20 & 21 & 13.7 & 13.33 & \\
\hline \multirow{3}{*}{$\begin{array}{l}\text { MYARMO } \\
\text { C1 }\end{array}$} & \multirow{3}{*}{2.222 .1} & \multirow{3}{*}{2.2309} & \multirow[t]{3}{*}{$0.40 \%$} & 0.444 & 83.75 & 10 & 21.7 & 18.86 & \\
\hline & & & & 0.359 & 68.15 & 10 & 21.7 & 18.98 & $-15.42 \%$ \\
\hline & & & & 0.377 & 64.90 & 10 & 21.7 & 17.21 & \\
\hline \multirow{3}{*}{$\begin{array}{l}\text { NO } 410 \\
\text { D1 }\end{array}$} & \multirow[t]{3}{*}{2.2843} & \multirow[t]{3}{*}{2.3337} & \multirow[t]{3}{*}{$2.16 \%$} & 0.527 & 102.60 & 11 & 18.7 & 17.70 & \\
\hline & & & & 0.426 & 82.25 & 11 & 18.7 & 17.55 & $-6.34 \%$ \\
\hline & & & & 0.461 & 87.70 & 11 & 18.7 & 17.29 & \\
\hline \multirow{3}{*}{$\begin{array}{l}\text { NO MI } 418 \\
\text { E1 }\end{array}$} & \multirow[t]{3}{*}{2.0775} & \multirow[t]{3}{*}{2.1075} & \multirow[t]{3}{*}{$1.44 \%$} & 0.478 & 25.00 & 9 & 7.5 & 5.81 & \\
\hline & & & & 0.501 & 26.60 & 9 & 7.5 & 5.90 & $-22.59 \%$ \\
\hline & & & & 0.478 & 24.55 & 9 & 7.5 & 5.71 & \\
\hline \multirow{3}{*}{$\begin{array}{l}\text { MEL } 228 \\
\text { F1 }\end{array}$} & \multirow[t]{3}{*}{2.4215} & \multirow[t]{3}{*}{2.4332} & \multirow{3}{*}{$0.48 \%$} & 0.427 & 82.85 & 11 & 21.7 & 17.64 & \\
\hline & & & & 0.429 & 80.65 & 11 & 21.7 & 17.09 & $-20.89 \%$ \\
\hline & & & & 0.422 & 77.85 & 11 & 21.7 & 16.77 & \\
\hline & & 500 HRS & 3 IN R-14 & $3 a->24 H$ & RS@30 & $2 F$ & & & \\
\hline NO/MY/NO & 4.3650 & 4.3636 & $.0 .03 \%$ & 0.387 & $13, .00$ & 21 & 17.4 & 16.86 & \\
\hline$A 2$ & & & & 0.359 & 130.70 & 21 & 17.4 & 17.34 & $-0.40 \%$ \\
\hline & & & & 0.449 & 167.80 & 21 & 17.4 & 17.80 & \\
\hline$D A / M Y / D A$ & 4.2803 & 4.2778 & $.0 .06 \%$ & 0.434 & 121.40 & 21 & 13.7 & 13.32 & \\
\hline$B 2$ & & & & 0.453 & 133.90 & 21 & 13.7 & 14.08 & $-3.05 \%$ \\
\hline & & & & 0.392 & 102.50 & 21 & 13.7 & 12.45 & \\
\hline MYLAR MO & 2.1159 & 2.1171 & $0.06 \%$ & 0.471 & 97.35 & 10 & 21.7 & 20.67 & \\
\hline $\mathrm{C} 2$ & & & & 0.485 & 96.70 & 10 & 21.7 & 19.94 & $-6.76 \%$ \\
\hline & & & & 0.488 & 98.05 & 10 & 21.7 & 20.09 & \\
\hline NOMEX 410 & 2.6514 & 2.6510 & $-0.02 \%$ & 0.522 & 106.60 & 11 & 18.7 & 18.56 & \\
\hline D2 & & & & 0.509 & 91.70 & 11 & 18.7 & 16.38 & $-5.58 \%$ \\
\hline & & & & 0.493 & 97.75 & 11 & 18.7 & 18.03 & \\
\hline NO/MI 418 & 2.3856 & 2.3892 & $0.15 \%$ & 0.499 & 25.85 & 9 & 7.5 & 5.76 & \\
\hline & & & & 0.488 & 25.65 & 9 & 7.5 & 5.84 & $-21.99 \%$ \\
\hline & & & & 0.499 & 26.75 & 9 & 7.5 & 5.96 & \\
\hline MEL 228 & 2.3440 & 2.3424 & $-0.07 \%$ & 0.530 & 104.10 & 11 & 21.7 & 17.86 & \\
\hline$F 2$ & & & & 0.538 & 101.00 & 11 & 21.7 & 17.07 & $-18.44 \%$ \\
\hline & & & & 0.484 & 96.75 & 11 & 21.7 & 18.17 & \\
\hline
\end{tabular}




\begin{tabular}{|c|c|c|c|c|c|c|c|c|}
\hline \multicolumn{8}{|c|}{ After 500 hour exposure @ $140^{\circ} \mathrm{F}\left(60^{\circ} \mathrm{C}\right)$} & \multirow[b]{2}{*}{$\begin{array}{c}\text { VISUAL } \\
\text { EXP }\end{array}$} \\
\hline $\mathrm{DD}$ & $\begin{array}{c}\text { STRETCH } \\
\text { (inch) }\end{array}$ & $\begin{array}{c}\% \\
\text { ELONG }\end{array}$ & $\begin{array}{l}\text { BASE } \\
\text { ELONG } \\
\text { (AVE) }\end{array}$ & $\begin{array}{c}\text { ELONG } \\
\% \\
\text { CHANGE }\end{array}$ & $\begin{array}{c}\text { BASE } \\
\text { DIE } \\
\text { (AVE) }\end{array}$ & EXPDIE & $\begin{array}{c}\text { DIE \% } \\
\text { CHANGE }\end{array}$ & \\
\hline \multirow{3}{*}{$\begin{array}{l}\mathrm{NO} / \mathrm{MY} / \mathrm{NO} \\
\mathrm{A} 1\end{array}$} & .26 & \#\#\#\#\# & $20.0 \%$ & & $>18.97$ & $>14.23$ & & \multirow[t]{3}{*}{$\mathrm{N} / \mathrm{C}$} \\
\hline & 1.18 & $29.5 \%$ & $20.0 \%$ & $40.0 \%$ & $>18.97$ & $>14.66$ & flash & \\
\hline & 1.06 & $26.5 \%$ & $20.0 \%$ & & $>18.97$ & $>14.40$ & & \\
\hline \multirow{3}{*}{$\begin{array}{l}\text { DA/MY/DA } \\
\text { B1 }\end{array}$} & 0.56 & $28.0 \%$ & $46.0 \%$ & & $>15.27$ & $>12.60$ & & \multirow[t]{3}{*}{$\mathrm{N} / \mathrm{C}$} \\
\hline & 0.49 & $24.5 \%$ & $46.0 \%$ & $-45.3 \%$ & $>15.27$ & $>13.49$ & flash & \\
\hline & 0.46 & $23.0 \%$ & $46.0 \%$ & & $>15.27$ & $>12.69$ & & \\
\hline \multirow{3}{*}{$\begin{array}{l}\text { MYLAR MO } \\
\text { C1 }\end{array}$} & 2.74 & $137.0 \%$ & $131.0 \%$ & & $>14.91$ & $>1347$ & & \multirow[t]{3}{*}{$\mathrm{N} / \mathrm{C}$} \\
\hline & 2.96 & $148.0 \%$ & $131.0 \%$ & $-6.6 \%$ & $>14.91$ & $>11.90$ & flash & \\
\hline & 1.64 & $82.0 \%$ & $131.0 \%$ & & $\geq 14.91$ & $\geq 14.13$ & & \\
\hline \multirow{3}{*}{$\begin{array}{l}\text { NO } 410 \\
\text { D1 }\end{array}$} & 0.64 & $16.0 \%$ & $17.0 \%$ & & 10.67 & 10.62 & & \multirow[t]{3}{*}{$N / C$} \\
\hline & 0.58 & $14.5 \%$ & $17.0 \%$ & $-15.2 \%$ & 10.67 & 9.46 & $-1.3 \%$ & \\
\hline & 0.51 & $12.8 \%$ & $17.0 \%$ & & 10.67 & 11.50 & & \\
\hline \multirow{3}{*}{$\begin{array}{l}\text { NO MI } 418 \\
\text { E1 }\end{array}$} & 0.09 & $2.3 \%$ & $4.0 \%$ & & 10.23 & 9.90 & & \multirow[t]{3}{*}{$\mathrm{N} / \mathrm{C}$} \\
\hline & 0.08 & $2.0 \%$ & $4.0 \%$ & $-47.9 \%$ & 10.23 & 8.90 & $-9.7 \%$ & \\
\hline & 0.08 & $2.0 \%$ & $4.0 \%$ & & 10.23 & 8.90 & & \\
\hline \multirow{4}{*}{$\begin{array}{l}\text { MEL } 228 \\
F 1\end{array}$} & 3.32 & $166.0 \%$ & $160.0 \%$ & & $>14.22$ & $>13.31$ & & \multirow[t]{3}{*}{$\mathrm{N} / \mathrm{C}$} \\
\hline & 2.92 & $146.0 \%$ & $160.0 \%$ & $-2.6 \%$ & $>14.22$ & $>13.23$ & flash & \\
\hline & 3.11 & $155.5 \%$ & $160.0 \%$ & & $>14.22$ & $>14.49$ & & \\
\hline & \multicolumn{7}{|c|}{ After 500 hour exposure plus a 24 hour airbake at $302^{\circ} \mathrm{F}(150$} & ${ }^{\circ} \mathrm{C}$ \\
\hline \multirow{3}{*}{$\begin{array}{l}\mathrm{NO} / \mathrm{MY} / \mathrm{NO} \\
\mathrm{A} 2\end{array}$} & 0.81 & $20.3 \%$ & $20.0 \%$ & & $>18.97$ & $>12.46$ & & \multirow[t]{3}{*}{$\mathrm{N} / \mathrm{C}$} \\
\hline & 0.76 & $19.0 \%$ & $20.0 \%$ & $6.7 \%$ & $\geq 18.97$ & $>14.36$ & flash & \\
\hline & 0.99 & $24.8 \%$ & $20.0 \%$ & & $>18.97$ & $>14.34$ & & \\
\hline \multirow{3}{*}{$\begin{array}{l}D A / M Y / D A \\
B 2\end{array}$} & 0.52 & $26.0 \%$ & $46.0 \%$ & & $>15.27$ & $>13.61$ & & \multirow[t]{3}{*}{$\mathrm{N} / \mathrm{C}$} \\
\hline & 0.62 & $31.0 \%$ & $46.0 \%$ & $-42.0 \%$ & $>15.27$ & $>13.43$ & flash & \\
\hline & 0.46 & $23.0 \%$ & $46.0 \%$ & & $\geq 15.27$ & $>13.53$ & & \\
\hline \multirow{3}{*}{$\begin{array}{l}\text { MYLAR MO } \\
\text { C2 }\end{array}$} & 3.22 & $161.0 \%$ & $131.0 \%$ & & $>14.91$ & $>11.02$ & & \multirow[t]{3}{*}{$N / C$} \\
\hline & 2.81 & $140.5 \%$ & $131.0 \%$ & $16.4 \%$ & $>14.91$ & $>11.68$ & flash & \\
\hline & 3.12 & $156.0 \%$ & $131.0 \%$ & & $>14.91$ & $>13.25$ & & \\
\hline \multirow{3}{*}{$\begin{array}{l}\text { NOMEX } 410 \\
\text { D2 }\end{array}$} & 0.60 & $15.0 \%$ & $17.0 \%$ & & 10.67 & 11.44 & & \multirow[t]{3}{*}{$\mathrm{N} / \mathrm{C}$} \\
\hline & 0.36 & $9.0 \%$ & $17.0 \%$ & $-25.0 \%$ & 10.67 & 11.05 & $5.0 \%$ & \\
\hline & 0.57 & $14.3 \%$ & $17.0 \%$ & & 10.67 & 11.13 & & \\
\hline \multirow{3}{*}{$\begin{array}{l}\text { NO/MI } 418 \\
\text { E2 }\end{array}$} & 0.08 & $2.0 \%$ & $4.0 \%$ & & 10.23 & 9.15 & & \multirow[t]{3}{*}{$N / C$} \\
\hline & 0.09 & $2.3 \%$ & $4.0 \%$ & $-43.8 \%$ & 10.23 & 9.30 & $-10.3 \%$ & \\
\hline & 0.10 & $2.5 \%$ & $4.0 \%$ & & 10.23 & 9.09 & & \\
\hline MEL 228 & 2.98 & $149.0 \%$ & $160.0 \%$ & & $>14.22$ & $>14.14$ & & $\mathrm{~N} / \mathrm{C}$ \\
\hline $\mathrm{F} 2$ & 3.26 & $163.0 \%$ & $160.0 \%$ & $2.6 \%$ & $>14.22$ & $>13.55$ & flash & \\
\hline & 3.61 & $180.5 \%$ & $160.0 \%$ & & $>14.22$ & $>13.92$ & & \\
\hline
\end{tabular}


SLEEVING

\begin{tabular}{|c|c|c|c|c|}
\hline & \multicolumn{4}{|c|}{500 HRS IN R-143a @ 140 F } \\
\hline & WT & EXPWT & $\begin{array}{c}\% \\
\text { CHANGE }\end{array}$ & EXP VS \\
\hline $\begin{array}{l}\text { A } 1 \\
\text { NOMEX }\end{array}$ & 0.5480 & 0.5670 & $3.47 \%$ & $N / C$ \\
\hline $\begin{array}{l}\text { B1 } \\
\text { MYLAR }\end{array}$ & 0.5110 & 0.0191 & $1.59 \%$ & $\mathrm{~N} / \mathrm{C}$ \\
\hline $\begin{array}{l}\mathrm{C} 1 \\
\mathrm{NO} / \mathrm{MY}\end{array}$ & 0.4000 & 0.4076 & $1.90 \%$ & $\mathrm{~N} / \mathrm{C}$ \\
\hline & $R-143 a \cdots$ & 4 hrs @ 30 & $2 F$ & \\
\hline $\begin{array}{l}\text { A2 } \\
\text { NOMEX }\end{array}$ & 0.5548 & 0.5584 & $0.65 \%$ & $\mathrm{~N} / \mathrm{C}$ \\
\hline $\begin{array}{l}\text { B2 } \\
\text { MYLAR }\end{array}$ & 0.4442 & 0.4470 & $0.63 \%$ & \begin{tabular}{|c|} 
Some delamination \\
pockets \\
"see photo \\
\end{tabular} \\
\hline $\begin{array}{l}\mathrm{C} 2 \\
\mathrm{NO} / \mathrm{MY}\end{array}$ & 0.3948 & 0.3968 & $0.51 \%$ & \begin{tabular}{|c|} 
Pockects where \\
mylar pulled away \\
"see photo \\
\end{tabular} \\
\hline
\end{tabular}




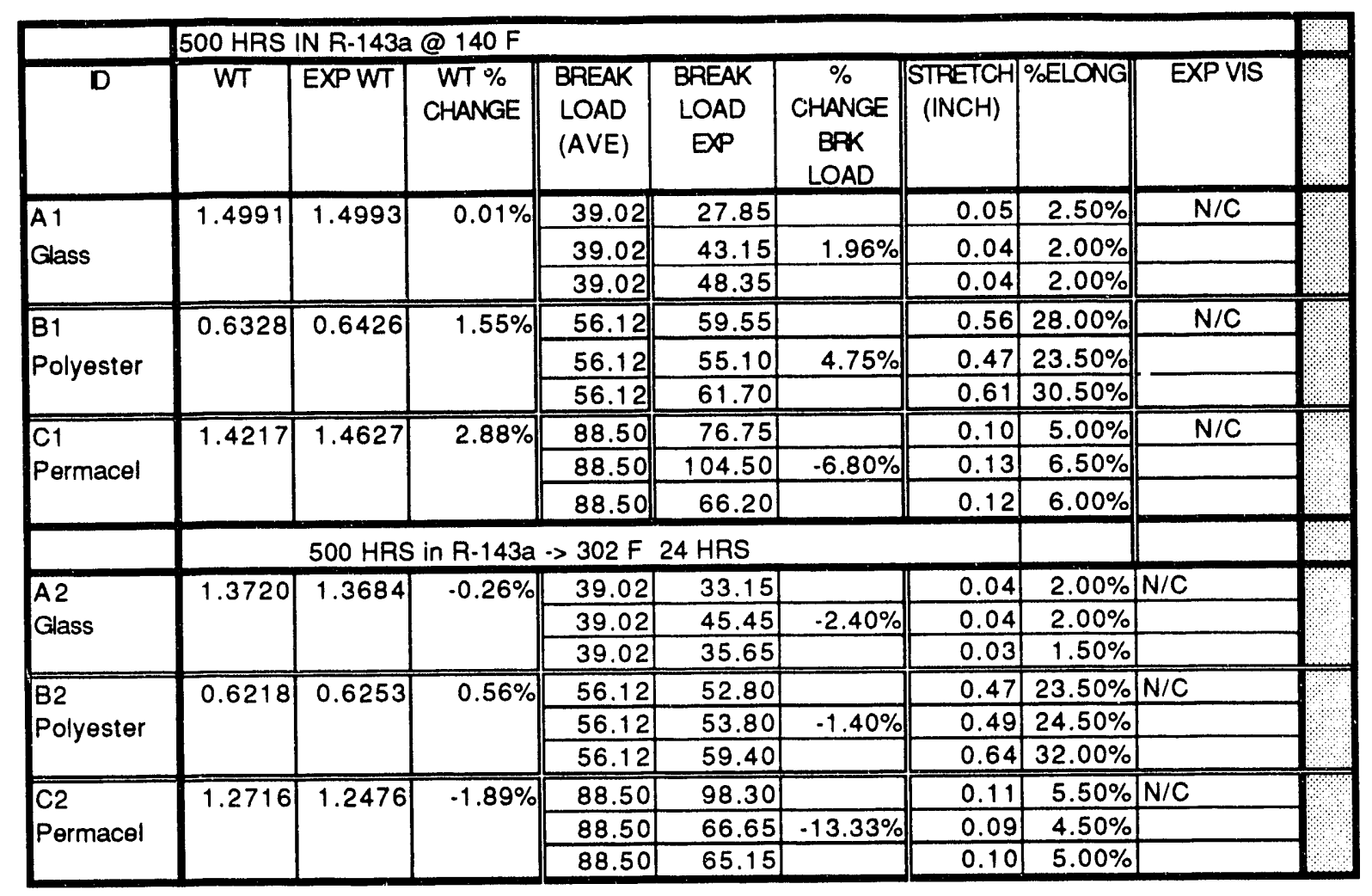


TIE CORDS

\begin{tabular}{|c|c|c|c|c|c|c|c|c|c|}
\hline \multicolumn{10}{|c|}{500 HRS IN R-143a@140F } \\
\hline ID & WT & EXPWT & $\begin{array}{c}\text { WT \% } \\
\text { CHANGE }\end{array}$ & \begin{tabular}{|l|} 
EXP \\
VIS
\end{tabular} & $\begin{array}{l}\text { BREAK } \\
\text { LOAD } \\
\text { (AVE) }\end{array}$ & $\begin{array}{c}\text { BREAK } \\
\text { LOAD } \\
\text { EP } \\
(\text { ave })\end{array}$ & \begin{tabular}{|c|}
$\%$ \\
CHANGE \\
BPK \\
LOAD
\end{tabular} & $\begin{array}{c}\text { STRETCH } \\
\text { (Inch) }\end{array}$ & $\begin{array}{c}\% \\
\text { ELONG }\end{array}$ \\
\hline \multirow{4}{*}{ A1. } & \multirow{3}{*}{0.2283} & \multirow{3}{*}{0.2362} & \multirow{3}{*}{$3.46 \%$} & $\mathrm{~N} / \mathrm{C}$ & 28.36 & 28.80 & & 0.26 & $13.0 \%$ \\
\hline & & & & & 28.36 & 38.15 & \multirow{2}{*}{$12.83 \%$} & 0.30 & $15.0 \%$ \\
\hline & & & & & 28.36 & 29.05 & & 0.26 & $13.0 \%$ \\
\hline & \multicolumn{9}{|c|}{500 HRS IN R-143a -> 24 HRS @ 302F } \\
\hline \multirow[t]{3}{*}{ A2 } & \multirow[t]{3}{*}{0.2406} & \multirow[t]{3}{*}{0.241} & \multirow[t]{3}{*}{$0.166 \%$} & $\mathrm{~N} / \mathrm{C}$ & 28.36 & 33.65 & & 0.45 & $22.5 \%$ \\
\hline & & & & & 28.36 & 33.10 & $14.19 \%$ & 0.51 & $25.5 \%$ \\
\hline & & & & & 28.36 & 30.40 & & 0.48 & $24.0 \%$ \\
\hline
\end{tabular}




\begin{tabular}{|c|c|c|c|c|c|c|c|}
\hline & 500 HRS IN F & 143a@14 & $O F$ & & & & \\
\hline ID & WT & EXPWT & $\begin{array}{c}\text { WT \% } \\
\text { CHANGE }\end{array}$ & EXP VIS & $\begin{array}{c}\text { BASE DIE } \\
\text { (AVE) }\end{array}$ & EXP DIE & $\begin{array}{c}\text { DIE\% } \\
\text { CHANGE }\end{array}$ \\
\hline DMD & 4.0528 & 4.0641 & $0.28 \%$ & $\mathrm{~N} / \mathrm{C}$ & 9.61 & 7.59 & \\
\hline$A 1$ & & & & & 9.61 & 9.10 & $-11.93 \%$ \\
\hline & & & & & 9.61 & 8.70 & \\
\hline DTMD & 4.4995 & 4.5150 & $0.34 \%$ & $\mathrm{~N} / \mathrm{C}$ & 9.95 & 9.96 & \\
\hline B1 & & & & & 9.95 & 7.70 & $-5.59 \%$ \\
\hline & & & & & 9.95 & 10.52 & \\
\hline & $R-143 a \rightarrow>2$ & HRS@30 & & & & & \\
\hline DMD & 4.0372 & 4.0378 & $0.01 \%$ & $\mathrm{~N} / \mathrm{C}$ & 9.61 & 9.30 & \\
\hline A2 & & & & & 9.61 & 8.91 & $-4.02 \%$ \\
\hline & & & & & 9.61 & 9.46 & \\
\hline DTMD & 4.3000 & 4.3006 & $0.01 \%$ & $\mathrm{~N} / \mathrm{C}$ & 9.95 & 9.72 & \\
\hline $\mathrm{B} 2$ & & & & & 9.95 & 10.13 & $-5.46 \%$ \\
\hline & & & & & 9.95 & 8.37 & \\
\hline
\end{tabular}




\section{Appendix M}

Experimental Data for HFC-245ca Exposure at $121^{\circ} \mathrm{C}\left(250^{\circ} \mathrm{F}\right)$ 


\begin{tabular}{|c|c|c|c|c|c|c|c|c|c|c|}
\hline & 500 HRS IN & $\mathrm{R}-245 \mathrm{ca}$ & $250^{\circ} \mathrm{F}(12$ & $\left.1^{\circ} \mathrm{C}\right)$ & & & & & & \\
\hline ID & WT & EXPWT & $\begin{array}{c}\text { WT \% } \\
\text { CHANGE }\end{array}$ & EXP VIS & $\begin{array}{c}\text { BASE BRN } \\
\text { OUT } \\
\text { (AVE) }\end{array}$ & $\begin{array}{c}\text { EXP BRN } \\
\text { OUT }\end{array}$ & $\begin{array}{c}\text { BRN OUT } \\
\% \\
\text { CHANGE }\end{array}$ & $\begin{array}{c}\text { BASE DIE } \\
\text { (AVE) }\end{array}$ & EXPDIE & $\begin{array}{c}\text { DIE \% } \\
\text { CHANGE }\end{array}$ \\
\hline A1 & 23.9544 & 23.9664 & $0.050 \%$ & $\mathrm{~N} / \mathrm{C}$ & 576 & 434 & & 15.80 & 13.49 & \\
\hline & & & & & 576 & 403 & $-27.0 \%$ & 15.80 & 14.04 & $-11.8 \%$ \\
\hline & & & & & 576 & 425 & & 15.80 & 14.26 & \\
\hline B1 & 24.5981 & 24.6922 & $0.383 \%$ & $\mathrm{~N} / \mathrm{C}$ & 736 & 725 & & 11.62 & 11.63 & \\
\hline & & & & & 736 & 728 & $-1.2 \%$ & 11.62 & 12.55 & $3.6 \%$ \\
\hline & & & & & 736 & 728 & & 11.62 & 11.93 & \\
\hline $\mathrm{C} 1$ & 23.0305 & 23.0417 & $0.049 \%$ & $N / C$ & 579 & 438 & & 16.58 & 11.70 & \\
\hline & & & & & 579 & 433 & $-17.6 \%$ & 16.58 & 12.49 & $-24.2 \%$ \\
\hline & & & & & 579 & 561 & & 16.58 & 13.50 & \\
\hline & $500 \mathrm{hr}$ in $\mathrm{R}$ & $245 \mathrm{ca}$ plus & $24 \mathrm{hr} \mathrm{a}$ & $302 F$ & & & & & & \\
\hline $\mathrm{A} 2$ & 23.7141 & 23.7230 & $0.038 \%$ & $N / C$ & 576 & 500 & & 15.80 & 9.87 & \\
\hline & & & & & 576 & 517 & $-13.5 \%$ & 15.80 & 16.29 & $-18.2 \%$ \\
\hline & & & & & 576 & 477 & & 15.80 & 12.63 & \\
\hline$B 2$ & 24.8296 & 24.8501 & $0.083 \%$ & $\mathrm{~N} / \mathrm{C}$ & 736 & 724 & & 11.62 & 11.67 & \\
\hline & & & & & 736 & 730 & $-1.2 \%$ & 11.62 & 10.76 & $-5.7 \%$ \\
\hline & & & & & 736 & 727 & & 11.62 & 10.43 & \\
\hline $\mathrm{C} 2$ & 22.8833 & 22.8906 & $0.032 \%$ & $N / C$ & 579 & 578 & & 16.58 & 14.34 & \\
\hline & & & & & 579 & 593 & $-2.7 \%$ & 16.58 & 15.33 & $-15.7 \%$ \\
\hline & & & & & 579 & 519 & & 16.58 & 12.27 & \\
\hline
\end{tabular}




\begin{tabular}{|c|c|c|c|c|c|c|c|c|c|c|c|}
\hline \multicolumn{12}{|c|}{ HOURS IN R-245ca @ 250 $\mathrm{F}\left(121^{\circ} \mathrm{C}\right)$} \\
\hline & VARN & $\begin{array}{l}\text { TWISTED } \\
\text { PAIR WT }\end{array}$ & $\begin{array}{l}\text { Exp Pair } \\
\text { weight }\end{array}$ & $\begin{array}{c}\text { WT \% } \\
\text { CHANGE }\end{array}$ & $\begin{array}{l}\text { EXP } \\
\text { VIS }\end{array}$ & BASE DIE & EXPDIE & $\begin{array}{c}\text { DIE \% } \\
\text { CHANGE }\end{array}$ & $\begin{array}{c}\text { BASE } \\
\text { BURN } \\
\text { OUT }\end{array}$ & $\begin{array}{l}\text { EXP } \\
\text { BURN } \\
\text { OUT }\end{array}$ & $\begin{array}{c}\text { BRNOUT } \\
\% \\
\text { CHANGE }\end{array}$ \\
\hline \multirow{3}{*}{$\begin{array}{l}P \\
O \\
L\end{array}$} & \multirow{3}{*}{$\begin{array}{l}\text { U-475 } \\
\text { A } 1\end{array}$} & \multirow[t]{3}{*}{22.8170} & \multirow[t]{3}{*}{22.8873} & \multirow[t]{3}{*}{$0.308 \%$} & \multirow[t]{3}{*}{$\mathrm{N} / \mathrm{C}$} & 16.24 & 14.18 & & 430 & 545 & \\
\hline & & & & & & 16.24 & 12.67 & $-16.77 \%$ & 430 & 368 & $-0.85 \%$ \\
\hline & & & & & & 16.24 & 13.70 & & 430 & 366 & \\
\hline \multirow{3}{*}{$\begin{array}{l}Y \\
E \\
S\end{array}$} & \multirow{3}{*}{$\begin{array}{l}-390 \\
B 1\end{array}$} & \multirow[t]{3}{*}{24.7034} & \multirow[t]{3}{*}{24.7978} & \multirow[t]{3}{*}{$0.382 \%$} & $\mathrm{~N} / \mathrm{C}$ & 18.77 & 19.99 & & 510 & 414 & \\
\hline & & & & & & 18.77 & 17.45 & $1.99 \%$ & 510 & 521 & $-6.86 \%$ \\
\hline & & & & & & 18.77 & 19.99 & & 510 & 490 & \\
\hline \multirow{4}{*}{$\begin{array}{l}T \\
E \\
R\end{array}$} & ER-610 & \multirow[t]{3}{*}{22.0534} & \multirow[t]{3}{*}{22.0842} & \multirow[t]{3}{*}{$0.140 \%$} & $\mathrm{~N} / \mathrm{C}$ & 15.57 & 15.09 & & 442 & 337 & \\
\hline & \multirow{2}{*}{$C_{1}$} & & & & & 15.57 & 14.52 & $-13.40 \%$ & 442 & 381 & $-20.89 \%$ \\
\hline & & & & & & 15.57 & 10.84 & & 442 & 331 & \\
\hline & \multirow{3}{*}{$\begin{array}{l}\text { Y-833 } \\
D 1\end{array}$} & \multirow[t]{3}{*}{23.2259} & \multirow[t]{3}{*}{23.2277} & \multirow[t]{3}{*}{$0.008 \%$} & $\mathrm{~N} / \mathrm{C}$ & 12.04 & 11.46 & & 578 & 569 & \\
\hline \multirow{2}{*}{$\begin{array}{l}P \\
O\end{array}$} & & & & & & 12.04 & 10.44 & $-16.75 \%$ & 578 & 549 & $-3.29 \%$ \\
\hline & & & & & & 12.04 & 8.17 & & 578 & 559 & \\
\hline \multirow{3}{*}{$\begin{array}{l}\mathrm{L} \\
\mathrm{Y} \\
\mathrm{A}\end{array}$} & \multirow{3}{*}{$\begin{array}{l}923 \\
E 1\end{array}$} & 24.1274 & 24.2176 & $0.374 \%$ & $\mathrm{~N} / \mathrm{C}$ & 16.76 & 17.74 & & 606 & 538 & \\
\hline & & & & & & 16.76 & 19.15 & $2.98 \%$ & 606 & 550 & $-10.01 \%$ \\
\hline & & & & & & 16.76 & 14.89 & & 606 & 548 & \\
\hline$M$ & ISO-800 & 23.8215 & 23.8599 & $0.161 \%$ & $\mathrm{~N} / \mathrm{C}$ & 19.08 & 13.89 & & 580 & 574 & \\
\hline 1 & $\mathrm{~F} 1$ & & & & & 19.08 & 19.73 & $-21.91 \%$ & 580 & 514 & $-3.22 \%$ \\
\hline$D$ & & & & & & 19.08 & 11.08 & & 580 & 596 & \\
\hline$E$ & & $24 \mathrm{HOUR}$ & S AT $302 \mathrm{~F}$ & & & & & & & & \\
\hline & U-475 & 22.7834 & 22.8114 & $0.123 \%$ & $\mathrm{~N} / \mathrm{C}$ & 16.24 & 13.42 & & 430 & 374 & \\
\hline 1 & A2 & & & & & 16.24 & 14.43 & $-16.15 \%$ & 430 & 568 & $16.20 \%$ \\
\hline$M$ & & & & & & 16.24 & 13.00 & & 430 & 557 & \\
\hline 1 & $Y-390$ & 24.0036 & 24.0449 & $0.172 \%$ & $\mathrm{~N} / \mathrm{C}$ & 18.77 & 12.25 & & 510 & 445 & \\
\hline$D$ & B2 & & & & & 18.77 & 12.89 & $-30.85 \%$ & 510 & 392 & $-18.95 \%$ \\
\hline$E$ & & & & & & 18.77 & 13.80 & & 510 & 403 & \\
\hline & ER-610 & 21.6249 & 21.6387 & $0.064 \%$ & $\mathrm{~N} / \mathrm{C}$ & 15.57 & 12.60 & & 442 & 334 & \\
\hline & $\mathrm{C} 2$ & & & & & 15.57 & 13.45 & $-18.75 \%$ & 442 & 369 & $-22.02 \%$ \\
\hline & & & & & & 15.57 & 11.90 & & 442 & 331 & \\
\hline & $Y-833$ & 23.3424 & 23.3301 & $-0.053 \%$ & $\mathrm{~N} / \mathrm{C}$ & 12.04 & 9.61 & & 578 & 499 & \\
\hline & $D 2$ & & & & & 12.04 & 11.94 & $-12.15 \%$ & 578 & 492 & $-9.86 \%$ \\
\hline & & & & & & 12.04 & 10.18 & & 578 & 572 & \\
\hline & 923 & 23.7579 & 23.8077 & $0.210 \%$ & $\mathrm{~N} / \mathrm{C}$ & 16.76 & 18.70 & & 606 & 467 & \\
\hline & E2 & & & & & 16.76 & 11.90 & $-5.99 \%$ & 606 & 582 & $-11.06 \%$ \\
\hline & & & & & & 16.76 & 16.67 & & 606 & 568 & \\
\hline & ISO-800 & 22.2331 & 22.2484 & $0.069 \%$ & $\mathrm{~N} / \mathrm{C}$ & 19.08 & 12.66 & & 580 & 552 & \\
\hline & $\mathrm{F} 2$ & & & & & 19.08 & 17.17 & $-29.19 \%$ & 580 & 576 & $-0.06 \%$ \\
\hline & & & & & & 19.08 & 10.70 & & 580 & 611 & \\
\hline
\end{tabular}




\begin{tabular}{|c|c|c|c|c|c|c|c|c|c|c|c|}
\hline \multicolumn{12}{|c|}{ HOURS IN R-245ca @ 250 $\mathrm{F}\left(121^{\circ} \mathrm{C}\right)$} \\
\hline & VARN & $\begin{array}{l}\text { TWISTED } \\
\text { PAIR WT }\end{array}$ & $\begin{array}{c}\text { EXP T } \\
\text { PAIR WT }\end{array}$ & $\begin{array}{c}\text { WT \% } \\
\text { CHANGE }\end{array}$ & $\begin{array}{l}\text { EXP } \\
\text { VIS }\end{array}$ & $\begin{array}{c}\text { BASE } \\
\text { DIE }\end{array}$ & $\begin{array}{l}\text { EXP } \\
D I E\end{array}$ & $\begin{array}{c}\text { DIE \% } \\
\text { CHANGE }\end{array}$ & $\begin{array}{l}\text { BASE } \\
\text { BUPN } \\
\text { OUT }\end{array}$ & \begin{tabular}{|c|} 
EXP \\
BURN \\
OUT
\end{tabular} & $\begin{array}{c}\text { BRNOUT } \\
\% \\
\text { CHANGE }\end{array}$ \\
\hline \multirow{3}{*}{$\begin{array}{l}P \\
O \\
L\end{array}$} & \multirow{3}{*}{$\begin{array}{l}\text { U-475 } \\
\text { A } 1\end{array}$} & 26.3285 & 26.5326 & $0.78 \%$ & $\mathrm{~N} / \mathrm{C}$ & 13.32 & 16.09 & & 746 & 739 & \\
\hline & & & & & & 13.32 & 18.61 & $19.64 \%$ & 746 & 741 & $-0.98 \%$ \\
\hline & & & & & & 13.32 & 13.11 & & 746 & 736 & \\
\hline \multirow{3}{*}{$\begin{array}{l}Y \\
E \\
S\end{array}$} & \multirow{3}{*}{$\begin{array}{l}Y-390 \\
B 1\end{array}$} & 26.4158 & 26.5817 & $0.63 \%$ & $\mathrm{~N} / \mathrm{C}$ & 12.28 & 11.47 & & 755 & 740 & \\
\hline & & & & & & 12.28 & 7.67 & $-29.70 \%$ & 755 & 742 & $-1.81 \%$ \\
\hline & & & & & & 12.28 & 6.76 & & 755 & 742 & \\
\hline \multirow{3}{*}{$\begin{array}{l}T \\
E \\
R\end{array}$} & \multirow{3}{*}{$\begin{array}{l}\text { ER-610 } \\
\text { C1 }\end{array}$} & 26.3762 & 26.5056 & $0.49 \%$ & $\mathrm{~N} / \mathrm{C}$ & 12.73 & 14.26 & & 734 & 731 & \\
\hline & & & & & & 12.73 & 13.69 & $13.56 \%$ & 734 & 729 & $-0.59 \%$ \\
\hline & & & & & & 12.73 & 15.42 & & 734 & 729 & \\
\hline \multirow{3}{*}{\multicolumn{2}{|c|}{$\begin{array}{l}Y-833 \\
D 1\end{array}$}} & 24.2096 & 24.3013 & $0.38 \%$ & $\mathrm{~N} / \mathrm{C}$ & 12.49 & 10.99 & & 734 & 728 & \\
\hline & & & & & & 12.49 & 12.10 & $-2.43 \%$ & 734 & 728 & $-0.59 \%$ \\
\hline & & & & & & 12.49 & 13.47 & & 734 & 733 & \\
\hline \multirow{3}{*}{$\begin{array}{l}L \\
Y \\
A\end{array}$} & \multirow{3}{*}{$\begin{array}{l}923 \\
E 1\end{array}$} & 27.0503 & 27.2093 & $0.59 \%$ & $\mathrm{~N} / \mathrm{C}$ & 14.38 & 19.73 & & 742 & 753 & \\
\hline & & & & & & 14.38 & 15.69 & $28.07 \%$ & 742 & 749 & $0.99 \%$ \\
\hline & & & & & & 14.38 & 19.83 & & 742 & 746 & \\
\hline \multirow{3}{*}{$\begin{array}{l}M \\
1 \\
D\end{array}$} & \multirow{3}{*}{$\begin{array}{l}\text { ISO-800 } \\
\text { F1 }\end{array}$} & \multirow[t]{3}{*}{25.3057} & 25.4436 & $0.54 \%$ & $\mathrm{~N} / \mathrm{C}$ & 12.29 & 12.21 & & 747 & 737 & \\
\hline & & & & & & 12.29 & 13.31 & $8.60 \%$ & 747 & 734 & $-1.65 \%$ \\
\hline & & & & & & 12.29 & 14.52 & & 747 & 733 & \\
\hline$E$ & & \multicolumn{10}{|c|}{ R-245ca $-->24$ hours @ 302 F } \\
\hline \multirow{3}{*}{$\begin{array}{l}1 \\
M\end{array}$} & U-475 & 25.0593 & 25.0915 & $0.13 \%$ & $\mathrm{~N} / \mathrm{C}$ & 13.32 & 11.79 & & 746 & 725 & \\
\hline & \multirow{2}{*}{$\mathrm{A} 2$} & & & & & 13.32 & 12.10 & $-11.24 \%$ & 746 & 720 & $-2.59 \%$ \\
\hline & & & & & & 13.32 & 11.58 & & 746 & 735 & \\
\hline \multirow{3}{*}{$\begin{array}{l}I \\
D \\
E\end{array}$} & \multirow{3}{*}{$\begin{array}{l}Y-390 \\
B 2\end{array}$} & 25.7646 & 25.8467 & $0.32 \%$ & $\mathrm{~N} / \mathrm{C}$ & 12.28 & 11.63 & & 755 & 752 & \\
\hline & & & & & & 12.28 & 16.08 & $12.65 \%$ & 755 & 746 & $-0.79 \%$ \\
\hline & & & & & & 12.28 & 13.79 & & 755 & 755 & \\
\hline & \multirow{3}{*}{$\begin{array}{l}\text { ER-610 } \\
\text { C2 }\end{array}$} & 26.3366 & 26.3556 & $0.07 \%$ & $\mathrm{~N} / \mathrm{C}$ & 12.73 & 12.06 & & 734 & 730 & \\
\hline$E$ & & & & & & 12.73 & 12.16 & $-8.04 \%$ & 734 & 725 & $-0.77 \%$ \\
\hline$P$ & & & & & & 12.73 & 10.90 & & 734 & 730 & \\
\hline \multirow{4}{*}{$\begin{array}{l}0 \\
X \\
Y\end{array}$} & \multirow{3}{*}{$\begin{array}{l}\mathrm{Y}-833 \\
D 2\end{array}$} & 24.5875 & 24.5805 & $-0.03 \%$ & $\mathrm{~N} / \mathrm{C}$ & 12.49 & 12.17 & & 734 & 739 & \\
\hline & & & & & & 12.49 & 10.46 & $-5.98 \%$ & 734 & 738 & $-1.04 \%$ \\
\hline & & & & & & 12.49 & 12.60 & & 734 & 702 & \\
\hline & \multirow{3}{*}{$\begin{array}{l}923 \\
E 2\end{array}$} & 26.4211 & 26.4948 & $0.28 \%$ & $N / C$ & 14.38 & 12.45 & & 742 & 748 & \\
\hline$G$ & & & & & & 14.38 & 13.31 & $-14.39 \%$ & 742 & 735 & $0.81 \%$ \\
\hline$L$ & & & & & & 14.38 & 11.17 & & 742 & 728 & \\
\hline A & ISO-800 & 25.4682 & 25.5096 & $0.16 \%$ & & 12.29 & 10.40 & & 747 & 743 & \\
\hline$S$ & & & & & $\mathrm{~N} / \mathrm{C}$ & 12.29 & 12.34 & $-3.1 .7 \%$ & 747 & 739 & $-0.80 \%$ \\
\hline$S$ & & & & & & 12.29 & 12.96 & & 747 & 739 & \\
\hline
\end{tabular}




\begin{tabular}{|c|c|c|c|c|c|c|c|c|c|c|c|}
\hline \multicolumn{12}{|c|}{ HOURS IN R-245ca@250 $\mathrm{F}\left(121^{\circ} \mathrm{C}\right)$} \\
\hline & VARN & $\begin{array}{l}\text { TWVISTED } \\
\text { PAIR WT }\end{array}$ & $\begin{array}{c}\text { EXPT } \\
\text { PAIR WT }\end{array}$ & $\begin{array}{c}\text { WT \% } \\
\text { CHANGE }\end{array}$ & $\begin{array}{l}\text { EXP } \\
\text { VIS }\end{array}$ & $\begin{array}{c}\text { BASE } \\
\text { DIE }\end{array}$ & $\begin{array}{l}\text { EXP } \\
\text { DIE }\end{array}$ & $\begin{array}{c}\text { DIE \% } \\
\text { CHANGE }\end{array}$ & \begin{tabular}{|l|} 
BASE \\
BURN \\
OUT \\
\end{tabular} & \begin{tabular}{|c|} 
EXP \\
BURN \\
OUT
\end{tabular} & $\begin{array}{c}\text { BRNOUT } \\
\% \\
\text { CHANGE }\end{array}$ \\
\hline \multirow{3}{*}{\begin{tabular}{l|}
$P$ \\
$O$ \\
L
\end{tabular}} & \multirow{3}{*}{$\begin{array}{l}\text { U-475 } \\
\text { A } 1\end{array}$} & 23.2373 & 23.3192 & $0.352 \%$ & $\mathrm{~N} / \mathrm{C}$ & 15.10 & 14.90 & & 469 & 500 & \\
\hline & & & & & & 15.10 & 14.34 & $-3.77 \%$ & 469 & 290 & $.17 .56 \%$ \\
\hline & & & & & & 15.10 & 14.35 & & 469 & 370 & \\
\hline \multirow{3}{*}{\begin{tabular}{l|l}
$Y$ & $Y$ \\
$E$ & $E$ \\
$S$
\end{tabular}} & \multirow{3}{*}{$\begin{array}{l}\text { Y-390 } \\
\text { B1 }\end{array}$} & 24.5239 & 24.6104 & $0.353 \%$ & $\mathrm{~N} / \mathrm{C}$ & 18.24 & 14.35 & & 473 & 619 & \\
\hline & & & & & & 18.24 & 19.94 & $-3.23 \%$ & 473 & 585 & $25.93 \%$ \\
\hline & & & & & & 18.24 & 18.66 & & 473 & 583 & \\
\hline \multirow{4}{*}{$\begin{array}{l}T \\
E \\
R\end{array}$} & \multirow{3}{*}{$\begin{array}{l}\text { ER-610 } \\
\text { C1 }\end{array}$} & 21.3455 & 21.3740 & $0.134 \%$ & $\mathrm{~N} / \mathrm{C}$ & 14.53 & 10.44 & & 494 & 407 & \\
\hline & & & & & & 14.53 & 11.09 & $-24.68 \%$ & 494 & 338 & $-29.49 \%$ \\
\hline & & & & & & 14.53 & 11.30 & & 494 & 300 & \\
\hline & \multirow{3}{*}{$\begin{array}{l}Y-833 \\
D 1\end{array}$} & 23.1436 & 23.1455 & $0.008 \%$ & $\mathrm{~N} / \mathrm{C}$ & 11.38 & 10.00 & & 557 & 461 & \\
\hline 1 & & & & & & 11.38 & 10.78 & $-8.93 \%$ & 557 & 594 & $-9.87 \%$ \\
\hline$M$ & & & & & & 11.38 & 10.31 & & 557 & 451 & \\
\hline \multirow{4}{*}{$\begin{array}{l}D \\
E\end{array}$} & \multirow{3}{*}{$\begin{array}{l}923 \\
E 1\end{array}$} & 23.5125 & 23.5899 & $0.329 \%$ & $\mathrm{~N} / \mathrm{C}$ & 15.85 & 14.26 & & 503 & 552 & \\
\hline & & & & & & 15.85 & 15.37 & $3.64 \%$ & 503 & 425 & $-3.91 \%$ \\
\hline & & & & & & 15.85 & 19.65 & & 503 & 473 & \\
\hline & \multirow{3}{*}{$\begin{array}{l}\text { ISO-800 } \\
F 1\end{array}$} & \multirow[t]{3}{*}{22.4032} & \multirow[t]{3}{*}{22.4405} & \multirow[t]{3}{*}{ \#VALUE! } & $\mathrm{N} / \mathrm{C}$ & 14.75 & 16.02 & & 632 & 578 & \\
\hline$P$ & & & & & & 14.75 & 15.93 & $13.92 \%$ & 632 & 537 & $-12.13 \%$ \\
\hline \multirow{2}{*}{$\begin{array}{l}0 \\
L \\
v\end{array}$} & & & & & & 14.75 & 18.46 & & 632 & 551 & \\
\hline & & \multicolumn{10}{|c|}{ R-245ca $-->24$ hours@ @ $302 F$} \\
\hline$Y$ & \multirow{3}{*}{$A 2$} & 23.4377 & 23.4685 & $0.131 \%$ & $\mathrm{~N} / \mathrm{C}$ & 15.10 & 11.57 & & 469 & 394 & \\
\hline$A$ & & & & & & 15.10 & 14.29 & $-12.85 \%$ & 469 & 342 & $-15.14 \%$ \\
\hline$M$ & & & & & & 15.10 & 13.62 & & 469 & 458 & \\
\hline \multirow{4}{*}{$\begin{array}{l}I \\
D \\
E\end{array}$} & \multirow{3}{*}{$\begin{array}{l}Y-390 \\
B 2\end{array}$} & 24.2281 & 24.2701 & $0.173 \%$ & $\mathrm{~N} / \mathrm{C}$ & 18.24 & 19.24 & & 473 & 590 & \\
\hline & & & & & & \begin{tabular}{|l|}
18.24 \\
\end{tabular} & 17.97 & $-0.90 \%$ & 473 & 515 & $19.66 \%$ \\
\hline & & & & & & 18.24 & 18.18 & & 473 & 593 & \\
\hline & \multirow{3}{*}{$\begin{array}{l}\text { ER-610 } \\
\text { C2 }\end{array}$} & 21.3143 & 21.3240 & $0.046 \%$ & $\mathrm{~N} / \mathrm{C}$ & 14.53 & 14.05 & & 494 & 415 & \\
\hline 1 & & & & & & 14.53 & 13.58 & $-10.85 \%$ & 494 & 441 & $-17.61 \%$ \\
\hline$M$ & & & & & & 14.53 & 11.23 & & 494 & 365 & \\
\hline \multirow{9}{*}{$\begin{array}{l}1 \\
D \\
E\end{array}$} & \multirow{3}{*}{$\begin{array}{l}\mathrm{Y}-833 \\
D 2\end{array}$} & 23.2352 & 23.2275 & $-0.033 \%$ & $N / C$ & 11.38 & 11.46 & & 557 & 435 & \\
\hline & & & & & & 11.38 & 11.49 & $0.91 \%$ & 557 & 602 & $-5.98 \%$ \\
\hline & & & & & & 11.38 & 11.50 & & 557 & 534 & \\
\hline & \multirow{3}{*}{$\begin{array}{l}923 \\
E 2\end{array}$} & \multirow[t]{3}{*}{23.0656} & 23.1038 & $0.166 \%$ & $\mathrm{~N} / \mathrm{C}$ & 15.85 & 19.92 & & 503 & 508 & \\
\hline & & & & & & 15.85 & 19.73 & $23.66 \%$ & 503 & 538 & $9.01 \%$ \\
\hline & & & & & & 15.85 & 19.15 & & 503 & 599 & \\
\hline & ISO-800 & 21.6721 & 21.6843 & $0.056 \%$ & $\mathrm{~N} / \mathrm{C}$ & 14.75 & 14.40 & & 632 & 579 & \\
\hline & $F 2$ & & & & & 14.75 & 13.30 & $0.97 \%$ & 632 & 571 & $-8.97 \%$ \\
\hline & & & & & & 14.75 & 16.98 & & 632 & 576 & \\
\hline
\end{tabular}


HELICAL COILS $/$ IIRE A-.-HFC-245ca @ $121^{\circ} \mathrm{C}\left(250^{\circ} \mathrm{F}\right)$

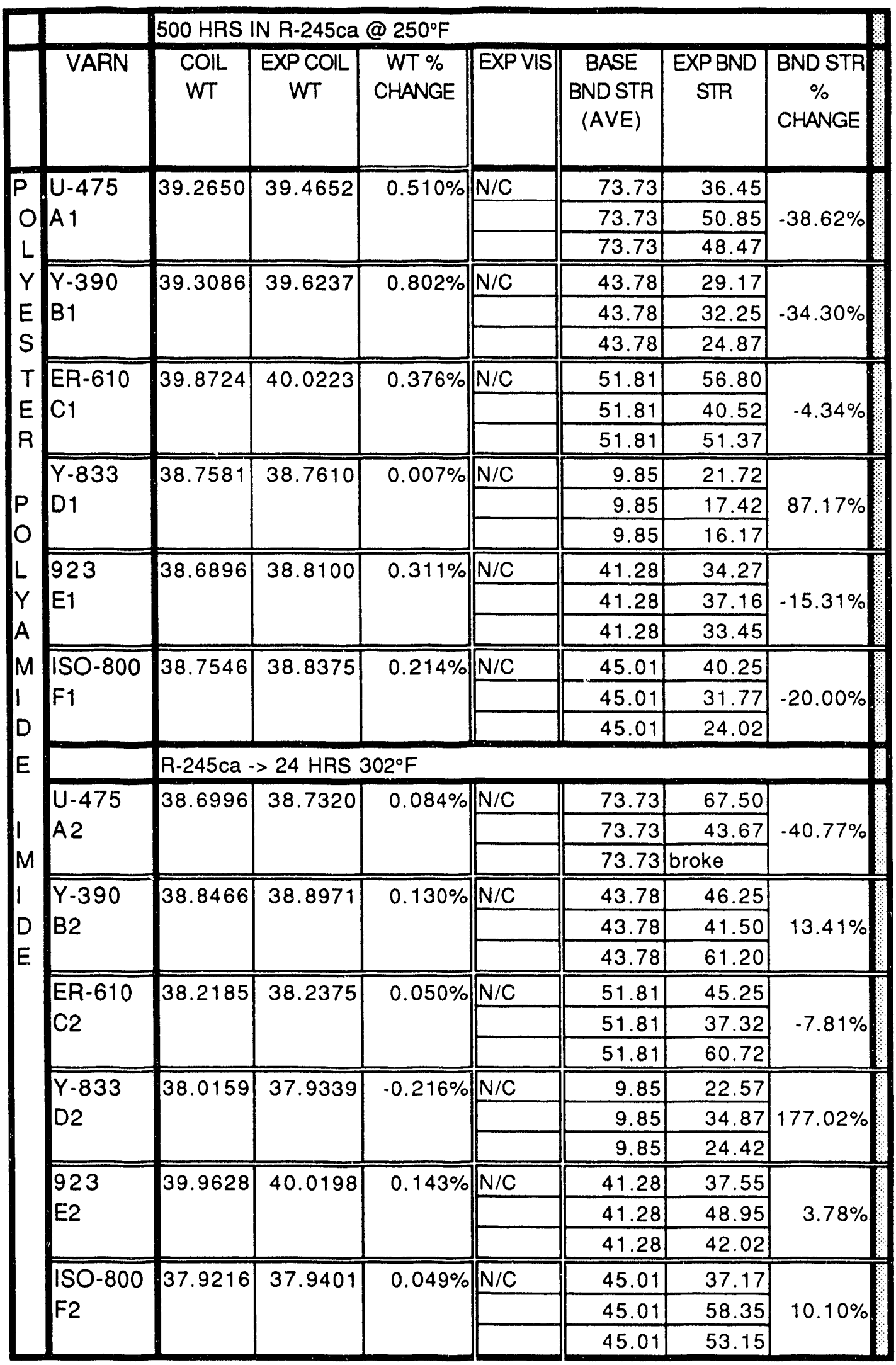

) 
HELICAL COILS W:PE B--.HFC-245c a @ $121^{\circ} \mathrm{C}\left(250^{\circ} \mathrm{F}\right)$

\begin{tabular}{|c|c|c|c|c|c|c|c|c|}
\hline & & $500 \mathrm{HRS}$ & N R-245 & @ 250 $\mathrm{F}$ & & & & \\
\hline & VARN & $\begin{array}{l}\text { COIL } \\
\text { WT }\end{array}$ & $\begin{array}{c}\text { EXP COIL } \\
W T\end{array}$ & $\begin{array}{c}\text { WT \% } \\
\text { CHANGE }\end{array}$ & EXP VIS & $\begin{array}{c}\text { BASE } \\
\text { BND STR } \\
(\text { AVE })\end{array}$ & $\begin{array}{c}\text { EXPBND } \\
\text { STR }\end{array}$ & $\begin{array}{c}\text { BND STR } \\
\% \\
\text { CHANGE }\end{array}$ \\
\hline $\mathrm{P}$ & $U-475$ & 36.5423 & 36.7338 & $0.524 \%$ & $\mathrm{~N} / \mathrm{C}$ & 40.14 & 42.85 & \\
\hline 0 & A1 & & & & & 40.14 & 44.35 & $4.57 \%$ \\
\hline$L$ & & & & & & 40.14 & 38.72 & \\
\hline$Y$ & $\overline{Y-390}$ & 38.2599 & 38.2702 & $0.027 \%$ & $\mathrm{~N} / \mathrm{C}$ & 36.12 & 40.60 & \\
\hline$E$ & B1 & & & & & 36.12 & 33.17 & $3.70 \%$ \\
\hline $\mathrm{s}$ & & & & & & 36.12 & 38.60 & \\
\hline$T$ & ER-610 & 37.3413 & 37.4752 & $0.359 \%$ & $\mathrm{~N} / \mathrm{C}$ & 35.96 & 37.55 & \\
\hline$E$ & & & & & & 35.96 & 29.77 & $-6.73 \%$ \\
\hline $\mathrm{R}$. & & & & & & 35.96 & 33.30 & \\
\hline & $\overline{Y-833}$ & 37.8417 & 37.8724 & $0.081 \%$ & $\mathrm{~N} / \mathrm{C}$ & 33.14 & 40.60 & \\
\hline$P$ & D1 & & & & & 33.14 & 43.77 & $27.76 \%$ \\
\hline 0 & & & & & & 33.14 & 42.65 & \\
\hline$L$ & 923 & 38.5834 & 38.6841 & $0.261 \%$ & $\mathrm{~N} / \mathrm{C}$ & 40.52 & 36.15 & \\
\hline$Y$ & E1 & & & & & 40.52 & 37.55 & $-3.61 \%$ \\
\hline$A$ & & & & & & 40.52 & 43.47 & \\
\hline M & $150-800$ & 38.5317 & 38.6353 & $0.269 \%$ & $\mathrm{~N} / \mathrm{C}$ & 20.20 & 24.02 & \\
\hline 1 & $\mathrm{~F} 1$ & & & & & 20.20 & 22.35 & $9.03 \%$ \\
\hline$D$ & & & & & & 20.20 & 19.70 & \\
\hline$E$ & & R-245ca-> & 24 HRS & $02^{\circ} \mathrm{F}$ & & & & \\
\hline & U-475 & 35.7003 & 35.7459 & $0.128 \%$ & $\mathrm{~N} / \mathrm{C}$ & 40.14 & 30.47 & \\
\hline 1 & $A 2$ & & & & & 40.14 & 37.40 & $-6.27 \%$ \\
\hline$M$ & & & & & & 40.14 & 45.00 & \\
\hline 1 & $Y-390$ & 38.8799 & 38.9132 & $0.086 \%$ & $\mathrm{~N} / \mathrm{C}$ & 36.12 & 36.90 & \\
\hline$D$ & $B 2$ & & & & & 36.12 & 32.57 & $-6.08 \%$ \\
\hline$E$ & & & & & & 36.12 & 32.30 & \\
\hline & ER-610 & 37.2912 & 37.3114 & $0.054 \%$ & $\mathrm{~N} / \mathrm{C}$ & 35.96 & 32.57 & \\
\hline$E$ & $\mathrm{C} 2$ & & & & & 35.96 & broken & $-9.43 \%$ \\
\hline$P$ & & & & & & 35.96 & broken & \\
\hline 0 & $\overline{Y-833}$ & 38.8639 & 38.7674 & $-0.248 \%$ & $\mathrm{~N} / \mathrm{C}$ & 33.14 & 18.95 & \\
\hline$x$ & D2 & & & & & 33.14 & 19.62 & $-36.76 \%$ \\
\hline$Y$ & & & & & & 33.14 & 24.30 & \\
\hline & 923 & 38.9499 & 38.9958 & $0.118 \%$ & $\mathrm{~N} / \mathrm{C}$ & 40.52 & 33.70 & \\
\hline$G$ & E2 & & & & & 40.52 & 34.27 & $-18.73 \%$ \\
\hline$L$ & & & & & & 40.52 & 30.82 & \\
\hline A & $150-800$ & 38.7774 & 38.7929 & $0.040 \%$ & $\mathrm{~N} / \mathrm{C}$ & 20.20 & 18.82 & \\
\hline$S$ & $\mathrm{~F} 2$ & & & & & 20.20 & 9.58 & $-21.20 \%$ \\
\hline$S$ & & & & & & 20.20 & 19.35 & \\
\hline
\end{tabular}


HELICAL COILSMIRE C--.HFC-245ca @ $121^{\circ} \mathrm{C}\left(250^{\circ} \mathrm{F}\right)$

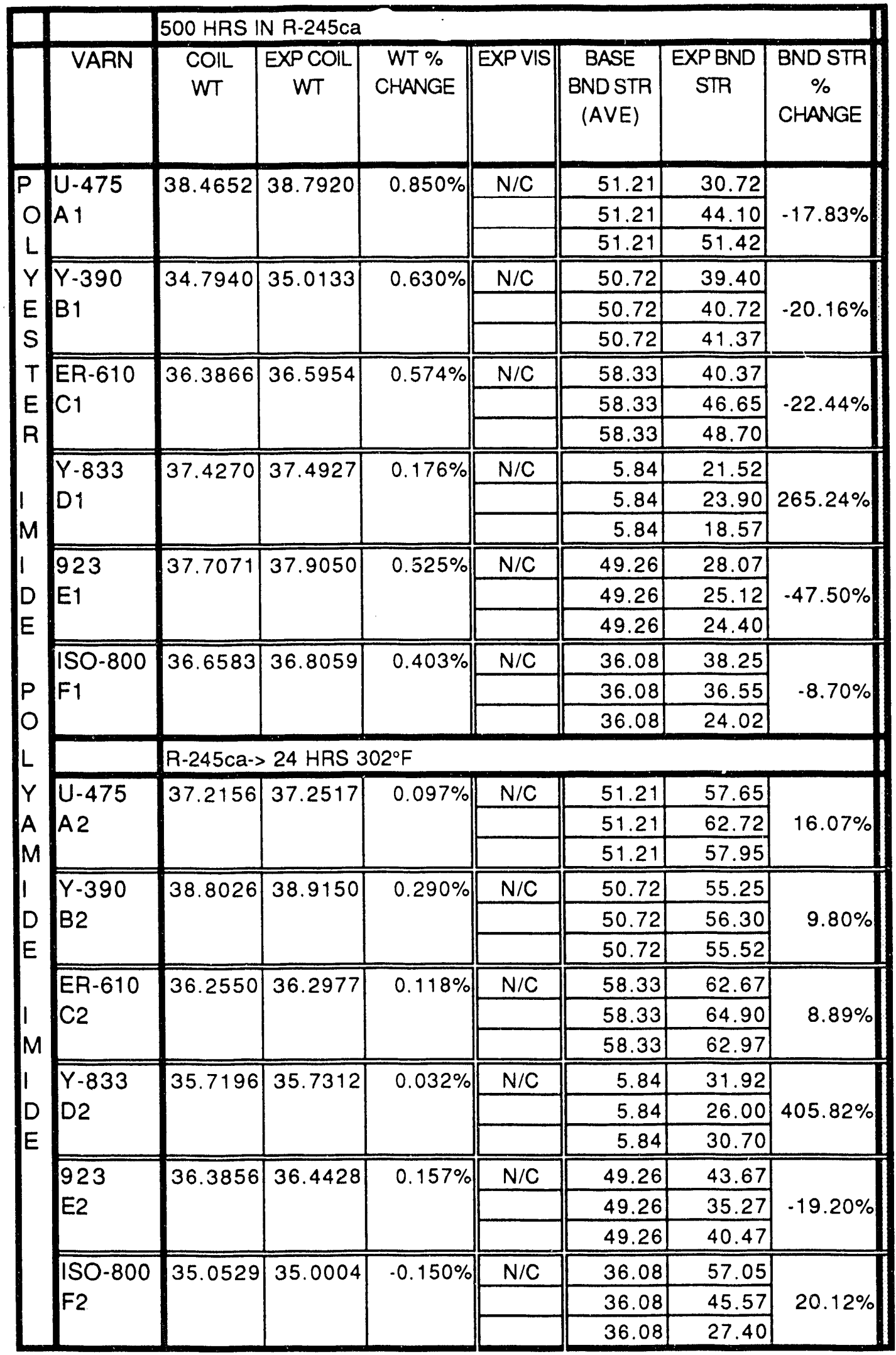




\begin{tabular}{|c|c|c|c|c|c|}
\hline & 500 HRS IN & R-245@2 & $0^{\circ} \mathrm{F}\left(121^{\circ} \mathrm{C}\right)$ & & \\
\hline ID & WT & EXPWT & $\begin{array}{l}\text { WT \% } \\
\text { CHANGE }\end{array}$ & EXP VIS & EXPFLEX \\
\hline A1 & 4.0839 & 4.0853 & $0.034 \%$ & $\mathrm{~N} / \mathrm{C}$ & \\
\hline & & & & & YES \\
\hline$\overline{\mathrm{B1}}$ & 4.1603 & 4.174 & $0.329 \%$ & $\overline{N / C}$ & \\
\hline & & & & & NO \\
\hline C1 & 4.1346 & 4.1365 & $0.046 \%$ & $\mathrm{~N} / \mathrm{C}$ & \\
\hline & & & & & YES \\
\hline & $R-245->3$ & $2^{\circ} \mathrm{F}$ for 24 & ARS & & \\
\hline A2 & 3.8376 & 3.8387 & $0.029 \%$ & $\mathrm{~N} / \mathrm{C}$ & \\
\hline & & & & & YES \\
\hline$\overline{\mathrm{B} 2}$ & 4.1982 & 4.2005 & $0.055 \%$ & $\mathrm{~N} / \mathrm{C}$ & \\
\hline & & & & & YES \\
\hline$\overline{\mathrm{C} 2}$ & 4.1817 & 4.1835 & $0.043 \%$ & $\bar{N} / \mathrm{C}$ & \\
\hline & & & & & YES \\
\hline & & & & & \\
\hline
\end{tabular}




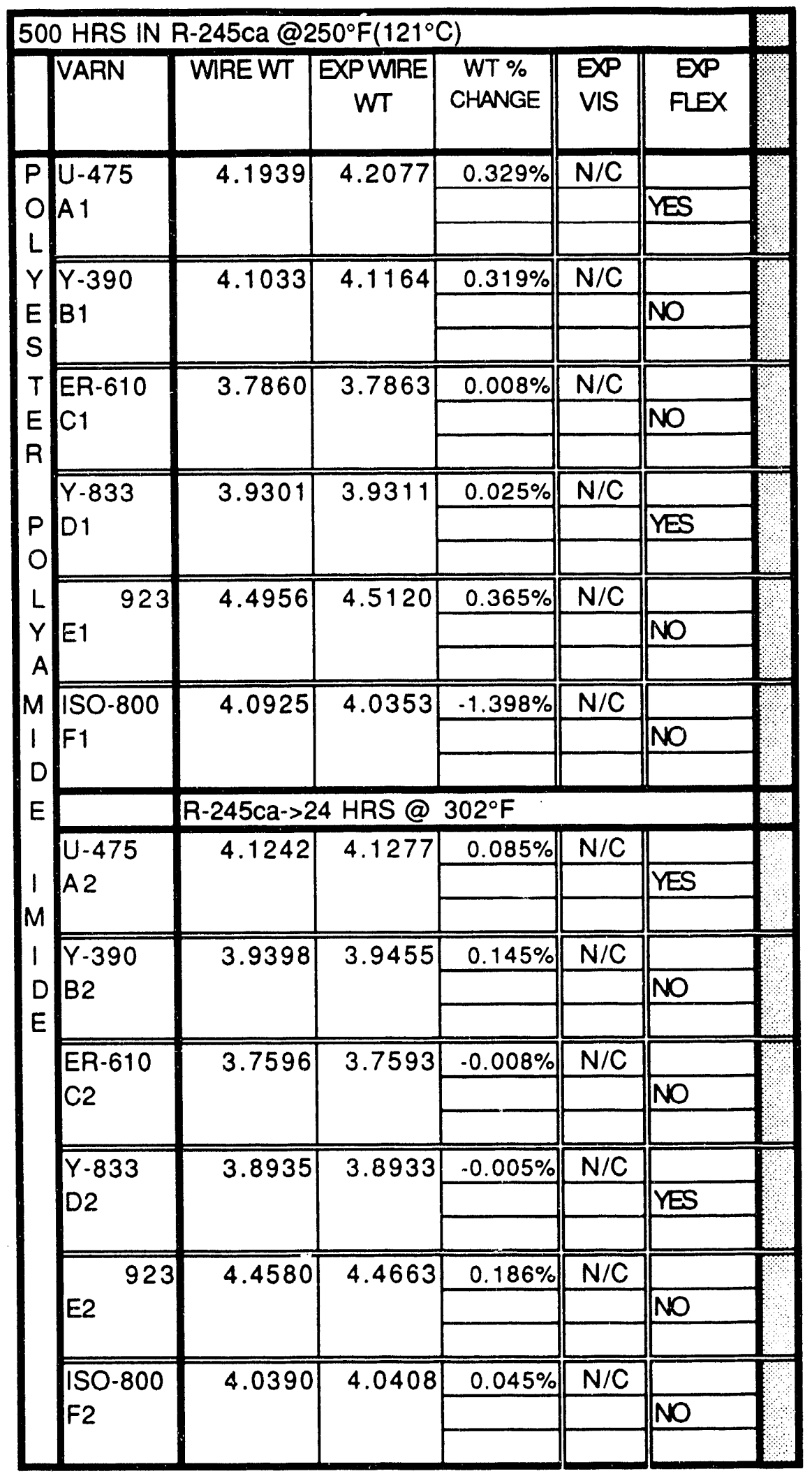




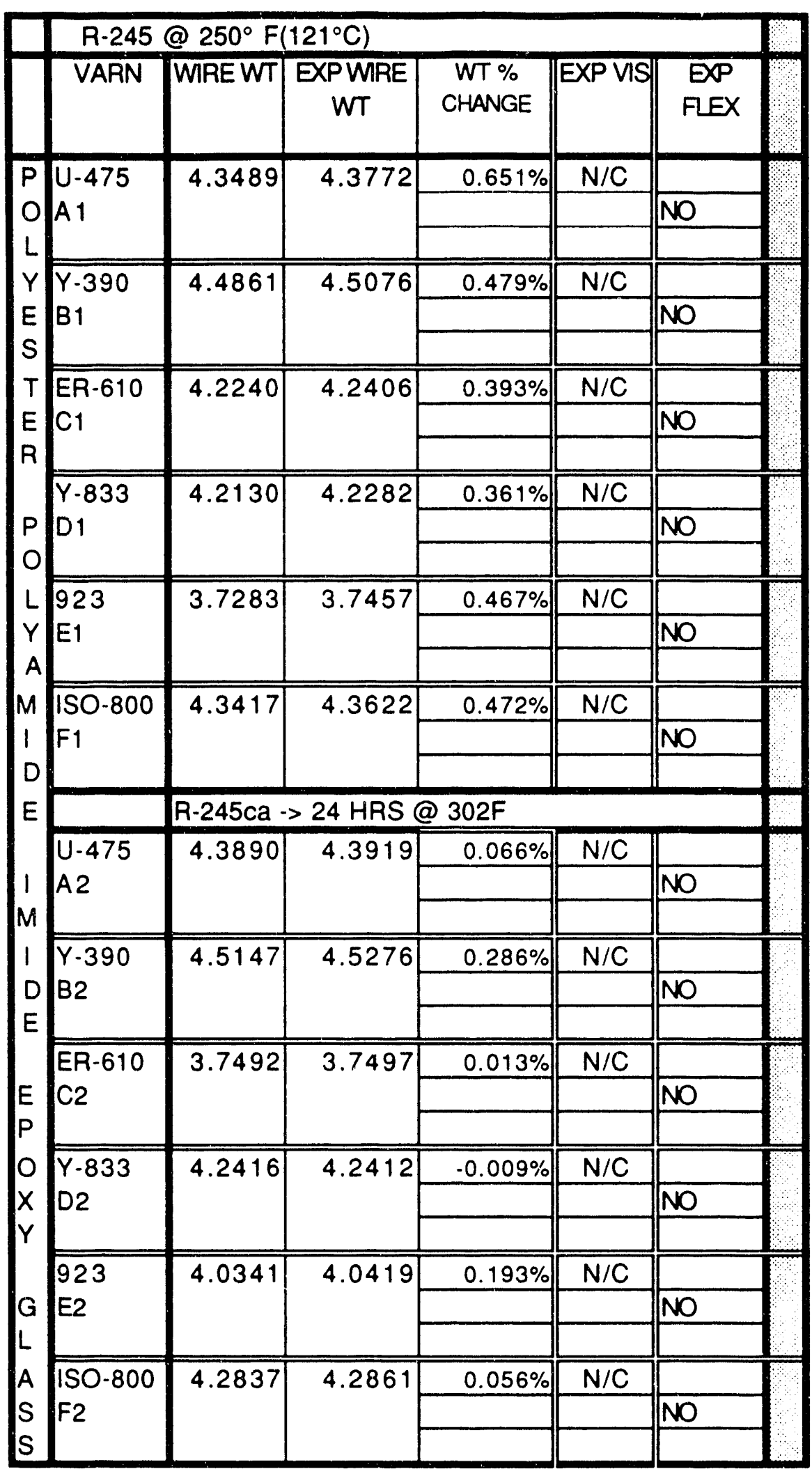




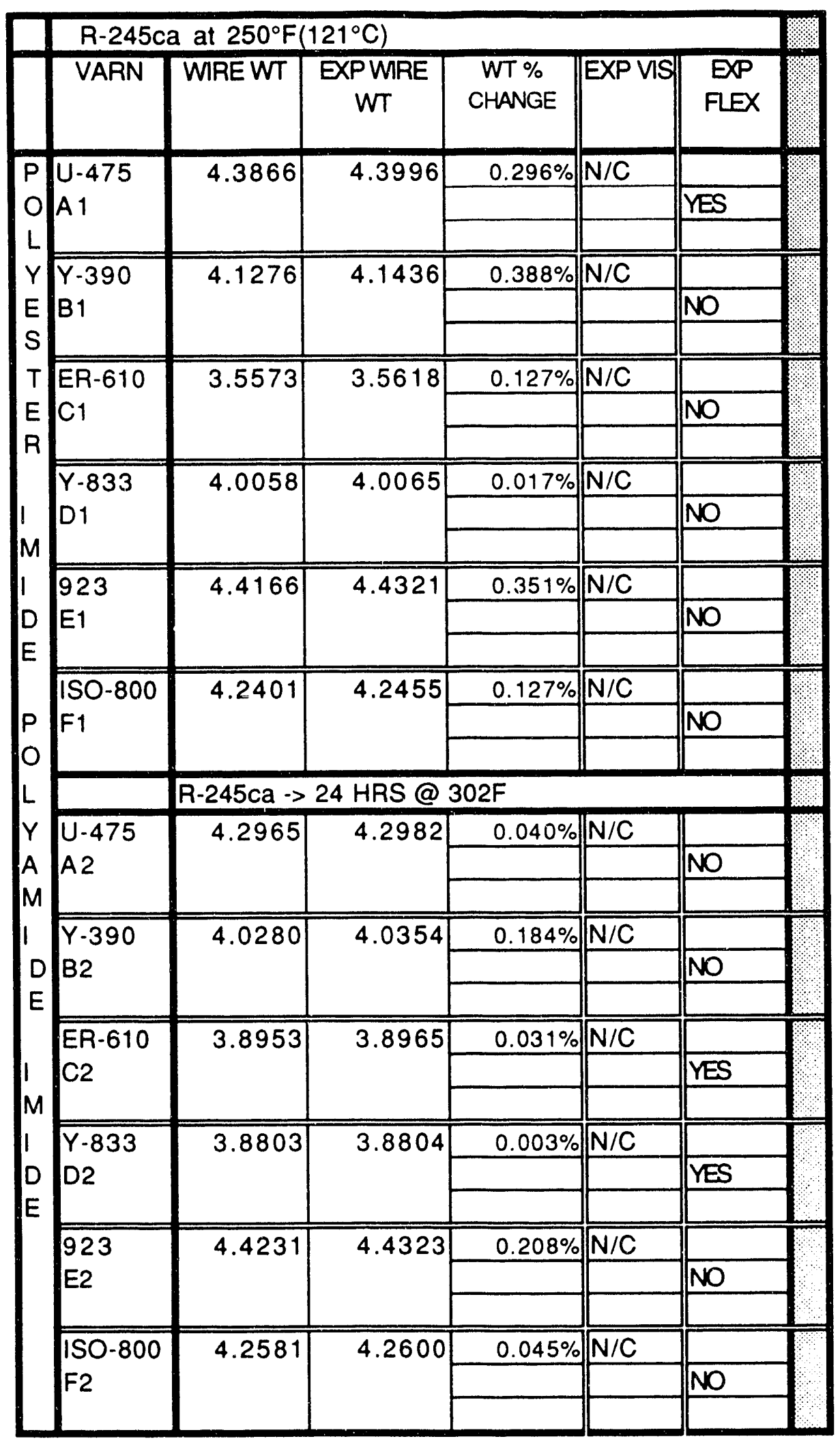




\begin{tabular}{|c|c|c|c|c|c|c|c|c|c|}
\hline \multicolumn{10}{|c|}{500 HR IN R-245ca @ 250F } \\
\hline ID & WT & EXPWT & $\begin{array}{c}\text { WT \% } \\
\text { CHANGE }\end{array}$ & $\begin{array}{l}\text { SAMPLE } \\
\text { WIOTH }\end{array}$ & $\begin{array}{l}\text { BREAK } \\
\text { LOAD }\end{array}$ & $\begin{array}{c}\text { SAMPLE } \\
\text { THICKNESS } \\
\text { Mils }\end{array}$ & $\begin{array}{c}\text { TENSILE } \\
\text { STR } \\
\text { BASE } \\
\end{array}$ & $\begin{array}{l}\text { TENSILE } \\
\text { STREXP }\end{array}$ & $\begin{array}{c}\% \\
\text { CHANGE } \\
\text { TENSILE }\end{array}$ \\
\hline \multirow{3}{*}{$\begin{array}{l}\text { NO/MY/NO } \\
\text { A1 }\end{array}$} & \multirow[t]{3}{*}{6.1323} & \multirow[t]{3}{*}{7.0830} & \multirow[t]{3}{*}{$15.50 \%$} & 0.384 & 128.40 & 21 & 17.4 & 15.92 & \\
\hline & & & & 0.448 & 152.10 & 21 & 17.4 & 16.17 & $.7 .66 \%$ \\
\hline & & & & 0.475 & 160.70 & 21 & 17.4 & 16.11 & \\
\hline \multirow{3}{*}{$\begin{array}{l}\mathrm{DA} / \mathrm{MY} / \mathrm{DA} \\
\mathrm{B} 1\end{array}$} & \multirow[t]{3}{*}{4.3157} & \multirow[t]{3}{*}{4.4206} & \multirow[t]{3}{*}{$2.43 \%$} & 0.396 & 101.20 & 21 & 13.7 & 12.17 & \\
\hline & & & & 0.398 & 105.90 & 21 & 13.7 & 12.67 & $-11.20 \%$ \\
\hline & & & & 0.482 & 118.00 & 21 & 13.7 & 11.66 & \\
\hline \multirow{3}{*}{$\begin{array}{l}\text { MYLAR MO } \\
\text { C1 }\end{array}$} & \multirow[t]{3}{*}{2.2516} & \multirow[t]{3}{*}{2.3441} & \multirow[t]{3}{*}{$4.11 \%$} & 0.417 & 79.25 & 10 & 21.7 & 19.00 & \\
\hline & & & & 0.515 & 102.50 & 10 & 21.7 & 19.90 & $-13.83 \%$ \\
\hline & & & & 0.461 & 79.25 & 10 & 21.7 & 17.19 & \\
\hline \multirow{3}{*}{$\begin{array}{l}\text { NO } 410 \\
\text { D1 }\end{array}$} & \multirow[t]{3}{*}{2.1666} & \multirow[t]{3}{*}{2.5562} & \multirow[t]{3}{*}{$17.98 \%$} & 0.525 & 102.50 & 10 & 18.7 & 19.52 & \\
\hline & & & & 0.442 & 84.70 & 10 & 18.7 & 19.16 & $3.92 \%$ \\
\hline & & & & 0.505 & 99.05 & 10 & 18.7 & 19.61 & \\
\hline \multirow{3}{*}{$\begin{array}{l}\text { NO MI } 418 \\
\text { E1 }\end{array}$} & \multirow[t]{3}{*}{2.3018} & \multirow[t]{3}{*}{2.3378} & \multirow[t]{3}{*}{$1.56 \%$} & 0.468 & 23.35 & 10 & 7.5 & 4.99 & \\
\hline & & & & 0.495 & 25.90 & 10 & 7.5 & 5.23 & $-31.28 \%$ \\
\hline & & & & 0.478 & 25.05 & 10 & 7.5 & 5.24 & \\
\hline \multirow{3}{*}{$\begin{array}{l}\text { MEL } 228 \\
\text { F1 }\end{array}$} & \multirow[t]{3}{*}{2.3996} & \multirow[t]{3}{*}{2.4931} & \multirow[t]{3}{*}{$3.90 \%$} & 0.454 & 88.80 & 11 & 21.7 & 17.78 & \\
\hline & & & & 0.496 & 101.40 & 11 & 21.7 & 18.59 & $.18 .03 \%$ \\
\hline & & & & 0.468 & 87.50 & 11 & 21.7 & 17.00 & \\
\hline & & $500 \mathrm{HF}$ & NR-2 & ca @250 & $j^{\circ} \mathrm{F}->24$ & HRS@30 & & & \\
\hline NO/MY/NO & 5.1528 & 5.1479 & $-0.10 \%$ & 0.400 & 137.60 & 21 & 17.4 & 16.38 & \\
\hline$A 2$ & & & & 0.455 & 146.40 & 21 & 17.4 & 15.32 & $-6.53 \%$ \\
\hline & & & & 0.460 & 165.10 & 21 & 17.4 & 17.09 & \\
\hline$D A / M Y / D A$ & 4.5752 & 4.4454 & $-2.84 \%$ & 0.418 & 117.40 & 21 & 13.7 & 13.37 & \\
\hline$B 2$ & & & & 0.458 & 130.70 & 21 & 13.7 & 13.59 & $-1.42 \%$ \\
\hline & & & & 0.488 & 138.90 & 21 & 13.7 & 13.55 & \\
\hline MYLAR MO & 2.1640 & 2.1447 & $-0.89 \%$ & 0.350 & 70.35 & 10 & 21.7 & 20.10 & \\
\hline $\mathrm{C} 2$ & & & & 0.433 & 85.05 & 10 & 21.7 & 19.64 & $-8.65 \%$ \\
\hline & & & & 0.406 & 80.10 & 10 & 21.7 & 19.73 & \\
\hline NOMEX 410 & 2.3839 & 2.4154 & $1.32 \%$ & 0.479 & 102.10 & 10 & 18.7 & 21.32 & \\
\hline D2 & & & & 0.412 & 83.70 & 10 & 18.7 & 20.32 & $11.24 \%$ \\
\hline & & & & 0.503 & 104.50 & 10 & 18.7 & 20.78 & \\
\hline NO/MI 418 & 2.4346 & 2.4522 & $0.72 \%$ & 0.485 & 27.50 & 10 & 7.5 & 5.67 & \\
\hline E2 & & & & 0.510 & 29.10 & 10 & 7.5 & 5.71 & $-24.10 \%$ \\
\hline & & & & 0.490 & 27.95 & 10 & 7.5 & 5.70 & \\
\hline MEL 228 & 2.4680 & 2.4631 & $-0.20 \%$ & 0.463 & 92.25 & 10 & 21.7 & 19.92 & \\
\hline $\mathrm{F} 2$ & & & & 0.508 & 97.75 & 10 & 21.7 & 19.24 & $-10.17 \%$ \\
\hline & & & & 0.480 & 92.70 & 10 & 21.7 & 19.31 & \\
\hline
\end{tabular}




\begin{tabular}{|c|c|c|c|c|c|c|c|c|}
\hline & After 500 & hour $\theta$ & ure @ & $121^{\circ} \mathrm{C}(2$ & $\left.250^{\circ} \mathrm{F}\right)$ & & & \\
\hline ID & $\begin{array}{c}\text { STRETCH } \\
\text { (inch) }\end{array}$ & \begin{tabular}{c||}
$\%$ \\
ELONG
\end{tabular} & $\begin{array}{l}\text { BASE } \\
\text { ELONG } \\
\text { (AVE) }\end{array}$ & \begin{tabular}{|c||} 
ELONG \\
$\%$ \\
CHANGE
\end{tabular} & \begin{tabular}{|c|} 
BASE \\
DEE \\
$(A \vee E)$ \\
\end{tabular} & EXP DIE & $\begin{array}{c}\text { DIE \% } \\
\text { CHANGE }\end{array}$ & VISUAL EXP \\
\hline NO/MY/NO & 0.62 & $15.5 \%$ & $20.0 \%$ & & $>18.97$ & flash & & $N / C$ \\
\hline A1 & 0.25 & $6.3 \%$ & $20.0 \%$ & $-37.5 \%$ & $>18.97$ & flash & flash & \\
\hline & 0.63 & $15.8 \%$ & $20.0 \%$ & & $>18.97$ & flash & & \\
\hline DA/MY/DA & 0.59 & $29.5 \%$ & $46.0 \%$ & & $>15.27$ & flash & & slighlty \\
\hline & 0.45 & $22.5 \%$ & $46.0 \%$ & $-41.7 \%$ & $>15.27$ & flash & flash & warped \\
\hline & 0.57 & $28.5 \%$ & $46.0 \%$ & & $>15.27$ & flash & & yellowed \\
\hline MYLAR MO & 2.12 & $106.0 \%$ & $131.0 \%$ & & $>14.91$ & flash & & $\mathrm{N} / \mathrm{C}$ \\
\hline$c_{1}$ & 1.89 & $94.5 \%$ & $131.0 \%$ & $.21 .8 \%$ & $>14.91$ & flash & flash & \\
\hline & 2.14 & $107.0 \%$ & $131.0 \%$ & & $>14.91$ & flash & & \\
\hline NO 410 & 0.38 & $9.5 \%$ & $17.0 \%$ & & 10.67 & 9.34 & & $\mathrm{~N} / \mathrm{C}$ \\
\hline D1 & 0.13 & $3.3 \%$ & $17.0 \%$ & $-60.8 \%$ & 10.67 & 10.96 & $-4.3 \%$ & \\
\hline & 0.29 & $7.3 \%$ & $17.0 \%$ & & 10.67 & 10.34 & & \\
\hline NO Ml 418 & 0.04 & $1.0 \%$ & $4.0 \%$ & & 10.23 & 10.28 & & $\mathrm{~N} / \mathrm{C}$ \\
\hline & 0.04 & $1.0 \%$ & $4.0 \%$ & $.75 .0 \%$ & 10.23 & 10.46 & $-2.1 \%$ & \\
\hline & 0.04 & $1.0 \%$ & $4.0 \%$ & & 10.23 & 9.31 & & \\
\hline MEL 228 & 2.99 & $149.5 \%$ & $160.0 \%$ & & $>14.22$ & flash & & $\mathrm{N} / \mathrm{C}$ \\
\hline $\mathrm{F} 1$ & 3.71 & $185.5 \%$ & $160.0 \%$ & $5.9 \%$ & $>14.22$ & flash & flash & \\
\hline & 3.47 & $173.5 \%$ & $160.0 \%$ & & $>14.22$ & flash & & \\
\hline & After 500 & hour ex & posure plu & us a 24 a & airbake & at $150^{\circ} \mathrm{C}$ & $\left(302^{\circ} \mathrm{F}\right)$ & \\
\hline NOIMY/NO & 0.39 & $9.8 \%$ & $20.0 \%$ & & $\geq 18.97$ & flash & & $\mathrm{N} / \mathrm{C}$ \\
\hline A2 & 0.30 & $7.5 \%$ & $20.0 \%$ & $.51 .7 \%$ & $>18.97$ & flash & flash & \\
\hline & 0.47 & $11.8 \%$ & $20.0 \%$ & & $>18.97$ & flash & & \\
\hline $\mathrm{DA} / \mathrm{MY} / \mathrm{DA}$ & 0.47 & $23.5 \%$ & $46.0 \%$ & & $>15.27$ & flash & & slighlty \\
\hline$B 2$ & 0.42 & $21.0 \%$ & $46.0 \%$ & $-50.7 \%$ & $>15.27$ & flash & flash & warped \\
\hline & 0.47 & $23.5 \%$ & $46.0 \%$ & & $>15.27$ & flash & & yellowed \\
\hline 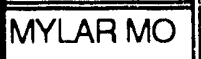 & 1.66 & $83.0 \%$ & $131.0 \%$ & & $>14.91$ & flash & & $\mathrm{N} / \mathrm{C}$ \\
\hline C2 & 1.46 & $73.0 \%$ & $131.0 \%$ & $.41 .0 \%$ & $>14.91$ & flash & flash & \\
\hline & 1.52 & $76.0 \%$ & $131.0 \%$ & & $>14.91$ & flash & & \\
\hline NOMEX 410 & 0.43 & $10.8 \%$ & $17.0 \%$ & & 10.67 & 10.52 & & $N / C$ \\
\hline D2 & 0.41 & $10.3 \%$ & $17.0 \%$ & $-39.2 \%$ & 10.67 & 9.60 & $-3.3 \%$ & \\
\hline & 0.40 & $10.0 \%$ & $17.0 \%$ & & 10.67 & 10.83 & & \\
\hline NO/MI 418 & 0.03 & $0.8 \%$ & $4.0 \%$ & & 10.23 & 9.75 & & $\mathrm{~N} / \mathrm{C}$ \\
\hline E2 & 0.05 & $1.3 \%$ & $4.0 \%$ & $.75 .0 \%$ & 10.23 & 8.19 & $-10.1 \%$ & \\
\hline & 0.04 & $1.0 \%$ & $4.0 \%$ & & 10.23 & 9.66 & & \\
\hline MEL 228 & 1.51 & $75.5 \%$ & $160.0 \%$ & & $>14.22$ & flash & & $\mathrm{N} / \mathrm{C}$ \\
\hline F2 & 1.73 & $86.5 \%$ & $160.0 \%$ & \begin{tabular}{|l|}
$-50.5 \%$ \\
\end{tabular} & $>14.22$ & flash & flash & \\
\hline & 1.51 & $75.5 \%$ & $160.0 \%$ & & $>14.22$ & flash & & \\
\hline
\end{tabular}


Varnish Disks----HFC-245ca at $250^{\circ} \mathrm{F}\left(121^{\circ} \mathrm{C}\right)$

\begin{tabular}{|c|c|c|c|c|c|}
\hline & 500 HRS IN & १-245ca@ & $0^{\circ} \mathrm{F}$ & & \\
\hline ID & WT & EXPWT & $\begin{array}{l}\text { WT \% } \\
\text { CHANGE }\end{array}$ & EXP VIS & EXPFLEX \\
\hline$U-475$ & 2.6637 & 2.8546 & $7.17 \%$ & $N / C$ & $\mathrm{~N} / \mathrm{C}$ \\
\hline$Y-390$ & 2.0726 & 2.1681 & $\mid 4.61 \%$ & $N / C$ & $N / C$ \\
\hline & & & & & \\
\hline $\begin{array}{l}\text { ER-610 } \\
\text { C1 }\end{array}$ & 2.4971 & 2.7365 & $9.59 \%$ & \begin{tabular}{|l|} 
slightly \\
darkened \\
\end{tabular} & $\mathrm{N} / \mathrm{C}$ \\
\hline & & & & & \\
\hline $\begin{array}{l}Y-833 \\
D 1\end{array}$ & 2.7800 & 3.0132 & $8.39 \%$ & $\frac{\text { light }}{\text { green }}$ & $\mathrm{N} / \mathrm{C}$ \\
\hline & & & & color & \\
\hline$\overline{923}$ & 2.0604 & 2.1257 & $3.17 \%$ & slightly & $\mathrm{N} / \mathrm{C}$ \\
\hline E1 & & & & warped & \\
\hline$\overline{150-800}$ & 2.2249 & 2.2750 & $2.25 \%$ & |Slightly & $N / C$ \\
\hline & & & & warped & \\
\hline & $\mathrm{B}-245 \mathrm{ca}$ & $\$ 24$ hour at & $302^{\circ} \mathrm{F}$ & & \\
\hline U-475 & 2.1442 & 2.1805 & $1.69 \%$ & Darkened & $\mathrm{N} / \mathrm{C}$ \\
\hline A2 & & & & & \\
\hline$Y-390$ & 1.7675 & 1.8033 & $2.03 \%$ & darkened & $N / C$ \\
\hline B2 & & & & & \\
\hline ER-610 & 2.3400 & 23934 & $2.28 \%$ & darkened & $N / C$ \\
\hline $\mathrm{C} 2$ & 2.0700 & & & & \\
\hline & & & & & \\
\hline$Y-833$ & 3.4539 & 3.6606 & $5.98 \%$ & slightly & $\mathrm{N} / \mathrm{C}$ \\
\hline D2 & & & & orange & \\
\hline$\overline{923}$ & 1.8354 & 1.8738 & $2.09 \%$ & darkened & $\mathrm{N} / \mathrm{C}$ \\
\hline E2 & & & & warped & \\
\hline & & & & & \\
\hline ISO-800 & 2.3839 & 2.3604 & $-0.99 \%$ & slightly & $N / C$ \\
\hline & & & & warped & \\
\hline
\end{tabular}


7

\begin{tabular}{|c|c|c|c|c|}
\hline & \multicolumn{4}{|c|}{500 HRS IN R-245ca @ 250 F } \\
\hline & WT & EXPWT & $\begin{array}{c}\% \\
\text { CHANGE }\end{array}$ & EXPVIS \\
\hline $\begin{array}{l}1 \\
\text { NOMEX }\end{array}$ & 0.5549 & 0.6767 & $21.95 \%$ & Darkened \\
\hline $\begin{array}{l}\text { B1 } \\
\text { MYAR }\end{array}$ & 0.4522 & 0.4809 & $6.35 \%$ & $\mathrm{~N} / \mathrm{C}$ \\
\hline \begin{tabular}{|l|}
$\mathrm{C1}$ \\
$\mathrm{NO} / \mathrm{MY}$
\end{tabular} & 0.4013 & 0.4354 & $8.50 \%$ & $\mathrm{~N} / \mathrm{C}$ \\
\hline & R-245ca@ & $50^{\circ} \mathrm{F} \cdots>24$ & hrs @ 30 & \\
\hline $\begin{array}{l}\text { A2 } \\
\text { NOMEX }\end{array}$ & 0.5572 & 0.5546 & $-0.47 \%$ & $\mathrm{~N} / \mathrm{C}$ \\
\hline \begin{tabular}{|l|} 
B2 \\
MYLAR
\end{tabular} & 0.4999 & 0.4952 & $-0.94 \%$ & $\begin{array}{l}\text { Some small } \\
\text { pockets } \\
\text { distorsions }\end{array}$ \\
\hline $\begin{array}{l}\mathrm{C2} \\
\mathrm{NO} / \mathrm{MY}\end{array}$ & 0.3989 & 0.3938 & $-1.28 \%$ & \begin{tabular}{|l|} 
Pockects where \\
mylar pulled away \\
\end{tabular} \\
\hline
\end{tabular}




\begin{tabular}{|c|c|c|c|c|c|c|c|c|c|}
\hline & \multicolumn{9}{|c|}{500 HRS IN R-245ca } \\
\hline D & WT & EXPWT & $\begin{array}{c}\text { WT \% } \\
\text { CHANGE }\end{array}$ & $\begin{array}{l}\text { BREAK } \\
\text { LOAD } \\
\text { (AVE) }\end{array}$ & $\begin{array}{c}\text { BREAK } \\
\text { LOAD } \\
\text { EXP }\end{array}$ & $\begin{array}{c}\% \\
\text { CHANGE } \\
\text { BPK } \\
\text { LOAD } \\
\end{array}$ & $\mid \begin{array}{c}\text { STRETCH } \\
\text { (INCH) }\end{array}$ & $\%$ ELONG & EXP VIS \\
\hline \multirow{3}{*}{$\begin{array}{l}\text { A } 1 \\
\text { Glass }\end{array}$} & \multirow[t]{3}{*}{1.4628} & \multirow[t]{3}{*}{1.4717} & \multirow[t]{3}{*}{$0.61 \%$} & 39.02 & 77.15 & & 0.07 & $3.50 \%$ & $\mathrm{~N} / \mathrm{C}$ \\
\hline & & & & 39.02 & 50.60 & $45.35 \%$ & 0.07 & $3.50 \%$ & \\
\hline & & & & 39.02 & 42.40 & & 0.08 & $4.00 \%$ & \\
\hline \multirow{3}{*}{$\begin{array}{l}\text { B1 } \\
\text { Polyester }\end{array}$} & \multirow[t]{3}{*}{0.7395} & \multirow[t]{3}{*}{0.7693} & \multirow[t]{3}{*}{$4.03 \%$} & 56.12 & 58.65 & & 0.67 & $33.50 \%$ & $\mathrm{~N} / \mathrm{C}$ \\
\hline & & & & 56.12 & 52.60 & $0.35 \%$ & 0.51 & $25.50 \%$ & \\
\hline & & & & 56.12 & 57.70 & & 0.66 & $33.00 \%$ & \\
\hline \multirow{4}{*}{$\begin{array}{l}\text { C1 } \\
\text { Permacel }\end{array}$} & \multirow[t]{3}{*}{1.3366} & \multirow[t]{3}{*}{1.3678} & \multirow[t]{3}{*}{$2.33 \%$} & 88.50 & 65.40 & & 0.09 & $4.50 \%$ & Darkened \\
\hline & & & & 88.50 & 104.90 & $-5.01 \%$ & 0.14 & $7.00 \%$ & \\
\hline & & & & 88.50 & 81.90 & & 0.11 & $5.50 \%$ & \\
\hline & \multicolumn{7}{|c|}{$500 \mathrm{HRS}$ in R-245ca $\rightarrow 302^{\circ} \mathrm{F} 24 \mathrm{HRS}$} & & \\
\hline \multirow{3}{*}{$\begin{array}{l}\text { A2 } \\
\text { Glass }\end{array}$} & \multirow[t]{3}{*}{1.8105} & \multirow[t]{3}{*}{1.8127} & \multirow[t]{3}{*}{$0.12 \%$} & 39.02 & 41.80 & & 0.05 & $2.50 \%$ & $\mathrm{~N} / \mathrm{C}$ \\
\hline & & & & 39.02 & 59.00 & $30.76 \%$ & 0.05 & $2.50 \%$ & \\
\hline & & & & 39.02 & 52.27 & & 0.05 & $2.50 \%$ & \\
\hline \multirow{3}{*}{$\begin{array}{l}\text { B2 } \\
\text { Polyester }\end{array}$} & \multirow[t]{3}{*}{0.6814} & \multirow[t]{3}{*}{0.6794} & \multirow[t]{3}{*}{$-0.29 \%$} & 56.12 & 54.30 & & 0.55 & $27.50 \%$ & $N / C$ \\
\hline & & & & 56.12 & 55.40 & $-2.82 \%$ & 0.66 & $33.00 \%$ & \\
\hline & & & & 56.12 & 53.92 & & 0.50 & $25.00 \%$ & \\
\hline \multirow{3}{*}{$\begin{array}{l}\text { C2 } \\
\text { Permacel }\end{array}$} & \multirow[t]{3}{*}{1.8628} & \multirow[t]{3}{*}{1.8678} & \multirow[t]{3}{*}{$0.27 \%$} & 88.50 & 135.10 & & 0.15 & $7.50 \%$ & $N / C$ \\
\hline & & & & 88.50 & 98.40 & $12.77 \%$ & 0.10 & $5.00 \%$ & \\
\hline & & & & 88.50 & 65.90 & & 0.08 & $4.00 \%$ & \\
\hline
\end{tabular}


TIE CORD-..-HFC-245ca@ $121^{\circ} \mathrm{F}\left(250^{\circ} \mathrm{F}\right)$

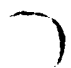

\begin{tabular}{|c|c|c|c|c|c|c|c|c|c|}
\hline \multicolumn{10}{|c|}{500 HRS IN R-245ca @ 250 F } \\
\hline ID & WT & EXP WT & $\begin{array}{c}\text { WT \% } \\
\text { CHANGE }\end{array}$ & $\begin{array}{l}\text { EXP } \\
\text { VIS }\end{array}$ & $\begin{array}{l}\text { BREAK } \\
\text { LOAD } \\
\text { (AVE) }\end{array}$ & $\begin{array}{l}\text { BREAK } \\
\text { LOAD } \\
\text { EXP } \\
(\operatorname{av} \theta)\end{array}$ & $\begin{array}{c}\% \\
\text { CHANGEE } \\
\text { BPK } \\
\text { LOAD }\end{array}$ & $\begin{array}{c}\text { STRETCH } \\
\text { (Inch) }\end{array}$ & $\begin{array}{c}\% \\
\text { ELONG }\end{array}$ \\
\hline \multirow[t]{4}{*}{ A1 1} & \multirow[t]{3}{*}{0.3348} & \multirow[t]{3}{*}{0.3428} & \multirow[t]{3}{*}{$2.39 \%$} & $\mathrm{~N} / \mathrm{C}$ & 28.36 & 32.20 & & 0.49 & $24.5 \%$ \\
\hline & & & & & 28.36 & 31.35 & $8.19 \%$ & 0.46 & $23.0 \%$ \\
\hline & & & & & 28.36 & 28.50 & & 0.42 & $21.0 \%$ \\
\hline & \multicolumn{9}{|c|}{500 HRS IN R-245ca $>24$ HRS @ 302 $\mathrm{F}$} \\
\hline \multirow[t]{3}{*}{ A2 } & \multirow[t]{3}{*}{0.3348} & \multirow[t]{3}{*}{0.3315} & \multirow[t]{3}{*}{$-0.986 \%$} & $\mathrm{~N} / \mathrm{C}$ & 28.36 & 34.62 & & 0.49 & $24.5 \%$ \\
\hline & & & & & 28.36 & 22.87 & $2.56 \%$ & 0.38 & $19.0 \%$ \\
\hline & & & & & 28.36 & 29.77 & & 0.44 & $22.0 \%$ \\
\hline
\end{tabular}

;) 


\begin{tabular}{|c|c|c|c|c|c|c|c|}
\hline & 500 HRS IN & 245ca@25 & $50^{\circ} \mathrm{F}\left(121^{\circ}\right.$ & & & & \\
\hline ID & WT & EXPWT & $\begin{array}{c}\text { WT \% } \\
\text { CHANGE }\end{array}$ & EXP VS & $\begin{array}{c}\text { BASE DIE } \\
\text { (AVE) }\end{array}$ & EXP DIE & $\begin{array}{c}\text { DIE\% } \\
\text { CHANGE }\end{array}$ \\
\hline DMD & 3.9876 & 4.0470 & $1.49 \%$ & $\mathrm{~N} / \mathrm{C}$ & 9.61 & 7.93 & \\
\hline A1 & & & & & 9.61 & 9.29 & $-10.58 \%$ \\
\hline & & & & & 9.61 & 8.56 & \\
\hline DTMD & 4.3524 & 4.4402 & $2.02 \%$ & $\mathrm{~N} / \mathrm{C}$ & 9.95 & 9.01 & \\
\hline B1 & & & & & 9.95 & 7.82 & $-15.08 \%$ \\
\hline & & & & & 9.95 & 8.52 & \\
\hline & $R-245 \mathrm{ca} \rightarrow 2$ & HRS@30 & $2^{\circ} \mathrm{F}$ & & & & \\
\hline DMD & 4.0289 & 4.0205 & $-0.21 \%$ & $\mathrm{~N} / \mathrm{C}$ & 9.61 & 9.64 & \\
\hline A2 & & & & & 9.61 & 8.29 & $-8.67 \%$ \\
\hline & & & & & 9.61 & 8.40 & \\
\hline DTMD & 4.2809 & 4.2721 & $-0.21 \%$ & $\mathrm{~N} / \mathrm{C}$ & 9.95 & 6.50 & \\
\hline $\mathrm{B} 2$ & & & & & 9.95 & 9.54 & $-17.96 \%$ \\
\hline & & & & & 9.95 & 8.45 & \\
\hline
\end{tabular}



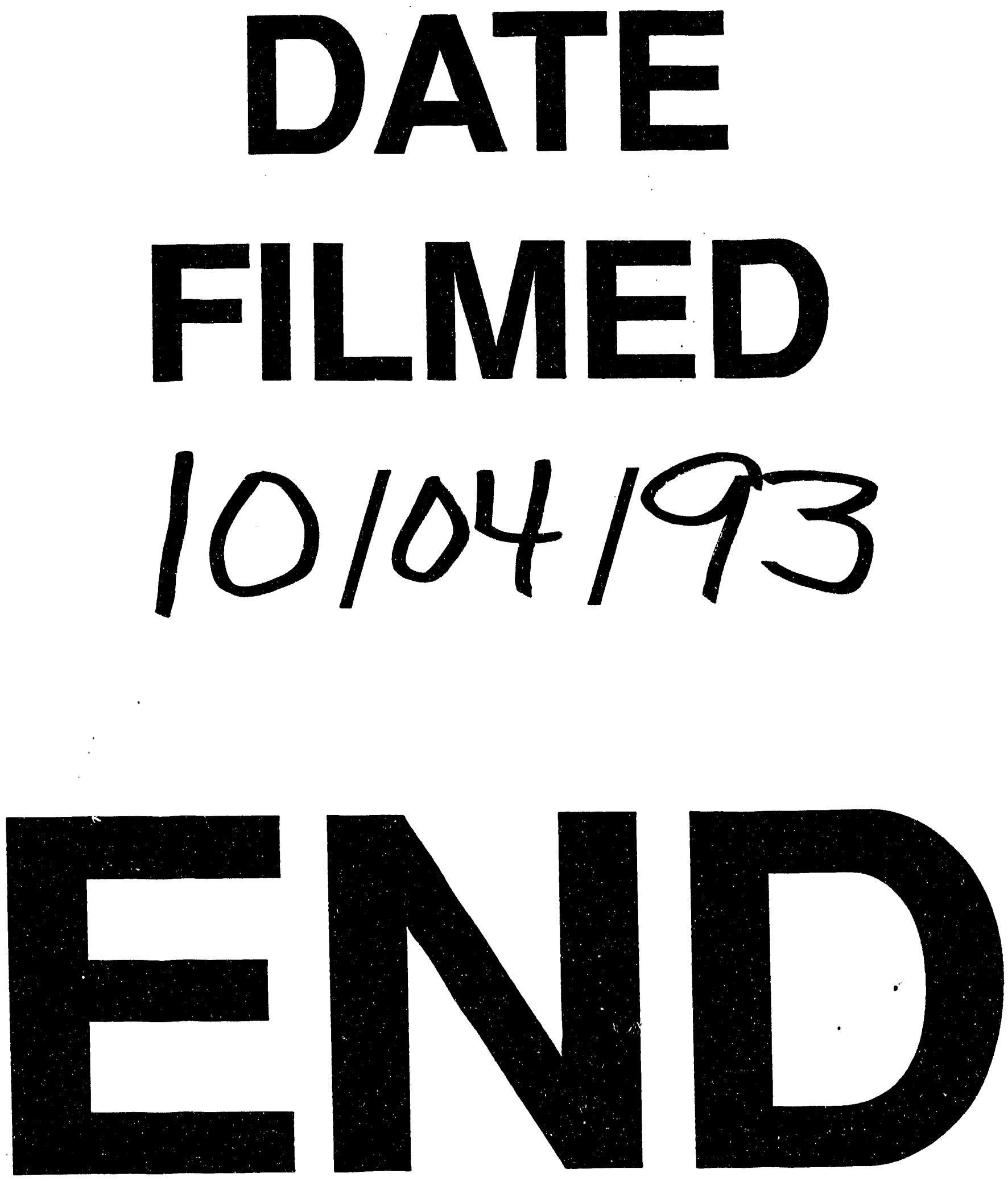
